\title{
SEX HORMONES AND ATHEROSCLEROSIS
}

\section{JANE ESTHER YEEND-DAHLSTROM}

A thesis submitted for the degree of Doctor of Philosophy of The Australian National University

December 1991 


\section{STATEMENT}

All of the experimental work reported in this thesis was performed by the author except for the following:

The processing of cells for electron microscopy (Chapter 2) (Ms Leslie Maxwell);

The immunocytochemical staining for estrogen receptors on HUV endothelial cells (Chapter 3) (Dr Anne Jackson);

The PCR to amplify ECGF cDNA (Chapter 3) (Dr Ming Chu Fung); and Gas chromatography of LDL samples (Chapter 6) (Mr Mohaneeshwar Singh).

None of the material has been presented previously for the purpose of obtaining any other degree.

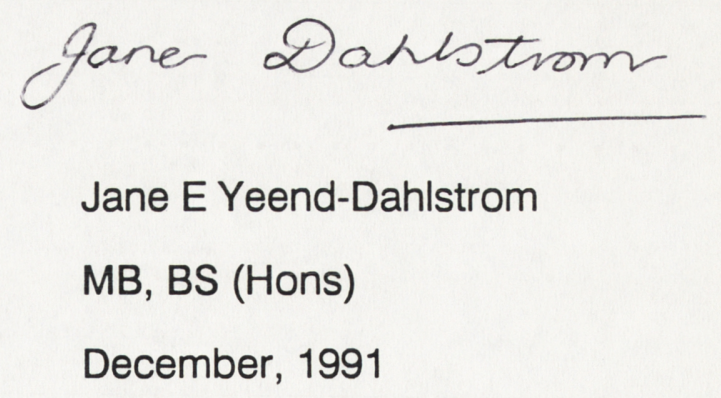




\section{ACKNOWLEDGEMENTS}

I wish to thank my supervisor Dr Neville Ardlie for his wisdom and support throughout these studies, and my advisers, Dr. A. Hapel and Dr. M. Selley. I am very grateful also to my family, especially my parents, children and Stephen, and my friends, for their understanding and patience over the past few years. I also thank Dr M Bassett, Dr M Baker and Dr W Cowden of the John Curtin School of Medical Research, Australian National University, Canberra, for reading and criticizing portions of this thesis, and the following for valuable assistance, particularly in teaching me various laboratory techniques: Sue Elsbury, Julie McGuiness, Ming Chu Fung, Jennifer Pratt, Lynette Preston, Louise Skipsey, Jennifer Stapleton and Erika van de Pol. I am grateful to Dr D Kelker, Department of Statistics and Applied Probability, University of Alberta, Edmonton, who helped me with the statistics, Mrs Elaine Wellington for locating the difficult references, Chris Treadell for typing many of the references, and Ludwig Heinrich for designing the computer program to collate the references. I am also indebted to the Photography Department at the John Curtin School of Medical Research. Finally, I wish to acknowledge financial support from the National Heart Foundation, the Australian National University (PhD Scholarship), and the Division of Clinical Sciences, John Curtin School of Medical Research, where these studies were conducted.

I dedicate this thesis to my mother, my father and Mark. 


\section{ABBREVIATIONS USED IN THIS THESIS}

\begin{tabular}{|c|c|}
\hline ADP & adenosine diphosphate \\
\hline AdR & tritiated adenine \\
\hline ATP & adenosine triphosphate \\
\hline AHF & anti haemophillic factor \\
\hline $\mathrm{Ag}$ & antigen \\
\hline AMI & acute myocardial infarction \\
\hline ATPase & adenosine triphosphatase \\
\hline apo B & apolipoprotein B \\
\hline bp & base pair \\
\hline BSA & bovine serum albumin \\
\hline $\mathrm{BPB}$ & p-bromophenacyl bromide \\
\hline$\beta-V L D L$ & beta very low density lipoprotein \\
\hline$C D_{n}$ & cluster of differentiation (antigen), $n=$ number \\
\hline CDNA & complementary deoxyribonucleic acid \\
\hline CHD & coronary heart disease \\
\hline $\mathrm{Ci}$ & curie \\
\hline CMF HBSS & $\begin{array}{l}\text { calcium- and magnesium-free Hanks balanced salt } \\
\text { solution }\end{array}$ \\
\hline $\mathrm{CO}_{2}$ & carbon dioxide \\
\hline $\mathrm{cpm}$ & counts per minute \\
\hline$\left(\mathrm{cpm} \times 10^{-3}\right)$ & counts per million \\
\hline DDT & dithiothreitol \\
\hline DEPC & diethylpyrocarbonate \\
\hline DMSO & dimethyl sulfoxide \\
\hline
\end{tabular}




$\begin{array}{ll}\text { ECGF } & \text { endothelial cell growth factor } \\ \text { ECGS } & \text { endothelial cell growth supplement } \\ \text { EDTA } & \text { ethylenediaminetetraacetic acid } \\ \text { ELAM-1 } & \text { endothelial leukocyte adhesion molecule 1 } \\ \text { FACS } & \text { fluorescent activated cell sorter } \\ \text { F.I.T.C. } & \text { fluoroscein isothiocyanate } \\ \text { FCS } & \text { fetal calf serum } \\ \text { aFGF } & \text { acidic fibroblast growth factor } \\ \text { bFGF } & \text { basic fibroblast growth factor } \\ \text { FVIII } & \text { clotting factor VIII } \\ \text { g } & \text { grams } \\ \text { Xg } & \text { gravitational acceleration } \\ \text { G-CSF } & \text { granulocyte colony stimulating factor } \\ \text { GM-CSF } & \text { granulocyte-macrophage colony stimulating factor } \\ \text { GSH } & \text { glutathione } \\ \text { [3H] } & \text { tritiated } \\ \text { h } & \text { hours } \\ \text { HBSS } & \text { Hank's balanced salt solution } \\ \text { HDL } & \text { high density lipoproteins } \\ \text { HUV } & \text { human umbilical vein } \\ \text { ICAM } & \text { intercellular adhesion molecule } \\ \text { IL } & \text { interleukin } \\ \text { L } & \text { litres } \\ \text { LDL } & \text { low density lipoproteins } \\ \text { LPS } & \text { lipopolysaccharide, bacterial endotoxin } \\ \text { LPDS } & \text { lipoprotein-deficient serum } \\ \text { kb } & \text { M }\end{array}$




\begin{tabular}{|c|c|}
\hline $\min$ & minutes \\
\hline $\mathrm{ml}$ & millilitres \\
\hline mRNA & messenger ribonucleic acid \\
\hline$\eta \mathrm{g} / \mathrm{ml}$ & nanograms per millilitre \\
\hline OD & optical density \\
\hline PBS & phosphate-buffered saline \\
\hline PCR & polymerase chain reaction \\
\hline PDGF & platelet-derived growth factor \\
\hline $\mathrm{PGI}_{2}$ & prostacyclin \\
\hline PgP-1 & CD44 or Hermes-lymphocyte homing receptor \\
\hline PPP & platelet poor plasma \\
\hline PRF & phenol red-free \\
\hline PRP & platelet rich plasma \\
\hline PUFA & polyunsaturated fatty acids \\
\hline$r$ & recombinant \\
\hline $\mathrm{RD}$ & reagent diluent \\
\hline RPMI & Rosewell Park Memorial Institute \\
\hline SE & standard error of the mean \\
\hline SD & standard deviation \\
\hline SDS & sodium dodecyl sulphate \\
\hline TBA & thiobarbituric acid \\
\hline TdR & tritiated thymidine \\
\hline vWf & Von Willebrand factor \\
\hline vWf : Ag & Von Willebrand factor related antigen \\
\hline WHHL & Watanabe heritable hyperlipidemic \\
\hline$\mu \mathrm{g} / \mathrm{ml}$ & micrograms per millilitre \\
\hline $\mathrm{U}$ & units \\
\hline V & voltage \\
\hline VLDL & very low density lipoproteins \\
\hline
\end{tabular}


standard additives in this thesis refers to glutamine $(2 \mathrm{mM})$, heparin sodium $(20 \mathrm{U} / \mathrm{ml})$ and endothelial cell growth supplement (ECGS) $(50 \mu \mathrm{g} / \mathrm{ml})$.

sex steroids in this thesis refers to $17 \beta$-estradiol $\left(10^{-5} \mathrm{M}\right)$, progesterone $\left(10^{-5}\right.$ $M)$, the combination of $17 \beta$-estradiol $\left(10^{-5} \mathrm{M}\right)$ with progesterone $\left(10^{-5} \mathrm{M}\right)$, progesterone $\left(10^{-9} \mathrm{M}\right), 17 \beta$-estradiol $\left(10^{-10} \mathrm{M}\right)$, and the combination of progesterone $\left(10^{-9} \mathrm{M}\right)$ with $17 \beta$-estradiol $\left(10^{-10} \mathrm{M}\right)$. 


\section{A note on style:}

The style conforms to that required by the Australian National University for PhD theses (1991). Abbreviations used for publications in the references are those used in Indexus Medicus (1991). Abbreviations for terms were used only where the term was used frequently. 


\section{ABSTRACT}

Coronary heart disease (CHD) is an important complication of atherosclerosis and a major cause of mortality in Western society. Epidemiological studies suggest that female sex steroids are an important protective factor in CHD. Premenopausal women have only one third the rate of CHD compared to men, and post menopausal women on estrogen replacement therapy have up to a $40-50 \%$ reduction in $\mathrm{CHD}$ risk compared to untreated women of a similar age. The apparent protection of estrogen against CHD is often incorrectly attributed solely to favourable changes in the lipid profile. However, female sex steroids can alter many other cellular functions which are important in CHD. This thesis examines the effects of $17 \beta$-estradiol and progesterone on certain endothelial cell functions considered important in atherosclerosis using human umbilical vein (HUV) endothelial cells. It also examines the effects of $17 \beta$-estradiol and progesterone on platelet and monocyte binding to endothelial cells and on oxidation of LDL. The studies have shown that low concentrations of $17 \beta$ estradiol (10-10 M) increased endothelial cell proliferation and mRNA production for PDGF in endothelial cells but did not alter the other endothelial cell functions studied, while low concentrations of progesterone $\left(10^{-9} \mathrm{M}\right)$ inhibited the proliferative effect of $17 \beta$-estradiol (10-10 M), increased mRNA for PDGF in endothelial cells and inhibited vWf expression on endothelial cells. High concentrations of $17 \beta$-estradiol $\left(10^{-5} \mathrm{M}\right)$ and progesterone $\left(10^{-5}\right.$ M) altered many endothelial cell functions studied. $17 \beta$-estradiol $\left(10^{-5} \mathrm{M}\right)$ and progesterone $\left(10^{-5} \mathrm{M}\right)$ individually and in combination inhibited endothelial cell proliferation, increased mRNA expression for PDGF and reduced the proliferative effects of native LDL on endothelial cell proliferation. $17 \beta$ estradiol $\left(10^{-5} \mathrm{M}\right)$ alone inhibited $\mathrm{PGI}_{2}$ release and platelet binding, and these effects were prevented by the addition of progesterone $\left(10^{-5} \mathrm{M}\right)$. Both high 
and low concentrations of $17 \beta$-estradiol and progesterone increased the ability of monocytes to bind to endothelial cells. Finally, 17 $\beta$-estradiol (10-10 M) was found to inhibit LDL oxidation which is considered to be very important in atherosclerosis lesion formation. It is suggested that low concentrations of estrogen may retard atherosclerosis by promoting endothelial cell proliferation at sites of injury, and by inhibiting LDL oxidation, thereby inhibiting foam cell formation. On the other hand, high concentrations of estrogen may promote atherosclerosis by inhibiting endothelial cell proliferation, increasing cytokine production, inhibiting $\mathrm{PGl}_{2}$ release and increasing monocyte binding to endothelial cells. Of particular interest is the modulation by progesterone of some of the changes induced by $17 \beta$-estradiol in endothelial cells. These studies show that sex steroids have diverse effects on cellular functions important in atherosclerosis and have implications for the relationship between atherosclerosis and gender, oral contraceptive use and hormone replacement therapy. 


\section{PUBLICATIONS}

(1) Dahlstrom, J.E., Hapel, A.J. and Ardlie, N.G. Effect of sex steroids on proliferation of cultured endothelial cells. J. Cell. Biochem. 1991; Supplement 14E: 203

(2) Dahlstrom, J.E., Hapel, A.J. and Ardlie, N.G. Sex steroids increase monocyte binding to endothelial cells. J. Cell. Biochem. 1992; Supplement 16A: A207

(3) Dahlstrom, J.E., Hapel, A.J., Kelker, D. and Ardlie, N.G. Effect of sex steroids on monocyte binding to endothelial cells (in preparation).

(4) Dahlstrom, J.E. and Ardlie, N.G. Effect of native, minimally oxidized and oxidized LDL on HUV endothelial cell proliferation (in preparation).

(5) Dahlstrom, J.E., Hapel, A.J. and Ardlie, N.G. Effect of sex steroids on HUV endothelial cell proliferation (in preparation). 


\section{TABLE OF CONTENTS}

Title page

Statement

Acknowledgements iii

Abbreviations iv

A note on style viii

Abstract

Publications xi

Table of contents xii

CHAPTER 1: SEX HORMONES AND ATHEROSCLEROSIS

1.1 ATHEROSCLEROSIS: A MAJOR PROBLEM

\subsection{PATHOLOGY OF ATHEROSCLEROSIS}

1.2.1 Normal artery 3

1.2.2 Atherosclerotic artery 3

1.3 MAJOR HYPOTHESES OF ATHEROSCLEROSIS 5

1.3.1 Injury hypothesis $\quad 5$

$\begin{array}{ll}\text { 1.3.1.1 Role of the endothelial cell } & 7\end{array}$

1.3.1.2 Role of the platelet 9

$\begin{array}{ll}\text { 1.3.1.3 Role of growth factors } & 11\end{array}$

1.3.1.4 Role of the monocyte /macrophage 20

1.3.1.5 Role of the smooth muscle cell $\quad 21$

$\begin{array}{ll}\text { 1.3.2 Lipid hypothesis } & 22\end{array}$

1.3.3 Immunological theory of atherogenesis 26

1.3.4 Transformation hypothesis (monoclonal theory) 27 
1.4 RISK FACTORS, ATHEROSCLEROSIS AND CORONARY HEART DISEASE 30

1.4.1 Major risk factors 31

1.4.2 Gender 38

1.4.3 Endogenous hormones 40

1.5 EXOGENOUS SEX HORMONES AND ATHEROSCLEROSIS 42

1.5.1 Effect of estrogens and progestins on lipid metabolism 46

1.5.2 Role of estrogens and progestins in platelet aggregation 49 and thrombosis

1.5.3 Experimental evidence for the involvement of estrogens and progestins in atherosclerosis

1.5.3.1 Sex steroid receptors and steroid metabolism 54

1.5.3.2 Effects of estrogens and progestins on endothelial cell $\quad 58$ proliferation and angiogenesis

1.5.3.3 Influence of estrogens and progestins on platelets and thrombosis

1.5.3.3.1 Effects of sex hormones on $\mathrm{PGl}_{2}$ production

1.5.3.3.2 Effects of sex hormones on vWf production and release

1.5.3.4 Influence of estrogens and progestins on release of growth factors

1.5.3.5 Effects of estrogens and progestins on monocyte function

1.5.3.6 Influence of estrogens and progestins on fatty streak formation 

plaque formation

\section{CHAPTER 2: CULTURE AND CHARACTERIZATION OF HUMAN UMBILICAL VEIN ENDOTHELIAL CELLS}

\subsection{INTRODUCTION}

2.1.1 Isolation and culture of endothelial cells

2.1.2 Factors affecting endothelial cell proliferation

2.1.3 Endothelial cell characterization

\subsection{MATERIALS}

2.2.1 Buffers and solutions 78

2.2.2 Reagents 79

\subsection{METHODS}

2.3.1 Endothelial cell retrieval from human umbilical vein

2.3.2 Passaging of HUV endothelial cells

2.3.3 Counting and assessing viability of HUV endothelial cells

2.3.4 Freezing, storage and thawing of HUV endothelial cells

2.3.5 vWf : Ag immunofluorescence microscopy of

HUV endothelial cells

\subsubsection{Electron microscopy of HUV endothelial cells}

2.3.6.1 Transmission electron microscopy

2.3.6.2 Scanning electron microscopy

2.3.7 The effect of two commercial media on HUV endothelial cell proliferation 
proliferation

2.3.9 Effect of FCS on HUV endothelial cell proliferation 88

2.3.10 Effect of ECGS on HUV endothelial cell proliferation 88

2.3.11 Effect of heparin on HUV endothelial proliferation 89

2.3.12 The effects of different cell densities and time on HUV 89 endothelial response to sex steroids

2.3.13 Statistical analysis

90

\subsection{RESULTS}

2.4.1 HUV endothelial cell culture 90

2.4.2 vWf : Ag immunofluorescence microscopy of 91 HUV endothelial cells

2.4.3 Electron microscopy of HUV endothelial cells 91

2.4.4 The effect of two different media on 92

HUV endothelial cell proliferation

2.4.5 Effect of phenol red in Medium 199 on 92

HUV endothelial cell proliferation

2.4.6 Effect of FCS on HUV endothelial cell proliferation 92

2.4.7 Effect of ECGS on HUV endothelial cell proliferation 93

2.4.8 Effect of heparin on HUV endothelial cell proliferation 93

2.4.9 The effects of different cell densities and time on HUV 94 endothelial response to sex steroids 


\section{CHAPTER 3: EFFECTS OF SEX STEROIDS ON HUV}

ENDOTHELIAL CELL PROLIFERATION AND ON THE EXPRESSION OF mRNA FOR PDGF IN HUV ENDOTHELIAL CELLS

3.1 INTRODUCTION

3.1.1 Concentrations of sex hormones in human plasma 103

3.1.2 Effect of 17ק-estradiol and progesterone on cell proliferation 106

3.1.3 Factors influencing the proliferative response of cells to 110 $17 \beta$-estradiol and progesterone

3.1.4 Effect of steroids on endothelial cell proliferation

3.1.5 Characterization and regulation of PDGF release from endothelial cells

3.1.6 Effect of steroids on expression of PDGF in cells

3.1.7 Effect of PDGF on endothelial cell proliferation

3.1.8 Characterization and expression of ECGF

\subsection{MATERIALS}

3.2.1 Reagents 116

3.2.2 Solutions

\subsection{METHODS}

3.3.1 Presence of estrogen receptor-related antigen in HUV endothelial cells

3.3.2 Effect of sex steroids on HUV endothelial cell proliferation

3.3.3 Isolation of total RNA from HUV endothelial cells

3.3.3.1 HUV endothelial cell culture 
3.3.3.4 Assessment of integrity of RNA

$\begin{array}{ll}\text { 3.3.4 Northern blot preparation } & 125\end{array}$

$\begin{array}{ll}\text { 3.3.5 RNA slot blots } & 127\end{array}$

3.3.6 Prehybridization and hybridization of northern and slot blots

$\begin{array}{ll}\text { 3.3.6.1 Prehybridization } & 127\end{array}$

3.3.6.2 Probe preparation by random primer synthesis 128

$\begin{array}{ll}\text { 3.3.6.3 Hybridization } & 129\end{array}$

$\begin{array}{ll}\text { 3.3.6.4 Washing } & 129\end{array}$

3.3.6.5 Repeated hybridization of blots 129

$\begin{array}{ll}\text { 3.3.7 Preparation of specific DNA probes } & 130\end{array}$

3.3.8 Isolation of DNA from low melting point agarose gels 130

3.3.9 Characterization of CDNA probes and calculation of 131 their concentration

3.3.10 Preparation of labelled DNA marker 132

3.3.11 Effect of PDGF on HUV endothelial cell proliferation 132

$\begin{array}{ll}\text { 3.3.12 Statistical analysis } & 133\end{array}$

\subsection{RESULTS}

3.4.1 Presence of estrogen receptor-related antigen in 133 HUV endothelial cells

3.4.2 Effect of sex steroids on HUV endothelial cell proliferation

3.4.3 Expression of mRNA for PDGF-A and PDGF-B in HUV endothelial cells

3.4.4 Expression of mRNA for ECGF in HUV endothelial cells

3.4.5 Effect of PDGF on HUV endothelial cell proliferation 


\section{CHAPTER 4: EFFECTS OF SEX STEROIDS ON ENDOTHELIAL CELLS: PLATELET ADHESION, PROSTACYCLIN RELEASE AND VON WILLEBRAND FACTOR PRODUCTION}

\subsection{INTRODUCTION}

4.1.1 Antithrombotic and prothrombotic features of endothelial cells 146

4.1.2 Role of platelet binding to endothelial cells in atherogenesis 148

4.1.3 Role of exogenous sex steroids in platelet binding and aggregation

4.1.4 $\mathrm{PGl}_{2}$ production and release by endothelial cells 149

4.1.5 Effect of exogenous sex steroids on $\mathrm{PGI}_{2}$ production 151 and release

4.1.6 VWf : Ag expression by endothelial cells 152

4.1.7 Effect of exogenous sex steroids on the production 154 and release of vWf

\subsection{METHODS}

4.3.1 $\mathrm{PGI}_{2}$ release by HUV endothelial cells exposed to sex steroids

4.3.1.1 Treatment of HUV endothelial cells 159

4.3.1.2 Measurement of $\mathrm{PGI}_{2} \quad 160$

4.3.2 Binding of platelets to HUV endothelial cells exposed to 
sex steroids

4.3.2.1 Preparation and labelling of washed platelets for 160 adhesion studies

4.3.2.2 Preparation and labelling of PRP and PPP for adhesion studies

4.3.2.3 Treatment of HUV endothelial cell preparation

4.3.2.4 Platelet-HUV endothelial cell monolayer adhesion assay 162

4.3.3 vWf : Ag immunofluorescence of HUV endothelial cells exposed to sex steroids

4.3.3.1 Treatment of HUV endothelial cells 163

4.3.3.2 Immunofluorescence and flow cytometry 164

4.3.4 Effect of supernatant from HUV endothelial cells exposed to sex steroids on ristocetin-induced platelet aggregation

4.3.4.1 Treatment of HUV endothelial cells

4.3.4.2 Ristocetin-induced platelet aggregation

4.3.5 Measurement of vWf : Ag from supernatants of HUV endothelial cells exposed to sex steroids

4.3.5.1 Treatment of HUV endothelial cells 166

$\begin{array}{ll}\text { 4.3.5.2 Immunoelectrophoresis } & 167\end{array}$

$\begin{array}{lr}\text { 4.3.6 Statistical analysis } & 167\end{array}$

\subsection{RESULTS}

4.4.1 Platelet binding to HUV endothelial cells exposed to 168 sex steroids

4.4.2 $\mathrm{PGI}_{2}$ release by HUV endothelial cells exposed to sex steroids

4.4.3 vWf : Ag immunofluorescence of HUV endothelial cells exposed to sex steroids

4.4.4 Effect of supernatants from HUV endothelial cells exposed to sex steroids on ristocetin-induced platelet aggregration 


\section{CHAPTER 5: INFLUENCE OF SEX STEROIDS ON MONOCYTE BINDING TO ENDOTHELIAL CELLS}

\subsection{INTRODUCTION}

5.1.1 Monocyte adhesion to endothelial cells

5.1.2 Factors influencing monocyte binding to

endothelial cells

5.1.3 Effect of estrogens and progestins on monocyte binding to endothelial cells

\subsection{MATERIALS}

\subsection{METHODS}

5.3.1 Treatment of HUV endothelial cells 193

5.3.2 Monocyte isolation and labelling $\quad 194$

5.3.3 Monocyte adhesion assay 195

5.3.4 Non-specific esterase stain for monocyte identification 196

5.3.5 Detection of endotoxin in solutions used in monocyte studies 196

$\begin{array}{ll}\text { 5.3.6 Statistical analysis } & 196\end{array}$

\subsection{RESULTS}

5.4.1 Monocyte adhesion to gelatin-coated wells 197

5.4.2 Incubation of HUV endothelial cells with sex steroids. 198 Effect on monocyte adhesion to HUV endothelial cells 
5.4.3 Incubation of monocytes with sex steroids. Effect on monocytes and monocyte adhesion to HUV endothelial cells

5.4.4 Detection of endotoxin in solutions used in monocyte studies 202

5.5 DISCUSSION 202

5.6 SUMMARY 206

\section{CHAPTER 6: THE EFFECT OF SEX STEROIDS ON LOW DENSITY LIPOPROTEINS AND THE VASCULAR ENDOTHELIUM}

$\begin{array}{ll}6.6 \text { INTRODUCTION } & 208\end{array}$

$\begin{array}{ll}\text { 6.1.1 Modification of LDL } & 209\end{array}$

6.1.2 Oxidative changes to LDL 210

6.1.3 Cytotoxicity of LDL for endothelial cells 212

6.1.4 Lipid peroxidation and the role of antioxidants 215

6.1.5 Sex steroids, LDL oxidation and atherosclerosis 218

$\begin{array}{lr}\text { 6.2 MATERIALS } & 219\end{array}$

\subsection{METHODS}

6.3.1 Preparation of native LDL 223

6.3.2 Modified Lowry protein assay 225

6.3.3 Determination of fatty acid content of LDL preparations by 225 gas-liquid chromatography

6.3.4 The thiobarbituric acid (TBA) assay 226

6.3.5 Polyacrylamide gel electrophoresis of LDL preparations 227

6.3.6 Agarose gel electrophoresis of LDL preparations 227

6.3.7 Effects of native and oxidised LDL on HUV endothelial 228 
cell proliferation

6.3.8 Lipoprotein oxidation by copper sulphate 230

6.3.9 Oxidation of LDL by HUV endothelial cells 231

6.3.10 Statistical analysis 231

\subsection{RESULTS}

6.4.1 Fatty acid profiles of LDL preparations as assessed by 232 gas-liquid chromatography

6.4.2 Extent of lipid peroxidation in LDL preparations 233 using TBA assay

6.4.3 Presence of conjugated dienes in LDL preparations 234

6.4.4 Electrophoresis of LDL samples in agarose 235 and polyacrylamide gels

6.4.5 Effect of native and oxidized LDL on

HUV endothelial cell proliferation 236

6.4.6 Modulation by sex steroids of the effects of native 238 and oxidized LDL on HUV endothelial cell proliferation

6.4.7 Inhibitory effect of $17 \beta$-estradiol on peroxidation of LDL by 239 copper sulphate.

6.4.8 Influence of sex steroids on oxidative modification of LDL caused by HUV endothelial cells 


\section{CHAPTER 7: SEX HORMONES AND ATHEROSCLEROSIS: THE CURRENT STUDIES IN PERSPECTIVE}

7.1 HUV endothelial cells as a model of arterial endothelial cell function

7.2 Effects of $17 \beta$-estradiol and progesterone on HUV endothelial 256 cell functions: The importance of concentration and time

7.3 Effect of $17 \beta$-estradiol and progesterone on monocyte binding 259 and LDL oxidation

7.4 Implications for the use of $17 \beta$-estradiol and progesterone 260 in hormone replacement therapy and oral contraceptive use

7.5 Future directions of research 261

APPENDIX 1: COMPOSITION OF TISSUE CULTURE 263 MEDIA (MEDIUM 199)

APPENDIX 2: EFFECT OF SEX STEROIDS ON THE 265 REQUIREMENT OF HUV ENDOTHELIAL CELLS FOR FCS, ECGS AND HEPARIN 


\section{CHAPTER 1}

\section{SEX HORMONES AND ATHEROSCLEROSIS}




\subsection{ATHEROSCLEROSIS: A MAJOR PROBLEM}

Atherosclerosis and its complications account for approximately $50 \%$ of the overall mortality in most Western countries (Olson, 1979; Kannel et al, 1984). The clinical manifestations of atherosclerosis are multiple. The most prevalent in our community are coronary heart disease (CHD), cerebrovascular disease and peripheral vascular disease. The most important complication of atherosclerosis, in terms of mortality, is CHD. Although the prevalence of $\mathrm{CHD}$ is greater in men at all ages, the occurrence of CHD in women after the age of 65 is very high (Furman, 1986; Levy et al, 1990). Moreover, while the incidence of CHD in men is decreasing (Kannel et al, 1984; Sytkowski et al, 1990), some studies have suggested no change (Johansson et al, 1984) or an increasing incidence of CHD in women (Elveback et al, 1986). CHD accounts for $23 \%$ of all deaths and $52 \%$ of all cardiovascular deaths in women (Kannel and Abbott, 1987). It is the leading cause of death in women over 50 years of age in most Western countries although there are striking differences in the mortality rates between countries (Thelle, 1990). In women aged $30-39$ years, CHD is second only to breast cancer as the major cause of death (Becker and Corrao, 1990). One risk factor for CHD specific for women in the premenopausal age group is the oral contraceptive (Oliver, 1970; Mann and Inman, 1975; Mann et al, 1975a ; b; Inman et al, 1970; Rosenberg et al, 1976a; Royal College of General Practitioners' Oral Contraceptive Study, 1977; Vessey et al, 1977, Vessey and Mann, 1978; Jick et al, 1978a; Shapiro et al, 1979). In contrast, the use of estrogen in post menopausal women has been shown to reduce the risk of CHD by $40-50 \%$ compared with women who do not receive such therapy (Bush, 1990; Stampfer et al, 1991). The possible role of female sex steroids in modifying CHD risk is thus apparent. 
This Chapter will review atherosclerosis in terms of its pathology, the major hypotheses of atherogenesis, the major known risk factors for the development of atherosclerosis in relation to $\mathrm{CHD}$ (with special reference to the importance of these risk factors in women), and finally the possible roles of estrogens and progestins in the development of atherosclerosis.

\subsection{PATHOLOGY OF ATHEROSCLEROSIS}

\subsubsection{Normal artery}

Normal large and medium sized arteries consist of three components:

(a) A tunica intima which consists of a single continuous layer of endothelial cells supported by a small amount of underlying loose connective tissue including smooth muscle cells (Parker and Oland, 1966);

(b) A tunica media, which consists of varying proportions of smooth muscle cells and elastic tissue with the first elastic sheet being called the internal elastic lamina; and

(c) A tunica adventitia, which consists of fibroblasts intermixed with smooth muscle cells loosely arranged between bundles of collagenous and elastic fibres (Ross and Glomset, 1976). It is usually separated from the media by an external elastic lamina. The tunica adventitia also contains the small blood vessels, called vasa vasorum, as well as autonomic and sensory nerve fibres (Gardner et al, 1975).

\subsubsection{Atherosclerotic artery}

The lesions of atherosclerosis have been classified into three general stages: the fatty streak, the fibrous plaque, and the complicated lesion (Ross, 1981b). 
Fatty streaks appear to represent the earliest lesions of atherosclerosis and are macroscopically visible in the arteries of children (Stary, 1991). They appear as yellow non-raised spots on the luminal surface which enlarge and coalesce to form irregular yellow streaks. Microscopically, the fatty streak consists of an accumulation of lipid droplets in both macrophages and to a lesser extent smooth muscle cells lying beneath the endothelium (Anderson, 1980; Ross and Glomset, 1976; Ross, 1981b).

The fibrous plaque is the hallmark of advancing atherosclerosis. This is an elevated yellow or white lesion which protrudes into the lumen of the artery. The fibrous plaque consists of a further accumulation of lipid in macrophages and smooth muscle cells surrounded by extracellular lipid and relatively large amounts of connective tissue. The cells, including smooth muscle cells, monocytes, T-lymphocytes (Hanssen and Bondjers, 1987) and increased connective tissue matrix form a fibrous cap that covers a large deep deposit of extracellular lipid and cell debris. This fibrous tissue is provided, at least in part, by proliferating smooth muscle cells (Anderson, 1980; Ross, 1981b). An intermediate lesion has also been described in children after puberty and in young adults. This lesion is histologically and chemically between the early stage of increased intimal lipid and the advanced lesion. These intermediate lesions occur in the same region as the advanced lesion (Stary, 1991).

The complicated atherosclerotic lesion occurs if the fibrous layer breaks down resulting in ulceration of the plaque. A mural thrombus is then likely to form on the ulcerated surface. Another common change is the deposition of calcium salts which may convert the plaque to a hard brittle lesion (Lansing et al, 1950). There may also be thinning of the tunica media, and in some cases extension of the plaque into the adjacent tunica media. This may result in an aneurysm. Small blood vessels may grow into the atheromatous plaque from 
the adventitia and some vessels may also grow from the luminal surface (Anderson, 1980, Ross, 1981b).

\subsection{MAJOR HYPOTHESES OF ATHEROSCLEROSIS}

Steinberg (1987b) stated that " the etiology and pathogenesis of the human arterial lesion is multiple and interactive, rather than single and direct". The major hypotheses of atherogenesis are:

\subsubsection{Injury hypothesis}

This hypothesis was first proposed by Rokitansky in 1852 (Duguid, 1946; Olson, 1979). He believed that the lesions of atherosclerosis arose from deposition of fibrin on an injured intimal surface. Later Duguid (1946) expanded this theory. Ross and Glomset (1976) also proposed that atheroma began with injury, followed by platelet adhesion and release of a platelet mitogen, platelet-derived growth factor (PDGF), (refer to Section 1.3.1.3 and 3.1.5) with subsequent proliferation of smooth muscle cells. The "response to injury " hypothesis has since been modified by Nilsson (1986) to incorporate the findings of increased endothelial replication and permeability rather than cell loss following endothelial cell injury as proposed by Ross (1981c and 1986). Ross's hypothesis postulates that the endothelial cells normally form a continuous nonreactive layer which lines the artery and mediates metabolic exchange through the artery wall. Agents may interact with the endothelium leading to changes such as separation between endothelial cells, altered endothelial permeability, or frank denudation of the endothelial cells. If the injury is severe enough platelet adhesion occurs resulting in the formation of a mural thrombus, with or without adhesion of blood monocytes, which subsequently migrate into the tissue. The interactions between the substances derived from platelets, monocytes, and 
plasma (e.g. lipoproteins or hormones) may stimulate the migration of smooth muscle cells from the media of the artery into the intima, and induce the proliferation of these migrating cells. Eventually blood flow could be compromised by partial narrowing of the arterial lumen and the lesion may also be complicated by thrombosis. However, if the injury were limited this process could be reversed and the integrity of the artery wall could be reconstituted (Ross, 1981b and Ross, 1986). Agents considered to be injurious to endothelial cells include mechanical forces (Bjorkerud and Bondjers, 1971; Helin et al, 1971, Moore, 1981; Ramsay et al, 1982), radiation (Tracy et al, 1974; Eldor et al, 1989), chemical agents such as lipoproteins (Henriksen et al, 1977; 1979a; 1979b; Hessler et al, 1979; Tauber et al, 1980; Evensen et al, 1983; Morel et al, 1983a), various toxins such as homocystine (Harker et al, 1974; 1976) and nicotine (Booyse et al, 1981b), immunologic injury such as drug sensitivity (Saphir et al, 1965) and allergy, (Minik et al, 1966; Minick and Murphy, 1973) and viruses (Fabricant et al, 1978).

Evidence to support the "response to injury" hypothesis has been published by Faggiotto et al (1984) and Faggiotto and Ross (1984) who used pigtail monkeys to follow the changes in the aorta and iliac artery that result from a prolonged hypercholesterolemic diet. They demonstrated early monocyte attachment and entry into the subendothelial space, and the development of fatty streaks. By the third or fourth month junctions between some endothelial cells appeared to separate over fatty streaks principally at branches and bifurcations. In some cases platelet adhesion, aggregation, and mural thrombosis occurred at these sites. One or two months later similar sites contained advanced smooth muscle proliferative lesions. Considerable variation between monkeys as to the extent of the aortic changes was observed suggesting genetic susceptibility. Stemerman and Ross (1972) demonstrated a similar sequence following intravascular balloon catheter injury to iliac arteries, beginning with platelet adhesion followed by 
smooth muscle cell migration and fibrous plaque formation. Gerrity and colleagues (1980a; b; Gerrity et al, 1981a; b), however, demonstrated in pigs that dietary-induced hypercholesterolemia, while producing fatty streak lesions in the aortic arch, did not progress to more extensive lesions. Fatty streak formation has also been demonstrated in Carneau pigeons fed cholesterol (Jerome and Lewis, 1984), in rabbits following mechanical injury or a hypercholesterolemic diet (Poole and Florey, 1958; Goode et al, 1977; Rosenfeld et al, 1987a) and in rats after mechanical injury (Schwartz et al, 1975). Ramsay et al (1982) demonstrated that repair following endothelial injury in vivo in the rabbit could occur without significant platelet reaction or smooth muscle cell proliferation. This has been supported by the findings of Reidy and Silver (1985) in the rat aorta. Some other studies examining mechanical injury (Bjorkerud and Bondjers, 1971; Christensen and Garbarish, 1973) or chronic hyperlipidemia (Ross and Harker, 1976; Lewis and Kottke, 1977; Gerrity and Schartz, 1977; Gerrity et al, 1979) have shown that even if the monolayer is re-established there is an increased permeability and a tendency to accumulate lipids. In hyperlipidemia and hypertension an increase in endothelial cell turnover has been observed which may itself promote plaque formation (Florentin et al, 1969; Schwartz and Benditt, 1977; Schwartz et al, 1980; DiCorleto and Chisolm, 1986).

The "response to injury" hypothesis implicates the endothelial cell, the platelet, the monocyte / macrophage and the smooth muscle cell as being centrally involved in the pathogenesis of atherosclerosis.

\subsubsection{Role of the endothelial cell}

The endothelial cell has many important functions related to atherogenesis. These include: 
(a) Active transfer of metabolic substances, including lipoproteins, between the circulation and the surrounding tissues (Stein et al, 1973);

(b) Acting as a permeability barrier (Gimbrone, 1976; Gimbrone, 1981);

(c) Providing a non-thrombogenic luminal surface by possessing a net negative charge on its surface (Collen, 1980), by synthesizing prostacyclin $\left(\mathrm{PGI}_{2}\right.$ ) (Moncada and Vane 1979), secreting plasminogen activators (van Hinsbergh et al, 1987) and containing an ectoplasmic ATPase (Dieterle et al, 1978). Other antithrombotic properties of endothelial cells include production of 13-hydroxyoctadecadienoic acid (13-HODE) (Buchanan et al, 1985), endothelium-derived relaxing factor (EDRF) (Radomski et al, 1987; Sneddon and Vane, 1988), the presence of antithrombin III bound to endothelial cells which can inactivate thrombin (Marcum et al, 1984), and the presence of thrombomodulin which can modulate the activity of thrombin (Nievelstein and de Groot, 1988) (refer to Section 4.1 for more detail);

(d) Secreting collagen (Sage et al, 1984; Jaffe et al, 1985), elastin (Jaffe, 1985) and fibronectin (plasma cold-insoluble globulin) (Macarak et al, 1978) beneath their abluminal surface. These endothelial cell products help to maintain the integrity of the vascular lining and they can also interact with blood elements in the hemostatic process (Gimbrone, 1981);

(e) Synthesizing and releasing of procoagulant substances such as von Willebrand factor (VWf) (Jaffe et al, 1973), factor V (Jaffe, 1985), thrombospondin (Silverstein et al, 1986; Nievelstein and de Groot, 1988), platelet-activating factor (Prescott et al, 1984; Whatley et al, 1987) and thromboplastin (tissue factor) which promote hemostasis and thrombosis (Zeldis et al, 1972). Endothelial cells can also inhibit the degradation of fibrin by expression of plasminogen activator inhibitors (PAI) such as plasminogen activator inhibitor-1 and plasminogen activator inhibitor-2. The expression of these plasminogen activator inhibitors is altered by various cytokines (Scarpati and Salder, 1989); 
(f) Synthesizing a number of cytokines which have been demonstrated in vitro to have potential roles in atherogenesis (refer to Section 1.3.1.3); and (g) Binding low density lipoproteins (LDL) and modifying these so that they are recognized and ingested by cells such as macrophages (Steinberg, 1983). This is thought to be important in fatty streak formation. Human LDL are synthesized in the liver as spherical particles with a diameter of about 22 $\eta \mathrm{m}$ and an average MW of 2.5 million. LDL carries most of the lipids of normal human plasma (Esterbauer et al, 1990). LDL contains about $50 \%$ cholesterol and its esters and approximately $22 \%$ protein (Hatch and Lees, 1968). The major protein is called apolipoprotein B (apo B). There are two forms of apo B, apo B-48 and apo B-100. Apo B-100 is the form found in LDL (Young, 1990).

In vivo and in vitro studies have shown that, in response to injury, endothelial cells reendothelialize from the pre-existing marginal cells by migration and division (Poole et al, 1958; Bjorkerud and Bondjers, 1971; Spaet et al, 1975: Schwartz et al, 1975; Ramsay et al, 1982; Coomber and Gotlieb, 1990). Once an intact monolayer is formed the reparative response ceases and endothelial cell turnover returns to its normal slow rate (Gimbrone, 1981; Lindner et al, 1989). Walker and Bowyer (1984) demonstrated in rabbits that following superficial injury to aortic endothelium, reendothelialization occurred at the same rate in both normocholesterolemic and hypercholesterolemic animals. However, hypercholesterolemic animals accumulated lipid in areas of regeneration while normocholesterolemic animals did not.

\subsubsection{Role of the platelet}

The platelet has many potential roles in atherogenesis from binding to altered endothelium, interaction with the subendothelium resulting in 
thrombus formation and release of platelet contents (which are important in coagulation, thrombosis, proliferation of smooth muscle cells and macrophages, and lipid accumulation (Curtiss et al, 1987)).

In response to endothelial cell injury platelets adhere at the site, aggregate and release their contents (Lewis and Kottke, 1977; Eldor et al. 1981). These include, from the $\alpha$-granules, thromboxane $\mathrm{A}_{2}$ (Svensson and Fredolm, 1977), platelet factor 3, fibrinogen (Day and Solum, 1973), calcium (Mustard et al, 1981; Han et al, 1983), platelet factor 4 and $\beta$-thromboglobulin (Mustard et al, 1981) which are involved in coagulation and platelet aggregation. $\beta$ thromboglobulin can reduce $\mathrm{PGI}_{2}$ release from endothelial cells (Hope et al, 1979) while platelet factor 4 can bind to heparin sulphates and inhibit its anticoagulant activity (Rybak et al, 1989). Platelet factor 4 and $\beta$ thromboglobulin are also capable of inducing chemotaxis in monocytes (Niewiarowski and Thomas, 1969; Ross, 1986). Platelets also release adenosine nucleotides (ATP and ADP) (Niewiarowski and Thomas, 1969) and serotonin (5-hydroxytryptamine) (which can potentiate the response to ADP (Baumgartner and Born, 1968)) from the dense bodies of platelets (Chesterman and Berndt, 1984). Serotonin causes the release of endothelium-derived vasodilating substances from endothelial cells, (Haudenschild, 1990), and like ADP and thromboxane $A_{2}$ can also cause smooth muscle cells to contract (Ellis et al, 1976; Needleman et al, 1977). Kishi and Numano (1989) found that thromboxane $A_{2}$ and serotonin also cause direct endothelial cell damage as determined by $\left[{ }^{3} \mathrm{H}\right]$-adenine release. Platelets release PDGF (Kaplan et al, 1979), platelet-derived endothelial cell growth factor (PD-ECGF) (Usuki et al, 1989; Ishikawa et al, 1989) and transforming growth factor- $\beta$ (TGF- $\beta$ ) which have been shown to alter endothelial cell proliferation and migration (refer Section 1.3.1.3). Serotonin, transforming growth factor- $\beta$ and PDGF have all been shown to reduce bovine aortic endothelial cell proliferation (Fraater-Schroder et al, 1986; 
Takehara et al, 1987; Bell and Madri, 1989) and migration while norepinephrine and histamine, also released from platelets, increase endothelial cell proliferation but reduce endothelial cell migration (Bell and Madri, 1989). These same agents increase smooth muscle cell migration and proliferation (Bell and Madri, 1989). Platelets also release glycosidases, which can inhibit the anticoagulant effects of heparin (Kaplan et al, 1979; Ross, 1981c, Haudenschild, 1990), and release vWf (Zucker and Nachmias, 1985) and fibronectin, which are important in platelet aggregation and adhesion.

If platelets are removed from the circulation with antiplatelet serum (Moore et al. 1976; Friedman et al, 1977), or if platelet adherence and release of contents is prevented, for example, by the use of dipyridamole (Harker et al, 1976), the intimal proliferative response and, in particular, the proliferation of smooth muscle cells in response to endothelial cell injury is prevented.

\subsubsection{Role of growth factors}

Three growth factors are of particular importance in atherogenesis. These include PDGF, endothelium-derived growth factor (EDGF) and monocyte / macrophage-derived growth factor (MDGF).

PDGF is a protein which exists in several isomeric forms depending on the cell of origin. PDGF was first identified in alpha granules of platelets (Kaplan et al, 1979; Heldin et al, 1979). Human platelet PDGF is a $30 \mathrm{kD}$ protein comprising principally $(70 \%)$ a heterodimer (one A and one B chain, also termed PDGF-1 and PDGF-2 respectively (PDGF-AB) (Ross, 1989)). The subunit chains are linked by disulphide bonds (Chesterman and Berndt, 1986). Porcine platelets however contain homodimers of two $B$ chains (PDGF-BB) and human glioma cells produce PDGF-AA (Nister et al, 1988a; 
Reilly and Broski, 1989). Some cells produce PDGF-AA and PDGF-AB which are secreted, whereas PDGF-BB remains associated with the cells (Thyberg et al, 1990). The A and B chains of PDGF display a considerable degree of homology which amounts to $60 \%$ within the mature peptides (Ross et al, 1986; Heldin and Westermark, 1987 Westermark and Heldin C-H, 1989). The amino acid sequence of the B chain of PDGF is almost identical (93-96\% homology) (Heldin and Westermark, 1987; Ross, 1987; Ross, 1989) to that of the transforming protein of simian sarcoma virus (SSV) p28sis, encoded by the viral oncogene v-sis (Waterfield et al, 1983; Doolittle et al, 1983) and entirely homologous to the predicted product of its normal cellular homologue, C-sis (Johnsson et al, 1984). Simian sarcoma virus-transformed cells produce a mitogen that resembles PDGF immunologically and functionally (Owen et al, 1984) and thus may stimulate their own growth in an autocrine manner by the PDGF receptor (Nilsson, 1986). The A chain appears to have two forms, one isolated from the glioma cells, the other secreted by arterial endothelial cells (Tong et al, 1987; Collin et al, 1987; Ross, 1989). Different PDGF isoforms have been shown to have different effects on smooth muscle cells and fibroblasts (Bywater, 1988; Reilly and Broski, 1989). This may be in part due to the composition of the PDGF receptor. Human PDGF receptor consists of two subunits, designated a and $\mathrm{b}$, that are brought together by one of the three isoforms of PDGF to form the mature PDGF B-type receptor or PDGF A-type receptor (Ross, 1989; Claesson - Welsh et al, 1989). The overall similarity between the two PDGF receptor molecules is $44 \%$ (Claesson - Welsh et al, 1989). There are two PDGF receptor genes (Matsui et al, 1989). The a-subunit of the receptor can bind either PDGF-A of PDGF-B, whereas the B-subunit can bind only to PDGF-B (Claesson-Welsh et al, 1988; 1989). Therefore, the PDGF B-type receptor can bind PDGF-BB with high affinity, and PDGF-AB with lower affinity, but not PDGF-AA (Claesson-Welsh et al, 1989) while the PDGF A-type receptor can bind all three PDGF isoforms with high affinity (Claesson-Welsh 
et al, 1989). Different cell types have different numbers of the two receptor subunits (e.g. human dermal fibroblasts contain more b-subunits than asubunits, while mouse 3T3 cells contain equal amounts of $a$ and $b$ subunits). This means that PDGF-BB is more mitogenic for human dermal fibroblasts than PDGF-AA, but that these isoforms are equally mitogenic for mouse 3T3 cells (Ross, 1989).

PDGF is also produced by activated arterial endothelial cells in vitro (DiCorleto and Bowen-Pope, 1983), placental cytotrophoblasts (Goustin et al, 1985), macrophages but not monocytes (Shimokado et al, 1985), vascular smooth muscle cells (Seifert et al, 1984; Barrett et al, 1985), several human tumour cell lines including human glioma cells (Pantazis et al, 1985; Nister et al, 1988a, Hermansson et al, 1988), sarcoma cells (Heldin et al, 1980; Betsholtz et al, 1983; Pantazis et al, 1985), mammary carcinoma cells (Bronzert et al, 1987; Perez et al, 1987), lung carcinoma cells (Betsholtz et al, 1987) and an anaplastic thyroid carcinoma cell line (Heldin et al, 1988). PDGF-A and PDGF-B expression are found in atherosclerotic plaques using CDNA probes. In these plaques PDGF-A was expressed predominantly by the smooth muscle cells in the plaque while PDGF-B expression correlated with macrophages (Barrett and Benditt, 1988).

PDGF induces proliferation and migration of arterial smooth muscle cells (Reilly and Broski, 1989) and fibroblasts (Ross and Vogel, 1978; Thorgeirsson and Robertson, 1978; Ross, 1987; Pierce et al, 1989) by affecting the cycling fractions of these cells (Chen and Rabinovitch, 1989). PDGF has also been shown to bind and stimulate growth of microvessel endothelial cells from bovine fat, mouse brain (Bar et al, 1989) and cloned endothelial cells from fetal bovine bone at a concentration of $2 \mathrm{\eta g} / \mathrm{ml}$ (Streeten et al, 1989). In contrast, Bell and Madri (1989) found that PDGF (1.0-2.5 IU / ml) decreased bovine calf endothelial cell migration on collagen and inhibited proliferation of 
these cells. Wall et al (1978) also found that platelet released factors decreased the proliferation rate of HUV endothelial cells. PDGF has also been shown to have no effect on human arterial endothelial cell proliferation Wall et al, 1978; Thorgeirrson and Robertson, 1978; Thorgeirrsson et al, 1979; Weinstein and Weng, 1986) or bovine aortic endothelial proliferation (Davies and Ross, 1978; Kazlauskas and DiCorleto, 1985). Some of these endothelial cells, such as cloned endothelial cells from fetal bovine bone, express PDGF receptors (Streeten et al, 1989) while other endothelial cells (e.g. HUV endothelial cells (Heldin et al, 1981), bovine aortic endothelial cells and rat heart endothelial cells (Davies and Ross, 1978; Kazlauskas and DiCorleto, 1985, Westermark and Heldin, 1989) appear to lack detectable amounts of PDGF receptors. Therefore it is uncertain whether the effects observed by Wall et al (1978) and Bell and Madri (1989), for example, were receptor or nonreceptor mediated. PDGF does not appear to share receptors with epidermal growth factor, FGF or insulin in fibroblasts (Heldin et al, 1981). Some investigators have suggested that the apparent absence of receptors may in fact be due to competition with an endogenously produced competitor (Bowen-Pope and Ross, 1982; Bell and Madri, 1989).

In culture, binding of PDGF to its receptor stimulates tyrosine protein kinase activity of the receptor. If the amino acid sequence for the tyrosine kinase is deleted from the PDGF receptor the mitogenic component of PDGF function is lost but not other functions (Escobedo and Williams, 1988a). Other cellular events following stimulation of quiescent cells with PDGF include alteration of cytoskeletal elements, increased turnover of phosphatidylinosital, alteration in ion transport, in particular iron, and alteration in expression of both insulin-like growth factor and its receptor in fibroblasts (Yarden et al, 1986; Hart et al, 1988). PDGF has been shown to increase the rate of pinocytosis in cultured arterial smooth muscle cells and bovine endothelial cells (Davies and Ross, 1978) and has also been shown to stimulate LDL binding and degradation in 
cultured aortic smooth muscle cells by increasing the number of available LDL receptors (Chait et al, 1980). In smooth muscle cells PDGF has been shown to increase phospholipid metabolism leading to the formation of increased amounts of arachidonic acid which is important in $\mathrm{PGl}_{2}$ synthesis (Ross, 1981c). PDGF also stimulates protein synthesis, including collagen and proteoglycan synthesis, by smooth muscle cells (Ross, 1981b; Antoniades et al, 1987) and activates monocytes to generate superoxide, degranulate, aggregate and increase their binding to plastic surfaces (Tzeng et al, 1985). PDGF is also chemotactic for monocytes (Pierce et al, 1989). In fibroblasts, PDGF can increase interleukin-1 (IL-1) receptor density and responsiveness of these cells to IL-1 (Bonin and Singh, 1988). PDGF treatment of cells has been shown to alter receptors for other growth factors such as insulin-like growth factor I (IGF-1) and epidermal growth factor (EGF) (Hannink and Donoghue, 1989). PDGF is able to regulate gene expression. Two proto-oncogenes, c-myc and c-fos are activated soon after PDGF treatment (Kelly et al, 1983; Muller et al, 1984) in NIH 3T3 cells, a fibroblast cell line. Chen and Rabinovitch (1989) showed that PDGF regulates the proportion of cells capable of entering the cell cycle from the quiescent state in fibroblasts with an initial lag phase of 14-16 $\mathrm{h}$. Some of these effects are potentially atherogenic while others could be protective. Whether these events occur in vivo however, is uncertain (Steinberg, 1987b).

Endothelium-derived growth factor is released from many types of endothelial cells into culture medium (Gajdusek et al, 1980). It comprises at least two mitogens which are separable by biochemical and immunological methods (DiCorleto et al, 1983; DiCorleto and Chisolm, 1986). One mitogen which comprises about $25 \%$ of the activity is PDGF (DiCorleto and BowenPope, 1983; Dicorleto et al, 1983; Schweigerer et al, 1987; Vlodavsky et al, 1987a) while the other is basic fibroblast growth factor (bFGF). bFGF but not PDGF is released when endothelial cells are mechanically wounded (McNeil 
et al, 1989). The release of bFGF by endothelial cells has been shown to promote their own growth and motility in vitro (Schweigerer et al, 1987; Sato and Rifkin, 1988; Klagsbrun and Edelman, 1989 Vlodavsky et al, 1987b; Hannan et al, 1988) while antibodies to bFGF prevent the migration of bovine endothelial cells into denuded areas (Sato and Rifkin, 1988). bFGF has also been isolated from bovine pituitary (Gospodarowicz, 1975) and bovine brain (Gospodarowicz et al, 1977; Gospodarowicz et al, 1978a; Wall et al, 1978). Gospodarowicz et al (1978b) have reported that the addition of purified FGF $(1 \eta \mathrm{g} / \mathrm{ml})$ but not FGF $(1 \mu \mathrm{g} / \mathrm{ml})$ and human thrombin $(1 \mu \mathrm{g} / \mathrm{ml})$ to cultures of HUV endothelial cells prevented the premature senescence of endothelial cells and stimulated a high mitotic index. The effect of thrombin seemed to be to sensitize the cells to lower concentrations of mitogens rather than to affect directly the cycling time of the cells (Gospodarowicz et al, 1978). Vlodavsky et al (1979b) demonstrated that FGF is also involved in controlling the differentiation and phenotypic expression of vascular endothelial cells.

Endothelial cells release other growth factors including interleukin-8 (IL-8) (Strieter et al, 1989b; Pober and Cotran, 1990), growth inhibitors such as heparin and IL-1 (refer below) which are important in monocyte and smooth muscle function (Ross, 1986; Mantovani and Dejana, 1989) and transforming growth factor- $\beta$. Transforming growth factor $-\beta$ has been shown to inhibit proliferation of vascular endothelial cells and smooth muscle cells in vitro and is strongly chemotactic for macrophages in vivo (Stein and Stein, 1990). Transforming growth factor- $\beta$, in the presence of bFGF, inhibits the mitogenic activity of bFGF on HUV endothelial cells in a noncompetitive manner (Baird and Durkin, 1986; Fraater-Schroder, 1986).

Endothelial cells also release colony stimulating factors such as granulocyte colony stimulating factor (G-CSF), granulocyte-macrophage colony stimulating factor (GM-CSF) and macrophage colony stimulating factor (M- 
CSF) in response to IL-1, tumour necrosis factor- $\alpha$, lipopolysaccharide (LPS or endotoxin) and minimally modified LDL (Mantovani and Dejana, 1989; Rajavashisth et al, 1990). G-CSF and GM-CSF have been shown to increase endothelial cell proliferation and migration (Bussolino et al, 1989a) and may be responsible for increasing circulating monocyte numbers by stimulating proliferation and differentiation of hematopoietic precursors of monocytes.

Macrophage-derived growth factor is released by macrophages and stimulates proliferation of fibroblasts (Leibovich and Ross, 1976; Martin et al, 1981), smooth muscle cells and arterial endothelial cells in vitro (Martin et al, 1981). Macrophage-derived growth factor has been shown to comprise bFGF (Baird et al, 1985) and also a PDGF-like mitogen (Shimokado et al, 1985). Vascular endothelial cell proliferation factor (VEPF) has been isolated from macrophage supernatants and found to be mitogenic to endothelial cells (Okabe and Takaku, 1986). Vascular endothelial cell growth factor (VEGF) has also been shown to be produced by monocytes and bovine pituitary follicular cells and has a similar structure to the B chain of PDGF (Keck et al, 1989; Leung et al, 1989). The production of these growth factors has not been demonstrated in vivo (Steinberg, 1987b).

Monocytes also release a number of other cytokines with potential importance in atherogenesis. These include IL-1 (Vilette et al, 1990; Saegusa et al, 1990), tumour necrosis factor- $\alpha$ (TNF- $\alpha$ ), and transforming growth factor- $\beta$. IL-1 is a pleiotropic inflammatory mediator produced not only by macrophages but also by fibroblasts, B-lymphocytes, endothelial cells, smooth muscle cells and epithelial cells (Dinarello, 1988). In macrophages, IL-1 consists of two major polypeptides, interleukin- $1 \alpha$ and interleukin-1 $\beta$ (Pober and Cotran, 1990). Cells have different affinities for the two polypeptides although they tend to act in a similar manner on cells (Dejana et al, 1987; Pober and Cotran, 1990). IL-1 alters many functional properties of 
vascular cells including the production of $\mathrm{PGl}_{2}$ (Rossi et al, 1985; Kawakami et al, 1986; Dejana et al, 1987; Zavoico et al, 1989; Breviario et al, 1990), promoting prothrombotic changes in endothelial cells by increasing the production of platelet-activating factor (Bussolino et al, 1986), stimulating expression of tissue factor-like procoagulant activity (Bevilacqua et al, 1984; Nawroth et al, 1986) and suppressing anticoagulant activity by increasing plasminogen activator inhibitor-1 synthesis (Emeis and Kooistra, 1986; Scleef et al, 1988; Pober and Cotran, 1990). IL-1 increases vascular endothelial cell permeability to macromolecules (Royall et al, 1989). IL-1 also promotes monocyte and neutrophil binding to endothelial cells by increasing expression of adhesion molecules such as endothelial leukocyte adhesion molecule-1 (ELAM-1) (Bevilacqua et al, 1985). IL-1 inhibits the growth of endothelial cells but promotes the growth of smooth muscle cells and fibroblasts (Bonin et al, 1989; Mantovani and Dejana, 1989) and stimulates the synthesis of collagen, collagenases and several growth factors. In endothelial cells, IL-1 induces Cfos protooncogene expression (Colotta et al, 1988) and the production of PDGF, colony stimulating factors, interleukin-6 (IL-6), IL-8 and itself (Pober and Cotran, 1990). IL-1 also stimulates PDGF release from smooth muscle cells and endothelial cells (Mantovani and Dejana, 1989).

Tumour necrosis factor- $\alpha$ and tumour necrosis factor- $\beta$ (lymphotoxin) have been shown to have a spectrum of effects on vascular cells very similar to those of IL-1, some being potentially important in atherosclerosis. Tumour necrosis factor- $\alpha$ has been shown to induce migration but inhibit endothelial cell proliferation in vitro especially in cells with $70-80 \%$ of their life span complete (Shimada et al, 1990; Pober and Cotran, 1990). In vivo, tumour necrosis factor- $\alpha$ has been associated with endothelial cell injury following its injection into mice (Shalaby et al, 1989). The effects of tumour necrosis factor- $\alpha$ are augmented by interferon- $\gamma$ (IFN- $\gamma$ ) (Mantovani and Dejana, 1989; Shimada et al, 1990; Vilette et al, 1990). Tumour necrosis factor- $\alpha$ also 
augments production of coagulant activity from endothelial cells (Pober, 1988) and induces phospholipase $A_{2}$ activity in bovine endothelial cells (Clark et al, 1988). Tumour necrosis factor- $\alpha$ has been shown to reduce endothelial cell fibrinolytic activity (Schleef et al, 1988), which is important in thrombosis, and to stimulate endothelial cells to produce PDGF (Hajjar et al, 1987), IL-1, IL-6 and GM-CSF (Kaushansky, 1989; Mantovani and Dejana, 1989). The latter are important in hematopoiesis and cell proliferation and migration. Tumour necrosis factor- $\alpha$ also stimulates increased expression of intercellular adhesion molecules on endothelial cells (Mantovani and Dejana, 1989). In vivo, tumour necrosis factor- $\beta$ has been shown to induce angiogenesis (Mantovani and Dejana, 1989).

Other mitogens which may influence atherosclerosis by stimulating endothelial cell proliferation include interferon gamma (IFN- $\gamma$ ), epidermal growth factor (EGF), platelet-derived endothelial cell growth factor (PDECGF), transforming growth factor- $\alpha$ (TGF- $\alpha$ ) (Schreiber et al, 1986), insulinlike growth factors (Weinstein and Weng, 1986), vascular permeability factor (NPF) and acidic fibroblast growth factor (aFGF). Interferon- $\gamma$ has been shown to cause endothelial cells to proliferate at low concentrations (10-100 $\mathrm{U} / \mathrm{ml}$ ) but inhibit proliferation at high concentrations (1000 U /ml). Interferon- $\gamma$ has been shown to augment expression of MHC class II antigens which play a role in integrating the immunological functions of endothelial cells (Mantovani and Dejana, 1989). Epidermal growth factor, first isolated by Cohen (1962) from submaxillary glands, also stimulates the proliferation of HUV endothelial cells (Weinstein and Weng, 1986), a response which can be potentiated by thrombin (Gospodarowicz et al, 1978). Platelet-derived endothelial cell growth factor is mitogenic and chemotactic for endothelial cells but not other cells (Usuki et al, 1989; Ishikawa et al, 1989). Plateletderived endothelial cell growth factor also has angiogenic activity in vivo. The structural and functional characteristics of platelet-derived endothelial cell 
growth factor are distinct from heparin-binding FGFs (Miyazono et al, 1987) (refer to Section 2.1.2). Transforming growth factor- $\alpha$ shares sequence homology with epidermal growth factor and promotes growth of endothelial cells and fibroblasts (Derynck, 1988). Growth factors, such as transforming growth factor- $\alpha$ and epidermal growth factor, increase endothelial cell survival in vitro while transforming growth factor- $\beta$ has been shown to reduce endothelial cell survival (Etoh et al, 1989). Vascular permeability factor has a similar amino acid sequence to vascular endothelial cell proliferation factor (Keck et al, 1989). Finally, aFGF also termed heparin-binding growth factor $I$, is produced by smooth muscle cells and in vitro both smooth muscle cells and endothelial cells proliferate in response to this mitogen suggesting both paracrine and autocrine control of proliferation of these major components of the vasculature (Winkles et al, 1987) (refer to Section 2.1.2).

In summary, the potential role of growth factors in atherogenesis is complex. They provide communication signals between cells and appear to modulate many cell functions in vitro. The relative importance of each growth factor in vivo is largely unknown.

\subsubsection{Role of the monocyte/macrophage}

The monocyte-derived macrophage has an important role at several stages of atherogenesis. Firstly, these cells play a key role in the development of the fatty streak (Gerrity and Naito, 1980a; b). Circulating monocytes adhere to the endothelium (DiCorleto and de la Motte, 1985), penetrate the subendothelium (Navab et al, 1988) and can become loaded with cholesterol esters to develop into foam cells (Fowler et al, 1979; Gerrity et al, 1979; Schaffner et al, 1980; Gerrity, 1981a; Faggiotto et al, 1984). They are the predominant cell in early lesions of atherosclerosis. Secondly, in vitro experiments indicate that the monocyte /macrophage is capable of secreting 
growth factors that stimulate fibroblasts, smooth muscle cells, and possibly endothelial cells to multiply in culture (Liebovich and Ross, 1976) (refer to Section 1.3.1.3). This may be important in contributing to the proliferative components of plaque development. Thirdly, activated monocytes may contribute to vascular wall injury by generating cytotoxic factors (such as tumour necrosis factor, IL-1, reactive oxygen intermediates or oxidized LDL (Cathcart et al, 1985; Parthasarathy et al, 1986a) which damage neighbouring cells. Cathcart et al (1985) showed that oxidized LDL from macrophages was cytotoxic to fibroblasts while Peri et al (1990) showed that activated monocytes were cytotoxic in vitro to endothelial cells but not by the above proposed mechanisms. As foam cells, macrophages may also contribute to vascular wall injury by physically damaging the endothelium during their migration from the intima (Gerrity, 1981b).

\subsubsection{Role of the smooth muscle cell}

The smooth muscle cell is important in the progression of atherosclerotic lesions. Medial smooth muscle cells have been shown to comprise the early neointimal cells in the aorta following mechanical injury (Spaet et al, 1975; Eldor et al, 1981) and are the predominant cell in fibrous plaques (Ross, 1986). They have at least three potential roles in atherogenesis, including cell proliferation, accumulation of lipid, and synthesis of collagen, elastin and proteoglycans. Smooth muscle cells proliferate in response to PDGF, endothelial-derived growth factor, macrophage-derived growth factor and LDL (Ross, 1981c). In culture, smooth muscle ceils can assume a resting "contractile state" where they are not responsive to PDGF and do not produce connective tissue (Dilley et al, 1987). However in response to reduced density in culture or injury to the endothelium in vivo they become sensitive to PDGF, produce connective tissue, proliferate and migrate from the media to the intima. This is called a "synthetic state" (Chamley-Campbell 
et al, 1981a; b; Campbell and Campbell, 1985; Nilsson, 1986; Dilley et al, 1987). These smooth muscle cells increase their synthetic organelles and reduce the amount of myosin containing filaments (Campbell and Campbell, 1986). Smooth muscle cells have also been shown to form proteoglycans in vivo (Wight and Ross, 1975), to accumulate lipid (Parker and Odland, 1966; Newman et al, 1971; Fowler et al, 1979), modify LDL (Henriksen et al, 1982a; Heinecke et al, 1984; Morel et al, 1984) (refer to Section 1.3.2) and produce $\mathrm{PGI}_{2}$ (DeWitt et al, 1983). Nilsson et al (1985) has also demonstrated that rat smooth muscle cells produce a PDGF-like protein (A-chain) in culture suggesting possible autocrine regulation of smooth muscle cell growth. Smooth muscle cells have also been shown to produce endothelial cell growth factor (ECGF) and aFGF, to which they also respond (Winkles et al, 1987).

In summary, platelets, endothelial cells, monocytes and smooth muscle cells each have a number of functions which are potentially atherogenic in that they are thrombogenic, growth stimulating, or they release factors that influence cellular phenotype (Haudenschild, 1990). Equally, these same cells can release substances under certain circumstances which may be antiatherogenic by virtue of their growth inhibitory, antithrombotic, or vasodilating properties. It is the balance of these properties which determines whether a plaque develops.

\subsubsection{Lipid hypothesis}

This hypothesis was first proposed by Virchow in 1856 (Olson, 1979) and expanded by others since (Gofman et al, 1950; Anitschkow, 1954; Stamler et al, 1970). It states that infiltration of the arterial wall by serum lipids, particularly LDL-cholesterol and $\beta$-very low density lipoprotein ( $\beta$-VLDL) cholesterol from the blood stream gives rise to deposits of cholesterol. These 
act as an irritant, causing inflammation and proliferation of cells thus giving rise to atheroma lesion formation (Benditt, 1977). Furthermore it is postulated that dietary fat and cholesterol are the principal factors regulating serum lipids (Olson, 1979).

Evidence to support this hypothesis includes the following observations:

(a) Accumulation of cholesterol in the arterial wall is a hallmark of both experimental and human atherosclerosis (Steinberg, 1987b; Roberts, 1988);

(b) Atherosclerosis, in particular fatty streak lesions, can be induced in animals by cholesterol feeding (Walker and Bowyer, 1984);

(c) High plasma cholesterol (this being greater than $150 \mathrm{mg} / \mathrm{dl}$ for long periods of time), particularly in the LDL fraction, increases the incidence of CHD in Western countries especially in those persons under 50 years of age (Kannel et al, 1979; Consensus Conference, 1985; Roberts, 1988);

(d) Patients with familial hypercholesterolemia who have a genetic defect resulting in a deficiency of LDL receptors (Goldstein and Brown, 1977) have a high plasma cholesterol and a high incidence of premature atherosclerosis (Roberts, 1988);

(e) Intervention studies show that lowering plasma cholesterol (specifically, LDL-cholesterol) reduces the risk of CHD (Consensus Conference, 1985; Steinberg et al, 1989); and

(f) Atherosclerotic plaques regress when high blood cholesterol levels are lowered (Blankenhorn et al, 1987).

However, the foam cell which is characteristic of fatty streak lesions is not formed by simple accumulation of native LDL. Goldstein et al (1979a) showed that macrophages in culture take up native LDL at a slow rate via the classic LDL receptor pathway and that incubating macrophages with high concentrations of native LDL does not convert them to foam cells. Work by Mahley et al (1979), Goldstein et al (1980), Fogelman et al (1980) and 
Henriksen et al (1981 and 1983) have shown that by chemically modifying LDL (refer to Section 6.1.1) it is converted to a form which can be taken up more rapidly by the macrophage via a "scavenger" receptor (Goldstein et al, 1979a) which allows foam cell formation. Endothelial cells (Henriksen et al, 1981), smooth muscles cells (Henriksen et al, 1982a) and macrophages (Parthasarathy et al, 1986a) can all modify LDL. This change in LDL is thought to be an oxidative modification of LDL (Steinbrecher et al, 1984; Parthasarathy et al, 1985), and a number of studies (Sato et al, 1979; Tappel et al, 1980; Goldstein et al, 1981; Hoff and Morton, 1985; Tsuchida et al, 1985; Mowri et al, 1986; Daugherty et al, 1988) provide evidence that modification of LDL can occur in vivo. There are four lines of evidence to show that oxidized LDL is generated in vivo. Firstly, antibodies against modified LDL show immunostaining in rabbit aortic atherosclerotic lesions, but not normal lesions (Haberland et al, 1988 and Palinski et al, 1989; Boyd et al, 1989). Secondly, LDL which has been eluted from aortic lesions in Watanabe heritable hyperlipidemic (WHHL) rabbits shows cross reactivity with antibodies specific to malondialdehyde-conjugated LDL (Palinski et al, 1989). Thirdly, plasma of WHHL rabbits and human subjects has been shown to contain autoantibodies which react with various forms of oxidized LDL (Palinski et al, 1989). Tertov et al (1989b) have also shown that LDL isolated from patients with CHD results in increased deposition of lipids in smooth muscle cells compared to healthy subjects, and suggested that this LDL may be modified in some way. A fourth line of evidence is that treatment of WHHL rabbits with probucol (an antioxidant and cholesterol-lowering agent) has been shown to inhibit the progression of atherosclerosis (Carew et al, 1987; Kita et al, 1987) which could not be solely attributed to its cholesterol-lowering effect. However, probucol has also been found to inhibit IL-1 release from macrophages, increase cholesteryl ester transfer protein and perhaps interfere with the ability of macrophages to take up and degrade 
lipoproteins (Steinberg, 1991). This makes it difficult to attribute its inhibitory effect on atherosclerosis to its antioxidant properties alone.

Oxidized LDL is potentially important in the formation of atherosclerotic plaques for the following reasons:

(a) It promotes foam cell formation by virtue of uptake through the scavenger LDL receptor (Henriksen et al, 1981; 1983; Parthasarathy et al, 1986b);

(b) It favours the recruitment of monocytes because oxidatively modified LDL is a potent chemoattractant for monocytes (Quinn et al, 1985; Quinn et al, 1987). The chemotactic activity of oxidized LDL has been shown to be in the lipid fraction; it as been attributed to lysophosphatidlycholine (lysolecithin) generated during the conversion of LDL to its oxidized form (Quinn et al, 1988; Steinberg et al, 1989);

(c) It blocks the exit of resident arterial macrophages by inhibiting migration of these cells (Quinn et al, 1985). The active component again is in the lipid fraction of oxidized LDL, but not in native LDL (Quinn et al, 1985);

(d) It damages endothelial cells leading to endothelial denudation and other processes induced by damage to the endothelial lining of blood vessels (Ross and Glomset, 1976; Henriksen et al, 1979b; Tauber et al, 1980; Hessler et al, 1983; Morel et al. 1984);

(e) It can stimulate the release of a monocyte chemotactic factor (monocyte chemotactic protein 1) (Cushing et al, 1990) and induce the expression of GCSF and GM-CSF from endothelial cells in culture (Rajavashisth et al, 1990); and

(f) It is highly immunogenic so that it may contribute to atherogenesis by forming immune complexes that can then be taken up rapidly by macrophages (Steinberg et al, 1989).

Steinberg (1987a: b) proposed a hypothetical sequence of events by which an elevated plasma level of LDL could cause fatty streak formation : 
Increased LDL levels

$\Downarrow$

LDL infiltration of intima

$\Downarrow$

Oxidative modification of LDL

$\Downarrow$

Recruitment of circulating monocytes

by oxidatively modified LDL

$\Downarrow$

Phenotypic modulation of monocytes

to resident macrophages

$\Downarrow$

Retention of macrophages due to

inhibitory effect of oxidized LDL on motility

$\Downarrow$

Uptake of oxidized LDL via acetyl LDL receptor

$\Downarrow$

Foam cell and fatty streak formation

In summary, oxidized LDL is endowed with many properties which could be very important in the pathogenesis of atherosclerosis.

\subsubsection{Immunological theory of atherogenesis}

Klimov et al (1979) proposed the autoimmune theory of atherosclerosis. This theory states that autoantibodies to apo B containing lipoproteins are formed which result in the formation of circulating lipoprotein-antibody immune complexes. These may injure the arterial wall resulting in an influx of the complexes and free apo B-containing lipoprotein into the intima of 
arteries. This may result in uncontrolled uptake by arterial wall macrophages of lipoprotein-antibody immune complexes and the transformation of these cells to foam cells. Autoantibodies to lipoproteins were first detected in myeloma patients (Lewis and Page, 1965) and later Szondy et al (1983) detected anti-LDL activity in patients with ischaemic heart disease. There is evidence for immune complex complement fixation in atherosclerotic plaques of rabbits (Klimov et al, 1979). Furthermore, treatment with an immunosuppressant (cyclophosphamide) inhibited complement fixation (Klimov et al, 1979).

The immunological theory of atherosclerosis can be reconciled with the "response-to-injury" and the lipid hypothesis. The immune complexes may act as an injurious agent and set off the cascade of changes postulated by Ross (1986). However, the immunological hypothesis is not widely regarded as a major hypothesis.

\subsubsection{Transformation hypothesis (monoclonal theory)}

The transformation hypothesis was proposed by Benditt and Benditt (1973) who postulated that atheroma originates with the transformation of a clone of smooth muscle cells by a carcinogen or mutagen. They postulated three stages. First there was an initiation stage during which there was mutation in an artery-wall cell. Then, some factors (e.g. intrinsic genetic or extrinsic, such as viruses or chemicals) promoted the expression of the selective proliferative advantage conferred by the mutation, enlarging the mass of cells. Finally, there was a stage of complication, with a tendency for cells to degenerate and for lesions composed of those cells to break down and ulcerate (Benditt, 1977). The origins of this hypothesis come from the observation that the cells of early spontaneous plaques in chicken and suture-induced injury to arteries were different from normal artery wall cells. They were arrayed differently, 
they appeared smaller, to have few or no intercellular junctions, to develop vacuoles and manufacture large amounts of collagen. Benditt wondered whether the cell changes were genetic and caused by mutation, being confined to the smooth muscle cells, or a general response by many normal cells to a stimulus. Based on the work of Linder and Gartler (1965) who examined the X-linked enzyme glucose-6-phosphate dehydrogenase (G6PD) in benign uterine smooth muscle tumours, Benditt and Benditt (1973) observed that in some black women who were heterozygous for the $\mathrm{X}$-linked enzyme glucose-6-phosphate dehydrogenase, the normal intima contained both cells with the $A$ and the B-isoenzyme of glucose-6-phosphate dehydrogenase, but the atherosclerotic plaques consisted of the cells with only one or other isoenzyme (Benditt and Benditt, 1973). Pearson et al (1977) extended these studies to fatty streaks and then to small and large fibrous plaques (Pearson et al, 1978), finding that many, but not all, atherosclerotic lesions were monoclonal (that is, having only one type of enzyme). Benditt (1977) explained that not all plaques appeared to be monoclonal because of contamination by nearby blood cells rather than a polyclonal population of cells.

While it may explain some types of plaque formation, the monoclonal hypothesis fails to explain, for example, the migration of monocytes which is a feature of early lesions.

\subsubsection{Unifying hypothesis}

Haust (1970) proposed that all of the above theories could be "embraced" by, or accommodated within the concept of a single hypothesis. This "injury and repair" concept has been developed and expanded by many authors since. Injurious agents may come from three sources: the blood (its constituents or elements in transit), hemodynamics (of the circulation) and 
the arterial wall itself (Haust, 1987). Some injurious agents may be involved in the initiation, and others largely in the progression of the atherosclerotic lesions. The repair process that follows injury to the arterial intima is basically intended as a healing process but this process may proceed beyond the actual need for replacement of lost tissues. If this injury is continuous or repeated the repair process itself contributes to the development and progression of atherosclerotic lesions (Haust, 1987).

Steinberg $(1983 ; 1987 b)$ has proposed the following model for the pathogenesis of atherosclerosis combining the injury and lipid hypotheses:

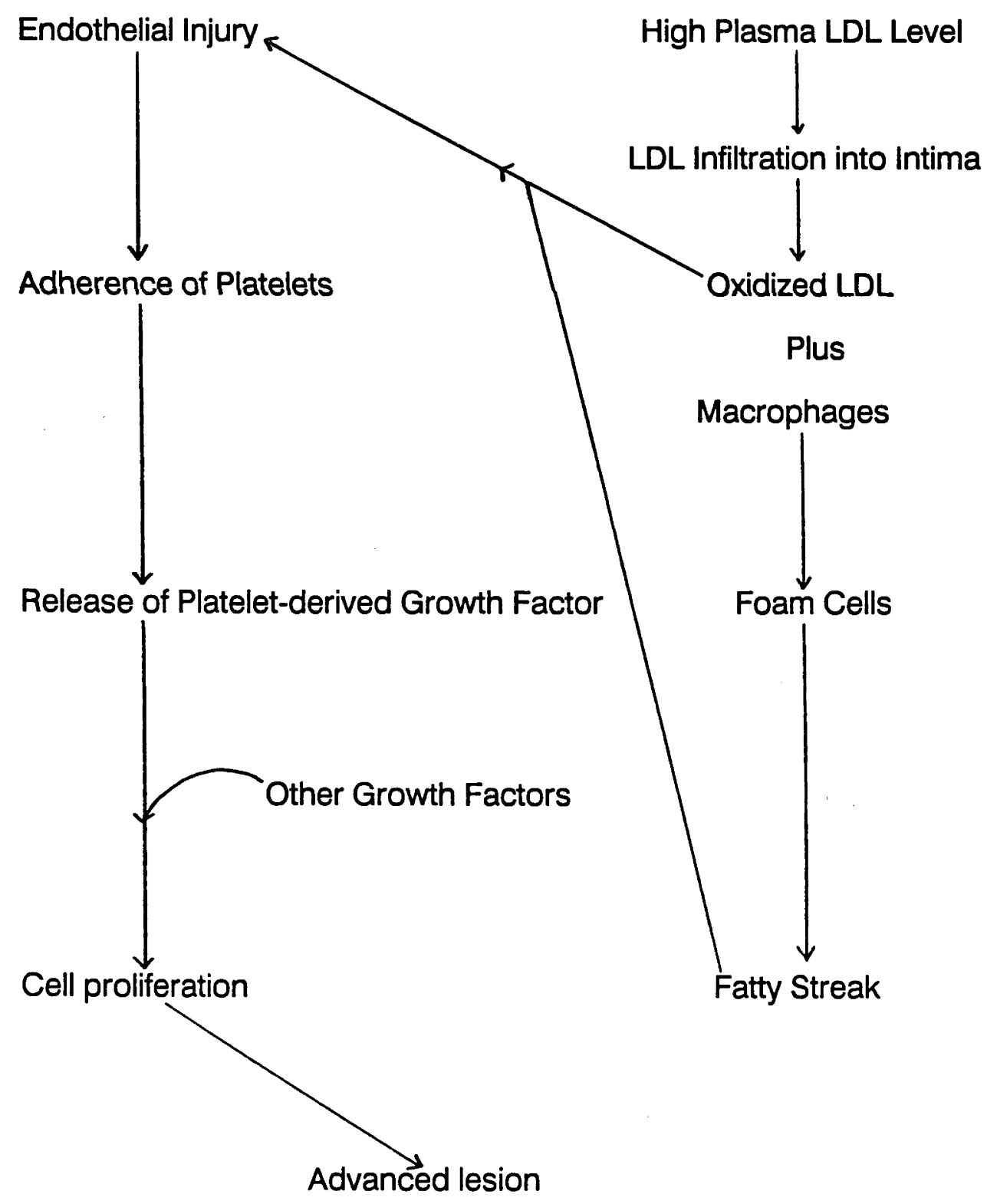


Several potential links between the two hypotheses have been proposed. Firstly, high concentrations of oxidized LDL in vitro have been shown to damage the endothelium. Secondly, foam cells formed beneath the endothelium may release toxic substances which could injure the endothelial cells. Thirdly, when smooth muscle cells proliferate in response to PDGF, they synthesize and secrete connective tissue matrix which may trap lipoproteins.

This unifying hypothesis incorporates the concept that atherogenesis is a disease of multiple etiologies rather than one specific agent as suggested by the transformation hypothesis.

\subsection{RISK FACTORS, ATHEROSCLEROSIS AND CORONARY HEART DISEASE}

Atherosclerosis is a disease of multiple etiologies in which the lesion arises as a result of a complex interaction between the arterial wall and the blood plasma (Olson, 1979). At least 246 risk factors have been associated with CHD (Hopkins and Williams, 1981). A risk factor can be defined as a factor that can be measured in individuals or groups, which discriminates among them, identifying those at increased risk (Last, 1983). Risk factors are however measures of association and not causation. Features that lend credence to a particular risk factor playing an etiologic role include:

(a) Presence of the factor prior to disease onset;

(b) Strong, dose-related association with the disease;

(c) Consistent prediction in several studies with varying population composition; and

(d) Pathogenic plausibility (Kannel and Sytkowski, 1987). 
Variation in the relative predictive value of a risk factor occurs, depending on the population studied and differences in risk factor definition (Hopkins and Williams, 1986). The probability of developing CHD increases with the number and severity of positive risk factors present in an individual (Editor, 1989). Important risk factors for CHD include age, a strong positive family history, plasma levels of LDL-cholesterol and high density lipoprotein (HDL)cholesterol, systolic and diastolic hypertension, hypercoagulation, cigarette smoking, truncal obesity, diabetes, and psychosocial factors. The relative importance of these risk factors varies with the age of the individual and gender (Stokes et al, 1987). Not all CHD can be attributed to known risk factors. In fact, CHD can occur at an accelerated rate in some individuals with little or no increase in generally accepted risk factors (Wissler, 1991). However, Crouse (1984) suggested that closer examination of these and other risk factors already known to be associated with CHD may improve our understanding of CHD.

This thesis focuses on the influence of estrogens and progestins on some cellular and biochemical events involved in the atherosclerotic process. The following section briefly discusses the importance of the major risk factors in relation to gender differences followed by a discussion of endogenous and exogenous hormones in relation to CHD.

\subsubsection{Major risk factors}

The development of a thrombus at the site of partly obstructive coronary atheromatous lesions is a major factor converting occult to clinically manifest disease (Oliver, 1986b; Davies and Thomas, 1985). Risk factors which impact either on atherosclerosis or thrombosis can therefore contribute to CHD. 
Age is one of the strongest risk factors for CHD (Hopkins and Williams, 1986). Atherosclerosis increases with age. In humans, only a few atherosclerotic lesions are present up until the second decade. In Western populations there is a rapid development in atherosclerotic lesions both in men and women, with men predominating in the third decade (Sternby, 1986). CHD does not become a major cause of morbidity and mortality in women until the age of 55 years (Corrao et al, 1990). There is an inverse correlation between longevity and most risk factors for CHD. Acute myocardial infarction (AMI) is one outcome of CHD which is often used in studies examining risk factors associated with CHD. All risk factors are less predictive of future AMI at older ages (Hopkins and Williams, 1986).

Family history of CHD is an independent risk factor. The importance of family history in CHD is most apparent in early-onset CHD (Rissanen, 1986). Other major risk factors, for example, LDL pathway defects, may be familial but fail to account fully for the familial component in CHD (Rissanen, 1986; Hopkins and Williams, 1986). Slack (1986) found that the genetic contribution to CHD was greater in females. The pattern of inheritance of recognised risk factors for CHD was mainly polygenic. Twin-studies show that the incidence of CHD was greater in monozygotic females than in any other combination of twins (Rissanen, 1986). There are few studies on the risks of CHD in siblings of female patients with CHD. Rosenberg et al (1983) found that a history of AMI or stroke before the age of 60 years in a mother or sibling was a risk factor for AMI in a woman before the age of 50 years. Rissanen (1986) found that CHD was more common among siblings of female patients than male patients. The risks for female first degree relatives of male patients is increased (Slack and Evans, 1966). Other studies have shown that CHD tends to aggregate not only in blood relatives but also in spouses (Kannel, 1976). The risk of CHD events in a wife has been found to be greatest in the 
few months after the coronary death of the husband. This may reflect stress or shared environmental influences, or both (Kannel, 1987).

Lipoproteins and altered lipoprotein metabolism are intimately associated with the development of atherosclerosis, especially the inherited forms of dyslipoproteinemia. Total serum cholesterol and LDL-cholesterol have been shown to be significant risk factors for CHD in multiple prospective, case control, autopsy, and angiographic studies (Hopkins and Williams, 1981). The potential roles of $L D L$ in atherogenesis were discussed in Section 1.3.2. Raised levels of VLDL or LDL, or subnormal concentrations of HDL (Gordon et al, 1977) are associated with increased atherosclerosis (Nikkila et al, 1986). Lipoprotein(a), an apo B-100-containing lipoprotein has also been shown to be a risk factor for CHD in hyperlipidemic people (Utermann, 1989; Young, 1990). The protein and lipid composition of lipoprotein(a) is similar to LDL. The distinguishing feature is the presence of the protein apo(a) (Scott, 1989). There is striking homology between apo(a) and plasminogen, which is important in fibrinolysis. Lipoprotein (a) competes with plasminogen for binding to the plasminogen receptor on endothelial cells (Scott, 1989) and this inhibition of fibrinolysis may contribute to its association with AMI.

It is well documented that high levels of HDL are associated with a reduced risk of CHD (Gordon et al, 1977). This is particularly true of both the $\mathrm{HDL}_{3}$ and $\mathrm{HDL}_{2}$ subfractions of $\mathrm{HDL}$ cholesterol (Stampfer et al, 1991). The biologic phenomena responsible for this are unclear. Glomset (1968) proposed that HDL serves as a carrier for the removal of cholesterol from peripheral tissues, transporting it back to the liver where it can be converted to bile salts and excreted. There is little in vivo proof for this proposed mechanism. Another proposed mechanism is that HDL may inhibit the uptake and degradation of LDL (Stein and Stein, 1976). A third possible mechanism by which HDL may inhibit atherosclerosis is as a carrier of 
antioxidants such as estradiol which can be transferred to LDL (Leszczynski and Schafer, 1991).

Generally, women have been shown to have higher levels of HDL lipids and lower concentrations of VLDL than men (Russ et al, 1951) but with no major differences in LDL levels between the sexes before middle age (Nikkila et al, 1986). Some studies have shown a cyclical change in plasma cholesterol and cholesterol ester during the menstrual cycle with a fall in total cholesterol and cholesterol esters at the time of ovulation (Oliver, 1986a) while others have not (Svanborg and Vikrot, 1967). After menopause the plasma HDL levels do not change despite the fall in estradiol (Cauley et al, 1990) while the LDL-cholesterol level increases. In men, autopsy studies have shown that total cholesterol minus HDL-cholesterol was significantly associated with increased coronary atherosclerosis while HDL-cholesterol was inversely associated with atherosclerosis (Reed et al, 1989).

Blood pressure is a major risk factor for CHD in both men and women especially in relation to AMI (McGill and Stern, 1979; Hopkins and Williams, 1986; Stokes et al, 1987). The fraction of CHD attributable to hypertension (diastolic blood pressure above $90 \mathrm{~mm} \mathrm{Hg}$ ) is approximately $20 \%$ (Hopkins and Williams, 1986). Hypertension has been shown to be a greater risk factor in women than in men in some studies (Master et al, 1939; Kannel, 1976). However, several large trials have found that lowering blood pressure does little to influence the mortality from CHD (Werko, 1987).

The development of thrombosis at the site of partly obstructive coronary atheromatous lesions is a very important trigger converting occult to clinically manifest CHD (Oliver, 1986b; Davies and Thomas, 1985). Davies and Thomas (1985) found that $95 \%$ of cases of AMI, sudden ischaemic death and crescendo angina were associated with fissuring of atherosclerotic 
plaques leading to intraintimal or intraluminal thrombus which occludes the artery or leads to plaque growth. Increased factor VII activity and an increased concentration of plasma fibrinogen have been shown to relate to an increased incidence of CHD in men and women (Meade et al, 1980b; Wilhelmsen et al, 1984; Kannel, 1987). Fibrinogen is important in thrombosis as it is a cofactor in platelet aggregation and it also increases blood viscosity (Oliver, 1986b). Other risk factors for CHD such as cigarette smoking, stress and hypertension are associated with increased platelet aggregation (refer below).

Cigarette smoking is the single most preventable cause of CHD. Cigarette smoking increases platelet adhesiveness and aggregation, decreases HDLcholesterol and interacts with oral contraceptives to increase the risk of AMI (Corrao et al, 1990). Smokers have a twofold increased risk of CHD (Levy et al, 1990). The carbon monoxide in cigarette smoke increases the risk of ventricular fibrillation which could lead to sudden death (Hopkins and Williams, 1986). Many studies have shown that the relative risk of CHD increases with the number of cigarettes smoked (Bengtsson, 1973; Kannel, 1976; Ross et al 1981a). Rosenberg et al (1983) found that in women the relative risk of $\mathrm{AMI}$ for current smokers (35 or more cigarettes per day) was 10 times that of women who had never smoked. The duration of the habit appeared to be a less important factor and therefore it has been suggested that smoking promotes coronary artery thrombosis rather than atherosclerosis itself (Anderson, 1980).

Obesity is also an important modifiable risk factor for CHD (Manson et al, 1990). However it is the distribution of obesity which appears to be a more significant risk factor than total obesity (Depres et al, 1990). It is central or abdominal obesity associated with high waist-to-hip ratios which is the better predictor of cardiovascular risk than total adiposity (Lapidus et al, 1984; 
Larsson et al, 1984). It is this pattern of obesity which is associated with disorders such as insulin resistance, type II diabetes, glucose intolerance, hypertension, and changes in the concentration and composition (e.g. smaller, denser, more oxidizable LDL particles) of plasma lipoproteins. Central obesity has been shown to predict lipoprotein levels (Wingard, 1990) with the relationship being strongest in women (Hopkins and Williams, 1986). The causal mechanism involved in these associations has not been clearly identified.

Type A personality is characterized by impatience, hostility, time and deadline consciousness, aggressiveness, and excessive competitiveness (Hopkins and Williams, 1986). Most prospective studies (including the Western Collaborative Group Study, the Framingham Study and the Recurrent Coronary Prevention Project) have estimated that the relative risk between type A and type B (absence of a chronic sense of time urgency and not easily aroused) (Friedman et al, 1982) is approximately two (Rosenman et al, 1975; Hopkins and Williams, 1986). Type A behaviour described by Friedman and Rosenman (1971) has been established as a risk factor for CHD in women as well as men (Bengtsson, 1973; Haynes, 1986). This is particularly true of initially healthy individuals rather than persons at increased risk of CHD due to previous myocardial infarction or elevations of traditional risk factors (Manuck et al, 1986). Epidemiological studies have shown that there are certain components of Type A personalities which are more strongly associated with CHD than others. These include feelings of hostility and expression of anger (Manuck et al, 1986; Henry, 1986). About $50 \%$ of angiographic studies have shown a relationship between vessel occlusion and Type A personality especially when Type A personality is assessed using a structured interview method (Manuck et al, 1986). However, angiographic studies which have assessed the relationship between hostility and angiographic changes in the coronary arteries have all shown a significant 
correlation (Manuck et al, 1986). Type A personalities have increased adrenocorticotropic hormone (ACTH) and catecholamine levels, more labile cholesterol levels (Friedman and Rosenman, 1971; Friedman, 1977) and acute rises in heart rate and blood pressure which may promote atherosclerosis and its complications by altering platelet aggregation, adversely affecting lipid metabolism and causing hemodynamic disturbances (Manuck et al, 1986).

Another predictor of CHD related to behaviour is reaction to life stress and social supports. In the Framingham study women employed in careers with nonsupportive bosses had an increased risk of CHD (Haynes, 1986). This has also been shown in similar studies involving men (Manuck et al, 1986). This type of stress results in changes in the neuroendocrine system similar to those described for Type A personalities (Manuck et al, 1986). In particular, increases in catecholamines, testosterone and cortisol are associated with stress (Henry, 1986). Stress induced in animals by physical restraint has been shown to cause an increase in endothelial cell turnover which may be important in the early stages of atherogenesis (Manuck et al, 1986).

Diabetes mellitus is a well established risk factor for CHD (Kannel, 1976; Ross et al 1981a; Hopkins and Williams, 1986). The age-specific prevalence of severe atherosclerosis in coronary arteries is higher in diabetic patients (Robertson and Strong, 1968; Reunanen, 1986). Pyorala and Laakso (1983) reviewed autopsy studies, some angiographic studies and major clinical studies and concluded that diabetes was an independent risk factor for CHD. Diabetes is a much more important risk factor for CHD in women than in men (Stokes et al, 1987). Premenopausal diabetic women are not protected from CHD in the way that premenopausal non-diabetic women are (Pyorala and Laakso, 1983; Reunanen, 1986). Diabetic patients (Silberbauer et al, 1979) and rats rendered experimentally diabetic (Harrison et al, 1978) have an 
accelerated rate of atherogenesis. Other risk factors such as hypertension, obesity and lipid disorders are also more common in diabetics. Fasting serum insulin levels have been shown to be an independent predictor of systolic blood pressure and they are inversely related to HDL (Donahue et al, 1987). Oxidative stress has been proposed as a common pathway linking diverse mechanisms in the pathogenesis of diabetic complications including atherosclerosis (Baynes, 1991). Mechanisms contributing to oxidative stress in diabetes may include increased nonenzymatic glycosylation, autoxidative glycosylation and changes in the level of inflammatory mediators and antioxidant defence mechanisms (Baynes, 1991).

In summary, as stated previously, the presence of one or more of these major risk factors does not predict the occurrence of $\mathrm{CHD}$ in a given individual although the probability of developing CHD increases with the number and severity of positive risk factors present in the individual (Nutrition Reviews, 1989).

\subsubsection{Gender}

Sex differences in atherosclerosis depend on the site of the vascular lesion and the age of the population examined (McGill and Stern, 1979). In general, men have more extensive and severe atherosclerosis in the coronary arteries. The male to female ratio of deaths from CHD is close to two (Kalin and Zumoff, 1990). In the Framingham Study, Kannel et al (1976) reported that, under sixty years of age, the frequency of cardiovascular events is $27.5 \%$ for men, but only $10.1 \%$ for women. After the age of sixty-five the frequency increases to $36.7 \%$ for men and $18 \%$ for women. The relative immunity of women to AMI and sudden death is graphically illustrated by a twenty-year lag in the incidence of these complications for women when compared to men. Pathological evidence also supports the marked difference between 
men and women (White et al, 1950, Ackerman et al, 1950). In women, the more rapid appearance of cardiovascular complications after menopause suggests that hormonal factors are important in the delayed progression of CHD (Robinson et al, 1959; Oliver and Boyd, 1959; Parrish et al, 1967; McNamara et al, 1978). Parrish et al (1967) found that it took an average of 14.4 yrs after bilateral oophorectomy before CHD became apparent. In contrast, Furman (1986) found that the age-specific death rate for CHD increases with age in both sexes, while there was diminishing acceleration of the male mortality rate beginning in the fifth decade, bringing the male and female mortality rates closer. The curves for men and women were qualitatively similar in shape with no upward discontinuity of the female curve at the menopause. Zumoff (1981) suggested that there are three possible explanations for these findings, as follows. Firstly, the factors that cause the rate to rise with age become less prominent in older individuals. Secondly, the susceptible young population dies off early leaving a less susceptible older population, and thirdly, protective factors appear increasingly with age.

Gender-related differences in platelet function including platelet responsiveness to aggregating agents (Johnson et al, 1975), platelet morphology (Milton and Frojmovic, 1983; Pinto et al, 1990) and production of various factors such as thromboxane $A_{2}$ (Pinto et al, 1990) and antithrombin III (Meade, 1990) have been observed which appear to increase the thrombotic risk in men. Differences in the lipid profile between men and women have also been found. Premenopausal women have lower levels of serum VLDL- and LDL-cholesterol and higher HDL-cholesterol levels than men of corresponding age (Crona et al, 1985; Seely, 1990). Men also have higher triglyceride levels throughout life (Crona et al, 1985). The level of serum lipid peroxides has been found to be higher in men than women and tends to increase with age (Yagi and Komura, 1986). Another gender-related 
difference which has ben linked to CHD risk is serum iron stores which are lower in premenopausal women than men of the same age (Sullivan, 1981).

Studies in primates have also shown differences between males and females. Kaplan et al (1984) observed that socially dominant female monkeys had less extensive and less severe coronary atherosclerosis than males or socially subordinate females which appeared to be related to behavioural and hormonal characteristics (more regular ovarian function) and not to plasma lipids.

There has been much debate as to the cause of the difference in CHD between men and women. There are at least five possible explanations:

(a) A lower prevalence of risk factors in women than in men;

(b) A better tolerance, by women, of risk factors when they are present;

(c) Hormonal and metabolic differences which give relative protection to women;

(d) A deleterious effect of maleness (Heller and Jacobs, 1978; Zumoff, 1981); and

(e) Differences in clinical presentation.

None of these explanations is, by itself, entirely adequate.

\subsection{Endogenous hormones}

Kalin and Zumoff (1990) recently reviewed the literature on endogenous hormone levels and the risk of CHD. They found that, in general, men with CHD who have never suffered an AMI have normal estradiol and testosterone levels while endogenous estradiol levels are elevated from immediately after an AMI for about 1 year post infarction (Phillips, 1976; Luria et al, 1982; Klaiber et al, 1982; Phillips et al, 1983; Goldberg et al, 1987). The mechanism 
for this increased estradiol level is not apparent but it may be a response to stress (Kalin and Zumoff, 1990). Endogenous testosterone levels are also decreased in men who have survived an AMI from soon after the infarction to about 1 year later. Progesterone levels show a stress-mediated increase in levels during the immediate post infarction period (Kalin and Zumoff, 1990). Men with high endogenous estrogen levels and low endogenous testosterone levels due to cirrhosis of the liver have a decreased risk of CHD (Kalin and Zumoff, 1990) while women with testosterone excess, associated with menstrual irregularities, appear to have an increased risk of CHD (La Vecchia et al, 1987).

There is much controversy as to whether or not menopause (natural or surgical) is a risk factor for CHD. Some studies report an increased risk (Kannel et al, 1976; Gordon et al, 1978; Rosenberg et al, 1981; Meade et al, 1983; Bengtsson et al, 1986; Witteman et al, 1989) while others do not (Ritterband et al, 1963; Jullien et al, 1977; Blanc et al, 1977; Colditz et al, 1987). Many of the studies of spontaneous menopause have been flawed by not controlling for cigarette smoking (Kannel et al, 1976) or the length of time of the post menopausal period (Colditz et al, 1987). Studies of surgical menopause have reported an increased risk of CHD (Oliver and Boyd, 1959; Parrish et al, 1967; Kannel et al, 1976; Gordon et al, 1978; Rosenberg et al, 1981; Colditz et al, 1987). Autopsy studies of the coronary arteries have also produced conflicting results, some showing more severe atherosclerosis (Novak and Williams, 1960; Parrish et al, 1967) while others have failed to confirm this (Novak and Williams, 1960). Some studies have found an association between menopause and various risk factors for $\mathrm{CHD}$ such as serum cholesterol (Hjortland et al. 1976; Shibata et al,1979), in particular, a fall in HDL-cholesterol and an increase in LDL-cholesterol (Matthews et al, 1989), serum triglycerides (Shibata et al,1979), smoking habits (Bengtssen et al, 1986), coagulation, and in particular an increase in antithrombin III activity 
(Meade et al, 1983; 1990), haemoglobin (Elwood et al, 1970; Hjortland et al, 1976; Shibata et al, 1979; Meade et al, 1983) and blood pressure (Stokes et al, 1987). Hjortland et al (1976) and Matthews et al (1989) however, did not find an association between blood pressure and natural menopause. Early menopause appears to give rise to increased serum cholesterol and triglyceride levels (Kannel et al, 1976; Bengtsson et al, 1986), but a reduced blood pressure and body weight (Bengtssen et al, 1986).

Thus, the relation between menopause and risk factors for $\mathrm{CHD}$ is complex. However it is known that post menopausal women have a higher incidence of CHD than aged matched premenopausal women (Rudy, 1990) and that hormone replacement therapy markedly reduces cardiovascular mortality (Hunt et al, 1990) (refer to Section 1.5).

\subsection{EXOGENOUS SEX HORMONES AND ATHEROSCLEROSIS}

Exogenous estrogen and progestins have been shown to influence a number of known risk factors for CHD such as hypertension (Lim et al, 1970; Wallace, 1971; Spellacy and Birk, 1972; Ramcharan and Pellegrin, 1975; Fisch and Frank, 1977; Weir and Robertson, 1986), smoking (Jick et al, 1978a; Petitti et al, 1979; Rosenberg, 1986; Bush et al, 1986a), carbohydrate metabolism (Ajabar et al, 1972; Wynn, 1983; 1986; Bardin et al, 1983), lipid metabolism (refer to Section 1.5.1) and hypercoagulation (refer to Section 1.5.2). Most of this information in humans comes from studies examining oral contraceptive use and estrogen replacement therapy in women, and estrogen treatment in men with prostatic cancer.

There are two major types of estrogens used in hormonal therapy. The first is the natural estrogens and includes conjugated equine estrogens, estrone sulphate and $17 \beta$-estradiol. The second class is the synthetic agents 
including ethinylestradiol and mestranol, the methyl ether of ethinyl estradiol (Shearman, 1986). The clinical response to these estrogens depends on their metabolism (Wren et al, 1982). Estradiol can be converted to the less active form of estrone which can then be metabolized to estriol or 2hydroxyestrone (Wren et al, 1982). There are also two major classes of synthetic progestins. The first is the 19-nortestosterone derivatives (norgestrel, norethindrone acetate and norethisterone) which are strongly androgenic, and the second includes the hydroxyprogesterone derivatives (medroxyprogesterone acetate and progesterone) (Bush, 1986b). The effects of exogenous sex steroids vary according to the type and concentration of the sex steroid given and whether it is in combination with another steroid.

There appears to be an increased risk of CHD in current users of the oral contraceptive (Oliver, 1970; Mann and Inman, 1975; Mann et al, 1975a ; b ; Inman et al, 1970; Rosenberg et al, 1976a; Royal College of General Practitioners' Oral Contraceptive Study, 1977; Vessey et al, 1977, Vessey and Mann, 1978; Jick et al, 1978a; Shapiro et al, 1979; Rosenberg et al, 1990), but not in past users (Mann et al, 1975a ; b; Mann and Inman, 1975; Royal College of General Practitioners' Oral Contraceptive Study, 1977; Shapiro et al, 1979; Stampfer et al, 1988; Rosenberg et al, 1990). Many of the above studies examined the risk of $\mathrm{CHD}$ in oral contraceptive users in which the concentration of synthetic estrogen was higher than is currently used. The increase in CHD events has been attributed to estrogen use and is probably due to an increase in thromboembolic events (Stadel, 1981a: b). Reducing the dose of estrogen has been shown to reduce the incidence of $\mathrm{CHD}$ (Meade et al, 1980a). Angiographic (Dear and Jones, 1971) and autopsy studies (Weiss, 1965) have shown that AMI in oral contraceptive users is due to segmental occlusions in the coronary arteries, which were otherwise normal. The progestin component (especially if highly androgenic such as 
norgestrel) in the oral contraceptive has also been shown to be a risk factor for AMI (Meade et al, 1980a; Stadel 1981a: b). There have been other studies however which have failed to observe an association between oral contraceptive use and AMI (Petitti et al, 1979). Some studies have shown an association of AMI and contraceptive use only in cigarette smokers (Croft and Hannaford, 1989).

Studies on post menopausal estrogen use generally show a protective effect of estrogen against CHD (Burch et al, 1974; Hammond et al, 1979; Nachtigall et al, 1979; Ross et al, 1981a; Stampfer et al, 1985; Bush et al, 1983; 1986a; Ross et al, 1989; McFarland et al, 1989; Hazzard, 1989b), especially in surgically induced menopause (Kalin and Zumoff, 1990). One case control study found that the use of estrogen replacement therapy reduced the rate of CHD by as much as $45 \%$ (Stampfer, 1991). In most of these studies the patients were taking estrogen alone (usually a natural conjugated form at a relatively low concentration). Conjugated estrogens (e.g. "Premarin", Ayerst Laboratories) are much less potent than synthetic estrogens (e.g. ethinyl estradiol) used in oral contraceptives. For example, $0.02 \mathrm{mg}$ ethinyl estradiol has about the same effect on suppressing menopausal symptoms as 62.5 times this concentration $(1.25 \mathrm{mg})$ of conjugated estrogens (McGill and Stern, 1979). Although most studies have shown a protective effect of post menopausal estrogen against CHD some studies have not shown a significant effect (Rosenberg et al, 1976b; 1980; Adams et al, 1981; Bain et al, 1981). Indeed, a few studies have suggested an increased risk of CHD with post menopausal estrogen use (Gordon et al, 1978; Jick et al, 1978a; b). Gordon et al (1978) for example, found a significant increase in angina but not AMI. Bush et al (1986a) reviewed those papers which reported an increased risk and concluded that in each the study design was flawed. Many of the studies had a high preponderance of smokers and a small number of subjects. A recent large study of 48,470 
post-menopausal women followed for up to 10 years revealed a significant reduction in major coronary disease (relative risk 0.56 ) in those women who were using estrogen therapy, but no change in the risk of stroke (Stampfer et al, 1991). The findings were similar in a low risk sub-group which excluded women who reported current cigarette smoking, diabetes, hypertension or hypercholesterolemia.

Information about the use of progestins in studies on estrogen replacement therapy is often not provided or is minimal (Kalin and Zumoff, 1990). In one study in which all the patients were taking progestins along with estrogen the risk of CHD was unchanged (Nachtigall et al, 1979), whereas in another study where no progestins were used there was a decreased risk of CHD (Lafferty and Helmuth, 1985). Barrett-Connor et al (1989) reported no statistically significant differences in the effects of estrogen ("Premarin") alone compared with estrogen and medroxyprogesterone acetate ("Provera", Upjohn), with both formulations resulting in a decreased risk of CHD.

In summary, the evidence favours an increased risk of $\mathrm{CHD}$ with the oral contraceptive, although this depends on the type of oral contraceptive, whereas with post menopausal estrogen replacement therapy, estrogen appears to have a protective effect.

Estrogen used experimentally in men to prevent recurrent AMI has been reported to be associated with an increased risk of CHD particularly when high doses of conjugated estrogens (5-10 mg) were administered (Stamler et al, 1963; The Coronary Drug Project Research Group, 1970 ; 1973; Byar, 1973). On the other hand some investigators have shown a reduced risk of recurrent $A M I$ in men with low dose $(1.25-2.5 \mathrm{mg})$ conjugated estrogen (Marmosstom et al, 1962; Stamler et al, 1963), but not with ethinyl estradiol (0.05-1.5 mg) (Marmosston et al, 1962). 


\subsubsection{Effect of estrogens and progestins on lipid metabolism}

The influence of estrogens and progestins on the metabolism of all three major plasma lipoproteins has been studied extensively. Estrogen causes a decrease in the concentration of LDL in post menopausal women (Russ et al, 1955; Gustafson and Svanborg 1972; Wallace et al, 1977a; Tikkanen et al, 1978; Bush et al, 1986a; Hazzard, 1986; Sonnendecker et al, 1989; Applebaum-Bowden et al, 1989; Barrett-Connor et al, 1989) and in men with prostatic cancer (Eriksson et al, 1989). The lowering of plasma LDL concentration is probably due to increased catabolism of LDL particles as a result of enhanced binding and uptake of LDL particles in the liver (Kovanen et al, 1979; Eriksson et al, 1989) rather than decreased production. Estrogen causes a change in the composition of LDL by increasing the triglyceride and phospholipid content relative to that of cholesterol (Russ et al, 1955). The

addition of a progestin such as norethisterone acetate or norgestrel with $17 \beta-$ estradiol also results in a reduction in LDL-cholesterol by reducing apo $B$ production (Jensen and Christiansen, 1987; Wolfe and Huff, 1989).

Estrogen replacement therapy has been shown not to influence plasma triglyceride or VLDL levels in some studies (Tikkanen et al, 1978; Knopp et al, 1981), but has been shown to influence levels in others (Robinson and LeBeau, 1965; Hazzard, 1986). This appears to depend on whether the estrogen is alkylated or not (Enk et al, 1986) and the route of administration of the estrogen (Basdevant et al, 1983). Non-alkylated estrogens (such as estradiol valerate) do not increase triglycerides and VLDL while alkylated estrogens such as 17 -estradiol do (Crona et al, 1985; Enk et al, 1986; Colvin et al, 1990) by stimulating hepatic metabolism (Chan et al, 1976; Schaefer et al, 1983; Colvin et al, 1990). Basdevant et al (1983) found that oral administration of $17 \beta$-estradiol resulted in a significant increase in 
triglyceride and VLDL-triglyceride levels while percutaneous estradiol induced a significant decrease in triglyceride and VLDL-triglyceride levels. The addition of a progestin such as norethindrone resulted in a significant lowering of total triglyceride and VLDL levels probably by increasing the clearance of triglycerides (Glueck et al, 1972), while medroxyprogesterone, which has low androgenic activity, did not have this effect (Nikkila et al, 1986; Sonnendecker et al, 1989).

Post menopausal estrogen use causes a significant increase in HDLcholesterol compared with subjects not taking estrogens (Furman et al, 1958; Gustafson and Svanborg 1972; Wallace et al, 1977a; Bradley et al, 1978; Sonnendecker et al, 1989; Applebaum-Bowden, et al, 1989), probably by down-regulating hepatic lipase activity (Tikkanen and Nikkila, 1987; Applebaum-Bowden, et al, 1989). Applebaum-Bowden (1989) found in women that $17 \alpha$-ethinyl estradiol and a high cholesterol diet resulted in an increase in apo A-1, the major apolipoprotein in HDL. Haberland (1991) found that sequentially combined hormone replacement therapy induced an increase in HDL-cholesterol during estrogen therapy and a decrease during combined therapy. However, no cyclical changes in HDL-cholesterol were seen with continuously combined estrogen replacement therapy. The reduction in HDL-cholesterol and apo A-1 was equivalent with 17hydroxyprogesterone derivatives and 19-nortestosterone derivatives. Other studies have also shown that highly androgenic progestins result in a decrease in HDL-cholesterol levels in women (Tikkanen et al, 1981; Bradley et al, 1978). Estrogenic hormones also cause changes in the composition of HDL, with an increase in the triglyceride component (Knopp et al, 1981).

Oral contraceptives have been shown to increase plasma triglyceride, plasma cholesterol, VLDL-cholesterol and LDL-cholesterol levels (Aurell et al, 1966, Wynn et al, 1966; Wynn et al, 1969; Alfin-Slater and Aftergood, 1971; 
Stokes and Wynn, 1971; Wallace et al, 1977a; b). Other studies, however, have failed to show changes in serum cholesterol, LDL or apo B levels (Dyerberg and Horne, 1972). These different findings probably reflect differences in contraceptive formulations. The most estrogenic contraceptive pills are associated with the highest triglyceride levels while the most progestational pills are associated with the highest cholesterol values (Stokes and Wynn, 1971). Increased LDL-cholesterol levels occur in association with highly androgenic progestins (Aurell et al, 1966; Wahl et al, 1983). The effect of oral contraceptives on HDL also depends on the androgenic activity of the progestin used (Bradley et al, 1978, Knopp et al, 1981). If the oral contraceptive contains a highly androgenic progestin there is an associated fall in HDL (Castelli et al, 1977; Wahl et al, 1983). When the androgenic activity is low there is an associated increase in HDL (Wallace et al, 1979; Wynn, 1986).

Estrogens have been shown to lower plasma levels of LDL in men (Bulusu et al, 1982; Eriksson et al, 1989) and increase HDL levels (Bulusu et al, 1982; Oliver and Boyd, 1956; Aro et al, 1990) while androgen treatment results in a fall in HDL and a rise in LDL levels (Russ et al, 1955; Oliver and Boyd, 1956) due to increased hepatic lipase activity (Tikkanen and Nikkila, 1987). Progesterone administration has no significant effect on serum lipid levels in men (Oliver and Boyd, 1956).

Oral contraceptives have also been shown to decrease the serum level of antioxidants such as vitamin $\mathrm{E}$, vitamin $\mathrm{C}$ and $\beta$-carotene which are important in the prevention of oxidation of lipoproteins (Gavaltia and Renaud, 1991). Vitamin $E$ and $\beta$-carotene are transported by lipoproteins in the circulation. Decreases in serum vitamin $E$ have not been observed in all studies, the level depending on the composition of the oral contraceptive (as described above) and on dietary intake of polyunsaturated fatty acids. Some studies have also 
reported that levels of lipid peroxides in blood are increased in women receiving oral contraceptives (Ciavatti and Renaud, 1991). Interestingly, pregnancy has also been shown to be associated with increased lipid peroxide levels in blood and reduced antioxidant activity (Ciavatti and Renaud, 1991). Ciavatti and Renaud (1991) suggested that these changes may be one factor responsible for the hyperaggregability and increased platelet reactivity seen with oral contraceptive use and in pregnancy (refer to Section 1.5.2).

In summary, the adverse effects on lipid profile and the potential increase in risk of $\mathrm{CHD}$ associated with sex hormone use varies greatly, being dependent on the type, combination and concentration of sex hormones used (Knopp et al, 1982).

\subsubsection{Role of estrogens and progestins in platelet aggregation and thrombosis}

The oral contraceptive increases the risk of coronary thrombosis (Inman and Vessey, 1968; Inman et al, 1970), cerebral artery thrombosis (Inman and Vessey, 1968; Vessey and Doll, 1968; Collaborative Group for the Study of Stroke in Young, 1973), as well as pulmonary and deep-venous thrombosis (Vessey and Doll, 1968; Inman and Vessey, 1968; Sartwell et al, 1969; Inman et al, 1970; Boston Collaborative Drug Surveillance Programme, 1973). Not all studies have, however, found a relationship between contraceptive use and coronary thrombosis (Vessey and Doll, 1968; 1969, Royal College of General Practitioners, 1977). The incidence of coronary thrombotic disease seems to increase as the concentration of estrogen increases in the oral contraceptive (Inman et al, 1970; Stolley et al, 1975, Stadel, 1981b). Some progestins appear to increase the risk of superficial venous thrombosis (Royal College of General Practitioners' Oral Contraceptive study, 1978) but 
not coronary thrombosis (Inman et al, 1970). Other progestins are thought to protect against the increased thrombotic risk associated with estrogens (Inman et al, 1970; Elam et al, 1980) although there may be individual variation (Bierenbaum et al, 1979). Sabra and Bonnar (1983) found that oral contraceptive preparations with ethinyl estradiol (30 $\mu \mathrm{g})$ combined with levonorgestrel caused smaller changes in coagulation in women than ethinyl estradiol $(30 \mu \mathrm{g})$ with norethindrone acetate. However, in the studies referred to above the absolute number of cases of thrombosis was generally small and the problem of obtaining reliable information in relation to oral contraceptive use and thrombotic complications makes interpretation difficult. Some authors have reported enhanced platelet reactivity and suggested that this could be due to increased lipid peroxides in plasma known to occur in association with oral contraceptive treatment (Wynn et al, 1979, Ciavatti et al, 1989). However the enhanced aggregation was only observed in women consuming polyunsaturated fat-rich diets, and was inhibited by vitamin $E$, glutathione or catalase (Ciavatti and Renaud, 1991).

Estrogen causes changes in the coagulation system (Dugdale and Masi, 1969; Coope et al, 1975; Bonnar et al, 1976). A review by Beller and Ebert (1985) reported that oral contraceptives (especially if the ethinylestradiol concentration was above $50 \mu \mathrm{g}$ ) alter both coagulation factors (increasing fibrinogen, Factor VII, Factor $X$ and reducing antithrombin III activity) and the fibrinolytic system (increasing plasminogen). Coope et al (1975) found similar changes in coagulation factors in menopausal patients prescribed natural estrogens, and Bonnar et al (1976) also reported these changes in menopausal women administered mestranol and norethisterone. Lindberg et al (1989) compared the effects of ethinyl estradiol and the natural estrogen, estradiol valerate, in post menopausal women and found that ethinyl estradiol $(10 \mu \mathrm{g})$ increased the levels of Factor VII : Ag, Factor VIII : C and $\beta$ thromboglobulin while estradiol valerate $(2 \mathrm{mg})$ did not. Both estrogens were 
shown to decrease antithrombin III activity. Hessel (1987) suggested that the increase in coagulation activity was usually balanced by an increase in fibrinolytic activity but in a sub-group of women this counterbalance may be insufficient, resulting in thrombosis.

Beaumont and Beaumont (1986) found that women who had developed CHD while taking the oral contraceptive had a high prevalence of serum antiethinylestradiol antibodies regardless of the concentration of estrogen, which often persisted for up to a year after the oral contraceptive was ceased. Smokers with this antibody were most at risk of CHD. These authors proposed that the resulting immune complexes could damage the vascular endothelium, promoting intimal proliferation, and interfering with coagulation factors (Beaumont and Beaumont, 1987). Interestingly, natural equine estrogens did not induce antiethinylestradiol antibodies (Beaumont and Beaumont, 1987).

In summary, estrogen causes changes which may increase the risk of CHD, including alterations to carbohydrate metabolism and clotting factors, while at the same time increasing HDL and reducing LDL levels which would tend to protect against CHD. Progestins tend to have the opposite effect but this depends on their androgenicity. The net effect is likely to be related to the relative concentration and composition of estrogen and progestin used, other risk factors present and the age group examined.

\subsubsection{Experimental evidence for the involvement of estrogens and progestins} in atherosclerosis

The previous section reviewed clinical and epidemiological studies concerning sex steroids and atherosclerosis. In this section experimental 
studies on the effects of exogenous estrogens and progestins on various aspects of atherogenesis and thrombosis will be reviewed.

Numerous animal studies have shown that estrogen administration tends to result in fewer atherosclerotic lesions (Pick et al, 1952; Prichard et al, 1966; Souadjian et al, 1968; Hanash et al, 1972; Hough and Zilversmit, 1986; Adams et al , 1987; Foegh et al, 1987; Henriksson et al, 1989; Williams et al, 1990). Pick et al (1952a) showed that daily injections of estradiol benzoate (1 $\mathrm{mg}$ / day) to cockerels fed a cholesterol-supplemented diet for up to thirteen weeks resulted in reduced coronary atherosclerosis while the aorta was not protected. Prichard et al (1966) found the same pattern in white Carneau pigeons. Adams et al (1987) found that a persistent elevation of plasma 17 $\beta$ estradiol but not serum progesterone in pregnant monkeys was associated with a reduced extent of coronary artery atherosclerosis when compared to non-pregnant monkeys. Adams et al (1989a; b, 1990) subsequently showed that monkeys fed a moderately atherogenic diet and supplemented with $17 \beta$ estradiol and progesterone or $17 \beta$-estradiol alone (giving rise to an average plasma concentration of $17 \beta$-estradiol of $8.1 \times 10^{-10} \mathrm{M}$ and progesterone of 3 $\left.x 10^{-8} \mathrm{M}\right)$ for 30 months had less coronary artery atherosclerosis than animals given no hormone treatment. Progesterone had no additional effect compared to $17 \beta$-estradiol alone. LDL accumulation in coronary arteries in estrogen treated monkeys was less (Wagner et al, 1989). In contrast, ovariectomy in cynomolgus monkeys resulted in increased coronary artery atherosclerosis (Clarkson et al, 1987). Monkeys given an oral contraceptive (11 $\mu \mathrm{g}$ ethinyl estradiol, $110 \mu \mathrm{g}$ norgestrel) combined with a cholesterolcontaining diet had less plaque development in the coronary arteries than monkeys treated with an intravaginal ring ( $38 \mu \mathrm{g} 17 \beta$-estradiol and $87 \mu \mathrm{g}$ levonorgestrel) or untreated monkeys (Clarkson et al, 1987). This occurred despite the observation that the oral contraceptive lowered HDL-cholesterol (Clarkson et al, 1990). Williams et al (1990) treated ovariectomized monkeys 
on a cholesterol diet with $17 \beta$-estradiol for just over two years, using concentrations in the replacement range (average plasma concentration $9 \times$ 10-10 M), and found that plaque development was less in the estrogentreated monkeys than controls. McGill et al (1977) and Kushwaha et al (1991) however, did not find statistically significant differences in atherosclerotic lesions in the coronary arteries of ovariectomized baboons fed an atherogenic diet and given estrogen (17 $\alpha$-ethinyl estradiol or estrone sulfate) for two years (McGill et al, 1977) or when estrogen alone, progesterone alone, or the combination of these was administered for 18 months (Kushwaha et al, 1991). Kushwaha et al (1991) however found that hormone treatment significantly reduced lesions in the aorta and carotid arteries with the combination of estrogen and progesterone being the most effective in reducing fatty streaks but that progesterone alone increased fatty streak formation in these vessels. The differences in the findings between Kushwaha et al (1991) and McGill et al (1977), and Williams et al (1990) and Adams et al (1990) probably relates to the different types and concentrations of estrogen given. McGill et al (1977) used an oral estrogen with a diet containing $1.5 \mathrm{mg}$ cholesterol / kcal, while Kushwaha et al (1991) used intramuscular estrogen $(100 \mu \mathrm{g} / \mathrm{kg} /$ week) and a similar high fat diet. In contrast, Williams et al (1990) and Adams et al (1990b) used subcutaneous estrogen combined with a cholesterol diet (0.25 mg cholesterol /cal).

Henriksson et al (1989) reported that administration of high concentrations of estrogens to cholesterol-fed male rabbits retarded development of arterial lesions, while Souadjian et al (1968) showed that high concentrations of estrogen ( $0.252 \mathrm{mg} / \mathrm{kg} /$ day) decreased spontaneous aortic atherosclerosis in the young white Carneau pigeon. Low concentrations of estrogen had no statistically significant effect. Prichard et al (1966) observed a decrease in severity of coronary arterial lesions with no effect on aortic atherosclerosis in young male Carneau pigeons fed cholesterol and treated with estradiol 
valerate. Hanash et al (1972) found that a high concentration of estrogen $(0.252 \mathrm{mg} / \mathrm{kg} /$ day $)$ did not alter the size of atherosclerotic lesions in the aorta but it did reduce lipid accumulation in the plaques of mature Carneau pigeons fed a normal diet. There was no difference in the incidence of CHD in the control and treated pigeons. From these studies it would appear that high concentrations of estrogen administered to young pigeons on a normal diet can reduce the extent of atherosclerotic plaques in the aorta, whereas when high concentrations of estrogen are given to adult pigeons there is no regression of established plaques although development of further plaques is prevented.

\subsubsection{Sex steroid receptors and steroid metabolism}

Of potential importance in understanding the relationship between steroid hormones and the development of atherosclerosis has been the detection of steroid receptors in the arterial wall. Estradiol receptors have been detected in rabbit aorta (Fischer et al, 1985b), in baboon cardiac interstitial cells, some endothelial cells (16\%), smooth muscle cells and fibroblasts (McGill and Sheridan, 1981: Lin et al, 1986), rat aortic smooth muscle cells (Harder and Coulson, 1979; Nakao et al, 1981), HUV endothelial cells and rabbit aortic endothelial cells (Colburn and Buonassisi, 1978), bovine aortic endothelial cells (Alexander et al, 1989a) and canine aorta, predominantly on smooth muscle cells (Horwitz and Horwitz, 1982). Cytoplasmic progesterone receptors have been detected in baboon aorta (Lin et al, 1982), on endothelial cells in the thymus (Fujii-Hanamoto et al, 1990) and in $62 \%$ of endothelium of human aorta, internal carotid and coronary arteries (Ingegno et al, 1988). No nuclear accumulation of progesterone has been detected in the wall of blood vessels or in endothelial cells (Stumpf, 1990). Androgen receptors have also been detected in the cardiovascular system (Lin et al, 1981; McGill and Sheridan, 1981). The presence of sex steroid receptors in 
cells of the arterial wall implies a role for sex steroids in the regulation of the function of these cells.

The interaction of steroid hormones with intracellular receptors in target cells can be best explained on the basis of a "two-step mechanism" which has been proposed by Jensen et al (1968) and Gorski et al (1968). According to this concept, target cells for steroid hormones contain specific high affinity receptors called type I sites in the cytoplasm. Steroid hormones diffuse into target cells to bind to these receptors (Wren et al, 1981). Binding of the steroid causes the cytoplasmic receptor to be activated. The estrogenreceptor complex is translocated to the nucleus where it stimulates the transcription of responsive genes by interacting with regulatory DNA sequences. DNA sites which interact with the receptor are called hormone responsive elements. This concept has been demonstrated for estradiol in such tissues as the uterus, pituitary and mammary glands in a variety of species (Jensen and DeSombre, 1973). The regulated genes include enzymes, secretory products, and structural proteins (Chan et al, 1976; Mulvihill and Palmiter, 1980), as well as growth factors (Dickson et al, 1986b; Walker et al 1981; Wiseman et al, 1986; Dickson et al, 1988) and protooncogenes (Norris et al, 1984; Murphy et al, 1987, Fink et al, 1988; Weisz et al, 1988a; b). A second class of nuclear and cytoplasmic estrogen binding sites (called type II) has been reported. The cytoplasmic type II sites are of lower affinity and remain in the cytoplasm (Katzenellenbogen, 1980). The type II nuclear binding sites have a lower affinity for estradiol than type I but are present at a higher concentration and may be occupied when pharmacological concentrations of estradiol are used (Katzenellenbogen, 1980). Yamamoto (1985) found that steroid hormone binding and activation results in a greater than 10-fold increase in affinity for nonspecific DNA sequences compared to specific DNA sequences, which makes detection of specific DNA binding sites difficult. 
Receptors for glucocorticoid and mineralocorticoid hormones, progesterone, and androgens are closely related to each other in structure and function and differ from estrogen receptors (Kooistra et al, 1990). Estrogen and progesterone have both been shown to regulate the levels of each other's receptors (Kooistra et al, 1990). Estrogen has been shown to increase the concentration of progesterone receptors in the uterus and breast while progesterone has been shown to reduce estrogen receptor concentration in these tissues (Katzenellenbogen and Norman, 1990). Lin et al (1986) showed that the treatment of ovariectomized baboons with $17 \beta$ estradiol resulted in a redistribution of arterial intracellular estrogen receptors from the cytoplasmic fraction to the nuclear fraction, and an increase in the cytoplasmic concentration of progesterone receptors. Horwitz and Horwitz (1982) reported that progesterone receptors could be induced in dogs by treatment for one week with estradiol $\left(3.7 \times 10^{-10} \mathrm{M}\right)$. The net effect (either synergism or antagonism), is determined by the estrogen : progesterone ratio (Katzenellenbogen, 1980). Colburn and Buonassisi (1978) have shown that progesterone, and to a lesser extent testosterone, appeared to compete with estradiol for the binding site on HUV endothelial cells when the experiments were performed by labelling the receptor in the intact cell but not after extraction of receptor from the cell (Colburn and Buonassisi, 1978). Buonassisi and Colburn (1980) suggested that the apparent competition in the intact cell may be due to conversion of progesterone and testosterone to a derivative which can compete with estradiol for the receptor, or by competition for transport into the cell. In contrast, Nakao et al (1981) showed that estradiol binding in smooth muscle cells exhibited a high degree of specificity and that progesterone had no affinity for estradiol binding sites. Progesterone has been shown to control the proliferative response of estrogen in the endometrium by initiating the production of two enyzmes, estradiol dehydrogenase and estrogen sulfotransferase (Wren et al, 1981). 
Estradiol dehydrogenase converts estradiol to the less active estrone while estrogen transferase conjugates estrogens to estrogen sulphates which are cellularly inactive (Wren et al, 1981).

When steroid hormones are present at physiological levels their interaction is generally restricted to the specific receptor protein for that class of hormone. However, when hormones are administered in pharmacological concentrations they may interact with another receptor (Katzenellenbogen, 1980). For example, a pharmacological concentration of androgen (10-6 M) has been shown to deplete both androgen and estrogen receptors, increase MCF-7 cell growth, and stimulate synthesis of progesterone receptors, while androgens at a lower concentration (10-8 M) deplete only androgen receptors and do not stimulate growth (Zava and McGuire, 1978).

Steroid metabolizing enzymes are present in endothelial cells. These include $5 \alpha$-reductase, 17 $\beta$-hydroxysteroid oxidreductase, $3 \alpha$-hydroxysteroid oxidreductase and $3 \beta$-hydroxysteroid oxidoreductase (Milewich et al, 1989). Seillan et al (1983) observed that testosterone was largely converted to estrogens by female aortic endothelial cells. HUV endothelial cells have been shown to metabolise radiolabelled progesterone and androstenedione via steroid $5 \alpha$-reductase. Estrogens (17 $\beta$-estradiol and estrone) however, were not detected as products of androstenedione metabolism (Milewich et al, 1987). In post-menopausal women, estrogen is derived mainly from plasma 4-androstenedione as a result of extraglandular activity. 4-androstenedione is formed from progesterone or 17-hydroxyprogesterone and is an important intermediate in testosterone biosynthesis (Gower, 1988). Longcope et al (1969) demonstrated that androgens such as testosterone are converted to estrone and 17k-estradiol in the blood of men and women and that the estrogens, estrone and $17 \beta$-estradiol are interconvertible. 
In summary, endothelial cells possess both sex steroid receptors and steroid metabolizing enzymes. This needs to be considered when evaluating experimental data in relation to the effects of sex steroids on endothelial cell function. The sex steroid level in serum may be only a guide to the concentration of sex steroids to which individual cells may be exposed.

\subsubsection{Effects of estrogens and progestins on endothelial cell proliferation and angiogenesis}

Sex steroids can influence endothelial cell proliferation. Harrison and McKee (1984a) have shown that $17 \beta$-estradiol $\left(7.3 \times 10^{-9} \mathrm{M}\right)$ increased the rate of HUV endothelial cell proliferation over three days in the presence of 2 \% FCS, assessed by an increase in DNA content. Johannisson (1986) also found that 17 -estradiol (3.7-14.7 $\left.\times 10^{-8} \mathrm{M}\right)$ stimulated DNA synthesis in human endometrial endothelial cells whereas progesterone $\left(1.6-6.4 \times 10^{-8} \mathrm{M}\right)$ decreased DNA synthesis in the presence of $20 \% \mathrm{FCS}$ as assessed by [ $\left.{ }^{3} \mathrm{H}\right]-$ thymidine incorporation over six days. Corvazier et al (1984) however found that $17 \beta$-estradiol $\left(10^{-8} \mathrm{M}\right)$ and progesterone $\left(10^{-8} \mathrm{M}\right)$, in the absence of serum, had no effect on HUV endothelial cell proliferation over three days. Streeten et al (1989) found that clonal endothelial cells from fetal bovine bone proliferated in the presence of progesterone $\left(24-100 \times 10^{-12} \mathrm{M}\right)$ but not estradiol ( $\left.1 \times 10^{-12} \mathrm{M}-1 \times 10^{-6} \mathrm{M}\right)$, in the absence of serum.

$17 \alpha$-hydroxyprogesterone and hydrocortisone have been shown to greatly inhibit angiogenesis in the presence of heparin (Crum et al, 1985) while testosterone and estrone have only minimal anti-angiogenic activity and progesterone had no influence on angiogenesis. Progesterone, estradiol, estriol and testosterone have been shown to have no effect on endothelial cell morphology (Maca et al, 1978). However, pharmacological concentrations of 
ethinylestradiol administered to female rats have been shown to increase aortic permeability, possibly due to changes in the intercellular cement (Almen et al, 1975).

In summary, in the presence of serum, $17 \beta$-estradiol $\left(10^{-7}-10^{-8} \mathrm{M}\right)$ has been shown to enhance endothelial cell proliferation in some cultures while progesterone $\left(10^{-8} \mathrm{M}\right)$ decreases endothelial cell proliferation, but not in a serum free medium. Progesterone $\left(10^{-9} \mathrm{M}\right)$ may cause endothelial cells to proliferate in the absence of serum.

\subsubsection{Influence of estrogens and progestins on platelets and thrombosis}

Estrogen treatment has been shown to inhibit platelet aggregation in some studies (Mitchell, 1974; Johnson et al, 1977; Subbiah et al, 1981), increase platelet aggregation in other studies (Elam et al, 1980), while having no significant effect on platelet aggregation in the remaining studies (Coope et al, 1975; Subbiah et al, 1981). These differences appear to be due to the type of estrogen tested, the experimental design, and in particular the agonist used to induce platelet aggregation. For example, Emms and Lewis (1985) found that treatment of rats for 6 weeks with $17 \beta$-estradiol resulted in decreased platelet aggregation induced by collagen but not ADP, arachidonic acid or $\mathrm{PGI}_{2}$. Mitchell (1974) showed in rabbits that estrogens (diethylstilbestrol, equine estrogens and estradiol cypionate) had a depressive effect on platelet aggregation in response to ADP, while Subbiah et al (1981) showed that 17 $\beta$ estradiol decreased platelet aggregation in response to arachidonic acid in female pigeons. However, collagen-induced aggregation of platelets was not affected, nor was platelet adhesiveness tested with a platelet adhesive device (Subbiah et al, 1981). 
A similar range of results has been demonstrated for progesterone. Some studies have shown that progesterone causes decreased platelet aggregration in vitro (Johnson et al, 1977), with no effect in a rabbit model (Mitchell, 1974) or in vitro (Subbiah et al, 1981), or in women prescribed the progesterone-only pill (Poller et al, 1969). The oral contraceptive has been shown not to alter platelet aggregration in some studies (Shaude et al, 1978; Elan et al, 1980), while other studies (Poller et al, 1969; Sartwell et al, 1969) have shown enhanced platelet aggregation. Ciavatti et al (1987) found that thrombin-induced platelet aggregation was increased when female rats were fed a combination of a high polyunsaturated diet and an oral contraceptive. In a later study (Ciavatti et al, 1989) they found that the enhanced response of platelets could be inhibited by adding antioxidants and concluded that in rats oral contraceptive treatment increased lipid peroxides which stimulated platelets. Elam et al (1980) reported increased in vivo platelet aggregation in rabbits following administration for 7-14 days of ethinyl estradiol, which was inhibited by the progestin, norethindrone. They proposed that the increase in aggregation was due to a reduction $\mathrm{PGI}_{2}$-like formation in steroid-treated rabbits.

\subsection{Effects of sex hormones on $\mathrm{PGI}_{2}$ production}

$\mathrm{PGI}_{2}$ is an important inhibitor of platelet aggregation and a potent vasodilator. $\mathrm{PGI}_{2}$ inhibits platelet aggregation by stimulating adenylate cyclase, leading to an increase in cAMP levels in platelets (Vane et al, 1982). The effect of sex steroids on $\mathrm{PGI}_{2}$ production and release is controversial. Karpati et al (1980) found that in rats injected with ethinyl estradiol (10 $\mu \mathrm{g}$ $/ 100 \mathrm{~g})$ and ethinyl estradiol-norethisterone for 30 days there was a significant increase in $\mathrm{PGI}_{2}$-like activity suggesting that sex steroids may inhibit platelet aggregation and protect against atherosclerosis. Similar observations were made by Roncaglioni et al (1979) in the rat. Subbiah et al 
(1981) observed that injections of $17 \beta$-estradiol increased the synthesis of $\mathrm{PGI}_{2}$ in the aorta of female pigeons. Seillan et al (1983) found that the addition of $17 \beta$-estradiol $\left(10^{-8} \mathrm{M}\right)$ or testosterone $\left(10^{-8} \mathrm{M}\right)$ to female piglet aortas induced marked stimulation of $\mathrm{PGl}_{2}$ after $72 \mathrm{~h}$ of treatment. No change in $\mathrm{PGI}_{2}$ release was observed in male piglets. In apparent contrast, Pomerantz et al (1980) found that ovariectomized rats released six-fold more 6 -keto-PGF ${ }_{1 \alpha}$ than intact female rats and that $17 \beta$-estradiol $(100 \mu \mathrm{g} / \mathrm{kg})$ suppressed by $50 \%$ the increase in 6-keto-PGF $1 \alpha$. Progesterone elevated 6keto-PGF ${ }_{1 \alpha}$ while testosterone had no effect. Subbiah (1981) demonstrated in female pigeons that testosterone increased $\mathrm{PGl}_{2}$ synthesis. Elan et al (1980) reported that $\mathrm{PGI}_{2}$ like activity was decreased in aortas of both ethinyl estradiol treated and ethinyl estradiol-norethisterone treated rabbits compared to controls, supporting the findings of Pomerantz et al (1980). Fogelberg et al (1990) showed that in cholesterol-fed rabbits treated with pharmacological doses of estrogen (50 $\mu \mathrm{g}$ ethinylestradiol /day and $10 \mathrm{mg}$ polyestradiol phosphate /week), there was an increase in $\mathrm{PGI}_{2}$ production and a decrease in thromboxane in urine. The ratio of in vivo $\mathrm{PGI}_{2}$ to thromboxane formation increased 2-3 fold during estrogen therapy. However, Fogelberg et al (1990) were unable to demonstrate these changes under in vitro conditions. This was probably due to the presence of other factors in vivo such as transcortin which is known to increase the availability of arachidonic acid for $\mathrm{PGI}_{2}$ production (David et al, 1989). Furthermore, in vivo, there are sources of endoperoxides other than the endothelial cell, for example, platelets and smooth muscle cells. Thus, $P G I_{2}$ synthesis in vivo is a complex process. The above observations appear to depend on the gender of the animal, the route of administration of the sex steroid and the animal model used.

Chang et al (1981) observed in cultured rat aortic smooth muscle cells that estradiol cypionate $\left(10^{-9} \mathrm{M}\right)$ caused marked stimulation of $\mathrm{PGI}_{2}$ biosynthetic 
activity which was maximal after five days of treatment. They showed that estradiol stimulated fatty acid cyclooxygenase and $\mathrm{PGI}_{2}$ synthetase activity (Chang et al, 1980b; 1981) probably by binding to estradiol receptors (Nakao et al, 1981). Chang et al (1981) also reported that estradiol did not affect thromboxane biosynthesis in platelets. Shakhov et al (1988) found that in rabbit and human smooth muscle cells coincubated with lipoproteins, estradiol $\left(10^{-7} \mathrm{M}\right)$ stimulated the release of $\mathrm{PGI}_{2}$ while progesterone $\left(10^{-7} \mathrm{M}\right)$ inhibited $\mathrm{PGI}_{2}$ release. Wakasugi et al (1989) observed increased $\mathrm{PGI}_{2}$ synthesis using aortic preparations from rats following estradiol $\left(10^{-7}, 10^{-9} \mathrm{M}\right)$ treatment, whereas aortas from testosterone treated females exhibited reduced $\mathrm{PGI}_{2}$ synthesis. Testosterone $\left(10^{-10}-10^{-6} \mathrm{M}\right)$ has also been shown to inhibit $\mathrm{PGI}_{2}$ production in rat aortic smooth muscle cells over 5 days (Nakao et al, 1981b). In conclusion, estradiol appears to stimulate $\mathrm{PGI}_{2}$ release while progesterone and testosterone inhibit $\mathrm{PGI}_{2}$ release in cultured smooth muscle cells.

In HUV endothelial cells, Needleman and Parks (1982) demonstrated that preincubation with estradiol $\left(20 \times 10^{-6} \mathrm{M}\right)$ and one of its metabolites, 2hydroxyestradiol $\left(20 \times 10^{-6} \mathrm{M}\right)$, inhibited $\mathrm{PGI}_{2}$ release caused by bradykinin, arachidonic acid and calcium ionophore. However, when thrombin was used as a stimulus, estradiol enhanced and 2-hydroxyestradiol inhibited $\mathrm{PGI}_{2}$ formation. Preincubation of HUV endothelial cells with lower (nanomolar) concentrations of estradiol did not alter $\mathrm{PGI}_{2}$ release (Needleman and Parks, 1982). Corvazier et al (1984) found that $17 \beta$-estradiol $\left(10^{-8} \mathrm{M}\right)$ did not alter $\mathrm{PGI}_{2}$ release after 3 days following stimulation with the calcium ionophore A23187. Witter and DiBlasi (1984) found that when HUV endothelial cells were incubated with physiological concentrations of ethinyl estradiol, progesterone or testosterone for $30 \mathrm{~min}$ at $37^{\circ} \mathrm{C}$ there was no significant change in the production of 6-keto-PGF ${ }_{1 \alpha}$. Lewis et al (1986) also found that preincubation of HUV endothelial cells with progesterone $\left(10^{-6} \mathrm{M}\right.$ or $\left.10^{-10} \mathrm{M}\right)$ 
for 2-48 $\mathrm{h}$ did not alter histamine stimulated $\mathrm{PGI}_{2}$ release. Berge et al (1990) found that when HUV endothelial cells were preincubated with $17 \beta$-estradiol (7.3-36.7 $\left.\times 10^{-10} \mathrm{M}\right)$ and the combination of $17 \beta$-estradiol and progesterone (3.2-16 $\left.\times 10^{-8} \mathrm{M}\right)$, there was an inhibition of $\mathrm{PGI}_{2}$ release caused by thrombin. David et al (1989) demonstrated that when HUV endothelial cells were stimulated with adenosine triphospate $(1 \mathrm{mg} / \mathrm{ml}), 17 \alpha$-ethinyl estradiol (30 $\eta \mathrm{g}$ $/ \mathrm{ml}$ ) decreased $\mathrm{PGI}_{2}$-synthesis by $11 \%$ and $\mathrm{PGI}_{2}$-release by $32 \%$. Lewis et al (1986) found that progesterone and testosterone did not alter $\mathrm{PGI}_{2}$ release from HUV endothelial cells that had been stimulated with histamine, bradykinin, calcium ionophore or mechanical agitation. Makila et al (1982) found that exposure to $17 \beta$-estradiol $\left(3.7-37 \times 10^{-6} \mathrm{M}\right.$ ) stimulated $\mathrm{PGI}_{2}$ formation in umbilical cords superfused with Eagle's Medium but had no effect on thromboxane $A_{2}$ generation. Progesterone (1.6-16 $\times 10^{-6} \mathrm{M}$ ) caused no change in $\mathrm{PGl}_{2}$ or thromboxane $\mathrm{A}_{2}$ generation (Makila et al, 1982). However, the addition of progesterone abolished the stimulatory effect of estradiol (Makila et al, 1982). In summary, it seems that estradiol alone can influence $\mathrm{PGI}_{2}$ release from HUV endothelial cells in vitro while progesterone can influence the estrogen response. The response is dependent on the concentration of estradiol and the agonist used.

Ylikorkala et al (1982) found that prolonged use of oral contraceptives (1.5 $\mathrm{mg}$ estradiol with $0.150 \mathrm{mg}$ desogestrel) in women led to decreased serum levels of $\mathrm{PGI}_{2}$ while progestin-only pills had no suppressive effects. Postmenopausal therapy using estradiol $(1.5 \mathrm{mg})$ and desogestrel $(0.150 \mathrm{mg})$ however, did not alter $\mathrm{PGI}_{2}$ excretion (Ylikorkala et al, 1990). Hull et al (1991) showed that conjugated estrogen $(0.6 \mathrm{mg} / \mathrm{kg} /$ day $)$ given to men significantly reduced endothelial cell $\mathrm{PGI}_{2}$ production.

In conclusion, the ability of estrogen and progestin to affect $\mathrm{PGI}_{2}$ metabolism may vary with the type and combination of estrogen and 
progestin and the concentration, as well as the cell or species studied. In the endothelial cell and in humans there is a tendency for estrogen to reduce $\mathrm{PGI}_{2}$ production, while in the intact rat or rabbit production of $\mathrm{PGI}_{2}$ is increased when they are injected with or fed estrogen.

\subsection{Effects of sex hormones on vWf production and release}

VWf is important in platelet binding to the subendothelium. Pregnancy and the use of oral contraceptives have been associated with elevated levels of vWf (Schiffman and Rapaport, 1966; Crowell et al, 1971; Leone et al, 1975). Estrogen in vivo has been shown to increase vWf production (Alperin, 1982). Harrison et al (1981b) reported that when HUV endothelial cells were treated with $17 \beta$-estradiol $\left(1.83-7.34 \times 10^{-9} \mathrm{M}\right)$ there were increased levels of $\mathrm{VWf}$ in media as assessed by ristocetin-induced platelet aggregation, radioreceptor platelet assay and DNA content (Harrison et al, 1984a). Johannisson (1986) reported that $17 \beta$-estradiol $\left(3.7 \times 10^{-8} \mathrm{M}\right)$ increased FVIII immunofluorescence intensity in endometrial endothelial cells whereas progesterone (1.6$6.35 \times 10^{-8} \mathrm{M}$ ) failed to influence fluorescence. This observation suggests that $17 \beta$-estradiol (at these concentrations) may be prothrombotic by increasing endothelial expression and release of vWf.

\subsubsection{Influence of estrogens and progestins on release of growth factors}

Steroid hormones regulate expression of genes in target cells at the level of transcription (Fink et al, 1988) by binding to specific intracellular receptors. Estrogen has been shown to regulate the release of growth factors such as transforming growth factor- $\alpha$-like substances, insulin-like growth factor-1related material, epidermal growth factor, transforming growth factor- $\beta$ and PDGF from breast cancer cell lines (Dickson et al, 1986a; b; Walker et al 1981; Wiseman et al, 1986; Dickson et al, 1987; 1988). Bronzert et al (1987) 
also showed that $17 \beta$-estradiol $\left(10^{-8} \mathrm{M}\right)$ can increase PDGF release from endothelial cells in gliomas. The secretion of the cytokines interferon- $\beta_{2}$ (IFN$\beta_{2}$ )/IL-6 by human endometrial stromal cells has been shown to be inhibited by $17 \beta$-estradiol $\left(1 \times 10^{-9} \mathrm{M}\right)$ in serum-free conditions (Tabibzadeh et al, 1989) while the secretion of insulin-like growth factor-1 is increased by $17 \beta-$ estradiol $\left(1 \times 10^{-9} \mathrm{M}\right)$ in some osteoblasts but not others (Schmid et al, 1989).

Barak et al (1986) found that pretreatment of progenitor cells with estradiol results in an increase in granulocyte-macrophage colonies in response to colony-stimulating factor. Estradiol did not act synergistically with colonystimulating factor nor did it induce colony-stimulating factor secretion. They concluded that estradiol augmented the effect of colony-stimulating factor on granulocyte-macrophage colonies. Gaunt and Pierce (1986) also found that estradiol sulfate did not alter the in vitro growth of granulocyte-macrophage colonies while in $17 \beta$-estradiol treated mice the number of granulocytemacrophage colonies in the humoral marrow decreased (Gaunt and Pierce, 1985). Maoz et al (1985) found that estradiol, but not progesterone, increased monomyelocytic colony formation in vitro, and tamoxifen (an antiestrogen) did not inhibit this.

Many of these cytokines have been shown to modulate endothelial cell functions. For example, G-CSF and GM-CSF induce endothelial cells to migrate and proliferate (Bussolino et al, 1989a), and some of these cytokines are produced by endothelial cells. These include IL-6 and GM-CSF (Mantovani and Dejana, 1989). This suggests another potential role for sex steroids in atherosclerosis through effects on cytokine release from cells associated with atherogenesis. 


\subsubsection{Effects of estrogens and progestins on monocyte function}

Danel et al (1983) reported that estrogen binds to human monocytes. They demonstrated a single class of receptor for estradiol. Gulshan et al (1990) showed that a human monocyte leukaemic-derived cell line had two estrogen receptors but that there was no binding of monoclonal antibodies to the classical estrogen receptor.

Estrogens have been shown to have numerous effects on macrophages. They increase phagocytic activity (Vernon-Roberts, 1969; Gotzos et al, 1981), increase or decrease production of IL-1 depending on concentration (Flynn, 1984; 1986; Polan et al, 1988; Hu et al, 1988; Stock et al, 1989), increase the number of myelomonocytic colonies in human blood (Barak et al, 1986) and stimulate monocyte proliferation (Gotzos et al, 1981). Testosterone has not been reported to have these effects Nernon-Roberts, 1969; Flynn, 1984, 1886). Progesterone does not inhibit increased phagocytic activity of macrophages induced by $17 \beta$-estradiol (Nernon-Robert, 1969). Indeed, progesterone mildly stimulates phagocytosis by sinusoidal macrophages (Vernon-Roberts, 1969). Polan et al (1988) showed that estradiol (10-9 M-10$10 \mathrm{M}$ ) and progesterone $\left(10^{-8} \mathrm{M}-10^{-9} \mathrm{M}\right)$ resulted in significant stimulation of IL-1 secretion from human peripheral monocytes, while estradiol $\left(10^{-7} \mathrm{M}\right)$ and progesterone (10-7 M-10-5 M) reduced IL-1 secretion. Polan et al (1989) subsequently demonstrated that estradiol $\left(<10^{-5} \mathrm{M}\right.$ ) and progesterone (< $10^{-5} \mathrm{M}$ ) altered IL-1 $\beta$ levels by altering its mRNA. Pacifici et al (1989) demonstrated that treating post menopausal women with estrogen and progesterone decreased IL-1 activity in their blood monocytes. Hu et al (1988) found that the combination of estradiol and lipopolysaccharide in vitro enhanced secretion of IL-1 by macrophages even more than estradiol alone. Pfeifer and Patterson (1985) demonstrated that $17 \beta$-estradiol and its major 
metabolite activate macrophages (measured in terms of macrophage cytostasis against tumour cells) when macrophages are induced by $T$ cell lymphokine.

In summary, estradiol and progesterone appear to influence monocyte function although the effects are diverse and depend on the concentration, type and combination of sex hormones. Sex hormones have the potential to modulate functions of monocytes which may influence atherogenesis.

\subsubsection{Influence of estrogens and progestins on fatty streak formation}

It has been shown that when cholesterol-fed rabbits are administered 17estradiol cypionate, development of arterial lesions is retarded despite there being no change in plasma cholesterol concentration (Hough and Zilversmit, 1986). In this study cholesteryl ester influx into the aortic wall, and metabolism of the ester, were lower in estrogen-treated rabbits compared with control animals. Haarbo et al (1991b) examined the effects of $17 \beta-$ estradiol and $17 \beta$-estradiol in combination with norethisterone acetate or levonorgestrel in rabbits and found no significant difference in aortic accumulation of cholesterol between hormone groups but less plaque development than in an untreated group. Kushwaha et al (1991) found in ovariectomized monkeys that the combination of estrogen and progesterone resulted in fewer fatty streaks, while progesterone treated monkeys had the most developed fatty streaks. They suggested that these differences were due to the effects of sex hormones on plasma lipoproteins since progesterone increased VLDL and LDL-cholesterol levels while VLDL and the LDL / HDL-cholesterol ratio were lower in the monkeys administered estrogen and progesterone than in the monkeys administered progesterone alone. Henriksson et al (1989) also demonstrated that estrogen reduced plasma VLDL and increased plasma HDL-cholesterol levels in cholesterol-fed rabbits. 
Further investigations have shown that sex steroids may alter the cellular uptake of LDL by changing LDL receptor density and affinity. In vitro studies by Alexander et al (1989a) showed that estradiol $\left(1.8 \times 10^{-10} \mathrm{M}\right)$ does not affect the surface binding of LDL to cultured bovine aortic endothelial cells but enhances LDL uptake (25 $\mu \mathrm{g}$ protein $/ \mathrm{ml}$ ) without affecting its degradation. Estrogen-treated endothelial cells have been shown to have a greater binding capacity and a lower binding affinity for LDL (Alexander et al, 1989b). Dexamethasone, but not progesterone $\left(2.5 \times 10^{-8} \mathrm{M}\right)$, inhibited LDL receptor activity by both HUV endothelial cells and macrophages (Hirsch and Mazzone, 1986). Dexamethasone $\left(2.5 \times 10^{-8} \mathrm{M}\right)$ however increased acetylated-LDL degradation in macrophages but not in endothelial cells (Hirsch and Mazzone, 1986). These effects were time and concentration dependent. Maziere et al (1991) found that estradiol (5-50 x 10-6 M) could inhibit LDL degradation by $J 774$ macrophage-like cells. Corsini et al (1988) showed that progesterone increases HDL binding to human skin fibroblasts in the absence of a cholesterol load, in a concentration dependent manner up to $10^{-4} \mathrm{M}$ by inhibiting the acyl-CoA cholesterol acyltransferase activity of the cells (Schmitz et al, 1985).

Estrogen has also been shown to have antioxidant properties which may be important in inhibiting oxidation of LDL (refer to Section 1.3.2). Sugioka et al (1987) showed that estradiol $\left(5-30 \times 10^{-6} \mathrm{M}\right)$, estrone and estriol but not testosterone inhibit lipid peroxidation of liposomes as assessed by fatty acid composition and oxygen consumption using an Fe $3+$-ADP-adriamycin complex system. This has been supported by Maziere et al (1991) and Adelman et al (1991), the former finding that estradiol and to a lesser extent estrone and estriol $\left(5-50 \times 10^{-6} \mathrm{M}\right)$ inhibited copper and cellular induced modification of LDL over $24 \mathrm{~h}$ as assessed by production of thiobarbituric acid reactive substances (TBARS). Testosterone (Maziere et al, 1991) and 
progestin (Adelman et al, 1991) were shown to have no inhibitory effect on LDL oxidation. Huber et al (1990) showed that oxidation of LDL in the presence of copper sulphate could be inhibited for up to 80 minutes in the presence of $17 \beta$-estradiol $\left(2.5 \times 10^{-6} \mathrm{M}\right)$ but not testosterone by examining the appearance of conjugated dienes at $234 \mathrm{~nm}$ and the formation of fluorochromic lipid-protein conjugates. They found that $17 \beta$-estradiol appeared to be more potent than probucol (an antioxidant) at low molar ratios in inhibiting the formation of cholesteryl esters in macrophages. Nakano et al (1987) found that 2-hydroxyestradiol and 2-hydroxyestrone (major metabolites of estradiol and estrone) suppress lipid peroxidation even more avidily than estrone and estradiol as assessed by oxygen consumption. However when 2-hydroxyestradiol was depleted, oxidation proceeded at the same rate as in the absence of antioxidant (Niki and Nakano, 1990). Yagi and Komura (1986) showed that estradiol, estrone, and estriol suppressed the peroxidation of methyl linoleate caused by UV irradiation.

Estrogens may act as a chain breaking antioxidant like vitamin $E$ (refer equation in Section 6.1.5) (Niki and Nakano, 1990), although the mode of inhibition is different since the lag phase before achieving maximal inhibition of oxidation is much less for estrogens than for vitamin E (Sugioka et al, 1987). Estrogens contain a phenolic structure. Such aromatic compounds are known to react rapidly with hydroxyl radicals (Halliwell and Gutteridge, 1986) to produce a mixture of hydroxylated products. Estradiol has also been shown to bind to the core components of lipoproteins, particularly $\mathrm{HDL}_{3}$ and form esters (Leszczynski and Scafer, 1991). This may explain some of the observed antioxidant effects of HDL.

In summary, the observed reduction in fatty streak formation with estrogen treatment may be due, in part, to changes in transport and metabolism of 
LDL, and to inhibition of oxidative modification of LDL in addition to changes in serum lipid levels as outlined in Section 1.5.1.

\subsubsection{Influence of estrogens and progestins on fibrous plaque formation}

Fischer and Swain (1977) showed that in castrated male rats administered estrogen, total collagen, the percentage of collagen, total elastin, and the collagen /elastin ratio were significantly lower, and that the elastin content was increased when compared to rats receiving testosterone. In noncastrated male rats, administration of estrogen had the same effect. Fischer (1972) has also shown that in ovariectomized female rats the administration of estradiol reduces the accumulation of both collagen and elastin in aorta. These observations have been confirmed by Wolinsky (1973). This study was subsequently repeated in ovariectomized rabbits (Fischer et al, 1985a) and again there was a reduction in collagen synthesis. Fischer et al (1985b) also showed that female rabbits fed an atherogenic diet treated with progesterone for 10 weeks developed more atherosclerosis and a greater accumulation of collagen and elastin in the aorta than control animals not treated with progesterone. Fischer et al (1981) have also shown that in female rabbits fed an atherogenic diet and treated with an oral contraceptive (mestranol-norethynodrel), a lower rate of collagen synthesis occurs, with less deposition of cholesterol in the aorta (Beledekas et al, 1979). These changes in collagen synthesis associated with sex steroid treatment have not been shown in humans (Levene and Poole, 1962).

Pharmacological concentrations of estradiol have also been reported to lead to a lipoprotein-induced arterial smooth muscle cell proliferation in vitro (Rhee et al, 1978; Fischer-Dzoga et al, 1983), and inhibition of myointimal proliferation associated with mechanical endothelial injury (Rhee et al, 1974; Drouet et al, 1978; Weigensberg et al, 1984). Belbekas et al (1974) found 
that $17 \beta$-estradiol $\left(10^{-5} \mathrm{M}-10^{-12} \mathrm{M}\right.$ ) inhibited synthesis of collagen in cultured smooth muscle cells.

Thus, the evidence suggests that sex steroids can modify potentially important reactions in fibrous plaque formation.

\subsection{SUMMARY}

Differences in the established cardiovascular risk factors can only partly explain the sex differences in CHD (McGill and Stern, 1979; Kalin and Zumoff, 1990). Although there is no direct proof that circulating estrogens, progestins or androgens at physiological levels can either accelerate or inhibit atherosclerosis (McGill and Stern, 1979), there is ample evidence that sex hormones profoundly influence biochemical and cellular events leading to atherosclerosis. It is probable that sex steroids act at many different sites, with different effects depending on the type and concentration of sex steroid, and interaction with other factors. The role of exogenous sex steroids in preventing atherosclerosis, and the mechanism for such an effect, is still being evaluated.

In this thesis human umbilical vein (HUV) endothelial cells have been used as a model to further examine possible ways in which $17 \beta$-estradiol and progesterone alter endothelial cell function and related aspects which may be of importance in the pathogenesis of atherosclerosis. 


\section{CHAPTER 2}

CULTURE AND CHARACTERIZATION OF HUMAN UMBILICAL VEIN ENDOTHELIAL CELLS 


\subsection{INTRODUCTION}

The human umbilical vein (HUV) endothelial cell has been chosen as an endothelial cell model for the studies reported in this thesis and in this Chapter the methodology for the isolation, culture and characterization of endothelial cells and the rationale for using this model are reviewed. The reasons for using this model are discussed later in the Chapter.

\subsubsection{Isolation and culture of endothelial cells}

Endothelial cells were first isolated from umbilical cord veins by Maruyama (1963). The vein was perfused with a trypsin solution and isolated cells formed monolayers in cell culture for up to 14 days but did not replicate and had degenerated by 21 days. Fryer et al (1966), using Maruyama's method, obtained endothelial cells from umbilical arteries. These cultures were rapidly overgrown by spindle-shaped contaminant cells and the endothelial cells did not replicate. Jaffe extensively modified these techniques and was able to isolate umbilical cord vein endothelial cells that could be passaged (Jaffe et al, 1972a; b; 1973; Jaffe, 1984). This work forms the basis of the technique used in the studies reported in this thesis. In the original description of the modified technique, Jaffe perfused undamaged veins with collagenase, instead of trypsin, for 15 min (Jaffe et al, 1972a; b; 1973a) and collected the collagenase perfusate containing the endothelial cells in a tube with fetal calf serum (FCS). He originally cultured the cells in Medium 199 with $20 \%$ FCS in petri dishes. The endothelial cells were passaged by incubating them with 0.1 \% collagenase- $0.01 \%$ ethylenediamine tetraacetic acid (EDTA) for $5 \mathrm{~min}$ at 37 ${ }^{\circ} \mathrm{C}$. Jaffe has refined the technique over the last 12 years. In Chapter 2 of "The Biology of Endothelial Cells" (1984), he describes perfusing the umbilical cord for only 10 min with collagenase, culturing endothelial cells on $0.2 \%$ 
gelatin coated flasks, passaging endothelial cells with $0.1 \%$ collagenase- 0.01 \% EDTA-0.25 \% bovine serum albumin, supplementing cultures with endothelial cell growth supplement (ECGS) from bovine brain, and culturing cells using $20 \%$ pooled human serum instead of fetal calf serum. His culture technique was modified by Gimbrone et al (1974) who used $0.25 \%$ trypsin$0.05 \%$ EDTA in modified Puck 's saline for 2-3 min to passage cells.

\subsubsection{Factors affecting endothelial cell proliferation}

The terms proliferation and cell growth are often used interchangeably, growth indicating cell division and thus proliferation. Wall et al (1978) and Jaffe (1984) showed that proliferation of preconfluent endothelial cells was directly related to the concentration of whole blood serum or cell free plasmaderived serum and was maximal at $35-50 \%$. Once confluent, endothelial cells stop dividing and do not synthesize DNA in response to blood serum or mitogens (Gimbrone et al, 1974; Haudenschild et al, 1976; Wall et al, 1978; Thorgeirsson et al, 1979). Low density HUV endothelial cells do not proliferate in the presence of serum alone suggesting that the mitogenic components present in serum are not sufficient for optimal HUV endothelial cell growth (Gimbrone et al, 1974; Haudenschild et al, 1976; Maciag et al, 1984). It was found that human vascular endothelial cells can be grown only at very high cell densities for short periods of time in serum-containing media (Jaffe et al, 1973a; Gimbrone et al, 1974; Gimbrone and Cotran, 1975; Gimbrone, 1976).

Using Jaffe's technique, large scale long term culture of HUV endothelial cells was impractical until Maciag et al (1979) showed that extracts of bovine hypothalamus prepared at neutral $\mathrm{pH}$ stimulated HUV endothelial cell growth in vitro. This represented the discovery of a new growth factor, which was named endothelial cell growth factor (ECGF), and is now included under the 
category of aFGF (Joseph-Silverstein and Rifkin, 1987). Maciag et al (1982) showed that there are two forms of ECGF found in the bovine hypothalamus, a high molecular weight form (greater than 70000 daltons), and a low molecular weight form (17 000-20 000 daltons). The high molecular weight form can be converted into the low molecular weight form by mild treatment with acetic acid (Maciag et al, 1982; Burgess et al, 1986). The two polypeptides of ECGF are called $\alpha$-ECGF (17000 MW) and $\beta$-ECGF (20 000 MW) (Burgess et al, 1985). They are acid and heat labile proteins (Maciag et al, 1979). The mitogenic effect of ECGF is independent of HUV endothelial cell plating density and acts in a synergistic manner with the mitogenic components present in serum (Maciag et al, 1979), thus allowing endothelial cells to be cultured at low plating densities. ECGF is mitogenic for HUV endothelial cells at a concentration of $15-300 \mu \mathrm{g} / \mathrm{ml}$ in the presence of $20 \%$ FCS (Maciag et al, 1984). Other mitogens that influence HUV endothelial cell proliferation are described in Section 1.3.1.3. Thorton et al (1984) subsequently found that the addition of heparin greatly potentiated the stimulatory effect of ECGF on the proliferation of HUV endothelial cells. For endothelial cells grown on a gelatin matrix with Medium 199 and $20 \%$ FCS, (crude preparation) maximal growth was achieved with ECGF at a concentration of $25 \mu \mathrm{g} / \mathrm{ml}$ in the presence of heparin $(90 \mu \mathrm{g} / \mathrm{ml})$. In the absence of heparin, $100-200 \mu \mathrm{g}$ /ml ECGF was required for maximal growth. Heparin alone did not support cell growth. The addition of heparin with ECGF also reduced the doubling time from 2-3 days to 17-21 $\mathrm{h}$ and increased the life-span from 27-34 population doublings to $42-79$ population doublings. These observations have been confirmed by Maciag et al (1984), Hasegawa et al (1988) and Shimado et al (1990), the latter group finding that HUV endothelial cells can be passaged about 60 times at a $1: 2$ split ratio before their life span is complete (50-55 population doubling levels). Barzu et al (1986a) demonstrated that there was an interaction between purified ECGF and heparin. Heparin has also been reported to increase the affinity of ECGF for 
its receptor on endothelial cells (Barzu et al, 1986a), stimulate the internalization of ECGF (Barzu et al, 1986a) and increase its stability as assessed by proliferation ability (Mueller et al, 1989). ECGF activity has been demonstrated in vivo and heparin significantly increases ECGF binding to intact vascular endothelium (Rosengart et al, 1988). Injury to the endothelium results in an increase in ECGF binding to the deendothelialized segment and this is decreased in the presence of heparin (Rosengart et al, 1988). Barzu et al (1986b) and Rosenbaum et al (1986) showed that heparin alone inhibits HUV endothelial cell proliferation in a concentration-dependent manner. Inhibition was found to be maximal with heparin in the presence of $1 \%$ serum, and this inhibitory effect is abolished in the presence of $10 \%$ serum. Cariou et al (1988) have confirmed these findings.

In summary, HUV endothelial cells can be successfully harvested and grown for long periods of time. They require a matrix on which to grow and a complex medium containing serum and growth factors.

\subsubsection{Endothelial cell characterization}

Cultured HUV endothelial cells have been characterized by phase contrast microscopy as monolayers of closely apposed, homogeneous, large (30-40 $\mu \mathrm{m} \times 60-95 \mu \mathrm{m})$, polygonal cells with an oval, centrally located nucleus containing two or three prominent nucleoli surrounded by a perinuclear granular region, and a broad, thin peripheral cytoplasm (Jaffe et al, 1973a; Gimbrone et al, 1974 Haudenschild et al, 1975). Using transmission electron microscopy HUV endothelial cells have been shown to contain Weibel-Palade bodies (Jaffe et al, 1973a; Gimbrone et al, 1974; Haudenschild et al, 1975; Elgjo et al, 1975). These structures were originally described by Weibel and Palade (1964) in endothelial cells from pulmonary arteries. They are rodshaped cytoplasmic inclusion bodies $(1-2 \mu \times 3.2 \mu)$ which contain between 
6-26 small cylindrical tubules (150-200 $\AA$ in diameter and $40 \AA$ thick), embedded in a relatively dense matrix (Weibel and Palade, 1964). The Weibel-Palade body is thought by some (Sengel and Stoebner, 1970) but not others (Parry and Abramovich, 1972) to originate from the Golgi complex. Jaffe et al (1973a) found that $30 \%$ of cultured HUV endothelial cells contain Weibel-Palade bodies while Gimbrone et al (1974) found these bodies in 30 $70 \%$ of cultures up to the eighth passage. Weibel-Palade bodies contain vWf (Wagner et al, 1982) and GMP-140, a platelet $\alpha$-granule membrane protein (McEver et al, 1989; Bonfanti et al, 1989).

Another criterion used to identify HUV endothelial cells is the presence of VWf : Ag as revealed by immunofluorescence microscopy (Hoyer et al, 1973; Jaffe et al, 1973b; Jaffe, 1977). These three criteria (typical light microscopic appearance, Weibel-Palade bodies, and VWf : Ag) are specific to HUV endothelial cells and are not found in cultures of either smooth muscle cells or fibroblasts, the most common contaminants in HUV endothelial cell cultures (Hoyer el al, 1973 Jaffe et al, 1973a; b; Gimbrone et al, 1974; Haudenschild et al, 1975). Two other features of HUV endothelial cells occasionally used for identification are the presence of angiotensin I-converting enzyme, also present in fibroblasts (Weinberg et al, 1982; Rubin et al, 1982), and the presence of ABO blood group antigens (Jaffe et al, 1973a). HUV endothelial cells have many other exciting properties and functions, some of which are highlighted in the following Chapters.

Endothelial cells have been cultured from a wide variety of donor animals and tissues. Some common tissues are bovine aorta (Booyse et al, 1975; Eskin et al, 1978), calf pulmonary arteries (Ryan et al, 1978), bovine umbilical vein (Gospodarowicz et al, 1977), bovine saphenous vein (Eskin et al, 1978), human iliac arteries (Glassberg et al, 1982), pulmonary arteries, veins and aorta (Johnson, 1980), human adult saphenous veins (Watkins et al, 1984), 
porcine aorta (Slater and Sloan, 1975; Dickinson and Slakey, 1982), inferior vena cava, pulmonary veins and arteries (Hennig et al, 1985), rabbit aorta (Buonassissi and Venter, 1976), marginal vessels of the rabbit ear (Davison et al, 1980), guinea pig aorta and portal veins (Blose and Chako, 1975), and canine jugular veins (Ford et al, 1981). Some of the work carried out by investigators using these tissues will be referred to in following Chapters. It has become apparent that endothelial cells from different sources behave differently to HUV endothelial cell cultures. The rationale for choosing HUV endothelial cells, and the reasons for some differences in behaviour of endothelial cells from different tissues, will be discussed later in this Chapter.

\subsection{MATERIALS}

\subsubsection{Buffers and solutions}

Fetal calf serum (FCS) Hybriserum (Commonwealth Serum Laboratories, Melbourne, Australia) was dispensed into $100 \mathrm{ml}$ aliquots and stored at -20 ${ }^{\circ} \mathrm{C}$.

Gelatin (BDH, UK). A $0.1 \%$ solution was made up in distilled water, autoclaved and stored at room temperature.

Medium 199 with Earle's salts, without sodium bicarbonate, without Lglutamine and without phenol red (PRF) (Cytosystems Pty Ltd, Castle Hill, Australia) was made up from powder in $10 \mathrm{~L}$ batches to which sodium bicarbonate and $\mathrm{HCl}$ were added to obtain a $\mathrm{pH}$ of 7.4 and osmolarity of 292$295 \mathrm{mOsm} / \mathrm{L}$. It was then filtered and stored at $4^{\circ} \mathrm{C}$.

Medium 199 with Earle's salts without sodium bicarbonate and with L- 
glutamine (Flow Laboratories, Irvine, Scotland) was prepared in the same manner as above.

Phosphate buffered saline (PBS). This comprised NaCl ( $8 \mathrm{~g} / \mathrm{L}), \mathrm{Na}_{2} \mathrm{HPO}_{4}(1$ $\mathrm{g} / \mathrm{L}), \mathrm{Na}_{2} \mathrm{PO}_{4} \mathrm{H}_{2} \mathrm{O}(0.4 \mathrm{~g} / \mathrm{L})$ made up to $1 \mathrm{~L}$ with distilled water, $\mathrm{pH} 7.4$ and stored at room temperature.

$0.1 \%$ Trypsin-versene. $5 \mathrm{ml}$ of stock trypsin (10\%) (Sigma Chemical Company, St Louis, USA) was added to $50 \mathrm{ml}$ of stock versene. This was made up to $500 \mathrm{ml}$ with distilled water, $\mathrm{pH} 7.2$, filtered and stored in $5 \mathrm{ml}$ aliquots at $-20{ }^{\circ} \mathrm{C}$. The versene comprised $\mathrm{NaCl}(16 \mathrm{~g}), \mathrm{KCl}(0.4 \mathrm{~g}), \mathrm{KH}_{2} \mathrm{PO}_{4}$ $(0.4 \mathrm{~g}), \mathrm{Na}_{2} \mathrm{HPO}_{4}(2.3 \mathrm{~g}), \mathrm{NaOH}(0.134 \mathrm{~g})$, EDTA Na $2(0.4 \mathrm{~g})$ made up to 200 $\mathrm{ml}$ in distilled water. $1 \mathrm{ml}$ of $1 \%$ phenol red was added. The versene was filtered and stores in $50 \mathrm{ml}$ aliquots.

\subsubsection{Reagents}

Acetone (propanone) AR Grade (May and Baker Australia Pty Ltd, Footscray, Australia) was used without dilution and stored at room temperature.

Absolute ethanol (alcohol, AR grade) (May and Baker Pty Ltd, Victoria, Australia). This was stored at $4^{\circ} \mathrm{C}$. Alcohol was used to dissolve sex steroids. In experiments it was used as a control at a final concentration of $0.05 \%$ or $0.1 \%$.

Bovine serum albumin (BSA) Fraction V (Sigma Chemical Company, St Louis, USA) was stored at $4^{\circ} \mathrm{C}$.

Collagenase IV (Cooper Biomedical, Malvern, PA or Worthington Biochemical 
Corporation, New Jersey). Collagenase was dissolved in Medium 199 to a concentration of $50 \mathrm{U} / \mathrm{ml}$, filtered and stored in $15 \mathrm{ml}$ aliquots at $-20^{\circ} \mathrm{C}$.

Dimethyl sulfoxide (DMSO) AR grade (Mallinckrodt Australia Pty. Ltd, South Oakleigh, Australia) was stored at room temperature in the dark.

Endothelial cell growth supplement (ECGS) (Sigma Chemical Company, St Louis, USA). $15 \mathrm{mg}$ was dissolved in $7.5 \mathrm{ml}$ of Medium 199 to give a final concentration of $2 \mathrm{mg} / \mathrm{ml}$ and stored at $4^{\circ} \mathrm{C}$. This is a crude preparation of endothelial cell growth factor.

17 $\beta$-estradiol (Lot 35F-0210) (Sigma Chemical Company, St Louis, USA) was dissolved in absolute ethanol to obtain a $0.01 \mathrm{M}$ solution. Ten-fold dilutions were made in absolute ethanol to obtain stock concentrations ranging from $10^{-2}-10^{-7} \mathrm{M}$ which were stored at $-20^{\circ} \mathrm{C}$. The final concentration of absolute ethanol in experiments did not exceed $0.1 \%$.

E-toxate kit (limulus amebocyte lysate) (Sigma Chemical Company, St Louis, USA).

F.I.T.C conjugated anti goat IgG (whole molecule) (Mile-Yella LTD, Blough, England). It was diluted $1: 2$ in PBS and stored at $-90{ }^{\circ} \mathrm{C}$. A fresh $1: 32$ solution was made up with $1 \%$ BSA in PBS immediately before use.

Gentamicin Sulphate (Gentamicin) (David Bull Laboratories, Victoria, Australia) (80 $000 \mathrm{U} / 2 \mathrm{ml}$ ) was diluted $1: 4$ in Medium 199, dispensed into 3 $\mathrm{ml}$ aliquots and stored at $-20^{\circ} \mathrm{C}$.

L- Glutamine (Sigma Chemical Company, St Louis, USA) was dissolved in PBS at $29.2 \mathrm{~g} / \mathrm{L}(0.2 \mathrm{M})$, filtered, dispensed into $4 \mathrm{ml}$ aliquots and stored at $20^{\circ} \mathrm{C}$. 
Glycerol (Ajax Chemical, Auburn, Australia) was stored at room temperature.

Goat antiserum to human factor VIII (Miles Scientific, Naperville, IL or ICN Immunobiologicals, Lisle, Illinois, USA). A $1: 2$ dilution was made in PBS and stored in $50 \mu \mathrm{l}$ aliquots at $-90^{\circ} \mathrm{C}$. A 1 : 16 working solution was made up immediately before use with $1 \%$ BSA in PBS for staining.

Heparin sodium (heparin) (David Bull Laboratories, Victoria, Australia) was stored in $5 \mathrm{ml}$ aliquots $(1000 \mathrm{U} / \mathrm{ml})$ at $4^{\circ} \mathrm{C}$.

Phenol red (BDH Ltd, Poole, England) was dissolved in phenol red-free Medium 199 to give a concentration of $34 \mathrm{mg} / \mathrm{L}$ and stored at room temperature.

Progesterone (4-pregnene-3,20-dione) (lot 32F-0102) (Sigma Chemical Company, St Louis, USA) was dissolved in absolute ethanol to obtain a 0.01 M solution. Tenfold dilutions were then made as described for $17 \beta$-estradiol.

[Methyl ${ }^{3} \mathrm{H}$ ]-Thymidine (New England Nuclear, Boston, USA or Amersham, Buckinghamshire, England) $(5.0 \mathrm{mCi} / 5 \mathrm{ml} ; 6.7 \mathrm{Ci} / \mathrm{mmol})$. A 1 in 50 dilution was made up in Medium 199 without phenol red in the presence and absence of FCS and stored at $4^{\circ} \mathrm{C} .25 \mu$ of this was added to each well to achieve a final concentration of $0.5 \mu \mathrm{Ci}$.

Trypan-blue (Sigma Chemical Company, St Louis, USA). A $0.1 \%$ solution was made up in PBS, filtered 3 times Watermann paper and stored at room temperature. 


\subsection{METHODS}

\subsubsection{Endothelial cell retrieval from human umbilical vein}

Endothelial cells were obtained from HUV by a modification of the method of Jaffe et al (1973). Sterile techniques were used. All areas of the umbilical cord with clamp marks were discarded to avoid harvesting damaged cells or contaminating cells from damaged areas. One end of the cord vein was cannulated with a size 10 Foley catheter. The vein was perfused with $100 \mathrm{ml}$ of warmed PBS $\left(37^{\circ} \mathrm{C}\right)$ to wash out residual blood. Collagenase $(50 \mathrm{U} / \mathrm{ml})$ was perfused into the umbilical vein and the distal end of the vein clamped with forceps. After incubation at $37^{\circ} \mathrm{C}$ for $15 \mathrm{~min}$ the collagenase perfusate containing the endothelial cells was flushed from the cord by perfusion with $20 \mathrm{ml}$ Medium 199 (containing $20 \%$ FCS and gentamicin $60 \mathrm{U} / \mathrm{ml}$ ). The cell suspension was centrifuged (Beckman Model TJ-P centrifuge) at $200 \times \mathrm{g}$ for 8 min at room temperature. The pellet was resuspended into $2 \mathrm{ml}$ of Medium 199 (with $20 \%$ FCS and gentamicin $60 \mathrm{U} / \mathrm{ml})$. To this, glutamine $(2 \mathrm{mM})$, heparin sodium $(20 \mathrm{U} / \mathrm{ml})$ and ECGS $(50 \mu \mathrm{g} / \mathrm{ml})$ were added. These will subsequently be referred to as the "standard additives". The suspension was then plated into two wells of a gelatinized 24 well plate (Falcon Plastic, Becton Dickson, Australia or Nunclon, Kamstrup, Denmark). The cells were incubated in a $5 \% \mathrm{CO}_{2}-95 \%$ air atmosphere at $37^{\circ} \mathrm{C}$ (Heraeus). They were "fed" twice a week with a complete change of medium and standard additives.

\subsubsection{Passaging of HUV endothelial cells}

Detachment of cells from the culture surface was achieved using $0.1 \%$ trypsin-versene added to a confluent monolayer of cells for $3 \mathrm{~min}$ before dislodging the cells by a sharp tap to the side of the flask. Medium 199 (with 
$20 \%$ FCS and gentamicin $60 \mathrm{U} / \mathrm{ml}$ ) was added to neutralize the trypsin. The suspension was centrifuged ( $200 \times \mathrm{g}$ for $8 \mathrm{~min}$ at room temperature), and the cell pellet resuspended and diluted to the appropriate volume with Medium 199 (with $20 \%$ FCS, gentamicin $60 \mathrm{U} / \mathrm{ml}$ and the standard additives). A 1:1 dilution of the cell suspension was used for passages $1-3$, whereas a $1: 2$ dilution was used for subsequent passages. The suspension was transferred into gelatinized flasks and incubated in $5 \% \mathrm{CO}_{2}-95 \%$ air atmosphere at 37 ${ }^{\circ} \mathrm{C}$ until a confluent monolayer formed, normally within 3-4 days.

\subsubsection{Counting and assessing viability of HUV endothelial cells}

A $20 \mu$ sample of the cell suspension was diluted $1: 1$ with a $0.1 \%$ trypan blue solution. The cells were counted on a haemocytometer (Reichert, USA) and the viability (structural integrity) assessed by the exclusion of trypan blue from the cells (Patterson, 1979).

\subsubsection{Freezing, storage and thawing of HUV endothelial cells}

The endothelial cells were passaged as described in Section 2.3.2. The cell suspension was then washed twice by resuspending in Medium 199 (with 20 \% FCS and gentamicin $60 \mathrm{U} / \mathrm{ml}$ ) and centrifuged $(200 \times \mathrm{g}$ for $8 \mathrm{~min}$ at room temperature). The supernatant was removed and the pellet placed on ice for $30 \mathrm{~min}$. The cells were resuspended in $1 \mathrm{ml}$ of freezing medium (70\% Medium 199, $20 \%$ FCS and $10 \%$ DMSO) at $4{ }^{\circ} \mathrm{C}$ and placed into a precooled cryotube. This was then placed in a polystyrene box and stored in a $-70^{\circ} \mathrm{C}$ freezer overnight. The cells were finally transferred to liquid nitrogen for long term storage.

To thaw cells the ampoule was placed in a $37^{\circ} \mathrm{C}$ water bath. The contents were transferred to a $50 \mathrm{ml}$ centrifuge tube and warmed Medium 199 (with 20 
\% FCS) was gradually added over 10 min to allow a gradual change in osmolality. The suspension was then centrifuged $(200 \times \mathrm{g}$ for $8 \mathrm{~min}$ at room temperature). The pellet was resuspended in $20 \mathrm{ml}$ of Medium 199 and rewashed twice to remove the DMSO before being resuspended in $10 \mathrm{ml}$ of Medium 199 (with $20 \%$ FCS, gentamicin $60 \mathrm{U} / \mathrm{ml}$ and the standard additives) and placed into a $75 \mathrm{~cm}^{2}$ gelatinized flask (Corning, NY or Nunclon, Kamstrup, Denmark). The viability of the cells was assessed as described in Section 2.3.3. The flask was then placed in a humidified incubator set at $5 \% \mathrm{CO}_{2}-95 \%$ air atmosphere at $37^{\circ} \mathrm{C}$.

\subsection{5 vWf: Ag immunofluorescence microscopy of HUV endothelial cells}

The method used was a modification of the method of Jaffe et al (1975). A confluent monolayer of endothelial cells was passaged as described in Section 2.3.2 and resuspended in $10 \mathrm{ml}$ of Medium 199 (with $20 \%$ FCS, gentamicin $60 \mathrm{U} / \mathrm{ml}$ and the standard additives). Two drops of the cell suspension from a pasteur pipette were placed on $13 \mathrm{~mm}$ glass coverslips (Chance, Australia) and incubated overnight in $5 \% \mathrm{CO}_{2}-95 \%$ air atmosphere at $37^{\circ} \mathrm{C}$. The cells on the coverslips were washed twice in Medium 199 and then fixed in cold acetone for $10 \mathrm{~min}$. The cells were rehydrated in PBS. Two drops of antiserum to human factor VIII were added to the cells (using a 1 : 16 dilution of the stock) and incubated for $30 \mathrm{~min}$ at room temperature. The cells were then washed in five changes of $200-500 \mathrm{ml}$ of PBS. The second antibody, F.I.T.C-conjugated antigoat IgG was added ( $1: 32$ dilution of the stock) for $30 \mathrm{~min}$ at room temperature in the dark. The cells were then washed in five changes of 200-500 ml PBS. The coverslips were then mounted using one drop of $10 \%$ glycerol : $90 \%$ PBS and examined using an Olympus $\mathrm{BH}-2$ microscope with a fluorescence attachment. 


\subsubsection{Electron microscopy of HUV endothelial cells}

\subsubsection{Transmission electron microscopy}

HUV endothelial cells were grown to confluence in gelatinized $260 \mathrm{ml}$ flasks. The HUV endothelial cells were detached from the flask with $0.1 \%$ trypsinversene to which $15 \mathrm{ml}$ of Medium 199 (20\% FCS and gentamicin $60 \mathrm{U} / \mathrm{ml}$ ) were added. The cells were centrifuged (200 $\mathrm{x} \mathrm{g}$ for $8 \mathrm{~min}$ at room temperature), the pellet was washed twice in PBS and resuspended in $0.5 \mathrm{ml}$ of PBS, and the cells were placed on ice.

Specimens were fixed using $2 \%$ gluteraldehyde in $0.1 \mathrm{M}$ Cacodylate buffer $\mathrm{pH} 7.4$ for $1 \mathrm{~h}$. The specimens were washed three times for $15 \mathrm{~min}$ in $0.1 \mathrm{M}$ Cacodylate buffer $\mathrm{pH}$ 7.4. They were post-fixed in $3 \%$ Osmium tetroxide in $0.1 \mathrm{M}$ Cacodylate buffer, $\mathrm{pH} 7.4$ for $1 \mathrm{~h} 30 \mathrm{~min}$ and "en bloc" stained with $2 \%$ aqueous uranyl acetate for $1 \mathrm{~h} 30 \mathrm{~min}$.

Specimens were then dehydrated in graded alcohols from $30 \%, 50 \%, 70$ $\%, 90 \%, 95 \%$ and absolute alcohol, $15 \mathrm{~min}$ in each with 3 changes at the absolute alcohol stage. They were then placed in a mixture of $1: 1$ absolute alcohol and "Spurs" resin (low viscosity) for $2 \mathrm{~h}$ with $10 \mathrm{~min}$ agitations. Finally, samples were placed in $100 \%$ "Spurs" resin for $2 \mathrm{~h}$ with $15 \mathrm{~min}$ agitations, and then embedded. Sections were cut with a Reichert Ultracut ultramicrotome, stained with "Reynolds" Lead Citrate and subsequent micrographs taken with a Philips 301 electron microscope.

\subsubsection{Scanning electron microscopy}

The HUV endothelial cells were prepared in the same way as described in 
Section 2.3.6.1. Specimens were fixed using $2 \%$ gluteraldehyde in $0.1 \mathrm{M}$ Cacodylate buffer $\mathrm{pH} 7.4$ for $2 \mathrm{~h}$ and washed in $0.1 \mathrm{M}$ Cacodylate buffer with $4 \times 15$ min changes. They were then post-fixed in $1 \%$ Osmium tetroxide in $0.1 \mathrm{M}$ Cacodylate buffer for $2 \mathrm{~h}$ and washed in distilled water for $2 \times 10 \mathrm{~min}$. Specimens were dehydrated and critical point dried, and subsequent photomicrographs were taken with a H7000 SEM attachment.

\subsubsection{The effect of two commercial media on HUV endothelial cell proliferation}

The composition of the two culture media examined is listed in Appendix 1. A 1 in 2 dilution of phenol red containing Medium 199 (Flow Laboratories) (with $20 \%$ FCS and gentamicin $60 \mathrm{U} / \mathrm{ml}$ ) was made in PRF Medium 199 (Cytosystems) (with $20 \%$ FCS and gentamicin $60 \mathrm{U} / \mathrm{ml}$ ) in gelatinized 96 well plates to which HUV endothelial cells $\left(5 \times 10^{3}\right.$ cells $/$ well) and the standard additives in PRF Medium 199 were added. The cells were incubated for $24 \mathrm{~h}$ in $5 \% \mathrm{CO}_{2}-95 \%$ air atmosphere at $37{ }^{\circ} \mathrm{C}$ and $\left[{ }^{3} \mathrm{H}\right]$-thymidine $(0.5 \mu \mathrm{Ci} /$ well $)$ was then added. The cells were incubated for a further $18 \mathrm{~h}$ in $5 \% \mathrm{CO}_{2}-95 \%$ air atmosphere at $37^{\circ} \mathrm{C}$, washed twice in Medium 199, trypsinized for $30 \mathrm{~min}$ and harvested onto glass fibre paper (Titerteck, Skatron, Norway) using a Dynatech $\mathrm{CH}-103$ cell harvester. The discs were dried overnight at $37^{\circ} \mathrm{C}$ and added to $5 \mathrm{mls}$ of non-aqueous scintillation fluid or four drops of "Ultima Gold" scintillant and counted for 2 minutes in a Parkcard Tricarb $460 \beta$-scintillation counter.

A second series of experiments was performed in which FCS was absent and the endothelial cells $\left(1.25 \times 10^{4} /\right.$ well) were incubated for $18 \mathrm{~h}$ in $5 \%$ $\mathrm{CO}_{2}-95 \%$ air atmosphere at $37{ }^{\circ} \mathrm{C}$ in the presence of $\left[{ }^{3} \mathrm{H}\right]$-thymidine $(0.5$ $\mu \mathrm{Ci} /$ well) with different concentrations of "Flow Laboratories" Medium 199 
diluted in "Cytosystems" Medium 199. The cells were then washed, trypsinized and harvested as described above.

The E-toxate kit was used to detect endotoxin in the two media. This test was conducted according to the manufacturer's instructions.

\subsubsection{Effect of phenol red in Medium 199 on HUV endothelial cell proliferation}

Phenol red has been shown to exert estrogenic effects on different cell types (Berthois and Katzenellenbogen, 1986; Rajendran et al, 1987; Parikh et al, 1987; Ernst et al, 1988), enhancing proliferation of estrogen responsive cells. As phenol red is present in many media recommended for endothelial cell culture its effect on HUV endothelial cell proliferation was assessed. The methodology for the proliferation assays used below was modified from the method of Sakamoto et al (1987).

A 1 in 2 titration of phenol red (initial concentration $17 \mathrm{mg} / \mathrm{L}$ ) in Medium 199 (with $20 \%$ FCS and gentamicin $60 \mathrm{U} / \mathrm{ml}$ ) was made in phenol red free Medium 199 (PRF Medium 199) (with $20 \%$ FCS and gentamicin $60 \mathrm{U} / \mathrm{ml}$ ) in $0.1 \%$ gelatinized-96 well plates (Nuncon, Denmark). HUV endothelial cells (1 $\times 10^{4}$ cells /well) were added to these wells suspended in PRF Medium 199 with standard additives. The cells were then incubated for $48 \mathrm{~h}$ in $5 \% \mathrm{CO}_{2}$ $95 \%$ air atmosphere at $37{ }^{\circ} \mathrm{C}$ and $\left[{ }^{3} \mathrm{H}\right]$-thymidine $(0.5 \mu \mathrm{Ci} /$ well) was then added. The cells were then incubated for $18 \mathrm{~h}$ in $5 \% \mathrm{CO}_{2}-95 \%$ air atmosphere at $37{ }^{\circ} \mathrm{C}$ before being washed, trypsinized and harvested as described in Section 2.3.7.

The proliferative effect of phenol red on HUV endothelial cells was also assessed in the absence of FCS. In these experiments a 1 in 2 titration of phenol red (initial concentration $17 \mathrm{mg} / \mathrm{L}$ ) containing Medium 199 was made 
in PRF Medium 199 to which was added HUV endothelial cells $\left(1.25 \times 10^{4}\right.$ cells /well) suspended in PRF Medium 199 with the standard additives and $\left[{ }^{3} \mathrm{H}\right]$-thymidine $(0.5 \mu \mathrm{Ci} /$ well $)$. The cells were then incubated for $18 \mathrm{~h}$ in $5 \%$ $\mathrm{CO}_{2}-95 \%$ air atmosphere at $37^{\circ} \mathrm{C}$ before being washed, trypsinized and harvested as described above.

\subsubsection{Effect of FCS on HUV endothelial cell proliferation}

FCS (final concentration range 0-50 \%) was diluted in PRF Medium 199. HUV endothelial cells ( $1.25 \times 10^{4}$ cells / well), suspended in PRF Medium 199 with half the usual concentration of standard additives and $\left[{ }^{3} \mathrm{H}\right]$-thymidine ( $0.5 \mu \mathrm{Ci} /$ well), were pipetted into gelatinized 96 well plates in the presence of variable concentrations of FCS. The cells were then incubated for $18 \mathrm{~h}$ in $5 \%$ $\mathrm{CO}_{2}-95 \%$ air atmosphere at $37{ }^{\circ} \mathrm{C}$ before being washed, trypsinized and harvested as described in Section 2.3.7.

\subsubsection{Effect of ECGS on HUV endothelial cell proliferation}

To assess the influence of ECGS (Sigma) on HUV endothelial cell proliferation, serial dilutions of ECGS in gelatinized 96 well plates were made in PRF or phenol red containing Medium 199 (with $20 \%$ FCS and gentamicin $60 \mathrm{U} / \mathrm{ml}$ ). The maximum final concentration of ECGS varied between 400$1000 \mu \mathrm{g} / \mathrm{ml}$. HUV endothelial cells $\left(5 \times 10^{3} /\right.$ well) suspended in PRF Medium 199 (with 20 \% FCS, gentamicin $60 \mathrm{U} / \mathrm{ml}$, glutamine $2 \mathrm{mM}$ and heparin $20 \mathrm{U}$ $/ \mathrm{ml}$ ) were added to the wells. Plates were incubated for $48 \mathrm{~h}$ in $5 \% \mathrm{CO}_{2}-95$ $\%$ air atmosphere at $\left.37^{\circ} \mathrm{C} .{ }^{3} \mathrm{H}\right]$-thymidine $(0.5 \mu \mathrm{Ci} /$ well $)$ was then added and the cells incubated for a further $18 \mathrm{~h}$ before being washed, trypsinized and harvested as described in Section 2.3.7. This experiment was repeated in the absence of FCS over $18 \mathrm{~h}$ with $1.25 \times 10^{4}$ cells /well. 


\subsubsection{Effect of heparin on HUV endothelial cell proliferation}

Experiments were designed to assess the proliferative effect of heparin in combination with ECGS. Serial dilutions of heparin in gelatinized 96 well plates were made in PRF Medium 199 or phenol red containing Medium 199 (with $20 \%$ FCS and gentamicin $60 \mathrm{U} / \mathrm{ml}$ ). The maximum final concentration of heparin sodium was $200 \mathrm{U} / \mathrm{ml}$. HUV endothelial cells $\left(5 \times 10^{3}\right.$ cells /well) were suspended in PRF Medium 199 or phenol red containing Medium 199 (with $20 \%$ FCS, gentamicin $60 \mathrm{U} / \mathrm{ml}$, glutamine $2 \mathrm{mM}$ and ECGS $50 \mu \mathrm{g} / \mathrm{ml}$ ). The plates were incubated for $48 \mathrm{~h}$ in $5 \% \mathrm{CO}_{2}-95 \%$ air atmosphere at $37{ }^{\circ} \mathrm{C}$ and then $[3 \mathrm{H}]$-thymidine $(0.5 \mu \mathrm{Ci} /$ well) was added. The cells were incubated for a further $18 \mathrm{~h}$ in $5 \% \mathrm{CO}_{2}-95 \%$ air atmosphere at $37 \mathrm{OC}^{\mathrm{C}}$ before being washed, trypsinized and harvested as described in Section 2.3.7.

\subsubsection{The effects of different cell densities and time on HUV endothelial response to sex steroids}

These experiments were designed to establish the optimal parameters for use in the subsequent detailed proliferative experiments using HUV endothelial cells exposed to sex steroids. HUV endothelial cells were plated onto gelatinized 96 well plates at three densities $\left(1.25 \times 10^{3}\right.$ cells /well, $2.5 \times$ $10^{3}$ cells /well and $5 \times 10^{3}$ cells /well) in PRF Medium 199 and phenol red containing Medium 199 (with $20 \%$ FCS, gentamicin $60 \mathrm{U} / \mathrm{ml}$, ECGS $50 \mu \mathrm{g}$ $/ \mathrm{ml}$, heparin $20 \mathrm{U} / \mathrm{ml}$ and glutamine $2 \mathrm{mM}$ ) for $18 \mathrm{~h}, 42 \mathrm{~h}$ or $66 \mathrm{~h}$ in the presence of Medium 199 and sex steroids. In these studies the term sex steroids refers to $17 \beta$-estradiol $\left(10^{-5} \mathrm{M}\right)$, progesterone $\left(10^{-5} \mathrm{M}\right)$, the combination of $17 \beta$-estradiol $\left(10^{-5} \mathrm{M}\right)$ with progesterone $\left(10^{-5} \mathrm{M}\right)$, progesterone $\left(10^{-9} \mathrm{M}\right), 17 \beta$-estradiol $\left(10^{-10} \mathrm{M}\right)$, and the combination of progesterone $\left(10^{-9} \mathrm{M}\right)$ with $17 \beta$-estradiol $\left(10^{-10} \mathrm{M}\right)$ unless otherwise stated. A 1 in 1000 dilution of the stock $17 \beta$-estradiol or progesterone was made in 
Medium 199 (with $20 \%$ FCS, gentamicin $60 \mathrm{U} / \mathrm{ml}$, ECGS $50 \mu \mathrm{g} / \mathrm{ml}$, heparin $20 \mathrm{U} / \mathrm{ml}$ and glutamine $2 \mathrm{mM}$ ). Two controls were used in all experiments, Medium 199 alone and the vehicle used to dissolve the sex steroids, absolute ethanol (a/cohol). Alcohol was diluted in Medium 199 to give a $0.1 \%$ solution. $\left[{ }^{3} \mathrm{H}\right]$-thymidine $(0.5 \mu \mathrm{Ci} /$ well $)$ was added to cell cultures at $0 \mathrm{~h}, 24$ $\mathrm{h}$ and $48 \mathrm{~h}$. Following a further $18 \mathrm{~h}$ incubation in $5 \% \mathrm{CO}_{2}-95 \%$ air atmosphere at $37^{\circ} \mathrm{C}$ the cells were washed, trypsinized and harvested as described in Section 2.3.7.

\subsubsection{Statistical analysis}

Individual experiments were examined statistically using analysis of variance and presented graphically as the mean $\pm S D$. The multiple $t$ comparison (Bonferroni method) was then used to test for a significant difference between treatments. The Minitab programme (Data analysis software, 1987) was used for the above analyses. A p value of 0.05 or less was taken to represent a statistically significant difference.

\subsection{RESULTS}

\subsubsection{HUV endothelial cell culture}

Endothelial cells were discarded after 10 passages. Cells passaged between 5 and 10 times were used in the experiments outlined in the following Chapters (this corresponded to $8-16 \%$ of cell life span). Typical photomicrographs of HUV endothelial cells are illustrated in Figure 2.1 taken using a Leitz Diavert microscope. Endothelial cells divided until they formed a monolayer of polygonal, closely packed cells. A confluent monolayer had approximately $1 \times 10^{5}$ cells $/ \mathrm{cm}^{2}$ as previously reported by Gimbrone et al (1974). 


\subsection{2 vWf: Ag immunofluorescence microscopy of HUV endothelial cells}

Endothelial cell cultures were routinely screened for the presence of vWf : $\mathrm{Ag}$ as a measure of their purity. The purity of cultures was always greater than $98 \%$. The contaminants, if present, had a fibroblast like appearance and did not stain positively for vWf : Ag. These contaminated cultures were discarded. Figure 2.2 illustrates the typical immunofluorescence of an endothelial cell culture observed through a Leitz Orthoplan microscope.

\subsubsection{Electron microscopy of HUV endothelial cells}

Transmission electron microscopy was performed on randomly-selected endothelial cell cultures to confirm the typical ultrastructural characteristics and in particular the presence of Weibel-Palade bodies. Electron microscopy was also used to check for mycoplasma contamination of cultures. All cultures examined were negative for mycoplasma. Figure 2.3 illustrates the typical ultrastructural appearance of HUV endothelial cells at passage 3 and passage 8 . The cells were characterised by oval or flattened nuclei, pinocytotic vesicles, a perinuclear region containing Golgi complexes, a small array of endoplasmic reticulum, mitochondria, filaments which were most prominent at the periphery, and many Weibel-Palade bodies. The inset photomicrograph shows a Weibel-Palade body. It is rod-shaped with a single limiting membrane and contains about 14 tubules (range 6-20), each approximately $150 \AA$ in width.

A typical scanning electron photomicrograph of HUV endothelial cells is illustrated in Figure 2.4. The typical features include veiling and the presence of projections from the HUV endothelial cell surface as previously described by Ryan and Ryan (1981). 


\subsubsection{The effect of two different media on HUV endothelial cell proliferation}

Experiments were performed to determine whether Medium 199 obtained from two different sources had different effects on HUV endothelial cell proliferation. Medium 199 obtained from Flow Laboratories had a significant enhancing effect on HUV endothelial cell proliferation when compared to Medium 199 obtained from Cytosystems, in the absence of FCS (Figure 2.5). In the presence of $20 \%$ FCS there was no difference in the proliferative effects of the two different media on HUV endothelial cells (Figure 2.6). Endotoxin was only detected in Medium 199 obtained from Cytosystems. Semiquantitation showed this to be very small $(0.4 \rho \mathrm{g} / \mathrm{ml})$. The composition of the two different media is detailed in Appendix 1.

\subsection{Effect of phenol red in Medium 199 on HUV endothelial cell proliferation}

The presence of phenol red in Medium 199 (with or without FCS) had no statistically significant effect on HUV endothelial cell proliferation when compared to cells not exposed to phenol red (Figures 2.7 and 2.8).

\subsubsection{Effect of FCS on HUV endothelial cell proliferation}

Two batches of FCS were initially used in the studies reported here. Batch A had an optimal proliferative effect at concentrations greater than $10 \%$, while batch $B$ had an optimal proliferative effect at concentrations between 2.5-10 $\%$ (Figure 2.9). Using concentrations greater than $10 \%$ for batch B caused significant inhibition of HUV endothelial cell proliferation. 


\subsubsection{Effect of ECGS on HUV endothelial cell proliferation}

The optimal concentration of ECGS was found to be between $25-100 \mu \mathrm{g} / \mathrm{ml}$ when the heparin concentration was $20 \mathrm{U} / \mathrm{ml}$ and the concentration of FCS was $20 \%$ (Figure 2.11). Concentrations of ECGS greater than $100 \mu \mathrm{g} / \mathrm{ml}$ or less than $25 \mu \mathrm{g} / \mathrm{ml}$ resulted in sub-optimal HUV endothelial cell proliferation, although it was always significantly more than in the absence of ECGS. In the absence of FCS, ECGS at concentrations between $50-500 \mu \mathrm{g} / \mathrm{ml}$ caused optimal HUV endothelial cell proliferation (Figure 2.10). These responses did not depend on the passage number of the cells or the batch of ECGS used.

In the absence of heparin higher concentrations of ECGS were required for optimal proliferation, as previously observed (refer to Section 2.1.2) (Figure 2.12). Concentrations of 10,20 and $40 \mathrm{U} / \mathrm{ml}$ of heparin did not alter the proliferative response at various concentrations of ECGS (Figure 2.12). Alcohol had no effect on the requirement for ECGS or heparin relative to Medium 199 alone.

\subsubsection{Effect of heparin on HUV endothelial cell proliferation}

Heparin caused optimal HUV endothelial cell proliferation in the presence of ECGS $(50 \mu \mathrm{g} / \mathrm{ml})$ at concentrations between 12.5-50 U /ml (Figure 2.13). There were minor differences in the proliferative effects of heparin between batches. Heparin at a concentration of $20 \mathrm{U} / \mathrm{ml}$ was used unless otherwise stated. Both high concentrations of heparin (50-200 U / $/ \mathrm{ml}$ ) and low concentrations (less than $6.25 \mathrm{U} / \mathrm{ml}$ ) resulted in sub-optimal proliferation of HUV endothelial cells. 


\subsubsection{The effects of different cell densities and time on HUV endothelial response to sex steroids}

The proliferative response of HUV endothelial cells exposed to sex steroids was compared to two controls, Medium 199 and alcohol. In the presence of FCS the time of exposure to the sex steroids appeared to be important. When HUV endothelial cells $\left(5 \times 10^{3}\right.$ cells/ well) were exposed to $17 \beta$ estradiol $\left(10^{-5} \mathrm{M}\right)$ for increasing time intervals there was a significant increase in the percentage inhibition of proliferation relative to control ( $14 \%$ at $18 \mathrm{~h}, 47$ $\%$ at $42 \mathrm{~h}$ and $56 \%$ at $66 \mathrm{~h}$ ) (Figures 2.14, 2.15 and 2.16). A similar pattern of inhibition was observed when HUV endothelial cells were exposed to $17 \beta$ estradiol $\left(10^{-5} \mathrm{M}\right)$ at other cell densities (Figures 2.14, 2.15 and 2.16). Progesterone $\left(10^{-5} \mathrm{M}\right)$ and the combination of $17 \beta$-estradiol $\left(10^{-5} \mathrm{M}\right)$ with progesterone $\left(10^{-5} \mathrm{M}\right)$ also inhibited HUV endothelial cell proliferation, and this was also more pronounced with increasing time. There were no significant differences in proliferation when HUV endothelial cells were exposed to $17 \beta$-estradiol $\left(10^{-10} \mathrm{M}\right)$, progesterone (10-9 $\left.\mathrm{M}\right)$, the combination of $17 \beta$-estradiol $\left(10^{-10} \mathrm{M}\right)$ with progesterone $\left(10^{-9} \mathrm{M}\right)$ or alcohol at any of the time intervals examined (Figures 2.14, 2.15 and 2.16). A more detailed examination of the effects of sex steroids on HUV endothelial cell proliferation is presented in Section 3.4.2.

The response of HUV endothelial cells to sex steroids was independent of cell density (Figures 2.14, 2.15 and 2.16). However, the optimal standard deviation for $\beta$-scintillation counting (less than $1 \%$ for a 2 minute counting time) was achieved with a cell density of either $5 \times 10^{3}$ cells /well or $2.5 \times 10^{3}$ cells /well in $20 \%$ FCS when cells were incubated for $66 \mathrm{~h}$. As a consequence, $2.5 \times 10^{3}$ cells / well were used in proliferation assays where 20 $\%$ FCS was employed. 


\subsection{DISCUSSION}

In this Chapter techniques for the isolation, culture and characterization of HUV endothelial cells have been reviewed, as well as factors important in sustaining endothelial cell growth.

Medium 199 from Flow Laboratories, in the absence of FCS, had a significant proliferative effect when compared with Medium 199 from Cytosytems. The composition of each Medium is detailed in Appendix 1. The major difference between the two media is the presence of phenol red (17 $\mathrm{mg}$ /L) in the Flow Laboratories preparation. There are some other differences in the salt composition. However, phenol red at a concentration of $17 \mathrm{mg} / \mathrm{L} \mathrm{did}$ not affect HUV endothelial cell proliferation (see below). Only Medium 199 obtained from Cytosystems contained endotoxin at a concentration unlikely to alter proliferation. The explanation for the increased proliferative effect of Flow Laboratories Medium 199 is therefore unclear.

Phenol red was not observed to alter HUV endothelial cell proliferation in the current studies, which is important, as it has been shown to have proliferative effects in other cell culture systems. Ernst et al (1988) showed that phenol red $(16.8 \mu \mathrm{M})$ bound to the estrogen receptor, and caused a significant increase in proliferation of osteoblast-like cells and that tamoxifen ( $1 \mu \mathrm{M}$ ) abolished this stimulation. In MCF-7 cells (a breast cancer cell line) phenol red did not compete with $[3 \mathrm{H}]$-estradiol for estrogen binding sites, but it did activate a cytoplasmic estrogen receptor by interacting with a site distinct from the steroid binding site (Rajendran et al, 1987). Berthois et al (1986) found that phenol red had significant estrogenic activity at concentrations in the range $15-45 \mu \mathrm{M}$. They also observed that cell proliferation was highest at low concentrations of FCS and substantially 
reduced when the concentration of FCS was $20 \%$. The maximum concentration of phenol red in Medium 199 used in the current studies to test the effect of phenol red on HUV endothelial cell proliferation was $17 \mathrm{mg} / \mathrm{L}$ (48 $\mu \mathrm{M})$. This is the stated amount in Flow Laboratories Medium 199 which was used in some experiments. This was found not to influence HUV endothelial cell proliferation in the presence or absence of FCS. This may be because the estrogenic effect of phenol red fell in the concentration range (10-6-10-9 M) where $17 \beta$-estradiol was not found to influence HUV endothelial cell proliferation (refer to Section 3.4.2). Phenol red containing Medium 199 was however excluded (except where stated) from experiments assessing the effect of estrogens or antioxidants on various parameters of HUV endothelial cell function.

FCS was found to have a significant proliferative effect on HUV endothelial cell proliferation in vitro. It was batch dependent, as has been established by other investigators (refer to Section 2.1.2). Batch B, which inhibited HUV endothelial cell proliferation at concentrations greater than $5 \%$ was not used in the present experiments because previous investigators have shown that 20-50 \% FCS caused HUV endothelial cell to proliferate (Wall et al 1978; Jaffe, 1984). This suggests some inhibitor was present in batch B. No detailed analysis of this batch of FCS was performed since this was beyond the scope of this thesis. It was necessary to use serum in subconfluent monolayers, since in the absence of serum the cells had an abnormal microscopic appearance and did not proliferate as well as in the presence of $20 \%$ FCS, although they excluded trypan blue.

As discussed in Section 2.1.2, ECGS has been found to be mitogenic for HUV endothelial cells (Maciag et al, 1984). In the present studies, the optimal mitogenic concentration of ECGS was found to be within the range of 25-100 $\mu \mathrm{g} / \mathrm{ml}$. A concentration of $50 \mu \mathrm{g} / \mathrm{ml}$ was therefore chosen for use in most 
subsequent experiments based on these results and on observations by other investigators (refer to Section 2.1.2). A small variation in the optimal concentration of ECGS was found between batches but these differences were not significant. When heparin was omitted the optimal requirement for ECGS increased from $25 \mu \mathrm{g} / \mathrm{ml}$ to $200 \mu \mathrm{g} / \mathrm{ml}$, as previously reported by Thornton et al (1984), Maciag et al (1984) and Hasegawa et al (1988). In the presence of ECGS $(50 \mu \mathrm{g} / \mathrm{ml})$, heparin enhanced HUV endothelial cell proliferation within the concentration range of $12.5-50 \mathrm{U} / \mathrm{ml}$. Higher and lower concentrations of heparin resulted in reduced HUV endothelial cell proliferation relative to Medium 199 alone. The effect of heparin, in the absence of ECGS, on HUV endothelial cell proliferation was not formally assessed.

The sex steroids were prepared as stock solutions in ethanol in these experiments. Ethanol is a commonly used vehicle in studies assessing the effects of sex steroids on cell proliferation (Ishiwata et al, 1977; Rhee et al, 1978; Harder and Coulson, 1979; Seillan et al, 1983; Harrison and McKee, 1984a; Corvazier et al, 1984; Gray et al, 1987; Presta, 1988). Ethanol, diluted to a final concentration of $0.1 \%$, was shown not to modify cell proliferation at any cell plating density used in the current studies.

HUV endothelial cells were chosen as the endothelial cell model for investigating possible effects of sex steroids because they have been shown to provide an excellent model of human endothelial cell function in vivo (see below) and furthermore, they are readily available and technically easy to use. The factors that make HUV endothelial cells a suitable in vitro model for the study of atherogenesis include the following:

(a) They have been shown to have most of the characteristics of human arterial endothelial cells (Johnson, 1980; Weinstein and Weng, 1986); 
(b) They are preferable to culture of whole tissue which becomes overgrown with smooth muscle cells and fibroblasts (Jaffe, 1984);

(c) They are easy to obtain and can be maintained in culture, and continue to exhibit typical characteristics of endothelial cells for long periods of time; and (d) They have the ability to proliferate in response to the disruption of a confluent monolayer (Gimbrone et al, 1974; Haudenschild et al, 1976), consistent with the behaviour of endothelial cells in vivo (Poole et al, 1958).

Some possible limitations of the HUV endothelial cell model include the fact that HUV endothelial cells are derived from a fetal tissue which is "preprogrammed" to die. It has been suggested that their requirements for proliferation could therefore be different from cells derived from adult vascular tissues (Jaffe, 1984). Loss of replicative potential and morphological signs of cellular senescence are often observed in long-term umbilical vein cultures (Gimbrone, 1981). Nichols et al (1987) cytogenetically evaluated seven HUV endothelial cell lines and found that there was a rapid increase in the development of polyploidy in some lines which has been viewed as one signpost of in vitro senescence. They found $6-9 \%$ polyploid cells in HUV endothelial cell cultures with 8-16\% of their life span complete. The polyploidy did not appear to be related to maternal age of the donor or type of obstetric delivery. A second possible limitation is that HUV endothelial cells are derived from veins rather than arteries although umbilical veins have a well developed, pharmacologically reactive muscular coat (Gimbrone, 1981). Furthermore, oxygenated blood traverses the umbilical vein through the ductus venosus (Beischer and Mackay, 1976) thus simulating the environment which adult arteries are exposed to.

The use of human endothelial cells to study atherosclerosis is preferable to the use of endothelial cells from other species because there are many 
differences in the characteristics and metabolic functions of endothelial cells between species. These differences include the following:

(a) HUV endothelial cells produce a continuous endothelial monolayer and demonstrate density inhibition of growth (Gimbrone et al, 1974; Haudenschild, 1976) similar to endothelium in vivo (Gimbrone, 1976; Malezak and Buck, 1977). Post-confluent bovine endothelial cells, however, show focal areas of "sprouting". This is a one-cell layer beneath the confluent monolayer comprised of fusiform cells connected end to end to form a mycelium (Schwartz, 1978);

(b) HUV endothelial cells have a very low level of $\mathrm{PGI}_{2}$ release compared to pulmonary arterial cell cultures (Chung-Welch et al, 1978), nevertheless the level of release is 100 times more than from bovine aorta (Weksler et al, 1977);

(c) HUV endothelial cells respond to epidermal growth factor and FGF, in contrast to bovine endothelial cells which do not respond to epidermal growth factor (Gospodarowicz et al, 1978b);

(d) HUV endothelial cells require thrombin to be sensitized to the mitogenic effect of serum factors while bovine endothelial cells do not (Gospodarowicz et al, 1978b);

(e) HUV endothelial cells continue to produce vWf : Ag in culture, in contrast to porcine endothelial cells (Jaffe, 1984);

(f) HUV endothelial cells can modify LDL while bovine endothelial cells do not (Henriksen et al, 1982a; Morel et al, 1983b; 1984); and

(g) HUV endothelial cells are easier to identify than some other endothelial cells (e.g. bovine aortic endothelial cells) because of the consistent presence of Weibel-Palade bodies (Gospodarowicz et al, 1976; Schwartz, 1978).

In this Chapter techniques for the isolation, culture and characterization of HUV endothelial cells have been reviewed. HUV endothelial cell proliferation was shown to be influenced by ECGS, heparin, FCS, and the type of medium 
used, but proliferation was not influenced by phenol red. The techniques described here were used throughout the studies reported in this thesis. 


\section{Figure 2.1.}

Phase contrast photomicrograph of HUV endothelial cells.

Upper: A confluent monolayer of HUV endothelial cells (passage 3). The culture medium was removed to photograph cells. Magnification $608 \mathrm{x}$.

Lower: A confluent monolayer of HUV endothelial cells (passage 3). The culture medium was removed to photograph cells. Magnification $152 \mathrm{x}$. 

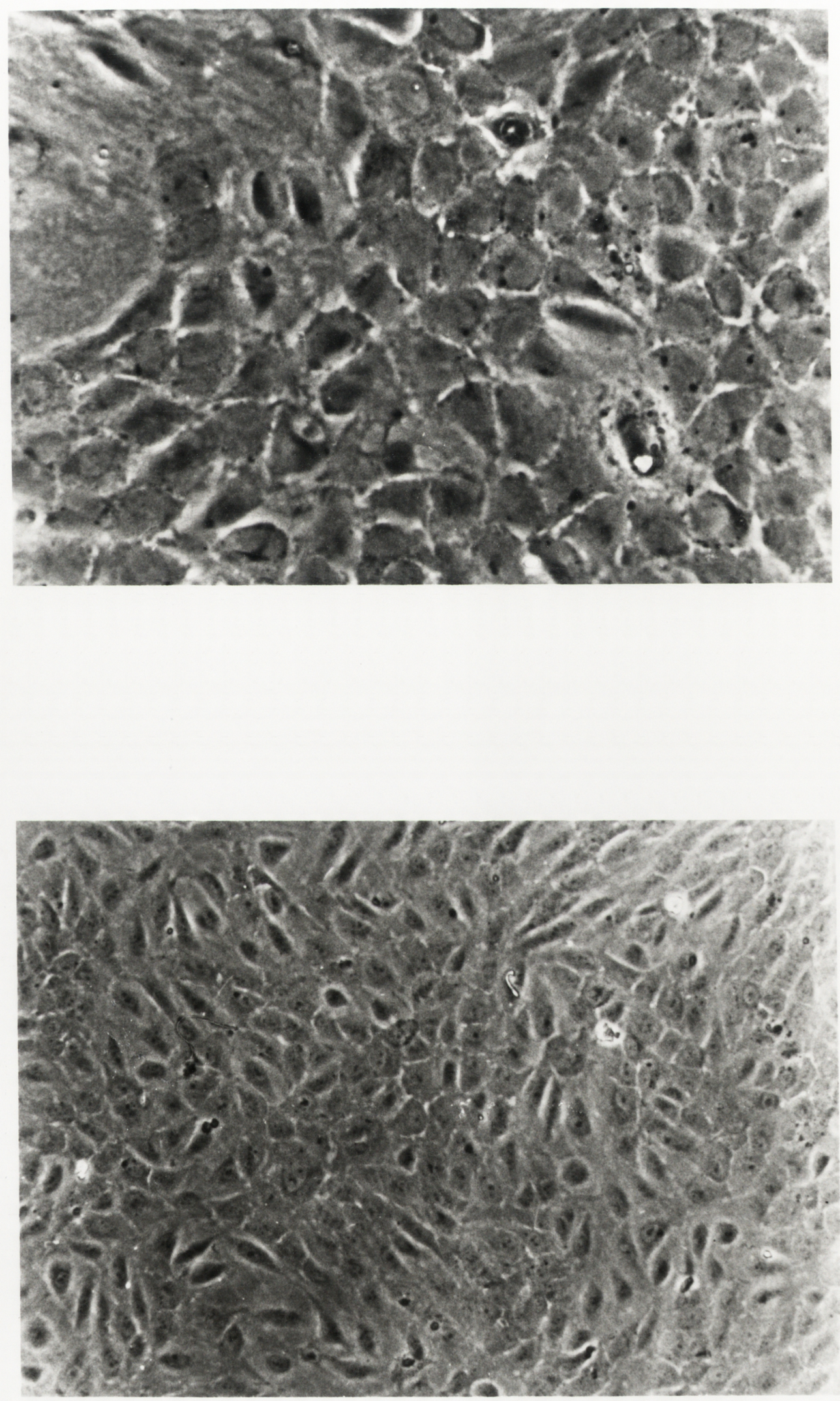


\section{Figure 2.2.}

Phase contrast fluorescent photomicrograph of vWf : Ag expression on HUV endothelial cells.

Upper: Presence of vWf : Ag on HUV endothelial cells (passage 7). Magnification $547 \mathrm{x}$.

Lower: Fluorescence on HUV endothelial cells (passage 7) with the omission of the primary antiserum. Magnification $547 \mathrm{x}$. 

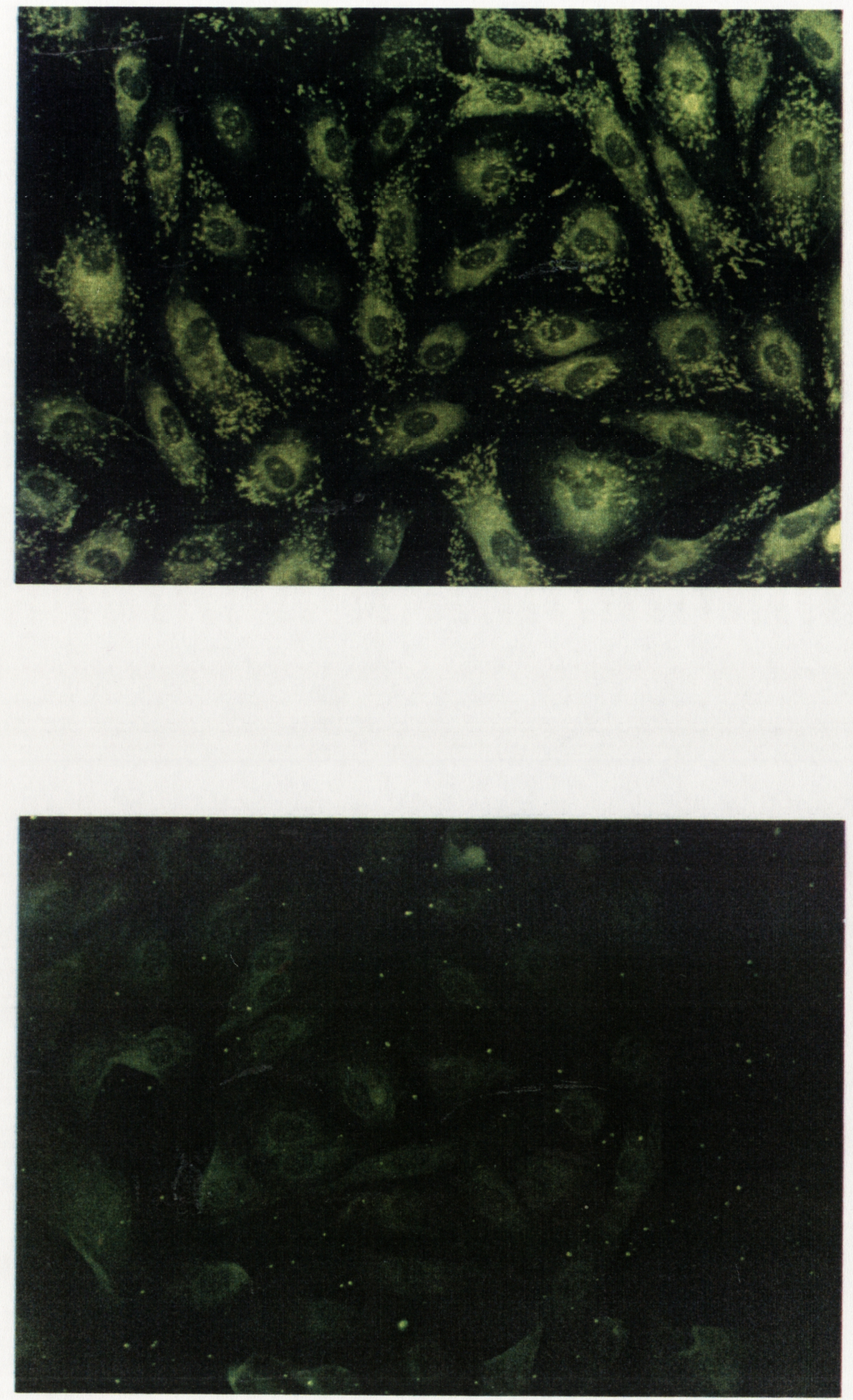


\section{Figure 2.3.}

Transmission electron photomicrograph of HUV endothelial cells. Upper: HUV endothelial cell (passage 8). Magnification $4600 \mathrm{x}$. Lower: HUV endothelial cell (passage 3). Magnification 2800 x Inset: A Weibel-Palade body. Magnification 102000 x.

Characteristic ultrastructural features of HUV endothelial cells include the presence of Weibel-Palade bodies, prominent nucleoli, perinuclear region rich in mitochondria, endoplasmic reticulum, Golgi complexes, attenuated peripheral cytoplasm containing free ribrosomes and macropinocytotic vesicles. The peripheral cytoplasm contains abundant numbers of microfilaments. 


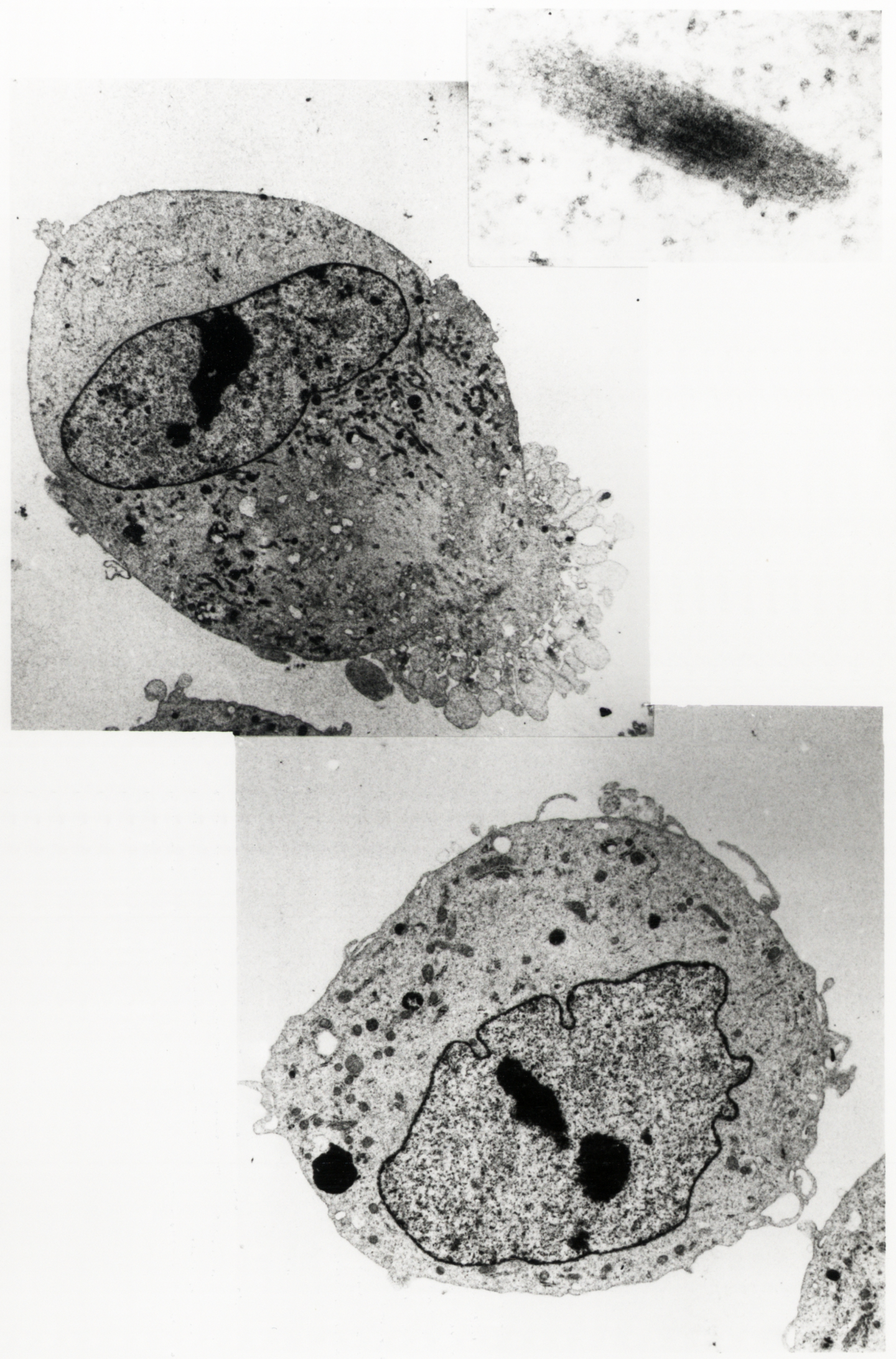




\section{Figure 2.4.}

Scanning electron photomicrograph of HUV endothelial cells.

Upper: HUV endothelial cell (passage 8). Magnification $6000 \mathrm{x}$.

Lower: HUV endothelial cell (passage 8). Magnification $5500 \mathrm{x}$.

These photomicrographs show the typical cytoplasmic projections on the endothelial cell surface. 

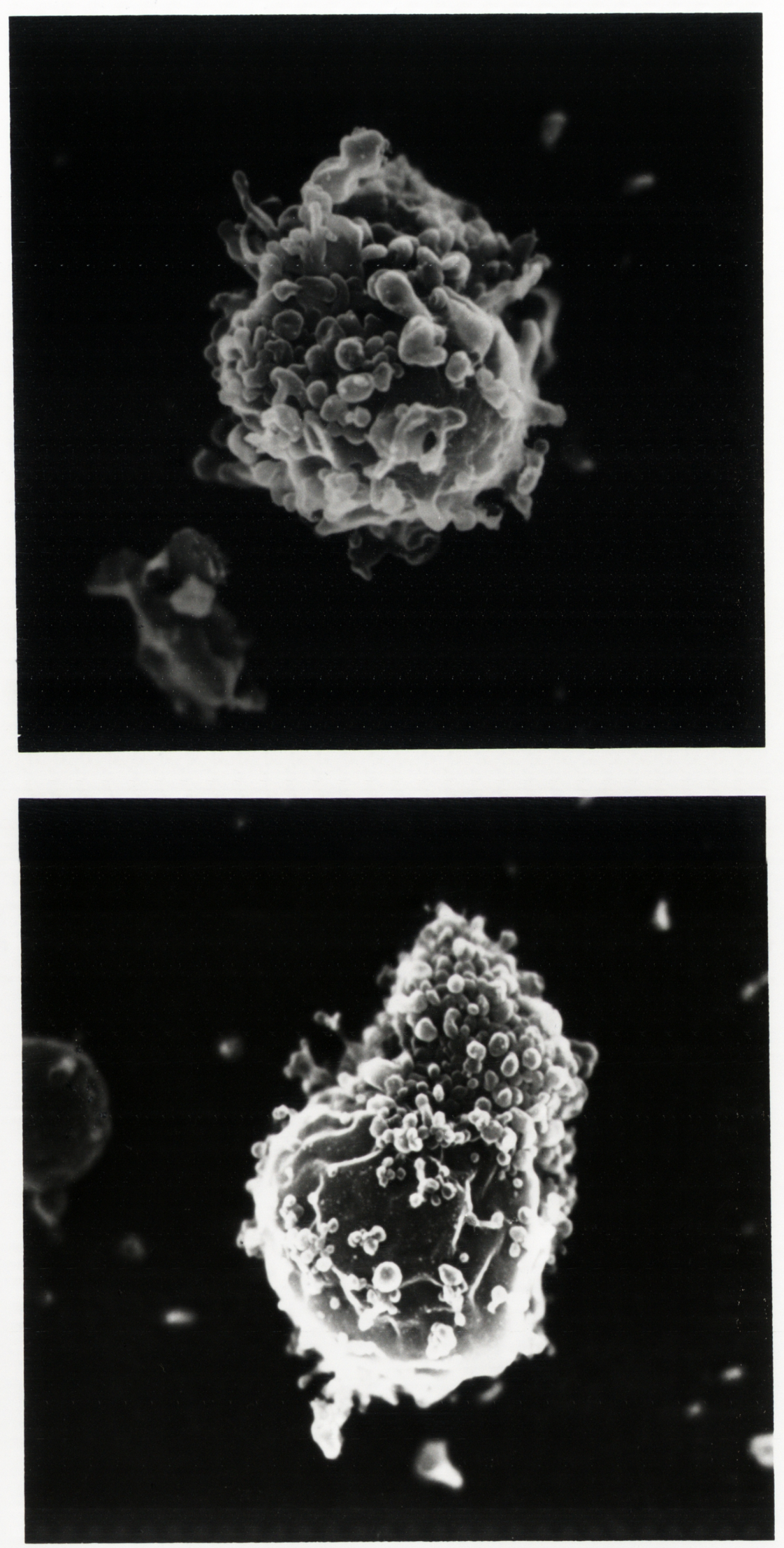
Figure 2.5 .

The effect of two commercial culture media on HUV endothelial cell proliferation in the absence of FCS. 1 in 2 dilutions of Medium 199 (Flow Laboratories) in Medium 199 (Cytosystem) were made to which endothelial cells (passage 8) were added and their proliferation assessed after $18 \mathrm{~h}$. The background represents endothelial cells grown solely in Medium 199 from Cytosystems in the absence of FCS while the " $x$ " axis represents different dilutions of Medium 199 from Flow Laboratories in Medium 199 from Cytosystems. The "y" axis indicates incorporation of $\left[{ }^{3} \mathrm{H}\right]$-thymidine (TdR) over $18 \mathrm{~h}$ by endothelial cells. This graph is representative of 2 experiments in triplicate. ${ }^{*}=p<0.05$

\section{Figure 2.6.}

The effect of two commercial culture media on HUV endothelial cell proliferation in the presence of FCS. 1 in 2 dilutions of Medium 199 (Flow Laboratories) in Medium 199 (Cytosystem) were made to which endothelial cells (passage 8) were added and their proliferation assessed after $66 \mathrm{~h}$. The background represents endothelial cells grown solely in Medium 199 from Cytosystems in the presence of FCS. The key to this figure is the same as for Figure 2.5. Note the difference in the scale of the "y" axis between Figures 2.5 and 2.6 because of different incubation time and the

presence of FCS in Figure 2.6. This graph is representative of 2 experiments in triplicate. 
EFFECT OF TWO COMMERCIAL MEDIA ON ENDOTHELIAL CELL PROLIFERATION

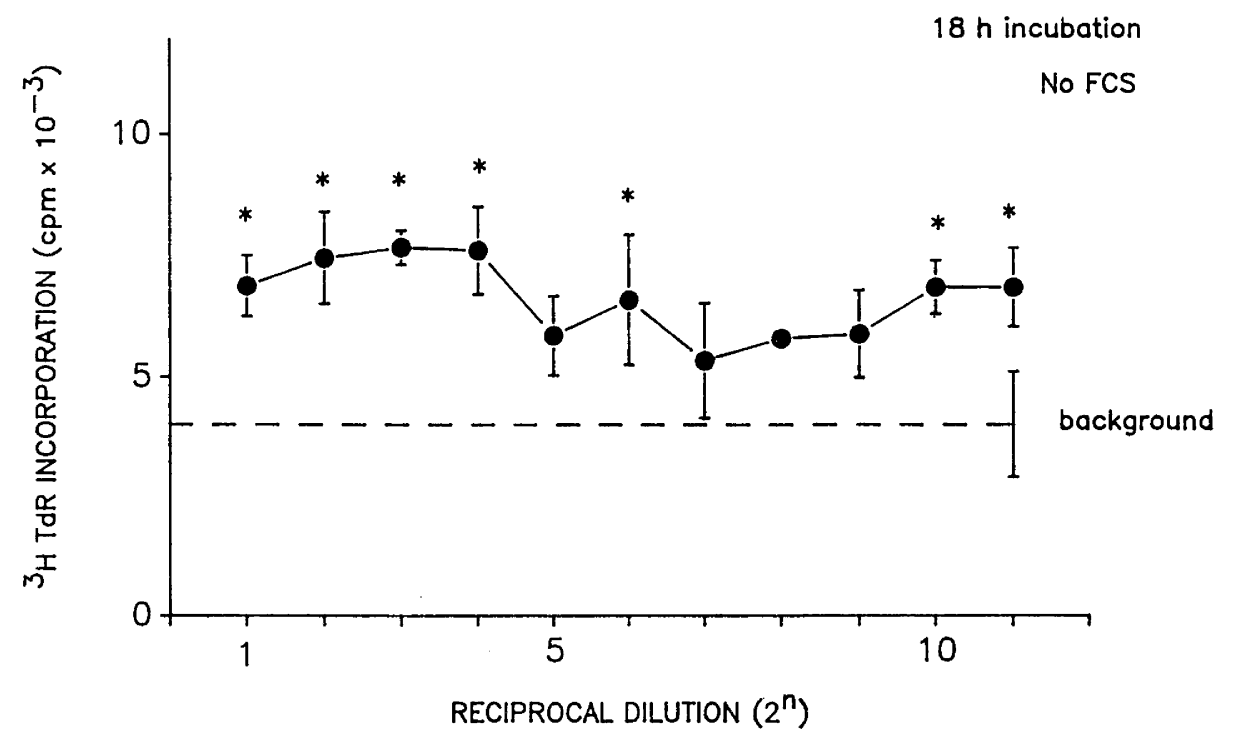

EFFECT OF TWO COMMERICIAL MEDIA ON ENDOTHELIAL CELL PROLIFERATION

$66 \mathrm{~h}$ incubation

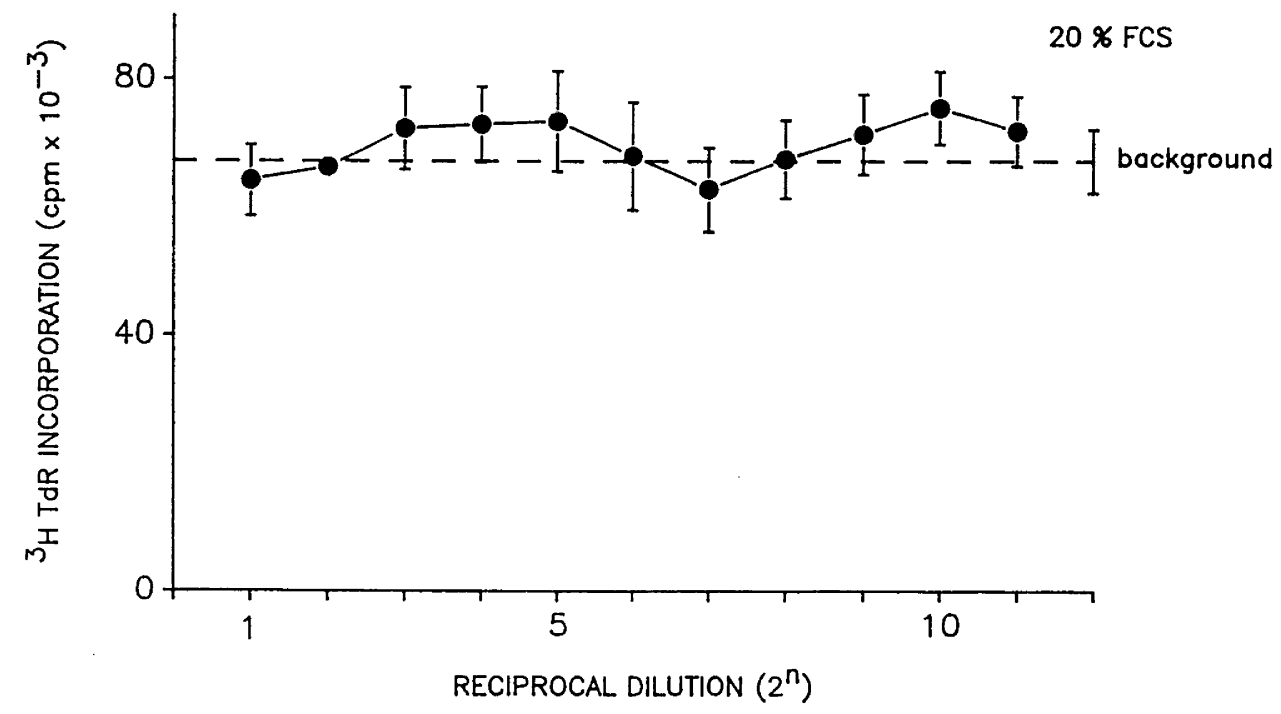




\section{Figure 2.7.}

Effect of phenol red in Medium 199 on HUV endothelial cell proliferation in the absence of FCS. 1 in 2 dilutions of phenol red (maximum concentration $17 \mathrm{mg} / \mathrm{L}$ ) in Medium 199 were made to which endothelial cells (passage 8) were added and their proliferation assessed over $18 \mathrm{~h}$. The background represents endothelial cells not exposed to phenol red. The "x" axis represents different concentrations of phenol red in Medium 199. This graph is representative of 2 experiments in triplicate.

\section{Figure 2.8.}

Effect of phenol red in Medium 199 on HUV endothelial cell proliferation in the presence of $20 \%$ FCS. 1 in 2 dilutions of phenol red (maximum concentration $17 \mathrm{mg} / \mathrm{L}$ ) in Medium 199 were made in PRF Medium 199 in the presence of $20 \%$ FCS in which endothelial cells (passage 8) were incubated for $66 \mathrm{~h}$. The background represents endothelial cells not exposed to phenol red. The key to this figure is the same as for Figure 2.7. Note the differences in the scale of the " $y$ " axis in the two figures because of the different incubation time and presence of FCS in Figure 2.8. This graph is representative of 2 experiments in triplicate. 
EFFECT OF PHENOL RED IN MEDIUM 199 ON ENDOTHELIAL CELL PROLIFERATION

$18 \mathrm{~h}$ incubation

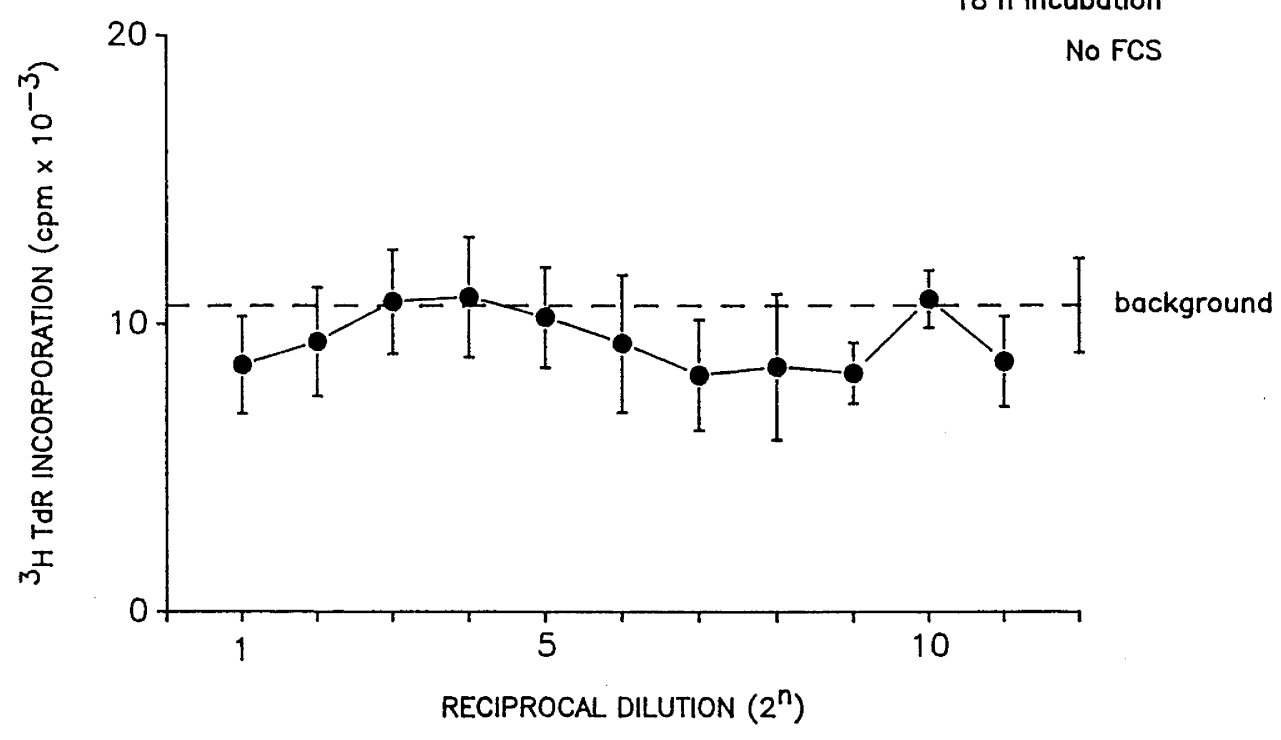

EFFECT OF PHENOL RED IN MEDIUM 199 ON ENDOTHELIAL CELL PROLIFERATION

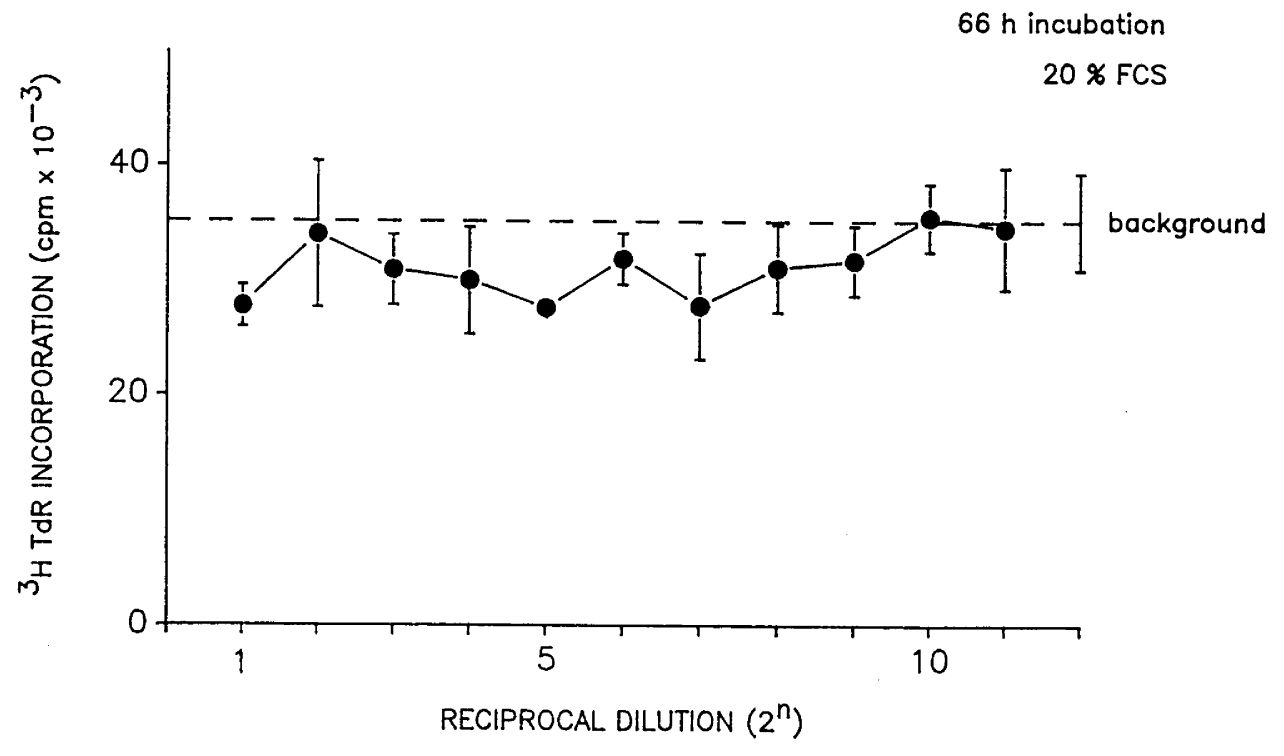




\section{Figure 2.9.}

Influence of FCS concentration on HUV endothelial cell proliferation. Endothelial cells (Passage 9) were exposed to varying concentrations of FCS (batch A or batch B) for $18 \mathrm{~h}$. The "y" axis indicates incorporation of $\left[{ }^{3} \mathrm{H}\right]$-thymidine $(\mathrm{TdR})$ over $18 \mathrm{~h}$ by endothelial cells as a percentage of maximum stimulation while the "x" axis represents different concentrations of FCS in Medium 199. This graph is representative of 2 experiments in triplicate. 
EFFECT OF FCS CONCENTRATION ON ENDOTHELIAL CELL PROLIFERATION

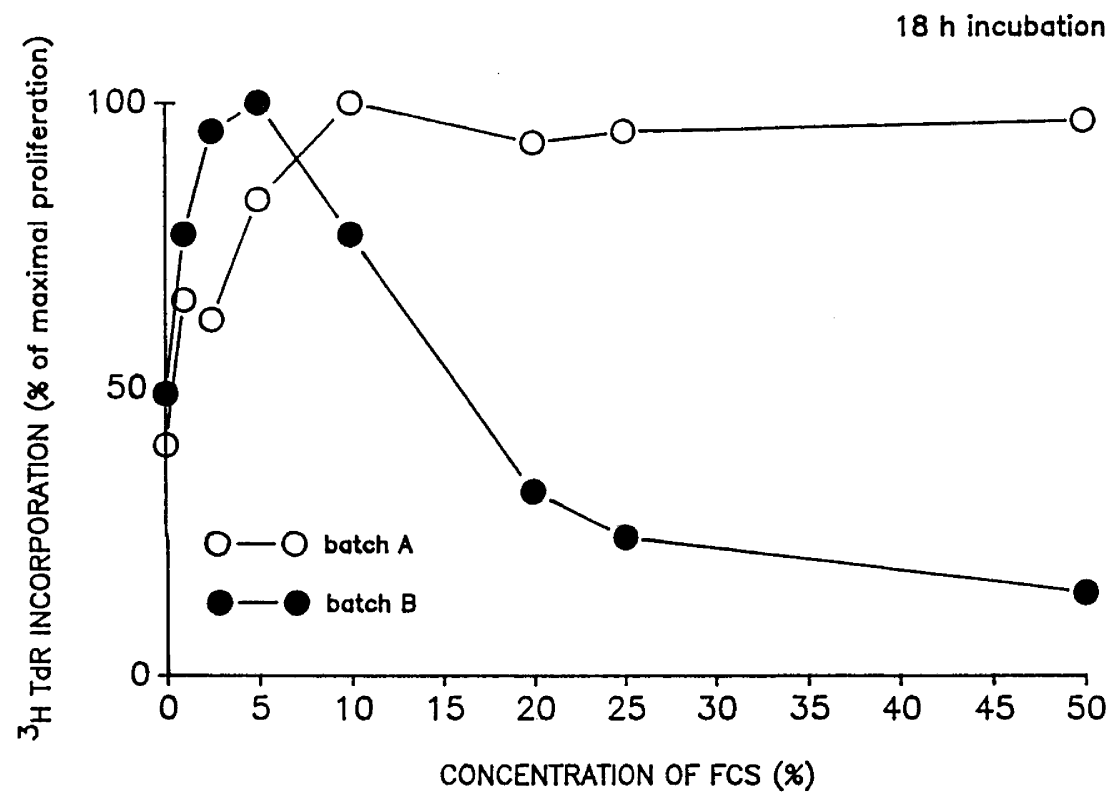




\section{Figure 2.10.}

Effect of different concentrations of ECGS on HUV endothelial cell proliferation in the absence of FCS. Endothelial cells (passage 7) were exposed to 1 in 2 dilutions of ECGS (maximum concentration $1 \mathrm{mg} / \mathrm{ml}$ ) over $18 \mathrm{~h}$ in the presence of heparin $20 \mathrm{U} / \mathrm{ml}$. Different concentrations of ECGS are shown on the "x" axis. This graph is representative of 2 experiments in triplicate.

\section{Figure 2.11.}

Effect of different concentrations of ECGS on HUV endothelial cell proliferation in the presence of $20 \%$ FCS. Endothelial cells (passage 7) were exposed to 1 in 2 dilutions of ECGS (maximum concentration $1 \mathrm{mg} / \mathrm{ml}$ ) over $66 \mathrm{~h}$ in the presence of $20 \% \mathrm{FCS}$ and heparin $20 \mathrm{U} / \mathrm{ml}$. The key to this figure is the same as for Figure 2.10. This graph is representative of 9 experiments. Note the difference in the scale of the " $y "$ axis between Figures 2.10 and 2.11 due to the different incubation time and presence of FCS in Figure 2.11. Each point is in triplicate. 
EFFECT OF ECGS ON ENDOTHELIAL CELL PROLIFERATION

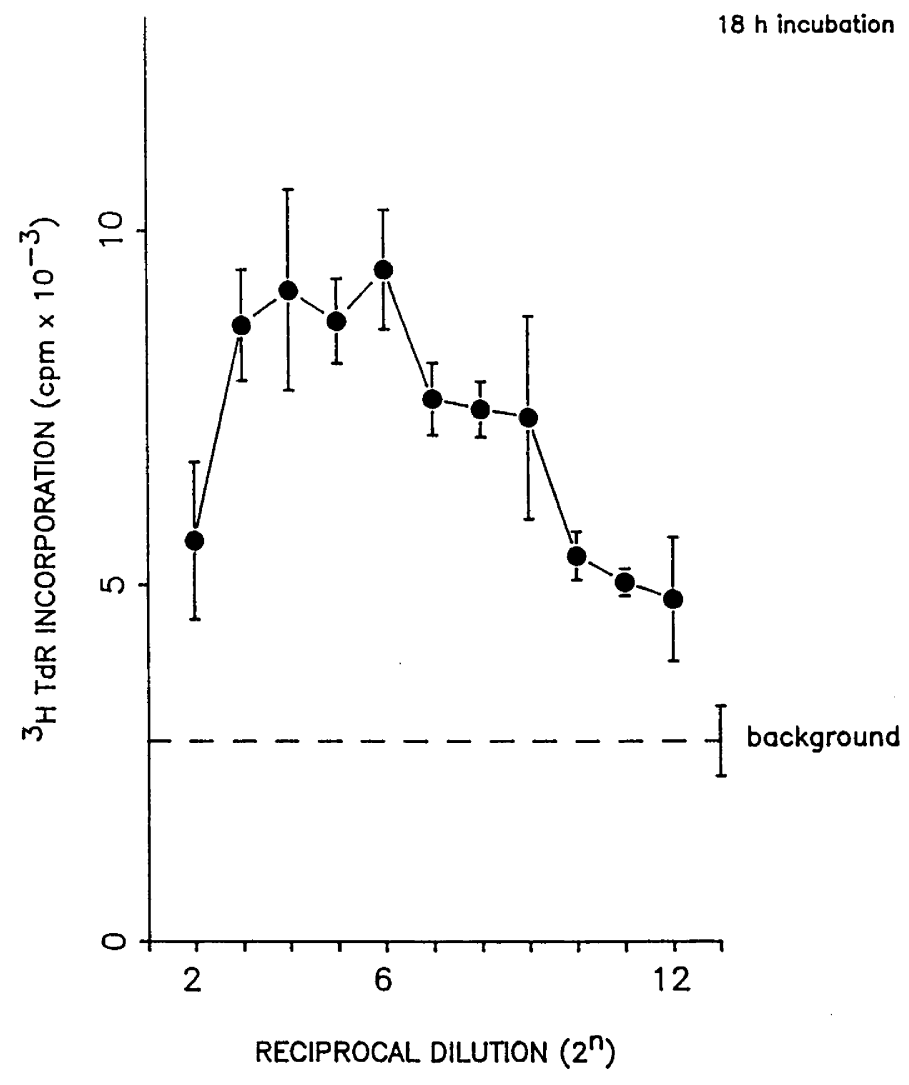

EFFECT OF ECGS ON ENDOTHELIAL CELL PROLIFERATION

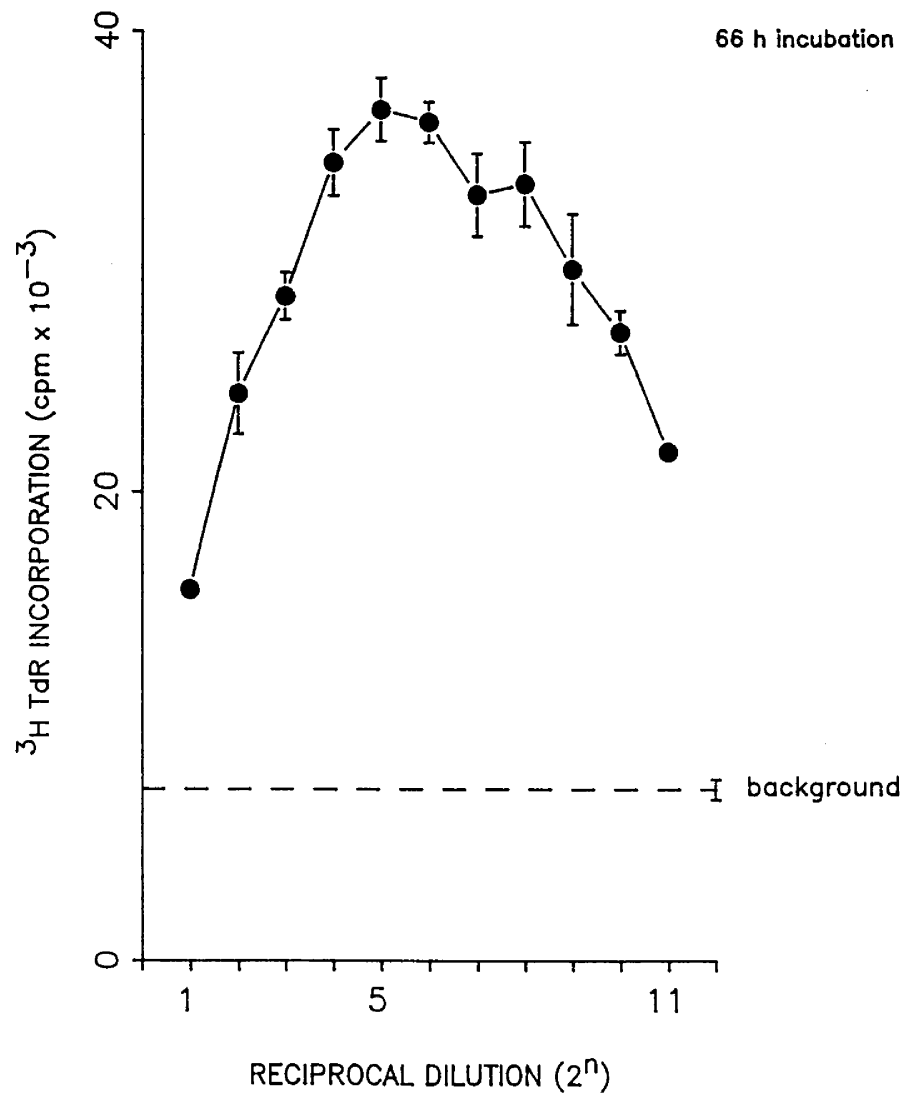




\section{Figure 2.12.}

Effect of different concentrations of ECGS on HUV endothelial cell proliferation in the presence of $20 \% \mathrm{FCS}$, alcohol $(0.1 \%)$ and different concentrations of heparin. Endothelial cells (passage 7) were exposed to 1 in 2 dilutions of ECGS (maximum concentration $400 \mu \mathrm{g} / \mathrm{ml}$ ) over $66 \mathrm{~h}$ in the presence of $20 \% \mathrm{FCS}$, heparin (0-20 U $/ \mathrm{ml}$ ) and alcohol (0.1\%). The background for endothelial cells in the absence of ECGS for cells exposed to alcohol averaged 1882 cpm. Different concentrations of ECGS are shown on the "x" axis. This graph is representative of 3 experiments. Each point is a single value.

Figure 2.13.

Effect of different concentrations of heparin on HUV endothelial cell proliferation. Endothelial cells (passage 7) were exposed to 1 in 2 dilutions of heparin (maximum concentration $200 \mathrm{U} / \mathrm{ml}$ ) in Medium 199 (with $20 \%$ FCS, gentamicin $60 \mathrm{U} / \mathrm{ml}$ and ECGS $50 \mu \mathrm{g} / \mathrm{ml}$ ) and incubated for $66 \mathrm{~h}$. Different concentrations of heparin are shown on the "x" axis. The background represents endothelial cells not exposed to heparin. This graph is representative of 3 experiments. Each point is a single value. 
EFFECT OF ECGS ON ENDOTHELIAL CELL PROLIFERATION

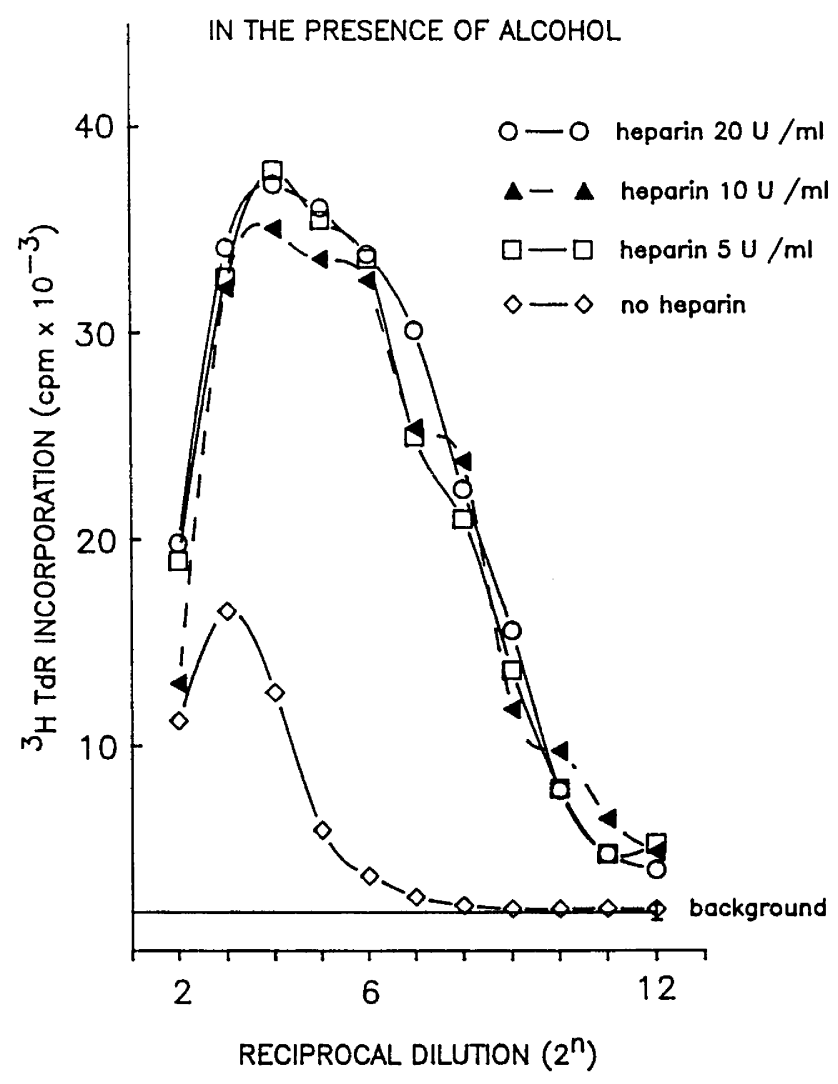

EFFECT OF HEPARIN ON ENDOTHELIAL CELL PROLFERATION

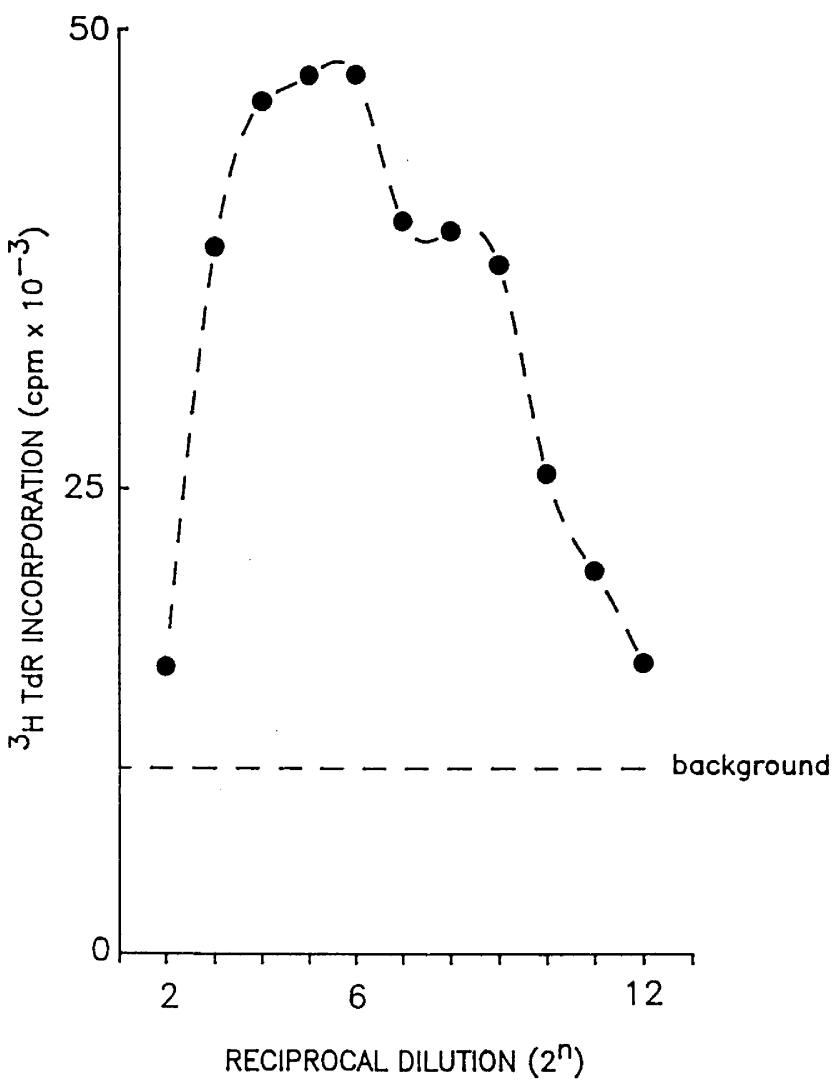




\section{Figure 2.14.}

The effect of sex steroids on HUV endothelial cell proliferation after $18 \mathrm{~h}$ incubation at different cell densities. Endothelial cells (passage 7) were used in this experiment which is representative of 2 experiments in triplicate. The hatched columns represent proliferation of endothelial cells plated at a density of $5 \times 10^{3}$ cells /well, the open columns represent endothelial cells plated at $2.5 \mathrm{x}$ $10^{3}$ cells /well while the filled columns represent endothelial cells plated at $1.25 \times 10^{3}$ cells /well. The abbreviations used here and elsewhere in the thesis for the sex steroids are: $E^{5}=17 \beta$-estradiol $\left(10^{-5} \mathrm{M}\right) ; E^{10}=17 \beta$-estradiol $\left(10^{-10} \mathrm{M}\right) ; \mathrm{P}^{5}=$ progesterone $\left(10^{-5}\right.$ $M) ; P^{9}=$ progesterone $\left(10^{-9} \mathrm{M}\right) ; E^{5} P^{5}=17 \beta$-estradiol $\left(10^{-5} \mathrm{M}\right)$ in combination with progesterone $\left(10^{-5} \mathrm{M}\right) ; \mathrm{E}^{10} \mathrm{P}^{9}=17 \beta$-estradiol $\left(10^{-10} \mathrm{M}\right)$ in combination with progesterone $\left(10^{-9} \mathrm{M}\right)$ : Alc = absolute ethanol and Control = Medium 199 alone.

\section{Figure 2.15.}

The effect of sex steroids on HUV endothelial cell proliferation at different cell densities after $42 \mathrm{~h}$ incubation. Endothelial cells (passage 7) were used in this experiment which is representative of 2 experiments in triplicate. The key to this figure is the same as for Figure 2.14. 


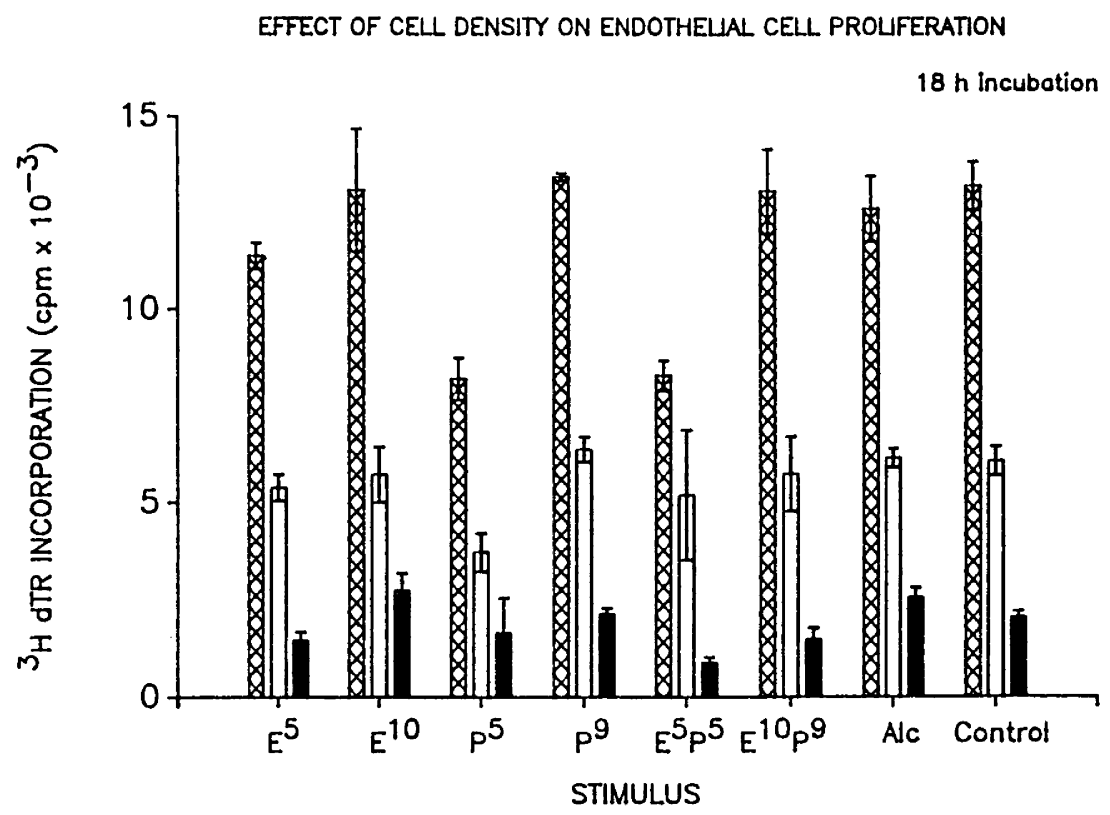

EFFECT OF CELL DENSITY ON ENDOTHELLAL. CELL PROLFERATION

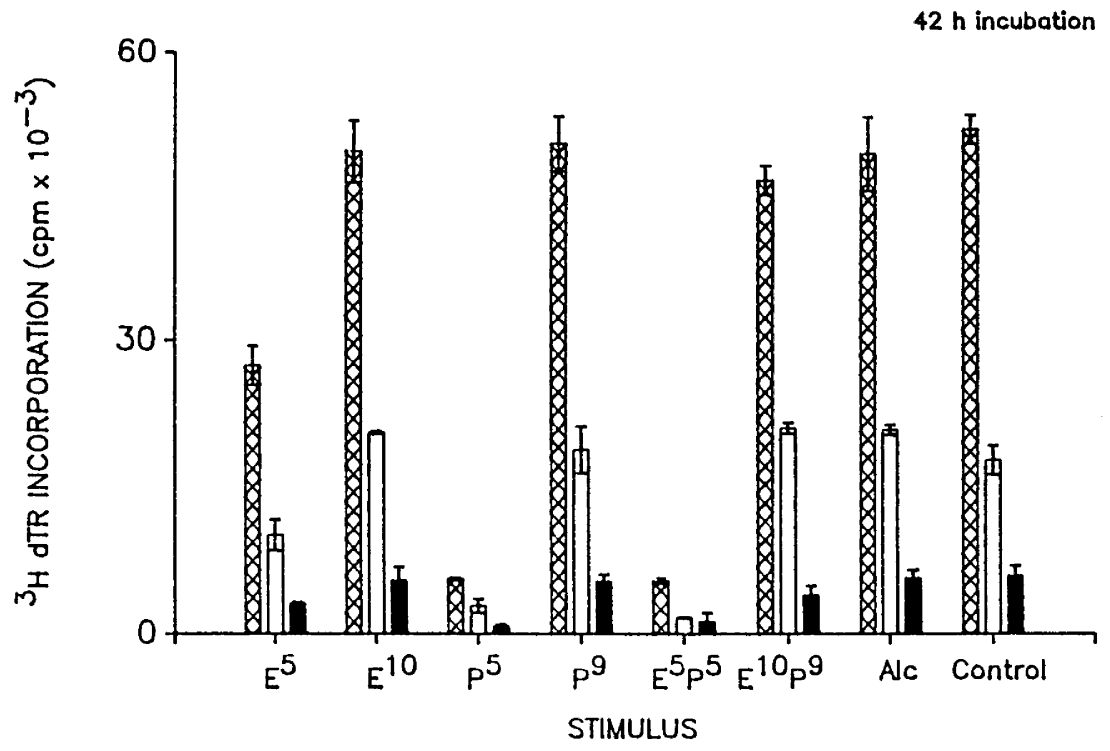


Figure 2.16.

The effect of sex steroids on HUV endothelial cell proliferation after $66 \mathrm{~h}$ incubation at different cell densities. Endothelial cells (passage 7) were used in this experiment which is representative of 2 experiments in triplicate. The key to this figure is the same as for Figure 2.14. Note the difference in the scale of the "y" axis between Figures $2.14,2.15$ and 2.16 reflecting increased proliferation with time. 
EFFECT OF CELL DENSITY ON ENDOTHELLAL CELL PROLFERATION

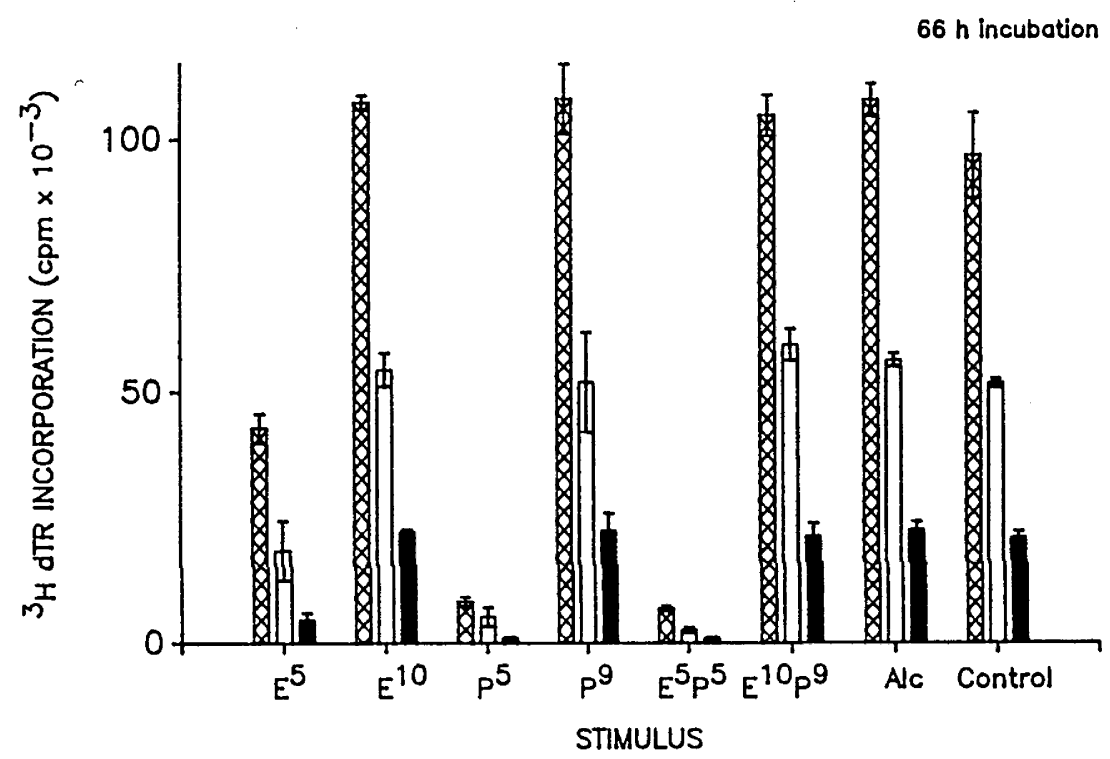




\section{CHAPTER 3}

EFFECTS OF SEX STEROIDS ON HUV ENDOTHELIAL CELL PROLIFERATION AND ON THE EXPRESSION OF mRNA FOR PDGF IN HUV ENDOTHELIAL CELLS 


\subsection{INTRODUCTION}

The endothelial cell monolayer of blood vessels acts as a semipermeable barrier between the underlying vessel wall and the blood stream. Endothelial cell turnover is not homogeneous in a blood vessel. Studies have shown that there are focal areas of endothelium where the replication rate is higher (Schwartz and Benditt, 1976). Caplan and Schwartz (1973) and Gimbrone (1976) have suggested that areas of high endothelial cell turnover may occur at sites in the arterial tree that are prone to the development of atherosclerosis. These focal areas of high endothelial cell turnover may be a consequence of injury to endothelium by various agents described in Section 1.3.1. This may result in activation of endothelial cells which could have a profound effect on many aspects of cellular regulation, such as increasing binding sites for monocytes and secretion of monocyte activators, secretion of oxygen free radicals that can modify LDL, and synthesis and secretion of PDGF-like proteins or other mitogens and chemoattractants for medial smooth muscle cells (DiCorleto and Chisolm, 1986) which are suppressed under normal physiological conditions.

Experimental studies (both in vivo and in vitro) have shown that endothelial cell injury and deendothelialization is followed by repair of the defect by spreading, migration and proliferation from the adjacent endothelial sheet (Ramsay et al, 1982; Coomber and Gotlieb, 1990). It has also been established that the processes of migration and cell proliferation are influenced by a number of factors. Some factors, for example hyperlipidemic serum, selectively inhibit movement of endothelial cells (Schwartz et al, 1981) while others such as tumour necrosis factor- $\alpha$ induce migration (Pober and Cotran, 1990) but inhibit endothelial cell proliferation (Hajjar et al, 1987; Saegusa et al, 1990; Shimada et al, 1990). Other factors known to influence 
migration or proliferation are outlined in Sections 1.3.1.3 and 2.1.2. Any factor influencing endothelial cell proliferation and the rate of reendothelialization may have a critical effect on atherogenesis because atherosclerotic lesions may regress once the overlying endothelium has regenerated (Stemerman and Ross, 1972). Furthermore, any alteration to the rate of loss and replacement of endothelial cells might alter permeability (Florentin et al, 1969). Lin et al (1989) found that endothelial cells undergoing division were associated with increased macromolecular leakage which may provide a significant site of entry for atherogenic LDL into the intima (Ross, 1981c; 1986).

The influence of the sex hormones $17 \beta$-estradiol and progesterone on endothelial cell proliferation and expression of specific growth factors in endothelial cells has not been extensively explored. The following Section will review the concentration of sex hormones that endothelial cells may be exposed to in vivo, the mechanism and effect of sex steroids on the regulation of cell proliferation and some factors which may influence the effects of sex steroids on cell behaviour in vitro.

\subsubsection{Concentrations of sex hormones in human plasma}

Only a very small fraction of each sex steroid hormone exists in plasma in the unbound state in humans. For estradiol and testosterone it is between 1 3 \% (Anderson, 1974; Leszczynski and Kakatnur, 1987). The circulating levels of free estradiol are determined by the amount bound to various proteins. Estradiol has been shown to bind avidly to albumin, less well to sex hormone binding globulin, and not significantly to corticosteroid-binding globulin (Anderson, 1974). Progesterone binds with high affinity to corticosteroid-binding globulin. Testosterone mainly binds to sex hormone binding globulin, and also to albumin but minimally to corticosteroid-binding 
globulin. Sex hormone binding globulin has approximately twice the affinity for testosterone as for estradiol. Estrogens stimulate and androgens inhibit sex hormone binding globulin production (Anderson, 1974). Sex steroid hormones can also bind to other proteins such as lipoproteins (Leszcynski and Schafer, 1991). Thus, the final concentration of sex steroid hormones that endothelial cells are exposed to is partly determined by the concentration of various proteins and other steroids present.

The primary products of estrogen biosynthesis are estradiol and estrone with estriol being a metabolite of these. Plasma estradiol and estrone levels in premenopausal women vary with the menstrual cycle. In the early follicular phase the plasma levels of each is $40-60 \mathrm{\rho g} / \mathrm{ml}\left(0.15-0.20 \times 10^{-9} \mathrm{M}\right)$, while in the late follicular phase the plasma estradiol level reaches $200-400 \rho g / m l$ $\left(0.73-1.5 \times 10^{-9} \mathrm{M}\right)$ and the plasma estrone level reaches $170-200 \rho \mathrm{g} / \mathrm{ml}$ $\left(0.62-0.73 \times 10^{-9} \mathrm{M}\right)$. In the luteal phase the plasma estradiol level is approximately $190 \rho \mathrm{g} / \mathrm{ml}\left(0.7 \times 10^{-9} \mathrm{M}\right)$ and the plasma estrone level is 30-70 $\rho \mathrm{g} / \mathrm{ml}\left(0.1-0.26 \times 10^{-9} \mathrm{M}\right)$ (Christensen et al, 1982). In contrast, in postmenopausal women the plasma estradiol range is $5-20 \rho \mathrm{g} / \mathrm{ml}(0.02-0.07 \mathrm{x}$ 10-9 M) (Christensen et al, 1982; Judd et al, 1974) while the plasma estrone range is $30-70 \mathrm{\rho g} / \mathrm{ml}\left(0.11-0.26 \times 10^{-9} \mathrm{M}\right)$. Lonning et al (1989) also showed substantial random variation in plasma levels of estrogens and androgens during the day in post menopausal women. In men the plasma estradiol range is $25-45 \mathrm{\rho g} / \mathrm{ml}\left(0.09-0.165 \times 10^{-9} \mathrm{M}\right)$ (Griffin and Wilson, 1989) and the plasma estrone range is $25-90 \mathrm{\rho g} / \mathrm{ml}\left(0.09-0.33 \times 10^{-9} \mathrm{M}\right)$. In pregnancy, the estrone level reaches $2-30 \mathrm{ng} / \mathrm{ml}\left(7-110 \times 10^{-9} \mathrm{M}\right)$ and the estradiol level reaches 6-30 $\mathrm{ng} / \mathrm{ml}\left(22-110 \times 10^{-9} \mathrm{M}\right)$ at term (Buster and Simon, 1989). Plasma progesterone levels in premenopausal women vary from 1-25 $\eta \mathrm{g} / \mathrm{ml}$ $\left(3-80 \times 10^{-9} \mathrm{M}\right)$ (Carr and Wilson, 1989) while in men and post-menopausal women the level is less than $2 \eta \mathrm{g} / \mathrm{ml}\left(6 \times 10^{-9} \mathrm{M}\right)$ (Wilson and Foster, 1985). 
In pregnancy, the plasma progesterone level reaches 100-300 $\mathrm{ng} / \mathrm{ml}$ (318$964 \times 10^{-9} \mathrm{M}$ ) at term (Buster and Simon, 1989).

It has also been shown that the serum concentration of exogenous sex steroids can vary enormously. Stadel et al (1980) showed that when women were given the identical oral contraceptive (mestranol) the serum concentration of ethinyl estradiol varied by $72 \%$ which was not explained on the basis of time since ingestion of the last oral contraceptive, day of menstrual cycle, age, weight, blood pressure, cigarette consumption, alcohol consumption, diurnal variation or lifetime use of oral contraceptives. The mean blood level of ethinyl estradiol was $83.3 \pm 39.7 \rho \mathrm{g} / \mathrm{ml}$ (range 0.06-0.86 $\left.\times 10^{-9} \mathrm{M}\right)$. This variability in serum estradiol level is also seen during estrogen replacement therapy. The serum levels of estrone and estradiol obtained with estrogen replacement therapy depend on the route of administration and type of estrogen used. For example, women treated with transdermal estradiol (100 $\mu \mathrm{g} /$ day) ("Estraderm", Ciba-Geigy) were found to have estradiol levels of $0.5 \pm 0.18 \times 10^{-9} \mathrm{M}$ and estrone levels of $0.64 \pm 0.06 \times 10^{-9}$ $M$, while women administered the equivalent oral form of estradiol $2 \mathrm{mg} /$ day ("Progynova", Schering, Weesp) had serum estradiol levels of $0.17 \pm 0.03 \times$ $10^{-9} \mathrm{M}$ and estrone concentrations of $1.29 \pm 0.2 \times 10^{-9} \mathrm{M}$ (Van Erpecum et al, 1991). The estrone levels during oral post-menopausal replacement therapy exceed those achieved with transdermal estrogen therapy, and both exceed the levels observed in premenopausal women (Powers et al, 1985). Some forms of estrogen replacement therapy (e.g. ethinyl estradiol $0.05 \mathrm{mg} /$ day) have also been shown to increase the serum concentration of sex hormone binding globulin (Helgasen et al, 1982) which may in turn alter the amount of free estradiol. Importantly, the concentrations of androgens and estrogens vary in different vascular systems (Judd et al, 1974). For instance, Judd et al (1974) showed that the mean concentrations of testosterone and $17 \beta$ - 
estradiol were significantly higher in the ovarian vein than in a peripheral vein in post-menopausal women.

Thus, in women, endothelial cells may be exposed to a wide range of concentrations of sex steroids depending on a number of factors, including whether the person is pre or post-menopausal, is receiving exogenous sex steroids, how those exogenous steroids are administered, the ability of the individual to metabolize sex steroids and the presence of other disease states which may influence the amount of unbound sex steroid present in the circulation.

The studies reported in this thesis have therefore utilized a range of concentrations of $17 \beta$-estradiol and progesterone. The lower concentrations of $17 \beta$-estradiol (10-8-10-10 $\mathrm{M}$ ) correspond to serum levels of estradiol seen in menstruating women and women receiving hormone replacement therapy, while the higher concentrations $\left(10^{-7}-10^{-8} \mathrm{M}\right)$ correspond to levels seen in pregnancy. The highest concentration $\left(10^{-5} \mathrm{M}\right)$ is in the pharmacological range. The lower concentrations of progesterone $\left(10^{-8-10^{-9}} \mathrm{M}\right)$ correspond to premenopausal serum levels while the higher concentrations of progesterone $\left(10^{-6}-10^{-7} \mathrm{M}\right)$ correspond to serum levels seen in pregnancy. Progesterone $\left(10^{-5} \mathrm{M}\right)$ is in the pharmacological range.

\subsubsection{Effect of 17 1 -estradiol and progesterone on cell proliferation}

The effects of sex steroids on cell proliferation have been extensively studied in a number of normal and cancer cell lines. Estrogen causes cell proliferation but the mechanism of action is a matter of debate (Gorski and Gannon, 1976; Soto and Sonnenschein, 1987). Estrogen has been reported to enhance proliferation through a direct interaction with the target cell (Gorski and Gannon, 1976; Rhee et al, 1978; Katzenellenbogen, 1980; Ernst 
et al, 1988; Westley and Rochefort, 1980) as described in Section 1.5.3.1. Autocrine stimulation of cell proliferation by estrogen and progesterone has been demonstrated in cancer cell lines (Dickson et al, 1986a: Murphy et al, 1987; Nishizawa et al, 1988). In breast cancer cell lines a strong correlation between the growth of breast cancers and their estrogen receptor content has been shown (Edwards et al, 1979). Autoregulation of steroid receptors by steroids has also been demonstrated (Okret et al, 1986).

In vivo, 17 -estradiol has been shown to induce n-myc and c-myc protooncogene expression in the rat uterus. C-myc is thought to be important in mediating the mitogenic signal in response to a number of growth factors (Murphy et al, 1987). Weisz et al (1988) found that estrogen induced c-fos and c-myc mRNA in the uterus. Since estrogen causes uterine cells to proliferate, Weiss et al (1988) suggested that a mechanism for mitogenesis by estrogen may be through the interaction of the estrogen-receptor complex with the c-fos regulatory region. Activation of c-myc was thought to be secondary to c-fos activation (Weisz and Brescuani, 1988). Santos et al (1988) found estradiol (10-9 M) had a biphasic effect on c-myc protooncogene mRNA expression in MCF-7 cells. There was an increase in mRNA within $1 \mathrm{~h}$ returning to baseline after 3-6 h, and increasing again after $24 \mathrm{~h}$ of exposure. Addition of cycloheximide inhibited the estradiol effect suggesting a post transcriptional mechanism for estradiol's action (Santos et al, 1988).

The interaction of the estrogen-receptor complex with nuclear acceptor sites on the chromatin may result in the synthesis and /or release of growth factor (s) which, in turn, could cause proliferation of the target cell in an autocrine fashion (Vignon et al, 1986; Nishizawa et al, 1988; Cavailles et al, 1988). Possible autocrine stimulation of $17 \beta$-estradiol responsive cells has been supported by the finding that transforming growth factor- $\alpha$ and transforming growth factor- $\beta$ are present in the conditioned medium obtained 
by culturing human breast cancer cell lines, and that the synthesis and secretion of these transforming growth factors was shown to be dependent on $17 \beta$-estradiol (Dickson et al, 1986a). Estrogen has been shown to induce pro-cathepsin D secretion from MCF-7 (a human mammary adenocarcinoma cell line) (Cavailles et al, 1988) and increase insulin-like growth factor-I mRNA expression in liver and uterus of rats, which is important in growth (Murphy and Friesen, 1988). 17 $\beta$-estradiol has also been shown to regulate epidermal growth factor receptors in the uterus and MCF-7 cells suggesting a role for epidermal growth factor in estrogen mediated growth (Lingham et al, 1988; Gardner et al, 1989; Berthois et al, 1989). This may be due to the fact that human estrogen receptors (Greene et al, 1986), human glucocorticoid receptors (Hollenberg et al, 1985) and progesterone receptors (Jeltsch et al, 1986) share a strong sequence homology with the v-erb-A oncogene, known to potentiate the action of v-erb-B, the latter being homologous with the epidermal growth factor receptor (Green et al, 1986).

Studies of estrogen receptors in MCF-7 cells have shown that the receptor may not be located at all times in a single subcellular compartment but may exist in a dynamic equilibrium between the plasma membrane, cytoplasm and nucleus (Parikh et al, 1987). However, many cell lines established from estrogen-sensitive tumours show estrogen-dependent growth in vivo, but not in vitro (Soule and McGrath, 1980). This dichotomy has led to another model in which an estrogen-induced growth factor (estromedins) stimulates adjacent (Cunha et al, 1983; Soto and Sonnenschein, 1987) or remote cells (Sirbasku et al, 1982), by so called paracrine or endocrine control, respectively. An indirect negative hypothesis that estrogen may inactivate the action of a growth-inhibiting factor present in serum has also been proposed (Soto and Sonnenschein, 1984; 1985).

Estradiol has been shown to stimulate proliferation of cell lines (e.g. a breast 
cancer cell line, ZR-75-1) at low concentrations (Darbre et al, 1983) but suppress to their growth at high concentrations in vitro. The latter effect has been demonstrated in endometrial cells (Ishiwata et al, 1977), cultures of bovine chrondocytes (Mackintosh and Mason, 1988), smooth muscle cells (Rhee et al, 1978), MCF-7 cells and HeLa cells (Seegers et al, 1989). Estrogen has also been shown to have growth inhibitory effects on some cell lines at low concentrations (Sato et al, 1984; Mactintosh and Mason, 1988). This led Nishizawa et al (1988) to propose that there are two pathways, one for stimulation and the other for inhibition, both induced by estrogen receptormediated events. Estradiol (10-7 $\mathrm{M}$ and $10^{-9} \mathrm{M}$ ) has been shown to downregulate estrogen receptors by suppression of receptor mRNA in MCF-7 cells with a maximal effect after $72 \mathrm{~h}$ (Saceda et al, 1988; Ree et al, 1989), while estradiol (10-10-10-11 M) causes an increase in mRNA for estrogen receptors (Ree et al, 1989). Estradiol (10-7-10-11 M) has also been shown to increase the mRNA level for progesterone receptors in the same cell line (Ree et al, 1989).

Progesterone has been shown to inhibit cell proliferation at high concentrations in a number of cell lines in vitro, including endometrial cells and epithelial cells, and to have no effect at low concentrations (Ishiwata et al, 1977; Cullingford and Pollard, 1988). The effects of progesterone are thought to be mediated via specific receptors which transfer the hormone to its active site in the nucleus. In several tissues including rat adipose tissue (Gray and Wade, 1979) the formation of such receptors requires the presence of estrogenic activity.

Estrogen and progesterone receptors have not always been detected on cell lines responsive to these steroids (Mactintosh and Mason, 1988). This may be due to these steroids binding to other receptors with which they share homology. For example, progesterone has been shown to have effects 
on cells following binding to corticosteroid hormone receptors (Dewey and Highfield, 1976). In contrast, human macrophages have been shown to have estrogen receptors but they are not stimulated to divide by $17 \beta$-estradiol (Gotzos et al, 1981). It therefore may be possible for estrogens and progestins to influence cell function through other receptors or perhaps by receptor independent means.

In summary, estrogen and progesterone appear to influence cellular events by binding to specific intracellular receptors as described in Section 1.5.3.1. The magnitude of the biologic effects of the hormones is determined by both the concentration of the hormone and by the receptor number (Kalinyak et al, 1987).

\subsubsection{Factors influencing the proliferative response of cells to $17 \beta$-estradiol}

\section{and progesterone}

The proliferative effect of sex steroids in vitro depends on cell density, concentration of serum and the presence or absence of other hormones. Nishizawa et al (1988) showed in B-1 cells, a mouse transformed Leydig cell line, that the proliferative effect of $17 \beta$-estradiol depended on cell density. At high cell density the proliferative effect was much less than when cells were plated at low density. The same effect was also demonstrated by Jozan et al (1985) in MCF-7 cells. In in vitro studies, the concentration of serum in the medium can influence the proliferative effect of sex steroids. Gray et al (1987) showed that $17 \beta$-estradiol $\left(10^{-10} \mathrm{M}\right)$ had no significant influence on proliferation of cultured UMR106 cells, a clonal osteoblastic cell line, in the presence of $5 \%$ FCS. However, in the absence of FCS, $17 \beta$-estradiol (10-8. 10-10 M) inhibited cell proliferation. Nishizawa et al (1988) showed that while $17 \beta$-estradiol $\left(10^{-8} \mathrm{M}\right)$ increased the proliferation of B-1 cells two-fold in the presence of $10 \%$ serum, it increased proliferation up to five-fold in the 
absence of serum. Fischer-Dzoga et al (1983) showed that estradiol (7.3 $\mathrm{x}$ $10^{-8} \mathrm{M}$ ) inhibited the proliferative effect of hyperlipemic serum on rabbit aortic medial cells but did not alter proliferation in the presence of $10 \%$ serum. Van der Burg et al (1988) demonstrated that the mitogenic effect of $17 \beta$-estradiol on MCF-7 cells was minimal in the absence of serum and that insulin in the culture medium was required for mitogenic activity.

Little is known about the effects of other hormones on the response of cells to estrogen and progesterone. Gray et al (1987) showed that 1,25dihydroxyvitamin $D_{3}$ antagonized the response of cultured UMR106 cells to 17k-estradiol. Nishizawa et al (1988) found that simultaneous addition of dexamethasone $\left(10^{-8} \mathrm{M}\right)$ and testosterone $\left(10^{-10} \mathrm{M}\right)$ did not alter the proliferative effect of $17 \beta$-estradiol on B-1 cells.

\subsubsection{Effect of steroids on endothelial cell proliferation}

The effect of sex steroids on endothelial cell proliferation has been reviewed in Section 1.5.3.2.

Steroids other than sex steroids also have an effect on endothelial cell proliferation. Several investigators have reported inhibition of endothelial cell proliferation by hydrocortisone or cortisone and heparin (Sakamuto et al, 1987, Folkman et al, 1983; Cariou et al, 1988). Glucocorticoids (1 $\mu \mathrm{M})$ have been found to inhibit cellular proliferation through a rapid decrease in the steady-state mRNA levels of the protooncogene c-myc (Vedeckis et al, 1987). Maca et al (1978) found that exposure of endothelial cells to glucocorticoids (in particular, dexamethasone) for 24 to $48 \mathrm{~h}$ changed their morphology but not their proliferative response. 


\subsubsection{Characterization and regulation of PDGF release from endothelial cells}

One mitogen produced by endothelial cells which has been shown to have an important role in atherosclerosis is PDGF (Ross, 1981c) (refer to Section 1.3.1.3). Endothelial cells express the genes for PDGF-A and PDGF-B both in vivo and in vitro and at similar levels (Barrett et al, 1984; Jaye et al, 1985; DiCorleto and Bowen-Pope, 1983; Barrett and Benditt, 1988, Hermansson et al, 1988; Suzuki et al, 1989). In vitro, expression of PDGF-B is found in proliferating but not in quiescent endothelial cells (Barrett et al, 1984; Jaye et al, 1985). Barrett et al (1984) found that confluent bovine aortic or HUV endothelial cells in vivo possess very low levels of mRNA for PDGF, whereas cultured endothelial cells from these sources contained an 83-fold (bovine) or 10-fold (HUV) increase in mRNA for PDGF, and formed PDGF on a continuous basis in culture (DiCorleto and Bowen-Pope, 1983). Thus Ross (1986) suggested that endothelial cells in culture were in an "injured state". Northern blot analysis of HUV endothelial cells transcribing the genes shows a major B chain transcript of about 3.6 kilobases $(\mathrm{kb})$ whereas A chain mRNA appears as three species, sized 1.7, 2.4 and $2.8 \mathrm{~kb}$, respectively (Collin et al, 1987; Barrett et al, 1984; Gay and Winkles, 1990). The gene for the A chain is present on chromosome 7 while the B-chain gene is on chromosome 22 (Bonthron et al, 1988; Ross, 1989).

The amount of mRNA for each chain of PDGF has been shown to vary with the stimulus. Gay and Winkles (1990) found that aFGF transiently increased the level of PDGF A-chain mRNA but not PDGF B-chain mRNA in endothelial cells within $2 \mathrm{~h}$. aFGF induction of PDGF A-chain mRNA expression occurred in the presence of cycloheximide, a protein synthesis inhibitor, but was inhibited by an RNA synthesis inhibitor actinomycin D. Phorbol 12-myristate 13-acetate (PMA), thrombin, IL-1, IL-6 and TNF- $\beta$ also increase both PDGF A- 
chain and PDGF B-chain mRNA levels in endothelial cells (Starksen et al, 1987; Gay and Winkles, 1990). However, Starksen et al (1987) showed that phorbol 12-myristate 13-acetate was most efficient in increasing PDGF-B chain mRNA while TGF- $\beta$ was most efficient for $A$ chain mRNA induction in human microvascular endothelial cells (Starksen et al, 1987). The effect of these agents could be blocked by agents that elevate CAMP. DiCorleto and Fox (1988) found that PDGF-B chain mRNA levels were not altered by oxidized or acetylated LDL although they inhibit PDGF release. They suggested post transcriptional regulation of PDGF production. Thus, secretion of PDGF may not require either protein synthesis or RNA synthesis (Gajdusek et al, 1986; Harlan et al, 1986). PDGF does not appear to be derived from a stored precursor pool since endothelial cell lysates contain little growth factor activity (Gajdusek et al, 1986; Harlan et al, 1986; Fox and DiCorleto, 1984). This suggests that secretion may involve modification of an inactive, stored precursor (Newby and Henderson, 1990).

The PDGF-like mitogen secreted by endothelial cells (DiCorleto and BowenPope, 1983; Barrett et al, 1984; Fox and DiCorleto, 1984; Vlodasky et al, 1987a; b; Sitaras et al, 1987) is predominantly PDGF-AA with a minor fraction being PDGF-AB (Gay and Winkles, 1990). PDGF synthesis is sensitive to changes in $\mathrm{pH}$ of the medium and is maximal at $\mathrm{pH}$ 8.5. The PDGF-like proteins secreted by endothelial cells have molecular weights of 31 and $35 \mathrm{kd}$ but under reducing conditions are converted to 15 and $16 \mathrm{kd}$ forms (Sitaras et al, 1987). Endothelial cell injury by phorbol esters or endotoxin results in an increased PDGF-like protein production (Fox and DiCorleto, 1984; DiCorleto and Chisolm, 1986). Fox and DiCorleto (1984) found that the increase in production of PDGF-like protein was accompanied by morphological changes in endothelial cells in culture. Other factors shown to increase PDGF release by endothelial cells include IL-1 (DiCorleto et al, 1983; Ross, 1987; Suzuki et al, 1989), TNF and LPS (Hajjar et al, 1987; Suzuki et al, 1989), thrombin and 
factor X (Gajdusek et al, 1986; Harlan et al, 1986; Daniel et al, 1986; Starksen et al, 1987), aFGF (Gay and Winkles, 1990), and TGF- $\beta$ (Starksen et al, 1987), while acetylated and oxidized LDL (but not native LDL) (Fox and DiCorleto, 1985; Fox and DiCorleto, 1986; DiCorleto and Fox, 1988) and interferon- $\gamma$ (Suzuki et al, 1989) decrease PDGF release from endothelial cells. Stimulation of endothelial cells to release PDGF-like proteins leads to two phases of secretion with a first peak at 3-4 $\mathrm{h}$ and a second peak at 15-17 $\mathrm{h}$ (Daniel et al, 1986; Gajdusek et al, 1986; Hajjar et al, 1987).

\subsubsection{Effect of steroids on expression of PDGF in cells}

Little is known about sex steroid regulation of PDGF. Bronzert et al (1987) found that the secretion of PDGF-like activity by MCF-7 cells was stimulated by $17 \beta$-estradiol $\left(10^{-8} \mathrm{M}\right)$ after 3 days exposure and that $17 \beta$-estradiol $\left(10^{-8}\right.$ M) induced a 2-3 fold increase in expression of MRNA for PDGF-A and PDGFB in these cells. Syms et al (1984) and Norris et al (1984) demonstrated in a smooth muscle tumour cell line that glucocorticoids can inhibit cell cycle progression by inhibiting the expression of $\mathrm{C}$-sis poly $\mathrm{A}+$ and that PDGF can reinitiate cell cycle progression. In contrast, androgen stimulates proliferation but this cannot be altered by the addition of PDGF.

\subsubsection{Effect of PDGF on endothelial cell proliferation}

This has been discussed in Section 1.3.1.3.

\subsubsection{Characterization and expression of ECGF}

As discussed in Chapter 2 ECGF is a polypeptide which is angiogenic in vivo and causes endothelial cells to proliferate in vitro. The biological activity of ECGF is mediated by a high-affinity polypeptide receptor on endothelial 
cells and its effects are potentiated by heparin. ECGF is found in neural tissue (Jaye et al, 1986) and platelets (Miyazono et al, 1987) but not in endothelial cells to date. In neural tissue the amino acid sequence has been deduced and the ECGF gene has been shown to be located on human chromosome 5 (Jaye et al, 1986). The molecular size of ECGF mRNA is 4.8 kb (Jaye et al, 1986). Interestingly, ECGF has $30 \%$ homology with IL-1 and IL-1 is thought to be cleaved from ECGF- $\beta$ (Thomas et al, 1985; Jaye et al, 1986). IL-1 is synthesized by a wide variety of cells including endothelial cells (Dinarello, 1988). It is therefore possible that mRNA for ECGF could exist in HUV endothelial cells but require induction by some factor as has been demonstrated for PDGF-B expression in U937 cells (monocyte-macrophage cell line) (Pantazis et al, 1986) and c-fos protoncogene expression in IL-1 treated HUV endothelial cells (Colotta et al, 1988). Pantazis et al (1986) showed that phorbol esters could induce expression of PDGF-B in U937 cells and that U937 cells secreted PDGF-like proteins. Steroid induction or regulation of ECGF expression in endothelial cells has not been demonstrated to date.

The aims of the studies reported in this Chapter were confirm that HUV endothelial cells contain cytoplasmic estrogen receptors, to determine whether sex steroids influence proliferation of endothelial cells, and whether sex steroids can alter expression of mRNA for PDGF or induce the expression of ECGF in HUV endothelial cells, two important mitogens which regulate endothelial cell function and which therefore have potential roles in atherosclerosis. 


\subsection{MATERIALS}

Materials used in this Chapter have been described in Section 2.2. Additional materials are listed below.

\subsubsection{Reagents}

Agarose (Sigma Chemical Company, St Louis, USA or Seakem, FMC Bioproducts, Rockland, USA) was made up in the appropriate solution and stored at room temperature.

$1 \mathrm{~Kb}$ DNA ladder (BRL, Grand Island, NY). $(1.1 \mu \mathrm{g} / \mu \mathrm{l})$ was stored at $-20^{\circ} \mathrm{C}$.

ECGF cDNA was obtained from M Jaye, Rorer Biotechnology, PA as a 2.859 $\mathrm{kb}$ EcoRI fragment in pMJ23a. The coding region of CDNA was then amplified from the pMJ23a plasmid using polymerase chain reaction (PCR) by Dr M-C Fung, JCSMR, ANU, ACT, Australia was used to produce an oligionucleotide comprising a $0.5 \mathrm{~kb}$ fragment used as the probe template.

Formamide (Fluka, Switzerland) was deionized as described by Maniatis et al (1982).

Hind III Lambda DNA (Pharmacia, Uppsala, Sweden) was stored at $-20^{\circ} \mathrm{C}$.

Recombinant human interleukin-1 $\alpha$ (Hoffmann-La Roche Inc, New Jersey, USA) was purified from E. Coli, Lot 14993-74/102 with an activity of $3 \times 10^{6}$ units $/ \mathrm{ml}$ at a protein concentration of $20 \mu \mathrm{g} / \mathrm{ml}$ was diluted in BSS and $10 \%$ heat inactivated FCS and stored at $-20^{\circ} \mathrm{C}$. 
Membranes (Zeta-probe and Nitrocellulose) (Biorad, Richmond, Australia).

Monoclonal antibody to estrogen receptor related antigen (ER-D5 antigen) (Amersham International, Buckinghamshire, England) was stored at $4^{\circ} \mathrm{C}$.

Non-aqueous scintillant. This comprised xylene $(200 \mathrm{~L})$ and PPO $(1 \mathrm{~kg})$.

PDGF-A CDNA was obtained from C Betsholtz, Uppsala, Sweden. This is a human CDNA isolated from a human glioma library (Betsholtz et al, 1986). The vector used was pUC-13 (amp') and the insert comprised a 1.3-kb EcoRI fragment. The restriction enzyme fragment isolated for use as a probe template was 802 bp Rsa1 fragment.

PDGF-B CDNA was obtained from C Belsholtz, Uppsala, Sweden. This is a human CDNA isolated from a human glioma library. The vector used was pUC-13 (amp') and the insert comprised 2.7-kb EcoRI fragment. The restriction enzyme fragment isolated for use as a probe template was $2082 \mathrm{bp}$ Bam HI fragment.

PDGF-AB was a gift from the Ludwig Institute for Cancer Research, Melbourne, Australia. Stock solution $(5 \mu \mathrm{g} / \mathrm{ml})$ was prepared by dissolving the growth factor in $0.5 \mathrm{M}$ Acetic acid. This was stored initially at $-20^{\circ} \mathrm{C}$ and then at $4^{\circ} \mathrm{C}$.

Phenol (AR grade, Mallinckrodt, USA) was stored at $-20^{\circ} \mathrm{C}$ and made up as described by Maniatis et al (1982).

[Q-32P]-dCTP (PB 10205, $3000 \mathrm{Ci} / \mathrm{mmol}, 10 \mathrm{mCi} / \mathrm{ml}$, Amersham, UK) was stored at $-20^{\circ} \mathrm{C}$. 
Poly-L-lysine (Sigma Chemical Company, St Louis, USA). A $0.1 \%$ solution was prepared and stored at $4^{\circ} \mathrm{C}$.

\subsubsection{Solutions}

BLOTTO. A $10 \%$ stock solution was made up and stored at $4^{\circ} \mathrm{C}$. This comprised non-fat milk powder ( $10 \%$ ) (Diploma) with $0.2 \%$ azide. This was stable for 2-3 weeks.

Buffer 1 comprised $0.25 \mathrm{M} \mathrm{NaCl}, 0.24 \mathrm{M} \mathrm{NaOAC}, \mathrm{pH} 7.5$ and $0.5 \%$ SDS (see below).

Buffer $B$ comprised $10 \mathrm{mM}$ Tris- $\mathrm{HCl}, \mathrm{pH} 8,100 \mathrm{mM} \mathrm{NaCl}, 10 \mathrm{mM} \mathrm{MgCl}, 1$ $\mathrm{mM} \beta$-mercaptoethanol (B-ME) (BDH chemicals, UK) and $100 \mu \mathrm{g} \mathrm{BSA} / \mathrm{ml}$.

Caesium chloride density cushion. This comprised $5.7 \mathrm{M}$ caesium chloride (BRL, Helena Lab, Australia), and $0.025 \mathrm{M}$ sodium acetate, pH 5 which was filtered through a $0.2 \mu \mathrm{M}$ filter (Millipore).

Denhardt's solution was made up as a $50 \mathrm{x}$ stock as described by Maniatis et al (1982) comprising $1 \%$ ficoll (Pharmacia, Uppsala, Sweden), 1\% polyvinylpyrrolidone (Sigma Chemical Company, St Louis, USA) and $1 \%$ BSA, stored at $-20^{\circ} \mathrm{C}$.

EDTA (Ajax Chemical, Auburn, Australia) was made up as a $0.5 \mathrm{M}$ stock solution in DEPC-treated distilled water and stored at room temperature.

Ethidium bromide (Sigma Chemical Company, St Louis, USA). A stock solution of $10 \mathrm{mg} / \mathrm{ml}$ was made up in DEPC-treated distilled water and kept at $4^{\circ} \mathrm{C}$ in the dark. 
Gel loading buffer comprised $50 \%$ glycerol, 20 mM EDTA, $0.15 \%$ xylene cyanol (Sigma Chemical Company, St Louis, USA) and $0.15 \%$ bromophenol blue (DBH, Poole, England).

Guanidine hydrochloride. This comprised 7.5 M guanidine hydrochloride (RNA grade) (Fluka, Switzerland) and $0.025 \mathrm{M}$ sodium acetate which was autoclaved. $0.5 \mathrm{mM}$ dithiothreitol (DTT) (Sigma Chemical Company, St Louis, USA) was added to $10 \mathrm{ml}$ immediately before use.

Guanidine isothiocyanate lysis buffer (lysis buffer) comprised $4 \mathrm{M}$ guanidine isothiocyanate (RNA grade) (Sigma Chemical Company, St Louis, USA), $0.025 \mathrm{M}$ sodium citrate, pH 7, $0.5 \%$ sarcosyl (Sigma Chemical Company, St Louis, USA) and $0.5 \%$ antifoam A (Sigma Chemical Company, St Louis, USA) which was then filtered through $0.45 \mu \mathrm{m}$ filter (Millipore). $0.1 \mathrm{M} \mathrm{2-}$ mercaptoethanol ( $72 \mu \mathrm{l}$ per $10 \mathrm{ml}$ of buffer) was added immediately before use.

Hybridization and prehybridization buffer comprised $50 \%$ formamide (refer above), $5 \times$ SSC (refer below), $5 \times$ Denhardts (refer above), $0.1 \%$ SDS (refer below) and $200 \mu \mathrm{g} / \mathrm{ml}$ salmon sperm DNA (refer below) made up to the required volume with diethylpyrocarbonate (DEPC) (Sigma Chemical Company, St Louis, USA) treated water.

RNA gel buffer. A $5 \times$ buffer was made up and stored at room temperature. This comprised $20 \mathrm{mM}$ triethanolamine, $\mathrm{pH} 7.4,2.5 \mathrm{mM}$ EDTA and $2.2 \mathrm{M}$ formaldehyde ( $37 \% \mathrm{w} / \mathrm{w}$ ) (Fluka, Buchs, Switzerland). The $\mathrm{pH}$ was adjusted to 7.4 with $4 \mathrm{~N} \mathrm{HCL}$ and stored in a fume hood. 
Salmon sperm DNA (Sigma Chemical Company, St Louis, USA). A $10 \mathrm{mg} / \mathrm{ml}$ stock suspension was made up and aliquoted as described by Maniatis et al (1982) was stored at $-20^{\circ} \mathrm{C}$. The salmon sperm DNA was boiled for 5 min then placed on ice prior to use.

Sample preparation buffer comprised 4 volumes of $5 \times$ gel buffer (see above) and 10 volumes of formamide (deionized).

Sodium pyrophosphate (Sigma Chemical Company, St Louis, USA) was made up as a $10 \%$ stock solution and stored at $-20^{\circ} \mathrm{C}$.

SSC. A $20 \times$ solution was made up comprising $3 \mathrm{M} \mathrm{NaCl}$ and $0.3 \mathrm{M}$ trisodium citrate, $\mathrm{pH} 7$ as described by Maniatis et al (1982).

Sodium dodecyl sulphate (SDS) (International Biotech, New Haven, Conn). A $10 \%$ stock solution was made up and stored at room temperature.

STE buffer comprised $0.1 \mathrm{M} \mathrm{NaCl}, 1 \mathrm{mM}$ EDTA, and $10 \mathrm{mM}$ Tris, $\mathrm{pH} 7.5$ made up to $1 \mathrm{~L}$ with distilled water and stored at room temperature.

TAE was made as a $20 \times$ solution comprising $800 \mathrm{mM}$ Tris, $400 \mathrm{mM}$ sodium acetate and $20 \mathrm{mM}$ EDTA, $\mathrm{pH}$ 7.4. This was stored at room temperature.

TBE was made as a $5 \times$ solution and comprised $0.5 \mathrm{M}$ boric acid, $0.5 \mathrm{M}$ Tris and $10 \mathrm{mM}$ EDTA, pH 8 made up to $500 \mathrm{ml}$ and stored at room temperature.

TE comprised $10 \mathrm{mM}$ Tris- $\mathrm{HCl}, \mathrm{pH} 8$ and $1 \mathrm{mM}$ EDTA, $\mathrm{pH} 8$ made up to $1 \mathrm{~L}$ with distilled water and stored at room temperature. 


\subsection{METHODS}

\subsubsection{Presence of estrogen receptor-related antigen in HUV endothelial cells}

HUV endothelial cells were grown to confluence in Medium 199 (with $20 \%$ FCS, gentamicin $60 \mathrm{U} / \mathrm{ml}$ and the standard additives). Cells were removed from flasks by trypsinization and washed 3 times in Medium 199 (centrifuged $200 \times \mathrm{g}$ for $8 \mathrm{~min}$ at room temperature) before being resuspended in Medium 199 with $20 \%$ FCS. The cells were incubated at room temperature for $1 \mathrm{~h}$. Cytocentrifuge slides were made using $2 \times 10^{4} \mathrm{HUV}$ endothelial cells in Medium 199 and 20 \% FCS on poly-L-lysine coated glass coverslips from primary passage cells through to passage 8 . Slides were dried at room temperature and then stored at $-20^{\circ} \mathrm{C}$.

The staining method used was based on that described by Hsu et al (1981) using a DAKO avidin-biotin-horseradish peroxidase complex (ABC-HRP) kit (Bioscientific Pty. Ltd., Gymea, Australia). ER-D5 is a monoclonal antibody raised against cytosolic estradiol receptor from human myometrium (Amersham, Buckinghamshire, England). Briefly, the slides were incubated with $10 \%$ normal rabbit serum (CSL, Australia) for $10 \mathrm{~min}$ and the serum was then tipped off without rinsing. The ER-D5 antibody ( in 5 dilution) was added and the slides incubated overnight at $4^{\circ} \mathrm{C}$. To evaluate non-specific binding, duplicate slides were incubated with control mouse serum rather than antibody, overnight. The slides were then washed 3 times in Tris buffer (Sigma Chemical Company, St Louis, USA) ( $5 \mathrm{~min}$ for each wash) before adding the biotinylated rabbit anti-mouse antibody (1 in 200 dilution) for 30 min. The slides were then washed as described above, ABC-HRP added and the slides were then incubated for $30 \mathrm{~min}$ at room temperature. Following 3 washes with Tris buffer (each of $5 \mathrm{~min}$ ) the slides were incubated with 
diaminobenzidine tetrahydrochloride (DAB) (Sigma Chemical Company, St Louis, USA) dissolved in Tris buffer with $0.03 \%$ hydrogen peroxide for $10 \mathrm{~min}$ before the slides were washed in tap water. The cells were then counterstained with haematoxylin (Histo-Lab, Pennant Hills, Australia), dehydrated and mounted in DPX (Ajax Chemicals, Australia).

\subsubsection{Effect of sex steroids on HUV endothelial cell proliferation}

A range of concentrations of $17 \beta$-estradiol $\left(10^{-5}-10^{-10} \mathrm{M}\right)$ and progesterone $\left(10^{-5}-10^{-9} \mathrm{M}\right)$ alone and in various combinations was prepared using PRF Medium 199 (with $20 \%$ FCS and gentamicin $60 \mathrm{U} / \mathrm{ml}$ ). HUV endothelial cells at passages 9-10 (2.5 $\times 10^{3}$ /well) suspended in Medium 199 (with $20 \%$ FCS, gentamicin $60 \mathrm{U} / \mathrm{ml}$ and the standard additives) were added to gelatinized 96 well plates to which sex steroids, alcohol or Medium 199 alone were added to achieve a final volume of $100 \mu \mathrm{l}$. Following $48 \mathrm{~h}$ incubation in $5 \% \mathrm{CO}_{2}-95 \%$ air atmosphere at $37^{\circ} \mathrm{C}, 25 \mu \mathrm{l}$ of $\left[{ }^{3} \mathrm{H}\right]$-thymidine $(0.5 \mu \mathrm{Ci} /$ well) was added. After $18 \mathrm{~h}$ the cells were washed twice in Medium 199, trypsinized and harvested as described in Section 2.3.7.

A second series of experiments examined the effects of sex steroids on HUV endothelial cell proliferation in the absence of FCS to determine whether FCS contained inhibitors or steroid-binding proteins which could interfere with the effects of sex steroids. In these experiments HUV endothelial cells ( $1.25 \mathrm{x}$ $10^{4}$ /well) were suspended in PRF Medium 199 containing half the standard additives with $\left[{ }^{3} \mathrm{H}\right]$-thymidine $(0.5 \mu \mathrm{Ci} /$ well) and incubated for $18 \mathrm{~h}$ in $5 \%$ $\mathrm{CO}_{2}-95 \%$ air atmosphere at $37^{\circ} \mathrm{C}$. The cells were then washed, trypsinized and harvested as described in Section 2.3.7.

In another experiment HUV endothelial cells were continuously exposed to $17 \beta$-estradiol (10-9 $\mathrm{M})$ in either Medium 199 or alcohol $(0.05 \%)$ for $66 \mathrm{~h}$, and 
progesterone (10-9 M) or alcohol $(0.05 \%)$ was added at $0 \mathrm{~h}, 12 \mathrm{~h}, 24 \mathrm{~h}, 30 \mathrm{~h}$ and $48 \mathrm{~h}$ to assess whether the duration of HUV endothelial cell exposure to progesterone altered the effect of estradiol on proliferation. $\left[{ }^{3} \mathrm{H}\right]$-thymidine was then added $(0.5 \mu \mathrm{Ci} /$ well) and the cells were incubated for $18 \mathrm{~h}$ in $5 \%$ $\mathrm{CO}_{2}-95 \%$ air atmosphere at $37{ }^{\circ} \mathrm{C}$ before being washed, trypsinized and harvested as described in Section 2.3.7.

A further series of experiments was performed using HUV endothelial cells (passage 6 or 7) in quadruplicate to determine whether varying the concentration of ECGS between $25 \mu \mathrm{g} / \mathrm{ml}$ and $50 \mu \mathrm{g} / \mathrm{ml}$ could influence the maximal proliferative response of HUV endothelial cells to $17 \beta$-estradiol $\left(10^{-10}\right.$ M). HUV endothelial cells $\left(2.5 \times 10^{3}\right.$ cells $/$ well or $5 \times 10^{3}$ cells /well) were exposed to either $25 \mu \mathrm{g} / \mathrm{ml}$ or $50 \mu \mathrm{g} / \mathrm{ml} \mathrm{ECGS}$ in the presence or absence of $17 \beta$-estradiol $\left(10^{-10} \mathrm{M}\right.$ ) diluted in Medium 199 (with $20 \%$ FCS, gentamicin 60 $\mathrm{U} / \mathrm{ml}$, glutamine $2 \mathrm{mM}$ and heparin $20 \mathrm{U} / \mathrm{ml}$ ) for $48 \mathrm{~h}$ in $5 \% \mathrm{CO}_{2}-95 \%$ air atmosphere at $37^{\circ} \mathrm{C}$. $\left[{ }^{3} \mathrm{H}\right]$-thymidine $(0.5 \mu \mathrm{Ci} /$ well) was then added and the cells incubated for a further $18 \mathrm{~h}$ before being washed, trypsinized and harvested as described in Section 2.3.7.

\subsubsection{Isolation of total RNA from HUV endothelial cells}

\subsubsection{HUV endothelial cell culture}

HUV endothelial cells (passage 8) were grown to confluence in phenol red containing Medium 199 (with $20 \%$ FCS and gentamicin $60 \mathrm{U} / \mathrm{ml}$ ) in gelatinized $75 \mathrm{~cm}^{2}$ flasks. The medium was removed and the cells washed twice with Medium 199 before the addition of sex steroids, IL-1 $(5 \mathrm{U} / \mathrm{ml})$ or alcohol each diluted in Medium 199 (with $20 \%$ FCS and gentamicin $60 \mathrm{U} / \mathrm{ml}$ ) with the control being Medium 199 alone (with $20 \%$ FCS and gentamicin $60 \mathrm{U}$ $/ \mathrm{ml}$ ). 5 flasks were used for each stimulus to give a cell count of $4 \times 10^{7}$ cells. 
The flasks were incubated for $19 \mathrm{~h}$ at $5 \% \mathrm{CO}_{2}-95 \%$ air atmosphere at 37 ${ }^{\circ} \mathrm{C}$. The cells were then lysed by the addition of $5 \mathrm{ml}$ of lysis buffer per 75 $\mathrm{cm}^{2}$ flask (refer to Section 3.2). The lysate was then transferred to new $50 \mathrm{ml}$ centrifuge tubes and stored at $-70^{\circ} \mathrm{C}$. The purity of the cells was assessed by microscopy and immunofluorescence for vWf expression and no contaminants were seen (refer to Section 2.3).

\subsubsection{General precautions}

Disposable rubber gloves were worn to protect RNA from RNases and to protect the operator from chemicals. Glassware was baked overnight in a hot oven $\left(250^{\circ} \mathrm{C}\right)$ to destroy RNases. Solutions and buffers which did not contain RNases inhibitors were treated overnight with $0.1 \%$ DEPC and then autoclaved.

\subsubsection{Isolation of total RNA by ultracentrifugation}

Total RNA was extracted by centrifugation through caesium chloride using a method modified from Chirgwin et al (1979). The cell lysate was thawed and $30 \mathrm{ml}$ was pipetted into polyallomer tubes (Beckman 326823). This was underlayed with $5 \mathrm{ml}$ of $5.7 \mathrm{M}$ caesium chloride. The tubes were centrifuged at $70090 \times \mathrm{g}$ (average radius) for $24 \mathrm{~h}$ at $15^{\circ} \mathrm{C}$ in SW27 rotor buckets (Beckman). The DNA-containing interphase and lysis buffer were removed by pipette and the caesium chloride cushion removed by inversion of the tube. The tubes were then cut with a heated scalpel blade below the interphase level (approximately $0.5 \mathrm{~cm}$ from the bottom of the tube). The RNA pellet was rinsed with cold absolute ethanol and allowed to drain in the inverted position for $10 \mathrm{~min}$. The RNA pellet in each tube was resuspended in $0.4 \mathrm{ml}$ of DEPC-treated distilled water and transferred into a Eppendorf tube. 
To this was added $2.6 \mathrm{ml}$ of $7.5 \mathrm{M}$ guanidine hydrochloride solution (refer above), $75 \mu \mathrm{l}$ of $1 \mathrm{M}$ acetic acid and $1.5 \mathrm{ml}$ of cold absolute ethanol. The solution was mixed by vortexing briefly and the RNA allowed to precipitate overnight at $-20^{\circ} \mathrm{C}$. RNA was then pelleted by centrifugation $(10000 \mathrm{x} \mathrm{g}$ for $15 \mathrm{~min}$ at $4^{\circ} \mathrm{C}$ ) in an Eppendorf centrifuge. The pellet was resuspended using $1.5 \mathrm{mls}$ (in $500 \mu \mathrm{l}$ aliquots) of DEPC-treated distilled water. To this was added $100 \mu \mathrm{l}$ of $3 \mathrm{M}$ sodium acetate and $4 \mathrm{ml}$ of cold absolute ethanol and the RNA allowed to precipitate at $-20^{\circ} \mathrm{C}$ overnight. RNA was then pelleted by centrifugation $\left(10000 \times \mathrm{g}\right.$ for $15 \mathrm{~min}$ at $4^{\circ} \mathrm{C}$ ). The pellet was resuspended in $1 \mathrm{ml}$ of DEPC-treated distilled water. $5 \mu \mathrm{l}$ of each sample was taken and dissolved in $1 \mathrm{ml}$ of distilled water to quantitate the amount of RNA in each preparation by measuring the absorbance of the sample at $260 \mathrm{~nm}$ using the method described by Maniatis et al (1982).

\subsubsection{Assessment of integrity of RNA}

The method was modified from that described by McGookin (1984). A $1 \%$ agarose gel was prepared in TBE buffer to which ethidium bromide (final concentration $0.5 \mu \mathrm{g} / \mathrm{ml}$ ) was added (refer Section to 3.2). $2 \mu \mathrm{g}$ of each RNA sample ( $9 \mu$ sample and $1 \mu \mathrm{l}$ gel loading buffer) was loaded with the $1 \mathrm{~kb}$ DNA ladder used as a size marker. The sample were electrophoresed dry for 10 min at $100 \mathrm{~V}$ and then the gel was covered with TBE and electrophoresed for a further $2 \mathrm{~h}$ at $80 \mathrm{~V}$ or until the dye front was $3 / 4$ of the way down the gel. The gel was then photographed to assess the presence of the $18 \mathrm{~s}$ and $28 \mathrm{~s}$ rRNAs in the samples.

\subsubsection{Northern blot preparation}

Single stranded RNA was separated on the basis of molecular size using the formaldehyde/ agarose gel denaturing electrophoresis system described 
by Davis et al (1986). The method used for Northern blot preparation was based on the method of Gurney (1984). A $1 \%$ agarose gel was prepared by melting $0.3 \mathrm{~g}$ of agarose in $24 \mathrm{ml}$ of DEPC-treated distilled water in an RNasefree flask. After cooling to $60^{\circ} \mathrm{C}, 6 \mathrm{ml}$ of $5 \times$ RNA gel buffer (refer to Section 3.2) was added. The gel was poured into an RNase-free horizontal minigel former (Pharmacia, Uppsala, Sweden) and allowed to set for $1 \mathrm{~h}$ before assembling in an electrophoresis chamber (Pharmacia, Uppsala, Sweden) containing $1 \times$ RNA gel. RNA samples $(20 \mu \mathrm{g})$ were prepared by overnight precipitation at $-20^{\circ} \mathrm{C}$ made up to $100 \mu$ with DEPC-treated distilled water to which $10 \mu \mathrm{l}$ of $3 \mathrm{M}$ sodium acetate and $250 \mu \mathrm{l}$ of cold absolute ethanol was added. The samples was then pelleted by centrifugation $(10000 \times \mathrm{g}, 30 \mathrm{~min}$ at $4^{\circ} \mathrm{C}$ ) in an Eppendorf centrifuge. The ethanol solution was decanted and the Eppendorf tube inverted and dried. The pellet was resuspended with $4 \mu \mathrm{l}$ of DEPC-treated distilled water to which $9.3 \mu$ l of freshly made sample preparation buffer was added (refer to Section 3.2). The RNA was denatured by heating to $65^{\circ} \mathrm{C}$ for $15 \mathrm{~min}$. Labelled DNA size markers were denatured under identical conditions. $1 \mathrm{~Kb}$ DNA ladder marker and a Hind III Lambda DNA were used as markers. $1 \mu \mathrm{l}$ of loading buffer (refer to Section 3.2) was added to each sample and then placed on ice. The samples were loaded dry and run for $15 \mathrm{~min}$ at $70 \mathrm{~mA}$ before the gel was covered with $1 \times$ RNA gel buffer and run at $40 \mathrm{~mA}$ for a further $4 \mathrm{~h}$ or until the marker band had migrated $2 / 3$ of the way along the gel. The gel was removed and the marker bands removed. These were stained in ethidium bromide $(0.5 \mu \mathrm{g} / \mathrm{ml})$ and destained in distilled water and then photographed under UV light. The gel was washed twice in $10 \times$ SSC in preparation for blotting as described by Maniatis et al (1982). A piece of Whatman 3 MM (Whatman Inc. New Jersey, USA) filter, soaked in $10 \times$ SSC, was suspended on a glass plate above a plastic tray containing $10 \times$ SSC. The filter ends were allowed to act as a wick from the reservoir. The gel was carefully laid (sample well openings face down) on the top of the filter paper and overlaid with nitrocellulose prewet in 
$10 \times$ SSC. Two pieces of wet Whatman $3 \mathrm{MM}$ were placed on top, followed by piles of paper towels ( $8 \mathrm{~cm}$ thick) and weights. The transfer of RNA to the nitrocellulose was allowed to proceed overnight $(16 \mathrm{~h})$ at room temperature by capillary action. The nitrocellulose was blotted dry and then placed between 2 sheets of Whatman $3 \mathrm{MM}$ filter paper and dried for $2 \mathrm{~h}$ at $80^{\circ} \mathrm{C}$ in a vacuum oven.

\subsubsection{RNA slot blots}

RNA was blotted onto nitrocellulose filters (Schleicher and Schuell) as outlined by Davis et al (1986). A slot blot apparatus was used (Minifold RII, Schleicher and Schuell, W Germany) and assembled as outlined by the manufacturer. A nitrocellulose filter and Whatman 3 MM paper were used soaked in DEPC-treated distilled water and then in $10 \times$ SSC. The slots were rinsed with $20 \times$ SSC. Dilutions of RNA in DEPC-treated distilled water were made. To this 1 volume of deionized formamide and 2 volumes of $20 \times$ SSC were added. The samples were then incubated at $65^{\circ} \mathrm{C}$ for $15 \mathrm{~min}$ before being placed on ice for $5 \mathrm{~min}$. 4 volumes of $10 \times$ SSC were loaded into each well and then the sample was added to the slots. A vacuum source was connected to blot the RNA onto the nitrocellulose. The apparatus was disassembled and the filter removed and baked in a gel dryer at $80^{\circ} \mathrm{C}$ for $2 \mathrm{~h}$ between 2 sheets of Whatman 3 MM filter paper.

\subsubsection{Prehybridization and Hybridization of northern and slot blots}

\subsubsection{Prehybridization}

Nitrocellulose filters from the slot blot or northern preparations were prehybridized overnight by gentle agitation at $50{ }^{\circ} \mathrm{C}$ in $15 \mathrm{ml}$ of 
prehybridization buffer (refer to Section 3.2) followed the method of Maniatis et al (1982).

\subsubsection{Probe preparation by random primer synthesis}

PDGF-A and PDGF-B CDNA probes were 5'end labelled with 32P using an Amersham multiprime DNA labelling kit (RPN 1601, Amersham, UK). The DNA fragment was dissolved $(25 \mathrm{\eta g} / \mathrm{ml}$ ) in $10 \mathrm{mM}$ Tris, $\mathrm{pH} 8,1 \mathrm{mM}$ EDTA solution. The DNA was denatured by heating to $95^{\circ} \mathrm{C}$ for $2 \mathrm{~min}$. After rapid chilling in iced water, $10 \mu \mathrm{l}$ of buffer ( $1 \mathrm{mM}$ each of dGTP, dTTP, dATP), $5 \mu \mathrm{l}$ of primer, $50 \mu \mathrm{Ci}[\alpha-32 \mathrm{P}] \mathrm{dCTP}$ and 10 units of DNA polymerase 1 were added to the DNA. After $2 \mathrm{~h}$ incubation at $37^{\circ} \mathrm{C}$ the labelled DNA was denatured by heating to $95^{\circ} \mathrm{C}$ for $2 \mathrm{~min}$ and then chilled on ice. 32P-labelled probe was then harvested by the spun column method (Maniatis et al, 1982). Briefly the bottom of a $1 \mathrm{ml}$ disposable syringe was plugged with sterile glass wool, and a $0.9 \mathrm{ml}$ column of Sepadex G-50 (Pharmacia, Uppsala, Sweden) in STE (refer above) packed and washed by centrifugation ( $1600 \times \mathrm{g}$ for $4 \mathrm{~min}$ at room temperature). DNA solution $(0.1 \mathrm{ml})$ was applied to the top of the column, the tube centrifuged ( $1600 \times \mathrm{g}$ for $4 \mathrm{~min}$ at room temperature) and $0.1 \mathrm{ml}$ of column effluent, containing the $32 \mathrm{P}$-labelled probe, was collected in an Eppendorf tube. Alternatively a NICK column (Pharmacia, Uppsala, Sweden) was used to collect the purified DNA. The procedure yielded specific activities of 3-6 $\times 10^{8} \mathrm{cpm}$ per $\mu \mathrm{g}$ of probe. This was assessed by taking $5 \mu$ of probe and counting its radioactivity on a $\beta$-scintillation counter.

ECGF probe was made by adding 10 pmoles of the oligonucleotide to $1 \mu \mathrm{l}$ of T4 polynucleotide kinase (Pharmacia, $5.3 \mathrm{U} / \mathrm{ml}$ ), $10 \mu \mathrm{l}$ (20 pmoles) of $\gamma$ 32P ATP (Amersham, $10 \mathrm{mCi} / \mathrm{ml}, 5000 \mathrm{Ci} / \mathrm{mmole}$ ) and $2 \mu \mathrm{l}$ of $10 \times$ kinase buffer $\left(670 \mathrm{mM}\right.$ Tris- $\mathrm{HCl}, \mathrm{pH} 8.3,100 \mathrm{mM} \mathrm{MgCl}_{2}$ and $100 \mathrm{mM}$ dithiothreitol (DTT)) made up to $20 \mu$ with DEPC-treated water. This was incubated at 37 
${ }^{\circ} \mathrm{C}$ for $30 \mathrm{~min}$, and then heated at $65^{\circ} \mathrm{C}$ for $5 \mathrm{~min}$ before being put through a NICK column as described below. The specific activities of the probes were assessed as described above.

\subsubsection{Hybridization}

The radiolabelled probe was boiled for $2 \mathrm{~min}$, chilled on ice and added to 15 $\mathrm{ml}$ of fresh prehybridization fluid which contained the filters. This was hybridized for $18 \mathrm{~h}$ at $50^{\circ} \mathrm{C}$ with gentle agitation.

\subsubsection{Washing}

Following hybridization the filters were washed at room temperature firstly rinsing in $2 \times$ SSC, and then sequentially in $2 \times$ SSC, $0.1 \%$ SDS for $15 \mathrm{~min}, 0.5$ $x$ SSC with $0.1 \%$ SDS for $30 \mathrm{~min}$, and $0.1 \times$ SSC with $0.1 \%$ SDS for $30 \mathrm{~min}$. For high background (assessed by placing a radioactive counter near the filter) a further wash with $0.1 \times$ SSC with $1 \%$ SDS for 30 min at $60^{\circ} \mathrm{C}$ was undertaken. Excess washing solution was blotted from the filters using Whatman 3 MM filter paper. Hybridized filters were covered with Saran Wrap (Dow Chemical Company, Indianapolis, Indiana) and secured inside an autoradiograph cassette. The filters were exposed to X-ray film (Kodak XAR5) with an intensifying screen (DuPont Lightning plus) at $-70^{\circ} \mathrm{C}$. The film was processed in Kodak RP X-omat rapid developer and fixer.

\subsubsection{Repeated hybridization of blots}

The filters were washed in $5 \mathrm{mM}$ Tris, $\mathrm{pH} 8,0.2 \mathrm{mM}$ EDTA, $0.05 \%$ pyrophosphate, and $0.1 \%$ Denhardts (refer above) made up to $200 \mathrm{ml}$ with DEPC-treated water for $2 \mathrm{~h}$ at $65^{\circ} \mathrm{C}$. The filter was then sealed in a bag and exposed to X-ray film (Kodak XAR-5) with an intensifying screen (DuPont 
Lightning plus) at $-70^{\circ} \mathrm{C}$ overnight to ensure that all of the probe had been removed. The filter was then stored between to pieces of Whatman $3 \mathrm{MM}$ paper at room temperature.

\subsubsection{Preparation of specific DNA probes}

PDGF-A. The pUC-13 (amp') plasmid containing CDNA fo the PDGF-A was digested with $15 \mathrm{U} / \mu$ l of Rsa I (Boehringer Mannheim, West Germany) in 400 $\mu l$ of digest buffer (low salt buffer with $100 \mathrm{mM} \beta$-mercaptoethanol, $1 \mathrm{M} \mathrm{KCl}, 1$ $\mu \mathrm{g} / \mu \mathrm{l}$ of BSA and distilled water) for $1 \mathrm{~h} 45 \mathrm{~min}$ at $37^{\circ} \mathrm{C}$.

PDGF-B. The pUC-13 (amp') plasmid containing CDNA for the PDGF-B chain was digested with $16 \mathrm{U} / \mu \mathrm{l}$ of BamH I (Pharmacia, Uppsala, Sweden) in $150 \mu$ of digest buffer (buffer B, Boehringer Mannheim, West Germany) and distilled water overnight at $37^{\circ} \mathrm{C}$.

Following digestion the cleaved DNA was separated by electrophoresis on $1 \%$ agarose gel with a suitable marker (such as $1 \mathrm{~kb}$ DNA ladder) to assess whether digestion was complete. The DNA was then extracted from a low melting point agarose gel (see below) and the amount of DNA was estimated on a DNA fluorometer or by electrophoresis (see below).

\subsubsection{Isolation of DNA from low melting point agarose gels}

A $1 \%$ low melting point agarose gel in TAE buffer was prepared according to the method of Maniatis et al (1982). Following digestion of the DNA sample (see above) $15 \mu \mathrm{l}$ of loading buffer (refer Section 3.2) was added to the digest. This was loaded into the wells of the gel $(940 \mu \mathrm{l} /$ well). A $1 \mathrm{~Kb}$ DNA ladder was used for sizing the DNA fragments. The gel was electrophoresed at $100 \mathrm{~V}$ for $5 \mathrm{~min}$ and then at $50 \mathrm{~V}$ for $3 \mathrm{~h}$ at $4^{\circ} \mathrm{C}$. The gel 
was stained with ethidium bromide $(0.5 \mu \mathrm{g} / \mathrm{ml})$ for $15 \mathrm{~min}$ and then photographed under low UV (wavelength $312 \mathrm{~nm}$ ). The required band was extracted from the gel. This DNA fragment was placed into a $5 \mathrm{ml}$ polypropylene tube to which buffer I (refer above) (up to $1 \mathrm{ml}$ ) was added. The sample was allowed to stand for $5 \mathrm{~min}$ at room temperature. The buffer was removed and the gel melted at $65^{\circ} \mathrm{C}$ for about $5 \mathrm{~min}$. An equal volume of buffer I was added to the gel and stood at $65^{\circ} \mathrm{C}$ for $15 \mathrm{~min}$. The DNA was extracted with stow addition of warmed phenol (1:1 with gel volume) while vortexing. The solution was allowed to stand for $2 \mathrm{~min}$ at $65^{\circ} \mathrm{C}$ before being centrifuged ( $10000 \times \mathrm{g}, 4^{\circ} \mathrm{C}$ for $5 \mathrm{~min}$ ). $1 / 20$ volume (gel volume) of $3 \mathrm{M}$ sodium acetato and 2.5 volumes (total volume) of cold ethanol was then added. The solution was centrifuged $(10000 \times \mathrm{g}, 30 \mathrm{~min}$ at room temperature) and the pellet was resuspended in $50 \mu$ of distilled water. A 1/2 volume of 7.5 sodium acetate and 2.5 volumes of ethanol was the added. The solution was centrifuged ( $10000 \times \mathrm{g}, 30 \mathrm{~min}$ at room temperature). The pellet was washed in $70 \%$ ethanol and finally dried under vacuum.

\subsubsection{Characterization of CDNA probes and calculation of their concentration}

A $1 \%$ agarose gel was made up in TAE (refer Section 3.2). Each sample was prepared by the addition of $1 \mu \mathrm{l}$ of test sample, $9 \mu$ of distilled water and $1 \mu$ l of loading buffer. Lambda Hind III was used as a size marker ( $1 \mu$ land 2 $\mu l$ samples were used). The samples were electrophoresed dry for $5 \mathrm{~min}$ at $100 \mathrm{~V}$ and then the well were covered with TAE and the samples electrophoresed for $2 \mathrm{~h}$ at $60 \mathrm{~V}$ or until the bromophenol blue band had migrated $3 / 4$ of the way down the gel. The gel was then stained with ethidium bromide $(0.5 \mu \mathrm{g} / \mu \mathrm{l})$ for $15 \mathrm{~min}$ and photographed under UV. The concentration of DNA was determined by comparing the fluorescence intensity of the sample with the marker bands. The concentration of the $1 \mathrm{~Kb}$ DNA ladder was $0.44 \mu \mathrm{g} / \mu \mathrm{l}$. 


\subsubsection{Preparation of labelled DNA marker}

This was prepared by Lynette Preston (JCSMR, ANU, ACT, Australia). A mixture of stock plasmid DNA ( $3 \mu \mathrm{g})$ was used and digested with EcoR1 ( 3 units) in $45 \mu$ of restriction digest buffer $(100 \mathrm{mM} \mathrm{NaCl}, 50 \mathrm{mM}$ Tris- $\mathrm{HCl}, \mathrm{pH}$ $7.5,10 \mathrm{mM} \mathrm{MgCl} 2$ and $1 \mathrm{mM} \mathrm{DTT}$ ) at $37^{\circ} \mathrm{C}$ for $1 \mathrm{~h} .20 \eta \mathrm{mol}$ of dTTP, 3.3 pmol of [ $\alpha-32$ P]-dATP (Amersham) and 5 units of E.Coli DNA polymerase 1 (Klenow fragment, Bresa) were added to the digest, which was then incubated for a further $30 \mathrm{~min}$ at room temperature and then made up to 300 $\mu l$ with $100 \mathrm{mM}$ Tris- $\mathrm{HCl}, \mathrm{pH} 7.5$ and $1 \mathrm{mM}$ EDTA. The resulting end labelled DNA was stored at $-20^{\circ} \mathrm{C}$ and used as size markers on Northern gels.

\subsubsection{Effect of PDGF on HUV endothelial cell preparation}

Serial dilutions of PDGF (maximum final concentration $25 \mathrm{ng} / \mathrm{ml}$ ) in gelatinized 96 well plates was made in Medium 199 (with $20 \%$ FCS and gentamicin $60 \mathrm{U} / \mathrm{ml})$. HUV endothelial cells $\left(5 \times 10^{3}\right.$ cells $/$ well) suspended in Medium 199 (with $20 \%$ FCS, gentamicin $60 \mathrm{U} / \mathrm{ml}$, heparin $10 \mathrm{U} / \mathrm{ml}$ and glutamine $2 \mathrm{mM}$ ) were added to the plates. The proliferative effect of PDGF was assessed in the presence and absence of ECGS (concentration range 50,25 and $5 \mu \mathrm{g} / \mathrm{ml}$ ). The HUV endothelial cells were incubated for $48 \mathrm{~h}$ in 5 $\% \mathrm{CO}_{2}-95 \%$ air atmosphere at $37{ }^{\circ} \mathrm{C}$. $\left[{ }^{3} \mathrm{H}\right]$-thymidine $(0.5 \mu \mathrm{Ci} /$ well) was added and the cells incubated for a further $18 \mathrm{~h}$ before being washed, trypsinized and harvested as described in Section 2.3.7. The same protocol as outlined above was used to assess whether $0.5 \mathrm{M}$ acetic acid diluted in Medium 199 (the vehicle used to dissolve PDGF) altered HUV endothelial cell proliferation. 


\subsubsection{Statistical analysis}

Individual experiments were examined statistically using analysis of variance and presented graphically as the mean \pm SD. Analysis of variance with regression was used for analysis of groups of experiments (Smart, 1970) where the experiment replicates were considered as a block effect and the treatments as fixed effects. The variance ratio ( $F$ test) was used to test for overall significance. The multiple t comparison (Bonferroni method) was then used to test for a significant difference between treatments. The Minitab programme (Data analysis software, 1987) was used for the above analyses. A p value of 0.05 or less was taken to represent a statistically significant difference.

\subsection{RESULTS}

\subsubsection{Presence of estrogen receptor related antigen in HUV endothelial cells.}

Estrogen receptor related antigen was detected in HUV endothelial cells when examined with a monoclonal antibody to ER-D5 antigen (Figure 3.1) over a range of passages (primary to passage 8). The amount of antigen appeared to vary from cell to cell being visualized as red-brown granules in the cytoplasm of the cells.

\subsubsection{Effect of sex steroids on HUV endothelial cell proliferation}

Preliminary studies on the effects of sex steroids on HUV endothelial cell requirements for FCS, ECGS and heparin were performed to determine the experimental conditions for the proliferation assays. The Methods and Results are presented in Appendix 2. 
Initial proliferation studies were performed in the presence of $20 \%$ FCS in PRF Medium 199. 17 $\beta$-estradiol $\left(10^{-5} \mathrm{M}\right)$ was found to significantly inhibit HUV endothelial cell proliferation ( $58 \pm 5.2 \%$ ) relative to control (Medium 199) (Figure 3.2). 17 3 -estradiol $\left(10^{-6}-10^{-9} \mathrm{M}\right)$ did not significantly alter HUV endothelial cell proliferation relative to control cells (Figure 3.2), while 17kestradiol (10-10 M) did not increase HUV endothelial cell proliferation (111 \pm $8.6 \%$ ) significantly compared to control cells (Figure 3.2).

Progesterone $\left(10^{-5} \mathrm{M}\right)$ alone inhibited HUV endothelial cell proliferation (14.5 $\pm 7.5 \%$ of control) (Figure 3.5). Progesterone $\left(10^{-6}-10^{-9} \mathrm{M}\right.$ ) did not significantly alter HUV endothelial cell proliferation compared to control cells (Figure 3.5). $17 \beta$-estradiol $\left(10^{-5} \mathrm{M}\right)$ in the presence of progesterone $\left(10^{-5}-10^{-}\right.$ $9 \mathrm{M}$ ) significantly inhibited HUV endothelial cell proliferation (Figures 3.2, 3.3 and 3.4). When combinations of these two sex steroids were examined it was found that $17 \beta$-estradiol (10-6-10-10 $\mathrm{M})$ combined with progesterone $\left(10^{-6}-10^{-}\right.$ $9 \mathrm{M}$ ) did not significantly alter HUV endothelial cell proliferation (Figures 3.2, 3.3 and 3.4).

17 $\beta$-estradiol (10-10 $\mathrm{M})$ had a significantly greater proliferative effect on HUV endothelial cells relative to control in the presence of $25 \mu \mathrm{g} / \mathrm{ml}$ ECGS (135.5 $\pm 7 \%$ ) compared to $50 \mu \mathrm{g} / \mathrm{ml}$ ECGS (122 $\pm 11 \%$ ), and at a cell density of $2.5 \times 10^{3}$ cells /well compared to a cell density of $5.0 \times 10^{3}$ cells (Figure 3.6).

Studies were also performed without FCS and with late passaged cells (passages 7-10). 17 3 -estradiol $\left(10^{-5}-10^{-6} \mathrm{M}\right)$ inhibited HUV endothelial cell proliferation (30 $\pm 9 \%$ relative to control) (Figure 3.7 ) more than in the presence of FCS. $17 \beta$-estradiol $\left(10^{-6}-10^{-7} \mathrm{M}\right)$ did not alter HUV endothelial cell proliferation relative to control cells, while $17 \beta$-estradiol $\left(10^{-8}-10^{-10} \mathrm{M}\right)$ enhanced HUV endothelial cell proliferation (122 $\pm 12 \%$ relative to control), although the difference was not statistically significant (Figure 3.7). In the 
presence of progesterone $\left(10^{-5}-10^{-6} \mathrm{M}\right)$ alone significant inhibition of HUV endothelial cell proliferation was observed ( $25 \pm 9 \%$ relative to control) (Figure 3.8). Progesterone $\left(10^{-6}-10^{-9} \mathrm{M}\right)$ did not significantly alter HUV endothelial cell proliferation compared to control cells (Figure 3.8). When combinations of these two sex steroids were examined it was found that $17 \beta$ estradiol (10-5 $\mathrm{M}$ ) combined with progesterone $\left(10^{-5} \mathrm{M}, 10^{-7} \mathrm{M}\right.$ and $\left.10^{-9} \mathrm{M}\right)$ significantly inhibited HUV endothelial cell proliferation (Figures 3.7 and 3.9), while $17 \beta$-estradiol $\left(10^{-7} \mathrm{M}\right)$ and progesterone $\left(10^{-5}, 10^{-7} \mathrm{M}\right.$ and $\left.10^{-9} \mathrm{M}\right)$ had no significant effect on HUV endothelial cell proliferation relative to controls. Estradiol $\left(10^{-10} \mathrm{M}\right)$ in combination with $10^{-7} \mathrm{M}$ progesterone but not $10^{-9} \mathrm{M}$ progesterone significantly inhibited HUV endothelial cell proliferation (Figures 3.7 and 3.9). The combination of progesterone (10-9 $\mathrm{M}$ ) and $17 \beta$-estradiol (10-10 M) did however result in significant inhibition of proliferation compared to HUV endothelial cells exposed to $17 \beta$-estradiol $\left(10^{-10} \mathrm{M}\right)$ alone (Figure 3.7).

The sequential addition of progesterone $\left(10^{-9} \mathrm{M}\right)$ to $17 \beta$-estradiol $\left(10^{-9} \mathrm{M}\right)$ did not affect the proliferative response of HUV endothelial cells when compared to continuous the proliferation of HUV endothelial cells continously exposed to both sex steroids (Figure 3.10).

\subsubsection{Expression of MRNA for PDGF-A and PDGF-B in HUV endothelial cells}

Hybridization of filters with ${ }^{32} \mathrm{P}$-labelled PDGF-A chain CDNA revealed three transcripts at $2.8 \mathrm{~kb}, 2.3 \mathrm{~kb}$ and $1.4 \mathrm{~kb}$ (Figure 3.11A) as reported by other investigators (Collin et al, 1987; Hermansson et al, 1988; Suzuki et al, 1989) while hybridization of filters with 32P-labelled PDGF-B chain showed a single band (Figure $3.11 \mathrm{C}$ ) at $3.6 \mathrm{~kb}$ as reported by other investigators (Collins et al, 1985; Gay and Winkles, 1990). A slot blot with the same RNA samples was hybridized with 32P-labelled PDGF-B chain. This showed that mRNA 
expression for PDGF-B was induced by all sex steroids and IL-1 compared to control (Medium 199 alone) (Figure 3.12A). Reprobing this blot with 32Plabelled PDGF-A chain showed similar induction of the PDGF-A chain by the

combinations of $17 \beta$-estradiol and progesterone (Figure 3.12B). This induction of PDGF mRNA expression by IL-1 has been previously demonstrated by Gay and Winkles (1990). The blot was not exposed for sufficient time which makes interpretation difficult. The integrity of the RNA used and equality of loading of samples is demonstrated in Figure 3.13.

\subsubsection{Expression of mRNA for ECGF in HUV endothelial cells}

The sex steroids did not induce expression of ECGF in HUV endothelial cells after $19 \mathrm{~h}$ stimulation (Figure 3.11B). A second northern blot was performed with the plasmid being used as the positive control and again no induction of ECGF expression was seen.

\subsubsection{Effect of PDGF on HUV endothelial cell proliferation}

There was an increase in HUV endothelial cell proliferation in the presence of 12.5 to $25 \mathrm{ng} / \mathrm{ml}$ of PDGF. This was best observed in the absence of ECGS but also was evident in the presence of $5 \mu \mathrm{g} / \mathrm{ml}$ of ECGS (Figure 3.14). Figure 3.15 demonstrates the significant increase in HUV endothelial cell proliferation in the absence of ECGS but not $25 \mu \mathrm{g} / \mathrm{ml}$ ECGS when the concentration of PDGF was 12.5-25 ng / $\mathrm{ml}$ (relative to Medium 199 alone). Acetic acid at various concentrations (the vehicle used to dissolve PDGF) did not alter HUV endothelial cell proliferation relative to control. 


\subsection{DISCUSSION}

The aim of the initial studies reported in this Chapter was to confirm the presence of estrogen receptors in HUV endothelial cells and to establish whether sex steroids altered HUV endothelial cell proliferation. In subsequent studies the influence of sex steroids on expression of mRNA for PDGF was assessed. Finally, the proliferative effect of PDGF on HUV endothelial cells was examined.

The major finding of these experiments was that sex steroids altered the proliferation of HUV endothelial cells in a concentration dependent manner. Low concentrations of 17 $\beta$-estradiol (10-9-10-10 M) increased HUV endothelial proliferation. This effect was statistically significant at lower concentrations of ECGS $(25 \mu \mathrm{g} / \mathrm{ml})$ probably because at a concentration of $50 \mu \mathrm{g} / \mathrm{ml}$ of ECGS, the HUV endothelial cells were already proliferating optimally so that any additionalproliferative effect of $17 \beta$-estradiol was masked. The proliferative effect of low concentrations of $17 \beta$-estradiol has been previously reported by Harrison and McKnee (1984b) and Johannisson (1986). The present study however shows that the enhancement of proliferation caused by $17 \beta$-estradiol was significantly inhibited when progesterone (10-5-10-9 $\mathrm{M}$ ) was also present. There are at least three possible explanations for this observation. Firstly, progesterone may compete with $17 \beta$-estradiol for its binding site, as proposed by Colburn and Buonassisi (1978). Secondly, progesterone may decrease expression of the estrogen receptor on HUV endothelial cells as seen in the uterus (Katzenellenbogen, 1980). Thirdly, progesterone may alter the expression of growth factor(s) released by $17 \beta$ estradiol which are responsible for the enhanced HUV endothelial cell proliferation. Progesterone (10-6-10-9 M) alone however did not alter HUV endothelial cell proliferation supporting the findings of Corvazier et al (1984). 
High concentrations of $17 \beta$-estradiol $\left(10^{-5} \mathrm{M}\right)$ and progesterone $\left(10^{-5} \mathrm{M}\right)$ alone, and in combination with each other, inhibited HUV endothelial cell proliferation. Such concentrations have also been shown to inhibit proliferation of some other cells in culture (Ishiwata et al, 1977; Rhee et al, 1978). These concentrations of sex steroids did not cause cell death as assessed by trypan blue exclusion. The mechanism of action of sex steroids on HUV endothelial cell proliferation was not studied although it may be at the postransciptional level through a low affinity receptor as discussed in Chapter 1 (refer to Section 1.5.3.1).

A number of factors (including FCS concentration, ECGS concentration, heparin concentration and formulation of Medium 199) altered the proliferative response of HUV endothelial cells as discussed in Chapter 2. Some of these factors were shown to alter the effect of sex steroids on the proliferative response of HUV endothelial cells. The proliferative and inhibitory effects of $17 \beta$-estradiol and progesterone were influenced, to some extent, by the concentration of FCS in the studies reported here. The presence of $20 \%$ FCS reduced the inhibition of HUV endothelial cell proliferation by $17 \beta$-estradiol $\left(10^{-5} \mathrm{M}\right)$ and progesterone $\left(10^{-5} \mathrm{M}\right)$. It also reduced the proliferative effect of $17 \beta$-estradiol (10-8-10-10 M) relative to Medium 199 alone. Some of the effects of FCS may be due to the presence of sex steroids in the FCS increasing the overall concentration of sex hormones, or perhaps the binding effect of proteins and the presence of other hormones such as 1,25-dihydroxyvitamin $D_{3}$ which have been shown to antagonize the response to $17 \beta$-estradiol in cultured UMR106 cells (Gray et al, 1987). Analysis of fetal bovine serum by Peek et al (1989) showed that there was measurable estrone activity present $\left(6.41 \times 10^{-10} \mathrm{M}\right)$ but not estradiol, estriol, progesterone, testosterone or sex hormone binding globulin. Endothelial cells are known to have 1,25-dihydroxyvitamin $D_{3}$ 
receptors, and 1,25-dihydroxyvitamin $D_{3}\left(10^{-8} \mathrm{M}\right)$ inhibits endothelial cell proliferation (Merke et al, 1989). Milo et al (1976) showed that FCS can alter the proliferative response of cells depending on the concentration of the sex steroid in the serum. Serum which best supported the growth of cloned cell lines from lung and foreskin was low in progesterone (less than $3.1 \times 10^{-9} \mathrm{M}$ ) and high in estradiol $\left(3.7 \times 10^{-10} \mathrm{M}\right)$. The addition of exogenous $17 \beta$-estradiol resulted in toxic effects on lung cell (at $3.7 \times 10^{-6} \mathrm{M}$ ) and foreskin cell (at $9 \times$ $10^{-6} \mathrm{M}$ ) lines. Progesterone was shown to completely inhibit cloning of a lung cell line at a concentration of $32 \times 10^{-9} \mathrm{M}$, and inhibit a foreskin line at a concentration of $327 \times 10^{-9} \mathrm{M}$. Darbre et al (1983) and Soto and Sonnenschein (1985) showed that charcoal-dextran treated fetal bovine serum altered the proliferative response of human breast cancer cell lines (e.g. MCF7 cells) to $17 \beta$-estradiol. Page et al (1983) found that non charcoaldextran treated fetal bovine serum was in fact required for MCF7 cells to respond to estradiol. Thus, the presence of serum can influence the proliferative response of cells to sex steroids although the effects vary depending on the cell type and the concentration of serum.

The sex steroids did not alter the growth requirement of endothelial cells for ECGS when heparin was used at a concentration of $20 \mathrm{U} / \mathrm{ml}$. There was no significant shift in heparin requirement by HUV endothelial cells with the addition of sex steroids. This suggests that there is no interaction between heparin and the sex steroids which were examined, which contrasts with the interaction between heparin and hydrocortisone (Folkman et al, 1983; Crum et al, 1985; Sakamuto et al, 1987).

A problem in interpreting in vitro data such as these is that endothelial cell proliferation in vivo is characterized by a high cell density, by the presence of plasma at a concentration of $100 \%$, and by a quiescent state with no more than a few cells per thousand replicating each day even in response to 
wounding and inflammation (Schwartz et al, 1981). However, the normally quiescent state of the endothelium does not rule out a potentially important role for sex steroids in endothelial cell replication in vivo in those regions of the vasculature where injury occurs or angiogenesis, and therefore it is relevant to study endothelial cell proliferation in vitro.

Sex steroids did not induce expression of mRNA for ECGF in HUV endothelial cells and therefore the observed differences in HUV endothelial cell proliferation following exposure to sex steroids cannot be attributed to ECGF induction and possible release of this mitogen which could then alter endothelial cell proliferation. However, mRNA expression for PDGF was increased in those cells exposed to sex steroids and rlL-1 relative to control and absolute alcohol. Secondly, PDGF-AB increased HUV endothelial cell proliferation relative to control when the concentration of ECGS was reduced. As discussed in Section 1.3.1.3 PDGF has been shown to either increase, decrease or have no apparent effect on endothelial cell proliferation. Some endothelial cells have been demonstrated to have PDGF receptors (Bar et al, 1989), while others such as HUV endothelial cells have not (Heldin et al, 1981). Heldin et al (1981) examined binding of 125/-labeled PDGF derived from platelets to assess the presence of PDGF receptors (Heldin et al, 1981), however this may have detected only the PDGF-A type of receptor as PDGFB type receptors only bind PDGF-AB with low affinity. It is possible that the proliferative effect of PDGF on HUV endothelial cells observed in the current studies is mediated through another growth factor receptor not yet identified as having specificity for PDGF. Bar et al (1989) found that recombinant PDGF $(A B$ and $B B$ ) was a potent stimulator of thymidine incorporation in microvessel endothelial cells at concentrations between $2-20 \mathrm{ng} / \mathrm{ml}$ which is in the same range as those reported in these studies. The magnitude of the proliferative effect of PDGF on HUV endothelial cells is unlikely to be due to another cell contaminating the culture (in particular, smooth muscle cells) 
although it is not possible to absolutely rule out minor $(<1 \%)$ cell contamination with the techniques used to characterize the cultures (refer to Chapter 2). The interaction of PDGF with other proteins or growth factors in relation to the biological effects of PDGF on the endothelial cell, and the potential importance of the cell phase in the action of PDGF have not been addressed in this thesis. Endothelial cells synthesize other growth factors such as insulin-like growth factor-1, bFGF and transforming growth factor- $\beta$ which are known to alter endothelial cell growth and may have supported PDGF activity (Bar et al, 1989). Gay and Winkles (1990), for example, have demonstrated that aFGF, which is used to maintain HUV endothelial cell cultures, can induce expression of PDGF A-chain mRNA but not B-chain and the release of PDGF-AA from HUV endothelial cells. In apparent contrast, Wall et al (1978) found that factors released from platelets by thrombin decreased the proliferation rate of HUV endothelial cells. This inhibitory effect on proliferation of released platelet factors may have therefore been due to other factors such as serotonin or transforming growth factor- $\beta$ which are known to inhibit endothelial cell proliferation. If endothelial cells in vivo could secrete PDGF-like molecules and also be a target for PDGF, an array of PDGF-endothelial cell interactions of autocrine, paracrine, and endocrine nature could conceivably occur.

From the current studies one could suggest a possible autocrine loop where the proliferative effect of $17 \beta$-estradiol $\left(10^{-10} \mathrm{M}\right)$ on HUV endothelial cells was due the increased release of PDGF from the HUV endothelial cells since PDGF was found to cause HUV endothelial cells to proliferate and $17 \beta$ estradiol (10-10 M) caused increased mRNA for PDGF. It is interesting that both the proliferative effect of PDGF and 17 $\beta$-estradiol (10-10 M) were demonstrated when the concentration of ECGS was reduced. One difficulty with this suggestion is that mRNA expression for PDGF was also increased in those cells exposed to the highest concentrations of $17 \beta$-estradiol $\left(10^{-5} \mathrm{M}\right)$ 
and progesterone $\left(10^{-5} \mathrm{M}\right)$. Sex steroids at these high concentrations caused marked inhibition of HUV endothelial cell proliferation. However, IL-1 has been shown to increase expression of PDGF-A and PDGF-B and also inhibit endothelial cell proliferation (Gay and Winkles, 1990). Raines et al (1989) have also demonstrated that IL-1 can increase PDGF-A chain expression in fibroblasts and increase release of PDGF from these cells. It remains to be determined whether sex steroids can cause an increase in PDGF-like mitogen release from the HUV endothelial cells or whether the change in mRNA expression is independent of PDGF release. If PDGF-like mitogen release is altered by sex steroids it may provide an interesting pathway by which these steroids can influence atherosclerosis. The present studies need to be expanded to further characterise the effects of sex steroids on mRNA expression of PDGF in endothelial cells (in terms of a time course of induction and a wider range of concentrations of sex steroids), and also in other cells involved in atherosclerosis. Sex steroids may alter the production and release of other mitogens such as bFGF or transforming growth factor- $\beta$. bFGF, for example, is produced by regenerating endothelial cells in vivo at high levels and it has been shown to be mitogenic and chemotactic to endothelial cells (Lindner et al, 1989; Klagsbrun and Edelman, 1989).

Finally, 17 $\beta$-estradiol and progesterone may alter HUV endothelial cell proliferation through a direct effect on cell division as proposed by some investigators (Gorski and Gannon, 1976; Rhee et al, 1978; Katzenellenbogen, 1980; Ernst et al, 1988). Endothelial cells are known to possess receptors for sex steroids (refer to Section 1.5.3.1). It has also been shown that pharmacological concentrations $\left(10^{-5}-10^{-8} \mathrm{M}\right)$ of estrogens can exert an inhibitory action on haemopoietic cell growth through type II estrogen binding sites which have a low affinity for estrogen but a higher binding capacity than type I estrogen receptors (Larocca et al, 1990). 
The present studies show that HUV endothelial cells possess cytoplasmic estrogen receptors, that estrogen and progesterone can alter HUV endothelial cell proliferation individually and that they can modulate the response to each other. Low concentrations of $17 \beta$-estradiol alone increase HUV endothelial cell proliferation, in the presence of reduced ECGS concentration, which may increase the rate of reendothelialization and therefore retard the atherogenic process. This proliferative effect is however prevented by the addition of progesterone. As progesterone and estrogen are both present in the circulation it seems likely that the net effect of these steroids is not to alter endothelial cell proliferation, as demonstrated in these studies. This observation may also have important implications for the use of progestins with estrogens in hormone replacement therapy. In contrast to the findings with physiological concentrations of sex steroids, the current studies showed that high concentrations of these sex steroids depress endothelial cell proliferation. Thus, in the event of endothelial cell injury and loss, the rate of re-endothelialization may be slowed, possibly allowing greater thrombus formation and increased migration of smooth muscle cells in response to PDGF release from platelets adherent to subendothelium and endothelial cells whose release of PDGF may have been increased by these sex steroids. Although it is not known what concentrations of sex steroids endothelial cells are exposed to in vivo, it seems very likely that endothelial cell proliferation and MRNA expression of PDGF are altered by exposure to $17 \beta$-estradiol and progesterone which are important in atherogenesis and angiogenesis. 


\subsection{SUMMARY}

In summary, the studies reported in this Chapter indicate that:

(1) HUV endothelial cells possess cytoplasmic estrogen receptors;

(2) Low concentrations of 17 -estradiol (10-9-10-10 M) increase endothelial cell proliferation, particularly at lower concentrations of ECGS;

(3) Progesterone $\left(10^{-5}-10^{-9} \mathrm{M}\right)$ reverse the effect of increased HUV endothelial cell proliferation induced by low concentrations of $17 \beta$-estradiol $\left(10^{-8}-10^{-10} \mathrm{M}\right)$;

(4) PDGF increase HUV endothelial cell proliferation in a concentration dependent manner;

(5) 17 -estradiol, progesterone and rIL-1 increase mRNA expression for PDGF over $19 \mathrm{~h}$;

(6) High concentrations of $17 \beta$-estradiol $\left(10^{-5} \mathrm{M}\right)$ and progesterone $\left(10^{-5} \mathrm{M}\right)$ alone and in combination inhibit HUV endothelial cell proliferation; and

(7) Sex steroids do not induce mRNA for ECGF expression in HUV endothelial cells over $19 \mathrm{~h}$. 
Figure 3.1.

Photomicrographs of cytospins of HUV endothelial cells (passage 3) which were stained for the presence of estrogen receptor related antigen using a monoclonal antibody to ER-D5 antigen. Magnification $672 \mathrm{x}$.

Upper: Presence of estrogen receptor related antigen in the cytoplasm of HUV endothelial cells.

Lower: HUV endothelial cells stained with the ABC-HRP kit method with the omission of the primary antiserum (monoclonal antibody). 

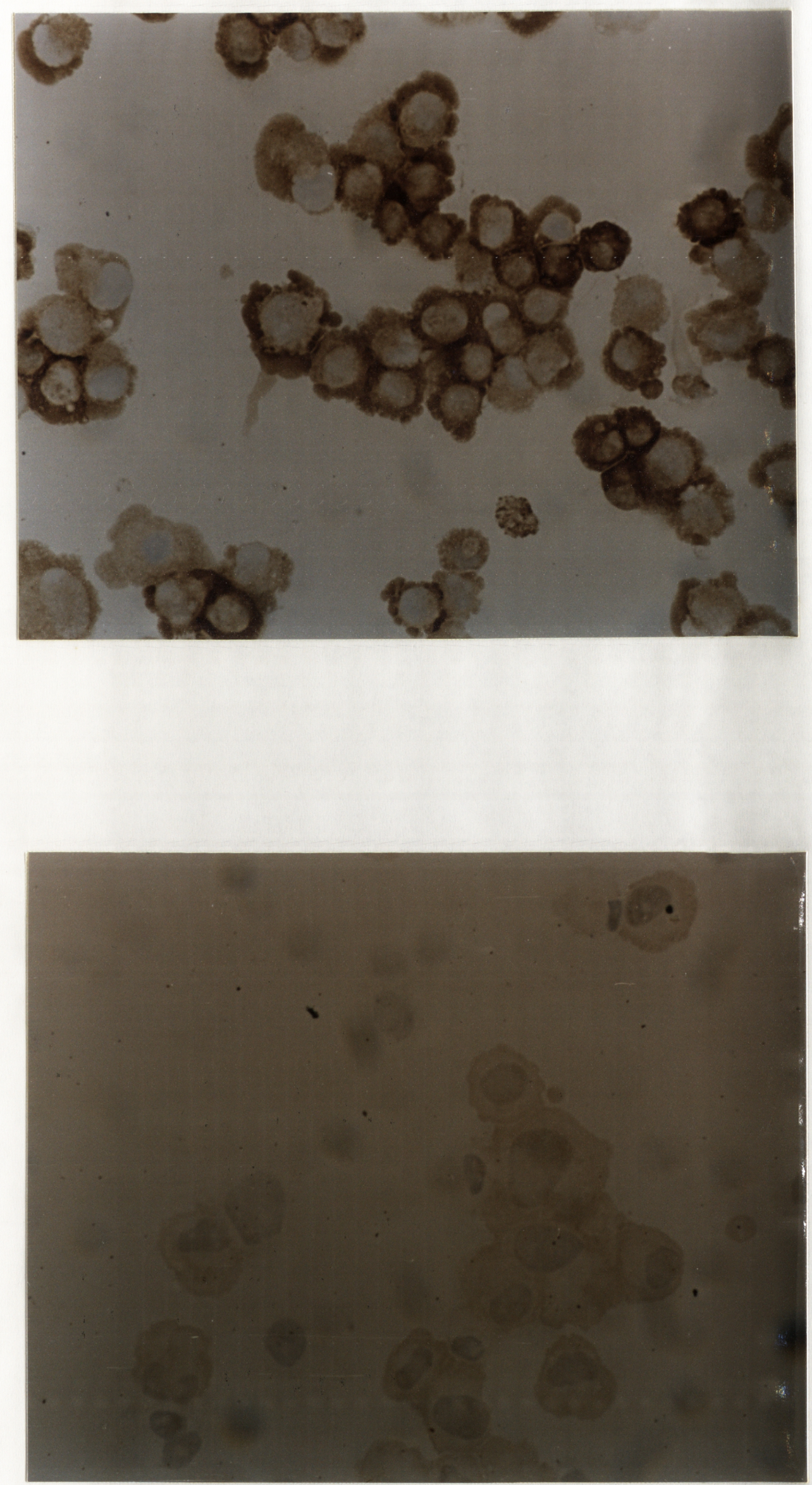


\section{Figure 3.2.}

The effect of $17 \beta$-estradiol and progesterone on HUV endothelial cell proliferation. Endothelial cells (passages 9 and 10) in Medium 199 (with $20 \%$ FCS, gentamicin $60 \mathrm{U} / \mathrm{ml}$, ECGS $50 \mu \mathrm{g} / \mathrm{ml}$, heparin $20 \mathrm{U} / \mathrm{ml}$ and glutamine $2 \mathrm{mM}$ ), in the presence of $17 \beta$-estradiol (10$\left.5_{-10^{-10}} \mathrm{M}\right)$ alone or progesterone $\left(10^{-9} \mathrm{M}\right)$ were incubated for $48 \mathrm{~h}$ and then $\left[{ }^{3} \mathrm{H}\right]$-thymidine was added. The endothelial cells were incubated for a further $18 \mathrm{~h}$ before being washed, trypsinized and harvested. The "y"-axis represents HUV endothelial cell proliferation as a percentage of control. The broken line represents cells exposed to Medium 199 alone (control). The graph represents the mean \pm SE of 4 experiments in at least triplicate. ${ }^{*}=p<0.05$.

\section{Figure 3.3.}

The effect of $17 \beta$-estradiol and progesterone on HUV endothelial cell proliferation. Endothelial cells (passages 9 and 10) in Medium 199 (with $20 \%$ FCS, gentamicin $60 \mathrm{U} / \mathrm{ml}$, ECGS $50 \mu \mathrm{g} / \mathrm{ml}$, heparin $20 \mathrm{U} / \mathrm{ml}$ and glutamine $2 \mathrm{mM}$ ), in the presence of $17 \beta$-estradiol $\left(10^{-5}-10^{-10} \mathrm{M}\right)$ and progesterone $\left(10^{-6}\right.$ or $\left.10^{-8} \mathrm{M}\right)$ were incubated for $48 \mathrm{~h}$ and then $\left[{ }^{3} \mathrm{H}\right]$-thymidine was added. The endothelial cells were incubated for a further $18 \mathrm{~h}$ before being washed, trypsinized and harvested. The key to this figure is the same as for Figure 3.2. The graph represents the mean \pm SE of 4 experiments in at least triplicate. ${ }^{*}=p<0.05$. 


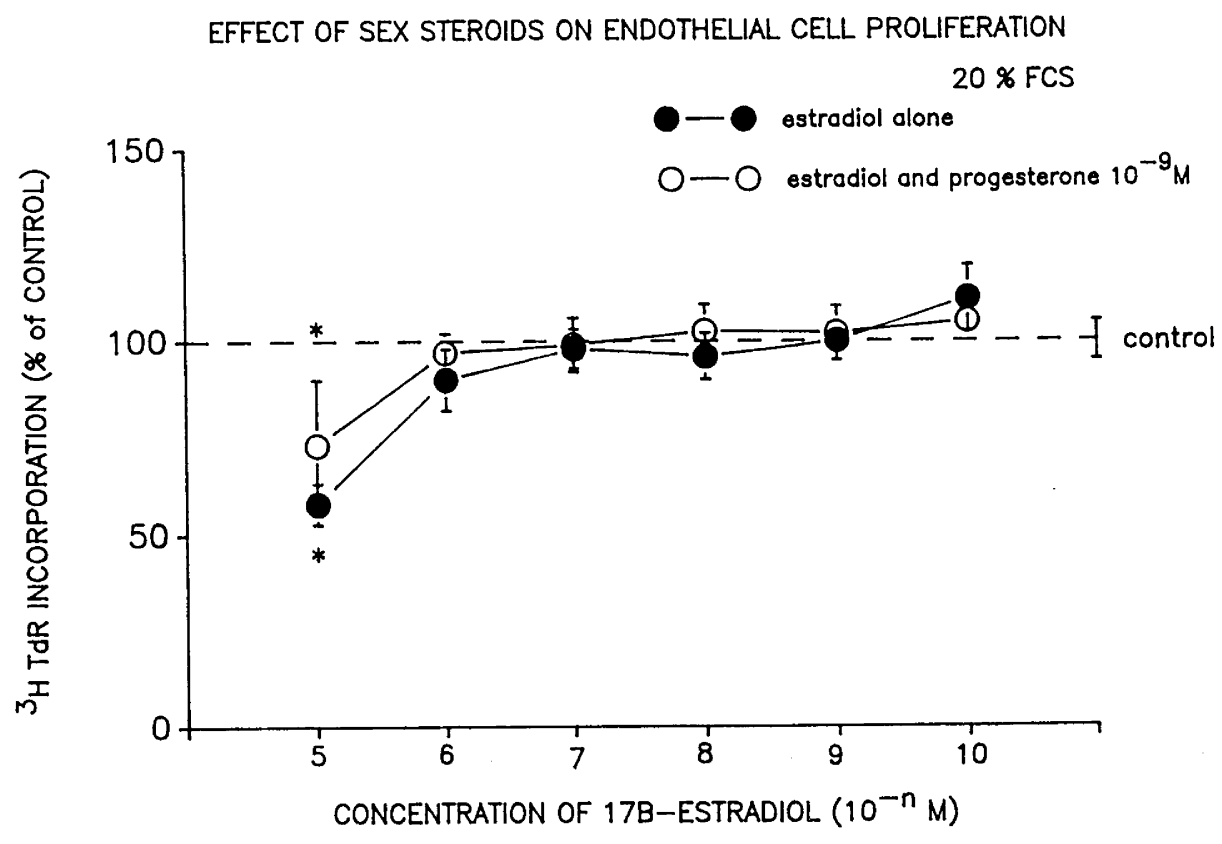

EFFECT OF SEX STEROIDS ON ENDOTHELIAL CELL PROLIFERATION

$20 \%$ FCS

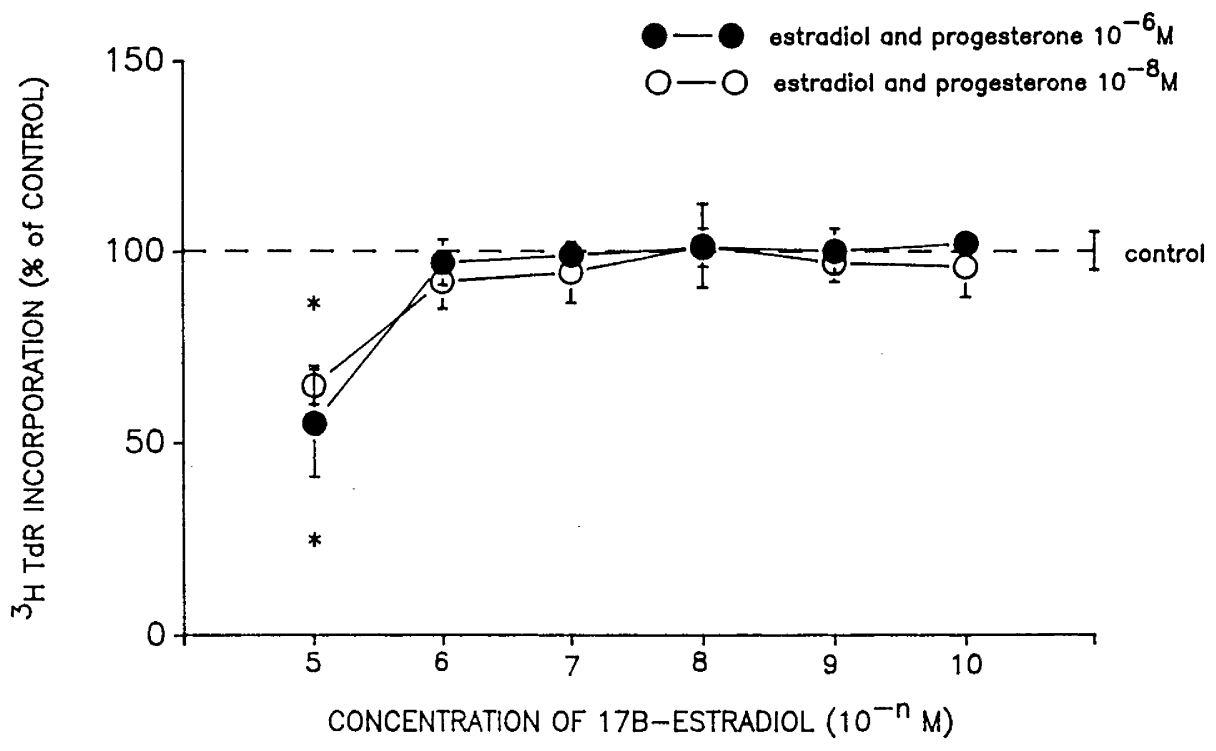




\section{Figure 3.4.}

The effect of $17 \beta$-estradiol and progesterone on HUV endothelial cell proliferation. Endothelial cells (passages 9 and 10) in Medium 199 (with $20 \%$ FCS, gentamicin $60 \mathrm{U} / \mathrm{ml}$, ECGS $50 \mu \mathrm{g} / \mathrm{ml}$, heparin $20 \mathrm{U} / \mathrm{ml}$ and glutamine $2 \mathrm{mM}$ ), in the presence of $17 \beta$-estradiol (10$\left.5_{-10^{-10}} \mathrm{M}\right)$ in combination with progesterone $\left(10^{-5}\right.$ or $\left.10^{-7} \mathrm{M}\right)$ were incubated for $48 \mathrm{~h}$ and then $\left[{ }^{3} \mathrm{H}\right]$-thymidine was added. The endothelial cells were incubated for a further $18 \mathrm{~h}$ before being washed, trypsinized and harvested. The key to this figure is the same as for Figure 3.2. The graph represents the mean \pm SE of 4 experiments in triplicate. ${ }^{*}=p<0.05$.

\section{Figure 3.5.}

The effect of progesterone on HUV endothelial cell proliferation. Endothelial cells (passages 9 and 10) in Medium 199 (with $20 \%$ FCS, gentamicin $60 \mathrm{U} / \mathrm{ml}$, ECGS $50 \mu \mathrm{g} / \mathrm{ml}$, heparin $20 \mathrm{U} / \mathrm{ml}$ and glutamine $2 \mathrm{mM})$, in the presence of progesterone $\left(10^{-5}-10^{-9} \mathrm{M}\right)$ were incubated for $48 \mathrm{~h}$ and then $\left[{ }^{3} \mathrm{H}\right]$-thymidine was added. The endothelial cells were incubated for a further $18 \mathrm{~h}$ before being washed, trypsinized and harvested. The broken line represents cells exposed to Medium 199 alone (control). The graph represents the mean \pm SE of 4 experiments in triplicate. ${ }^{*}=p<0.05$. 
EFFECT OF SEX STEROIDS ON ENDOTHELIAL CELL PROLIFERATION

$20 \%$ FCS

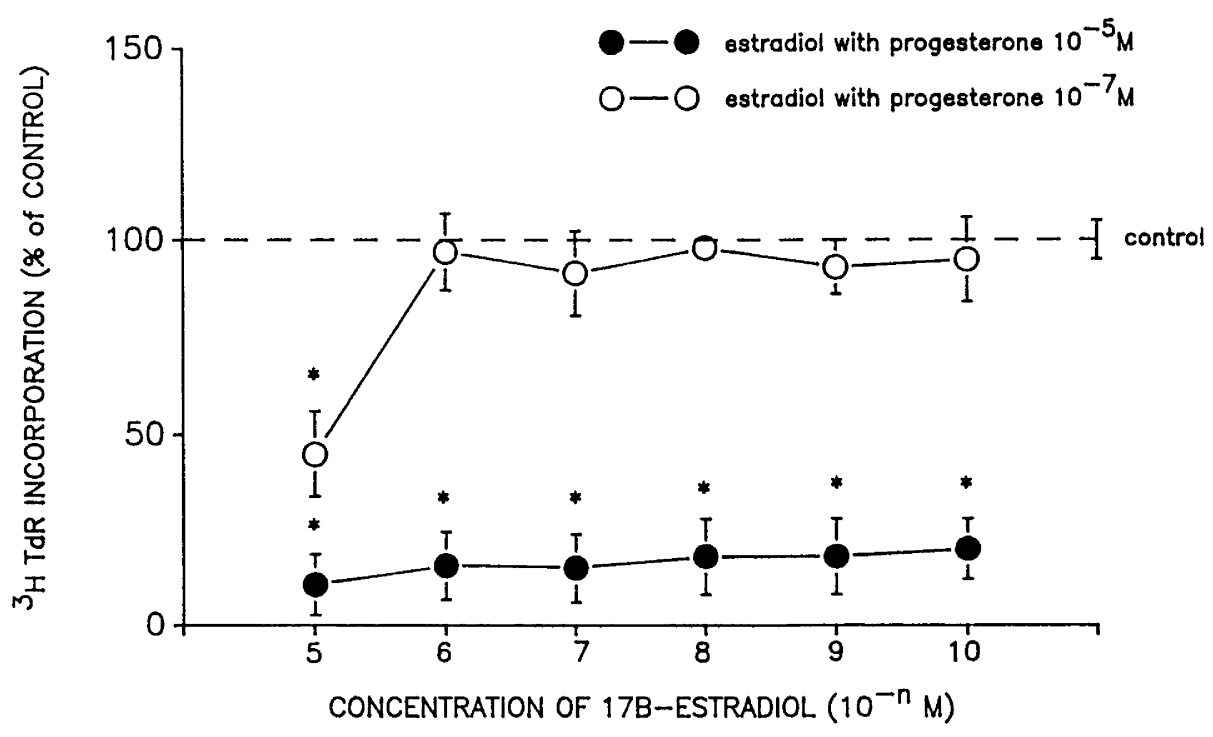

EFFECT OF SEX STEROIDS ON ENDOTHELIAL CELL PROLIFERATION $20 \%$ FCS

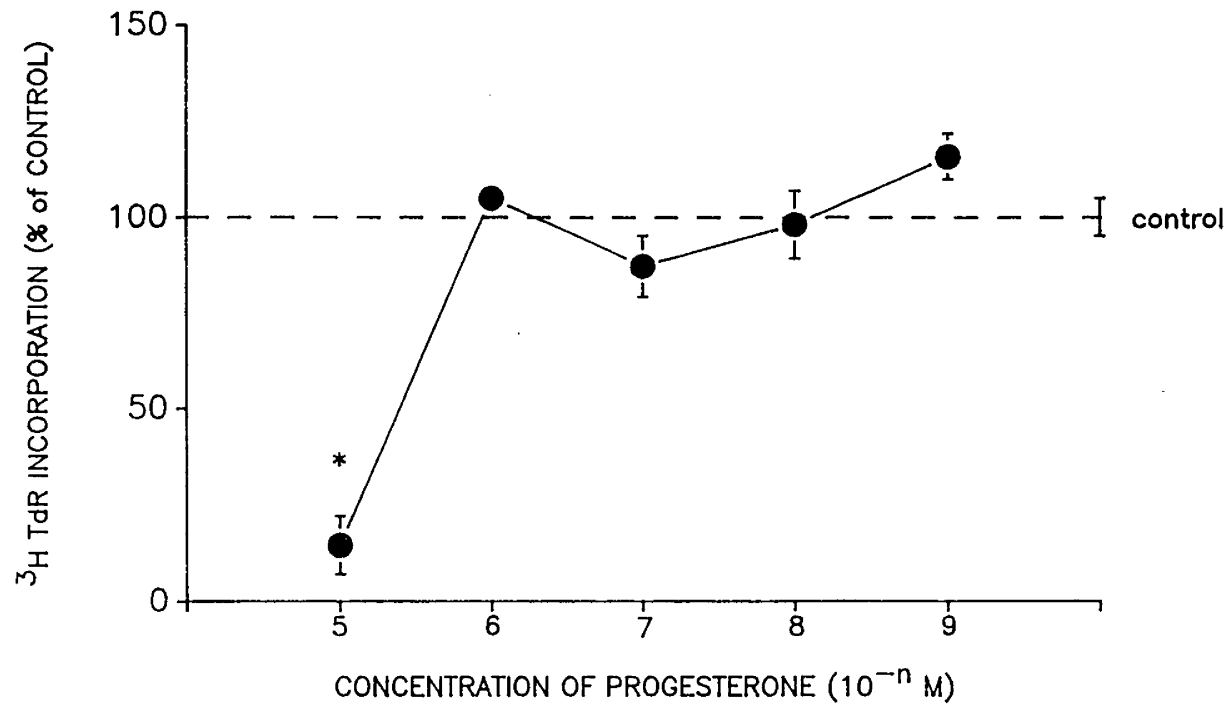




\section{Figure 3.6.}

Effect of different concentrations of ECGS on HUV endothelial cell proliferation in the presence of $20 \%$ FCS and $17 \beta$-estradiol $\left(10^{-10}\right.$ M). Endothelial cells (passage 6 and 7) were exposed to $17 \beta-$ estradiol $\left(10^{-10} \mathrm{M}\right)$ for $66 \mathrm{~h}$ in the presence of either $25 \mu \mathrm{g} / \mathrm{ml}$ or 50 $\mu \mathrm{g} / \mathrm{ml}$ ECGS at a cell density of either $2.5 \times 10^{3}$ cells /well or $5 \times$ $10^{3}$ cells /well. This graph is the mean \pm SE of two quintuplet experiments. ${ }^{*}=p<0.05$ refers to the comparison between the two cell densities. $+=p<0.05$ refers to the comparison between the proliferative effect of $17 \beta$-estradiol $\left(10^{-10} \mathrm{M}\right)$ and control (Medium 199). 
EFFECT OF ECGS CONCENTRATION ON ENDOTHELLAL CELL PROLIFERATION

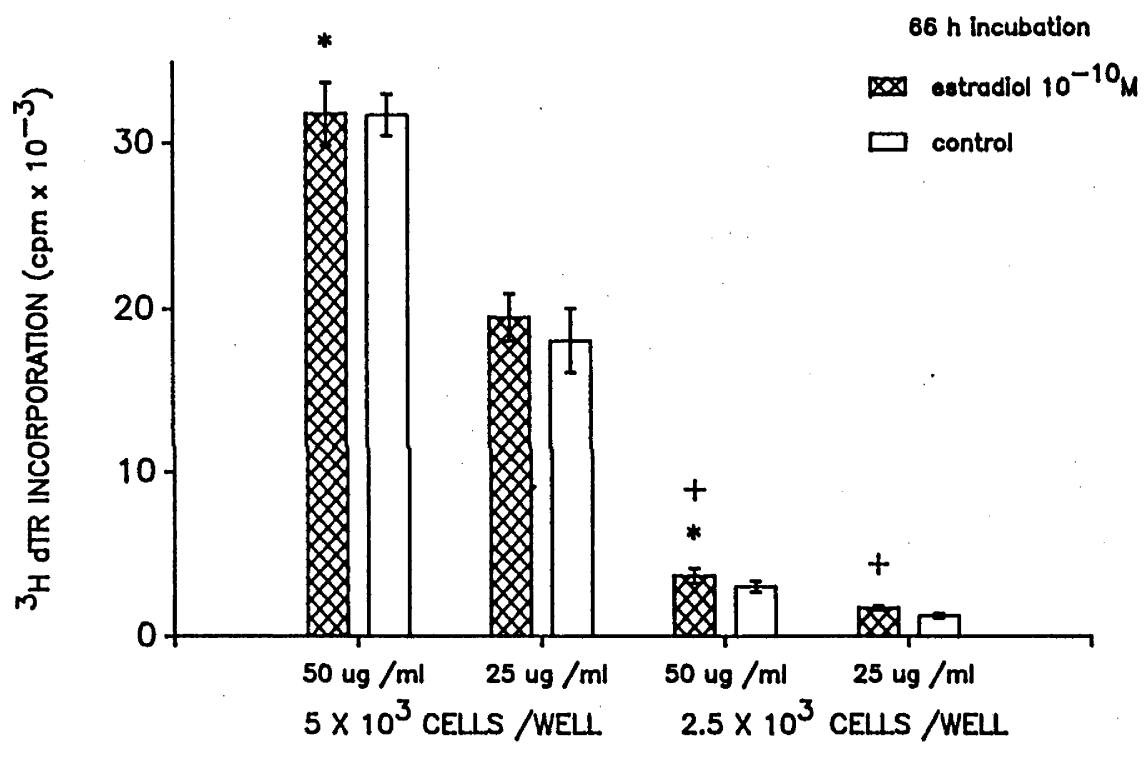




\section{Figure 3.7.}

The effect of $17 \beta$-estradiol and progesterone on HUV endothelial cell proliferation in the absence of FCS. Endothelial cells (passages 7-10) in Medium 199 (gentamicin $60 \mathrm{U} / \mathrm{ml}$, ECGS $25 \mu \mathrm{g} / \mathrm{ml}$, heparin $10 \mathrm{U} / \mathrm{ml}$ and glutamine $1 \mathrm{mM}$ ), in the presence of $17 \beta$ estradiol $\left(10^{-5}-10^{-10} \mathrm{M}\right)$ alone or $17 \beta$-estradiol $\left(10^{-5}-10^{-10} \mathrm{M}\right)$ with progesterone $\left(10^{-9} \mathrm{M}\right)$ were incubated for $18 \mathrm{~h}$ in the presence of $\left[{ }^{3} \mathrm{H}\right]$-thymidine. The endothelial cells were then washed, trypsinized and harvested. The key to this figure is the same as for Figure 3.2. The graph represents the mean \pm SE of 5 experiments in triplicate. $\star=p<0.05$.

\section{Figure 3.8.}

The effect of progesterone on HUV endothelial cell proliferation in the absence of FCS. Endothelial cells (passages 7-10) in Medium 199 (with gentamicin $60 \mathrm{U} / \mathrm{ml}$, ECGS $25 \mu \mathrm{g} / \mathrm{ml}$, heparin $10 \mathrm{U} / \mathrm{ml}$ and glutamine $1 \mathrm{mM})$, in the presence of progesterone $\left(10^{-5}-10^{-9}\right.$ M) were incubated for $18 \mathrm{~h}$ in the presence of $\left[{ }^{3} \mathrm{H}\right]$-thymidine. The endothelial cells were then washed, trypsinized and harvested. The key to this figure is the same as for Figure 3.5. The graph represents the mean \pm SE of five experiments in triplicate. ${ }^{*}=p<$ 0.05 . 
EFFECT OF SEX STEROIDS ON ENDOTHEUAL CELL PROLIFERATION

$18 \mathrm{~h}$ incubation

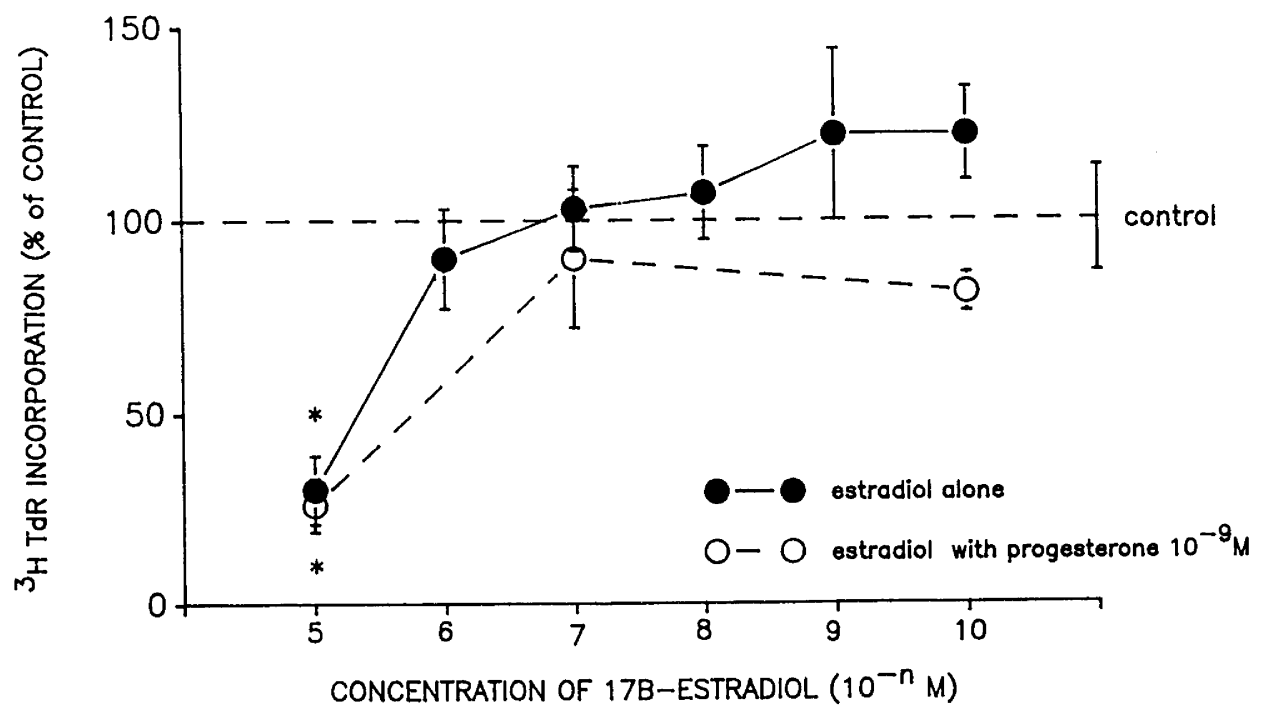

EFFECT OF SEX STEROIDS ON ENDOTHELIAL CELL PROLIFERATION

$18 \mathrm{~h}$ incubation

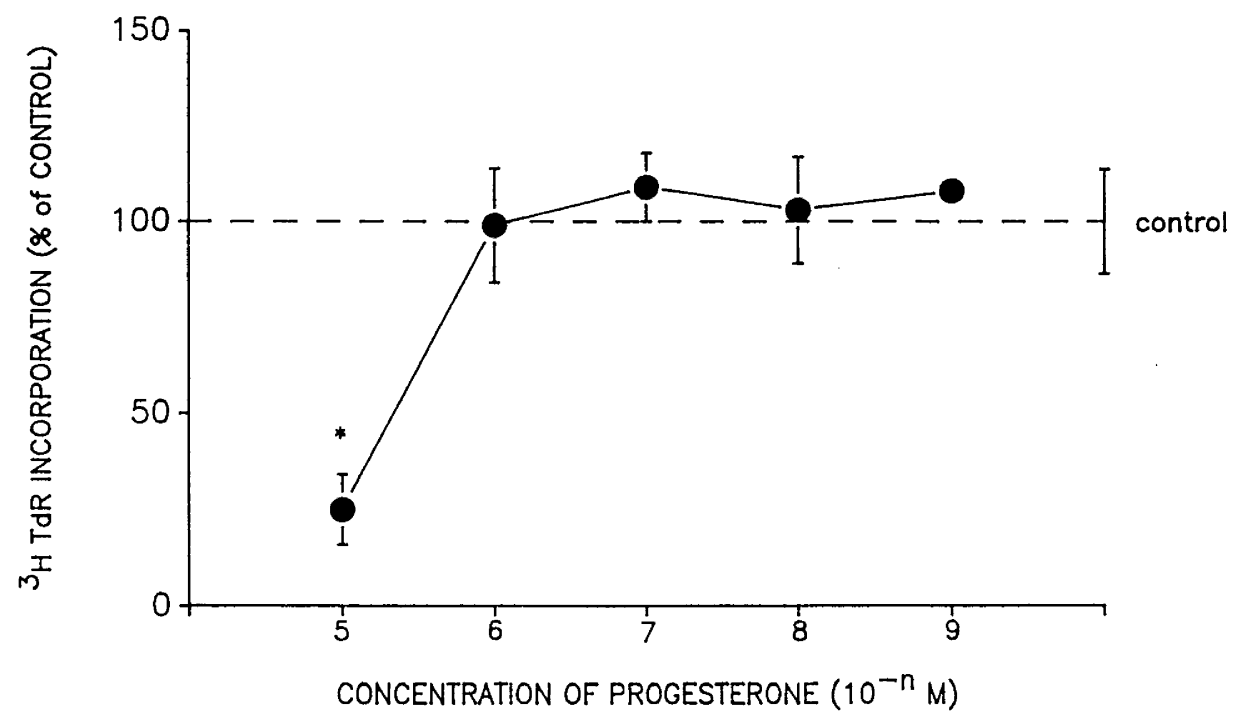




\section{Figure 3.9.}

The effect of $17 \beta$-estradiol and progesterone on HUV endothelial cell proliferation in the absence of FCS. Endothelial cells (passages 7-10) in Medium 199 (with gentamicin $60 \mathrm{U} / \mathrm{ml}$, ECGS $25 \mu \mathrm{g} / \mathrm{ml}$, heparin $10 \mathrm{U} / \mathrm{ml}$ and glutamine $1 \mathrm{mM}$ ), in the presence of $17 \beta$ estradiol $\left(10^{-5}-10^{-10} \mathrm{M}\right)$ in combination with progesterone $\left(10^{-5} \mathrm{M}\right.$ or $10^{-7} \mathrm{M}$ ) were incubated for $18 \mathrm{~h}$ in the presence of $\left[{ }^{3} \mathrm{H}\right]$ thymidine. The endothelial cells were then washed, trypsinized and harvested. The key to this figure is the same as for Figure 3.2. The graph represents the mean \pm SE of 5 experiments in triplicate. * $=$ $p<0.05$.

\section{Figure 3.10.}

The effect of sequential addition of progesterone to HUV endothelial cells exposed to $17 \beta$-estradiol. Endothelial cells (passage 8 ) were exposed to $17 \beta$-estradiol $\left(10^{-9} \mathrm{M}\right)$ for $66 \mathrm{~h}$ with the addition of alcohol $(0.05 \%)$ or progesterone $\left(10^{-9} \mathrm{M}\right)$ for $18 \mathrm{~h}, 30 \mathrm{~h}, 42 \mathrm{~h}, 52 \mathrm{~h}$ or $66 \mathrm{~h}$. The endothelial cells were incubated for $18 \mathrm{~h}$ in the presence of $\left[{ }^{3} \mathrm{H}\right]$-thymidine and then washed, trypsinized and harvested. The columns with diagonal lines represent cells exposed to $17 \beta$-estradiol $\left(10^{-9} \mathrm{M}\right)$ with the addition of progesterone $\left(10^{-9} \mathrm{M}\right)$ at the stated times. The open column represents the addition of alcohol $(0.05 \%)$ to $17 \beta$-estradiol $\left(10^{-9} \mathrm{M}\right)$ at the stated times. The solid columns represent cells exposed only to $17 \beta$ estradiol $\left(10^{-9} \mathrm{M}\right)$ while the wide cross-hatch represent cells exposed only to progesterone $\left(10^{-9} \mathrm{M}\right)$, the narrow cross-hatched column represent cells exposed to Medium 199 alone (control). This was a single experiment in quadruplicate. 


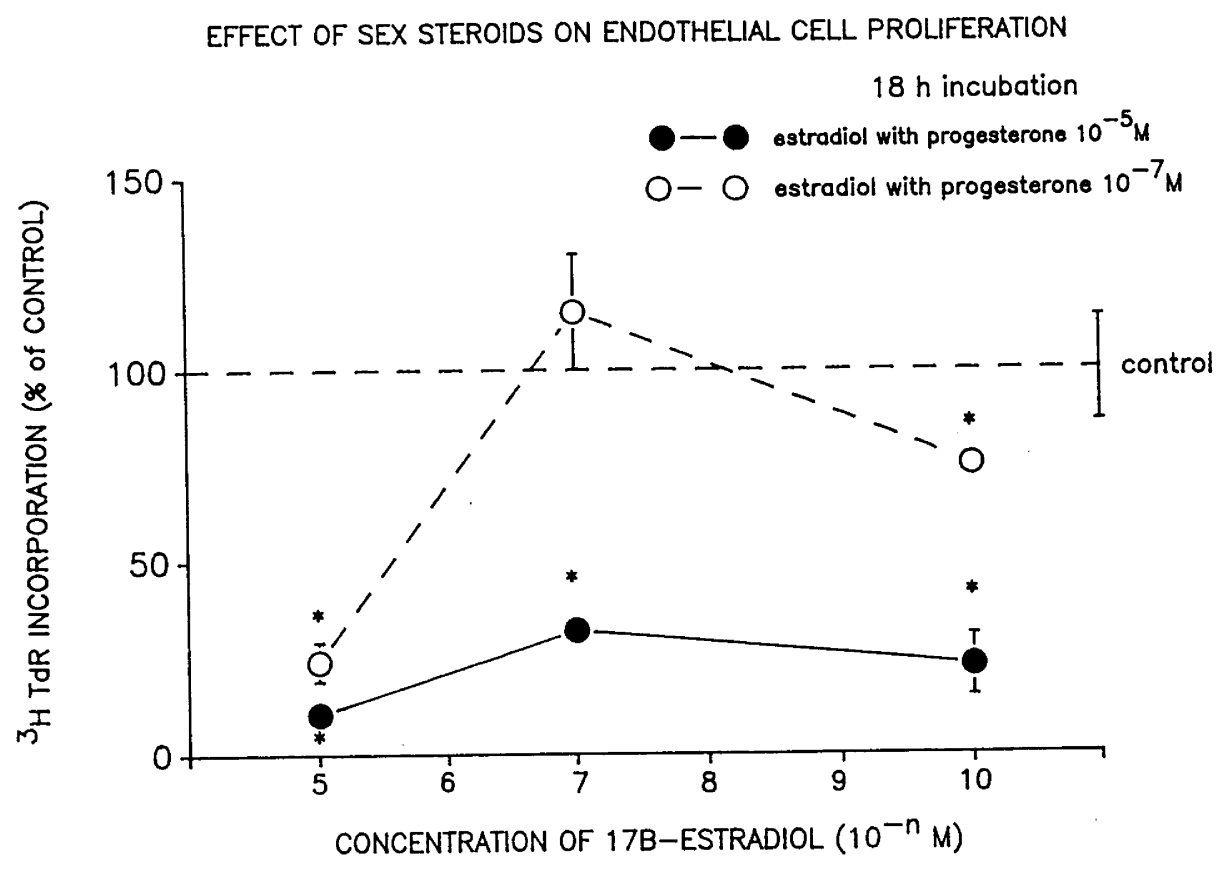

SEQUENTIAL ADDITION OF PROGESTERONE IN THE PRESENCE OF ESTROGEN EFFECT ON ENDOTHELIAL CELLL PROLIFERATION

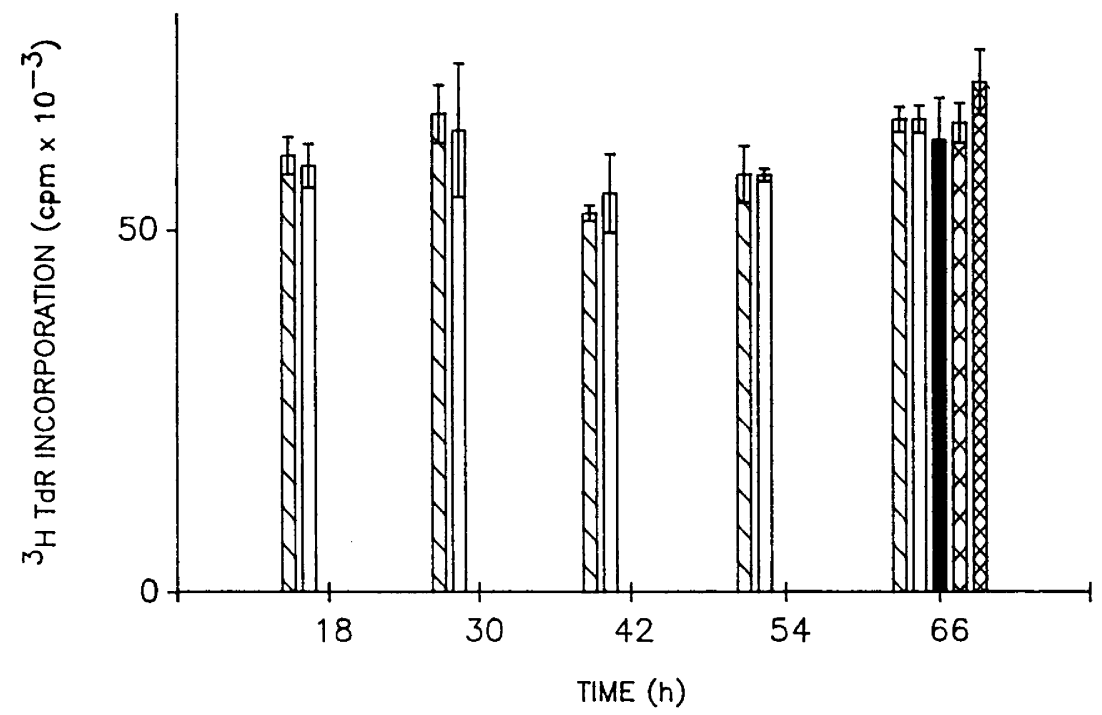




\section{Figure 3.11.}

Northern blot analysis of total RNA (20 $\mu \mathrm{g}$ per lane) showing expression of mRNA for PDGF-A, ECGF and PDGF-B in HUV endothelial cells exposed to various stimuli. Total RNA from cultured endothelial cells exposed to sex steroids, rIL-1, alcohol $(0.1$ \%) or Medium 199 alone (control) was electrophoresed, blotted to nitrocellulose and hybridized with (A) ${ }^{32} \mathrm{P}$-labelled PDGF-A chain CDNA probe, (B) ${ }^{32} \mathrm{P}$-labelled ECGF oligonucleotide probe and (C) ${ }^{32} \mathrm{P}$-labelled PDGF-B chain CDNA probe. The two unmarked lanes are the radioactive markers (refer above). Lane 1 represents control (Medium 199 alone), Lane 2 represents $17 \beta$-estradiol (10-10 $\mathrm{M})$ in combination with progesterone $\left(10^{-9} \mathrm{M}\right)$, Lane 3 represents progesterone $\left(10^{-9} \mathrm{M}\right)$, Lane 4 represents $17 \beta$-estradiol $\left(10^{-10} \mathrm{M}\right)$, Lane 5 represents absolute ethanol (alcohol), Lane 6 represents $17 \beta$-estradiol $\left(10^{-5} \mathrm{M}\right)$ in combination with progesterone $\left(10^{-5} \mathrm{M}\right)$, Lane 7 represents progesterone $\left(10^{-5} \mathrm{M}\right)$, Lane 8 represents $17 \beta$ estradiol $\left(10^{-5} \mathrm{M}\right)$, and Lane 9 represents $\mathrm{IL}-1(5 \mathrm{U} / \mathrm{ml})$. All filters were exposed to Kodak XAR-5 films at $-70^{\circ} \mathrm{C}$ overnight (B and $\mathrm{C}$ filters) then for 5 days (A filter). 

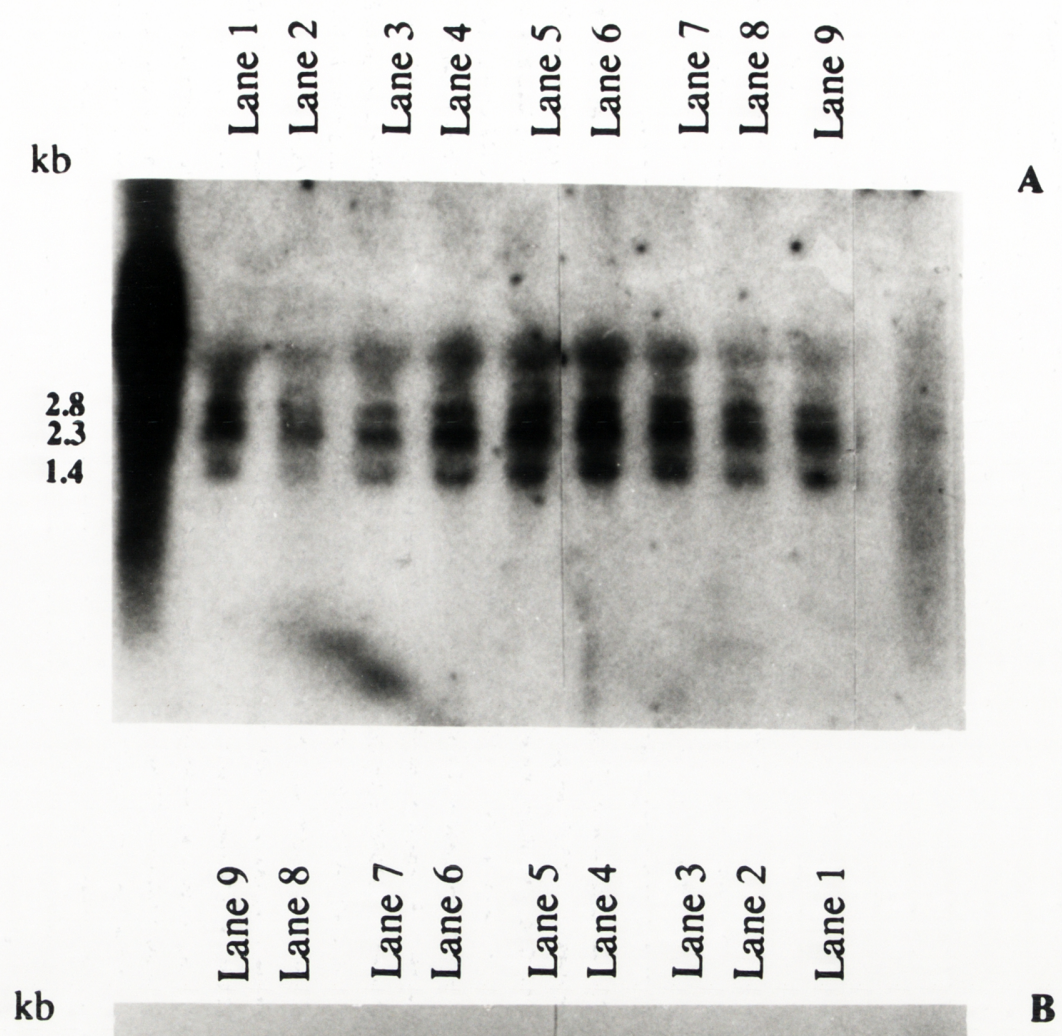

$\mathrm{kb}$

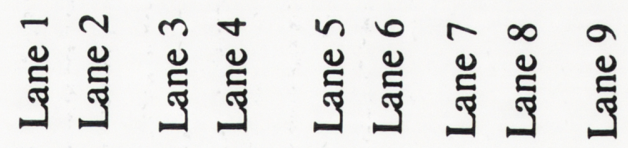

4.36

4.0

3.0

2.5

1.1

C 


\section{Figure 3.12.}

Slot blot analysis of total RNA ( $10 \mu \mathrm{g}, 3.3 \mu \mathrm{g}$ and $1 \mu \mathrm{g}$ per stimulus) showing the effect of sex steroids on the expression of mRNA for PDGF-B and PDGF-A in HUV endothelial cells. Total RNA from cultured endothelial cells exposed to sex steroids, rlL-1 $(5 \mathrm{U} / \mathrm{ml})$, alcohol $(0.1 \%)$ or Medium 199 alone (control) was transferred to nitrocellulose and hybridized with a ${ }^{32} \mathrm{P}$-labelled PDGF-B chain CDNA probe (A) and ${ }^{32} \mathrm{P}$-labelled PDGF-A chain CDNA probe (B). Lane 1 contains RNA from endothelial cells treated with IL-1 (5 U $/ \mathrm{ml})$; Lane 2, $17 \beta$-estradiol $\left(10^{-5} \mathrm{M}\right)$; Lane 3, $17 \beta$-estradiol $\left(10^{-10}\right.$ $\mathrm{M})$; Lane 4, progesterone $\left(10^{-5} \mathrm{M}\right)$; Lane 5 , progesterone $\left(10^{-9} \mathrm{M}\right)$; Lane $6,17 \beta$-estradiol $\left(10^{-5} \mathrm{M}\right)$ in combination with progesterone $\left(10^{-5} \mathrm{M}\right)$; Lane $7,17 \beta$-estradiol $\left(10^{-10} \mathrm{M}\right)$ in combination with progesterone $\left(10^{-9} \mathrm{M}\right)$; Lane 8 , absolute ethanol and Lane 9 , control (Medium 199 alone). Filters were exposed to Kodak XAR-5 films at $-70^{\circ} \mathrm{C}$ for 5 days. 


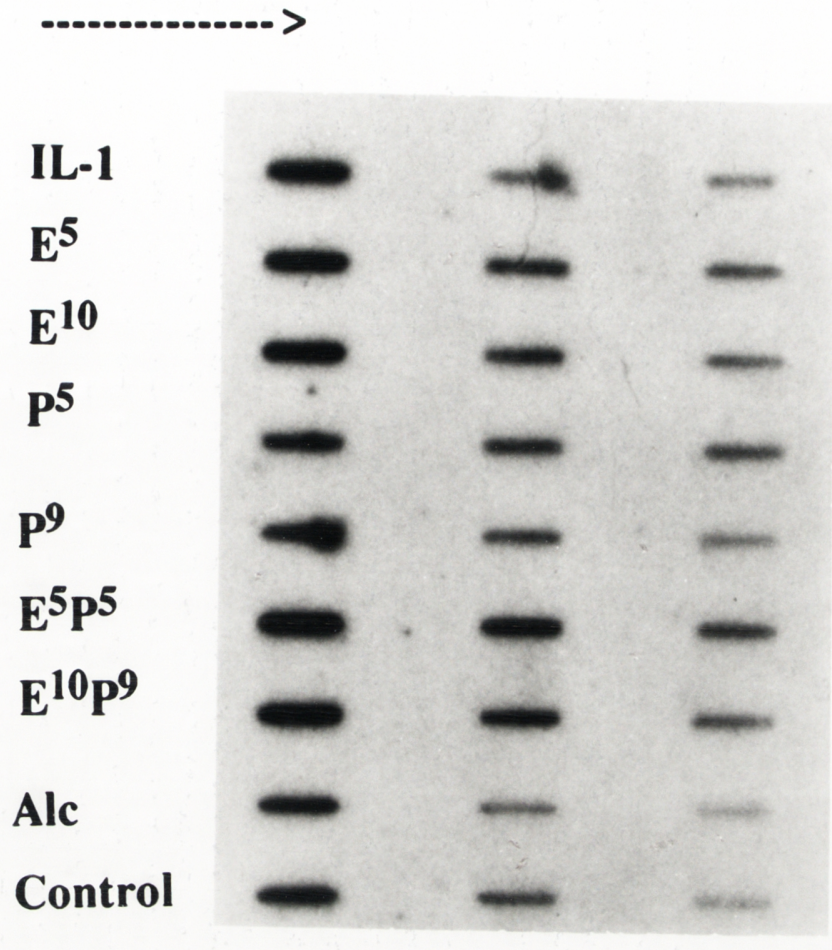

A

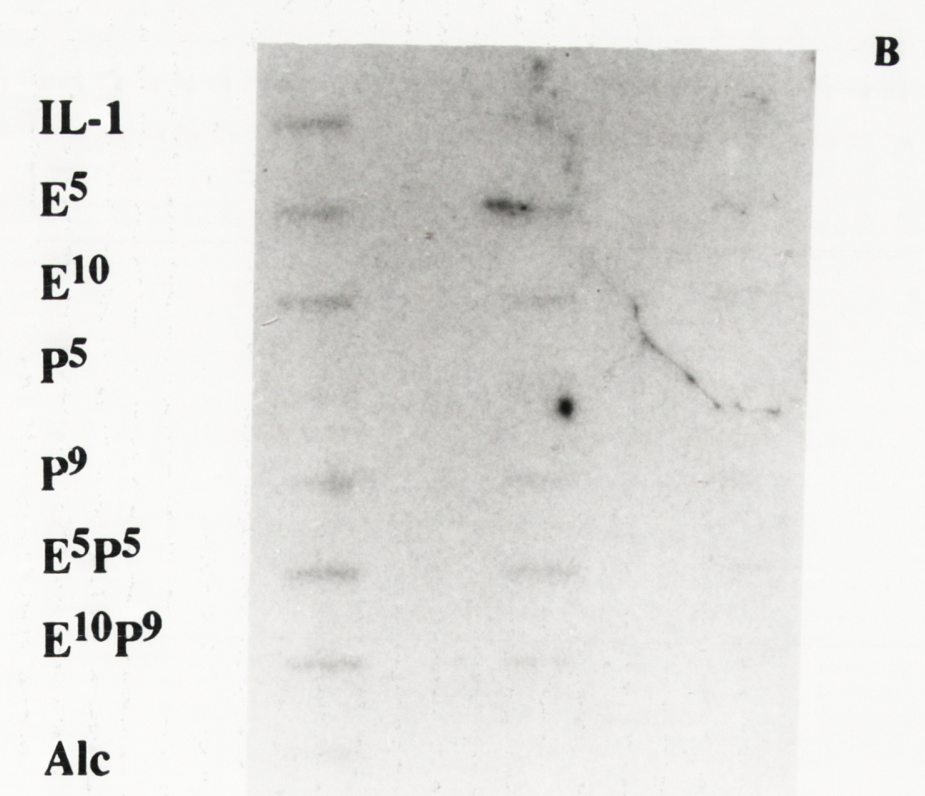

Control 
Figure 3.13.

A $1 \%$ agarose gel stained in ethidium bromide to demonstrate integrity of RNA samples from HUV endothelial cells using ribosomal RNA (28S and 18S) as a guide. Lane 1 represents $1 \mathrm{~kb}$ ladder $(1 \mu \mathrm{l})$, Lane 2 represents $17 \beta$-estradiol $\left(10^{-5} \mathrm{M}\right)$, Lane 3 represents $17 \beta$-estradiol $\left(10^{-10} \mathrm{M}\right)$, Lane 4 represents progesterone $\left(10^{-5} \mathrm{M}\right)$, Lane 5 represents progesterone $\left(10^{-9} \mathrm{M}\right)$, Lane 6 represents $17 \beta$-estradiol $\left(10^{-5} \mathrm{M}\right)$ in combination with progesterone $\left(10^{-5} \mathrm{M}\right)$, Lane 7 represents $17 \beta$-estradiol $\left(10^{-10} \mathrm{M}\right)$ in combination with progesterone $\left(10^{-9} \mathrm{M}\right)$, Lane 8 represents control (Medium 199 alone), Lane 9 represents IL-1 $(5 \mathrm{U} / \mathrm{ml})$, Lane 10 represents absolute ethanol and Lane 11 represents $1 \mathrm{~kb}$ ladder. 


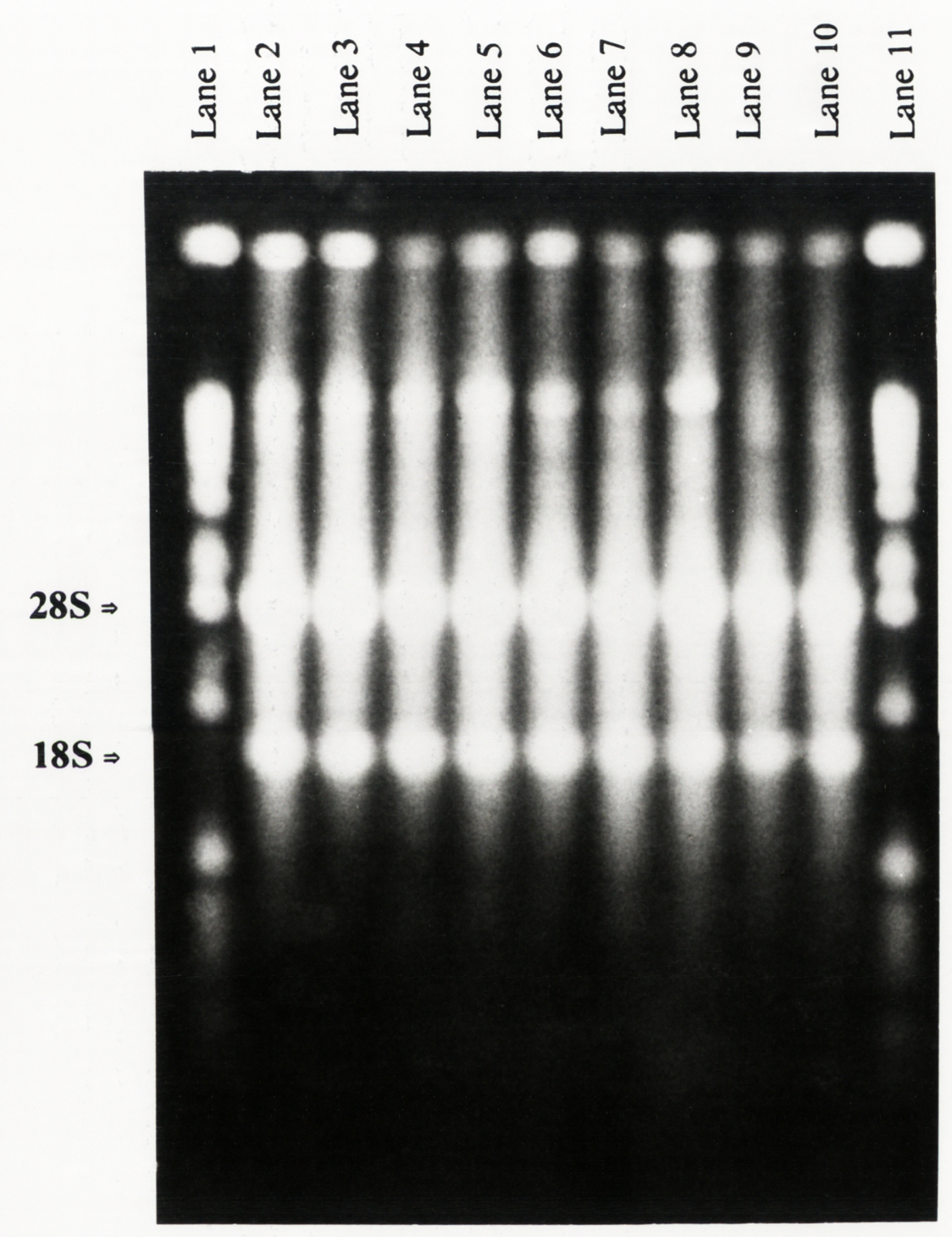


Figure 3.14.

Effect of PDGF on HUV endothelial cell proliferation. Endothelial cells were exposed to 1 in 2 dilutions of PDGF (maximum concentration $25 \mathrm{ng} / \mathrm{ml}$ ) over $66 \mathrm{~h}$ in the presence of $20 \%$ FCS, heparin $20 \mathrm{U} / \mathrm{ml}$ and variable concentrations of ECGS. The background is endothelial cells exposed to Medium 199 alone at a particular ECGS concentration. This graph is representative of 2 experiments in triplicate. ${ }^{*}=p<0.05$.

Figure 3.15.

Effect of PDGF on HUV endothelial cell proliferation. Endothelial cells were exposed to 1 in 2 dilutions of PDGF (maximum concentration $25 \mathrm{ng} / \mathrm{ml}$ ) over $66 \mathrm{~h}$ in the presence of $20 \% \mathrm{FCS}$, heparin $20 \mathrm{U} / \mathrm{ml}$ and in the presence or absence of ECGS $(25 \mu \mathrm{g}$ $/ \mathrm{ml}$ ). This graph is representative of 3 experiments each in triplicate. ${ }^{*}=p<0.05$. 

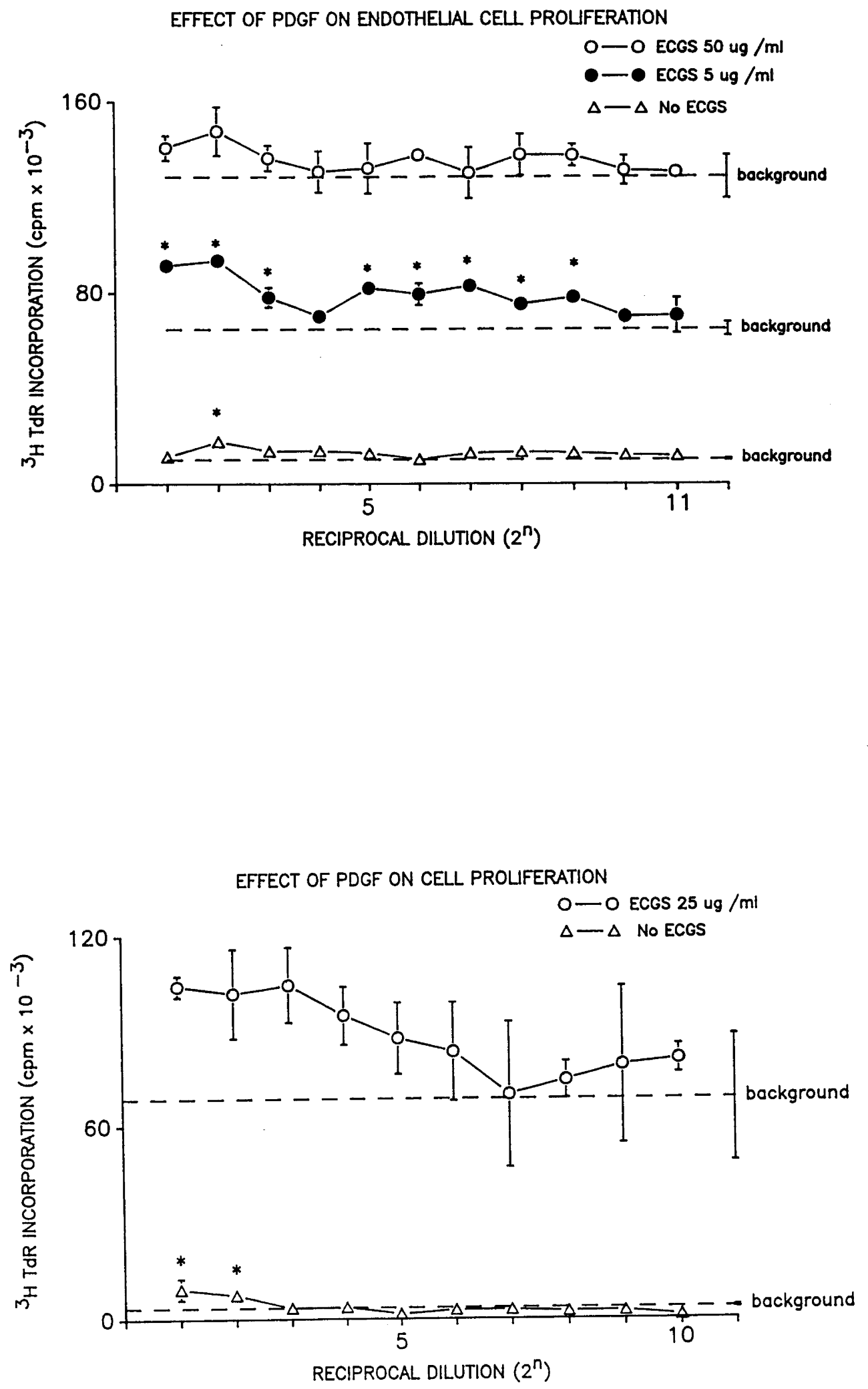
CHAPTER 4

EFFECTS OF SEX STEROIDS ON ENDOTHELIAL CELLS:

PLATELET ADHESION, PROSTACYCLIN RELEASE AND VON WILLEBRAND FACTOR PRODUCTION 


\subsection{INTRODUCTION}

Circulating blood platelets do not normally adhere to, or aggregate on, the vascular endothelial lining (Saba, 1973; Curwen et al, 1980; Dejana et al, 1980). Alteration to platelet or endothelial function may however lead to platelet adherence to endothelial cells and platelet aggregation, factors which may be important in the pathogenesis of atherosclerosis (Garrison et al, 1980).

\subsubsection{Antithrombotic and prothrombotic features of endothelial cells}

Endothelial cells possess properties which enable them to either promote or inhibit platelet binding depending on their environment. The properties which inhibit binding of platelets to the endothelial cell and platelet aggregation include:

(a) A net negative charge on the endothelial cell surface (Collen, 1980) attributable to the glycocalyx which consists of a high proportion of heparan sulfate (Keller et al, 1987) and sialic acid (Sprague et al, 1988), which are not reactive to platelets;

(b) The presence of an ectoplasmic ATPase that metabolizes platelet aggregating agents, e.g. nucleotides (Dieterle et al, 1978). Ectoplasmic ATPase activity converts ADP released from platelets to adenosine, a platelet inhibitor (Jaffe, 1985). In response to nonspecific injury endothelial cells also release ATP and ADP which can also be converted to adenosine (Pearson and Gordon, 1979);

(c) The synthesis (Weksler et al, 1977) and release of $\mathrm{PGI}_{2}$ (Moncada et al, 1976; 1979), a potent inhibitor of platelet aggregation;

(d) The presence of 13-hydroxyoctadecadienoic acid (13-HODE) (Buchanan et al, 1985). 13-hydroxyoctadecadienoic acid is a product of linoleic acid 
which decreases platelet adhesion and increases $\mathrm{PGI}_{2}$ production by some endothelial cells (Gryglewski et al, 1988);

(e) The production of endothelium-derived relaxing factor (EDRF) (Radomski et al, 1987; Sneddon and Vane, 1988) which has recently been identified as nitric oxide (Palmer et al, 1987) although nitric oxide may not be the sole or principal endothelium-derived relaxing factor (Myers et al, 1989). Endothelium-derived relaxing factor is a highly labile substance which causes smooth muscle cell relaxation and inhibits platelet aggregation and adhesion (Radomski et al, 1987; Snedden and Vane, 1988). Receptor mediated release of $\mathrm{PGI}_{2}$ and endothelium-derived relaxing factor may be coupled through the activation of phospholipase (de Nucci et al, 1988);

(f) The presence of antithrombin III, a glycoprotein, bound to endothelial cells which can inactivate thrombin (Marcum et al, 1984; Chesterman, 1988). Antithrombin III can also inhibit Factor Xa, IXa, Xla and XII; and

(g) The presence of thrombomodulin, which can modulate the activity of thrombin (Nievelstein and de Groot, 1988). When thrombin binds to thrombomodulin it activates protein C (Esmon and Esmon, 1984; Rodgers, 1988). Protein $C$ is a vitamin $K$ dependent protein synthesized by the liver which inactivates factors V and VIII by proteolysis (Jaffe, 1985). Protein C requires protein $S$ for optimal activity (Mustard et al, 1987; Nawroth and Stern, 1987).

The prothrombotic features of endothelial cells include:

(a) The synthesis and release of vWf into the plasma (Jaffe et al, 1973; Houdijk et al, 1986) or into the vascular subendothelial matrix (Rand et al, 1980). vWf is involved in the adhesion of platelets to the subendothelium especially at high shear flow (Chesterman and Berndt, 1986);

(b) The synthesis and release of thromboplastin (Zeldis et al, 1972). Thromboplastin reacts with factor VII and calcium and markedly accelerates the ability of factor VII to activate factor X (Jaffe, 1985); 
(c) Synthesis of platelet-activating factor (Prescott et al, 1984; Whatley et al, 1987). This is a potent autacoid that causes activation and aggregation of platelets. The production of platelet activating factor is dependent on the presence of extracellular calcium (Whatley et al, 1989) and is associated with a simultaneous increase in $\mathrm{PGI}_{2}$ (Whatley et al, 1990);

(d) Fibronectin synthesis (Houdijk et al, 1986; Mustard et al, 1987; Nievelstein and de Groot, 1988). Fibronectin is a large glycoprotein which has specific binding sites for collagen, fibrin and heparin, and probably functions as an adhesive protein for cell attachment (Mosher et al, 1984);

(e) Factor $V$ synthesis which accelerates the activation of prothrombin by factor Xa (Jaffe, 1985; Nievelstein and de Groot, 1988); and

(f) Thrombospondin synthesis (Silverstein et al, 1986; Nievelstein and de Groot, 1988). Thrombospondin influences the agglutination of erythrocytes by thrombin-treated human platelets. It promotes cell substratum adhesion (Tuszynski, 1987) and has been shown to cause rapid binding and activation of platelets on polymeric surfaces (Mosher et al, 1984).

\subsubsection{Role of platelet binding to endothelial cells in atherogenesis}

Platelets do not normally bind to intact endothelial cells. Increased platelet adhesion has been shown to occur as a result of in vitro injury to endothelium including treatment of the endothelium with epinephrine, serotonin, $0.15 \mathrm{M}$ $\mathrm{NaCl}$, trypsin, heat or scraping. As a consequence of these treatments, platelets come into contact with the exposed microfilaments between and /or beneath the endothelial cells (although not the endothelial cells themselves) (Booyse et al, 1975; Wechezak et al, 1975). Alteration to the endothelial cell surface by either 2-chloroacetaldehyde (Zetter et al, 1978), viral transformation (Gimbrone et al, 1976; Curwen et al, 1982), treatment with aspirin in the presence of thrombin (Czervionke et al, 1978), and thrombin alone (Kaplan et al, 1989) cause increased platelet binding to the endothelial 
cell. In addition, it is possible that activated platelets themselves may injure endothelial cells thus promoting further platelet adhesion (Kishi et al, 1989). Decreased platelet adhesion to aortic endothelial cells has been observed in pigs fed butterfat and fish oil, perhaps due to reduced thromboxane $B_{2}$ production (Foxall and Shwaery, 1990). Zwaginga et al (1990) showed that the thrombogenicity of endothelial cells in vitro depends on the source of the cells. HUV endothelial cells are amongst the least thrombogenic of endothelial cells studied.

In summary, there is evidence that alteration to the vascular endothelium may promote platelet adhesion, leading to activation of platelets and injury to the endothelium.

\subsubsection{Role of exogenous sex steroids in platelet binding and aggregation}

Although considerable information is available on the effect of exogenous estradiol and progesterone on platelet aggregation and thrombus formation (reviewed in Sections 1.5.2 and 1.5.3.3) little is known about the influence of these hormones on endothelial cells in relation to platelet binding. High concentrations of estradiol cypionate and progesterone have not been shown to have an effect on platelet adhesiveness (Mitchell and Williams, 1974).

Studies have however found that exogenous sex steroids can alter the production of factors such as antithrombin III, $\mathrm{PGI}_{2}$ and $\mathrm{vWf}$ which contribute to the antithrombogenic profile of the endothelial cell (detailed in Chapter 1).

\subsection{4 $\mathrm{PGI}_{2}$ production and release by endothelial cells}

$\mathrm{PGI}_{2}$ is the major prostanoid present in incubates of freshly isolated endothelial cells from large arteries and veins (Weksler et al, 1977). It is formed via the cyclooxygenase pathway, most commonly from arachidonic 
acid (Smith, 1986). It is released both from the luminal surface to prevent platelet aggregation (Eldor et al, 1981) and from the abluminal surface to relax underlying smooth muscle (Smith, 1986). $\mathrm{PGI}_{2}$ inhibits platelet aggregation by stimulating adenylate cyclase, leading to an increase in CAMP levels in platelets (Moncada and Vane, 1979; Vane et al, 1982).

The regulation of $\mathrm{PGl}_{2}$ synthesis by cultured endothelial cells is poorly understood. $\mathrm{PGI}_{2}$ synthesis by cultured vascular endothelial cells can be stimulated by arachidonic acid (Hong, 1980), calcium ionophore A23187 (Wesksler et al, 1978; Hong, 1980), thrombin (Wesksler et al, 1978; Czervionke et al, 1979b; Hong, 1980; Hallam et al, 1988), trypsin in some cultured endothelium (Wesksler et al, 1978; Hong, 1980), IL-1 (Rossi et al, 1985; Kawakami et al, 1986; Dejana et al, 1987; Zavoico et al, 1989; Breviario et al, 1990), tumour necrosis factor (Kawakami et al, 1986), epidermal growth factor and transforming growth factor- $\alpha$ (Ristimaki et al, 1988; Ristimaki, 1989), cyclosporin (Zojia et al, 1986), leukotriene C4 (Polage et al, 1984), histamine (Alhenc-Gelas et al, 1982), bradykinin (Hong, 1980; Alhenc-Gelas et al, 1982), angiotensin II (Lonchampt et al, 1981), oxidized LDL (Triau et al, 1988), phorbol 12-myristate 13-acetate (Wu et al. 1988), palmitoylcarnitine (Boeynaems et al, 1989) and high concentrations of native LDL (Holland et al, 1988). Balloon catheter trauma (Menta et al, 1982), radiation (Eldor et al, 1983a; Eldor et al, 1989), shear stress (Francos et al, 1985), or exposure to melittin (Johnson et al, 1985) also result in $\mathrm{PGI}_{2}$ release from endothelial cells. Other substances such as aspirin (Czervionke et al, 1979a; Dejana et al, 1980), plasmin (Schafer et al, 1989), low concentrations of LDL (Szczeklik and Gryglewski, 1980), picotamide (Landolfi et al. 1988), cyclosporin (Brown et al, 1990), heparin with ECGF (Boutherin-Falson and Blaes, 1989), glucocorticoids (Vane et al, 1990), 15-hydroperoxyarachidonic acid (15HPAA), a lipid peroxide (Vane et al, 1982) and selenium deficiency (Hampel et al, 1989) inhibit $\mathrm{PGl}_{2}$ production. Glucocorticoids, in particular 
dexamethasone $\left(10^{-11}-10^{-7} \mathrm{M}\right)$, inhibit $\mathrm{PGI}_{2}$ release from endothelial cells by stimulating the formation of lipocortin, an endogenous inhibitor of phospholipase $A_{2}$, which liberates arachidonic acid from membrane phospholipids (Lewis et al, 1986; Vane et al, 1990). Preincubation of endothelial cells with cycloheximide inhibits the effect of dexamethasone on $\mathrm{PGI}_{2}$ release (Lewis et al, 1986). Aspirin inhibits $\mathrm{PGI}_{2}$ release by acetylation of a serine residue at the active site of cyclooxygenase which converts arachidonic acid into prostaglandin endoperoxides (Vane et al, 1990). With some of these agents (e.g. arachidonic acid, angiotensin II) the response appears rapidly and lasts only a few minutes, while other substances (e.g. IL1, epidermal growth factor, phorbol 12-myristate 13-acetate) cause a delayed stimulation. Oxidized LDL causes a biphasic response with a rapid release within $\mathbf{3 0}$ minutes, followed by a second peak several hours later (Triau et al, 1988).

Although the production of $\mathrm{PGl}_{2}$ by the endothelium is not responsible for the lack of adhesion of platelets to the intact vascular lining (Czervionke et al, 1978; Curwen et al, 1980; Dejana et al, 1980), it is a potent inhibitor of subsequent platelet aggregation and thus the $\mathrm{PGl}_{2}$ pathway has been implicated as an important factor in the pathogenesis of atherosclerosis (Moncada et al, 1976). $\mathrm{PGI}_{2}$ is thought to act locally because the plasma concentrations are too low to elicit responses (Blair et al, 1982). Different catabolic systems in the lung, kidney and liver effectively prevent the passage of $\mathrm{PGI}_{2}$ through the circulation (Ferreira and Vane, 1967).

\subsubsection{Effect of exogenous sex steroids on $\mathrm{PGI}_{2}$ production and release}

This has been reviewed in Chapter 1 (refer to Section 1.5.3.3.1). 


\subsection{6 vWf: Ag expression by endothelial cells}

Coagulation Factor VIII circulates in plasma as a high molecular weight complex composed of two different glycoproteins (Jaffe, 1984). The major component is called vWf. vWf can be measured antigenically as vWf : Ag and is present in normal plasma at levels of about $10 \mu \mathrm{g} / \mathrm{ml}$ (Jaffe, 1984). vWf is found in the Weibel-Palade bodies of endothelial cells (Wagner et al, 1982) and has been identified in the cytoplasm, membrane and the endoplasmic reticulum. This is progressively released into the medium from cultured HUV endothelial cells (Jaffe et al, 1973b, Jaffe, 1977). The major known function of vWf is in platelet adhesion to the subendothelium (Zucker et al, 1969; Jaffe et al, 1973b; Bolhuis et al, 1981). Plasma vWf binds to subendothelial cell structures and this is followed by interaction with a specific receptor for vWf on the platelet membrane (Kao et al, 1979) which mediates platelet adhesion (Dejana et al, 1989). Under some circumstances, e.g. following thrombin or ADP activation, vWf also binds to a different platelet membrane glycoprotein which promotes platelet aggregation (Ruggeri et al, 1983). vWf is also an adhesion molecule for endothelial cells (Cheresh, 1987; Dejana et al, 1989). The adhesion of endothelial cells to vWf promotes the organisation of microfilaments and focal contacts in endothelial cells (Dejana et al, 1989).

Interestingly, Fuster et al (1978) demonstrated that pigs with von Willebrand's disease fed a high cholesterol diet develop intimal fatty streaks but do not develop the proliferative lesions of atherosclerosis. This is probably because platelets adhere poorly to subendothelial tissue in von Willebrand's disease (Fuster et al, 1978), and therefore do not release factors which promote migration and proliferation of smooth muscle into the intima.

The factors that regulate the synthesis and release of vWf are poorly understood. Exercise, adrenergic stimulation, growth hormone, thyroid 
hormone (Jaffe, 1984) and 1-deamino-8-D arginine vasopressin (DDAVP) (Mannucci et al, 1977; Hashemi et al, 1990) are associated with rises in vWf in vivo. However, epinephrine, 5-hydroxytryptamine, hydrocortisone, dexamethasone, growth hormone, and thyroxine do not alter the rate of release and accumulation of vWf by cultured HUV endothelial cells (Booyse et al, 1981a; Tuddenham et al, 1981). DDAVP possibly acts by stimulating monocytes to release a factor which subsequently stimulates endothelial cells to release vWf, since in vitro experiments have shown that DDAVP cannot directly stimulate vWf release from HUV endothelial cells (Hashemi et al, 1990). Radiation also induces the release of vWf protein from cultured endothelial cells (Sporn et al, 1984). Glucose has been shown to increase release of vWf : Ag (Mordes et al, 1983) while cytomegalovirus has been shown to reduce the amount of vWf in Weibel-Palade bodies (Bruggeman et al, 1988).

Two pathways for secretion of vWf have been identified. Firstly, there is a basal level of synthesis and release of the antigen which can be blocked by cycloheximide. Secondly, there is an additional release of $\mathrm{vWf}: \mathrm{Ag}$ in response to appropriate stimuli. The latter release occurs from storage granules in the cells and is dependent on extracellular calcium. It is independent of intracellular CAMP, involving a receptor-independent, nonlytic mechanism rather than an increase in synthesis and so it is not blocked by cycloheximide (Levine et al, 1981; 1982; Loesberg et al, 1983). Stimuli which can increase vWf release in this fashion include thrombin, calcium ionophore A23187, phorbol 12-myristate 13-acetate (Loesberg et al, 1983; Hashemi et al, 1990), lipopolysaccharide (Hashemi et al, 1990), IL-1 (Schorer et al, 1987; Breit and Green, 1988; Hashemi et al, 1990), tumour necrosis factor and recombinant human interferon- $\gamma$. Schorer et al (1987) found that IL-1 increased the release of $\mathrm{vWf}$ within $2 \mathrm{~h}$ but the residual cellular vWf : Ag was reciprocally lower in IL-1 treated endothelial cells at $24 \mathrm{~h}$ and $48 \mathrm{~h}$ indicating 
that a compensatory increase in synthesis of vWf : Ag did not occur during this time. de Groot et al (1987) also found that incubation with rlL-1 for $12 \mathrm{~h}$ or more caused a decline in the steady state level of vWf mRNA in HUV endothelial cells but also observed a decreased secretion into the culture medium and extravascular matrix over this time. Breit and Green (1988) and Schorer (1987) found that IL-1 caused more vWf : Ag to be released at $6 \mathrm{~h}$ than after $48 \mathrm{~h}$ which is in agreement with the decline in vWf mRNA observed by de Groot et al (1987). Zavoico et al (1989) found that IL-1 did not alter vWf secretion from HUV endothelial cells if they were pretreated for $24 \mathrm{~h}$. There does not appear to be a simple explanation for the different findings reported above for IL-1 secretion from endothelial cells apart from the fact that the composition of serum may alter vWf secretion from endothelial cells.

\subsubsection{Effect of exogenous sex steroids on the production and release of vWf}

This has been reviewed in Chapter 1 (refer to Section 1.5.3.3.2).

The aims of the experiments reported in this Chapter were to determine whether $17 \beta$-estradiol, progesterone or the combination of these sex steroids alters the ability of HUV endothelial cells to bind platelets, release $\mathrm{PGI}_{2}$, and release and express vWf, since both platelet binding and aggregation are considered to be important in the pathogenesis of atherosclerosis.

\subsection{MATERIALS}

Materials specific to the studies described in this Chapter were as follows:

Acid Citrate Dextrose $(A C D)$ consisted of trisodium citrate $(25 \mathrm{~g})$, citric acid $(14 \mathrm{~g})$ and dextrose $(20 \mathrm{~g})$ made up to $1.0 \mathrm{~L}$ in distilled water and stored at 4 ${ }^{\circ} \mathrm{C}$. 
O-Acetylsalicylic acid (Aspirin) (BDH Chemical Ltd, England) was made up immediately prior to each experiment as a $0.01 \mathrm{M}$ stock solution in PBS, pH 7.4 and filtered through a $0.22 \mu \mathrm{m}$ filter.

Adenine (Fluka AG, Buchs) was dissolved in HBSS at a working concentration of $7.5 \mathrm{mM}$ and stored at $4{ }^{\circ} \mathrm{C}$ in the dark.

[2-3 $\mathrm{H}$ ]-adenine (Amersham International, UK), 5 millicuries (185 megabq, specific activity $68 \mathrm{mCi} / \mathrm{mg}$ ) in $5 \mathrm{ml}$ of $2 \%$ alcohol. This was stored at $4{ }^{\circ} \mathrm{C}$.

Agarose (Seakem, FMC bioproducts, Rockland, USA). A $1 \%$ agarose gel was prepared in $0.05 \mathrm{M}$ barbital buffer (see below).

Antihemophilic factor (AHF) (fibrinogen-poor), human factor VIII for injection. $180 \mathrm{mg} \mathrm{(315} \mathrm{U)} \mathrm{of} \mathrm{factor} \mathrm{VIII} \mathrm{(CSL,} \mathrm{Melbourne,} \mathrm{Australia)} \mathrm{was} \mathrm{dissolved} \mathrm{in}$ PBS and stored at $4^{\circ} \mathrm{C}$.

Clotimmun Antihemophilic globulin (AHG)-associated protein (Factor VIIIrelated antigen) (Behring, Germany), Lot 105717A (antiserum raised in rabbits), and preserved in sodium azide and sodium p-ethyl-mercurymercapto-benzene-sulfonate. It was stored at $4^{\circ} \mathrm{C}$.

Antiserum to human Factor VIII (raised in goats) was obtained from Miles Scientific, Naperville, USA. Antiserum was diluted 1 in 2 in PBS and $50 \mu \mathrm{l}$ aliquots were stored in Eppendorf tubes at $-90^{\circ} \mathrm{C}$. This was diluted 1 in 8 in 1 $\%$ BSA in PBS just prior to use.

Apyrase (Sigma Chemical Company, St Louis, USA) was dissolved in PBS at a concentration of $30 \mathrm{mg} / \mathrm{ml}$ and stored at $-20^{\circ} \mathrm{C}$. 
Aqueous scintillant consisted of xylene (33 L), PPO (1.0 kg) and triton X (67 L) made up to $100 \mathrm{~L}$ and stored in a fume cupboard.

Assay reference plasma (ARP) (Helena Lab, Texas, USA). Reference value: Lot No.2005185 and Lot No. 5905185. This was reconstituted in Tris saline and kept at $-70^{\circ} \mathrm{C}$.

Barbital buffer. A $0.05 \mathrm{M}$ solution, $\mathrm{pH} 8.6$ was prepared by dissolving diethyl barbituric acid (2.76 g) (BDH Laboratory, Poole, UK) and sodium diethyl barbiturate (15.4 g) (BDH Laboratory, Poole, UK) in $1500 \mathrm{ml}$ of distilled water which was then stored at room temperature.

Buffered salt solution (BSS) comprised D-glucose (10 g), $\mathrm{KH}_{2} \mathrm{PO}_{4}(0.6 \mathrm{~g})$, $\mathrm{Na}_{2} \mathrm{PO}_{4} \cdot 2 \mathrm{H}_{2} \mathrm{O}(1.89 \mathrm{~g}), \mathrm{KCl}(4 \mathrm{~g})$ and $\mathrm{NaCl}(80 \mathrm{~g})$ made up to $500 \mathrm{ml}$ with distilled water combined with $\mathrm{MgCl}_{2} \cdot 6 \mathrm{H}_{2} \mathrm{O}(2 \mathrm{~g}), \mathrm{MgSO}_{4} \cdot 7 \mathrm{H}_{2} \mathrm{O}(2 \mathrm{~g})$ and $\mathrm{CaCl}_{2} \cdot 2 \mathrm{H}_{2} \mathrm{O}(1.4 \mathrm{~g})$ made up to $500 \mathrm{ml}$ with distilled water. This solution was then made up to $10 \mathrm{~L}$ with distilled water, filtered and stored at room temperature.

Cycloheximide (Sigma Chemical Company, St Louis, USA) was dissolved in absolute ethanol to obtain a solution of $20 \mu \mathrm{g} / \mathrm{ml}$ and stored at $-20^{\circ} \mathrm{C}$.

Decolourizer for the Laurell rocket immunoelectrophoresis comprised $40 \%$ methanol (May and Baker, Footscray, Victoria) and $10 \%$ glacial acetic acid (May and Baker, Footscray, Victoria) made up to $500 \mathrm{ml}$ with distilled water. This was stored at room temperature.

Murine ELAM-1 monoclonal antibody $H 18 / 7$ (prepared from ascites) was a 
gift from Dr M. Gimbrone and Dr M. Bevilacqua, Boston, MA, USA. A working solution of $1 / 2500$ in $1 \%$ BSA in PBS was used. This was stored at $4{ }^{\circ} \mathrm{C}$.

F.I.T.C.-conjugated antigoat IgG (raised in rabbits) (Miles-Yella, Ind, USA) was diluted $1 / 2$ in PBS and stored in $100 \mu$ aliquots in Eppendorf tubes at -90 ${ }^{\circ} \mathrm{C}$. This stock solution was diluted 1 in 16 in phenol red free BSS with $10 \%$ FCS prior to use.

F.I.T.C.-conjugated antimouse IgG (raised in sheep) (Silenus, Victoria, Australia) was stored at $4^{\circ} \mathrm{C}$. A $1 / 20$ dilution of the stock was dissolved in phenol red free BSS with $10 \%$ FCS just prior to use.

F.I.T.C-conjugated antirat IgG (raised in sheep) (Silenus, Victoria, Australia) was stored at $4^{\circ} \mathrm{C}$. A $1 / 20$ dilution of the stock was dissolved in phenol red free BSS with $10 \%$ FCS just prior to use.

Gel bond film agarose gel support medium (FMC bioproducts, Rockland, USA).

Hanks Balanced Salt Solution without calcium and magnesium (CMF HBSS). A 10 times concentrate comprising $\mathrm{KCl}(4 \mathrm{~g}), \mathrm{KH}_{2} \mathrm{PO}_{4}(0.6 \mathrm{~g}), \mathrm{NaCl}(80 \mathrm{~g})$, $\mathrm{NaHCO}_{3}(3.5 \mathrm{~g}), \mathrm{Na}_{2} \mathrm{PO}_{4}(0.475 \mathrm{~g})$ and D-glucose $(10 \mathrm{~g})$ was made up to 1.0 $\mathrm{L}$, filtered and stored at room temperature.

Hanks Balanced Salt Solution with calcium and magnesium (HBSS) comprised the same salts as detailed for CMF HBSS dissolved in $500 \mathrm{ml}$ of distilled water to which $\mathrm{CaCl}_{2} .2 \mathrm{H}_{2} \mathrm{O}(1.85 \mathrm{~g})$ and $\mathrm{MgSO}_{4} \cdot 6 \mathrm{H}_{2} \mathrm{O}(2 \mathrm{~g})$ made up separately in $500 \mathrm{ml}$ of distilled water were then added. The mixture was filtered and then stored at room temperature. 
Paraformaldehyde solution (BDH Chem Ltd, Poole, England). $2 \mathrm{~g}$ of paraformaldehyde was dissolved in $100 \mathrm{ml}$ of PBS, $\mathrm{pH}$ 7.4. This was stored in the dark at $4^{\circ} \mathrm{C}$.

PgP-1 monoclonal antibody IM7. This was a gift from Dr R. Ceredig, JCSMR, Australian National University, Canberra, Australia. This was stored at $-20^{\circ} \mathrm{C}$ and used without dilution.

Plasmin (American Diagnostic, NY, USA). A stock solution of $2 \mathrm{mg} / \mathrm{ml}$ in PBS was made and kept at $-20^{\circ} \mathrm{C}$. A $2 \mu \mathrm{g} / \mathrm{ml}$ solution was made in PBS just prior to use.

Prostacyclin $\left(\mathrm{PGI}_{2}\right)$ (Sigma Chemical Company, St Louis, USA) was dissolved in $0.5 \mathrm{M}$ Tris buffer ( $\mathrm{pH} 9.4$ ) to give a concentration of $0.5 \mathrm{mg} / \mathrm{ml}$.

Platelet washing solution comprised glucose $(0.1 \mathrm{~g})$, BSA $(0.3 \mathrm{~g})$ and $5 \mathrm{ml}$ of stock solution I diluted to $100 \mathrm{ml}$ with distilled water, $\mathrm{pH} 7.4$ and warmed to 37 ${ }^{\circ} \mathrm{C}$. Stock solution I consisted of $\mathrm{NaCl}(160 \mathrm{~g} / \mathrm{L}), \mathrm{KCl}(4 \mathrm{~g} / \mathrm{L}), \mathrm{NaHCO}_{3}(20$ $\mathrm{g} / \mathrm{L})$, and $\mathrm{Na}_{2} \mathrm{H}_{2} \mathrm{PO}_{4}(1 \mathrm{~g} / \mathrm{L})$ made up to $1.0 \mathrm{~L}$ with distilled water and stored at $4^{\circ} \mathrm{C}$.

RGDS-NH ${ }_{2}$ (Peptide Technology, Dee Why, Australia) was a gift from Dr W. Cowden, JCSMR, Australian National University, Canberra, Australia. A stock solution of $10 \mathrm{mg} / \mathrm{ml}$ was made up in PBS and stored at $4^{\circ} \mathrm{C}$.

Staining solution for Laurell-rocket Immunoelectrophoresis comprised $0.2 \mathrm{~g}$ Coomassie blue R-250 (ICl, Australia), $40 \%$ methanol and $10 \%$ glacial acetic acid made up to $100 \mathrm{ml}$ with distilled water and stored at room temperature. 
Ristocetin sulphate (Wellcome, Denmark) was dissolved in Tris saline buffer to obtain a stock solution of $10 \mathrm{mg} / \mathrm{ml}$ and stored at $4^{\circ} \mathrm{C}$.

\subsection{METHODS}

\subsection{1 $\mathrm{PGl}_{2}$ release by HUV endothelial cells exposed to sex steroids}

\subsubsection{Treatment of HUV endothelial cells}

HUV endothelial cells were cultured as outlined in Chapter 2 (refer to Section 2.3.1). They were plated at a density of $2 \times 10^{4}$ cells /well in gelatinized 96 well plates. Cells at passages 7-9 were used. A confluent monolayer was established over $3-4$ days in $5 \% \mathrm{CO}_{2}-95 \%$ air atmosphere at $37^{\circ} \mathrm{C}$. The medium was removed and the sex steroids, rlL-1 or alcohol each diluted in Medium 199 (with $20 \%$ FCS, gentamicin $60 \mathrm{U} / \mathrm{ml}$ and the standard additives). 10 wells were used for each stimulus. The control was Medium 199 (with $20 \%$ FCS, gentamicin $60 \mathrm{U} / \mathrm{ml}$ and the standard additives). Following incubation for either $30 \mathrm{~min}, 6 \mathrm{~h}$, or $24 \mathrm{~h}$ each supernatant was transferred into Eppendorf tubes and centrifuged for $2.5 \mathrm{~min}$. $100 \mu \mathrm{l}$ of supernatant was added to each assay tube undiluted. The HUV endothelial cell count following $24 \mathrm{~h}$ incubation with Medium 199 or various stimuli was 3.5-4.5 $\times 10^{5}$ cells $/ \mathrm{ml}$ of supernatant.

In other experiments the HUV endothelial celis were preincubated with cycloheximide $(10 \mu \mathrm{g} / \mathrm{ml})$ or aspirin $(0.1 \mathrm{mM})$ for $30 \mathrm{~min}$ before the addition of the sex steroids. 


\subsubsection{Measurement of $P G I_{2}$}

$\mathrm{PGI}_{2}$ in supernatants was measured using the method of Demers et al (1980) supplied as a kit (Amersham, Product code TRK.790). The method was based on the production of 6-keto-prostaglandin $F_{1 \alpha}$, the stable endproduct of $\mathrm{PGI}_{2}$. Briefly, the supernatant from HUV endothelial cells was incubated overnight with $\left[{ }^{3} \mathrm{H}\right]-6$-keto-prostaglandin $\mathrm{F}_{1 \alpha}$ and 6-ketoprostaglandin $F_{1 \alpha}$ antiserum. The following day activated charcoal was added to remove free label and the sample was then centrifuged. Finally, the supernatant, which contained the radiolabelled 6-keto-prostaglandin $F_{1 \alpha}$ bound to the antibody, was added to aqueous scintillant and counted in a $\beta-$ scintillation counter. The concentration of unlabelled 6-keto-prostaglandin $F_{1 \alpha}$ in the sample was then determined from a linear standard curve.

\subsubsection{Binding of platelets to HUV endothelial cells exposed to sex steroids}

\subsubsection{Preparation and labelling of washed platelets for adhesion studies}

The method used was a modification of the procedure developed by Mustard et al (1972). $50 \mathrm{ml}$ of whole blood was collected via a 19 gauge butterfly needle from healthy volunteers who had not ingested aspirincontaining medications in the past week and, in the case of women, were not using oral contraceptives. The blood was added to $8 \mathrm{ml}$ of acid citrate dextrose and mixed gently. This was centrifuged ( $100 \times \mathrm{g}$ for $15 \mathrm{~min}$ at room temperature). The platelet-rich plasma (PRP) was aspirated using a Pasteur pipette. $\mathrm{PGI}_{2}(300 \eta \mathrm{g} / \mathrm{ml})$ and apyrase $(300 \eta \mathrm{g} / \mathrm{ml})$ were added. [2-3 $\mathrm{H}$ ]adenine $(15 \mu \mathrm{Ci} / \mathrm{ml}$ ) was added ( 1 in 2 dilution of stock) and the PRP incubated for $30 \mathrm{~min}$ at $37^{\circ} \mathrm{C}$. The PRP was then centrifuged $(300 \times \mathrm{g}$ for 20 min at room temperature) and the sedimented platelets were resuspended in 
$10 \mathrm{ml}$ of platelet washing fluid $(\mathrm{pH} 7.4)$ with $\mathrm{PGl}_{2}(300 \mathrm{\eta g} / \mathrm{ml})$ and apyrase (300 $\eta \mathrm{g} / \mathrm{ml})$. This step was repeated and the platelets were finally resuspended in warmed HBSS and allowed to sit at $37^{\circ} \mathrm{C}$ for $40 \mathrm{~min}$ to inactivate $\mathrm{PGl}_{2}$. The supernatant obtained after the labelling of the PRP was retained for use as platelet poor plasma (PPP) $\left(<1.0 \times 10^{4}\right.$ platelets $\left./ \mathrm{ml}\right)$ and taken to represent non incorporated $[2-3 \mathrm{H}]$-adenine. It was made up to the appropriate volume using HBSS. This was used to assess whether the sex steroids, rIL-1 or alcohol influenced the uptake of [2-3H]-adenine by the HUV endothelial cells. Since PPP had $\mathrm{PGI}_{2}$ and apyrase added during preparation it was allowed to stand for at least $\mathbf{4 0} \mathrm{min}$ before use. The platelet count was determined using a Coulter counter (Model ZF).

The effect of cold adenine on inhibiting the uptake of $[2-3 \mathrm{H}]$-adenine by HUV endothelial cells was assessed by adding [2-3 $\mathrm{H}]$-adenine $(2 \mu \mathrm{Cl} / \mathrm{ml})$ for 30 min to a confluent monolayer of HUV endothelial cells in the presence or absence of cold adenine.

\subsubsection{Preparation and labelling of PRP and PPP for adhesion studies.}

The method used was a modification of the method of Curwen et al (1982). PRP was obtained as described in Section 4.3.2.1 and the platelet count determined with a Coulter counter. [2-3H]-adenine $(15 \mu \mathrm{Ci} / \mathrm{ml})$ was added and PRP was incubated for $30 \mathrm{~min}$ at $37^{\circ} \mathrm{C}$. At the end of the incubation PRP was centrifuged ( $550 \times \mathrm{g}$ for $10 \mathrm{~min}$ at room temperature) to obtain PPP ( $<1.0$ $\times 10^{4}$ platelets $/ \mathrm{ml}$ ). This was added to HUV endothelial cells immediately.

$10 \mu \mathrm{l}$ aliquots of PRP, PPP and washed platelet suspensions were added to plastic scintillation vials containing $10 \mathrm{ml}$ of aqueous scintillant and counted with a $\beta$-scintillation counter to provide a comparison of $[2-3 \mathrm{H}]$-adenine uptake by platelets between experiments. 


\subsubsection{Treatment of HUV endothelial cell preparation}

HUV endothelial cells between passages $5-8$ were plated out in gelatinized 24 well plates at $2 \times 10^{5}$ cells /well in Medium 199 (20\% FCS, gentamicin 60 $\mathrm{U} / \mathrm{ml}$ and the standard additives) and allowed to become confluent over 3-4 days in $5 \% \mathrm{CO}_{2}-95 \%$ air atmosphere at $37{ }^{\circ} \mathrm{C}$. The medium was removed and a solution of the sex steroids, rlL-1 or alcohol diluted in Medium 199 (with $20 \%$ FCS, gentamicin $60 \mathrm{U} / \mathrm{ml}$ and the standard additives) was added. The control was Medium 199 (with $20 \%$ FCS, gentamicin $60 \mathrm{U} / \mathrm{ml}$ and the standard additives). Incubation continued for either $6 \mathrm{~h}$ or $18 \mathrm{~h}$ before the HUV endothelial cells were washed.

\subsubsection{Platelet-HUV endothelial cell monolayer adhesion assay}

y

The HUV endothelial cell monolayer was washed three times in HBSS at 37 ${ }^{\circ} \mathrm{C}$ to remove serum and cell debris. $0.75 \mathrm{ml}$ of $[2-3 \mathrm{H}]$-adenine labelled washed platelets, PRP or PPP was added to each well and incubated for 30 min in $5 \% \mathrm{CO}_{2}-95 \%$ air atmosphere at $37{ }^{\circ} \mathrm{C}$ with gentle agitation. The monolayer was then examined microscopically.

After exposure to radiolabelled platelets the HUV endothelial cells were washed three times in HBSS at $37^{\circ} \mathrm{C}$ to remove nonadherent platelets. The cells were then trypsinised for $30 \mathrm{~min}$ in $0.1 \%$ trypsin-versene and pipetted into scintillation vials to which $10 \mathrm{ml}$ of aqueous scintillant was added. The vials were counted with a Packard Tricarb $\beta$-Scintillation Counter.

Unlabelled cold adenine $(0.1 \mathrm{mM})$ was added to the radiolabelled washed platelets, PRP and PPP just prior to incubation with the HUV endothelial cell 
monolayer to reduce the uptake of $\left[2-{ }^{3} \mathrm{H}\right]$-adenine by the HUV endothelial cells.

\subsection{3 vWf : $\mathrm{Ag}$ immunofluorescence of HUV endothelial cells exposed to sex steroids}

\subsubsection{Treatment of HUV endothelial cells}

HUV endothelial cells between passage 5-10 were plated out in gelatinized 24 well plates at a density of $2 \times 10^{5}$ cells /well and allowed to become confluent over $3-4$ days in $5 \% \mathrm{CO}_{2}-95 \%$ air atmosphere at $37{ }^{\circ} \mathrm{C}$. The medium was removed and sex steroids, rIL-1, or alcohol were each added in Medium 199 (20\% FCS, gentamicin $60 \mathrm{U} / \mathrm{ml}$ and the standard additives) and incubated for $18 \mathrm{~h}$ in $5 \% \mathrm{CO}_{2}-95 \%$ air atmosphere at $37^{\circ} \mathrm{C}$. The control was Medium 199 (20\% FCS, gentamicin $60 \mathrm{U} \mathrm{/ml}$ and the standard additives). Studies were also performed in which cycloheximide (10 $\mu \mathrm{g} / \mathrm{ml})$ was added to the HUV endothelial cells for $\mathbf{3 0} \mathrm{min}$ prior to the addition of the sex steroids, rlL-1, alcohol or Medium 199.

After $18 \mathrm{~h}$ the medium was removed, the cells were washed with Medium 199 to remove cell debris, and the adherent cells were trypsinized for $3 \mathrm{~min}$ at $37^{\circ} \mathrm{C}$, centrifuged ( $200 \times \mathrm{g}$ for $8 \mathrm{~min}$ at room temperature), and resuspended in PRF Medium 199. The cells were washed by recentrifugation $(200 \times \mathrm{g}$ for 8 $\mathrm{min}$ at room temperature) and finally resuspended in $0.5-1.0 \mathrm{ml}$ of PRF Medium 199 (with $20 \%$ FCS and gentamicin $60 \mathrm{U} / \mathrm{ml}$ ) containing the sex steroids, alcohol, rlL-1 or Medium 199 alone, and allowed to sit at room temperature for $1 \mathrm{~h}$. The cells were counted on a haemocytometer and cell integrity was assessed using trypan blue. Finally, the cells were plated into $\mathrm{V}$ bottom 96 well plates (Nunclon, Denmark) at a concentration of $1-2 \times 10^{5}$ cells /well. 
In other studies various concentrations of plasmin and $\mathrm{RGDS}-\mathrm{NH}_{2}$, rather than trypsin, were used to remove the HUV endothelial cells from the gelatin.

\subsubsection{Immunofluorescence and flow cytometry}

HUV endothelial cells were centrifuged ( $185 \times \mathrm{g}$ for 3 min at $4^{\circ} \mathrm{C}$ ) in a 96 well $\mathrm{V}$ shaped plate and incubated for $30 \mathrm{~min}$ on ice with a predetermined saturating concentration of antiserum to human FVIII. The cells were then underlayed with FCS and centrifuged ( $185 \times \mathrm{g}$ for $3 \mathrm{~min}$ at $4^{\circ} \mathrm{C}$ ). The supernatant was removed and F.I.T.C-conjugated antigoat IgG was added for 30 min on ice in the dark. FCS was underlayed and the cells centrifuged (185 $x \mathrm{~g}$ for $3 \mathrm{~min}$ at $4^{\circ} \mathrm{C}$ ). The cells were washed in PBS, centrifuged and resuspended in $150 \mu l 1 \%$ paraformaldehyde in PBS. The cell suspension was transferred to fluorescent activated cell sorter (FACS) tubes and analysed on a Becton-Dickinson FACS IV (Becton Dickinson, Mountain View, CA).

Studies were also performed to assess the specificity of the antiserum to human FVIII by preincubation of this antibody with various concentrations of AHF containing FVIII before adding the antibody to the HUV endothelial cells.

The expression of hermes $\mathrm{Ag}$ / lymphocyte homing receptor (H-CAM) and ELAM-1 on HUV endothelial cells was also examined to check the experimental design. The effects of trypsin on these antigens was also assessed. ELAM-1 is a cell surface glycoprotein expressed by cytokineactivated (e.g. IL-1) endothelium (Bevilacqua et al, 1989). ELAM-1 mediates the adhesion of neutrophils. H-CAM (also known as ECMRIII, P80, or CD44) is a human glycoprotein expressed on many tissues including HUV endothelial cells (Oppenheimer-Marks, 1990) that is involved in lymphocyte 
binding to high endothelial cell venules and has been implicated in other cell adhesion events (Zhou et al, 1989; Pals et al, 1989; Shimizu et al, 1989). It is identical to mouse phagocytic glycoprotein (Pgp-1 or Ly-24). The anti-Pgp-1 monoclonal antibody is the rat monoclonal antibody IM7 which cross-reacts with the human glycoprotein. For examination of this adhesion molecule on HUV endothelial cells using immunofluorescence, the primary antibody used was the rat monoclonal antibody, IM7 and the second antibody was F.I.T.Cconjugated antirat IgG. To examine expression of ELAM-1, the first antibody used was $\mathrm{H} 18 / 7$ and the second antibody was F.I.T.C-conjugated antimouse $\operatorname{lgG}$.

\subsubsection{Effect of supernatant from HUV endothelial cells exposed to sex steroids on ristocetin-induced platelet aggregration}

\subsubsection{Treatment of HUV endothelial cells}

HUV endothelial cells were plated out at an initial density of $10^{6}$ cells /260 $\mathrm{ml}$ gelatinized flask, and then grown to 1 day post confluence in $5 \% \mathrm{CO}_{2}-95$ $\%$ air atmosphere at $37^{\circ} \mathrm{C}$. The medium was removed and sex steroids, alcohol or rlL-1 were added in Medium 199 (20\% FCS, gentamicin $60 \mathrm{U} / \mathrm{ml}$ and the standard additives), and incubated for $48 \mathrm{~h}$ in $5 \% \mathrm{CO}_{2}-95 \%$ air atmosphere at $37{ }^{\circ} \mathrm{C}$. The control was Medium 199 alone (20 \% FCS, gentamicin $60 \mathrm{U} / \mathrm{ml}$ and the standard additives). The medium was then

centrifuged (200 x g for $8 \mathrm{~min}$ at room temperature) and the supernatant removed. This supernatant was concentrated 10 times using an Amicon concentrator, Minicon-B15 (W R Grace and Co, Danvers, MA) and then stored at $-70^{\circ} \mathrm{C}$. 


\subsubsection{Ristocetin-induced platelet aggregation}

Ristocetin aggregates human platelets in the presence of vWf (Jaffe, 1977), and therefore it can be used as an in vitro method of determining the presence of vWf in a sample. Platelets were harvested from PRP. The platelets were washed four times in Tris buffered saline and resuspended at a concentration of $200 \times 10^{9}$ cells /L. The assay was performed as detailed by the manufacturer (BioData corp, Hatboro, USA) from the method described by Howard and Firkin (1971) by adding $400 \mu$ of platelets, $50 \mu$ ristocetin (final concentration $1 \mathrm{mg} / \mathrm{ml}$ ), mixing and waiting 2 minutes. A stir bar was then added and the tube placed in an aggregometer (Chrono-log Corp.). 50 $\mu l$ of test supernatant or plasma was added and the platelet aggregation recorded on a chart recorder (Chrono-log Corp.). Platelet aggregation was assessed by light transmission $2 \mathrm{~min}$ after the addition of ristocetin. The slope of the steepest part of the aggregation curve over $30 \mathrm{sec}$ was used to obtain a measure of platelet aggregation. The baseline was set using Tris buffered saline and platelets. A standard curve was obtained by serial dilutions $(1 / 2-1 / 16)$ of pooled human plasma (20 donors). The external control was assay reference plasma (undiluted to $1 / 8$ ).

\subsubsection{Measurement of vWf: Ag from supernatants of HUV endothelial cells exposed to sex steroids}

\subsubsection{Treatment of HUV endothelial cells}

This has been described in Section 4.3.4.1. 


\subsubsection{Immunoelectrophoresis}

The Laurell rocket immunoelectrophoresis technique (Laurell, 1966) was used to measure vWf : Ag. Antihemophilic globulin (AHG)-associated protein (final concentration $0.3 \%$ ) was dissolved in $1 \%$ agarose gel solution, which was poured at $54^{\circ} \mathrm{C}$, and allowed to set on gelbond film for $10 \mathrm{~min}$ at room temperature, and then at $4^{\circ} \mathrm{C}$ for up to $1 \mathrm{~h}$. Wells were prepared in the gel using a vacuum apparatus (Gast, USA). Supernatants $(8 \mu l)$ from the treated HUV endothelial cells and appropriate controls (normal reference plasma diluted $1 / 2$ and $1 / 4$ ) and standards (assay reference plasma ranging from undiluted to $1 / 16$ ) were placed into the wells in the gel, the samples were electrophoresed at $120 \mathrm{~V}$ for $5 \mathrm{~min}$, and then at $20 \mathrm{~V}$ per plate for $18 \mathrm{~h}$ in barbitone buffer (refer to Section 4.2). The gel was placed in a saline bath for $24 \mathrm{~h}$ and then rinsed with distilled water. The gel was blotted and heavy even pressure applied for $1 \mathrm{~h}$. The gel was then dried at $50^{\circ} \mathrm{C}$ for $30 \mathrm{~min}$ or until clear. The dried gel was placed into the staining solution for $30 \mathrm{~min}$ and then destained for 1-2 min. The antigen-antibody reaction formed a precipitin line that had the appearance of a rocket ascending from the well. The concentration of antigen was directly proportional to the area of the rocket, but following convention the height of the rocket was measured and compared with appropriate controls.

\subsubsection{Statistical analysis}

The experimental design for the platelet binding studies involved $6-8$ separate plates with each plate containing a control and certain treatments. The uptake of $[2-3 \mathrm{H}]$-adenine by HUV endothelial cells was also assessed for each treatment on each plate. Each experiment was initially analysed separately. The uptake of $[2-3 \mathrm{H}]$-adenine by HUV endothelial cells was found 
not to be influenced by steroid treatment nor did it affect platelet binding. The experiments were then pooled and analysis of variance with regression was used for analysis where the experiment replicates were considered as a block effect and the treatments as fixed effects. Analysis of covariance was used for the immunofluorescence studies since the background uptake of fluorescence varied between and within experiments. The analysis used for $\mathrm{VWf}$ and $\mathrm{PGI}_{2}$ release from HUV endothelial cells was analysis of variance with regression. The variance ratio ( $F$ test) was used to test for overall significance. The multiple t comparison (Bonferroni method) was then used to test for significance difference between treatments. The Minitab programme (Data analysis software, 1987) was used for the above analyses. A $p$ value of 0.05 or less was taken to represent a statistically significant difference.

\subsection{RESULTS}

\subsubsection{Platelet binding to HUV endothelial cells exposed to sex steroids}

In preliminary studies total protein was assayed but this technique was found to be relatively insensitive. $[2-3 \mathrm{H}]$-adenine labelling of platelets according to the method of Curwen et al (1982) was then assessed. This technique had increased sensitivity and gave more reproducible results. Preliminary results showed that the optimal concentration of platelets for maximal adhesion was $10^{8}$ platelets $/ \mathrm{ml}$. The average platelet count was 1.4 $\times 10^{8}$ /well for the washed platelet experiments and $1.6 \times 10^{8}$ /well for platelets sedimented from PRP. Microscopically the platelets appeared to bind to the intercellular junction of HUV endothelial cells.

Preincubation of a confluent monolayer of HUV endothelial cells with sex steroids, alcohol or rlL-1 for $6 \mathrm{~h}$ did not alter the binding of washed platelets 
to endothelial cells relative to control (Medium 199 alone) (Figure 4.1). However, after preincubation of HUV endothelial cells with steroids, alcohol or rIL-1 for $18 \mathrm{~h}$, there was a significant inhibition of binding of washed platelets to HUV endothelial cells incubated with a high concentration of $17 \beta$-estradiol (10-5 M) (57 $\pm 19 \%$ ) (Figure 4.2), but not by a low concentration of $17 \beta$ estradiol (10-10 M). Other concentrations of sex steroids, alcohol or rll-1 did not alter platelet binding to HUV endothelial cells.

Since HUV endothelial cells take up free or unincorporated adenine it was necessary to measure this in each experiment, and also to minimize this uptake. In preliminary experiments, following the method of Curwen et al (1982), $0.1 \mathrm{mM}$ adenine was used just prior to the addition of labelled adenine to HUV endothelial cells, to inhibit the uptake of unincorporated $[2-3 \mathrm{H}]-$ adenine. This reduced incorporation of $[2-3 \mathrm{H}]$-adenine by a confluent monolayer of HUV endothelial cells (approx $2 \times 10^{5}$ cells /well) from an average count of $70812.5 \pm 3996 \mathrm{cpm}$ to $3725 \pm 225 \mathrm{cpm}$ for a $30 \mathrm{~min}$ incubation. The uptake of unincorporated $[2-3 \mathrm{H}]$-adenine by HUV endothelial cells was assessed using PPP harvested from labelled PRP (refer to Section 4.3.2.1). The uptake of $[2-3 \mathrm{H}]$-adenine by HUV endothelial cells was not influenced by preincubation of the cells with sex steroids, rlL-1 or alcohol at either $6 \mathrm{~h}$ or $18 \mathrm{~h}$ (Figures 4.1 and 4.2). Thus, the inhibitory effects of $17 \beta$ estradiol $\left(10^{-5} \mathrm{M}\right)$ on binding of washed platelets cannot be attributed to an effect of $17 \beta$-estradiol $\left(10^{-5} \mathrm{M}\right)$ on [2-3H]-adenine uptake by HUV endothelial cells. The average counts per minute for the washed platelet preparation in the control wells were $9600 \pm 1724$ while in the control wells containing PPP the counts were $4252 \pm 1044$ at $6 \mathrm{~h}$. At $18 \mathrm{~h}$ the counts per minute were 8 $562 \pm 1501$ for the PRP preparation and $2691 \pm 562$ for PPP (mean of 3 experiments in triplicate).

When platelets were sedimented from PRP but not washed there was no 
significant change in platelet binding relative to control cells (Medium 199 alone) when HUV endothelial cells were exposed to sex steroids or alcohol for $6 \mathrm{~h}$ or $18 \mathrm{~h}$ (Figures 4.3 and 4.4). In these experiments the average counts per minute obtained in the control wells with PRP were $18097 \pm 8553$ while the average counts per minute for PPP were $3342 \pm 2009$ at $6 \mathrm{~h}$ and 16 $284 \pm 8693$ and $2964 \pm 1669$ at $18 \mathrm{~h}$ respectively (mean of 3 experiments). There was a variation in absolute counts between experiments indicated by the wide standard error but only a small variation within each experiment. When HUV endothelial cells were preincubated with rIL-1 for 2, 6, 18, and 24 $h$ there was a significant increase in platelet binding at 6, 18 and $24 h$ (Figures 4.3, 4.4 and 4.5). The uptake of [2-3H]-adenine by HUV endothelial cells in this series of experiments was also assessed by the addition of PPP harvested from labelled PRP (refer to Section 4.3.2.2). The uptake of $[2-3 \mathrm{H}]-$ adenine was not influenced by preincubation of the HUV endothelial cells with sex steroids or alcohol at either $6 \mathrm{~h}$ or $18 \mathrm{~h}$ (Figures 4.3 and 4.4). However, there appeared to be an increase in $[2-3 \mathrm{H}]$-adenine uptake by HUV endothelial cells exposed to rIL-1 (Figure 4.5) relative to control (Medium 199 alone). For example, the average counts were $13665 \pm 12439$ for HUV endothelial cells exposed to rIL-1 and PPP for $24 \mathrm{~h}$ while the counts per minute were $56915 \pm 38993$ in wells with endothelial cells exposed to PRP and IL-1. This was in contrast to the average PPP and PRP counts per minute for the control wells as described above. Again, there was variation in absolute counts between experiments as indicated by the wide standard deviations but small variation within each experiment.

A comparison of the washed platelet preparation and the platelets sedimented from PRP shows that more platelets bound to endothelial cells if they were not washed. Thus, washing appears to remove a factor which promotes platelet adhesiveness. 


\subsection{2 $\mathrm{PGI}_{2}$ release by HUV endothelial cells exposed to sex steroids}

When HUV endothelial cells were exposed to sex steroids or alcohol for 30 min or $6 \mathrm{~h}$ there was no change in 6-keto-PGF ${ }_{1 \alpha}$ release into the supernatant (Figure 4.6) relative to control (Medium 199 alone). After $24 \mathrm{~h}$ exposure of HUV endothelial cells to sex steroids, a high concentration of $17 \beta$-estradiol (10-5 M) significantly reduced 6-keto $\mathrm{PGF}_{1 \alpha}$ release into the supernatant $(60 \pm$ $14 \%)$, while lower concentrations of $17 \beta$-estradiol $\left(10^{-6-10-10}\right)$ did not significantly alter $\mathrm{PGl}_{2}$ release from HUV endothelial cells (Figure 4.8). 17 $\beta$ estradiol $\left(10^{-10} \mathrm{M}\right)$, progesterone and the combination of $17 \beta$-estradiol and progesterone did not significantly alter 6-keto $\mathrm{PGF}_{1 \alpha}$ release (Figure 4.7). When HUV endothelial cells were exposed to rlL-1 $(5 \mathrm{U} / \mathrm{ml})$ 6-keto PGF $1 \alpha$ production was significantly increased (213 $\pm 35 \%)$ after $6 \mathrm{~h}$ (Figure 4.6) and after $24 \mathrm{~h}(260 \pm 17 \%)$ incubation (Figure 4.7$)$ as previously reported by Rossi et al (1985) and Dejana et al (1987). Alcohol alone had no significant effect on 6-keto-PGF $1 \alpha$ release from HUV endothelial cells.

Preincubation of HUV endothelial cells with aspirin before exposure to sex steroids, alcohol or rIL-1 for $24 \mathrm{~h}$ resulted in inhibition of 6-keto PGF $1 \alpha$ release. Since the values were generally below the detection level of the assay no particular pattern of 6-keto $\mathrm{PGF}_{1 \alpha}$ release could be determined.

There was marked variability in $\mathrm{PGI}_{2}$ production among identically treated cultures using the same cell passages. For example, one cell culture (passage 9) produced $41 \pm 9 \rho \mathrm{g}$ of 6-keto PGF $_{1 \alpha} /$ tube while another cell culture (passage 9) released $172.5 \pm 7.5 \rho g$ of 6 -keto PGF $_{1 \alpha} /$ tube in the control wells (Medium 199 alone), treated the same way and assayed with the same kit.

Preincubation of HUV endothelial cells with cycloheximide before exposure 
to sex steroids, alcohol or rlL-1 for $24 \mathrm{~h}$ resulted in a loss of the inhibition of release of 6-keto $\mathrm{PGF}_{1 \alpha}$ from HUV endothelial cells exposed to $17 \beta$-estradiol $\left(10^{-5} \mathrm{M}\right)$, and a loss of the increase in release of 6 -keto $\mathrm{PGF}_{1 \alpha}$ caused by rIL1 (Figure 4.7).

\subsection{3 $\mathrm{vWf:} \mathrm{Ag} \mathrm{immunofluorescence} \mathrm{of} \mathrm{HUV} \mathrm{endothelial} \mathrm{cells} \mathrm{exposed} \mathrm{to} \mathrm{sex}$ steroids}

A number of parameters were defined in preliminary experiments. Firstly, the type of agent used to remove the HUV endothelial cells from the gelatin was assessed. Three agents were tested: trypsin in versene $(0.1 \%)$, human plasmin $(2 \mu \mathrm{g} / \mathrm{ml})$ and RGDS-NH $2(2.5 \mathrm{mg} / \mathrm{ml})$, a synthetic fibronectin peptide (Pierschbacher et al, 1983; Hayman et al, 1985). The expression of vWf, PgP-1 and ELAM-1 on HUV endothelial cells was examined using the above three cleavage agents. Figures 4.9 and 4.10 illustrate the effects of the different cleavage agents on the expression of vWf and PgP-1 antigens as assessed by flow cytometry. RGDS- $\mathrm{NH}_{2}$ and plasmin caused a broader background fluorescence and higher nonspecific binding of the second antibody than $0.1 \%$ trypsin. The spread of fluorescence intensity was greater for both antigens when RGDS- $\mathrm{NH}_{2}$ or plasmin were used rather than trypsin. However, trypsin was found to be very efficient, requiring only $3 \mathrm{~min}$ at $37^{\circ} \mathrm{C}$ to remove $99 \%$ of HUV endothelial cells with an average viability of $98 \%$, as assessed by trypan blue exclusion. In contrast, RGDS- $\mathrm{NH}_{2}$ required at least $2 \mathrm{~h}$ to remove $50 \%$ of cells with an average viability of $88 \%$. Plasmin required $30 \mathrm{~min}$ at $37^{\circ} \mathrm{C}$ to remove $50 \%$ of cells with an average viability of $83 \%$. Trypsin was not only more efficient but it was also the least expensive of the three agents.

Expression of vWf appeared to be very sensitive to trypsinization, more so than the expression of PgP-1 or ELAM-1 (Figure 4.11). Storing the cells at 
room temperature for 1,2 or $4 \mathrm{~h}$ before staining did not improve the expression of vWf when compared to immediate staining (Figure 4.12). Placing HUV endothelial cells in overnight roller culture caused them to die. Increasing the storage time reduced the viability of the cells, as shown in Figure 4.12. When cells were stained immediately (Figure 4.12 C and F) there were only a few dead cells (as assessed by background fluorescent intensity in the region of 500 intensity units and by trypan blue exclusion), and few smaller cells in the forward scatter-side scatter profile. Following storage for 2 $h$ at room temperature (Figure $4.12 \mathrm{~B}$ and $\mathrm{E}$ ) there was an increase in numbers of smaller cells and an increase in background in the 500 intensity unit region. This was also confirmed by trypan blue exclusion. There was no change in viability during the course of the experiment if HUV endothelial cells were trypsinized and then stored at room temperature for $1 \mathrm{~h}$.

The effect of cell passage on vWf expression was also studied. There was no significant difference in vWf expression by HUV endothelial cells between passage 5 and passage 10 when stained on the same day (Figure 4.12A and B). However, the intensity of expression of immunofluorescence for vWf : Ag varied between preparations of HUV endothelial cells when the same antibody batches were used and stained on different days (Figure 4.13). There was also some variability in cell size and granularity between each HUV endothelial cell preparation which was not related to viability (Figure 4.14).

As no isotype matched control was available for the primary antibody the specificity of the antibody to $\mathrm{VWf}$ : Ag was assessed by preincubating AHF $(6.25-100 \mathrm{mg} / \mathrm{ml})$ with antiserum to human FVIII. AHF $(50 \mathrm{mg} / \mathrm{ml})$ was found to completely block the shift in fluorescence as shown in Figure 4.15.

The effect of sex steroids on vWf : Ag expression by HUV endothelial cells was assessed by flow cytometry (Figure 4.16). $17 \beta$-estadiol $\left(10^{-5} \mathrm{M}, 10^{-7} \mathrm{M}\right.$ 
and $\left.10^{-10} \mathrm{M}\right)$ did not alter vWf expression. However, progesterone $\left(10^{-5} \mathrm{M}\right.$ and $10^{-9} \mathrm{M}$ ) significantly inhibited vWf expression. The combination of $17 \beta$ estradiol $\left(10^{-5} \mathrm{M}\right)$ and progesterone $\left(10^{-5} \mathrm{M}\right)$ also significantly inhibited vWf expression. rIL-1 (which was used as a control) inhibited vWf expression (Figure 4.16). Although vWf : Ag expression was reduced in HUV endothelial cells exposed to progesterone alone or the combination of $17 \beta$-estradiol (10-5 M) and progesterone $\left(10^{-5} \mathrm{M}\right)$ when assessed by flow cytometry, immunofluorescent microphotographs demonstrated that vWf expression was still present in all of these cell cultures (Figure 4.18). Figure 4.18 (upper photo) shows a typical fluorescent micrograph of HUV endothelial cells exposed to $17 \beta$-estradiol $\left(10^{-10} \mathrm{M}\right)$ in combination with progesterone $\left(10^{-9} \mathrm{M}\right)$ and then stained for the presence of vWf : Ag. Figure 4.18 (middle photo) shows the effect of $17 \beta$-estradiol $\left(10^{-5} \mathrm{M}\right)$ in combination with progesterone $\left(10^{-5} \mathrm{M}\right)$, and Figure 4.18 (lower photo) shows the background level of fluorescence with the addition of the F.I.T.C-conjugated antibody alone to HUV endothelial cells exposed to $17 \beta$-estradiol $\left(10^{-5} \mathrm{M}\right)$ in combination with progesterone $\left(10^{-5} \mathrm{M}\right)$. The background level of fluorescence of HUV endothelial cells exposed to $17 \beta$-estradiol $\left(10^{-10} \mathrm{M}\right)$ in combination with progesterone $\left(10^{-9} \mathrm{M}\right)$ was similar. Figure 4.19 A demonstrates vWf : $\mathrm{Ag}$ expression on HUV endothelial cells exposed to progesterone (10-9 M) for 18 h compared to control (Medium 199 alone) using FACS analysis as described in Section 4.3.3.2 and Figure 4.19 B illustrates vWf : Ag expression on HUV endothelial cells exposed to progesterone $\left(10^{-5} \mathrm{M}\right)$ in combination with $17 \beta$ estradiol (10-5 M) for $18 \mathrm{~h}$ compared to control (Medium 199 alone). Thus, although vWf : Ag expression was altered by progesterone, no obvious differences were observed using fluorescent microscopy.

Preincubation of HUV endothelial cells with cycloheximide resulted in loss of the inhibitory effect of sex steroids and rlL-1 on vWf : Ag expression by HUV endothelial cells (Figure 4.17). There was no significant difference in vWf : Ag 
expression between control cells (Medium 199 alone) and cells exposed to sex steroids or $\mathrm{rlL}-1$ in the presence of cycloheximide. Figure 4.20 A demonstrates vWf : Ag expression on HUV endothelial cells preincubated with cycloheximide and then exposed to progesterone $\left(10^{-9} \mathrm{M}\right)$, while Figure 4.20 B demonstrates the effect of cycloheximide treatment of HUV endothelial cells exposed to the combination of $17 \beta$-estradiol $\left(10^{-5} \mathrm{M}\right)$ and progesterone $\left(10^{-5} \mathrm{M}\right)$. Inhibition of $\mathrm{vWf}: \mathrm{Ag}$ expression was lost. The experiments illustrated in Figures 4.19 and 4.20 were performed on the same day using the same antibodies.

\subsubsection{Effect of supernatants from HUV endothelial cells exposed to sex steroids on ristocetin-induced platelet aggregation}

The effect of sex steroids on ristocetin-induced platelet aggregation was compared to alcohol, since this was the vehicle used to dissolve the sex steroids. The results (Figure 4.21) showed that supernatants from HUV endothelial cells which had been exposed to sex steroids or rIL-1 did not influence platelet aggregation when compared to alcohol, but did cause inhibition of platelet aggregation when compared to Medium 199 alone (control). The value of the assay reference plasma used as an external control was within the range stated by the manufacturer (92.6 $\pm 22.6 \%)$.

\subsubsection{Laurell Rocket immunoelectrophoresis}

Laurell Rocket immunoelectrophoresis was used to measure the concentration of vWf in culture supernatants to assess release of vWf from HUV endothelial cells in the presence of sex steroids. $17 \beta$-estradiol $\left(10^{-5} \mathrm{M}\right)$ in combination with progesterone $\left(10^{-5} \mathrm{M}\right)$ and progesterone $\left(10^{-5} \mathrm{M}\right)$ alone were associated with significantly lower levels of vWf : Ag in the supernatants (Figure 4.22). Alcohol had no effect on vWf : Ag release while rlL-1 caused an 
increase in vWf : Ag release. Medium 199 (20\% FCS and gentamicin $60 \mathrm{U}$ /ml) contained no detectable $\mathrm{vWf}$ : Ag. The assay reference plasma used as an external control was within the range stated by the manufacturer (121.4 \pm $31.1 \%)$. Figure 4.23 illustrates a typical result obtained using Laurell Rocket immunoelectrophoresis. The standard curve is shown in lanes 1-4, the external standards in lanes 5-6 and the effects of sex steroids and rIL-1 in lanes 7-14. Lane 9 represents vWf in the supernatant of HUV endothelial cells exposed to progesterone $\left(10^{-5} \mathrm{M}\right)$ for $48 \mathrm{~h}$; the rocket in this line is shorter in height compared to control (Medium 199 alone) shown in lane 11.

\subsection{DISCUSSION}

These experiments have shown that: (1) exposure of HUV endothelial cells to high concentrations of $17 \beta$-estradiol $\left(10^{-5} \mathrm{M}\right)$ inhibit binding of washed platelets to HUV endothelial cells and inhibit release of $\mathrm{PGI}_{2}$ from endothelial cells; (2) progesterone $\left(10^{-5} \mathrm{M}\right.$ and $\left.10^{-9} \mathrm{M}\right)$ and progesterone $\left(10^{-5} \mathrm{M}\right)$ in combination with $17 \beta$-estradiol $\left(10^{-5} \mathrm{M}\right)$ inhibit $\mathrm{VWf}$ : Ag expression on HUV endothelial cells; and (3) progesterone alone $\left(10^{-5} \mathrm{M}\right)$ and progesterone $\left(10^{-5}\right.$ $M)$ in combination with $17 \beta$-estradiol $\left(10^{-5} \mathrm{M}\right)$ inhibit vWf : Ag release from HUV endothelial cells.

Platelets do not normally bind to endothelial cells but in response to endothelial cell injury and loss there is rapid binding of platelets to the subendothelium followed by release of their contents (Ross and Glomset, 1976; Lewis and Kottke, 1977). In the experiments reported here, less than 1 $\%$ of added washed platelets bound to untreated HUV endothelial cells. However this is still many platelets and perhaps reflects the "injured" state of HUV endothelial cells in culture (Ross, 1986). Platelets tended to bind to the edges of the cells. Microscopically, there appeared to be a confluent monolayer of endothelial cells but perhaps some platelets bound to exposed 
gelatin. Only exposure of HUV endothelial cells to $17 \beta$-estradiol $\left(10^{-5} \mathrm{M}\right)$ for $18 \mathrm{~h}$ altered platelet adhesion. This inhibition of platelet binding suggests that $17 \beta$-estradiol $\left(10^{-5} \mathrm{M}\right)$ is able to alter a thrombogenic property or properties of HUV endothelial cells through a mechanism requiring greater than $6 h$ exposure of the HUV endothelial cells to $17 \beta$-estradiol. Importantly, the addition of progesterone $\left(10^{-5} \mathrm{M}\right)$ inhibited the reduced platelet binding. Progesterone alone had no effect on platelet binding, nor did estrogen or progesterone in the physiological range. 17ß-estradiol $\left(10^{-5} \mathrm{M}\right)$ did not influence platelet binding to HUV endothelial cells when the platelets were harvested from PRP but not washed. This was probably due to the presence of other factors in the plasma, such as fibrinogen, which tend to increase platelet adhesion. This may also explain why more platelets bound to endothelial cells when sedimented from PRP than when washed. As expected there was no significant difference in the binding of platelets to HUV endothelial cells in the control wells over 6 or $18 \mathrm{~h}$ using either washed platelets or platelets sedimented from PRP.

$\mathrm{PGI}_{2}$ and $\mathrm{FVIII}$ are two very important factors produced by the endothelial cell which influence platelet aggregation. The studies reported here suggest that the release of $\mathrm{PGI}_{2}$ and $\mathrm{FVIII}$ from HUV endothelial cells can be modified by sex steroids. Exposure of HUV endothelial cells for $24 \mathrm{~h}$ to high concentrations of $17 \beta$-estradiol $\left(10^{-5} \mathrm{M}\right)$ but not low concentrations (10-6-10$10 \mathrm{M}$ ) reduced $\mathrm{PGI}_{2}$ release. The inhibition was delayed and was inhibited by cycloheximide, a known inhibitor of protein synthesis. The addition of progesterone $\left(10^{-5} \mathrm{M}\right)$ prevented the effect of $17 \beta$-estradiol $\left(10^{-5} \mathrm{M}\right)$. Progesterone $\left(10^{-5}\right.$ or $\left.10^{-9} \mathrm{M}\right)$ alone did not appear to influence $\mathrm{PGl}_{2}$ release from HUV endothelial cells.

The effect of $17 \beta$-estradiol and progesterone on $\mathrm{PGI}_{2}$ release is consistent with the findings of some investigators (Needleman and Parks, 1982; Makila 
et al, 1982; Corvazier et al, 1984; Witter and DiBlasi, 1984; Lewis et al, 1986) but not others (Makila et al, 1982; Berge et al, 1990; David et al, 1989) (refer to Section 1.5.3.3.1). The different findings probably reflect the different protocols used. For example, Berge et al (1990) demonstrated a significant inhibition of $\mathrm{PGI}_{2}$ release from HUV endothelial cell cultures which had been preincubated with $17 \beta$-estradiol $\left(7.3-36.7 \times 10^{-10} \mathrm{M}\right)$ alone or in combination with progesterone $\left(3.2-16 \times 10^{-8} \mathrm{M}\right)$ for $72 \mathrm{~h}$ and then stimulated with thrombin for $10 \mathrm{~min}$. This study therefore examined stimulated endothelial cells rather than cells in a "resting" state. David et al (1989), used first passage HUV endothelial cells exposed to $17 \alpha$-ethinyl estradiol $(30 \mathrm{\eta g} / \mathrm{ml})$, and demonstrated inhibition of $\mathrm{PGI}_{2}$ release but this was not time-dependent. They used a different isomer of estradiol and, furthermore, they used ATP to stimulate the release of $\mathrm{PGI}_{2}$ so that release would have been maximal rather than at the low basal level normally seen in vivo. They also combined cell lysates and supernatants and so they were therefore unable to differentiate between production and release of $\mathrm{PGl}_{2}$. Berge et al (1990) observed that under basal conditions confluent monolayers of HUV endothelial cells exposed to progesterone $\left(3.2-16 \times 10^{-8} \mathrm{M}\right)$ released less $\mathrm{PGI}_{2}$ relative to Medium 199 alone. This concentration of progesterone was not assessed in the current study.

$17 \beta$-estadiol $\left(10^{-5} \mathrm{M}\right)$ may reduce $\mathrm{PGI}_{2}$ release by partially inhibiting enzymes concerned with $\mathrm{PGI}_{2}$ production (e.g. cyclo-oxygenase or $\mathrm{PGI}_{2}$ synthetase) or possibly by stimulating the synthesis of an inhibitory protein produced by the endothelial cells. Evidence for the latter proposal derives from the observation that inhibition was blocked by cycloheximide and additionally that there was a lag time before the inhibitory effect of $17 \beta$ estradiol (10-5 M) became evident. In rat smooth muscle cells and uterus $17 \beta$-estradiol has been shown to regulate $\mathrm{PGI}_{2}$ synthetase and cyclooxygenase (Ham et al, 1975; Chang et al, 1980b). Further studies are 
required to determine whether there are low affinity estrogen receptors on HUV endothelial cells, and whether $17 \beta$-estradiol $\left(10^{-5} \mathrm{M}\right)$ affected $\mathrm{PGI}_{2}$ release through binding to such receptors. Alternatively, estradiol may have altered $P G I_{2}$ release through a non-receptor mediated mechanism such as perturbation of membrane lipids with secondary changes in fluidity (Needleman and Parks, 1982).

There was marked variability in $\mathrm{PGl}_{2}$ production among identically treated cultures using the same cell passages in the current studies. This variability has been reported by others (Witter and DiBlasi, 1984; Levine et al, 1984; Berge et al, 1990). There are many possible contributing factors. For instance, Ager et al (1982) and Evans et al (1984) reported that changing the growth medium caused a transient burst in production of $\mathrm{PGI}_{2}$. This may have differed between experiments and would not have been evident. Lahoua et al (1988) reported that the concentration of FCS altered $\mathrm{PGl}_{2}$ release from NRK49 cells and that this phenomenon was potentiated by adding oxysterols to the culture medium. However, in the current studies the same batch of FCS was used for all experiments. Although alcohol (the vehicle used to dissolve the sex steroids) has been shown to stimulate $\mathrm{PGI}_{2}$ release in vivo and in vitro at concentrations ranging between $0.04 \%$ and 1.2 \% (Landolfi and Steiner, 1984: Landolfi et al, 1988), and therefore could cause variability in $\mathrm{PGI}_{2}$ release, in the present experiments alcohol $(0.1 \%)$ was added to all treatments except rlL-1 and control. No difference was seen in $\mathrm{PGl}_{2}$ release from cells treated with alcohol or Medium 199 alone. Another factor which was controlled for in the experiments reported here and which is known to alter $\mathrm{PGl}_{2}$ release from endothelial cells is the degree of cell confluence (Ali et al, 1980; Eldor et al, 1983b; Ager et al, 1982; Evans et al, 1984). Firstly, $\mathrm{PGI}_{2}$ release by non-confluent HUV endothelial cell cultures decreases as cell density increases towards confluence (Evans et al, 1984). Secondly, Boutherin-Falson and Blaes (1989) found that the presence of 
heparin $(90 \mu \mathrm{g} / \mathrm{ml})$ with ECGF $(20 \mu \mathrm{g} / \mathrm{ml})$ in Medium 199 containing $20 \%$ FCS resulted in a decrease in $\mathrm{PGI}_{2}$ production by non-confluent primary passaged HUV endothelial cells. Similar results have also been obtained using endothelial cells passaged 10-16 times (Hasegawa et al, 1988). Only confluent monolayers were used in the studies described here. Chesterman et al (1983) and David et al (1989) have reported that early passaged HUV endothelial cells produce more $\mathrm{PGI}_{2}$ than serially cultured cells. Only a limited range of passaged cells was used in the present experiments to minimize this effect.

Any additional influence of sex steroids on $\mathrm{PGI}_{2}$ release by $\mathrm{HUV}$ endothelial cells in the presence of aspirin could not be assessed since the levels of 6 keto-PGF ${ }_{1 \alpha}$ were below the detection level of the assay. The inhibitory effect of aspirin has been described by Czervionke et al (1979a). Morikawa et al (1984) found that the inhibitory effect of aspirin was reduced in rats treated with estradiol and potentiated in rats treated with testosterone. The increase in $\mathrm{PGI}_{2}$ production by HUV endothelial cells treated with rlL-1 $(5 \mathrm{U} / \mathrm{ml})$ is in agreement with observations by Rossi et al (1985) and Zavoico et al (1989).

Progesterone $\left(10^{-5} \mathrm{M}\right)$ inhibited $\mathrm{VWf}$ : Ag expression on the cell surface and its release into the supernatant in the current experiments. This inhibition of vWf : Ag expression could be prevented by the addition of cycloheximide confirming that the effect of progesterone was mediated through inhibition of protein synthesis. The addition of $17 \beta$-estradiol $\left(10^{-5} \mathrm{M}\right)$ to progesterone did not alter this inhibition. $17 \beta$-estradiol alone had no effect on vWf expression or release from HUV endothelial cells. Although progesterone $\left(10^{-9} \mathrm{M}\right)$ inhibited vWf : Ag expression on HUV endothelial cells, progesterone did not significantly inhibit vWf : Ag release. No simple explanation is apparent. Ristocetin-induced platelet aggregation was also used to assess vWf : Ag release into the supernatant of HUV endothelial cells exposed to sex steroids, 
alcohol and $\mathrm{rlL}-1$, but the experiments were unable to demonstrate differences in vWf : Ag release because of the effect of alcohol and because of the wide standard deviations. The variability between experiments probably reflects biological variation of platelets donors as well as tissue culture and the wide variability seen with the test itself as demonstrated by the range given for the assay reference plasma used. Alcohol (used as the vehicle to dissolve the sex steroids) itself inhibited platelet aggregation. Alcohol is a known inhibitor of platelet aggregation and release of granule contents in response to many agonists (Rand et al, 1988b). Alcohol did not appear to influence vWf : $\mathrm{Ag}$ in the supernatant as assessed by Laurell Rocket immunoelectrophoresis. rIL-1 $(5 \mathrm{U} / \mathrm{ml})$ inhibited vWf : Ag expression but caused an increase in its release from HUV endothelial cells as previously reported by Schorer et al (1987) and Breit and Green (1988).

Inhibition of $\mathrm{VWf}: \mathrm{Ag}$ expression and release into the supernatant by progesterone suggests that it could play a role in inhibiting platelet adhesion at sites of injury. Little is known about the relationship between sex steroids and vWf : Ag expression and release. In endometrial endothelial cells, which may behave differently to vascular endothelial cells, progesterone (1.6-6.31 $\mathrm{x}$ 10-8 M) does not influence vWF : Ag expression (Johannisson, 1986) although it has been shown to inhibit platelet aggregation in the presence of estrogens (Elam et al, 1980). It is known that endothelial cells from different sources release different amounts of $\mathrm{vWf}$ and the amount released into the supernatant relative to the extracellular matrix also varies (Zwaginga et al, 1990). Pregnancy and oral contraceptive use have been associated with elevated levels of vWf (Schiffman and Rapaport, 1966; Crowell et al, 1971; Leone et al, 1975), although this has been attributed to estrogen. Harrison and McKee (1984a) found that incubation of HUV endothelial cells with 17 $\beta$ estradiol (1.83-7.34 $\left.\times 10^{-9} \mathrm{M}\right)$ but not $17 \beta$-estradiol $\left(9.2 \times 10^{-10} \mathrm{M}\right)$ for 3 days resulted in an increase in $\mathrm{vWf}:$ Ag release into the culture medium as 
assessed by ristocetin induced aggregation of platelets. In the studies reported here $17 \beta$-estradiol $\left(10^{-7}\right.$ and $10^{-10} \mathrm{M}$ ) did not influence the expression of vWf; the release of vWf into the supernatant of cells exposed to $17 \beta$-estradiol $\left(10^{-7}\right)$ was not assessed. The difference in the findings between the current studies and those of Harrison and McKee (1984a) probably reflects the lower concentration of alcohol used to dissolve estradiol, the lower concentration of FCS used and the longer duration of the experiments by Harrison and McKee (1984a).

$\mathrm{vWf}: \mathrm{Ag}$ is a peptide and therefore it is sensitive to trypsinization which is known to damage endothelial cells (McNeil et al, 1989). McNeil et al (1989) suggested that wounding by trypsinization resulted in the cytoplasm becoming accessible to exogenous probes, possibly allowing cytoplasmic molecules to diffuse out. Despite this, trypsin was used in the current studies to remove HUV endothelial cells from gelatin-coated plates because alternative agents which were assessed were found to be less efficient, caused reduced viability and were more expensive. vWf : Ag expression on HUV endothelial cells following trypsinization was however lower than for expression of PgP-1 or ELAM-1 on cells.

The time dependent increase in platelet binding to HUV endothelial cells preincubated with rIL-1, using platelets sedimented from PRP, illustrates yet another potential role for this important cytokine in atherogenesis. rIL-1 is known to have both prothrombotic and antithrombotic properties. IL-1 suppresses cell surface anticoagulant activity mediated by the thrombin protein C pathway (Nawroth et al, 1986) by reducing thrombomodulin expression (Pober and Cotran, 1990). IL-1 also induces the synthesis and expression of tissue factor (Pober and Cotran, 1990) and causes production of platelet-activating factor (Bussolino et al, 1986; Dejana et al, 1987) by endothelial cells (Bevilacqua et al, 1984). IL-1 also induces $P G I_{2}$ release 
(Rossi et al, 1985). It is the net balance of these factors which will determine the effect of IL-1 on coagulation. However, since $\mathrm{PGI}_{2}$ does not significantly inhibit platelet adhesion to endothelium, IL-1 in vitro would appear to mediate a shift towards coagulation and possibly thrombosis (Pober and Cotran, 1990). In vivo, IL-1 has also been shown to shift the balance of procoagulant and anticoagulant reactions on the endothelium towards clot formation (Nawroth et al, 1986). rIL-1 had no effect on platelet binding when washed platelets were used suggesting that some factor in plasma may be involved in the effect of rIL-1 on platelet binding.

The present experiments demonstrate that while high (in the pharmacological range) concentrations of $17 \beta$-estradiol inhibit binding of washed platelets to endothelial cells they also reduce $P G I_{2}$ release from endothelial cells confirming that $\mathrm{PGI}_{2}$ release does not alter platelet binding to HUV endothelial cells. Low concentrations of $17 \beta$-estradiol do not appear to influence platelet binding or $\mathrm{PGI}_{2}$ release from endothelial cells. High and low concentrations of progesterone, while not influencing platelet binding or $\mathrm{PGl}_{2}$ release from endothelial cells, inhibited the expression and release of vWf from endothelial cells. This suggests a possible protective role for progesterone in thrombosis and the progression of atherosclerosis by inhibiting platelet adhesion to the subendothelium especially at high shear stresses. If platelet adhesion is reduced there may be less degranulation and release of their contents, in particular PDGF, and perhaps less migration and proliferation of cells involved in atherosclerosis. These studies may also have important implications in relation to hormone replacement therapy and the oral contraceptive. As discussed in Chapter 1 the increased thrombosis associated with the oral contraceptive appears to be related to high dose estrogen use. This may be related, in part, to inhibition of $\mathrm{PGI}_{2}$ release which was observed in these studies with high dose estrogen. Estrogen replacement therapy does not appear to increase the risk of thrombosis and 
low concentrations of $17 \beta$-estradiol were not shown in the current studies to alter $\mathrm{PGI}_{2}$ release. From the current studies it would appear that the addition of high concentrations of progesterone could theoretically reduce the thrombotic risk associated with estrogen use by reducing the expression and release of $\mathrm{vWf}$ from endothelial cells. $\mathrm{PGI}_{2}$ and $\mathrm{vWf}$ are however just two of many factors present in HUV endothelial cells that influence platelet binding and aggregation which may be altered by sex steroids. The importance of sex steroids in influencing other properties remains to be assessed.

\subsection{SUMMARY}

The present studies here demonstrated that, under the experimental conditions used:

(1) $17 \beta$-estradiol at low concentrations $\left(10^{-10} \mathrm{M}\right)$ does not alter platelet binding to HUV endothelial cells, or influence $\mathrm{PGI}_{2}$ release by $\mathrm{HUV}$ endothelial cells;

(2) A high concentration of $17 \beta$-estradiol $\left(10^{-5} \mathrm{M}\right)$ inhibits binding of washed platelets to HUV endothelial cells and reduces $P \mathrm{PGl}_{2}$ release by HUV endothelial cells;

(3) a high concentration of progesterone $\left(10^{-5} \mathrm{M}\right)$ inhibits the effect of $17 \beta$ estradiol $\left(10^{-5} \mathrm{M}\right)$ on washed platelet binding;

(4) a high concentration of progesterone $\left(10^{-5} \mathrm{M}\right)$, but not $17 \beta$-estradiol, inhibits vWf expression and release from HUV endothelial cells; 
(5) A low concentration of progesterone (10-9 M) inhibits expression but not release of vWf from HUV endothelial cells; and

(6) rIL-1 increases binding to HUV endothelial cells of platelets in PRP. 


\section{Figure 4.1.}

Binding of washed platelets to HUV endothelial cells. A confluent monolayer of endothelial cells was exposed to Medium 199 (control), sex steroids, alcohol or $\mathrm{rlL}-1$ for $6 \mathrm{~h}$. The medium was removed, cells washed and labelled washed platelets or supernatant from the washed platelet preparation (refer to Section 4.3.2.1) was added for $30 \mathrm{~min}$ at $37^{\circ} \mathrm{C}$. The endothelial cell monolayer was then washed three times before the cells were removed from the plates by trypsinization. The cross-hatched histograms represent washed platelet binding $\left(\left[2-{ }^{3} \mathrm{H}\right]\right.$-adenine incorporation (AdR) to endothelial cells as a percentage of control (Medium 199 alone) (average radioactive cpm being 9 600) while the open histograms represent the uptake of unincorporated [2${ }^{3} \mathrm{H}$ ]-adenine by endothelial cells relative to control (average radioactive cpm being 4252 ). The abbreviations used for each of the stimuli in the " $x$ " axis of this figure are the same as for Figure 2.14. This graph represents the mean \pm SE of 5 experiments.

\section{Figure 4.2.}

Binding of washed platelets to HUV endothelial cells. A confluent monolayer of endothelial cells was exposed to Medium 199 (control), sex steroids, alcohol or rlL-1 for $18 \mathrm{~h}$. The experiment was then performed as described in the legend to Figure 4.1. The key to this figure is the same as for Figure 4.1. The average radioactive cpm for washed platelets bound to endothelial cells in the control wells was 8 562, and $2691 \mathrm{cpm}$ for unincorporated [2$\left.{ }^{3} \mathrm{H}\right]$-adenine uptake by endothelial cells. This graph represents the mean \pm SE of 4 experiments. ${ }^{*}=p<0.05$. 
BINDING OF WASHED PLATELETS TO ENDOTHELIAL CELLS EXPOSED TO VARIOUS STIMULI

$6 \mathrm{~h}$ incubation

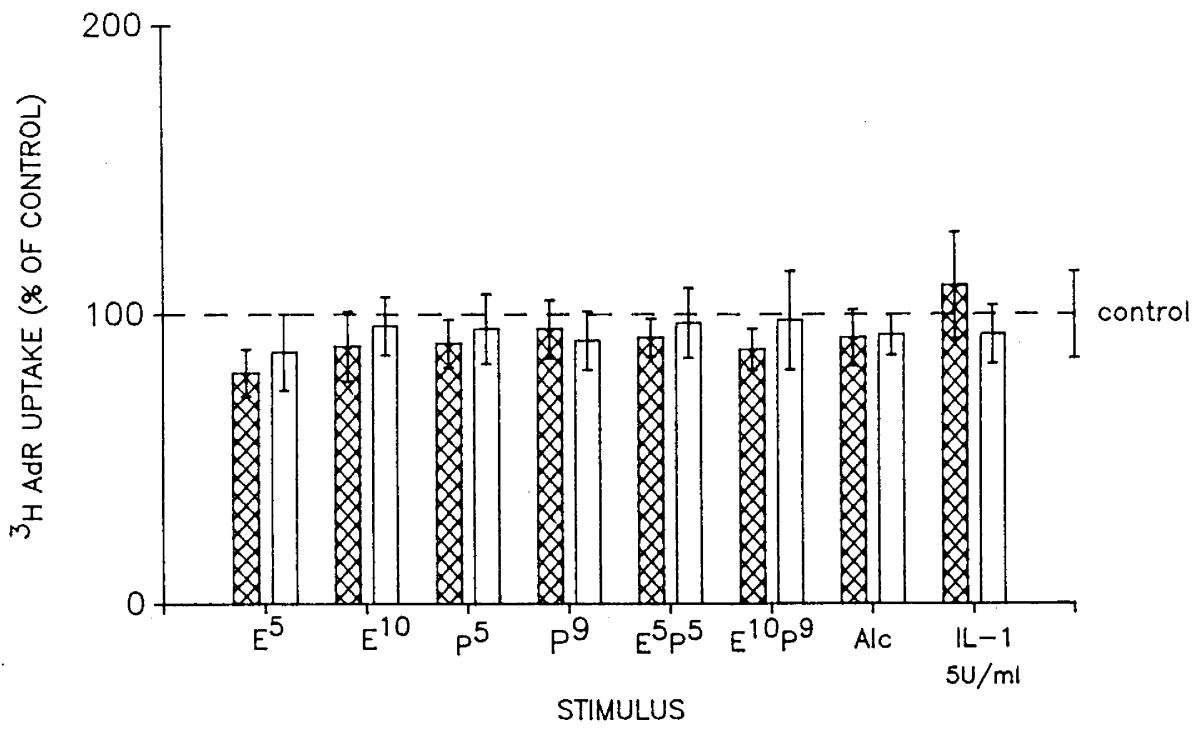

BINDING OF WASHED PLATELETS TO ENDOTHELIAL CELLS EXPOSED TO VARIOUS STIMULI

$18 \mathrm{~h}$ incubation

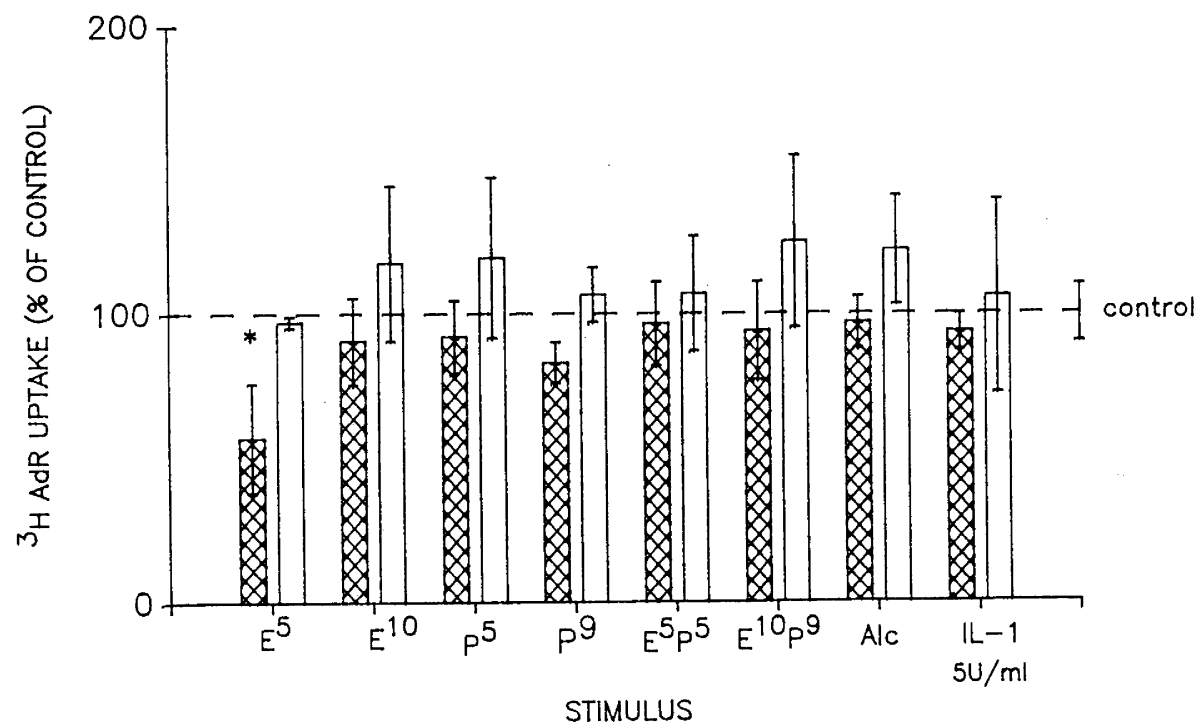




\section{Figure 4.3.}

Binding of platelets (sedimented from PRP) to HUV endothelial cells. A confluent monolayer of endothelial cells was exposed to Medium 199 (control), sex steroids, alcohol or rlL-1 for $6 \mathrm{~h}$. The medium was removed, the cells washed and labelled platelets sedimented from PRP or PPP (refer to Section 4.3.2.2) were added for $30 \mathrm{~min}$ at $37^{\circ} \mathrm{C}$. The experiment was then performed as described in the legend to Figure 4.1. The cross-hatched histograms represent binding of platelets (sedimented from PRP) to endothelial cells as a percentage of control (Medium 199 alone) (average radioactive $\mathrm{cpm}$ being 18 097) while the open histograms represent the uptake of unincorporated $\left[2-{ }^{3} \mathrm{H}\right]$-adenine by endothelial cells relative to control (average radioactive cpm being 3 342). This graph represents the mean $\pm S E$ of 3 experiments in triplicate. ${ }^{*}=p<0.05$

\section{Figure 4.4.}

Binding of platelets (sedimented from PRP) to HUV endothelial cells. A confluent monolayer of endothelial cells was exposed to Medium 199 (control), sex steroids, alcohol or rlL-1 for $18 \mathrm{~h}$. The experiment was then performed as described in the legend to Figure 4.3. The average radioactive $\mathrm{cpm}$ for platelets sedimented from PRP bound to endothelial cells in the control wells was 16284 , and 2964 for unincorporated $\left[2-{ }^{3} \mathrm{H}\right]$-adenine uptake by endothelial cells in the control wells. This graph represents the mean \pm SE of 3 experiments in triplicate. ${ }^{*}=p<0.05$. 
BINDING OF PLATELETS SEDIMENTED FROM PRP TO ENDOTHELIAL CELLS EXPOSED TO VARIOUS STIMULI

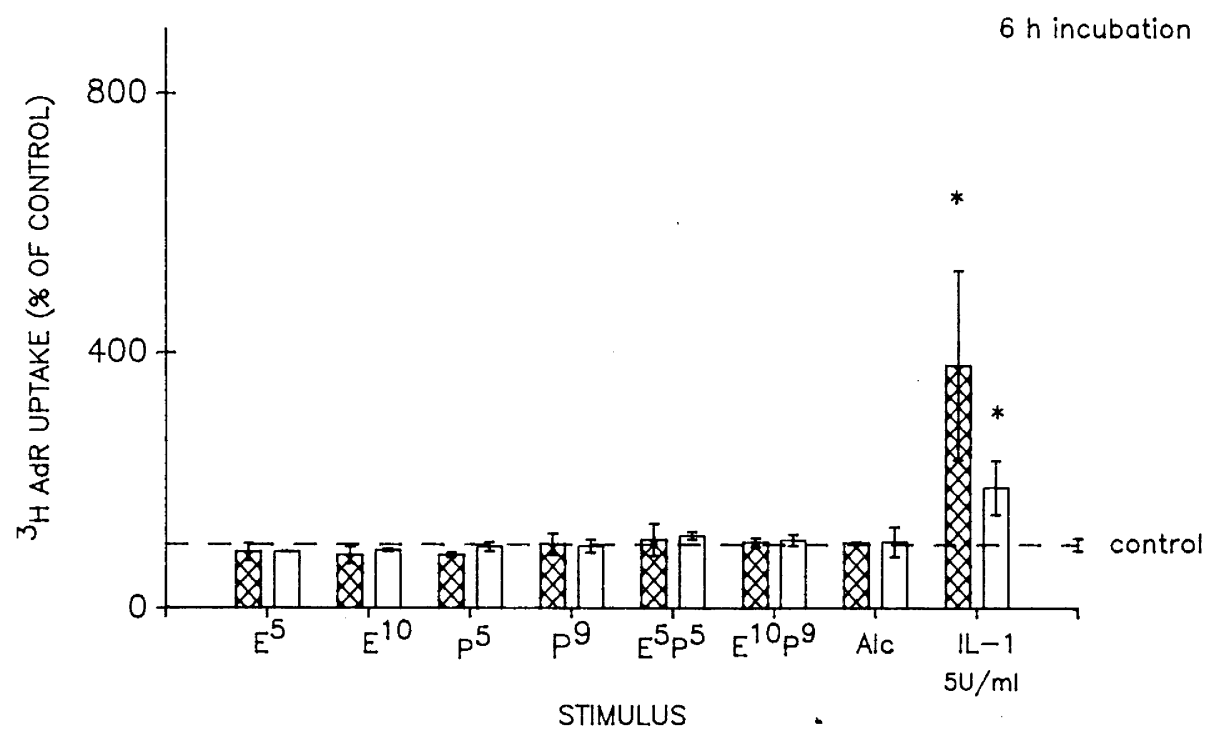

BINDING OF PLATELETS SEDIMENTED FROM PRP TO ENDOTHELIAL CELLS EXPOSED TO VARIOUS STIMULI

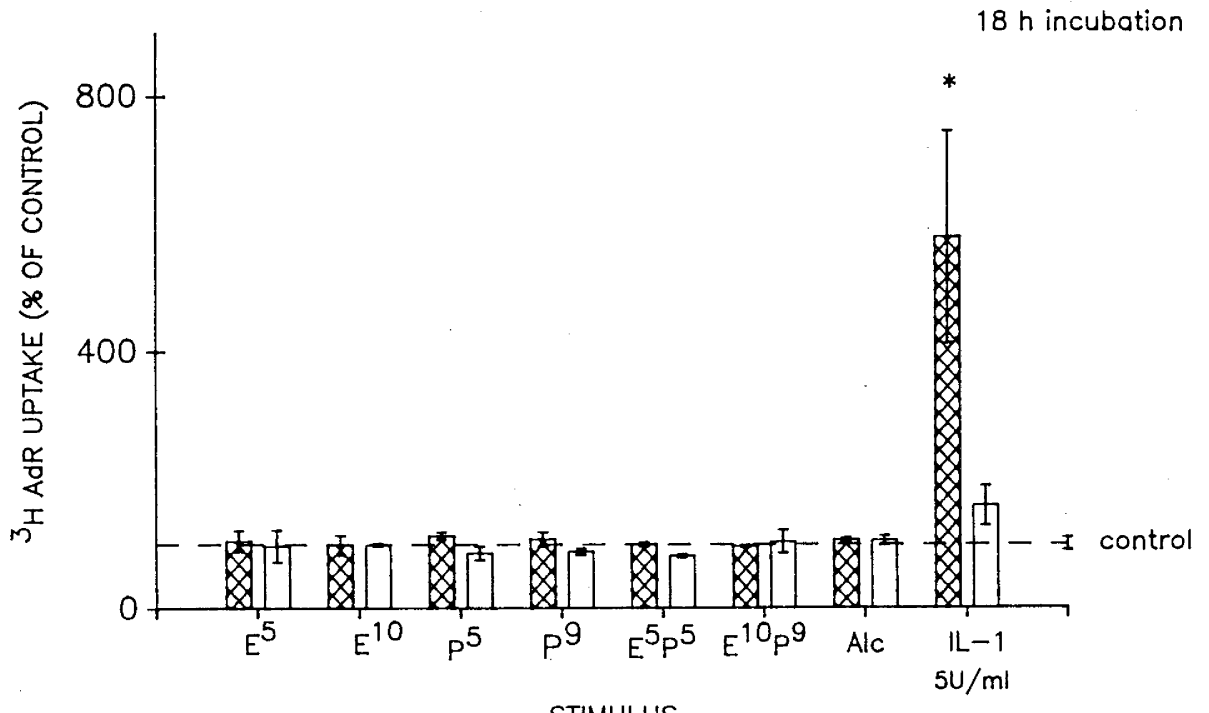

STIMULUS 


\section{Figure 4.5.}

Binding of platelets (sedimented from PRP) to HUV endothelial cells exposed to rIL-1. A confluent monolayer of endothelial cells was exposed to Medium 199 (control) or rlL-1 for 2, 6, 18 or $24 \mathrm{~h}$. The medium was removed, the cells washed and labelled platelets sedimented from the PRP or PPP were added for $30 \mathrm{~min}$ at $37^{\circ} \mathrm{C}$. The experiment was then performed as described in the legend to Figure 4.3. The cross-hatched histograms represent the binding of platelets to endothelial cells while the open histograms represent the uptake of unincorporated $\left[2-{ }^{3} \mathrm{H}\right]$-adenine by endothelial cells. This graph represents the mean \pm SE of 3 experiments in triplicate. $\star=p<0.05$.

Figure 4.6.

$\mathrm{PGI}_{2}$ release by $\mathrm{HUV}$ endothelial cells exposed to various stimuli. $A$ confluent monolayer of endothelial cells was exposed to Medium 199 (control), sex steroids, alcohol or rlL-1 for $30 \mathrm{~min}$ or $6 \mathrm{~h}$. The supernatant was removed and centrifuged. The supernatant was used to assess 6-keto $\mathrm{PGF}_{1 \alpha}$ release from endothelial cells. The cross-hatched histograms represent 6-keto $\mathrm{PGF}_{1 \alpha}$ release from endothelial cells after 30 min exposure to various stimuli (average radioactive cpm for control wells was 31.9) while the open histograms represent $6 \mathrm{~h}$ exposure to various stimuli (average radioactive $\mathrm{cpm}$ for control wells was 50.1 ). This graph represents the mean \pm SE of 3 experiments in duplicate. ${ }^{*}=p<0.05$. 
BINDING OF PLATELETS SEDIMENTED FROM PRP TO ENDOTHELIAL CELLS PREINCUBATED WITH IIL-1

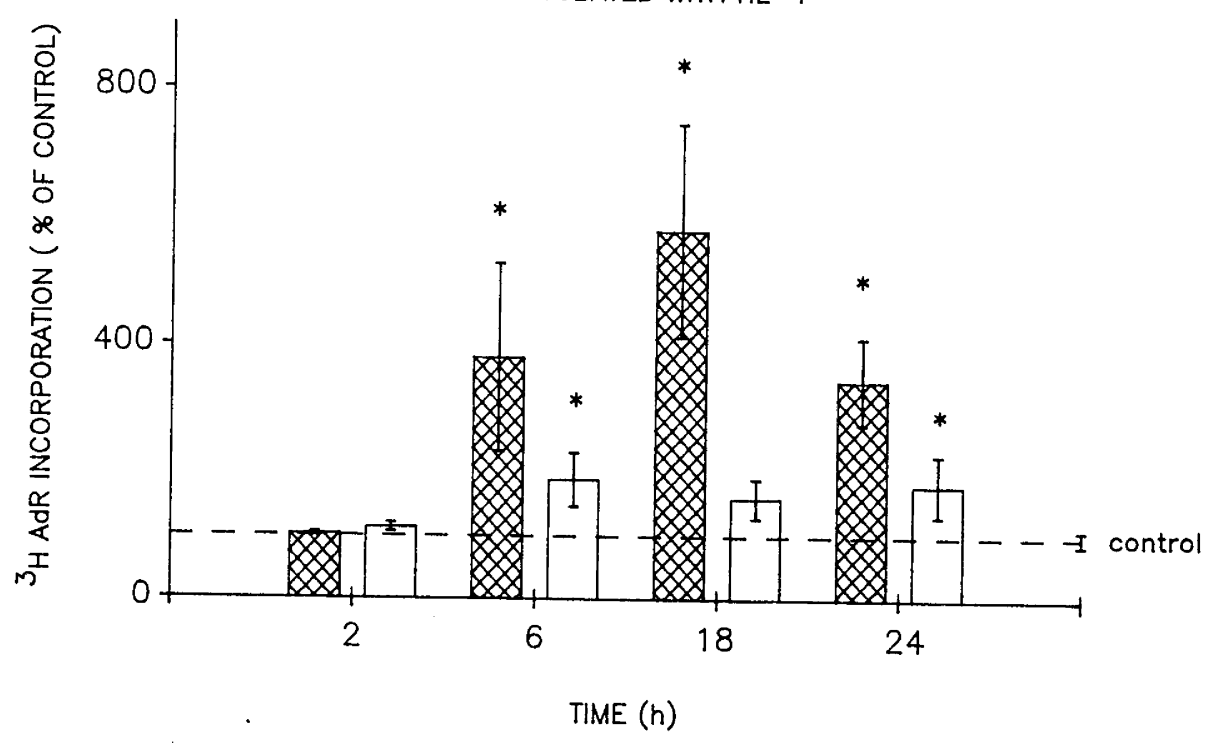

PROSTACYCLIN RELEASE BY ENDOTHELIAL CELLS EXPOSED TO VARIOUS STIMULI

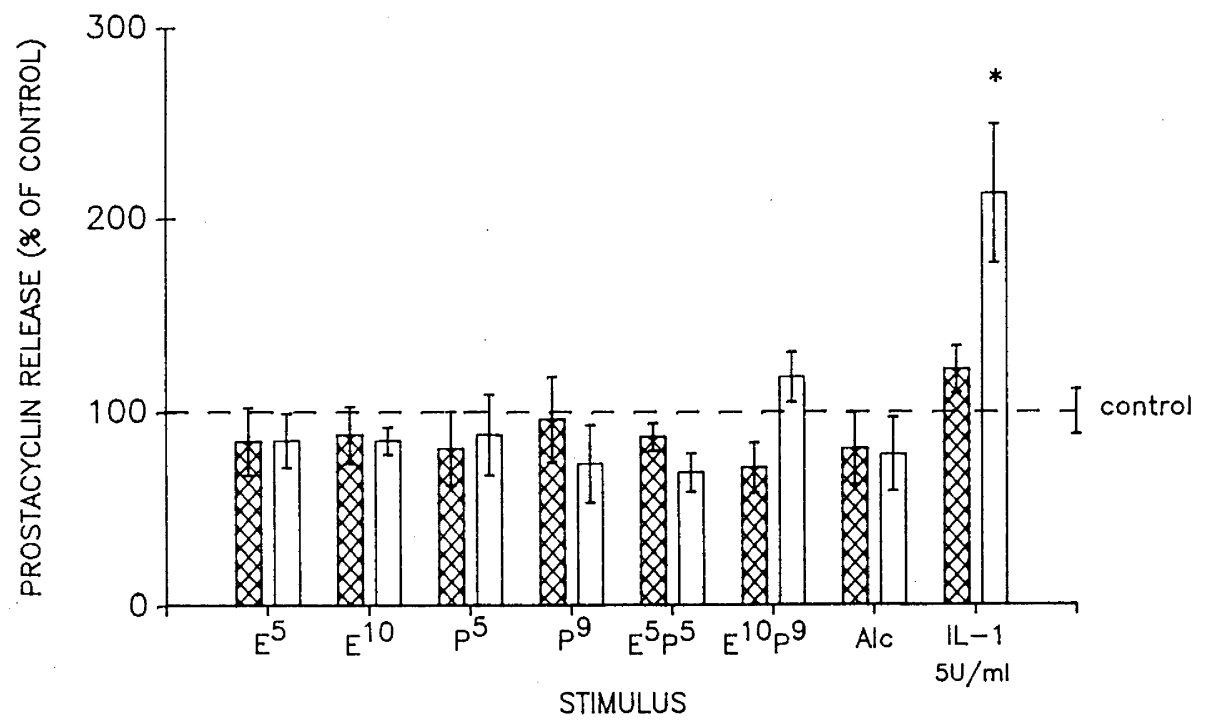




\section{Figure 4.7.}

$\mathrm{PGI}_{2}$ release by HUV endothelial cells exposed to various stimuli. A confluent monolayer of endothelial cells was exposed to sex steroids, alcohol or rlL-1 for $24 \mathrm{~h}$. In the same series of experiments some endothelial cells were preincubated with cycloheximide (10 $\mu \mathrm{g}$ $/ \mathrm{ml}$ ) and then exposed to the various stimuli. The experiment was then performed as described in the legend to Figure 4.6. The cross-hatched histograms represent 6-keto $\mathrm{PGF}_{1 \alpha}$ release from endothelial cells after $24 \mathrm{~h}$ exposure to various stimuli (average radioactive cpm for control wells was 83.2) while the open histograms represent endothelial cells preincubated with cycloheximide (average radioactive cpm for control well was 60.2) and then exposed to various stimuli for $24 \mathrm{~h}$. This graph represents the mean \pm SE of 10 experiments in duplicate for those cells not exposed to cycloheximide and the mean \pm SE of 3 experiments for the cycloheximide treated cells. ${ }^{*}=p<0.05$.

\section{Figure 4.8.}

$\mathrm{PGI}_{2}$ release by $\mathrm{HUV}$ endothelial cells exposed to various stimuli. A confluent monolayer of endothelial cells was exposed to Medium 199 (control) or different concentrations of $17 \beta$-estradiol for $24 \mathrm{~h}$. The supernatant was removed, centrifuged and assayed for 6-keto $\mathrm{PGF}_{1 \alpha}$ release from endothelial cells. This graph represents the mean \pm SE of 2 experiments in duplicate. * $=p<0.05$. 
PROSTACYCLIN RELEASE BY ENDOTHELIAL CELLS EXPOSED TO VARIOUS STIMULI

$24 \mathrm{~h}$ incubation

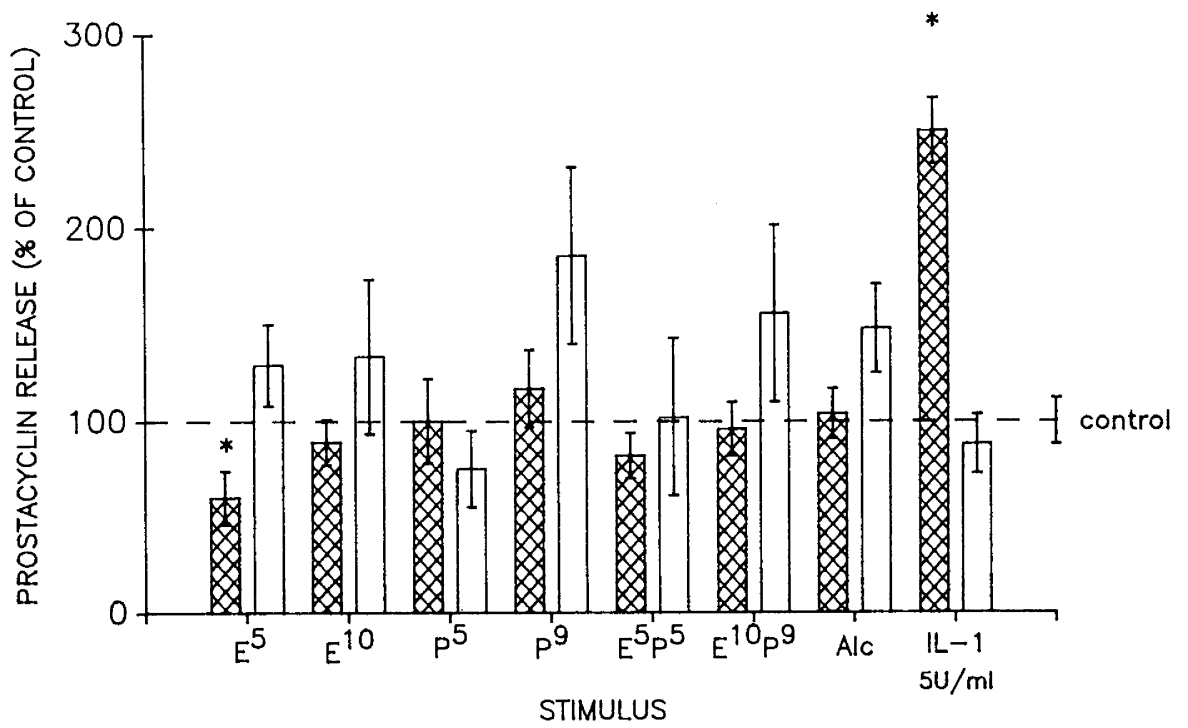

PROSTACYCLIN RELEASE BY ENDOTHELIAL CELLS EXPOSED TO ESTRADIOL

$24 \mathrm{~h}$ incubation

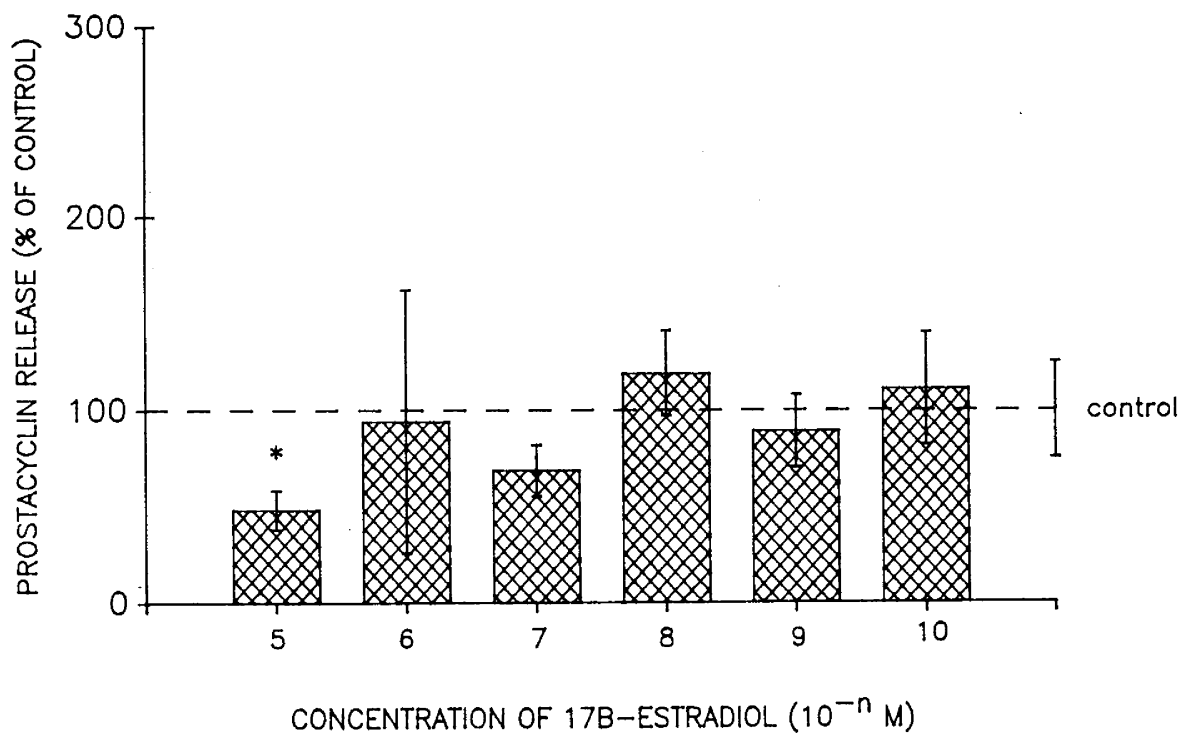




\section{Figure 4.9.}

Expression of vWf : Ag by HUV endothelial cells. A confluent monolayer of endothelial cells was established. The cells were removed from the gelatinized plates by (A) trypsin, (B) RGDS- $\mathrm{NH}_{2}$ or (C) plasmin, and stored at room temperature for $1 \mathrm{~h}$ before staining. The cells were then stained for the presence of vWf : Ag using antiserum to human Factor VIII (raised in goats) as the primary antibody and F.I.T.C.-conjugated antigoat IgG as the second antibody. Fluorescence was assessed by flow cytometry. The histograms have not been gated but were smoothed twice. The dotted line represents the addition of the F.I.T.C-conjugated antibody alone (background) while the closed line represents vWf : Ag expression on the endothelial cells. The instrument settings were the same for all samples. The histograms shown are representative of 3 experiments. 
FACTOR VIII IMMUNOFLUORESCENCE
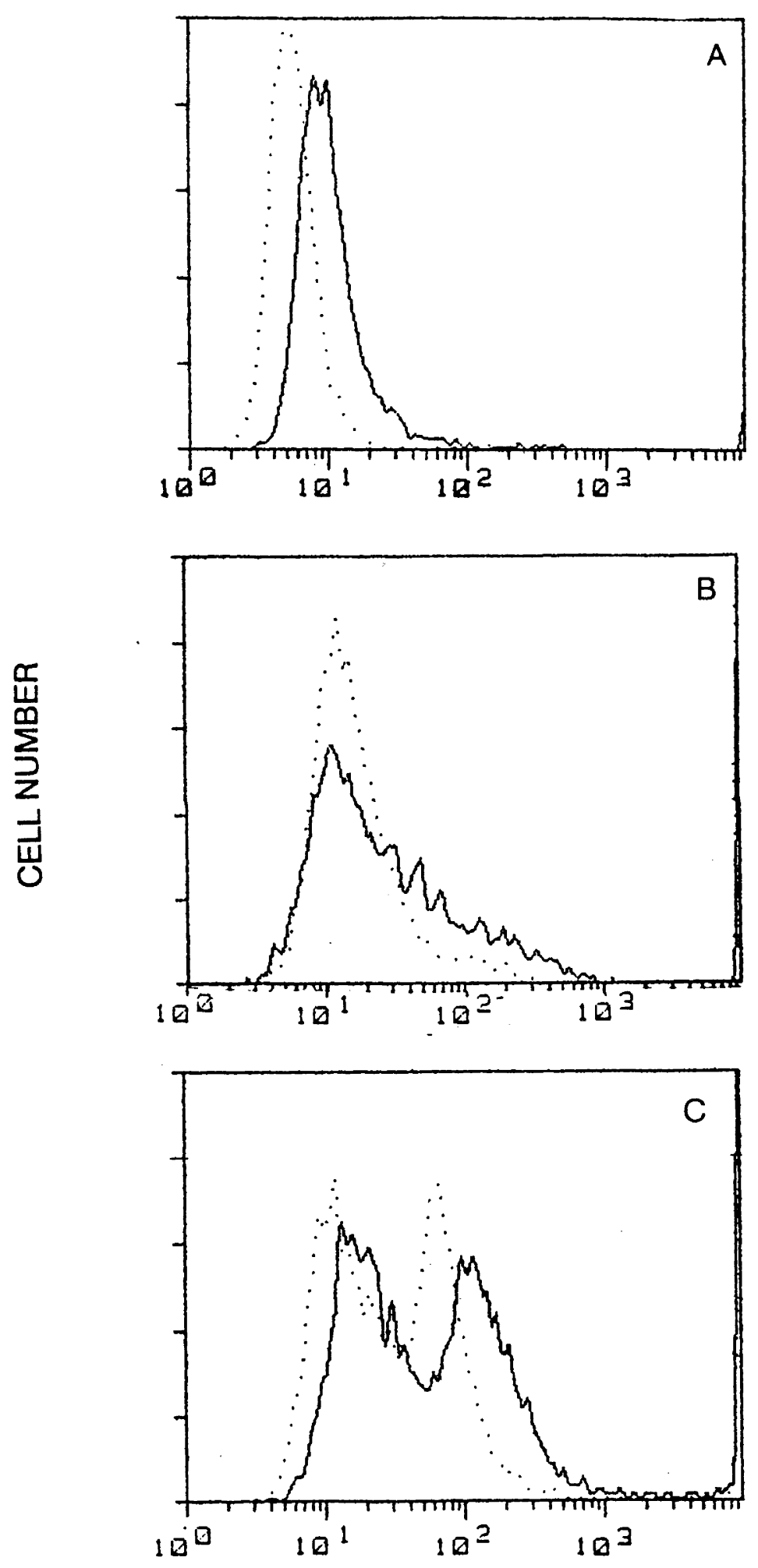

FLUORESCENCE INTENSITY 
Figure 4.10.

Expression of PgP-1 antigen by HUV endothelial cells. A confluent monolayer of endothelial cells was established. The cells were removed from the gelatinized plates by (A) trypsin, (B) RGDS- $\mathrm{NH}_{2}$, or (C) plasmin, and stored at room temperature for $1 \mathrm{~h}$ before staining. The cells were then stained for the presence of PgP-1 antigen using monoclonal antibody IM7 as the primary antibody and F.I.T.C.-conjugated antimouse IgG (raised in sheep) as the second antibody. Fluorescence was assessed by flow cytometry. The histograms were not gated but were smoothed twice. The dotted line represents the addition of the F.I.T.C.-conjugated antibody to the endothelial cells alone (background) while the closed line represents PgP-1 expression on the endothelial cells. The instrument settings were the same for all samples. The histograms shown are representative of 3 experiments. 


\section{PgP - 1 IMMUNOFLUORESCENCE}
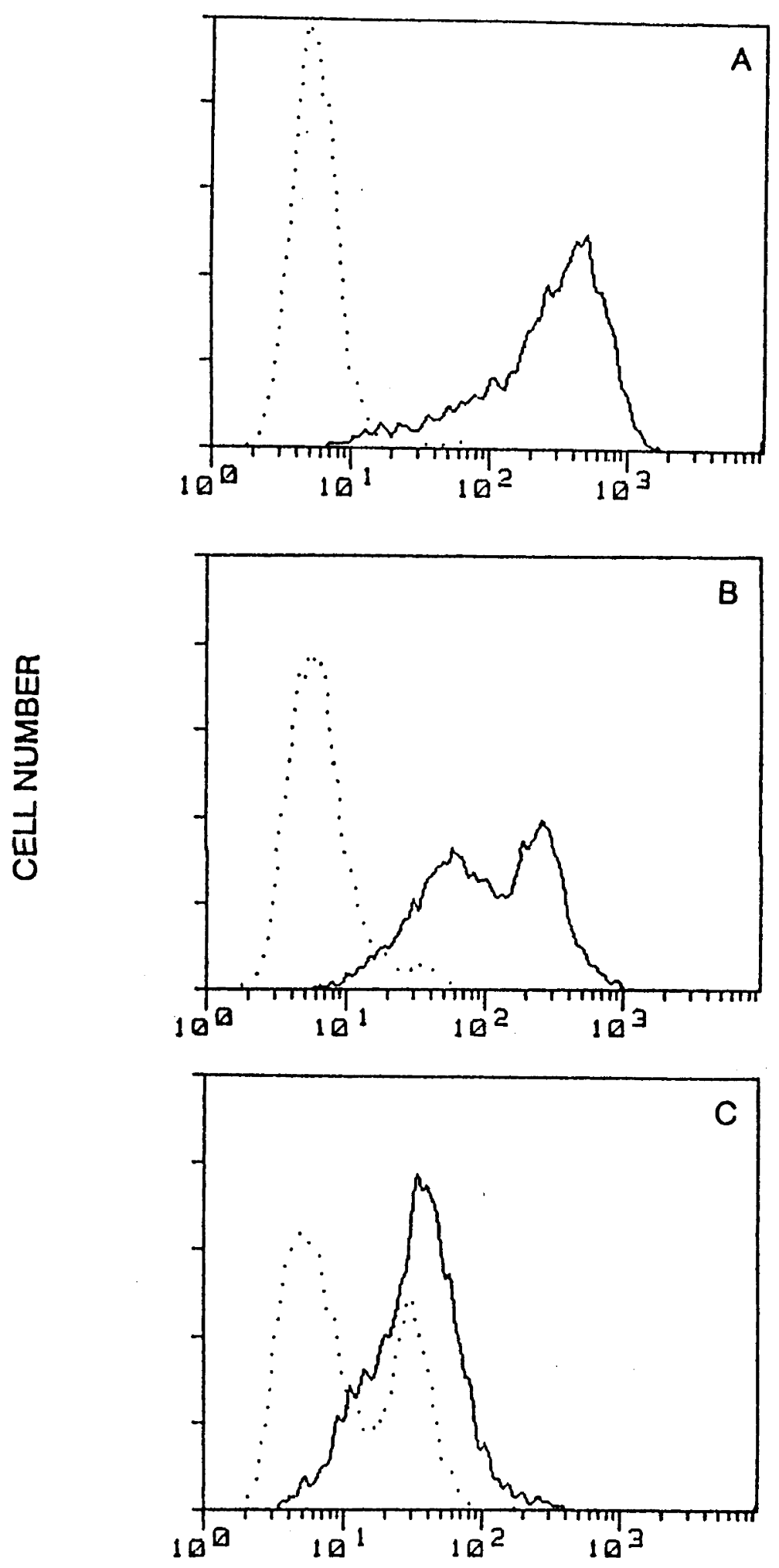

FLUORESCENCE INTENSITY 
Figure 4.11.

Expression of different antigens on HUV endothelial cells. A confluent monolayer of endothelial cells was established. The cells were removed from the gelatinized plates by trypsin and stained for the presence of (A) vWf : Ag, (B) PgP-1, or (C) ELAM-1 (the cells being preincubated with rlL-1 $(5 \mathrm{U} / \mathrm{ml})$. The presence of each antigen was assessed by flow cytometry. The histograms were not gated but were smoothed twice. The dotted line represents the addition of the F.I.T.C.-conjugated antibody to the endothelial cells alone (background) while the closed line represents expression of each antigen by endothelial cells. The histograms shown are representative of 3 experiments. 

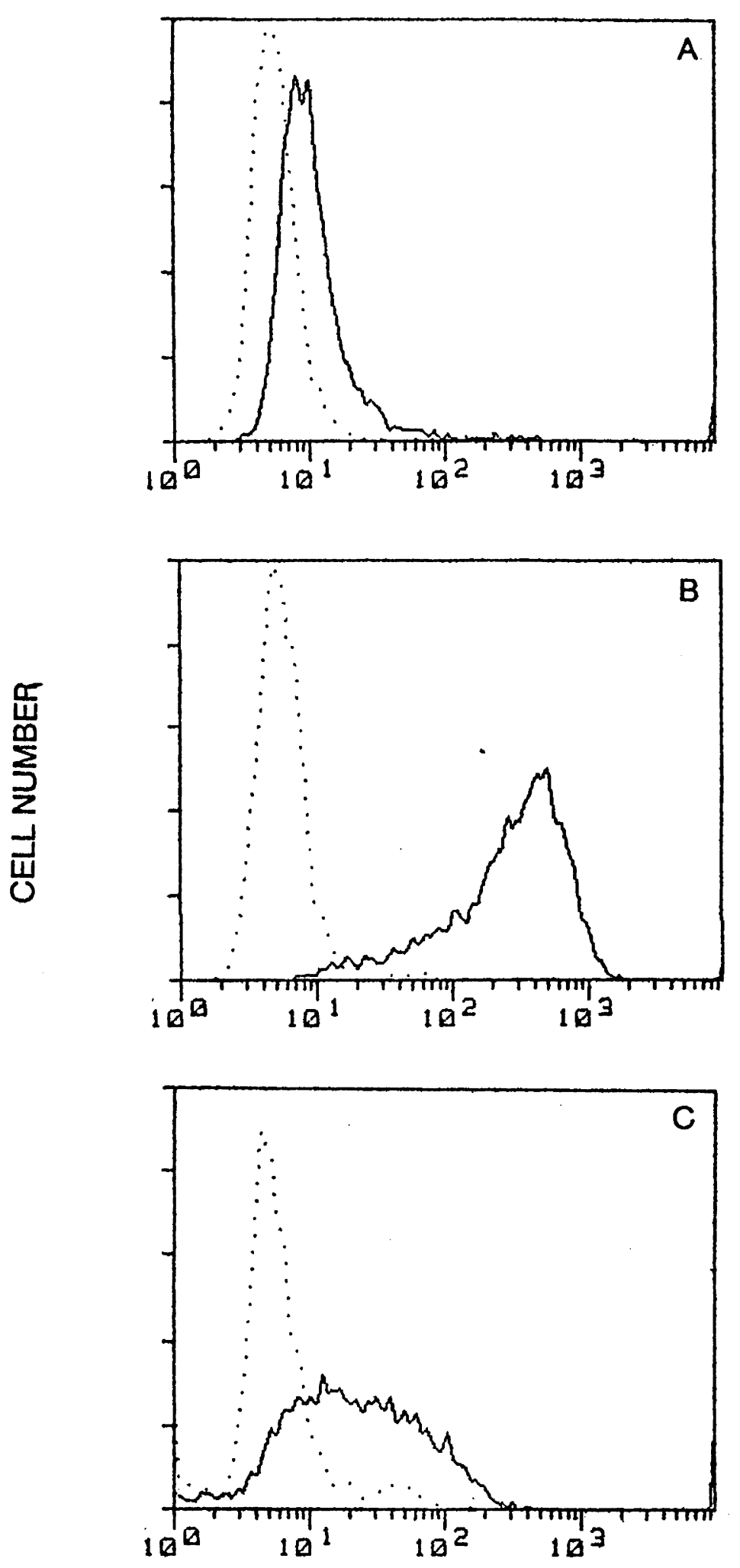

FLUORESCENCE INTENSITY 


\section{Figure 4.12.}

Effect on vWf expression of passaging and storage after trypsinization of HUV endothelial cells. Endothelial cells of different passages were grown to confluence in gelatinized 24 well plates. They were removed from the plates with trypsin and then washed 3 times. The cells were stained for the presence of $\mathrm{VWf}: \mathrm{Ag}$ as described in the legend to Figure 4.9. The dotted line represents the addition of the F.I.T.Cconjugated antibody alone (background) while the closed line represents VWf : Ag expression on HUV endothelial cells. The instrument settings were the same for all samples. (A) histogram of endothelial cells (passage 5) which were stained for the presence of $v W f$ after being stored at room temperature for $2 \mathrm{~h}$. (B) histogram of endothelial cells (passage 10) which were stained for the presence of $v W f$ after being stored at room temperature for $2 \mathrm{~h}$. (C) histogram of the same endothelial cells (passage 10) as in (B) which were stained immediately following trypsinization and washing. (D) forward scatterside scatter profile showing the size and granularity of the endothelial cells (passage 5), and (E and F) passage 10 endothelial cells stained immediately $(F)$ and after being stored at room temperature at $2 h(E)$. All of the above endothelial cells were processed on the same day with the same antibodies. Representative data from 2 experiments performed in duplicate are shown. 

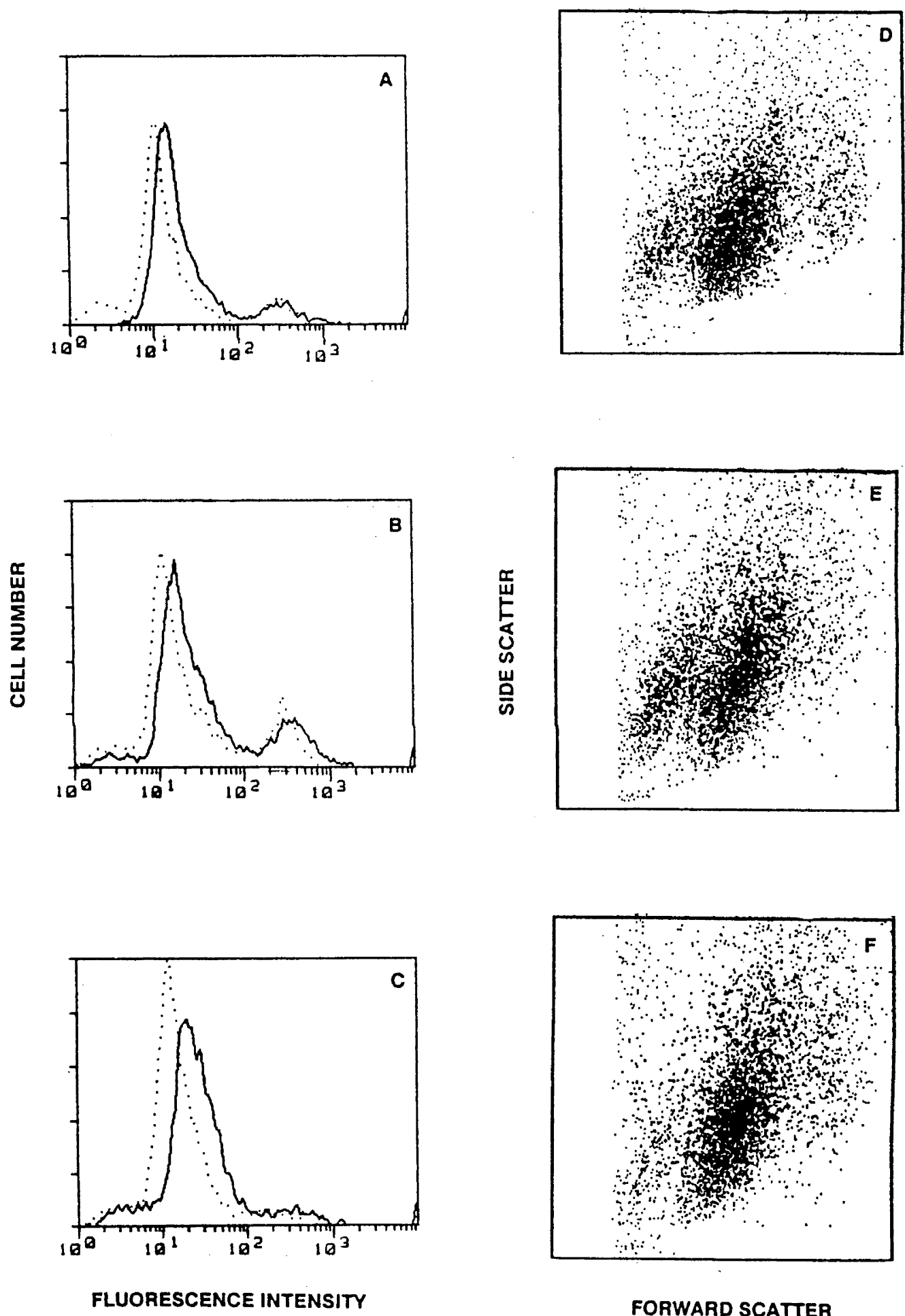

FORWARD SCATTER 
Figure 4.13.

The effect of passaging on vWf : Ag expression by HUV endothelial cells ( $A$ and $B$ ). The cells were removed from the gelatinized plates by trypsin, washed and stored at room temperature for $1 \mathrm{~h}$ before staining. The cells were then stained for the presence of $\mathrm{vWf}: \mathrm{Ag}$ as described in the legend to Figure 4.9. Fluorescence was assessed by flow cytometry. The histograms have not been gated but were smoothed twice. The dotted line represents the addition of the F.I.T.Cconjugated antibody to the endothelial cells alone (background) while the closed line represents vWf : Ag expression by endothelial cells. The instrument settings were the same for all samples. (A) represents endothelial cells at passage 5, (B) represents endothelial cells at passage 9. 


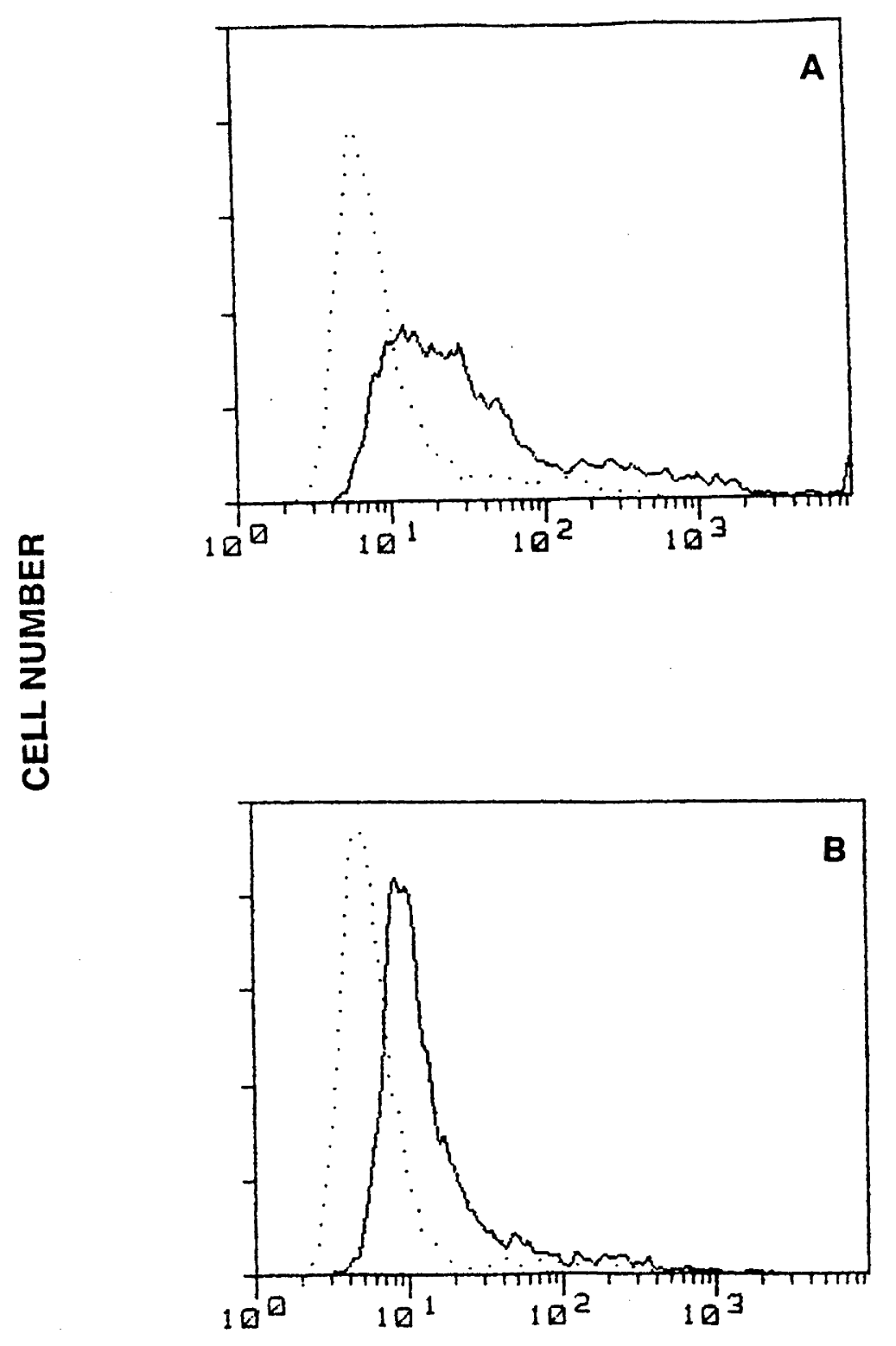

FLUORESCENCE INTENSITY 


\section{Figure 4.14.}

Effect of passaging on the size and granularity of HUV endothelial cells. Endothelial cells were prepared and stained for the presence of vWf : $\mathrm{Ag}$ as described in the legend to Figure 4.9. The forward scatter-side scatter profiles demonstrate the size (forward scatter) and granularity (side scatter) of endothelial cells with increasing passage. The profiles used the same primary and secondary antibody but were not all stained on the same day. 


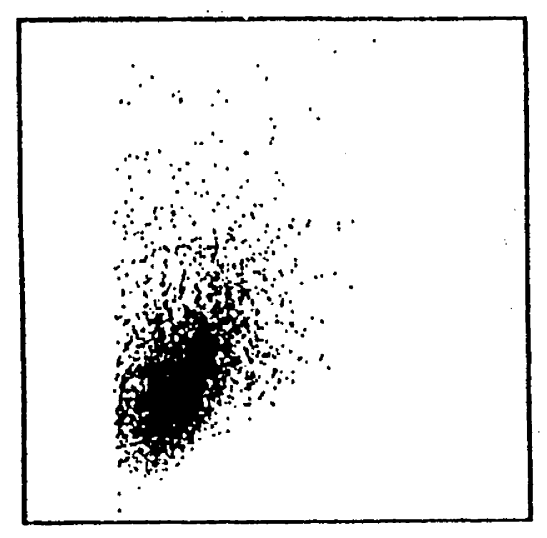

PASSAGE 5

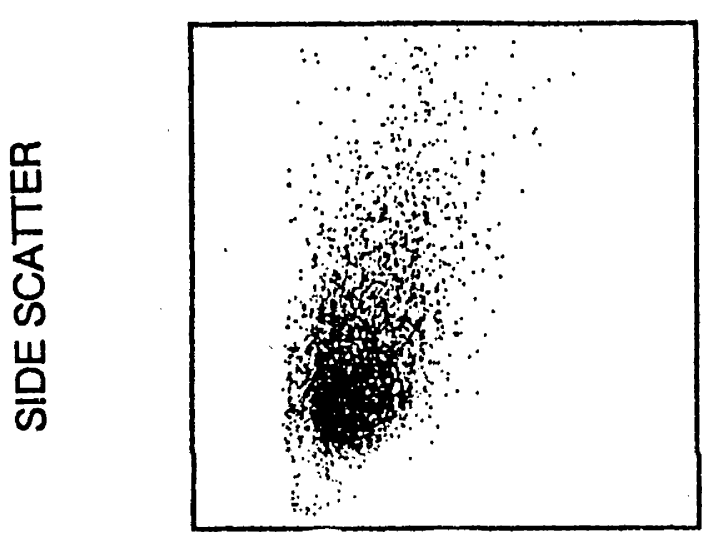

PASSAGE 7

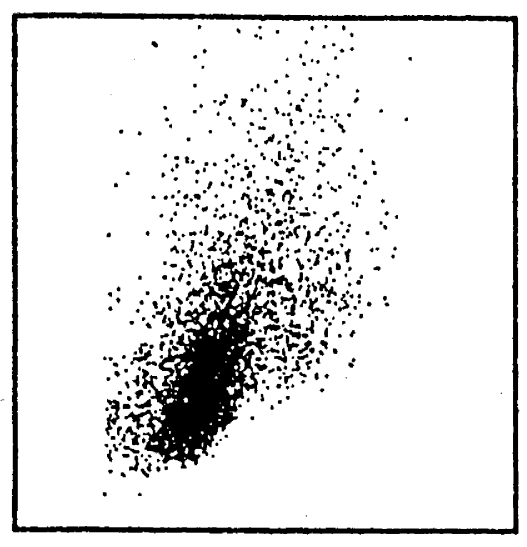

PASSAGE 9

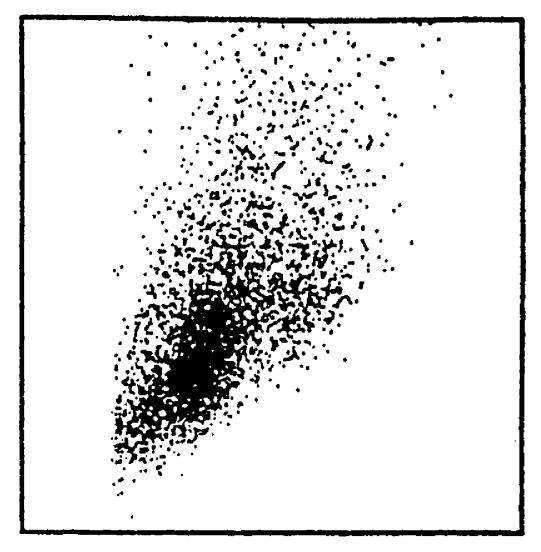

PASSAGE 6

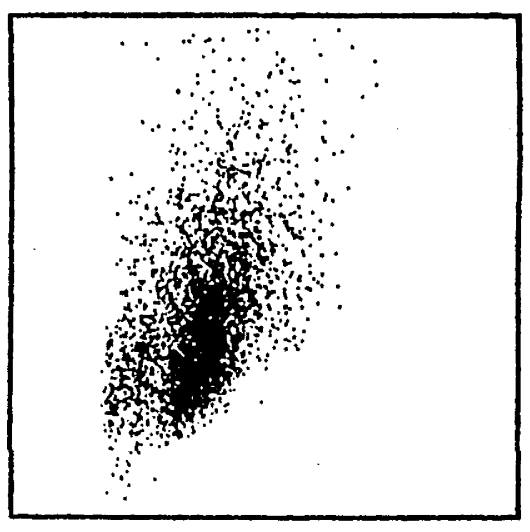

PASSAGE 8

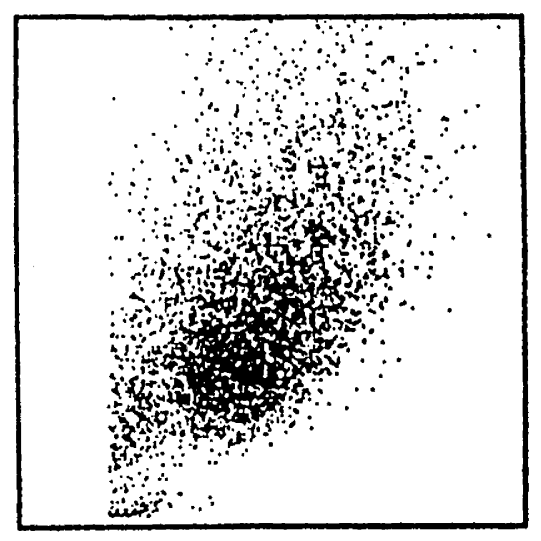

PASSAGE 10 


\section{Figure 4.15.}

Effect of preincubation of antiserum to human FVIII with AHF. A confluent monolayer of endothelial cells was established. The cells were trypsinized and stored at room temperature for $1 \mathrm{~h}$. Antiserum to human FVIII was preincubated with AHF $(50 \mathrm{mg} / \mathrm{ml})$ for $15 \mathrm{~min}$ at 4 ${ }^{\circ} \mathrm{C}$. This was then incubated with endothelial cells. Following the addition of F.I.T.C-conjugated antigoat IgG the shift in fluorescence was assessed by flow cytometry. The histogram was not gated but was smoothed twice. The broken line represents the addition of the F.I.T.C-conjugated antibody alone (background) to endothelial cells, while the dotted line represents vWf : Ag expression on endothelial cells and the closed line shows the effect of preincubating antiserum to FVIII with AHF. The histograms shown are representative of 3 experiments. 


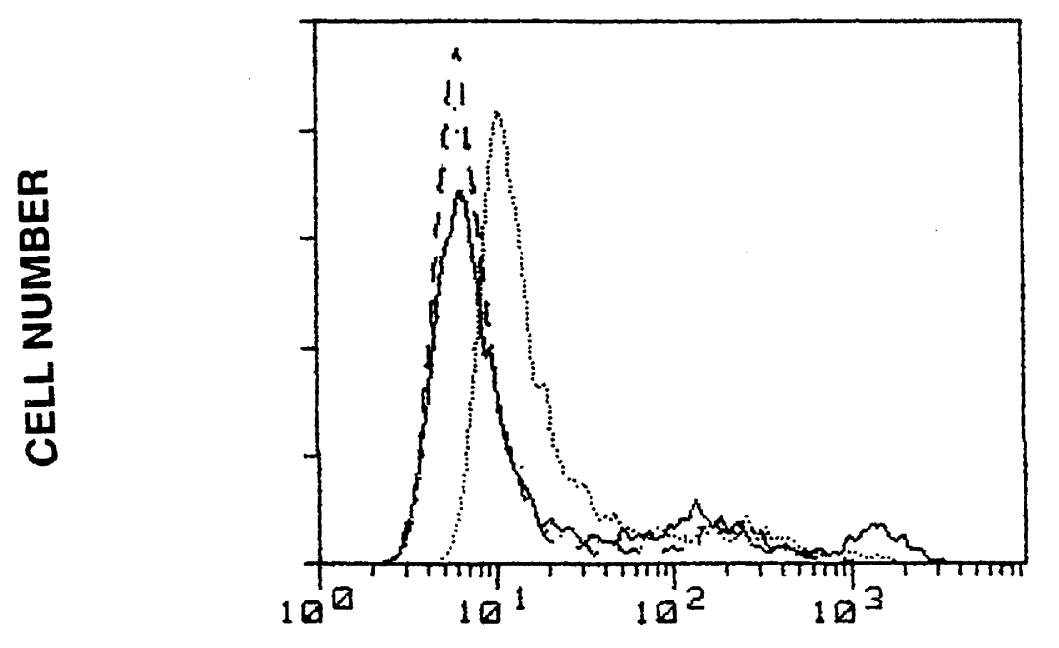

FLUORESCENCE INTENSITY 
Figure 4.16.

Effect of sex steroids on vWf : Ag expression by HUV endothelial cells. A confluent monolayer was established. The endothelial cells were then incubated with sex steroids, rIL-1 or Medium 199 (control). Following trypsinization and washing, the cells were resuspended in Medium 199 with the appropriate sex steroid or rIL1 and stored at room temperature for $1 \mathrm{~h}$. The expression of $\mathrm{vWf}$ : Ag was assessed by flow cytometry as described in the legend to Figure 4.9. This graph represents the mean \pm SE of 9 experiments in duplicate. ${ }^{*}=p<0.05$

\section{Figure 4.17.}

Effect of cycloheximide on vWf : Ag expression. A confluent monolayer of HUV endothelial cells was established. The cells were incubated with cycloheximide $(10 \mu \mathrm{g} / \mathrm{ml})$ for $30 \mathrm{~min}$ before the addition of the sex steroids, rlL-1 or Medium 199 (control) for $18 \mathrm{~h}$. The cells were trypsinized, and then washed and the expression of vWf : Ag was assessed by flow cytometry as described in the legend to Figure 4.9. This graph represents the mean \pm SE of 3 experiments in duplicate. 
EXPRESSION OF VWF ANTIGEN BY ENDOTHELIAL CELLS EXPOSED TO VARIOUS STIMULI

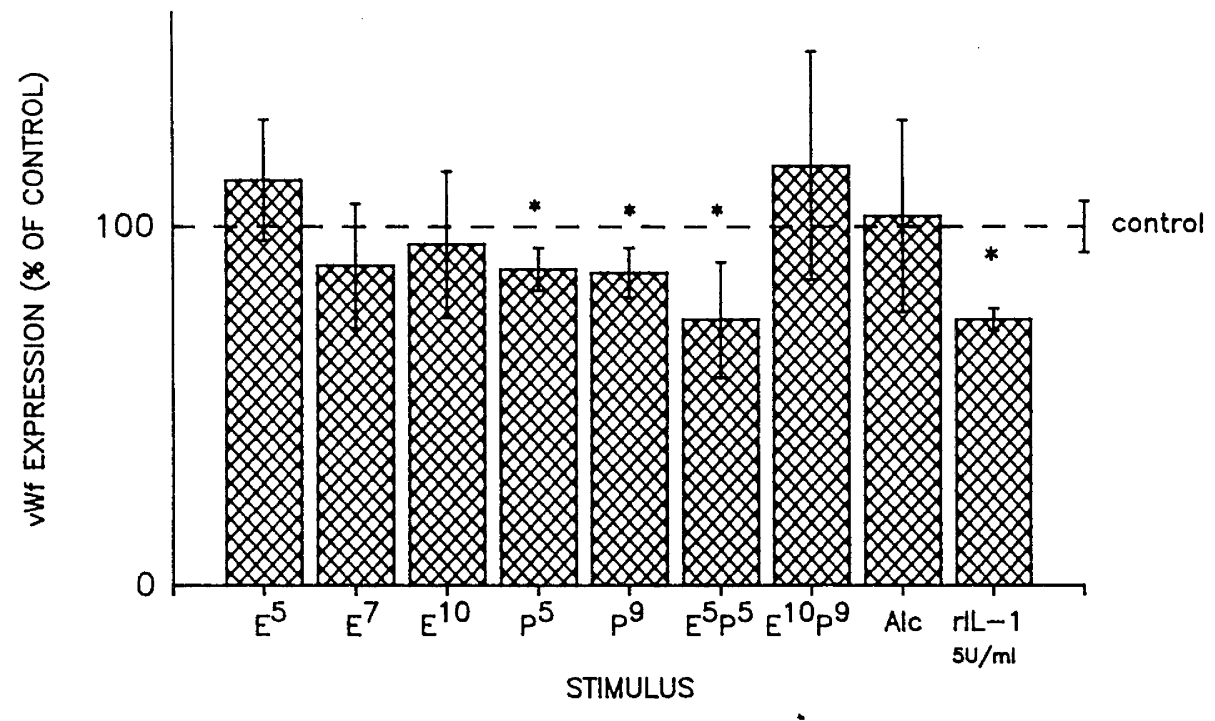

EXPRESSION OF VWF ANTIGEN BY ENDOTHELIAL CELLS EXPOSED TO VARIOUS STIMULI WHEN PREINCUBATED WITH CYCLOHEXIMIDE

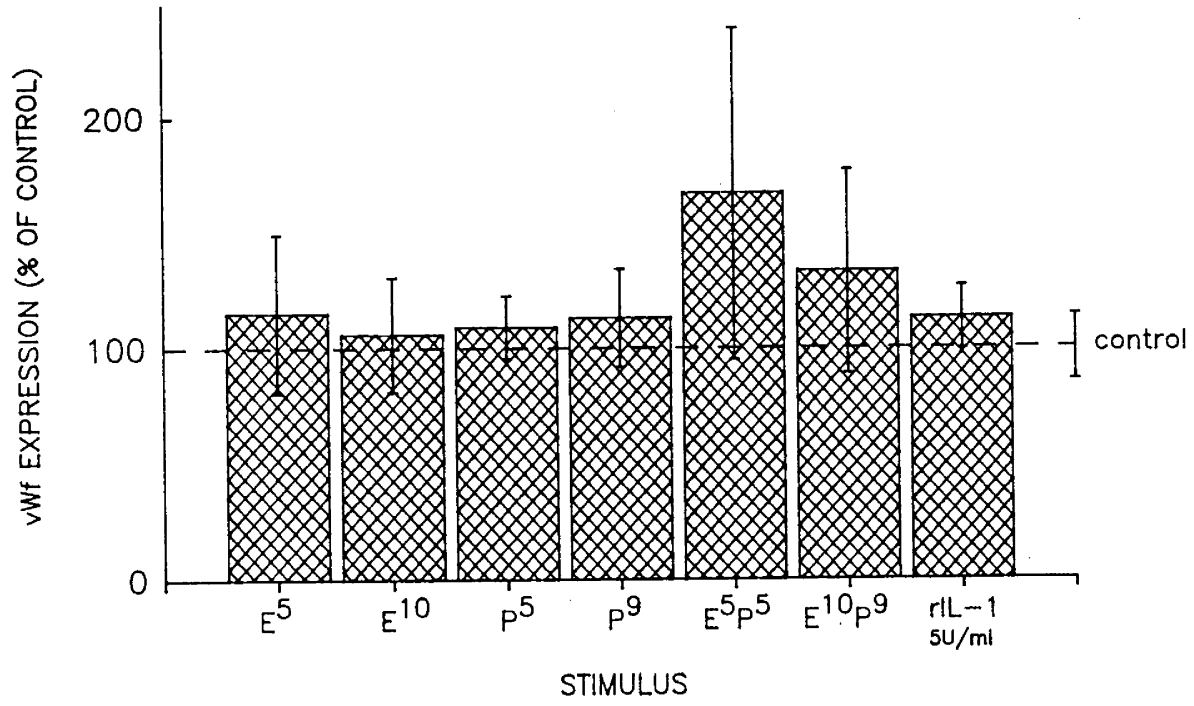




\section{Figure 4.18.}

vWf : Ag expression on endothelial cells treated with sex steroids. The upper photo demonstrates HUV endothelial cells exposed to $17 \beta$ estradiol $\left(10^{-10} \mathrm{M}\right)$ in combination with progesterone $\left(10^{-9} \mathrm{M}\right)$ and the middle photo represents $17 \beta$-estradiol $\left(10^{-5} \mathrm{M}\right)$ in combination with progesterone $\left(10^{-5} \mathrm{M}\right)$. The lower photo represents background fluorescence associated with the addition of the F.I.T.C-conjugated antigoat IgG alone to endothelial cells. These photomicrographs are representative of 4 experiments. Magnification $547 \mathrm{x}$. 

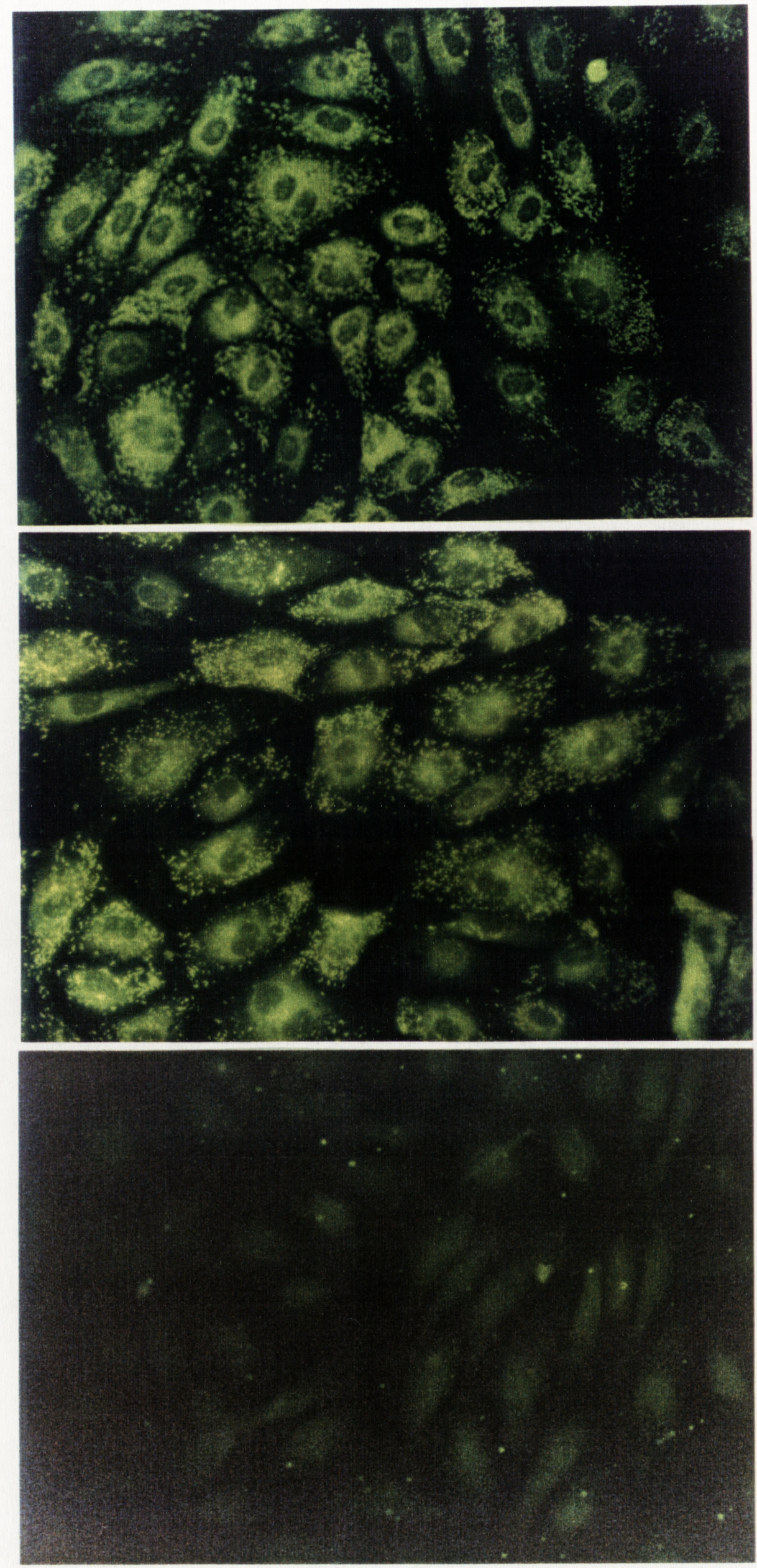


\section{Figure 4.19.}

Effect of sex steroids on vWf : Ag expression by HUV endothelial cells. The cells (passage 9) were prepared as described in the legend to Figure 4.9. In graph (A) the open dotted line represents the addition of the F.I.T.C-conjugated antigoat IgG alone (background) to cells treated with progesterone $\left(10^{-9} \mathrm{M}\right)$, while the continuous line represents VWf : Ag expression on cells treated with progesterone (10$9 \mathrm{M})$. The broken line represents background for control cells (Medium 199 alone) while the closed dotted line represents vWf : Ag expression by control cells (Medium 199 alone).

In graph (B) the open dotted line represents the background for cells treated with $17 \beta$-estradiol $\left(10^{-5} \mathrm{M}\right)$ in combination with progesterone $\left(10^{-5} \mathrm{M}\right)$, while the continuous line represents $\mathrm{VWf}: \mathrm{Ag}$ expression on cells treated with $17 \beta$-estradiol $\left(10^{-5} \mathrm{M}\right)$ and progesterone $\left(10^{-5} \mathrm{M}\right)$. The closed dotted line represents vWf : Ag expression on control cells (Medium 199 alone) and the broken line represents background for control cells (Medium 199 alone). These histograms are representative of 9 experiments. The histograms have not been gated but were smoothed twice.

\section{Figure 4.20.}

Effect of cycloheximide on vWf : Ag expression by HUV endothelial cells exposed to sex steroids. The method is described in the legend to Figure 4.9. These experiments were performed on the same preparation of cells as those described in Figure 4.19. The histograms shown are representative of 3 experiments. 


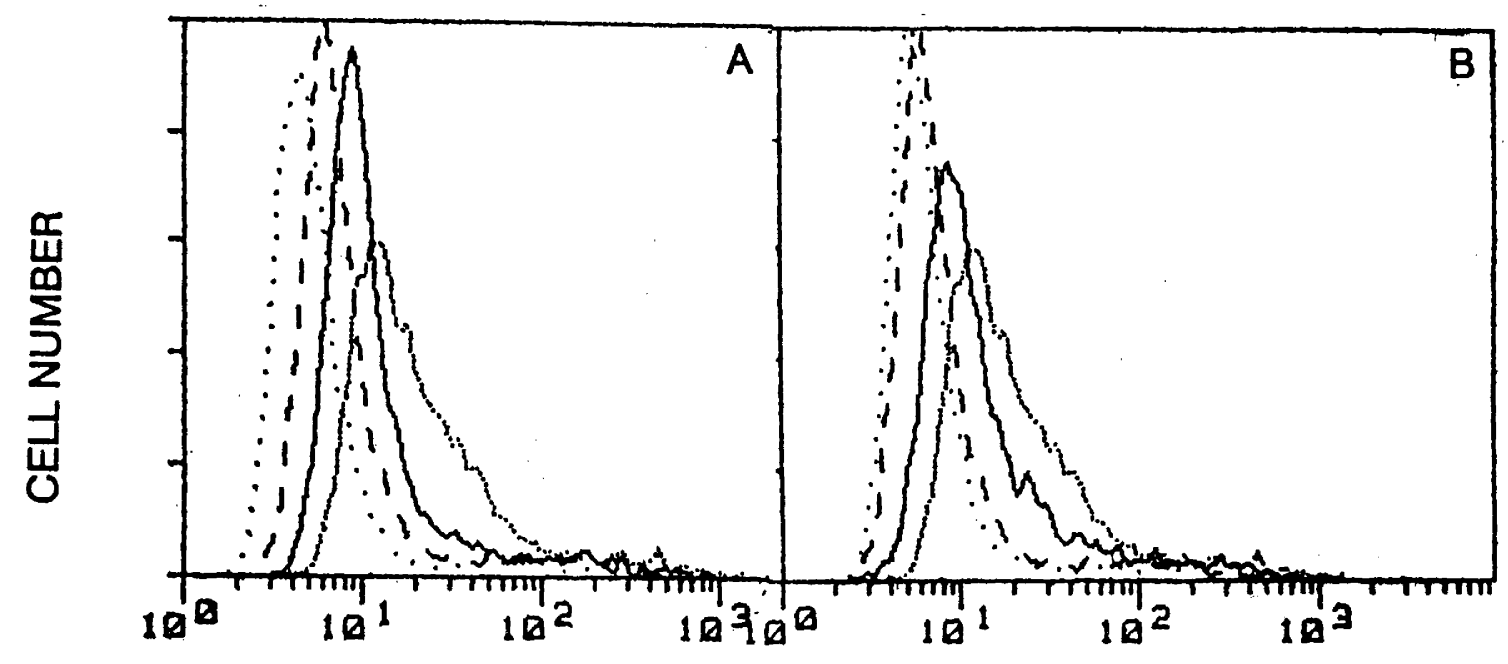

FLUORESCENCE INTENSITY

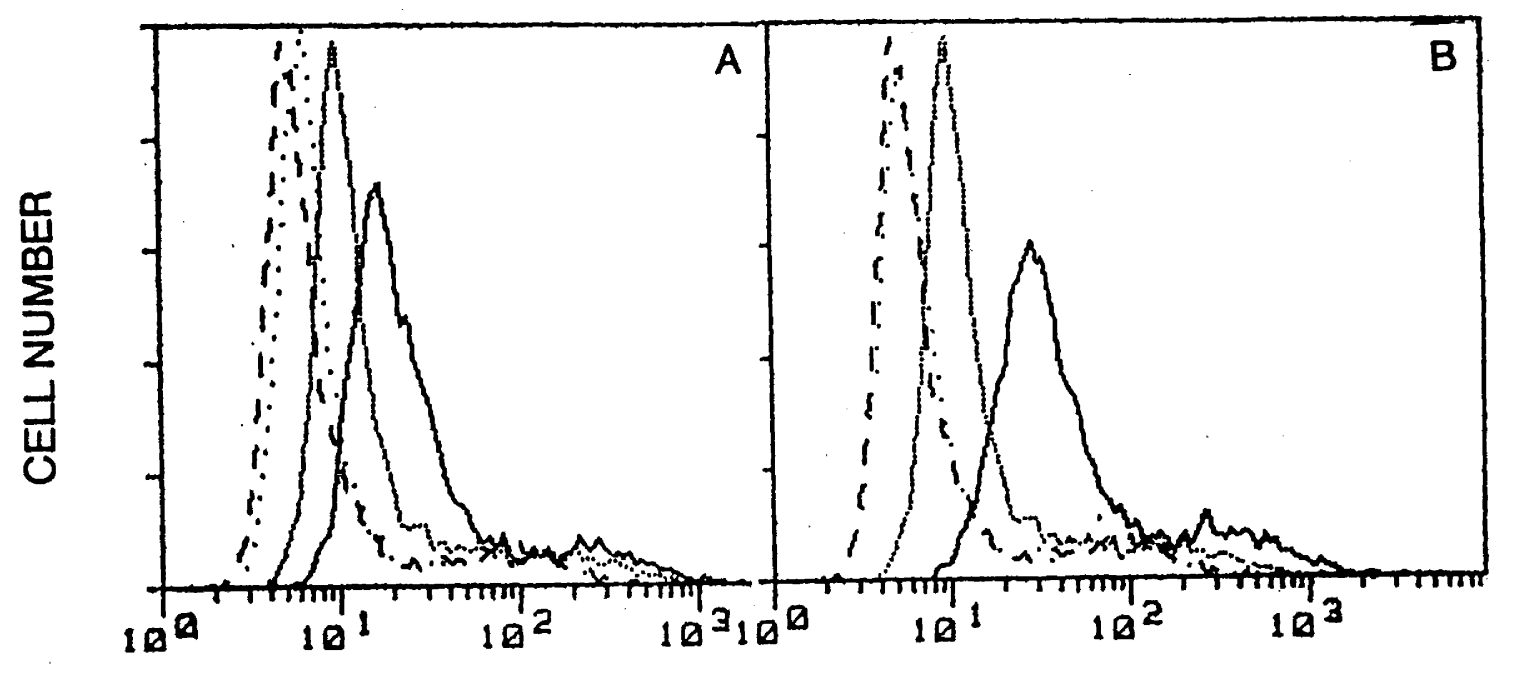

FLUORESCENCE INTENSITY 


\section{Figure 4.21.}

Effect of sex steroids on ristocetin-induced platelet aggregation. A confluent monolayer of HUV endothelial cells was established. The cells were incubated with Medium 199 (control), sex steroids, IL-1 or alcohol for $48 \mathrm{~h}$. The supernatant was removed and concentrated 10 times before being added to washed platelets. A.R.P. represents the assay reference plasma used as an external control. This graph is the mean \pm SE of 5 experiments, three in triplicate.

\section{Figure 4.22.}

Effect of sex steroids on vWf : Ag in culture supernatants from HUV endothelial cells. A confluent monolayer of HUV endothelial cells was exposed to Medium 199 (control), sex steroids, rlL-1 or alcohol for $48 \mathrm{~h}$. The supernatant was removed and concentrated 10 times. The amount of vWf : Ag was measured using Laurell Rocket immunoelectrophoresis. The data shown are the mean \pm SE of three supernatants in triplicate. 


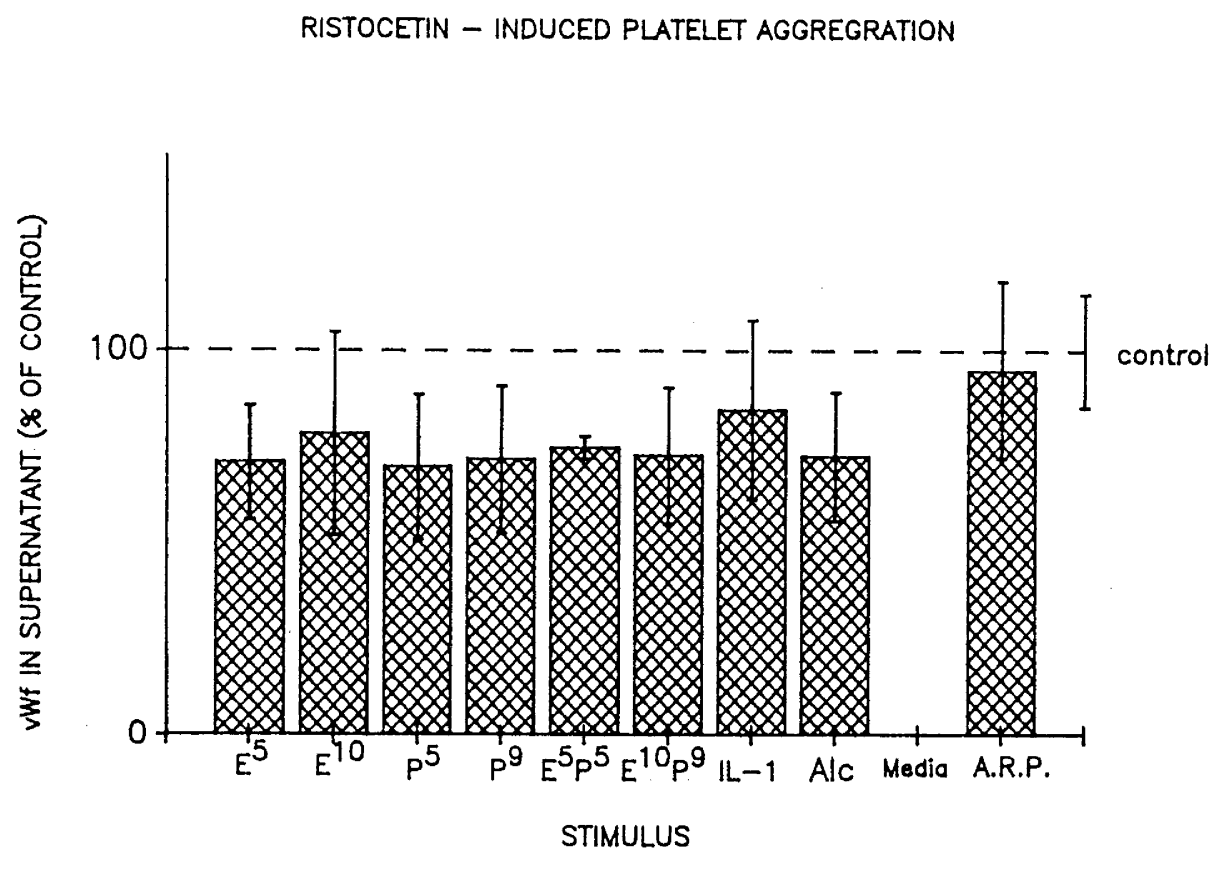

LAURELL ROCKET IMMUNOELECTROPHORESIS

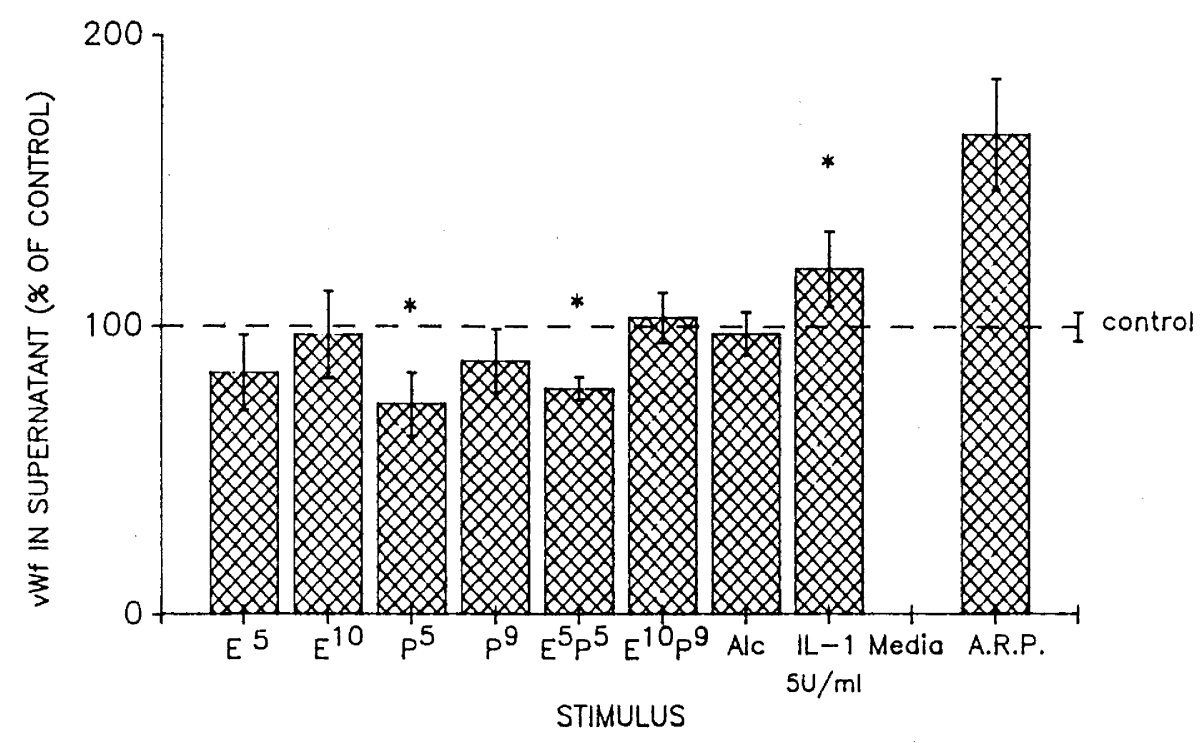


Figure 4.23.

Effect of sex steroids on vWf : Ag in culture supernatants from HUV endothelial cells. This is a representative immunoelectrophoretogram. Lanes 1-4 represent the standard curve derived from pooled human plasma, while lanes 5-6 show assayed reference plasma (1/2 and 1/4 dilution). All other lanes represent supernatants from endothelial cells exposed to various stimuli: Lane 7 is $17 \beta$-estradiol $\left(10^{-5} \mathrm{M}\right)$, Lane 8 is $17 \beta$-estradiol $\left(10^{-10} \mathrm{M}\right)$, Lane 9 is progesterone $\left(10^{-5} \mathrm{M}\right)$, Lane 10 is progesterone $\left(10^{-9} \mathrm{M}\right)$, Lane 11 is Medium 199 alone (control), Lane 12 is $17 \beta$-estradiol $\left(10^{-10} \mathrm{M}\right)$ in combination with progesterone $\left(10^{-9} \mathrm{M}\right)$, Lane 13 is rlL-1 $(5 \mathrm{U}$ $/ \mathrm{ml}$ ), and Lane 14 is alcohol. $17 \beta$-estradiol $\left(10^{-5} \mathrm{M}\right)$ in combination with progesterone $\left(10^{-5} \mathrm{M}\right)$ was not included on this gel. 


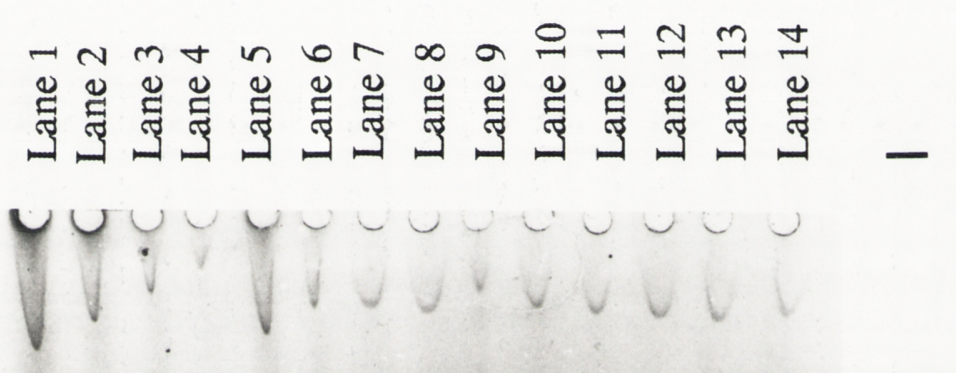




\section{CHAPTER 5}

\section{INFLUENCE OF SEX STEROIDS ON MONOCYTE BINDING}

TO ENDOTHELIAL CELLS 


\subsection{INTRODUCTION}

\subsubsection{Monocyte adhesion to endothelial cells}

As discussed in Chapter 1 (refer to Section 1.3.1.4), monocytes are important in the pathogenesis of atherosclerosis because of their role in foam cell formation, growth factor production, and vascular wall injury. A significant event in the atherosclerotic process is believed to be the adherence of monocytes to the endothelial cell surface (Arnaout, 1988) and their subsequent migration into the intima. Modification of monocyte adhesion may therefore be important in the pathogenesis of atherosclerosis.

Models of atherosclerosis such as the hypercholesterolemic pig (Gerrity, 1981a; b), monkey (Faggioto et al, 1984a), rat (Joris et al, 1983), rabbit (Poole and Florey, 1958) and pigeon (Lewis et al, 1982; Taylor and Lewis, 1986) have demonstrated focal adhesion of monocytes to the endothelium as an early event associated with cholesterol feeding. Gerrity et al (1985) showed that monocytes preferentially adhere to and penetrate areas of arteries which are predisposed to early atherosclerosis in hypercholesterolemic swine but not in normal swine. Similar findings were reported by Walker and Bowyer (1984) in rabbits.

There is in vitro evidence that monocytes bind selectively to the intercellular borders of individual endothelial cells and migrate at high basal rates between endothelial cells without disruption of the endothelial cell monolayer (Migliorisi et al, 1987 and Pawlowski et al, 1988). Under culture conditions 8-10 monocytes bind to the margins of one endothelial cell after 60-90 min incubation at $37^{\circ} \mathrm{C}$ (Pawlowski et al, 1985; 1988 ; Dicorleto et al, 1985; Beekhuizen et al, 1990). Monocyte binding is saturable and reaches an 
equilibrium after $15 \mathrm{~min}$ at $37^{\circ} \mathrm{C}$ and after $90 \mathrm{~min}$ at $4^{\circ} \mathrm{C}$ (DiCorleto and Chisolm, 1986). In hypercholesterolemic swine, monocytes bind at endothelial cell junctions but also invade the intima of lesion-prone areas of arteries, become phagocytic and accumulate lipid resulting in foam cell formation (Gerrity, 1981a: b). Gerrity (1981b) also demonstrated that these foam cells can migrate back into the bloodstream and cause endothelial cell damage. Beekhuizen et al (1990) studied the interaction of monocytes with both macrovascular and microvascular endothelial cells using microcinematographic video and found that the morphology of monocytes adherent to endothelial cells changed constantly, and that the percentage of endothelial cell-bound monocytes depended on the duration of incubation, the concentration of monocytes added to the endothelial cells, the density of the endothelial cell monolayer and the source of the endothelial cells.

Monocytes use different adhesion molecules to adhere to endothelial cells depending on whether the endothelial cell or monocyte is activated (Arnaout et al, 1988). Adhesion molecules on monocytes include lymphocyte functionassociated antigen-1 (LFA-1) (CD11a/ CD18) (Mentzer et al. 1986; 1987), macrophage antigen-1 (Mac-1) and p 150,95 antigens (Arnaout et al, 1988; Peri et al, 1990; Te Velde et al, 1987; Keizer et al, 1987; Gladwin et al, 1990). These three glycoproteins are composed of a structurally identical $95 \mathrm{kDa} \beta-$ subunit and different sized $\alpha$-subunits (Te Velde et al, 1987; Beekhuizen et al, 1990). Carlos et al (1990) showed that basal monocyte adhesion to endothelial cells is partly CD-18 independent. LFA-1, p 150,95 and CR3 receptors on macrophages and monocytes have also been shown to bind endotoxin (Wright and Jong, 1986). The molecules on endothelial cells which are important in macrophage adhesion include intercellular adhesion molecules ICAM-1 (CD54) and ICAM-2 (Staunton et al, 1989) although there are probably others. ICAM-1 on endothelial cells binds to leukocyte adhesive protein complex on monocytes (Staunton et al 1989). In summary, there are 
a number of antigens which are involved in the adhesion of monocytes to endothelial cells, but the precise role of these antigens in normal and pathological states is as yet uncertain.

\subsubsection{Factors influencing monocyte binding to endothelial cells}

In vitro studies have shown that adhesion of monocytes to HUV endothelial cell monolayers is cation dependent (Pawlowski et al, 1985; Bevilacqua et al, $1985 \mathrm{a} ; \mathrm{b}$ ), that adhesion is sensitive to trypsin (DiCorleto and de la Motte, 1984), and that binding is more avid to sparse rather than confluent endothelial cells (DiCorleto and de la Motte, 1984; de Bono and Green, 1984; DiCorleto et al, 1985; Alderson et al, 1986). When endothelial cells are treated with specific agents or when they are stimulated to migrate and divide in response to a "wound" in vitro (DiCorleto and de la Motte, 1984) they exhibit a greater ability to bind monocytes. Increased binding of monocytes can be induced by factors such as IL-1 (Bevilacqua et al, 1985 a; Goerdt et al, 1987; Peri et al, 1990), tumour necrosis factor (Goerdt et al, 1987) and lipopolysaccharide (Pohlman et al, 1985 a; b; Goerdt et al, 1987; Whisler et al, 1989; Territo et al, 1989; Berliner et al, 1990). Other factors which increase monocyte binding to endothelial cells include herpes virus infection (Span et al, 1989; Etingin et al, 1990), $\beta$-VLDL (Territo et al, 1987; 1989; Endermann et al, 1987), native LDL (however, no antioxidants were added to this preparation so it was may have been oxidized) (Alderson et al, 1986), minimally modified LDL (Berliner et al, 1990), oxidized LDL (Frostegard et al, 1990), cumene hydroperoxide (Molenaar et al, 1989), 14, 15epoxyeicosatrienoic acid, a metabolite of arachidonic acid (Pritchard et al, 1990), fibrinogen (Valente et al, 1984), phorbol 12-myristate 13-acetate, which is a protein kinase activator (DiCorleto and de la Motte, 1989), and thrombin (Saegusa et al, 1988; DiCorleto and de la Motte, 1989). IL-1, tumour necrosis factor and minimally modified LDL have been shown to induce expression of 
various monocyte chemotactic factors in endothelial cells (Strieter et al, 1989b). Interestingly, Wheeler et al (1988) found that endothelial cells generate a soluble leukocyte adhesion inhibitor (LAl) in response to stimulation by IL-1, tumour necrosis factor and lipopolysaccharide which significantly inhibited the adhesion of monocytes to activated, but not unactivated endothelial cells. Whisler et al (1989) found that when HUV endothelial cells were exposed to either lipopolysaccharide or IL-1 for 2-18 h, the stimulus acted directly on the HUV endothelial cells rather than on the monocyte to enhance monocyte adhesion to the endothelial cell. On the other hand, Doherty et al (1989) found that lipopolysaccharide stimulated human peripheral blood monocyte adherence to HUV endothelial cells and to serum-coated plastic surfaces suggesting an effect on the monocyte. This occurred within 25 min while stimulation of HUV endothelial cells to enhance monocyte adhesiveness required a minimum of $1.5-2 \mathrm{~h}$ exposure, as demonstrated by Whisler et al (1989). The differences between the studies of Doherty et al (1989) and Whisler et al (1989) can probably be attributed to the use of different adhesion assays, lipopolysaccharide concentrations and pretreatment protocols.

Another stimulus, formyl-methionyl-leucyl-phenylalanine (FMLP), a chemotactic peptide, acts on the monocyte but not the endothelial cell (Doherty et al, 1987; Whisler et al, 1989). IL-3 and GM-CSF also act on the monocyte to increase its adhesion to endothelial cells (Gamble et al, 1989; Elliott et al, 1990) as does zymosan-activated serum (Kamp et al, 1989) and hyperlipidemic serum (Foxall and Shwaery, 1990). Diabetic monocytes have been shown to have increased adhesiveness as a result of increased expression of fibronectin and Mo-1 receptors (Setiadi et al, 1987).

In summary, many factors can promote monocyte adhesion to endothelial cells. There are also factors which inhibit monocyte binding to endothelial 
cells, including phorbol esters such as phorbol 12-myristate 13-acetate (Kamp et al, 1989), $\mathrm{PGI}_{2}$ (Hassall et al, 1988), the presence of wheat germ agglutinin in the binding assay (DiCorleto and Chisolm, 1986), and preincubation of monocytes with fibrinogen or glycosylated fibrinogen (Valente et al, 1984). Phorbol 12-myristate 13-acetate has therefore been shown to act on the endothelial cell (DiCorleto and de la Motte, 1989) to increase monocyte binding but also to act on the monocyte to inhibit monocyte binding to the endothelial cell (Kamp et al, 1989). The different findings probably reflect the different techniques used to isolate the monocytes, the different numbers of monocytes added, and the different duration and temperature used in the adhesion assays.

\subsubsection{Effect of estrogens and progestins on monocyte binding to endothelial cells}

As discussed in Chapter 1 (refer to Section 1.5.3.5), sex steroids have been shown to modify a number of aspects of monocyte and macrophage function, but the effect of sex steroids on monocyte binding to endothelial cells does not appear to have been studied.

The aim of the experiments in this Chapter was to investigate whether sex steroids alter monocyte adhesion to endothelial cells through an effect on HUV endothelial cells, monocytes or both. Changes in monocyte adhesion to endothelial cells could account for different rates of progress of atherosclerosis, and could provide a link between gender, sex steroids and atherogenesis. 


\subsection{MATERIALS}

The composition and sources of media, solutions and chemicals used in this Chapter have been presented in Sections 2.2, 3.2 and 4.2. Additional materials are listed below.

Fibronectin was prepared by taking $50 \mathrm{ml}$ of $\mathrm{AB}$ serum (from a blood donor) and allowing it to clot at room temperature before being centrifuged ( $1000 \mathrm{x}$ $\mathrm{g}$ for $10 \mathrm{~min}$ at room temperature). The supernatant was collected and the sample heated to $56^{\circ} \mathrm{C}$ for $30 \mathrm{~min}$ before being stored in aliquots at $-20^{\circ} \mathrm{C}$.

Ficoll-hypaque (Pharmacia Fine Chemical, Sweden). This was stored at room temperature in the dark.

$\alpha$-Naphthyl acetate (Sigma Chemical Company, St Louis, USA).

Non-specific esterase-fixative comprised $\mathrm{Na}_{2} \mathrm{HPO}_{4},(20 \mathrm{mg}), \mathrm{KH}_{2} \mathrm{PO}_{4}(100$ $\mathrm{mg})$, distilled water $(30 \mathrm{ml})$, acetone $(45 \mathrm{ml})$ and formol $(25 \mathrm{ml}), \mathrm{pH} 6.6$.

Non-specific esterase incubation buffer comprised $0.067 \mathrm{M}$ phosphate buffer $(44.5 \mathrm{ml})$, hexatozotized pararosanilin $(3.0 \mathrm{ml})$ and $\alpha$-napthyl acetate $(50.0$ $\mathrm{mg}$ ) in $2.5 \mathrm{ml}$ 2-methoxy ethanol (Fluka AG, Buchs SG), pH 6.1.

Methyl Green (Gurr Ltd, England). A $1 \%$ solution was made up with methyl green $(1 \mathrm{~g})$, sodium acetate $(0.5 \mathrm{~g})$ and $100 \mathrm{ml}$ distilled water, $\mathrm{pH} 4.0$.

Pararosanilin (Sigma Chemical Company, St Louis, USA). Hexatozotized pararosanilin was prepared by adding $1.5 \mathrm{ml}$ of fresh $4 \% \mathrm{NaNO}_{2}$ to $75 \mathrm{mg}$ of pararosanilin in $1.2 \mathrm{ml}$ of distilled water and $0.3 \mathrm{ml}$ of concentrated $\mathrm{HCl}$. 
Phosphate buffer. This comprised $0.067 \mathrm{M} \mathrm{Na}_{2} \mathrm{HPO}_{4}(250 \mathrm{ml})$ and $0.067 \mathrm{M}$ $\mathrm{KH}_{2} \mathrm{PO}_{4}(1.0 \mathrm{~L}), \mathrm{pH} 7.4$.

RPMI 1640 medium without sodium bicarbonate but with glutamine (Flow Laboratories, Herts, UK) was prepared in $20 \mathrm{~L}$ batches to which sodium bicarbonate and $\mathrm{HCl}$ were added to achieve a $\mathrm{pH}$ of 7.2 and osmolarity of 293-295 mOsm / L. It was then filtered and stored at $4^{\circ} \mathrm{C}$.

\subsection{METHODS}

\subsubsection{Treatment of HUV endothelial cells}

Experiments were performed using either confluent or sub-confluent HUV endothelial cells, since other investigators have found differences in monocyte adhesion depending on whether the endothelial cells were confluent or not. HUV endothelial cells were cultured as outlined in Section 2.3.1. In the first series of experiments HUV endothelial cells were plated at an initial density of $10^{5}$ cells /well in gelatinized 24 well plates and grown to 1 day post confluency. In the second series of experiments the medium was removed $5 \mathrm{~h}$ after plating at the same density. Sex steroids, rlL-1 or alcohol were diluted in Medium 199 (with $20 \%$ FCS, gentamicin $60 \mathrm{U} / \mathrm{ml}$ and the standard additives). The control was Medium 199 with these additions. Following incubation for $6 \mathrm{~h}$ or $18 \mathrm{~h}$ the supernatant was removed, and the monolayer was then washed three times with HBSS at $37^{\circ} \mathrm{C}$.

The sex steroids which were added to HUV endothelial cell cultures were as described in Chapter 3 (refer to Section 2.3.12). Alcohol was used as one control because the sex steroids were dissolved in alcohol. rlL-1 was used as a positive control (refer to Section 5.1.2). 


\subsubsection{Monocyte isolation and labelling}

Monocytes were isolated from the peripheral blood of healthy donors according to the method of Fayle et al (1985). $70 \mathrm{ml}$ of whole blood was collected into 8 sterile tubes each containing $1.6 \mathrm{ml}$ of acid citrate dextrose. PRP was removed by centrifugation ( $100 \mathrm{xg}$ for $15 \mathrm{~min}$ at room temperature). The cells were then recentrifuged ( $200 \times \mathrm{g}$ for $10 \mathrm{~min}$ at room temperature), the buffy coat removed and diluted ( 1 in 2 ) with CMF HBSS. This was layered over Ficoll-hypaque $C(2: 3)$ in new $50 \mathrm{ml}$ centrifuge tubes and centrifuged ( $500 \times \mathrm{g}$ for $30 \mathrm{~min}$ at room temperature). The interface cells were collected, diluted in CMF HBSS $(4: 1)$ and washed twice by centrifugation $(225 \times \mathrm{g}$ for $10 \mathrm{~min}$ at $\left.4^{\circ} \mathrm{C}\right)$.

In experiments in which monocytes were not exposed to sex steroids, the cells were resuspended in $20 \mathrm{ml}$ of RPMI (with $10 \%$ FCS). The cells were then incubated in two $250 \mathrm{ml}$ gelatin-fibronectin coated flasks for $2 \mathrm{~h}$ in $5 \%$ $\mathrm{CO}_{2}-95 \%$ air atmosphere at $37^{\circ} \mathrm{C}$. When the monocytes were exposed to sex steroids they were resuspended in $22 \mathrm{ml}$ of RPMI (with $10 \%$ FCS). To each $125 \mathrm{ml}$ flask $2.5 \mathrm{ml}$ of cell suspension was added to $2.5 \mathrm{ml}$ of RPMI (with $10 \%$ FCS) containing the appropriate test substance and then incubated in 5 $\% \mathrm{CO}_{2}-95 \%$ air atmosphere at $37^{\circ} \mathrm{C}$ for $6 \mathrm{~h}$. The non adherent cells were removed by washing with CMF HBSS. The adherent cells were harvested by incubation for 10 min with $5 \mathrm{mM}$ EDTA in CMF HBSS and RPMI (1:1) containing $5 \%$ FCS. The cell suspension was centrifuged ( $145 \times \mathrm{g}$ for $10 \mathrm{~min}$ at $4^{\circ} \mathrm{C}$ ) and the cells were resuspended in $5 \mathrm{ml}$ of $\left[2-{ }^{-3} \mathrm{H}\right]$-adenine in CMF HBSS (final concentration $15 \mu \mathrm{Ci} / \mathrm{ml}$ ) (Curwen et al, 1982; Buchanan et al, 1983). The monocytes were incubated for $30 \mathrm{~min}$ at room temperature. They were then centrifuged $\left(145 \times \mathrm{g}\right.$ for $10 \mathrm{~min}$ at $4^{\circ} \mathrm{C}$ ) and resuspended in warmed HBSS. The monocyte poor supernatant $\left(<10^{4}\right.$ cells $\left./ \mathrm{ml}\right)$ was 
prepared to the same volume with warmed HBSS, this being used to correct for uptake of $[2-3 \mathrm{H}]$-adenine by HUV endothelial cells. A $10 \mu \mathrm{l}$ aliquot of each monocyte suspension and supernatant was added to $10 \mathrm{ml}$ of aqueous scintillant to allow a comparison of $\left[2-{ }^{3} \mathrm{H}\right]$-adenine uptake by monocytes between experiments.

A separate experiment was undertaken to assess the uptake of $[2-3 \mathrm{H}]$ adenine by monocytes. Aliquots of monocytes incubated with $[2-3 \mathrm{H}]$-adenine were removed at various time intervals and centrifuged $(8000 \times \mathrm{g}$ for $2 \mathrm{~min}$ at room temperature). The supernatant was removed and the cell pellet resuspended in HBSS. This was then added to aqueous scintillant and counted twice in a $\beta$-scintillation counter.

The monocyte cell count was determined using a haemocytometer (Reichert, USA). The viability of monocytes was assessed by trypan blue dye exclusion and purity was assessed by non specific esterase staining (Yam et al, 1971).

\subsubsection{Monocyte adhesion assay}

HUV endothelial cells and $0.75 \mathrm{ml}$ of [2-3H]-adenine labelled monocytes (to which $0.1 \mathrm{mM}$ of cold adenine had been added) were gently rocked at $37^{\circ} \mathrm{C}$ for 30 min (adapted from the methods of Valente et al, 1984; Alderson et al, 1986; Kamp et al, 1989). The endothelial cells were washed with warmed HBSS three times to remove non adherent cells, examined microscopically, and then $1.0 \mathrm{ml}$ of $0.1 \%$ trypsin-versene was added to each well. Following a further 30 min incubation in $5 \% \mathrm{CO}_{2}-95 \%$ air atmosphere at $37{ }^{\circ} \mathrm{C}$ the suspension was added to $10 \mathrm{ml}$ of aqueous scintillant and counted twice in a $\beta$-scintillation counter. The binding of monocytes to $0.1 \%$ gelatin was assessed by adding $0.75 \mathrm{ml}$ of $[2-3 \mathrm{H}]$-adenine labelled monocytes to gelatin- 
coated plates and then by following the protocol described above for the adhesion assay.

\subsubsection{Non-specific esterase stain for monocyte identification}

This stain was used to differentiate between monocytes and lymphocytes. Monocytes stain positively for esterase while lymphocytes do not. Cytocentrifuge slides were made using $2 \times 10^{4}$ cells in RPMI and $10 \%$ FCS on poly-L-lysine coated glass slides and stained according to the method of Yam et al (1971). Slides were air dried, fixed in fresh fixative for $45 \mathrm{sec}$ at 4 ${ }^{\circ} \mathrm{C}$, and then washed with water. Slides were then incubated for $60 \mathrm{~min}$ at room temperature in fresh incubation buffer (refer to Section 5.2). The slides were subsequently washed with running water and counter-stained with $1 \%$ methyl green for $5 \mathrm{~min}$, and finally washed with running water, air dried and mounted. The slides were photographed through a Zeiss Axioskop D-7082 microscope.

\subsubsection{Detection of endotoxin in solutions used in monocyte studies}

The E-toxate kit was used to detect and semiquantitate endotoxin in materials used. This test was conducted according to the manufacturer's instructions.

\subsubsection{Statistical analysis}

The experimental design for the monocyte binding studies involved 6-8 separate plates with each plate containing a control and certain treatments. The uptake of $[2-3 \mathrm{H}]$-adenine by HUV endothelial cells was also assessed for each treatment on each plate. Each experiment was initially analysed separately. The uptake of $[2-3 \mathrm{H}]$-adenine by HUV endothelial cells was found 
to influence the monocyte binding results when subconfluent monolayers were used but not confluent monolayers as found in Chapter 4 . The monocyte rich and monocyte poor preparations were analysed separately. The experiments were then pooled and analysis of variance with regression was used for analysis where the experiment replicates were considered as a block effect and the treatments as fixed effects. The variance ratio ( $F$ test) was used to test for overall significance. The multiple $t$ comparison (Bonferroni method) was then used to test for a significant difference between treatments. The Minitab programme (data analysis software, 1987) was used for the above analyses. A p value of 0.05 or less was taken to represent a statistically significant difference.

\subsection{RESULTS}

\subsubsection{Monocyte adhesion to gelatin-coated wells}

In preliminary experiments, ${ }^{51} \mathrm{Cr}$-labelled monocytes were used but this method was found to be relatively insensitive. Subsequently it was established that $[2-3 \mathrm{H}]$-adenine labelling of monocytes was suitable for adhesion assays. Initial studies showed that the maximum uptake of $[2-3 \mathrm{H}]-$ adenine by monocytes occurred after 30-45 min (Figure 5.1). Monocyte purity was assessed by morphology and non-specific esterase staining and was found to be $80 \%$ or better. Enzymic activity was seen as dark red granules, mainly in the cytoplasm of the monocyte (Figure 5.2 upper photo). The contaminating cells were mainly lymphocytes which did not stain for esterase activity (Figure 5.2 lower photo).

In order to quantitate monocyte adhesion to HUV endothelial cells it was necessary to determine monocyte adhesion to $0.1 \%$ gelatin-coated wells, upon which endothelial cells were grown. Monocytes bound to $0.1 \%$ gelatin 
within $10 \mathrm{~min}$ (as previously described by Pawlowski et al, 1985). The effect of preincubation of monocytes with sex steroids on monocyte adhesion to gelatin-coated wells was also examined. Binding of monocytes to gelatincoated wells was not increased significantly by sex steroids relative to Medium 199 alone (control) (Figure 5.3). Alcohol and rlL-1 had no effect on monocyte binding to $0.1 \%$ gelatin-coated wells relative to control (Figure 5.3). Fewer monocytes ( $16 \pm 9 \%$ of added cells) $(n=3)$ bound to $0.1 \%$ gelatin-coated wells than to confluent HUV endothelial monolayers.

Incubation of monocytes with sex steroids, rIL-1 or alcohol did not alter their ability to bind to fibronectin and did not affect their viability relative to the control cells. Monocyte viability (assessed with trypan blue) following exposure to the $[2-3 \mathrm{H}]$-adenine was greater than $95 \%$. The presence of sex steroids, rlL-1 or alcohol did not alter the monocyte cell count relative to control cells in Medium 199 (Figure 5.4). The average monocyte count in each well was $2.3 \pm 0.9 \times 10^{5}(n=9)$. The monocyte count was determined immediately prior to the addition of monocyte suspensions to the wells.

\subsubsection{Incubation of HUV endothelial cells with sex steroids.}

\section{Effect on monocyte adhesion to HUV endothelial cells}

These experiments were designed to determine whether monocyte adhesion to HUV endothelial cells was altered when HUV endothelial cells were preincubated with sex steroids. Monocytes bound avidly to HUV endothelial cells in the presence of HBSS at $37^{\circ} \mathrm{C}$. Within $10-20$ min of initial contact, the monocytes began to spread on the surface of HUV endothelial cells. No significant alteration in monocyte adhesion was found after preincubation of a subconfluent or confluent HUV endothelial cell monolayer with sex steroids compared to alcohol or Medium 199 alone (control) at either $6 \mathrm{~h}$ or $18 \mathrm{~h}$ (Figures 5.5, 5.6, 5.7 and 5.8). However, more monocytes (about 
3 times as many) bound to HUV endothelial cells and exposed gelatin in subconfluent monolayers than to the confluent monolayers, as described by DiCorleto and de la Motte (1984), DiCorleto et al (1985) and Alderson et al (1986). Microscopy confirmed that more monocytes bound to HUV endothelial cells in the subconfluent monolayers than confluent monolayers.

The average monocyte count in each well was $2.3 \times 10^{5}$ cells $\left(3.07 \times 10^{5}\right.$ monocytes $/ \mathrm{ml}$ ). At this concentration, phase-contrast microscopy revealed that, following three washes, 1-2 monocytes bound to each HUV endothelial cell after $\mathbf{3 0}$ min incubation in the control wells of the confluent monolayers. Preliminary experiments had found that $1.0 \times 10^{6}$ monocytes /well were needed for maximal monocyte binding (8-10 monocytes /endothelial cell as previously described by Pawlowski et al, 1985; 1988 and Dicorleto et al, 1985. Whole blood contains 2-8 $\times 10^{5}$ monocytes / $\mathrm{ml}$ (Dacie and Lewis, 1975). The amount of blood that would have been needed to obtain $1.0 \times 10^{6}$ monocytes /well would have exceeded $100 \mathrm{ml}$ since up to 48 wells were required for each experiment; this amount of blood would have been difficult to obtain from donors. Therefore a compromise was made, with the average count per well being $2 \times 10^{5}$ monocytes, which results in the binding of 1-2 monocytes/ endothelial cell. Such monocyte counts have also been used by others (Kamp et al, 1989; Berliner et al, 1990) to assess monocyte binding to endothelial cells. When HUV endothelial cells were incubated with rIL-1 (5 U $/ \mathrm{ml}$ ) there was, as previously shown by Bevilacqua et al (1985), a significant increase in monocyte binding to a confluent monolayer of HUV endothelial cells (Figures 5.5 and 5.6) but not to a subconfluent monolayer of cells (Figures 5.7 and 5.8).

Because HUV endothelial cells are known to take up [2-3 $\mathrm{H}]$-adenine despite the addition of cold adenine (Curwen et al, 1982), it was necessary to measure this uptake in each of the monocyte adhesion experiments. It was 
also necessary to determine whether steroids affected the uptake of unincorporated $[2-3 \mathrm{H}]$-adenine by HUV endothelial cells as described in Chapter 4 (refer to Section 4.4.1). The uptake of unincorporated [2-3H]adenine by confluent HUV endothelial cells was not altered by pre-exposure of these cells to sex steroids, rlL-1 or alcohol relative to Medium 199 (control cells) (Figures 5.9 and 5.10). This was assessed by incubating HUV endothelial cells with a monocyte poor suspension for $30 \mathrm{~min}$ at $37^{\circ} \mathrm{C}$ and then washing the HUV endothelial cell monolayer to remove unincorporated label. The average radioactive cpm between control wells for monocyte-rich preparations did not differ following $6 \mathrm{~h}$ or $18 \mathrm{~h}$ incubation. Similarly the average radioactive $\mathrm{cpm}$ between control wells for monocyte-poor preparations was not different at the two time points. The uptake of unincorporated $[2-3 \mathrm{H}]$-adenine by a subconfluent monolayer of HUV endothelial cells varied in relation to endothelial cell number. At $6 \mathrm{~h}$ (Figure 5.11) the uptake of unincorporated $[2-3 \mathrm{H}]$-adenine by HUV endothelial cells incubated with progesterone $\left(10^{-5} \mathrm{M}\right)$ or $17 \beta$-estradiol $\left(10^{-5} \mathrm{M}\right)$ combined with progesterone $\left(10^{-5} \mathrm{M}\right)$ was less than the control. By $18 \mathrm{~h}$ the cell count was altered as described in Chapter 3 with a reduction in cell count following exposure to $17 \beta$-estradiol $\left(10^{-5} \mathrm{M}\right)$, progesterone $\left(10^{-5} \mathrm{M}\right)$ and the combination of $17 \beta$-estradiol $\left(10^{-5} \mathrm{M}\right)$ with progesterone $\left(10^{-5} \mathrm{M}\right)$ (Figure 5.12). No change was observed with progesterone $\left(10^{-9} \mathrm{M}\right), 17 \beta$-estradiol $\left(10^{-10} \mathrm{M}\right)$ and the combination of $17 \beta$-estradiol $\left(10^{-10} \mathrm{M}\right)$ and progesterone (10-9 M). rIL-1 also appeared to inhibit HUV endothelial cell proliferation relative to control cells as previously demonstrated by Norioka et al (1987) and Raines et al (1989). However, these differences were not statistically significant (Figures 5.11 and 5.12) probably due to the cold adenine blocking the uptake of $[2-3 \mathrm{H}]$-adenine since the average radioactive counts per minute for control cells was only 1489 at $6 \mathrm{~h}$ and 1619 at $18 \mathrm{~h}$, while the counts following exposure to $17 \beta$-estradiol $\left(10^{-5} \mathrm{M}\right)$, progesterone $\left(10^{-5} \mathrm{M}\right)$ and the combination of $17 \beta$-estradiol $\left(10^{-5} \mathrm{M}\right)$ with progesterone $\left(10^{-5} \mathrm{M}\right)$ were 
substantially less with a percentage variation due to radioactive counting of \pm $2 \%$ when the sample was counted for $5 \mathrm{~min}$. These factors would have masked the proliferative effects of the sex steroids previously demonstrated in experiments reported in Chapter 3 (refer to Section 3.4.2).

\subsubsection{Incubation of monocytes with sex steroids. Effect on monocytes and monocyte adhesion to HUV endothelial cells}

These experiments were designed to assess whether the sex steroids altered the ability of monocytes to adhere to HUV endothelial cells when monocytes, rather than HUV endothelial cells were exposed to sex steroids.

Pre-incubation of monocytes with $17 \beta$-estradiol $\left(10^{-5} \mathrm{M}\right.$ or $10^{-10} \mathrm{M}$ ), progesterone $\left(10^{-5} \mathrm{M}\right)$ or the combination of $17 \beta$-estradiol $\left(10^{-10} \mathrm{M}\right)$ with progesterone (10-9 M) (Figures 5.13 and 5.14) regardless of whether or not HUV endothelial cells had previously been exposed to sex steroids resulted in an increase in monocyte binding to HUV endothelial cells. rIL-1 increased monocyte binding only when the endothelial cells had been exposed to rIL-1. Pre-treatment of monocytes with progesterone $\left(10^{-9} \mathrm{M}\right)$ did not significantly alter monocyte binding to HUV endothelial cells (not pre-exposed to progesterone) but incubation of both HUV endothelial cells and monocytes with progesterone $\left(10^{-9} \mathrm{M}\right)$ resulted in a significant increase in binding of monocytes relative to control. When monocytes, or the combination of monocytes and HUV endothelial cells, where exposed to the combination of $17 \beta$-estradiol $\left(10^{-5} \mathrm{M}\right)$ and progesterone $\left(10^{-5} \mathrm{M}\right)$ no significant change in monocyte binding was observed (Figures 5.13 and 5.14). Alcohol did not influence monocyte binding to HUV endothelial cells using either protocol. As expected, the average radioactive $\mathrm{cpm}$ in the control wells using either protocol was similar. 


\subsubsection{Detection of endotoxin in solutions used in monocyte studies}

The test was sensitive to $0.04 \rho \mathrm{g} / \mathrm{ml}$ of endotoxin. No endotoxin was detected in HBSS, CMF HBSS, distilled water, ficoll, FCS, acid citrate dextrose or AB serum. Endotoxin was detected in RPMI and Medium 199. However semiquantitation showed this to be very small $(0.4 \rho \mathrm{g} / \mathrm{ml})$. An inhibitor was present in Ficoll-hypaque as the addition of $4 \mu \mathrm{g} / \mathrm{ml}$ to Ficollhypaque failed to give a positive result.

\subsection{DISCUSSION}

The above studies have demonstrated that sex steroids increase monocyte binding to HUV endothelial cells when monocytes are preincubated with sex steroids or when both monocytes and HUV endothelial cells are each preincubated with sex steroids. Both low and high concentrations of $17 \beta-$ estradiol (10-5 $\mathrm{M}$ or $\left.10^{-10} \mathrm{M}\right)$, low and high concentrations of progesterone $\left(10^{-5}\right.$ or $\left.10^{-9} \mathrm{M}\right)$, and the combination of $17 \beta$-estradiol $\left(10^{-10} \mathrm{M}\right)$ and progesterone $\left(10^{-9} \mathrm{M}\right)$ increased monocyte binding to HUV endothelial cells. Preincubation of only HUV endothelial cells with sex steroids did not alter monocyte binding. Finally, sub-confluent monolayers of HUV endothelial cells bound more monocytes than confluent monolayers of HUV endothelial cells.

Monocyte binding to HUV endothelial cells is not influenced by the growth matrix (e.g. gelatin, fibronectin, plastic, collagen or amnion), added growth factors, or the suspending medium used in the binding assay (medium alone, serum, or plasma) (Pawlowski et al, 1988). Preincubation of HUV endothelial cells with sex steroids does not appear to alter the ability of confluent or subconfluent monolayers of HUV endothelial cells to bind monocytes. The addition of rIL-1 to the cultures increased monocyte adhesion when HUV 
endothelial cells were preincubated with rIL-1. This is in agreement with the observations of Bevilacqua et al (1987) who showed that the addition of IL-1 during the adhesion assay did not significantly alter adhesion, and that endothelial cells needed to be incubated with IL-1 for at least $4 \mathrm{~h}$ to observe an increase in monocyte adhesion. More monocytes bound to HUV endothelial cells in subconfluent monolayers than to confluent monolayers as described by DiCorleto and de la Motte (1984), DiCorleto et al (1985) and Alderson et al (1986).

Estrogen receptors have been identified on the surface of monocytes (Danel et al, 1983; Gulshan et al, 1990) and therefore it is likely that 17 $\beta$ estradiol, in the concentrations used in the present studies, bound to estrogen receptors and subsequently influenced cellular functions concerned with cell adhesion. There is little information available on whether monocytes have receptors for progesterone. A low concentration of progesterone (10-9 M) increased monocyte binding to HUV endothelial cells only when both the endothelial cell and the monocyte were exposed to progesterone, while a higher concentration of progesterone $\left(10^{-5} \mathrm{M}\right)$ increased monocyte binding to HUV endothelial cells when monocytes alone were exposed to the steroid. These observations may indicate a concentration-dependent effect of progesterone, and suggest that the effect of progesterone is dependent on an interaction between the HUV endothelial cell and the monocyte. If monocytes do not possess progesterone receptors, progesterone $\left(10^{-5} \mathrm{M}\right)$ may have exerted its effect through the estrogen receptor. Pharmacological concentrations of such hormones have been observed to interact with receptors for other steroid hormones (Zava and McGuire, 1978; Katzenellenbogen, 1980). Estrogen and progesterone have also been shown to regulate each other's receptors, particularly when pharmacological concentrations of sex steroids are used (refer to Section 3.1.3). The observation that higher concentrations of $17 \beta$-estradiol $\left(10^{-5} \mathrm{M}\right)$ and 
progesterone $\left(10^{-5} \mathrm{M}\right)$ in combination did not alter monocyte binding when either monocytes or HUV endothelial cells were exposed to these steroids suggests the possibility of down-regulation of the estrogen receptor.

The effects of sex steroids on monocyte adhesion may be due to cellular functions other than a specific alteration in monocyte adhesion molecules. The sex steroids may, for example, have caused the release from monocytes of IL-1 which is known to act on the endothelial cell to increase monocyte adhesion (Bevilacqua et al, 1985 a; Goerdt et al, 1987; Peri et al, 1990). Pharmacological concentrations of sex steroids have been shown to reduce IL-1 release (Flynn 1984) whereas physiological concentrations result in significant stimulation of IL-1 release (Polan et al 1988) from monocytes. IL-1 is known to alter endothelial cell adhesion molecules (but not monocyte adhesion molecules) to mediate its effect on monocyte binding to endothelial cells (Whisler et al, 1989) and it is known that at least $4 \mathrm{~h}$ is required for IL-1 to alter endothelial cell adhesion properties (Bevilacqua et al, 1987). The present experiments do not support a role for IL-1 in monocyte adhesion since the monocytes were not exposed to sex steroids during the adhesion assay and any IL-1 released during the $6 \mathrm{~h}$ incubation of sex steroids with monocytes would have been removed by washing prior to the adhesion assay. However, it is also possible that the sex steroids stimulated the release of some other cytokine (e.g. tumour necrosis factor) which continued to be released after washing of the cells and which could have rapidly altered monocyte binding to endothelial cells. A further possibility is that the enhanced binding of monocytes may be due to a contaminant in the monocyte preparation such as endotoxin which has been shown to increase monocyte binding to endothelial cells (Pohlman et al, 1985 a; b; Goerdt et al, 1987; Whisler et al, 1989; Territo et al, 1989; Berliner et al, 1990). However, endotoxin was only detected in the RPMI and Medium 199 used in these experiments, and this was much less than the amount of endotoxin (500 $\rho \mathrm{g}$ 
/ml) which has been shown to promote monocyte adhesion to endothelial cells (Berliner et al, 1990). Secondly, all monocytes were exposed to the same batch of RPMI and yet differences between steroid treatments were still detected. In summary, it seems unlikely, in the present studies, that the changes in monocyte adhesion to HUV endothelial cells were due to either IL1 release from monocytes or to a contaminant.

These studies have demonstrated that monocyte adhesion to endothelial cells is increased by certain sex steroids, suggesting the possibility of an adverse influence of sex steroids on atherogenesis. Monocyte adhesion is an important step in the development of atherosclerotic lesions, and sex steroids may promote atherosclerosis by increasing monocyte traffic into the vessel wall. These studies may also have important implications in relation to hormone replacement therapy and the oral contraceptive, because changes in monocyte adhesion were observed at both low and high concentrations of sex steroids. The net effect on monocyte adhesion is likely to be a complex summation of the variable effects of different sex steroids together with other factors which influence adhesion and migration, and, as demonstrated in these studies, probably depends on the concentration of the steroids. As discussed previously the actual concentration of sex steroids that monocytes and endothelial cells are exposed to in vivo in humans is uncertain. Furthermore, the net effect of sex steroids on atherosclerosis depends not only on the changes in monocyte adhesion, but also on other effects that sex steroids have on vascular cells and lipoproteins reported elsewhere in this thesis. 


\subsection{SUMMARY}

The present studies have demonstrated that, under the experimental conditions used:

1. The incubation of a confluent or subconfluent monolayer of endothelial cells with sex steroids does not alter monocyte binding to their surface;

2. The incubation of a confluent monolayer of endothelial cells with sex steroids does not alter the ability of HUV endothelial cells to accumulate unincorporated $[2-3 \mathrm{H}]$-adenine;

3. The incubation of monocytes with certain sex steroids causes an increase in monocyte binding to endothelial cells regardless of whether or not the endothelial cells have been exposed to sex steroids; and

4. Sub-confluent HUV endothelial cells bind more monocytes than confluent monolayers of HUV endothelial cells. 


\section{Figure 5.1.}

Uptake of $\left[2-{ }^{3} \mathrm{H}\right]$-adenine by monocytes. Monocytes were incubated with $\left[2-{ }^{3} \mathrm{H}\right]$-adenine for various time intervals and the uptake of $\left[2-{ }^{3} \mathrm{H}\right]$-adenine was assessed. Each point represents the mean of duplicates. The SD for all points was less than $4 \%$ for all but zero time. 
UPTAKE OF ${ }^{3}$ H-ADENINE BY MONOCYTES

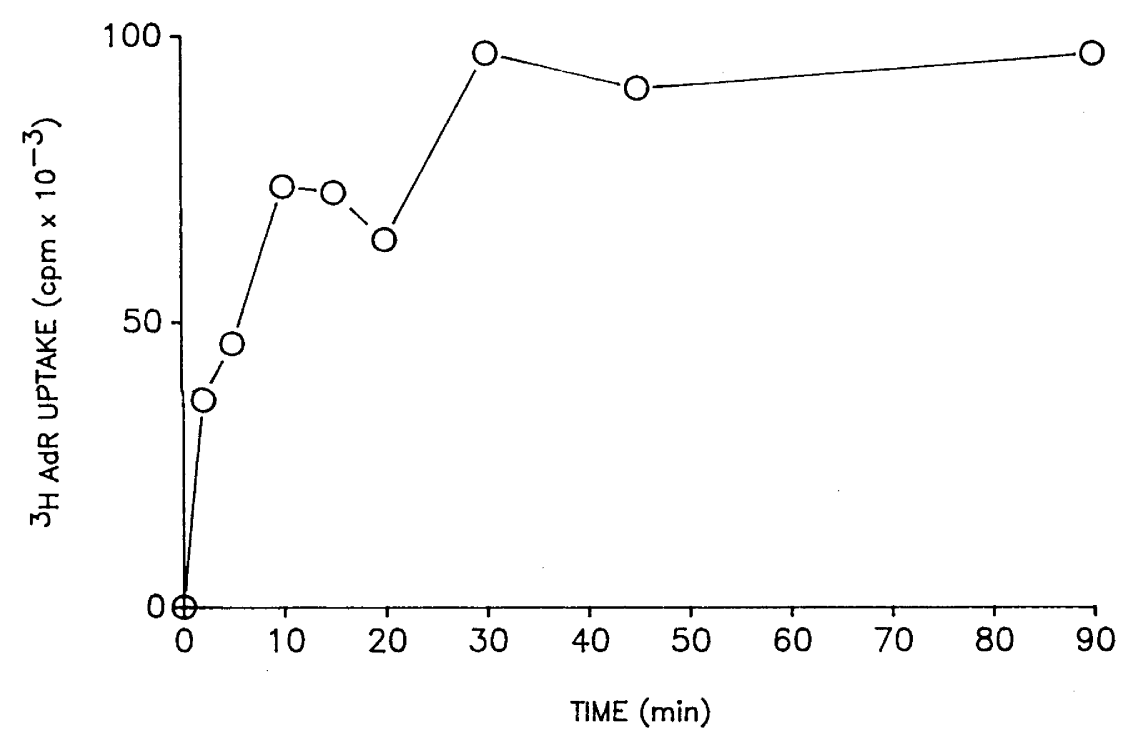


Figure 5.2.

Non-specific esterase staining of various cells.

Upper: Non-specific esterase staining of cells adherent to fibronectin. These are predominantly monocytes. The esterase activity can be seen as dark red granules. Magnification $672 \mathrm{x}$.

Lower: Non-specific esterase staining of cells non adherent to fibronectin. These are mainly lymphocytes which do not stain for esterase activity. Magnification 672x. 

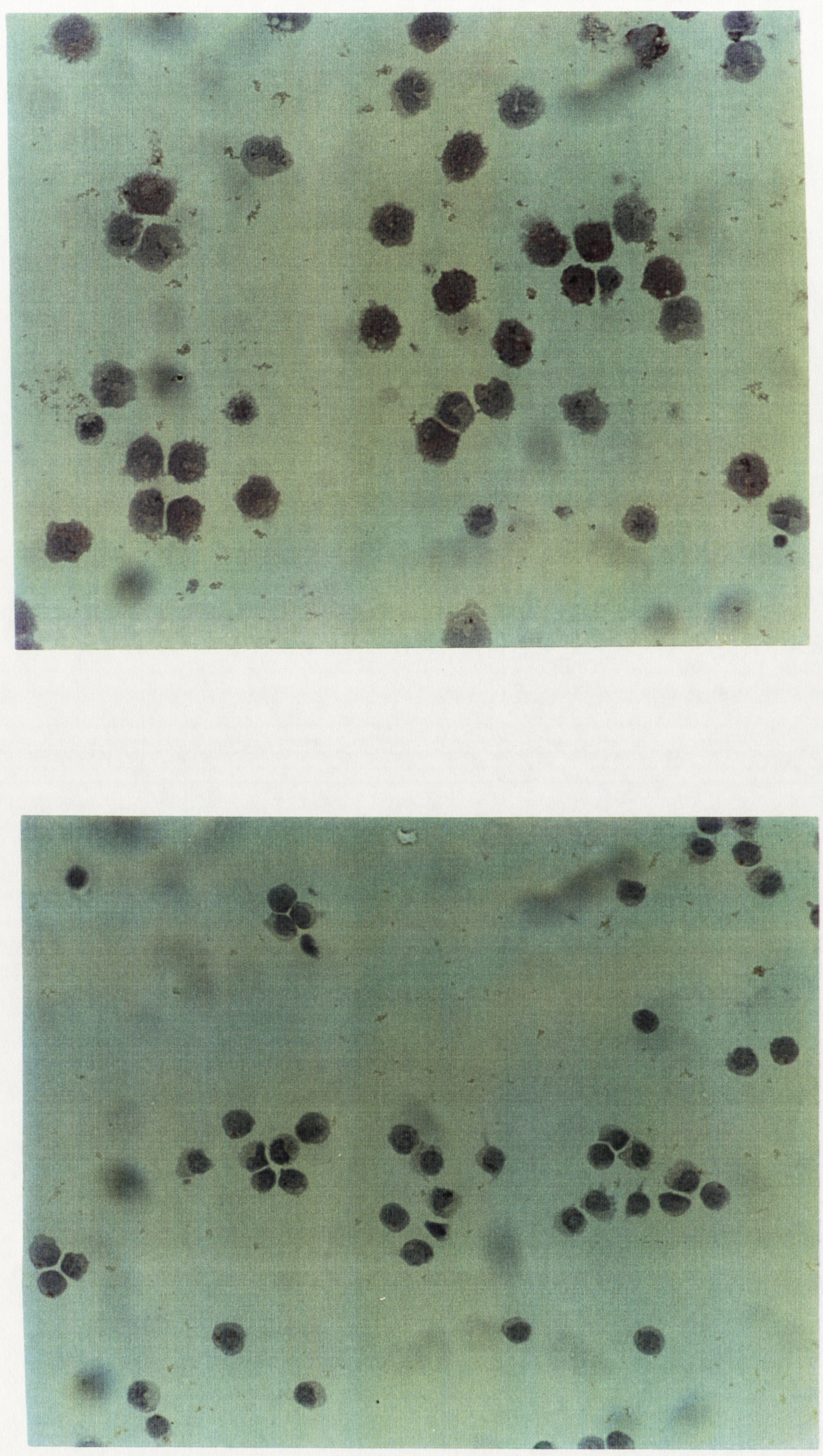


\section{Figure 5.3.}

Binding of monocytes exposed to various stimuli to $0.1 \%$ gelatin-coated wells. Monocytes were exposed to sex steroids, rlL-1, alcohol or Medium 199 (control) for $6 \mathrm{~h}$ and then labelled with $\left[2-{ }^{3} \mathrm{H}\right]$-adenine before being incubated on $0.1 \%$ gelatin-coated wells for $30 \mathrm{~min}$ at $37{ }^{\circ} \mathrm{C}$. The abbreviations used here are the same as in the legend to Figure 2.14. The average radioactive $\mathrm{cpm}$ in the control wells was 2073 . This graph represents the mean \pm SE of 3 experiments ( 2 in triplicate).

Figure 5.4.

Monocyte count, using the haemocytometer, following incubation with sex steroids, rIL-1 or alcohol relative to Medium 199 (control). The average monocyte count was $2.3 \pm 0.9 \times 10^{5}$ cells/ well. The graph represents the mean \pm SE of 6 experiments. 
BINDING OF MONOCYTES EXPOSED TO VARIOUS STIMULI TO $0.1 \%$ GELATIN - COATED WELLS

$30 \mathrm{~min}$ incubation

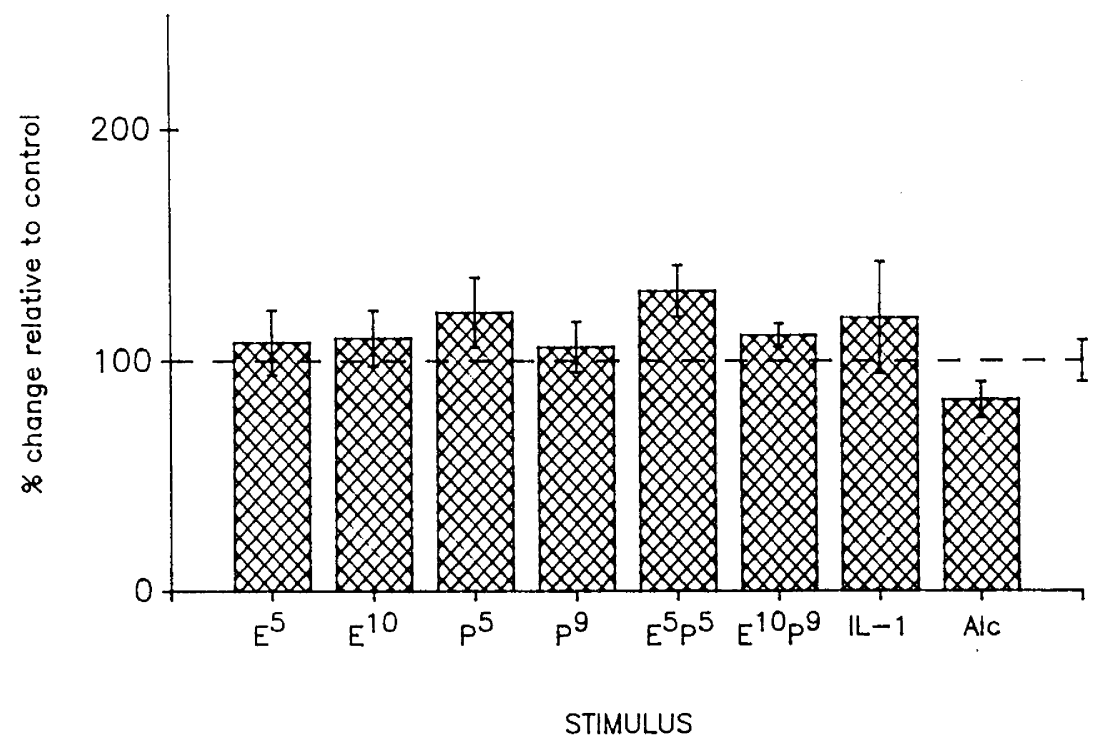

MONOCYTE COUNT FOLLOWING INCUBATION WITH VARIOUS STIMULI

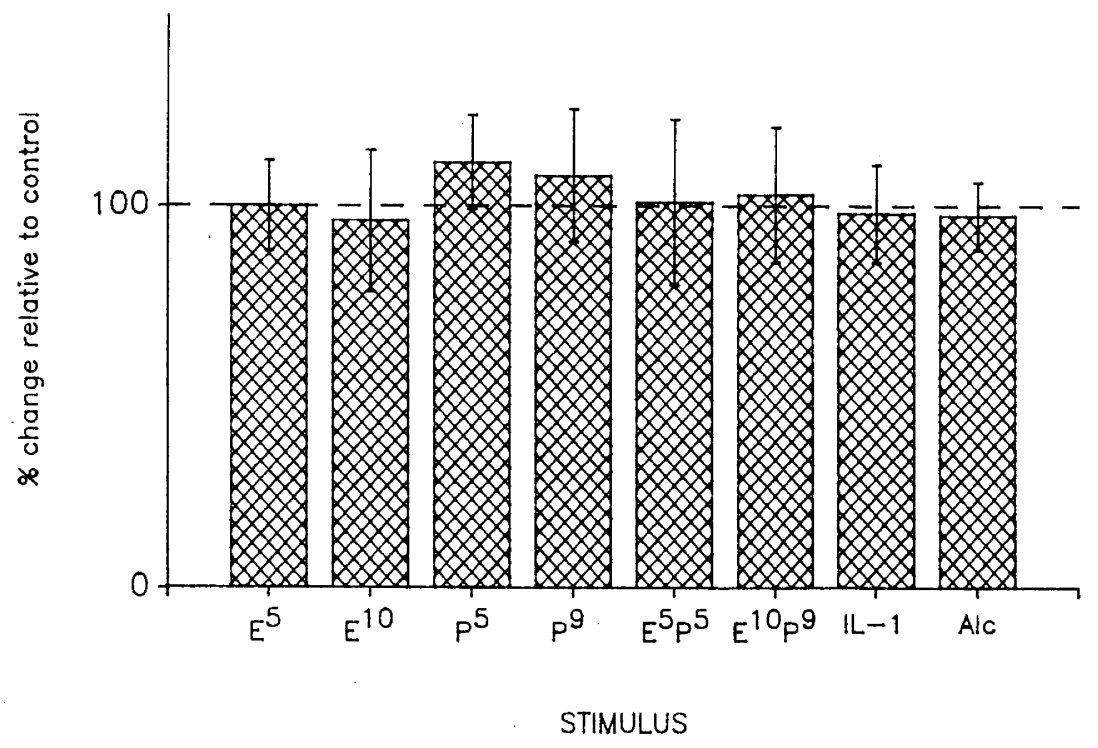


Figure 5.5.

Monocyte binding to a confluent monolayer of HUV endothelial cells exposed to various stimuli for $6 \mathrm{~h}$. A confluent monolayer was established and endothelial cells were exposed to sex steroids, rIL-1, alcohol or Medium 199 (control) for $6 \mathrm{~h}$. The endothelial cells were then washed with HBSS and labelled monocytes were added for $30 \mathrm{~min}$ at $37{ }^{\circ} \mathrm{C}$. The endothelial cells were washed to remove nonadherent monocytes and then trypsinized. The average radioactive cpm for control wells was 12879 . This graph represents the mean \pm SE of 3 experiments ( 2 in triplicate). $\star=p<0.05$.

\section{Figure 5.6.}

Monocyte binding to a confluent monolayer of HUV endothelial cells exposed to various stimuli for $18 \mathrm{~h}$. A confluent monolayer was established and endothelial cells were exposed to sex steroids, rIL-1, alcohol or Medium 199 (control) for $18 \mathrm{~h}$. The method described in the legend to Figure 5.5 was then followed. The average radioactive cpm for the control wells was 12828 . This graph represents the mean \pm SE of 3 experiments ( 2 in triplicate). ${ }^{*}=p<0.05$. 

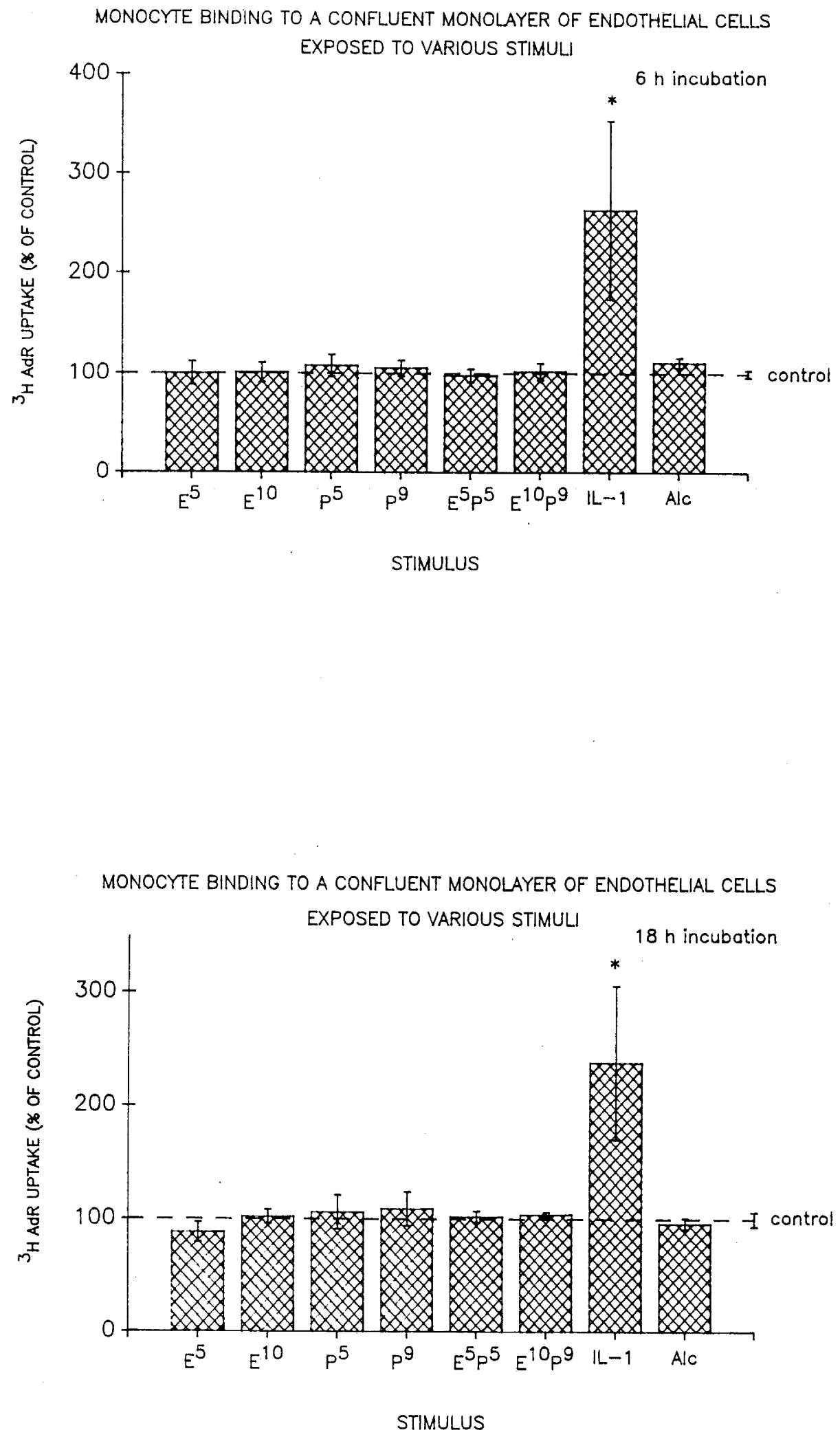


\section{Figure 5.7.}

Monocyte binding to a subconfluent monolayer of HUV endothelial cells exposed to various stimuli for $6 \mathrm{~h}$. Endothelial cells were plated at an initial density of $10^{5}$ cells /well and incubated at $37^{\circ} \mathrm{C}$ for $5 \mathrm{~h}$ before the addition of sex steroids, rlL-1, alcohol or Medium 199 (control) for $6 \mathrm{~h}$. The same protocol as described in the legend to Figure 5.5 was then followed. The average radioactive $\mathrm{cpm}$ for the control wells was 38362 . This graph represents the mean \pm SE of 2 experiments in triplicate.

\section{Figure 5.8.}

Monocyte binding to a subconfluent monolayer of HUV endothelial cells exposed to various stimuli for $18 \mathrm{~h}$. Endothelial cells were plated at an initial density of $10^{5}$ cells /well and incubated at $37^{\circ} \mathrm{C}$ for $5 \mathrm{~h}$ before the addition of sex steroids, rIL-1, alcohol or Medium 199 (control) for $18 \mathrm{~h}$. The same protocol as described in the legend to Figure 5.5 was then followed. The average radioactive $\mathrm{cpm}$ for the control wells was 37613 . This graph represents the mean \pm SE of 2 experiments in triplicate. 
MONOCYTE BINDING TO A SUBCONFLUENT MONOLAYER OF ENDOTHELIAL CELLS EXPOSED TO VARIOUS STIMULI

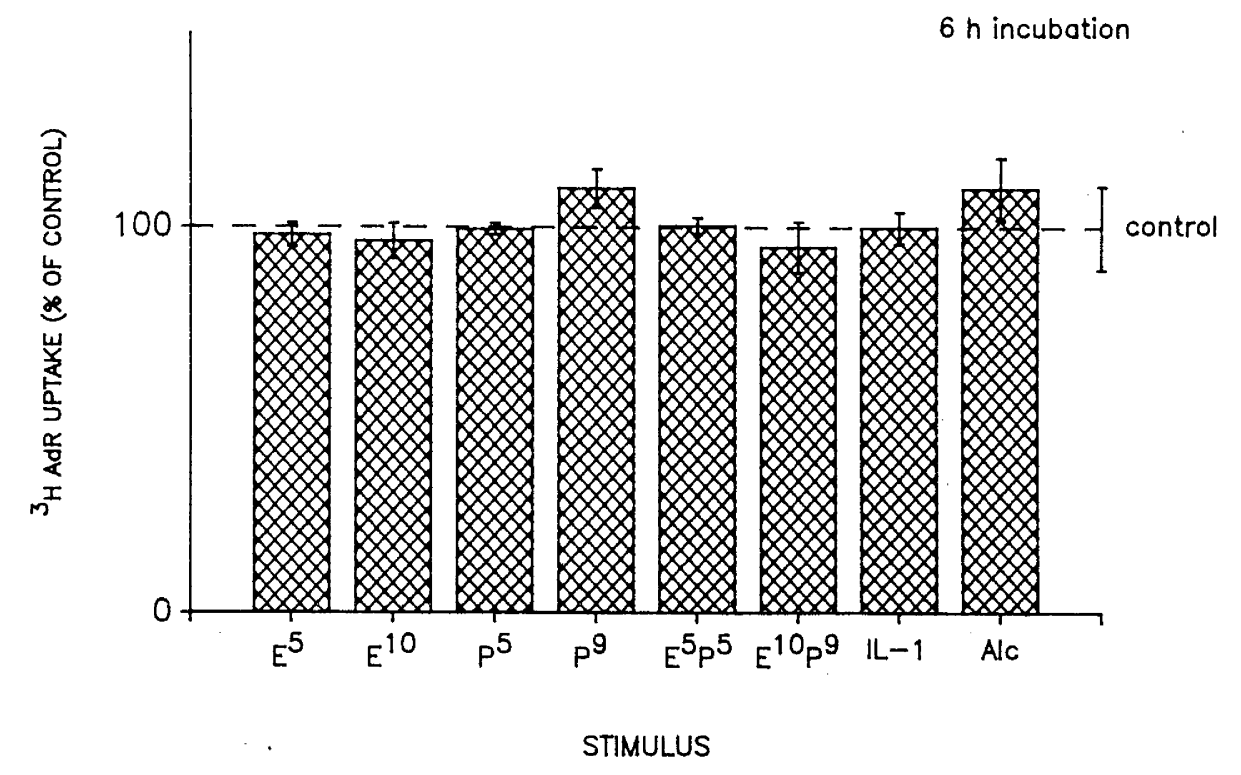

MONOCYTE BINDING TO A SUBCONFLUENT MONOLAYER OF ENDOTHELIAL CELLS EXPOSED TO VARIOUS STIMULI

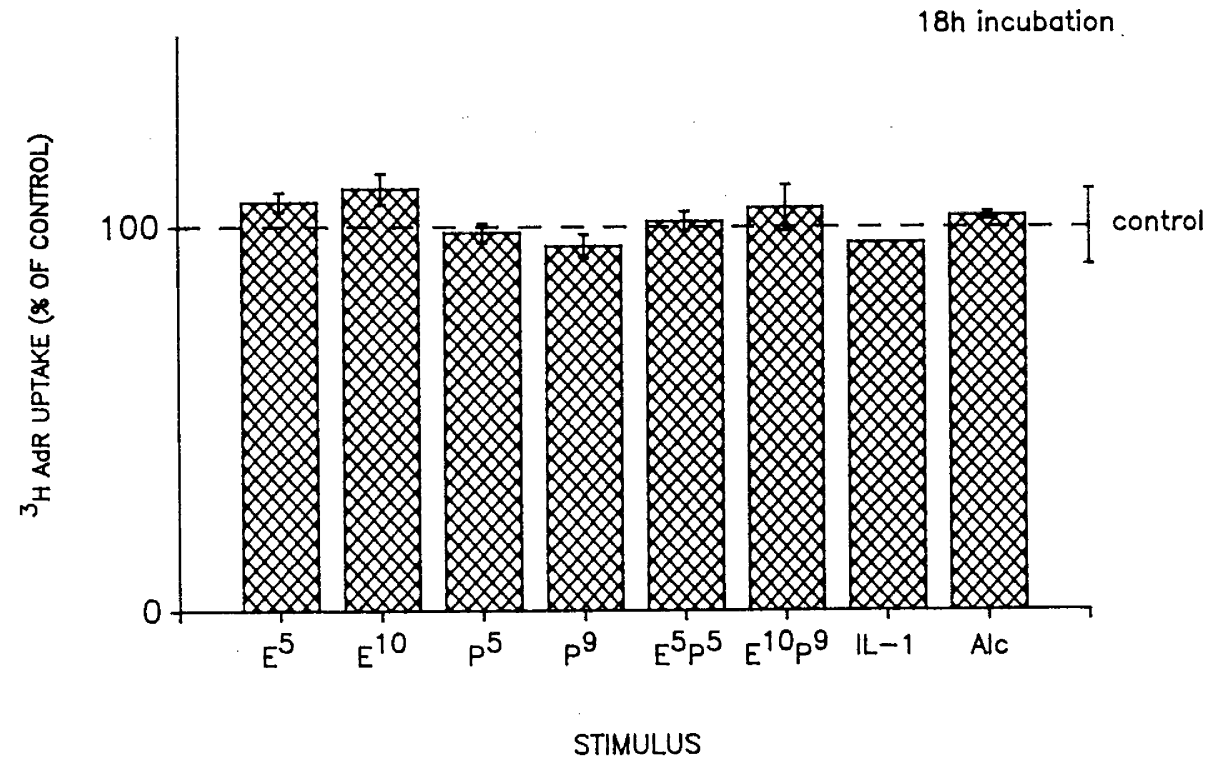


UPTAKE OF ${ }^{3}$ H-ADENINE BY A CONFLUENT MONOLAYER OF ENDOTHELIAL CELLS EXPOSED TO VARIOUS STIMULI

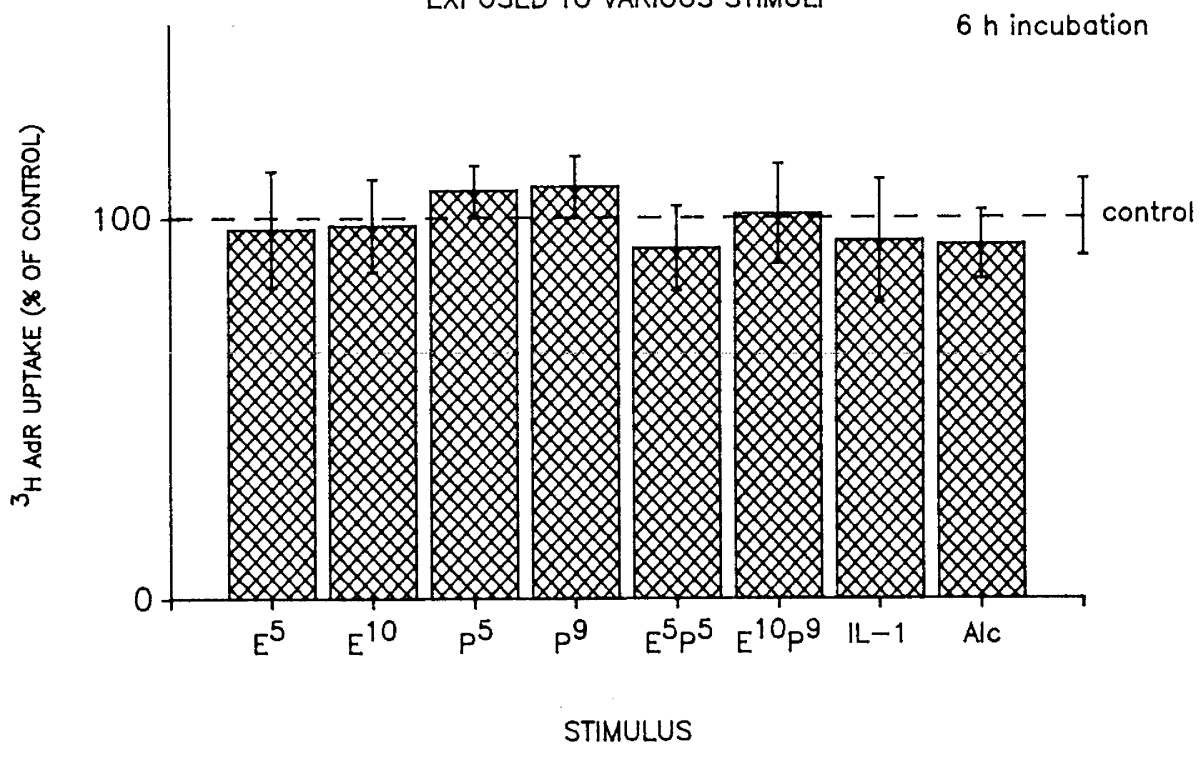

UPTAKE OF ${ }^{3}$ H-ADENINE BY A CONFLUENT MONOLAYER OF ENDOTHELIAL CELLS EXPOSED TO VARIOUS STIMULI

$18 \mathrm{~h}$ incubation

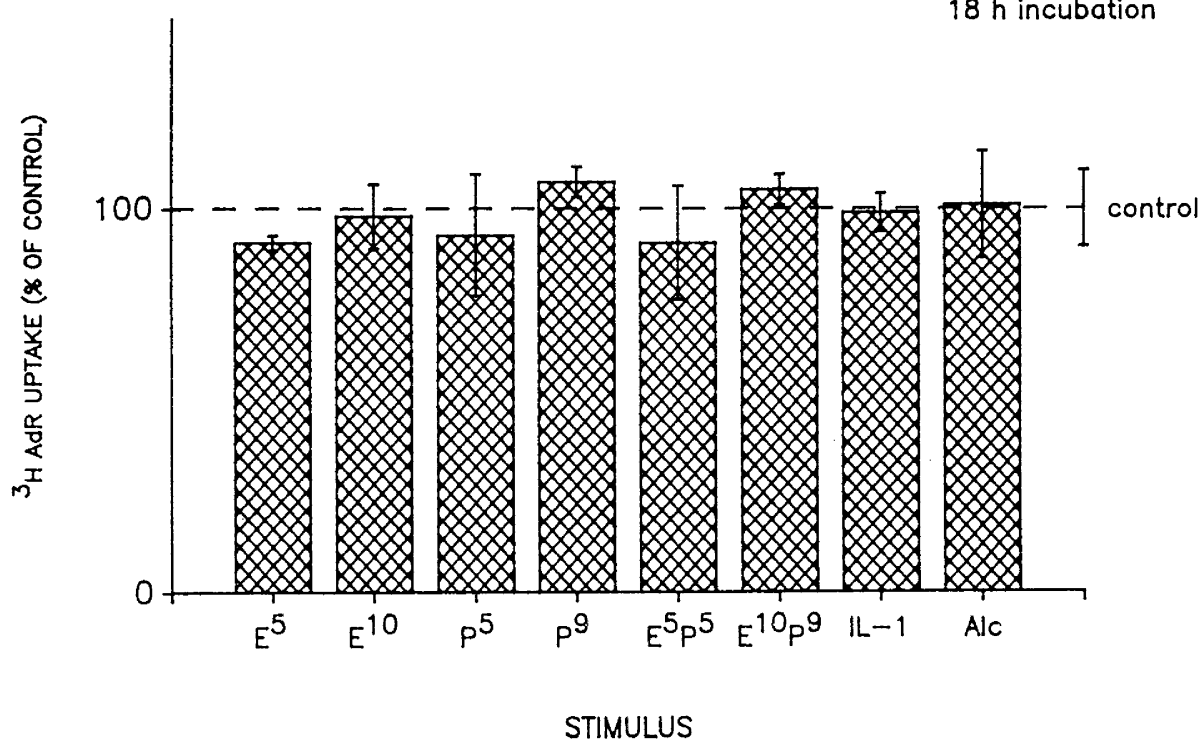




\section{Figure 5.9.}

Uptake of unincorporated $\left[2-{ }^{3} \mathrm{H}\right]$-adenine by a confluent monolayer of HUV endothelial cells. A confluent monolayer was established and the endothelial cells were exposed to sex steroids, rIL-1, alcohol or Medium 199 (control) for $6 \mathrm{~h}$. The endothelial cells were then washed with HBSS and the monocyte poor suspension was added for $30 \mathrm{~min}$ at $37^{\circ} \mathrm{C}$. The endothelial cells were washed again and then trypsinized. The average radioactive $\mathrm{cpm}$ for the control wells was 1489 . This graph represents the mean \pm SE of 3 experiments ( 2 in triplicate).

\section{Figure 5.10.}

Uptake of unincorporated $\left[2-{ }^{3} \mathrm{H}\right]$-adenine by a confluent monolayer of HUV endothelial cells. A confluent monolayer was established and the endothelial cells were exposed to sex steroids, rlL-1, alcohol or Medium 199 (control) for $18 \mathrm{~h}$. The same protocol as described in the legend to Figure 5.9 was then followed. The average radioactive $\mathrm{cpm}$ for the control wells was 1619 . This graph represents the mean \pm SE of 3 experiments (2 in triplicate). 


\section{Figure 5.11.}

Uptake of unincorporated $\left[2-{ }^{3} \mathrm{H}\right]$-adenine by a subconfluent monolayer of HUV endothelial cells exposed to various stimuli for $6 \mathrm{~h}$. Endothelial cells were prepared as described in the legend to Figure 5.6. The endothelial cells were then washed with HBSS and the monocyte poor suspension added for $30 \mathrm{~min}$ at $37^{\circ} \mathrm{C}$. The endothelial cells were washed again and then trypsinized. The average radioactive $\mathrm{cpm}$ for the control wells was 1344 . This graph represents the mean \pm SE of 3 experiments in triplicate. The changes did not reach statistical significance.

\section{Figure 5.12.}

Uptake of unincorporated $\left[2-{ }^{3} \mathrm{H}\right]$-adenine by a subconfluent monolayer of HUV endothelial cells exposed to various stimuli for $18 \mathrm{~h}$. Endothelial cells were prepared and treated as described in the legend to Figure 5.11. This graph represents the mean $\pm \mathrm{SE}$ of 2 experiments in triplicate. The average radioactive $\mathrm{cpm}$ in the control wells was 1577 . The changes did not reach statistical significance. The absolute counts were very low resulting in a wide SE in radioactive counts. 
UPTAKE OF ${ }^{3}$ H-ADENINE BY A SUBCONFLUENT MONOLAYER OF ENDOTHELIAL CELLS EXPOSED TO VARIOUS STIMULI

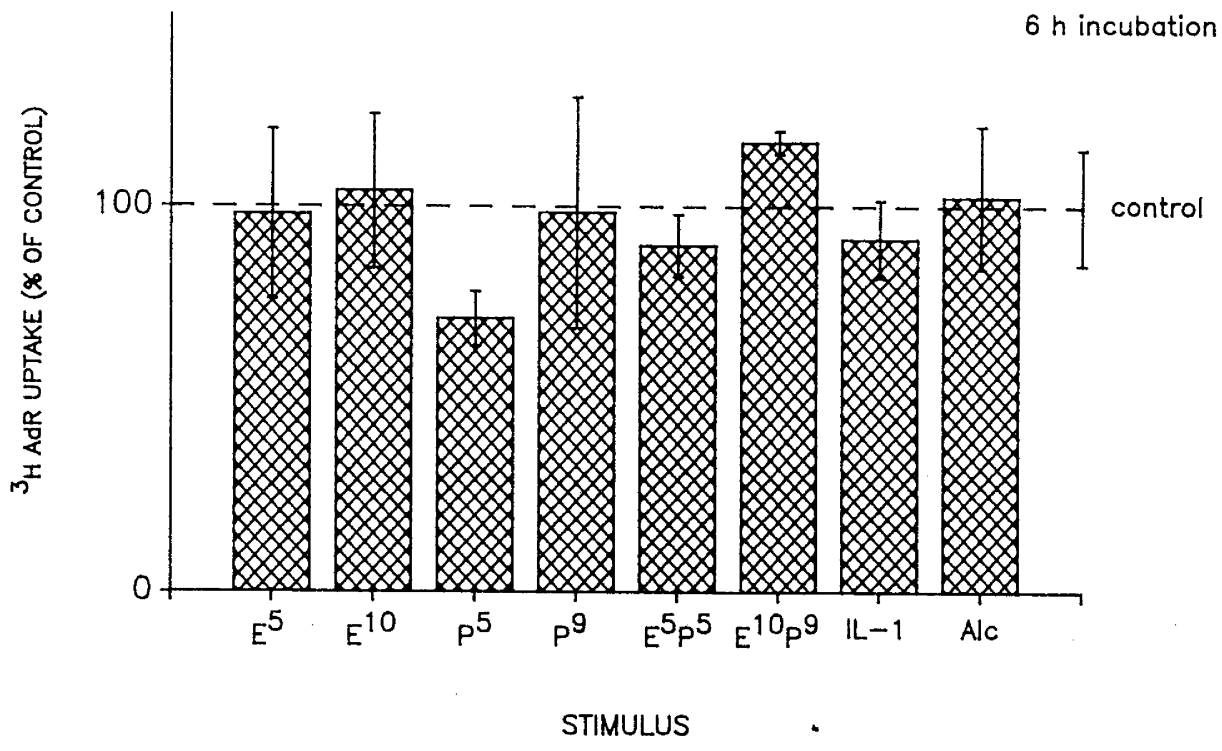

UPTAKE OF ${ }^{3}$ H-ADENINE BY A SUBCONFLUENT MONOLAYER OF ENDOTHELIAL CELLS EXPOSED TO VARIOUS STIMULI

$18 \mathrm{~h}$ incubation

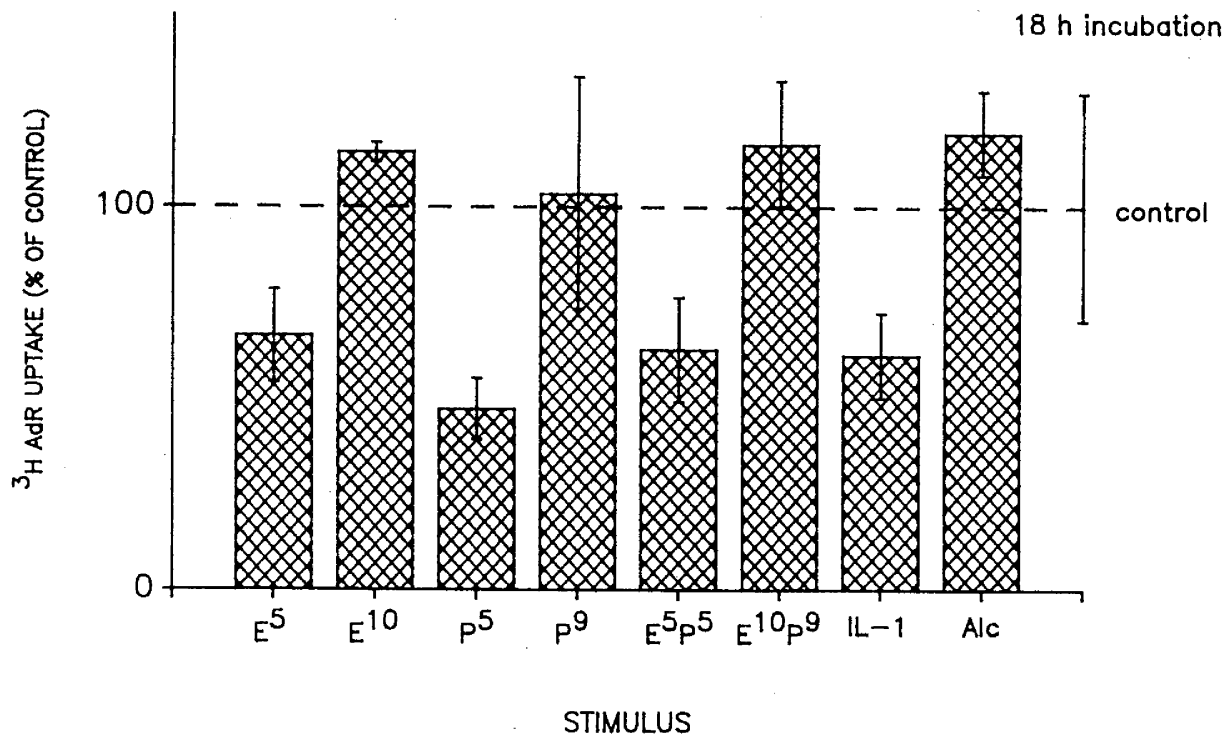




\section{Figure 5.13.}

Incubation of monocytes with various stimuli and the effect on monocyte binding to a confluent monolayer of HUV endothelial cells. Monocytes were incubated with various stimuli for $6 \mathrm{~h}$ in fibronectin-coated gelatinized flasks. Following incubation the nonadherent cells were washed from the flasks and the adherent cells removed with EDTA and resuspended in media containing the various stimuli. The cells were then washed and incubated with HBSS containing $\left[2-{ }^{3} \mathrm{H}\right]$-adenine for $30 \mathrm{~min}$. Following centrifugation the monocyte rich suspension was added to the endothelial cells and the monolayer was treated as described in the legend to Figure 5.5. The average radioactive cpm in the control wells was 18895 . This graph represents the mean \pm SE of three experiments in triplicate. $\star=p<0.05$.

\section{Figure 5.14.}

Incubation of both monocytes and endothelial cells with various stimuli demonstrating the effect on monocyte binding to a confluent monolayer of HUV endothelial cells. The same protocol as described in the legend to Figure 5.13 was followed except that the endothelial cells were also exposed to the same stimuli for $6 \mathrm{~h}$, prior to the addition of the monocytes to the endothelial cells. The average radioactive cpm in the control wells was 20506 . This graph represents the mean \pm SE of three experiments performed in triplicate. ${ }^{*}=p<0.05$. 
INCUBATION OF MONOCYTES WITH VARIOUS STIMULI

EFFECT ON MONOCYTE BINDING TO A CONFLUENT MONOLAYER OF ENDOTHELIAL CELLS

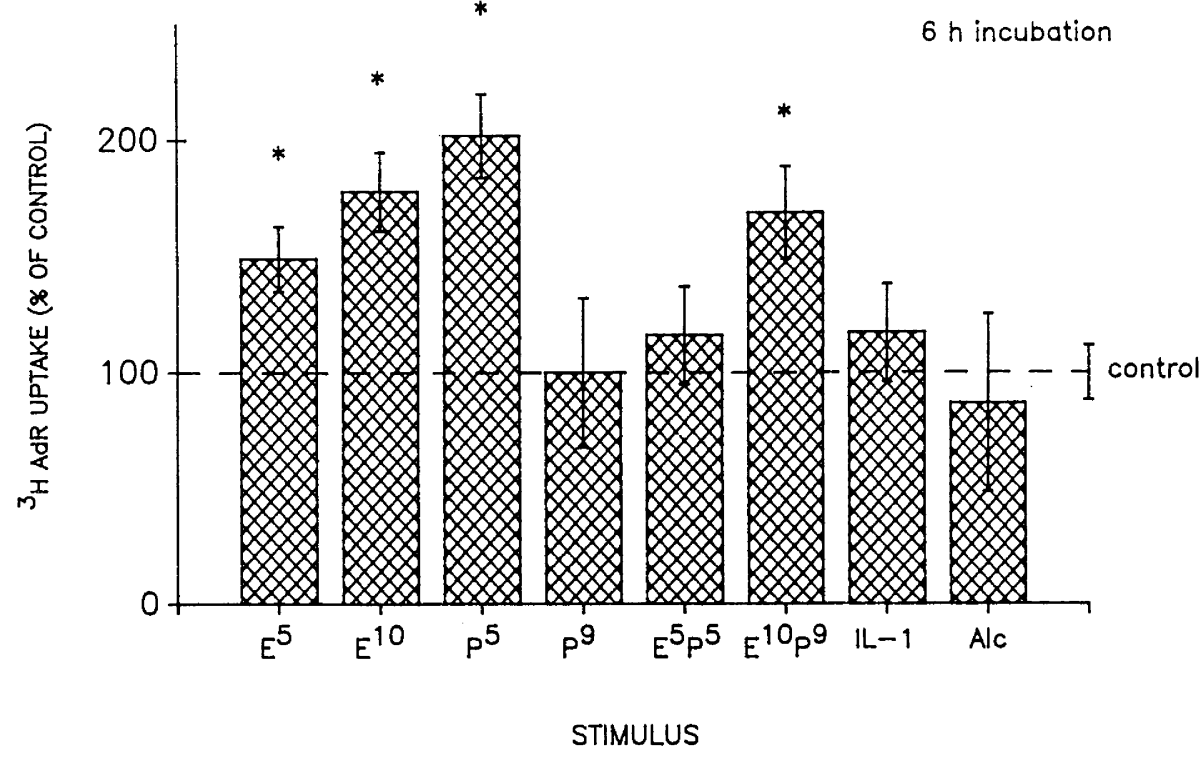

INCUBATION OF MONOCYTES AND ENDOTHELIAL CELLS WITH VARIOUS STIMULI

EFFECT ON MONOCYTE BINDING TO A CONFLUENT MONOLAYER OF ENDOTHELIAL CELLS

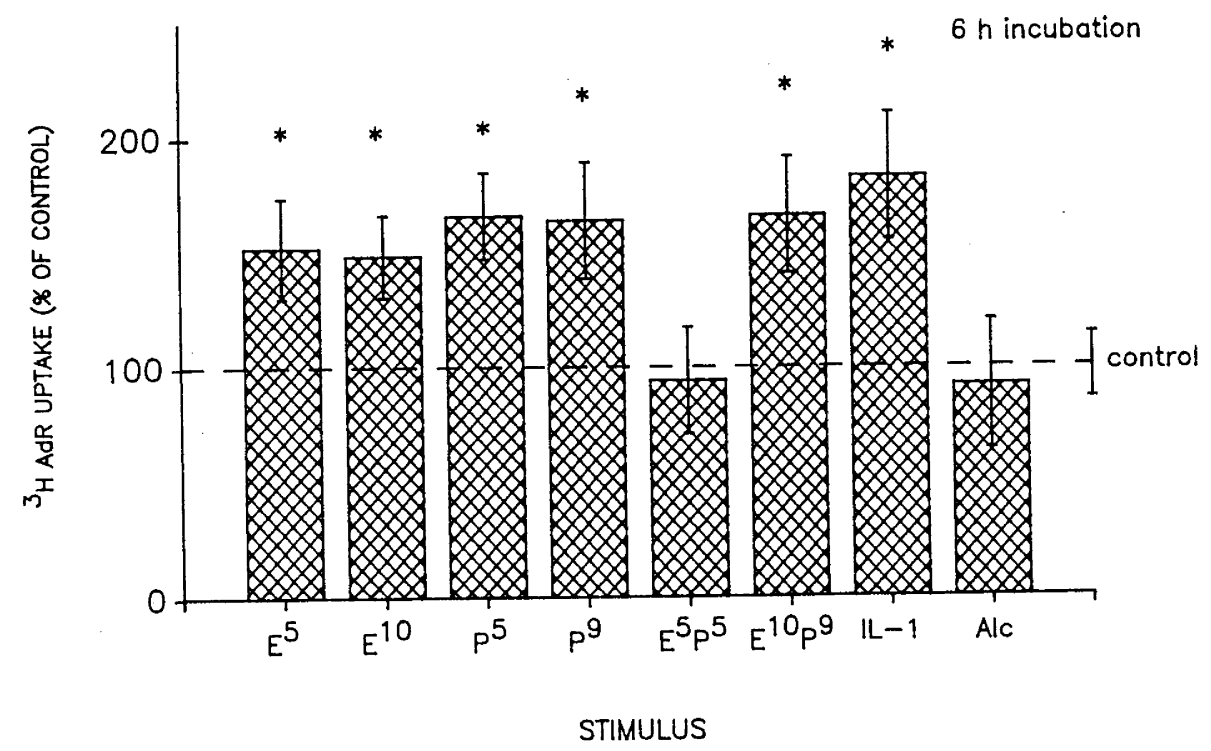




\section{CHAPTER 6}

THE EFFECT OF SEX STEROIDS ON LOW DENSITY LIPOPROTEINS

AND THE VASCULAR ENDOTHELIUM 


\subsection{INTRODUCTION}

An attractive hypothesis for atherogenesis is that LDL, the molecule which binds and transports cholesterol, has a key role in the pathogenesis of atherosclerosis (Steinberg, 1987; Steinberg et al, 1989b). Whether it is the high level of LDL itself which is important, oxidation of LDL, altered metabolism of LDL or some other abnormality remains unclear (Mahley, 1982; 1983; 1985; Steinberg, 1991).

LDL is present in the intima of healthy aortas at concentrations twice those found in serum (Smith and Staples, 1980; 1982). Human LDL comprises $20-$ $24 \%$ phospholipids, 9-10 \% free cholesterol, $40-44 \%$ cholesteryl esters, 3-5 $\%$ triglycerides, and $21-26 \%$ protein (Esterbauer et al, 1990). The neutral lipids (cholesteryl esters, triglycerides) form a hydrophobic core which is surrounded by a surface monolayer consisting of phospholipids and cholesterol. Embedded in the monolayer is a protein, apo B (MW approximately 500000 ). The fatty acid composition is variable, comprising about $50 \%$ polyunsaturated fatty acids (PUFAs), mainly linoleic acid (18:2) and arachidonic acid (20:4) (Esterbauer et al, 1990).

Foam cells containing cholesterol (Goldstein et al, 1979a; Mahley et al, 1979; Fogelman et al, 1980; Henriksen et al, 1981; Steinberg, 1983; Brown and Goldstein, 1983; Heinecke, 1987) are a prominent component of atherosclerotic plaques (Gerrity, 1981; Ross and Glomset, 1986). Most of the foam cells in the fatty streak are thought to be of macrophage-monocyte origin (Newman et al, 1971; Fowler et al, 1979; Gerrity, 1979; 1980; 1981; Schaffner et al, 1980; Faggiotto et al, 1984; Rosenfeld et al, 1987a and b) although they do not secrete detectable amounts of lysozyme and only a third of the cells display C3 receptors characteristic of macrophages (Fowler et al, 1979). Some other authors have suggested that foam cells arise from 
pre-existing arterial smooth muscle cells which acquire certain macrophagelike properties (Parker and Odland, 1966; Newman et al, 1971; Fowler et al, 1979). Carew et al (1987) however showed that most of the LDL degraded in fatty streaks took place in macrophages, whereas in non-lesioned aorta, degradation of LDL occurred almost exclusively in endothelial cells and smooth muscle cells.

As discussed in Section 1.3.2 macrophages have at least two types of receptors for the uptake of LDL. They have low levels of the classical highly regulated-LDL receptor (Goldstein and Brown, 1977) and therefore are unlikely to accumulate, via this receptor, sufficient lipid to become foam cells (Goldstein et al, 1979a; Haberland and Fogelman, 1987). The second type of receptor is the acetyl-LDL receptor or "scavenger" lipoprotein receptor (Goldstein et al, 1979a; Brown and Goldstein, 1983; Morton et al, 1986) which takes up modified lipoprotein (refer to Section 6.1.1) in an unregulated manner. Arai et al (1989) however has suggested that there are at least three receptors for modified LDL, since oxidized LDL only partially inhibits acetylLDL binding. It is this unregulated uptake of LDL that appears to be responsible for the accumulation of cholesterol esters and the development of foam cells (Goldstein et al, 1979a; Mahley et al, 1979, Fogelman et al, 1980, Henriksen et al, 1981; Steinberg, 1983; Heinecke, 1987).

\subsubsection{Modification of LDL}

Native LDL can be modified by a number of chemical processes, including:

(a) Acetylation (Goldstein et al, 1979a);

(b) Acetoacetylation (Weisgraber et al, 1978; Mahley et al, 1979);

(c) Malondialdehyde (MDA) and 4-hydroxynonenal (HNE) treatment (Fogelman et al, 1980; Schechter et al, 1981; Tertov et al, 1989b; Hoff et al, 1989; Jurgens et al, 1990); 
(d) Desialylation with sialidase (neuraminidase) (Orekhov et al, 1989; Tertov et al, 1989b);

(e) Methylation (Weisgraber et al, 1978; Suits et al, 1989);

(f) Maleylation (Weisgraber et al, 1978;

(g) Succinylation (Mahley et al, 1979);

(h) Carbamylation (Weisgraber et al, 1978);

(i) Glycosylation (Tertov et al, 1989); and

(j) Oxidation (Schuh et al, 1978; Steinbrecher et al, 1987; Zawadzki et al, 1989).

Of these various chemical modifications only two appear to occur in vivo, reaction with malondialdehyde and oxidation (Haberland et al, 1988; Steinberg, 1987).

\subsubsection{Oxidative changes to LDL}

There is growing evidence that oxidative modification of LDL occurs in vivo as well as in vitro. In vitro, oxidation can be induced by transition metal ions (e.g. copper and iron) (Schuh et al, 1978; Heinecke et al, 1984; Steinbrecher et al, 1987; Zawadzki et al, 1989) or it may be induced by the following cells:

(a) Endothelial cells (Henriksen et al, 1981; 1982a; b; 1983; Steinbrecher et al, 1984; Morel et al, 1984; Parthasarathy et al, 1985; 1989; Quinn et al, 1985; 1987);

(b) Arterial smooth muscle cells (Heinecke et al, 1984; Morel et al, 1984);

(c) Macrophages (Heinecke et al, 1984; Cathcart et al, 1985; Parthasarathy et al, 1986a);

(d) Polymorphonuclear leukocytes (PMN) (Cathcart, 1985); and

(e) Platelets (Aviram et al, 1990). 
Cell-induced oxidation of LDL in culture is promoted by micromolar concentrations of iron or copper in the culture medium. The rate of oxidation by metal ions is time and concentration dependent (Heinecke et al, 1984; Steinbrecher et al, 1984; Morel et al, 1984; Parthasarathy et al, 1985). However, Fong et al (1987) showed that the disappearance of apo B was much slower with copper ion-induced LDL modification than during endothelial cell induced LDL modification. Cell induced oxidative modification of $L D L$ is thought to result from the generation of oxygen free radicals in the cell which may involve a superoxide dependent pathway or perhaps a lipoxygenase pathway (Carew, 1989, Steinbrecher et al, 1990) (refer to Section 6.1.4). Lipoxygenases may act on the lipids present either in the cell membrane or in LDL particles themselves (Rankin et al, 1991). The evidence for in vivo oxidative modification of LDL has been discussed in Section 1.3.2.

Oxidative modification of LDL results in changes to the physico-chemical properties of LDL including:

(a) Increased buoyant density (Steinbrecher et al, 1990);

(b) Increased electrophoretic mobility in agarose (Henriksen et al, 1981; 1983; Steinberg, 1983; Morel et al, 1984; Steinbrecher et al, 1984; Nagelkerke et al, 1984; Van Hinsbergh et al, 1986);

(c) Phosphatidyl choline hydrolysis (Steinbrecher et al, 1984; Parthasarathy et al, 1985);

(d) Decreased content of cholesterol esters (Sternberg, 1983; Steinbrecher et al, 1989);

(e) Loss of polyunsaturated fatty acids (Nichols et al, 1961; Esterbauer et al, 1987);

(f) Fragmentation of apo B (Schuh et al, 1978; Parthasarathy et al, 1985; Zawadki et al, 1989);

(g) More rapid degradation of LDL by macrophages compared to native LDL (Steinbrecher et al, 1984); 
(h) Induction of expression of procoagulant activity (PCA) by monocytes and macrophages (Schuff-Weiner et al, 1989); and

(i) Modification of the pattern of LDL interactions with cell receptors with recognition of the scavenger receptor (Henriksen et al, 1981; 1983) and loss of recognition of the classical LDL receptor (Steinbrecher et al, 1987). Compared to native LDL, modified LDL is taken up 3-10 fold more rapidly by macrophages and at least partly by way of the scavenger receptor (Henriksen et al, 1981; 1983; Parthasarathy et al, 1985).

These modifications of LDL appear to promote atherosclerotic plaque formation by promoting foam cell formation, recruitment of monocytes, prevention of macrophage migration from the vessel wall and damage to endothelial cells as discussed in Section 1.3.2.

\subsubsection{Cytotoxicity of LDL for endothelial cells}

Plasma LDL is taken up by endothelial cells by both transcytosis and endocytosis. Most of the LDL transported across the endothelial cell is by transcytosis via plasmalemmal vesicles, and a relatively smaller amount is transported by endocytosis, both receptor-mediated (absorptive endocytosis) and receptor-independent (fluid endocytosis) (Vasile et al, 1983). Although transcytosis is the major mechanism for the transport of LDL through the endothelial cell, the mechanisms responsible for the localized accumulation of cholesterol and other lipids in atherogenesis are still not known (Poumay and Ronveaux-dupal et al, 1988). The interaction of endothelial cells and LDL has been studied in vivo and in vitro. In vitro, endothelial cells have been shown to have two classes of LDL binding sites, a high affinity, low capacity site (which saturates at LDL concentrations of 5 to $20 \mathrm{\eta g}$ protein $/ \mathrm{ml}$ ) and a low affinity site, which is not saturable (Reckless et al, 1978; van Hinsbergh et al, 1983), known as the "scavenger receptor" 
(Stein and Stein, 1980) which is identical to the receptor described by Goldstein et al (1979a) on macrophages.

A question which has concerned scientists is whether LDL is toxic to endothelial cells. LDL preparations were initially shown to exhibit concentration-related cytotoxicity to endothelial cell cultures (Henriksen et al, 1977; 1979a; 1979b; Hessler et al, 1979; Tauber et al, 1980; Evensen et al, 1983; Morel et al, 1983a). Tauber et al (1980) found that LDL had a biphasic effect on endothelial cell growth. It was found to be mitogenic for bovine vascular endothelial cells at low concentrations $(10-100 \mu \mathrm{g}$ protein $/ \mathrm{ml})$, but once physiological concentrations (600 to $800 \mu \mathrm{g}$ protein $/ \mathrm{ml}$ ) were reached the LDL became toxic to the cells. Gotlieb et al (1983) also showed that LDL at concentrations less than $100 \mu \mathrm{g} / \mathrm{ml}$ enhanced re-endothelialization of areas denuded of endothelial cells. Henriksen et al (1977) and Tauber et al (1980) found that the mitogenic effect of LDL was a function of the concentration of lipoprotein-deficient serum (LPDS) to which the cultures were exposed. The toxicity was greater when the concentration of LPDS was reduced. Henriksen et al (1977) also showed that the toxicity of LDL could be modified by co-incubation with HDL. This was confirmed by B $\phi$ rsum et al (1981) and Evensen et al (1983). Henriksen et al (1979b) did however notice that the protective effect of HDL was reduced by storage of the HDL, possibly because of oxidation.

Initially, LDL toxicity to cells was thought to be due to intracellular or membrane sterol overloading (Hessler et al, 1979). Henriksen et al (1979a) used a ${ }^{51} \mathrm{Cr}$ release assay to measure cellular injury in fibroblast cultures from a person with familial homozygous hypercholesterolemia, and demonstrated that the injury was independent of LDL receptors on fibroblasts. This was confirmed by Borsum et al (1981) using LDL-receptor negative endothelial cells. Chisolm et al (1981) concluded that the toxic effect of LDL was 
probably due to in vitro oxidation of LDL resulting in lipid peroxides which damaged the cells by causing peroxidation of the cell membrane (Halliwell and Gutteridge, 1986). This could be inhibited by EDTA or a suitable reducing agent. This proposed mechanism has been supported by the findings of others (Sacks et al, 1978; Weiss et al, 1981; Holland et al, 1988; Morel et al, 1983b; 1984; Hessler et al, 1983; Shingu et al, 1985; Shasby et al, 1985; Freeman et al, 1986). Cytotoxicity to endothelial cells secondary to oxidative changes to LDL has been confirmed by others (Steinbrecher et al, 1984; Evensen et al, 1983; Cathcart et al, 1985, Van Hinsberg et al, 1986).

Some studies have suggested that the induction of lipid peroxidation of cell membranes is not the mechanism by which oxidized LDL initially produces cell damage but rather that oxidant injury causes glutathione (GSH) depletion and metal ion release which may cause cell damage (Halliwell, 1987). Lipid peroxidation in the cell membrane may be important in spreading injury to adjacent cells (Halliwell, 1987). The susceptibility of endothelial cells to oxidized LDL has been shown to be dependent on intracellular glutathione levels and the phase of the cell cycle (Kuzuya et al, 1989; Kosugi et al, 1987; Kuzuya et al, 1991). Holland et al (1988) showed that although there was no overt cytotoxicity when antioxidants were added to LDL preparations there was still an increase in prostacyclin release indicative of endothelial cell perturbation. The toxic moiety of oxidized LDL appears to be the lipid hydroperoxide associated with the oxidized LDL particle (Kuzuya et al, 1991). Kuzuya et al (1991) have suggested that the toxicity of copper-oxidized LDL was due to copper binding to the lipoprotein to form a complex. Chelation of copper with EDTA inhibited the cytotoxicity of oxidized LDL. However other investigators have shown that minimally modified LDL is cytotoxic to certain endothelial cells. Minimally modified LDL is prepared by storing LDL (or by mild iron oxidation) rather than by exposure of LDL to high concentrations of copper (Berliner et al, 1990; Liao et al, 1991). In summary, there is increasing 
evidence that modified LDL is toxic for endothelial cells. The mechanism may involve a number of processes: oxidative modification of LDL, glutathione depletion, and metal ion release.

\subsubsection{Lipid peroxidation and the role of antioxidants}

Lipid peroxidation is broadly defined as oxidative deterioration of polyunsaturated lipids (Tappel, 1973). It is a free radical-mediated process (Slater, 1984) involving the reaction of oxygen with polyunsaturated lipids to form lipid-free radicals and semi-stable hydroperoxides, which then promote free radical chain oxidations (Bus and Gibson, 1979). The free radical chain reaction proceeds in three steps:

(a) Initiation, where singlet oxygen or hydroxyl radical is produced which can react with unsaturated fatty acids to abstract a hydrogen atom leading to the formation of a lipid radical $(L \cdot)$. This lipid radical $(L \cdot)$ can then react with oxygen to produce a lipid peroxyl radical (LOO.) which can then form lipid hydroperoxides (LOOH);

(b) Propagation, where the lipid hydroperoxides are decomposed to form additional radical products. This can be catalysed by trace amounts of transitional metal ions $\left(\mathrm{M}^{\mathrm{n}}\right)$ such as iron or copper resulting in the following reaction:

$$
\begin{aligned}
& \mathrm{LOOH}+\mathrm{Mn}^{n+} \\
& \mathrm{LOOH}+\mathrm{M}(\mathrm{n}+1)+\ldots
\end{aligned}
$$

(LO = lipid oxy radical; LOO = lipid peroxyl radical)

(c) Termination, which may occur when free radicals are either destroyed by reactions with themselves, intervention of antioxidants or when the substrate is depleted.

The lipid peroxidation process may be nonenzymic or enzymatically catalysed. Where the process involves a characterized enzyme that gives a 
defined set of products it is usually called a lipoxygenase type of activity (Slater, 1984).

A number of antioxidants inhibit LDL oxidation either in vivo, in vitro or both. Antioxidants of particular interest to this work include:

(a) D- $\alpha$-tocopherol (vitamin E), a lipid soluble antioxidant which can scavenge oxygen radicals and lipid free radicals by a reaction involving hydrogen, thus interrupting the free radical chain reaction (Bus and Gibson, 1979; Steinbrecher et al, 1984; Morel et al, 1984; Cathcart et al, 1985; Babiy et al, 1990). This can be summarized as:

$\mathrm{LOO}+\alpha \mathrm{TH}+-.-1-$

LOO + $\alpha$ T · --a

( $\alpha \mathrm{T}=\alpha$-tocopherol; $\alpha \mathrm{TQ}=\alpha$-tocopherol quinone);

(b) Glutathione (GSH) is a thiol compound and a substrate for an enzyme system for scavenging lipid peroxides. This can be summarized as:

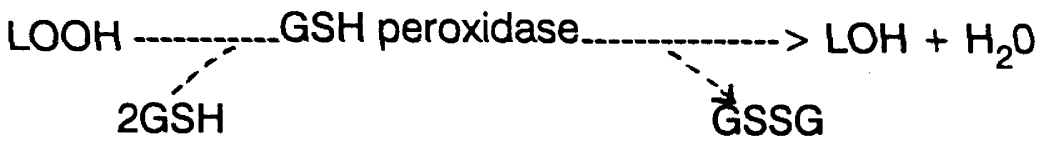

Glutathione peroxidase requires selenium (Machlin and Bendich, 1987). GSH reduces the selenium and it is the reduced form of the enzyme glutathione peroxidase which reacts with hydrogen peroxide or other peroxides. In cells, GSSG can then be reduced back to GSH by glutathione reductase (Schuh et al, 1978; Lee, 1980; Hessler et al, 1983; Cathcart et al, 1985; Kuzuya et al, 1989; Wang et al, 1989).

(c) EDTA and desferroxamine which chelate transitional metals (Ontko, 1970; Schuh et al, 1978 Chisolm et al, 1981; Morel et al, 1983; Fong et al, 1987; Esterbauer et al, 1990);

(d) p-Bromophenacylbromide (BPB), a site-specific irreversible inhibitor of phosopholipase $A_{2}$ which is an essential component in the modification of LDL by endothelial cells (Parthasarathy et al, 1985); 
(e) Butylated hydroxytoluene (BHT), a synthetic antioxidant which probably acts like D- $\alpha$-tocopherol (Schuh et al, 1978; Evensen et al, 1983; Steinbrecher et al, 1984; Cathcart et al, 1985; Parthasarathy et al, 1986a; Fong et al, 1987; Holland et al, 1988); and

(f) Probucol, a hypocholesterolemic drug, which has a structure similar to BHT (Jialal and Grundy, 1991) and has been shown in WHHL rabbits to reduce the size of atherosclerotic lesions (Carew et al, 1987; Kita et al, 1987) and to inhibit the oxidation of LDL by endothelial cells in vitro (Parthasarathy et al, 1986b; Steinberg et al, 1989a; Carew, 1989).

Known antioxidants in blood include vitamin $E$, ascorbate (vitamin $C$ ), carotene, peroxidases, superoxide dismutase, ceruloplasmin, transferrinlactoferrin, bilirubin, uric acid, albumin, proteins containing thiol groups and organic selenium compounds (Halliwell and Gutteridge, 1986; Steinbrecher et al, 1987; Cross et al, 1987; Machlin and Bendich, 1987; Wang et al, 1989). LDL contains its own antioxidants including $\alpha$-tocopherol, $\gamma$-tocopherol, $\beta$ carotene, lycopene (Esterbauer et al, 1990) and ubiquinol-10 (Stocker et al, 1991). Cells also contain their own antioxidants including superoxide dismutase and glutathione peroxidase although Vercellotti et al (1988) demonstrated that different cells within and between species utilize different antioxidant protective mechanisms when exposed to oxidants.

Some antioxidants prevent the initiation of lipid peroxidation (e.g. superoxide dismutase and carotenoid pigments) while others inhibit the propagation of free radical reactions (e.g. ascorbic acid, glutathione and tocopherol) (Bus and Gibson, 1979). Parthasarathy et al (1989) demonstrated that inhibitors of lipoxygenase activity such as nordihydroguaiaretic acid reduced endothelial cell oxidation of LDL by 70 to $85 \%$ while superoxide dismutase inhibited endothelial cell oxidation of LDL by only $21 \%$. However, superoxide dismutase is known to inhibit smooth 
muscle cell and macrophage oxidation of LDL suggesting that superoxide anions may be involved in oxidative changes caused by these cells (Heinecke et al, 1986; Hiramatsu et al, 1987; Parthasarathy et al, 1989).

In vivo, indices of lipid peroxidation (such as diene conjugation and the thiobarbituric acid (TBA) assay) confirm the presence of lipid peroxides in plasma, and in the aortic wall in rabbits with experimental hypercholesterolemia (Heinle and Liebrich, 1980), in plasma of vitamin E deficient rats (Higuchi, 1982), in human arteriosclerotic arteries (Glavind et al, 1952; Piotrowski et al, 1990), in rats rendered diabetic (Morel and Chisolm, 1989 ) and in the plasma of diabetic patients (Sato et al, 1979; Yagi, 1982).

In summary, the evidence supports lipid peroxidation of LDL with the formation of toxic aldehydes (Halliwell, 1987) as a possible cause of endothelial cell cytotoxicity. Thus, lipid peroxidation could initiate or promote the development of atherosclerotic lesions by directly damaging endothelial cells, by enhancing the adhesion and activation of monocytes, and by promoting aggregation of platelets (Hennig and Chow, 1988 Ardlie et al, 1989).

\subsubsection{Sex steroids, LDL oxidation and atherosclerosis}

The effects of sex steroids on plasma lipids was reviewed in Chapter 1 (refer to Section 1.5.1). Post-menopausally, estrogens have been shown to favourably alter serum lipoprotein levels (Wallace et al, 1979; Oliver and Boyd, 1956). This does not however adequately explain the apparent protective role of estrogen in CHD since estrogen can reduce atherosclerotic plaque formation even in the presence of elevated levels of circulating cholesterol (Pick et al, 1952; Souadjian et al, 1968; Hanash et al, 1972) (refer to Section 1.5.3). If oxidization of LDL is important in the pathogenesis of 
atherosclerosis estrogen may reduce atherosclerotic plaque formation by retarding LDL oxidation (as outlined in Section 1.5.3.6), possibly in conjunction with effects on serum lipids, $\mathrm{PGI}_{2}$ release and growth factor expression.

The studies reported in this Chapter focus on the interactions between LDL, sex steroids, and HUV endothelial cells, and the role that these interactions play in the pathogenesis of atherosclerosis. The aims of these studies were to determine whether native and oxidized LDL affect HUV endothelial cell proliferation, to examine the influence of sex steroids on this process, and to assess whether estradiol has antioxidant properties using two established models, copper sulphate oxidation of LDL and oxidation of LDL by HUV endothelial cells.

\subsection{MATERIALS}

Materials used which are specific to the studies described in this Chapter were as follows:

Acrylamide/Bis (30\% stock) (Biorad Laboratories, Richmond, Australia) was diluted in distilled water, stored at $4^{\circ} \mathrm{C}$ and degassed for $15 \mathrm{~min}$ at room temperature before use.

Ammonium persulfate (Sigma Chemical Company, St Louis, USA). A $10 \%$ solution was prepared in distilled water, and stored in $100 \mu$ aliquots for a maximum of 3 months at $-20^{\circ} \mathrm{C}$.

Biotinylated SDS-page standard kit, high range Avidin-HRP (Biorad Laboratories, Richmond, Australia) was stored at $-20^{\circ} \mathrm{C}$. A $1: 20$ dilution was prepared in sample buffer (see below) before use. 
Biotinylated SDS-page standard kit, low range Avidin-AP (Biorad Laboratories, Richmond, Australia) was stored at $-20^{\circ} \mathrm{C}$. A $1: 20$ dilution was prepared in sample buffer (see below) before use.

Boron trifluoride methanol complex (about $14 \% \mathrm{BF}_{3}$ ) (BDH Chemical Australia, Kilsyth, Australia).

p-Bromophenacyl bromide (BPB) (Koch-Light Laboratories, England) was dissolved in absolute ethanol to give a concentration of $11.12 \mathrm{mg} / \mathrm{ml}$ and stored at $4^{\circ} \mathrm{C}$ in the dark.

Butylated hydroxytoluene (BHT) (Sigma Chemical Company, St Louis, USA).

Coomassie blue R-250 (ICl, Australia). A $0.1 \%$ solution was prepared in $40 \%$ methanol (Ajax Chemicals, Sydney, Australia) and $10 \%$ acetic acid (May and Baker, Dagenham, England), and then stored at room temperature.

Cupric sulphate (BDH Laboratory, Poole, England) was dissolved in reagent diluent (RD) (see below) to give a $5 \mathrm{mM}$ stock solution.

Chloroform AR grade (Ajax Chemicals, Auburn, Australia).

Density solutions for lipoprotein separations by differential ultracentrifugation. The stock solution of $1.006 \mathrm{~g} / \mathrm{ml}$ comprised $\mathrm{NaCl}(11.42 \mathrm{mg} / \mathrm{ml}), \mathrm{GSH}(0.5$ $\mathrm{mg} / \mathrm{ml})$ and EDTA $(0.1 \mathrm{mg} / \mathrm{ml})$ and was stored at room temperature.

A $1.019 \mathrm{~g} / \mathrm{ml}$ solution was made by adding $1.85 \mathrm{~g}$ of solid $\mathrm{KBr}$ to $100 \mathrm{ml}$ of 1.006 density solution.

A $1.063 \mathrm{~g} / \mathrm{ml}$ solution was made by adding $8.33 \mathrm{~g}$ of solid $\mathrm{KBr}$ to $100 \mathrm{ml}$ of 1.006 density solution. 
Dialysis tubing (Selby Anax, Union Carbide Corporation, Chicago, USA) was boiled in $\mathrm{Na}_{2} \mathrm{CO}_{3}(30 \mathrm{~g})$ and EDTA $(1.1 \mathrm{~g})$ in distilled water $(3.0 \mathrm{~L})$ for $45 \mathrm{~min}$, and then washed in distilled water and stored in $50 \%$ ethanol.

Electrode buffer (for polacrylamide gel electrophoresis of LDL samples). A 5 $x$ concentrated stock solution was prepared and stored at $4^{\circ} \mathrm{C}$ and warmed to room temperature before use. It comprised Tris base ( $15 \mathrm{~g} / \mathrm{L})$, glycine (72 $\mathrm{g} / \mathrm{L})$ and SDS $(5 \mathrm{~g} / \mathrm{L})$, made up to $600 \mathrm{ml}$ in distilled water.

Ethylene diaminetetraacetic acid disodium salt (EDTA) (Ajax Chemicals, Auburn, Australia). A stock solution of $3.7 \mathrm{~g} / 100 \mathrm{ml}$ solution was prepared in distilled water and stored at $4^{\circ} \mathrm{C}$.

Folin-Ciocalteus Phenol reagent (Merk, Darmstadt) was kept in the dark at 4 ${ }^{\circ} \mathrm{C}$.

Glutathione (GSH) (Sigma Chemical Company, St Louis, USA) was stored at $4^{\circ} \mathrm{C}$.

n-Hexane (Ajax chemicals, Sydney Australia) Unichrom, especially purified for HPLC.

Modified Tyrode buffer comprised $50 \mathrm{ml}$ of RD made up to $1.0 \mathrm{~L}$ with EDTA (100 mg /L) and GSH (0.5 g /L).

Normal serum albumin (Commonwealth Serum Laboratories, Melbourne, Australia). Stock contained $200 \mathrm{~g} / \mathrm{L}$ of human plasma protein of which at least $95 \%$ was albumin. This was stored at $4^{\circ} \mathrm{C}$ and diluted in RD (see below) prior to use. 
Potassium bromide crystals (Ajax Chemicals, Auburn, Australia).

Reagent diluent (RD) comprised $50 \mathrm{ml}$ of stock 1 made up to $1.0 \mathrm{~L}$ with distilled water adjusted to $\mathrm{pH} 7.35$.

Sample buffer for agarose gel electrophoresis comprised glycerol (1.28 $\mathrm{ml})$ (Ajax Chemicals, Auburn, Australia), and $0.05 \%(\mathrm{w} / \mathrm{v})$ bromophenol blue $(0.64 \mathrm{ml})$ (Biorad, Richmond, CA) made up to $8 \mathrm{ml}$ with barbital buffer and stored at $4^{\circ} \mathrm{C}$.

Sample buffer for polyacrylamide gel electrophoresis comprised distilled water $(4.4 \mathrm{ml}), 0.5 \mathrm{M}$ Tris- $\mathrm{HCl}, \mathrm{pH} 6.8(1.0 \mathrm{ml})$, glycerol $(0.8 \mathrm{ml}), 10 \%(\mathrm{w} / \mathrm{v})$ SDS $(1.6 \mathrm{ml})$, and $0.05 \%(\mathrm{w} / \mathrm{v})$ bromophenol blue $(0.2 \mathrm{ml})$ stored at $4{ }^{\circ} \mathrm{C}$.

Sodium dodecyl sulfate (SDS) (Sigma Chemical Company, St Louis, USA). A $10 \%(w / v)$ stock was prepared and stored at room temperature.

Stock Solution 1 comprised $\mathrm{NaCl}(160 \mathrm{~g}), \mathrm{KCl}(4 \mathrm{~g}), \mathrm{NaHCO}_{2}(2 \mathrm{~g}), \mathrm{NaH}_{2}$ $\mathrm{PO}_{4}(1 \mathrm{~g})$ made up to $1.0 \mathrm{~L}$ with distilled water and kept at $4{ }^{\circ} \mathrm{C}$.

Sudan black (Sigma Chemical Company, St Louis, USA). A stock solution was made with $200 \mathrm{mg}$ of dye in $100 \mathrm{ml}$ of $60 \%$ ethanol. A 1 in 100 dilution was prepared in $60 \%$ ethanol.

TEMED (Biorad Laboratories, Richmond, Australia) was stored at $4^{\circ} \mathrm{C}$.

1,1,3,3 Tetra ethoxy propane (Sigma Chemical Company, St Louis, USA). 
Thiobarbituric acid (BDH Laboratory, Poole, England). A $0.375 \%(w / v)$ solution was prepared in TCA-HCl solution and mildly heated (to assist in the dissolution of thiobarbituric acid) to obtain a TCA-TBA-HCl solution.

Trichloroacetic acid (TCA) (Ajax Chemicals, Auburn Australia). A $15 \%(w / v)$ solution was prepared in $0.25 \mathrm{~N}$ hydrochloric acid (Ajax Chemicals, Auburn, Australia).

Tris- $\mathrm{HCl}$ (Biorad Laboratories, Richmond, Australia) was a $1.5 \mathrm{M}$ solution at $\mathrm{pH} 8.8$, stored at $4^{\circ} \mathrm{C}$.

Vitamin $E(D-\alpha$-tocopherol acetate type 111) (Sigma Chemical Company, St Louis, USA) was dissolved in absolute ethanol to give a stock solution of 100 $\mathrm{mg} / \mathrm{ml}$. It was stored at $4^{\circ} \mathrm{C}$ in the dark for up to 2 weeks.

\subsection{METHODS}

\subsubsection{Preparation of native LDL}

The method used was that of Ardlie et al (1989) except that blood from normolipidemic male patients only was used. Lipoproteins were prepared from freshly drawn blood anticoagulated with EDTA (63 mM) and GSH (1.63 mM). LDL was obtained by differential ultracentrifugal flotation (LDL 1.019 < $\left.d<1.063 \mathrm{~g} / \mathrm{cm}^{3}\right)$. Blood was centrifuged $\left(900 \times \mathrm{g}\right.$ for $20 \mathrm{~min}$ at $4^{\circ} \mathrm{C}$ ) to obtain plasma. The supernatant plasma was collected and vitamin E (0.2 $\mathrm{mM})$ and BPB $(40 \mu \mathrm{M})$ were added to prevent oxidation. The plasma density was adjusted to 1.019 by the addition of solid $\mathrm{KBr}$. The plasma was aliquoted $(25 \mathrm{ml})$ into Beckman polyallomer "quick-seal" centrifuge tubes $(38.5 \mathrm{ml}$ capacity) (Beckman Instruments Inc., Palo Alto, CA), layered with density solution 1.019 and then sealed with a Beckman tube sealer kit. The tubes 
were centrifuged in a L5-65B Ultracentrifuge (Beckman, Palo Alto, California) in a precooled $60 \mathrm{Ti}$ rotor $\left(74232 \times \mathrm{g}\right.$ for $17.6 \mathrm{~h}$ at $\left.4^{\circ} \mathrm{C}\right)$ to remove VLDL and intermediate density lipoproteins (IDL). The supernatants were discarded and the infranatants pooled. The infranatant density was adjusted to 1.063 with solid $\mathrm{KBr}$ and aliquoted into polyallomer quick seal tubes, layered with density solution 1.063 and centrifuged $\left(74232 \times \mathrm{g}\right.$ for $18.25 \mathrm{~h}$ at $\left.4^{\circ} \mathrm{C}\right)$. The supernatants containing the LDL were collected, aliquoted and washed by layering with density solution 1.063 and centrifuging $(74232 \times \mathrm{g}$ for $18.25 \mathrm{~h}$ at $4^{\circ} \mathrm{C}$ ). Preparations were then filtered through a $0.22 \mu \mathrm{m}$ Millipore membrane and concentrated using an Amicon membrane concentrator (Model 202). Preparations were washed and dialysed at $4{ }^{\circ} \mathrm{C}$ for $24 \mathrm{~h}$ against modified Tyrode buffer containing EDTA and GSH. The lipoprotein was then filtered, stored at $4^{\circ} \mathrm{C}$ and used within 3 weeks of collection. Before each centrifugation and following dialysis the following antioxidants were added: BPB $(40 \mu \mathrm{M})$, vitamin $\mathrm{E}(0.2 \mathrm{mM})$ and EDTA $(63 \mathrm{mM})$. Twelve batches of native LDL were prepared.

LDL was quantitated by measurement of protein content using a modified Lowry Protein assay (Method 6.3.2). Lipid peroxidation of samples was assessed using the TBA assay (Method 6.3.4), ultra violet spectrophotometric detection of diene conjugation absorption (Recknagel and Ghoshal, 1966), the loss of unsaturated fatty acid side-chains upon oxidation determined by gas-liquid chromatography (Method 6.3.3), and the composition of proteins in the lipoproteins assessed by polyacrylamide gel electrophoresis (Method 6.3.5). The amount of albumin present in the preparation was measured with an array protein system (Beckman). Finally, the mobility of protein and lipid in the lipoprotein preparations was assessed by agarose gel electrophoresis (Method 6.3.6). 


\subsubsection{Modified Lowry protein assay}

This method was based on the procedure described by Lowry et al (1951). The absorbance was read at $670 \mathrm{~nm}$ with a Hitachi U-2000 spectrophotometer.

\subsubsection{Determination of fatty acid content of LDL preparations by gas-liquid chromatography}

Lipid peroxidation results in the destruction of unsaturated fatty-acid sidechains. Separation of LDL fatty acids by gas-liquid chromatography (GLC) provides information on the oxidation of the LDL, especially the loss of polyunsaturated fatty acids (Halliwell and Gutteridge, 1986).

Lipids were extracted from $3 \mathrm{ml}$ aliquots of LDL with $9 \mathrm{ml}$ of chloroformmethanol $(2: 1 \mathrm{v} / \mathrm{v})$. After vigorous shaking, the mixture was centrifuged $\left(900 \times \mathrm{g}\right.$ for $5 \mathrm{~min}$ at $\left.20^{\circ} \mathrm{C}\right)$. The lower phase was evaporated under a gentle stream of nitrogen (oxygen-free). The fatty acids were then esterified using 1 $\mathrm{ml}$ of $14 \%$ boron trifluoride /methanol complex, and heated for $2 \mathrm{~min}$ at 100 ${ }^{\circ} \mathrm{C}$. The methyl esters were extracted into $5 \mathrm{ml}$ of hexane after the addition of $5 \mathrm{ml}$ of water and the top phase was then evaporated under nitrogen. Methyl esters were finally resuspended in $40 \mu \mathrm{l}$ of methylene chloride /butylated hydroxytoluene solution.

Fatty acid methylesters (FAMES) were separated by GLC using a $30 \mathrm{~m} \mathrm{x}$ $0.53 \mathrm{~mm}$ ID 50 \% cyanopropyl polysiloxane DB-23 megabore column ( $\mathrm{J}$ and W Scientific, Folsom, CA) and a flame ionization detector. Nitrogen was the carrier gas. Column conditions were optimised to separate peaks of interest. The temperature was programmed to run isothermally at $220^{\circ} \mathrm{C}$. Peaks were 
identified by comparison with chromatography of authentic FAMES mixtures. The classification follows that described by Nichols et al (1961). Standards of FAMES were obtained from Supelco, Inc. (Bellefonte, PA) and Alltech Associates (Deerfield, IL). A Hewlett Packard Model 3396A integrator was used to identify and measure the area of peaks. A coefficient of variation for measurement of the fatty acids was determined by processing the same sample 14 times on the same day and the patient variation assessed by processing serum from 5 donors each day for 5 days.

\subsubsection{The thiobarbituric acid (TBA) assay}

This assay was used to measure malondialdehyde (MDA) (a by-product of lipid peroxidation) according to the method of Buege and Aust (1978). A simplified scheme of MDA formation is:

$\mathrm{L} \cdot+\mathrm{O}_{2}$

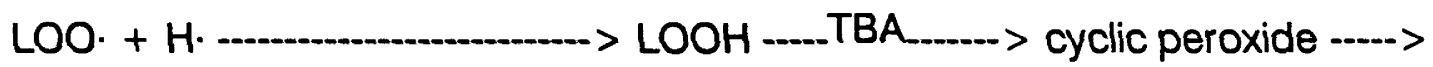

cyclic endoperoxide $+\mathrm{O}_{2}$--.-heat_-------> MDA.

MDA under acid conditions can react with TBA to generate a pink to red product which absorbs light at $535 \eta \mathrm{m}$ (Knight et al, 1988). A standard curve was determined using freshly prepared 1,1,3,3-tetraethoxypropane (TEP) which hydrolyses to MDA (Yagi, 1982). A working solution of $4.45 \mu \mathrm{M}$ TEP was prepared in $0.5 \mathrm{M} \mathrm{HCl}$. The range of the standards was from $0.45 \mu \mathrm{M}$ to $4.45 \mu \mathrm{M}$. As the TBA test is calibrated with MDA the results were expressed in terms of the amount of MDA (nmol MDA) produced per mg of protein. LDL preparations were generally assessed for the presence of MDA soon after preparation and following oxidation in copper sulphate. A time course of MDA production following oxidation in copper sulphate was also determined. Two batches of LDL stored at $4^{\circ} \mathrm{C}$ in antioxidants for 10 months were reassayed. 
The TBA assay is neither specific for the hydroperoxy group nor stoichiometric, as discussed by El-Saadani et al (1989), and linoleic acid, which comprises $98 \%$ of polyunsaturated fatty acids in LDL, does not produce MDA upon peroxidation (Halliwell and Gutteridge, 1986; Piotrowski et al, 1990). However the TBA test is still a useful measure of MDA formed from lipid peroxides and it allows a comparison between LDL preparations treated with antioxidants.

\subsubsection{Polyacrylamide gel electrophoresis of LDL preparations}

Discontinuous (Laemmli) polyacrylamide gels (10\% separating gel and a 4 $\%$ stacking gel) were prepared according to the instructions of the manufacturer (Biorad Laboratories, Richmond, Australia) based on the method of Laemmli (1970) under nonreducing conditions to assess the composition of the apoliprotein in the LDL preparations and the effect of oxidation on the protein composition of the LDL preparations.

\subsubsection{Agarose gel electrophoresis of LDL preparations}

Electrophoresis on agarose gels was used to assess the electrophoretic mobility of the lipoprotein preparations. The method was based on the technique of Noble (1968). A mini-protean II cell (Biorad Laboratories, Richmond, Australia) was used for preparation of the gel and electrophoresis of the samples. A $1 \%$ agarose gel was prepared by dissolving agarose in barbital buffer (refer to Section 5.2) and the gel was poured at $85^{\circ} \mathrm{C}$. The gel was allowed to set for $10 \mathrm{~min}$ at room temperature and then for $1 \mathrm{~h}$ at $4^{\circ} \mathrm{C}$. The barbital buffer was added to the upper and lower reservoirs before $10 \mu \mathrm{g}$ of sample made up to $10 \mu \mathrm{l}$ in sample buffer (refer to Section 6.2) was loaded into each well. Samples included freshly prepared LDL, minimally modified 
LDL (LDL stored in sterile plastic containers at $4^{\circ} \mathrm{C}$ for 6 to 10 months) and oxidized LDL (refer to Section 6.3.8). The gel was electrophoresed at $100 \mathrm{~V}$ for $5 \mathrm{~min}$ to ensure that all the samples had left each well, and then at $50 \mathrm{~V}$ for $2 \mathrm{~h}$ at $4{ }^{\circ} \mathrm{C}$. The gel was fixed using $5 \%$ glacial acetic acid in $75 \%$ ethanol for $30 \mathrm{~min}$, and then dried at $80^{\circ} \mathrm{C}$ on a glass plate for $1 \mathrm{~h}$ until completely transparent. The gel was finally stained in $0.2 \%$ Sudan Black for $1 \mathrm{~h}$, washed in water and blotted on filter paper soaked in $95 \%$ ethanol before being photographed. Alternatively, the gel was stained in $0.1 \%$ Coomassie blue R250 without prior fixing and destained for $1-3 \mathrm{~h}$ in $40 \%$ methanol and $10 \%$ acetic acid before being photographed.

\subsubsection{Effects of native and oxidized LDL on HUV endothelial cell proliferation}

An initial experiment was performed to assess the influence of FCS on the proliferative effect of native LDL on HUV endothelial cells. HUV endothelial cells (1.25 x $10^{4}$ cells /well) suspended in PRF Medium 199 (with standard additives) and [ $\left.{ }^{3} \mathrm{H}\right]$-thymidine $(0.5 \mu \mathrm{Ci} /$ well) were added to gelatinized 96 well plates. LDL $(1.0 \mathrm{mg} / \mathrm{ml})$ and FCS were then added and HUV endothelial cell proliferation was assessed over $18 \mathrm{~h}$ in $5 \% \mathrm{CO}_{2}-95 \%$ air atmosphere at 37 ${ }^{\circ} \mathrm{C}$. In order to determine whether the concentration of FCS influenced the effects of LDL, a range of concentrations of FCS was used (1-50\%). The cells were then washed, trypsinized and harvested as described in Section 2.3.7.

Following the initial experiments, HUV endothelial cells ( $5 \times 10^{3}$ cells /well) suspended in PRF Medium 199 (with $20 \%$ FCS, gentamicin $60 \mathrm{U} / \mathrm{ml}$ and the standard additives) were incubated for $18 \mathrm{~h}$ in $5 \% \mathrm{CO}_{2}-95 \%$ air atmosphere at $37^{\circ} \mathrm{C}$. The medium was removed and oxidized or native LDL was diluted in RD containing vitamin $\mathrm{E}(0.2 \mathrm{mM}), \mathrm{BPB}(40 \mu \mathrm{M})$ and $\mathrm{GSH}(1.63 \mathrm{mM})$. LDL concentrations between 0.125 and $2 \mathrm{mg} / \mathrm{ml}$ were chosen since the normal 
concentration of apo B in plasma is between 0.42 and $1.3 \mathrm{mg} / \mathrm{ml}$ (Young, 1990; Walmsley et al, 1991) and in type II hyperlipoproteinemias is higher. Controls included Medium 199 alone, RD alone, RD with vitamin E (final concentration $0.1 \mathrm{mM}$ ), BPB (final concentration $20 \mu \mathrm{M}$ ) and GSH (final concentration $0.815 \mathrm{mM}$ ). These were added to the cells for variable time intervals before washing the cells with Medium $199 .\left[{ }^{3} \mathrm{H}\right]$-thymidine $(0.5 \mu \mathrm{Ci}$ /well) in Medium 199 and $20 \%$ FCS with standard additives were then added. The system was incubated for $18 \mathrm{~h}$ in $5 \% \mathrm{CO}_{2}-95 \%$ air atmosphere at $37^{\circ} \mathrm{C}$. The cells were then washed, trypsinized and harvested as described in Section 2.3.7.

In a second series of experiments HUV endothelial cells $\left(1.25 \times 10^{4}\right.$ cells /well) were suspended in PRF Medium 199 with $2 \%$ FCS (final concentration), gentamicin $60 \mathrm{U} / \mathrm{ml}$, standard additives and $\left[{ }^{3} \mathrm{H}\right]$-thymidine ( $0.5 \mu \mathrm{Ci} /$ well). The LDL preparation and controls were the same as outlined above. The HUV endothelial cells were incubated for $18 \mathrm{~h}$ in $5 \% \mathrm{CO}_{2}-95 \%$ air atmosphere at $37^{\circ} \mathrm{C}$. The cells were then washed, trypsinized and harvested as described in Section 2.3.7. Similar experiments to those outlined above were performed in the absence of FCS. Using the same preparation of HUV endothelial cells proliferation was also assessed in the presence of 1 in 2 dilutions of different native, minimally modified and oxidized LDL batches to demonstrate variability of LDL preparations in terms of their effect on HUV endothelial cell proliferation over $18 \mathrm{~h}$.

The effect of sex steroids on the proliferative response of HUV endothelial cells to oxidized and native LDL in the presence and absence of FCS was assessed using the same protocols as outlined above. 


\subsubsection{Lipoprotein oxidation by copper sulphate}

Lipoprotein preparations were pipetted into dialysis tubing, placed in a measuring cylinder containing copper sulphate (final concentration $5 \mu \mathrm{M}$ ) in $\mathrm{RD}(\mathrm{pH} 7.2)$ and allowed to slowly rotate for $48 \mathrm{~h}$ at $37^{\circ} \mathrm{C}$. The total protein was reassayed using a modified Lowry protein assay (refer to Section 6.3.2) and the amount of MDA in the sample was then quantitated (refer to Section 6.3.4). The presence of conjugated dienes was also assessed. Agarose and polyacrylamide gel electrophoresis were performed to assess electromobility and the protein composition of LDL preparations (refer to Sections 6.3.6. and 6.3.5). Lipoprotein preparations oxidized by this method were used to study the effects of oxidized LDL on proliferation of HUV endothelial cells.

To study the effect of sex steroids on oxidative modification of LDL by copper sulphate, the LDL preparation was initially dialysed in RD for $18 \mathrm{~h}$ at 4 ${ }^{\circ} \mathrm{C}$ to remove BPB, EDTA and GSH. The protein concentration of the sample was then redetermined (refer to Section 6.3.2), and the sample was diluted in $\mathrm{RD}$ to a final concentration of $0.2 \mathrm{mg}$ protein $/ \mathrm{ml}$. LDL in the presence and absence of sex steroids (diluted in RD) was loaded into washed dialysis tubing, placed in a cylinder containing RD and copper sulphate $(5 \mu \mathrm{M})$ and slowly rotated for $9 \mathrm{~h}, 18 \mathrm{~h}$ or $24 \mathrm{~h}$ at $4^{\circ} \mathrm{C}$. The samples were removed from the copper sulphate solution and EDTA (63 mM), vitamin E (0.2 mM) and BPB $(40 \mu \mathrm{M})$ were then added. Controls included sex steroids in RD with no added LDL, RD with vitamin $\mathrm{E}(0.2 \mathrm{mM})$ and BPB $(40 \mu \mathrm{M}), \mathrm{RD}$ alone, LDL with vitamin $E(0.2 \mathrm{mM})$ and $L D L$ and alcohol $(0.1 \%)$. The total protein was reassayed using a modified Lowry protein assay. The amount of MDA in the sample was then quantitated. Agarose gel electrophoresis was performed on some occasions to assess electromobility of samples (refer to Section 6.3.6). 


\subsubsection{Oxidation of LDL by HUV endothelial cells.}

HUV endothelial cells were plated into gelatinized 24 well plates and grown to confluence in Medium 199 (with $20 \%$ FCS, gentamicin $60 \mathrm{U} / \mathrm{ml}$ and the standard additives).

Native LDL was dialysed in RD overnight at $4^{\circ} \mathrm{C}$ and the protein concentration was reassayed using a modified Lowry protein assay. LDL was diluted in RD to give a concentration of $0.4 \mathrm{mg}$ protein $/ \mathrm{ml}$ as described by Steinbrecher et al (1984). HUV endothelial cells were washed three times in Medium 199 and then incubated for $24 \mathrm{~h}$ at $37^{\circ} \mathrm{C}$ in the presence of LDL (final concentration $100 \mu \mathrm{g}$ protein $/ \mathrm{ml}$ ) and sex steroids, or sex steroids alone. The supernatant was removed and the samples were centrifuged to remove nonadherent cells and vitamin $E(0.2 \mathrm{mM})$ and BPB $(40 \mu \mathrm{M})$ was added. Controls included Medium 199 alone, Medium 199 with vitamin E (0.2 $m M)$, and LDL incubated in gelatinized plates in the absence of cells with or without vitamin $E(0.2 \mathrm{mM})$. The amount of MDA in the sample was quantitated (refer to Section 6.3.4).

\subsubsection{Statistical analysis}

Individual experiments were examined statistically using analysis of variance and is presented graphically as the mean \pm SD. Analysis of variance with regression was used for analysis of groups of experiments (Smart, 1970) where the experiment replicates were considered as a block effect and the treatments as fixed effects. The variance ratio ( $F$ test) was used to test for overall significance. The multiple t comparison (Bonferroni method) was then used to test for a significant difference between treatments. The Minitab programme (Data analysis software, 1987) was used for the above analyses. 
A $p$ value of 0.05 or less was taken to represent a statistically significant difference.

\subsection{RESULTS}

\subsubsection{Fatty acid profiles of LDL preparations as assessed by gas-liquid chromatography}

Fatty acid profiles of 5 batches of native LDL and oxidized LDL were determined using GLC. Figure 6.1 illustrates a GLC fatty acid profile of a freshly prepared sample of native LDL (batch 9). The percentage composition (relative to total fatty acid) was: saturated fatty acid palmitic acid (16:0) $39.15 \%$, stearic acid (18:0) $10.35 \%$, linoleic acid (18:2 cis and trans) $26.66 \%$, arachidonic acid (20:4) $6.98 \%$. GLC analysis of 5 batches of native LDL demonstrated an average percentage of linoleic acid to total fatty acid content of $27.8 \pm 3.11 \%$ (range 23.5 to $32.9 \%$ ) while the average percentage of arachidonic acid relative to total fatty acid content was $6.99 \pm$ $1.3 \%$ (range $5.04 \%$ to $9.11 \%$ ).

In contrast, GLC analysis of LDL which had been oxidized with copper sulphate showed a loss of polyunsaturated fatty acid (Figure 6.3). Arachidonic acid was found to be undetectable in 4 of 5 samples of oxidized LDL, with the fifth sample containing only $1.14 \%$ arachidonic acid relative to total fatty acid content. The percentage of linoleic acid relative to total fatty acid in the oxidized samples averaged $5.8 \pm 4.7 \%$ (range from $0.8 \%$ to 12.47 \%). Representative profiles of the fatty acid composition of native (batch 9) and oxidized LDL (batch 2 , which is the oxidized preparation from native LDL batch 9) samples are shown in Figures 6.1 and 6.3 with an appropriate reference chromatogram. The coefficient of variation for linoleic acid was $0.903 \%$ and for arachidonic acid was $2.77 \%$. 
Native LDL slowly oxidizes at $4^{\circ} \mathrm{C}$ despite the presence of antioxidants. This results in a loss of polyunsaturated fatty acids. For example, the linoleic acid content of batch 9 , when freshly prepared, comprised $26.66 \%$ of total fatty acids and, after storage for 3 months without antioxidants, was reduced to $17.10 \%$ of the total fatty acid content. The percentage of arachidonic acid decreased from $6.98 \%$ to $5.14 \%$ in the same time interval while saturated fatty acids increased as a percentage of the total but not in absolute amount (Figures 6.1 and 6.2). This LDL preparation has been termed minimally modified LDL (Berliner et al, 1990). This batch of LDL was reassessed 10 months later. A new column and extraction technique, however, were then in use making a direct comparison impossible.

\subsubsection{Extent of lipid peroxidation in LDL preparations using TBA assay}

Using the TBA assay, MDA was detected in 1 of the 9 batches of native LDL assessed. The MDA content of 8 samples of LDL oxidized in the presence of copper sulphate for $48 \mathrm{~h}$ at $37^{\circ} \mathrm{C}$ averaged $1.05 \pm 0.67 \mathrm{nmol} \mathrm{MDA} / \mathrm{mg}$ protein, ranging from 0.27 to $2.04 \mathrm{nmol} \mathrm{MDA} / \mathrm{mg}$ protein. Time courses of LDL oxidation by copper sulphate performed at $4^{\circ} \mathrm{C}$ also showed batch variability in the amount of MDA present in samples at different times and differences in the lag phase before MDA was detected despite using the same protein content in each experiment. One batch of LDL (batch 1), oxidized at $4^{\circ} \mathrm{C}$, had no MDA detectable in the native LDL preparation, 1.48 $\mathrm{nmol} \mathrm{MDA} / \mathrm{mg}$ protein at $8 \mathrm{~h}, 2.5 \mathrm{nmol} \mathrm{MDA} / \mathrm{mg}$ protein at $18 \mathrm{~h}$ and 6.7 $\mathrm{nmol} \mathrm{MDA} / \mathrm{mg}$ protein at $48 \mathrm{~h}$. In contrast, another batch (batch 3) of LDL, also oxidized at $4^{\circ} \mathrm{C}$, had no detectable MDA in the native preparation, 9.1 $\mathrm{nmol}$ MDA/ mg protein at $4 \mathrm{~h}, 18.7 \mathrm{nmol} M D A / m g$ protein at $9 \mathrm{~h}, 20 \mathrm{nmol}$ MDA/ mg protein at $18 \mathrm{~h}, 17.55 \mathrm{nmol}$ MDA/ mg protein at $24 \mathrm{~h}, 7.9 \mathrm{nmol}$ 
MDA/ mg protein at $36 \mathrm{~h}, 3.95 \mathrm{nmol} \mathrm{MDA} / \mathrm{mg}$ protein at $48 \mathrm{~h}, 3.3 \mathrm{nmol}$ $\mathrm{MDA} / \mathrm{mg}$ protein at $60 \mathrm{~h}$ and no MDA at $72 \mathrm{~h}$ (Figure 6.4).

Two samples of native LDL were stored at $4{ }^{\circ} \mathrm{C}$ with antioxidants for approximately 10 months and then reassessed for the presence of MDA. One sample (batch 9) showed an increase from no detectable MDA to 0.38 $\mathrm{nmol} \mathrm{MDA} / \mathrm{mg}$ protein in 10 months while the same sample oxidized at $37^{\circ} \mathrm{C}$ for $48 \mathrm{~h}$ in the presence of copper sulphate had $0.414 \mathrm{nmol}$ MDA / $\mathrm{mg}$ protein (batch 2). The second sample (batch 7) had no detectable MDA initially while 10 months later there was $0.23 \mathrm{nmol} \mathrm{MDA} / \mathrm{mg}$ protein. This same sample oxidized in the presence of copper sulphate at $37^{\circ} \mathrm{C}$ for $48 \mathrm{~h}$ had $0.79 \mathrm{nmol}$ MDA /mg protein (batch 3). Batches 7 and 9 were also assessed by electrophoresis to further demonstrate their modification with time (refer below).

\subsubsection{Presence of conjugated dienes in LDL preparations}

Conjugated dienes were assessed at $234 \mathrm{~nm}$ absorption. The absorbance range examined was $200-320 \mathrm{~nm}$. In native preparations of LDL (using 100 $\mu \mathrm{g} / \mathrm{ml}$ protein concentration) the average amount of conjugated dienes present was $0.434 \pm 0.068$ absorbance units. In minimally modified LDL preparations more conjugated dienes $(0.7205 \pm 0.2)$ were present while in the LDL samples oxidized for $48 \mathrm{~h}$ at $37^{\circ} \mathrm{C}$ the amount of conjugated dienes in the preparations was $1.16 \pm 0.44$ absorbance units. The presence of conjugated dienes in a pooled LDL preparation was also assessed over 5 days of oxidation with copper sulphate at $4^{\circ} \mathrm{C}$ (Figure 6.4). There was a gradual increase in conjugated dienes over the first $48 \mathrm{~h}$ beyond which the amount of conjugated dienes plateaued (Figure 6.4). The amount of conjugated dienes present in a solution containing only Vitamin E and BPB was small (0.087 absorbance units). 


\subsubsection{Electrophoresis of LDL samples in agarose and polyacrylamide gels}

Figure 6.4 demonstrates the different electrophoretic mobilities of a pooled LDL preparation exposed to copper sulphate at $4^{\circ} \mathrm{C}$ for various time intervals. There was an initial increase in electromobility of the samples with increasing time. From $18 \mathrm{~h}$ onwards the mobility of the LDL preparation did not change significantly. The presence of MDA and conjugated dienes was also assessed in these samples (refer above).

Figure 6.5 demonstrates the different electrophoretic mobilities of the proteins and lipid in various LDL preparations. LDL which has been oxidized is more electronegative (Lanes 1 and 2) than minimally modified LDL (Lanes 3, 4 and 5) and native LDL (Lanes 7 and 8 ) as previously reported by other investigators (Henriksen et al, 1981; 1983; Sternberg, 1983; Morel et al, 1984; Steinbrecher et al, 1984; Nagelkerke et al, 1984; Van Hinsbergh et al, 1986; Zawadzki et al, 1989). The LDL preparations contained only one band of lipid but did contain some protein which migrated a similar distance to human serum albumin (demonstrated in Lane 6 in Figure 6.5, Upper). These two gels were electrophoresed on the same day in the same gel apparatus for direct comparison. As expected no lipid was present in the human serum albumin preparation (Lane 6, Figure 6.5, Lower).

Polyacrylamide gel electrophoresis of various LDL preparations is shown in Figures 6.6 and 6.7. Electrophoresis was performed to further examine the composition of the LDL preparations used. In Figure 6.6 the electrophoretic pattern of LDL (Lane 1) with a band at about MW 500000 (probably apo B100 ) is quite distinct from HDL (Lane 4) in which protein bands at the same molecular weights as apo E (MW 37000 ) and apo A-1 (MW 28 300) can be seen as distinct bands. Also shown in Figure 6.6 are the electrophoretic 
patterns seen with oxidized LDL (Lane 2), oxidized HDL (Lane 5) and human serum albumin (Lane 7). Some protein did not appear to migrate at all. The nature of this protein (s) is uncertain. Figure 6.7 shows preparations of native LDL in Lanes 1 and 2. In these two preparations one major protein migrated with the same molecular weight as apo B (approximately 500 000). Other protein bands are also seen, in particular albumin (MW approximately 55000 ) (refer to Figure 6.6). Minimally modified LDL is seen in Lanes 4, 5 and 6. There appears to be less protein migrating at the same molecular weight as apo B. Following oxidation, there was minimal protein migrating at the same molecular weight as apo B (Lanes 8 and 9). Albumin in the oxidized LDL preparations has also been modified and migrates as a more diffuse band. The amount of albumin present in these preparations was assessed using an array protein system. The average amount of albumin present in the native LDL preparations was $111 \pm 3.9 \mathrm{mg} / \mathrm{L}$ while the albumin content of the HDL preparation was $515 \mathrm{mg} / \mathrm{L}$. Lanes 3 and 6 in Figure 6.6 are the molecular weight markers while in Figure 6.7 they appear in Lanes 3 and 7.

\subsubsection{Effect of native and oxidized LDL on HUV endothelial cell proliferation}

Initial experiments were performed to assess the influence of FCS on the proliferative effects of native LDL on HUV endothelial cells. In all experiments at least 3 batches of LDL were used unless stated otherwise, and experiments on each batch were performed in triplicate. The graphs shown were selected as representative of the experiments. FCS had an optimal proliferative effect at a concentration between $5-10 \%$ as previously reported in Chapter 2. The proliferative effect of native LDL $(1 \mathrm{mg} / \mathrm{ml})$ on HUV endothelial cells was observed only when the concentration of FCS was reduced to less than $10 \%$ (Figure 6.8). With this batch of LDL the maximal proliferative effect of native LDL $(1 \mathrm{mg} / \mathrm{ml})$ occurred in the presence of $5 \%$ FCS. HUV endothelial cell proliferation increased by $142 \%$ (Figure 6.8). In 
the absence of FCS, LDL ( $1 \mathrm{mg} / \mathrm{ml})$ increased proliferation of HUV endothelial cells by $97 \%$ compared to Medium 199 alone (Figure 6.8).

The effects of native and oxidized LDL on HUV endothelial cell proliferation were studied in the presence of FCS initially. There was no significant change in HUV endothelial cell proliferation when cells were exposed to native LDL over the range 0.25 to $2 \mathrm{mg} / \mathrm{ml}$ for $15 \mathrm{~min}, 1 \mathrm{~h}, 6 \mathrm{~h}$ or $18 \mathrm{~h}$ in the presence of $20 \%$ FCS (Figures 6.9 and 6.10). Following $42 \mathrm{~h}$ incubation HUV endothelial cell proliferation was significantly inhibited by LDL at $1 \mathrm{mg} / \mathrm{ml}(19 \%)$ and at 2 $\mathrm{mg} / \mathrm{ml}(69 \%)$ in the presence of $20 \%$ FCS in 2 out of 3 experiments (Figure 6.10). Figure 6.10 illustrates the data from one batch of LDL which caused inhibition of HUV endothelial cell growth at $42 \mathrm{~h}$ but not at $18 \mathrm{~h}$.

Oxidized LDL $(0.25-2 \mathrm{mg} / \mathrm{ml})$ after $15 \mathrm{~min}$ and $1 \mathrm{~h}$ resulted in a significant $(p<0.05)$ inhibition of HUV endothelial cell proliferation in the presence of 20 $\%$ FCS (Figures 6.11 and 6.12). After $18 \mathrm{~h}$ and $42 \mathrm{~h}$ there was also significant inhibition of HUV endothelial cell proliferation by oxidized LDL, the maximal effect being observed at $1.0-2.0 \mathrm{mg} / \mathrm{ml}$. In the above experiments the addition of vitamin E, BPB and RD separately and together had no effect on HUV endothelial cell proliferation relative to control (Medium 199 alone).

The effect of oxidized and native LDL was assessed in the presence of $2 \%$ FCS over $18 \mathrm{~h}$. Native LDL $(0.125-2 \mathrm{mg} / \mathrm{ml})$ did not significantly alter HUV endothelial cell proliferation in the presence of $2 \%$ FCS (Figure 6.13), while oxidized LDL significantly inhibited HUV endothelial cell proliferation at these same concentrations (Figure 6.14). Vitamin E, BPB and RD separately and together in the absence of LDL had no effect on HUV endothelial cell proliferation relative to control (Medium 199 alone). 
LDL on HUV endothelial cell proliferation in the absence of FCS. Experiments were conducted using 5 batches of native LDL and oxidized LDL. Concentrations of native LDL greater than $0.5 \mathrm{mg} / \mathrm{ml}$ significantly increased HUV endothelial cell proliferation (e.g. by up to $336 \%$ in the presence of $2 \mathrm{mg}$ /ml LDL) (Figure 6.13), while concentrations of oxidized LDL greater than $0.125 \mathrm{mg} / \mathrm{ml}$ significantly inhibited HUV endothelial cell proliferation (by up to $99.6 \%$ ) in the absence of FCS over $18 \mathrm{~h}$ (Figure 6.14). Microscopically, HUV endothelial cells exposed to oxidized LDL were contracted and many had lifted off the gelatin coated wells. The relative change in HUV endothelial cell proliferation compared to control cells varied from batch to batch of LDL, this variation being especially evident at concentrations greater than $1 \mathrm{mg} / \mathrm{ml}$. The effect of serial dilutions of different batches of native LDL and minimally modified LDL on the same preparation of HUV endothelial cells is illustrated in Figure 6.15. Serial dilutions of minimally modified LDL did not inhibit endothelial cell proliferation at any of the concentrations examined (Figure 6.15). Exposure of HUV endothelial cells to oxidized LDL at concentrations of $10-100 \mu \mathrm{g} / \mathrm{ml}$ caused endothelial cells to proliferate relative to control (medium 199 alone). At higher concentrations there was marked inhibition of HUV endothelial cell proliferation as detailed above (Figure 6.16).

\subsubsection{Modulation by sex steroids of the effects of native and oxidized LDL on HUV endothelial cell proliferation}

These experiments were carried out to examine the influence of sex steroids on the proliferative effects of different LDL preparations on HUV endothelial cells. In the absence of FCS, $17 \beta$-estradiol $\left(10^{-7}\right.$ and $\left.10^{-10} \mathrm{M}\right)$, progesterone $\left(10^{-9} \mathrm{M}\right)$, and the two steroids together did not alter the proliferative effect of native LDL ( 0.125 to $2 \mathrm{mg} / \mathrm{ml}$ ) on HUV endothelial cells (Figures 6.17, 6.18 and 6.19). However, high concentrations of $17 \beta$-estradiol $\left(10^{-5} \mathrm{M}\right)$, progesterone $\left(10^{-5} \mathrm{M}\right)$, and the two steroids together inhibited the 
proliferative effect of native LDL especially at the lower concentrations of LDL $(0.125-0.5 \mathrm{mg} / \mathrm{ml})$. The inhibitory effect of $17 \beta$-estradiol $\left(10^{-5} \mathrm{M}\right)$ on HUV endothelial cell proliferation was gradually reduced with increasing concentrations of native LDL, being overcome completely in the presence of native LDL at a concentration of $2 \mathrm{mg} / \mathrm{ml}$ (Figure 6.17). The same effect was also seen with LDL in the presence of progesterone $\left(10^{-5} \mathrm{M}\right)$, and $17 \beta$ estradiol $\left(10^{-5} \mathrm{M}\right)$ in combination with progesterone $\left(10^{-5} \mathrm{M}\right)$ (Figures 6.18 and 6.19). No enhancement of HUV endothelial cell proliferation was seen in the presence of $17 \beta$-estradiol $\left(10^{-10} \mathrm{M}\right)$ and native LDL.

$17 \beta$-estradiol $\left(10^{-10} \mathrm{M}\right)$, progesterone $\left(10^{-9} \mathrm{M}\right)$, and the two steroids together did not alter the depressive effect of oxidized LDL on HUV endothelial cell proliferation (Figures 6.20, 6.21 and 6.22). 17 $\beta$-estradiol (10-5 $M)$, progesterone $\left(10^{-5} \mathrm{M}\right)$, and the combination of these two steroids caused further inhibition of proliferation by oxidized LDL. Alcohol $(0.05 \%)$, which was used to dissolve the sex steroids, did not significantly alter HUV endothelial cell proliferation in the presence of various concentrations of native or oxidized LDL.

\subsubsection{Inhibitory effect of $17 \beta$-estradiol on peroxidation of LDL by} copper sulphate.

These experiments were performed to determine whether $17 \beta$-estradiol has antioxidant properties. After $9 \mathrm{~h}$ incubation of LDL with copper sulphate there was an increase in MDA content of LDL which had been incubated with 17 $\beta$ estradiol $\left(10^{-5} \mathrm{M}\right)$ and $17 \beta$-estradiol $\left(10^{-10} \mathrm{M}\right)$ in combination with progesterone $\left(10^{-9} \mathrm{M}\right)$. When $\mathrm{LDL}$ was incubated with vitamin $\mathrm{E}(100 \mu \mathrm{g} / \mathrm{ml})$ for $9 \mathrm{~h}$ there was a significant reduction in the MDA content of the preparation relative to LDL alone. There was no significant difference in the MDA content 
between control preparations (LDL alone) and LDL incubated with $17 \beta$ estradiol (10-10 $\mathrm{M}$ ) after $9 \mathrm{~h}$ (Figure 6.23).

After $18 \mathrm{~h}$, LDL which had been incubated with 17 -estradiol (10-10 M) contained significantly less MDA compared to LDL alone $(n=5)$. Inhibition of MDA content was also seen when LDL was incubated with 17 $\beta$-estradiol (10$10 M)$ in combination with progesterone $\left(10^{-9} M\right)(n=2)$, and when LDL was incubated with vitamin $E(100 \mu \mathrm{g} / \mathrm{ml})$. 17k-estradiol $\left(10^{-5} \mathrm{M}\right)$ had no significant effect on the MDA content of LDL relative to LDL alone $(n=5)$ (Figure 6.23) with 3 experiments showing an increase in MDA production and 2 experiments showing a decrease (Figure 6.23).

No significant reduction in MDA content in LDL preparations was observed after $24 \mathrm{~h}$ oxidation in the presence of any of the above treatments (Figure 6.23). To exclude the possibility that alcohol, $17 \beta$-estradiol or vitamin E may have influenced the TBA assay, LDL was omitted from samples and the MDA content assessed. No MDA was detected in RD, or in RD containing $17 \beta-$ estradiol, vitamin $\mathrm{E}$ or alcohol, or in $17 \beta$-estradiol added to a RD sample immediately prior to measuring MDA content. The inhibition of LDL oxidation by $17 \beta$-estradiol $\left(10^{-10} \mathrm{M}\right)$ and vitamin $\mathrm{E}$ was confirmed by agarose gel electrophoresis which showed reduced electromobility of LDL samples incubated with $17 \beta$-estradiol (10-10 M) and vitamin $E$ at $18 \mathrm{~h}$ (Figure 6.25). Preliminary experiments did not show any effect of progesterone $\left(10^{-5} \mathrm{M}\right.$ and $10^{-9} \mathrm{M}$ ) on oxidation. The effect of coincubation of $17 \beta$-estradiol $\left(10^{-5} \mathrm{M}\right)$ and progesterone $\left(10^{-5} \mathrm{M}\right)$ was similar to $17 \beta$-estradiol $\left(10^{-5} \mathrm{M}\right)$ alone. In summary, vitamin $E$ and $17 \beta$-estradiol (10-10 $\mathrm{M}$ ) inhibited LDL oxidation in a time dependent fashion. The effect of $17 \beta$-estradiol (10-10 M) was not influenced by the addition of progesterone. In contrast $17 \beta$-estradiol $\left(10^{-5} \mathrm{M}\right)$ caused an initial increase in the MDA content of the LDL samples examined. 
The level of MDA present in the LDL preparations incubated for the same time in copper sulphate varied between experiments as described in Section 6.4.2. In some experiments the amount of MDA in the LDL preparations increased with increasing time of oxidation with copper sulphate but generally the MDA content decreased after $18 \mathrm{~h}$ as demonstrated previously (Figures 6.4 and 6.25). The average MDA content of LDL alone following $9 \mathrm{~h}$ incubation with copper sulphate was $7.4 \pm 4.3 \mathrm{nmol}$ MDA $/ \mathrm{mg}$ protein, at $18 \mathrm{~h}$ it was $7.95 \pm 2.9 \mathrm{nmol} \mathrm{MDA} / \mathrm{mg}$ protein and at $24 \mathrm{~h} 5.22 \pm 3.1 \mathrm{nmol}$ MDA /mg protein.

\subsubsection{Influence of sex steroids on oxidative modification of LDL caused by HUV endothelial cells}

These experiments were performed to determine whether $17 \beta$-estradiol exhibited antioxidant properties in a biological system using HUV endothelial cells as an oxidant source. The TBA assay was used to assess oxidation of LDL by endothelial cells. Controls consisted of cells cultured without LDL and LDL incubated at $37 \circ \mathrm{C}$ on gelatin-coated plates without exposure to HUV endothelial cells.

In preliminary experiments the oxidation of LDL by HUV endothelial cells after $14 \mathrm{~h}$ and $18 \mathrm{~h}$ resulted in very low levels of MDA thus making interpretation impossible. However, after $24 \mathrm{~h}$ exposure of LDL to HUV endothelial cells the level of MDA was high enough to obtain reproducible results. The sex steroids, and in particular $17 \beta$-estradiol, did not inhibit oxidation of LDL by HUV endothelial cells (Figure 6.24). The average MDA content in the control wells for the three experiments was $8.26 \pm 3.4 \mathrm{nmol}$ MDA /mg protein (range 3.5 to $11.03 \mathrm{nmol}$ MDA /mg protein). Vitamin E inhibited oxidation by $20 \%$. LDL incubated in Medium 199 alone at $37^{\circ} \mathrm{C}$ with no cells also underwent some oxidation which varied between 
experiments $(3.8 \mathrm{nmol} \mathrm{MDA} / \mathrm{mg}$ protein in the first experiment, $0.8 \mathrm{nmol}$ MDA / $\mathrm{mg}$ protein in the second and $7 \mathrm{nmol}$ MDA / $\mathrm{mg}$ protein in the third experiment). The presence of lipid released from the HUV endothelial cells resulted in a background level of MDA in the absence of LDL. This was subtracted from the MDA level measured in LDL samples incubated with HUV endothelial cells.

\subsection{DISCUSSION}

The above studies have shown that: (1) native LDL stimulates HUV endothelial cell proliferation (depending on the concentration of FCS) in a concentration and time dependent manner; (2) oxidized LDL inhibits HUV endothelial cell proliferation in a concentration dependent manner; (3) native LDL appeared to undergo oxidative changes with time; (4) low concentrations of $17 \beta$-estradiol and progesterone do not influence the proliferative effects of native and oxidized LDL, while higher concentrations of $17 \beta$-estradiol and progesterone inhibit the proliferative effect of native LDL and augmented the inhibition of proliferation caused by oxidized LDL; (5) $17 \beta$-estradiol (10-10 M) reduces MDA formation in LDL preparations exposed to copper sulphate in a time and concentration dependent manner; but (6) has no effect on modification of LDL by HUV endothelial cells. Progesterone $\left(10^{-9} \mathrm{M}\right)$ did not alter the antioxidant effect of $17 \beta$-estradiol (10-10 M).

Native LDL preparations caused a significant increase in HUV endothelial cell proliferation in the presence and absence of FCS over $18 \mathrm{~h}$. The maximal proliferative response of HUV endothelial cells to native LDL was seen in the physiological range $(600$ to $800 \mu \mathrm{g} / \mathrm{ml}$ ) for most batches of LDL. This proliferative response to increased LDL can be attributed to the fact that LDL plays a regulatory role in cholesterol synthesis which is important in cell division and maintenance of cell membranes (Fielding and Fielding, 1984). In 
the current studies, no toxicity (assessed microscopically and in terms of endothelial cell proliferation) was seen at concentrations of native LDL as high as $10 \mathrm{mg} / \mathrm{ml}$ over $18 \mathrm{~h}$, supporting the findings of Steinbrecher et al (1984), Evensen et al (1983), Van Hinsberg et al (1986) and Triau et al (1988). Tauber et al (1980) used LDL preparations which contained no added antioxidants and found LDL to be mitogenic for bovine vascular endothelial cells at low concentrations (10-100 $\mu \mathrm{g}$ protein $/ \mathrm{ml})$, but once physiological concentrations (600 to $800 \mu \mathrm{g}$ protein $/ \mathrm{ml}$ ) were reached the LDL became toxic to the cells in the absence of FCS. This was also seen in the current studies when HUV endothelial cells were exposed to oxidized LDL. Prolonged exposure of HUV endothelial cells to native LDL at higher concentrations (1-2 $\mathrm{mg} / \mathrm{ml}$ ) also caused inhibition of endothelial cell proliferation. This may have been due to oxidative modification of the LDL by the HUV endothelial cells, despite the presence of vitamin E, BPB and GSH in the culture medium, although this was not formally assessed.

Oxidized LDL, in the current studies, caused significant inhibition of HUV endothelial cell proliferation at concentrations greater than $125 \mu \mathrm{g} / \mathrm{ml}$. This may represent a cytotoxic effect of $L D L$, which has been demonstrated by others (Henriksen et al, 1977; 1979a; 1979b; Hessler et al, 1979; Tauber et al, 1980; Evensen et al, 1983; Morel et al, 1983a). The mechanism of cell damage induced by oxidized LDL is unknown but the toxic component resides in the lipid phase of the LDL molecule (Hessler et al, 1983), with proliferating cells being more susceptible (Morel et al, 1983; Hessler et al, 1983). Kuzuya et al (1991) proposed that copper, bound to oxidized LDL, was responsible for LDL toxicity towards HUV endothelial cells. This cannot be excluded in the present studies since copper was not removed from the oxidized LDL samples following oxidation. However, the final concentration of copper (15-125 $\eta \mathrm{M}$, if one assumes that all the copper bound to the LDL, which is unlikely) used in the present experiments was much less than that (6 
$\mu \mathrm{M})$ used by Kuzuya et al (1991). Free radicals are involved in the oxidation of LDL, rendering it cytotoxic, but free radicals in the LDL preparations do not seem to be responsible for the cell damage since BHT does not inhibit cytotoxicity of oxidized LDL to endothelial cells (Morel et al, 1983).

In the current studies minimally oxidized LDL, despite being more electronegative, having less polyunsaturated fat relative to native LDL preparations and more conjugated dienes present than the native preparations, did not inhibit HUV endothelial cell proliferation over $18 \mathrm{~h}$ suggesting that such modifications to LDL do not significantly alter the proliferative ability of LDL in contrast to the effect of more oxidized LDL. Berliner et al (1990) also found that minimally modified LDL (5 to $120 \mu \mathrm{g} / \mathrm{ml}$ ) was not cytotoxic to HUV endothelial cells. In the current studies the antioxidants (in particular, vitamin E) added to the LDL preparations did not influence HUV endothelial cell proliferation as previously demonstrated by Huang et al (1988).

The addition of FCS masked the proliferative effect of native LDL in the current studies. Interestingly, even $2 \%$ FCS could mask the proliferative effect of many native LDL preparations. This was probably due to the presence of lipoproteins and other mitogens in the FCS. Kruth et al (1979) demonstrated, using an indirect immunofluorescence technique, that fibroblasts grown in a medium containing FCS bound little or no LDL. In the current studies, the inhibitory effect of oxidized LDL was reduced by the addition of FCS in a concentration dependent manner. This may be due to the presence of HDL in the FCS which enhances proliferation, or to sulfhydryl groups on proteins and other substances which bind to oxidized LDL, reducing its toxicity. This observation is supported by the findings of Henriksen et al (1977) who found that the mitogenic effect of LDL, in the absence of antioxidants, depended on the concentration of serum present. 
The sex steroids, and in particular $17 \beta$-estradiol $\left(10^{-10} \mathrm{M}\right)$, were unable to prevent the inhibitory effects of oxidized LDL on HUV endothelial cell proliferation suggesting that they cannot render it less toxic once it is oxidized. High concentrations of $17 \beta$-estradiol $\left(10^{-5} \mathrm{M}\right)$ and progesterone $\left(10^{-5} \mathrm{M}\right)$ augmented the inhibition of proliferation of HUV endothelial cells caused by oxidized LDL. This inhibition of proliferation by sex steroids is similar to that reported in Chapter 3 in the presence and absence of FCS. The addition of low concentrations of sex steroids did not alter the proliferative response of HUV endothelial cells to native LDL. However, native LDL was able to overcome the depressive effect of $17 \beta$-estradiol $\left(10^{-5} \mathrm{M}\right)$ and progesterone $\left(10^{-5} \mathrm{M}\right)$ on HUV endothelial cell proliferation more effectively than did $20 \%$ FCS (refer to Chapter 3). This may have been due, in part, to the incorporation of these sex steroids into the LDL structure which would then protect endothelial cells from high concentrations of these sex steroids. The uptake of steroids by lipoproteins has been demonstrated by Leszczynski and Schafer (1991).

In replicate experiments there was some variation in the effects of native and oxidized LDL on HUV endothelial cell proliferation, but good reproducibility was obtained within each experiment. Coetzee et al (1979), Van Hinsbergh et al (1983) and Poumay and Ronveaux-dupal (1988) have also reported this, and suggested that the differences were due to biological variation in the different cell cultures (as already reported in Chapter 3), in the LDL preparations or in both. This is supported by observations that different donors have different LDL fatty acid profiles as determined by GLC (Esterbauer et al, 1988; 1990). Low levels of linoleic acid in adipose tissue and in plasma cholesterol esters are associated with CHD, especially in those who smoke cigarettes (Wood et al, 1984; Kirkeby et al, 1972; Harats et al, 1989; Duthie et al, 1991). Patients may also have different plasma antioxidant 
levels (e.g. vitamin E and carotenoids), which could help explain the observed differences in fatty acid composition (Lee, 1980; Esterbauer et al, 1990).

The observed decrease in MDA content of some oxidized LDL preparations exposed to copper sulphate for several days suggests MDA becomes modified or metabolized so that it is no longer recognized by the TBA assay. Decreases in MDA with time have also been observed by Kim and LaBella (1987) and Malden et al (1991). The variable MDA content of native LDL preparations similarly treated may reflect the donors' diets and in particular ingestion of antioxidants and the type of lipids consumed.

Oxidized LDL may not only promote atherosclerosis by being taken up by the macrophage (and so cause fatty streak formation), but it may also cause endothelial cell damage which is important in atherogenesis (refer to Chapter 1). As a consequence any factor which can inhibit or retard the oxidation of LDL rendering it less cytotoxic may be important in inhibiting the progression of atherosclerosis. Estrogen may be one such factor. The studies reported in this Chapter show that $17 \beta$-estradiol $\left(10^{-10} \mathrm{M}\right)$ but not $17 \beta$-estradiol $\left(10^{-5}\right)$ was able to retard LDL oxidation caused by $5 \mu \mathrm{M}$ copper sulphate at $18 \mathrm{~h}$ but not at $9 \mathrm{~h}$ or $24 \mathrm{~h}$. The addition of progesterone $\left(10^{-9} \mathrm{M}\right)$ did not appear to alter the antioxidant effect of $17 \beta$-estradiol $\left(10^{-10}\right)$. This is not surprising since steroid hormones such as progesterone lack the phenolic A ring found in $17 \beta$-estradiol which is thought to give rise to its antioxidant properties (Adelman et al, 1991). The time dependence for oxidation probably reflects the way in which estradiol is thought to act. $17 \beta$-estradiol is thought to act as a chain breaking antioxidant probably by donating hydrogen atoms from phenolic hydroxyl groups to lipid peroxyradicals to terminate the chain 
reaction. A simplified scheme for the possible action of $17 \beta$-estradiol is depicted below:

Propagation : $\mathrm{LOOH}+\mathrm{Cu}^{2}+\ldots$

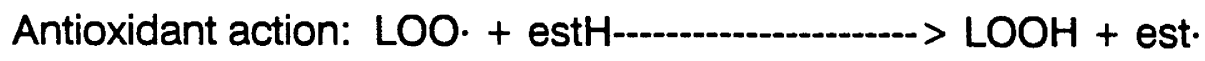

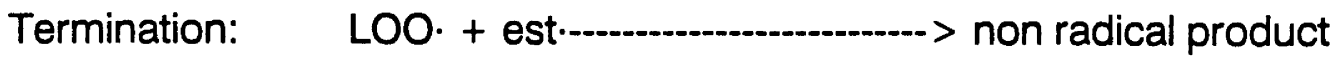
(estH $=17 \beta$-estradiol)

$17 \beta$-estradiol may also regenerate active vitamin $E$ by donating a hydrogen atom to the vitamin $\mathrm{E}$ radical, thereby terminating the chain of lipid peroxidation. Such a mechanism has been demonstrated in the maintenance of vitamin E levels by ascorbic acid (Niki, 1987a; b; Jialal and Grundy, 1991).

One possible reason for not observing the antioxidant effect of $17 \beta$-estradiol $\left(10^{-10} \mathrm{M}\right)$ at $9 \mathrm{~h}$ is the presence of antioxidants in the LDL molecule such as vitamin $E$ which must be consumed first to allow oxidation to commence. The time dependence of LDL oxidation is well established (Morel et al, 1984; Esterbauer et al, 1990). Esterbauer et al (1988) found that it took about $6 \mathrm{~h}$ under mild oxidative conditions for the vitamin $E$ and carotene content to decrease by $80 \%$ or more, and only then did lipid peroxidation commence, while it took just $2 \mathrm{~h}$ in the presence of copper $(2 \mu \mathrm{M})$ at $37^{\circ} \mathrm{C}$. The LDL preparations used in the present studies were prepared with the addition of antioxidants at each step so that variable oxidation at $9 \mathrm{~h}$ is consistent with the findings of other investigators (Henriksen et al, 1983; Jessup et al, 1990; Babiy et al, 1990). The antioxidant effect of $17 \beta$-estradiol $\left(10^{-10} \mathrm{M}\right)$ was overcome at $24 \mathrm{~h}$ in the studies reported in this Chapter. This suggests that $17 \beta$-estradiol was consumed or inactivated so that eventually oxidation of LDL was able to proceed. This observation is supported by the work of Huber et al (1990) and Niki and Nakano (1990) who both demonstrated that the antioxidant effect of $17 \beta$-estradiol could be overcome with time. 
An interesting observation was that $17 \beta$-estradiol $\left(10^{-5} \mathrm{M}\right)$ and the combination of $17 \beta$-estradiol $\left(10^{-10} \mathrm{M}\right)$ with progesterone $\left(10^{-9} \mathrm{M}\right)$ increased the MDA content in LDL preparations at $9 \mathrm{~h}$ but not after $18 \mathrm{~h}$. Liehr and Roy (1990) showed that pharmacological concentrations of estradiol could be oxidized to catechol estrogens. Catechol estrogens have been shown to participate in the formation of free radicals by redox cycling and thus could promote oxidation measured as increased MDA in the samples. However, chronic $17 \beta$-estradiol treatment of hamsters has not been shown to increase lipid peroxidation as assessed by malondialdehyde production measured by the TBA assay, although fluorescent products of lipid peroxidation increased (Roy and Liehr, 1989). This latter difference may reflect the lack of specificity of the TBA assay especially when used in an in vivo system (Knight et al, 1988). The reason for the loss of the pro-oxidant effect of $17 \beta$-estradiol (10-5 $\mathrm{M})$ and the combination of $17 \beta$-estradiol $\left(10^{-10} \mathrm{M}\right)$ with progesterone $\left(10^{-9} \mathrm{M}\right)$ at $18 \mathrm{~h}$ is not clear but may reflect consumption of the steroid. Thus, while a small amount of estrogen may act as an antioxidant, high concentrations of estrogen may promote free radical formation and lipid peroxidation.

Two recent studies suggest that $17 \beta$-estradiol at high concentrations (1-50 $\mathrm{x}$ $10^{-6}$ M) may act as an antioxidant (Maziere et al, 1991; Adelman et al, 1991). The study by Adelman et al (1991) has only been published in abstract form and is therefore difficult to evaluate because of lack of detail of experimental design. The study by Maziere et al (1991) differs from the present studies in that a higher concentration of oxidant was used, since Ham's F10 media itself generally contains about $10 \eta \mathrm{M}$ copper sulphate and $3 \mu \mathrm{M}$ iron sulphate to which a further $5 \mu \mathrm{M}$ copper sulphate was added. Whether antioxidants were used during preparation of LDL is not clear in the study by Maziere et al except that EDTA was added in the final stage of preparation. Therefore the composition of this LDL may well have differed from the LDL in the present studies, which might account for differences in oxidation. 
Oxidation of LDL by HUV endothelial cells was evident at $24 \mathrm{~h}$ as demonstrated by others (Morel et al, 1982; Van Hinsberg et al, 1986; Nagelkerke et al, 1984). The variable MDA content recorded between experiments has also been reported by others (Esterbauer et al, 1990). Possible reasons for this variability include differences in fatty acid composition, differences in the generation of MDA by damaged cells or differences in metabolism of MDA by cells (Esterbauer et al, 1990). Nevertheless, there was no significant effect of sex steroids, and in particular $17 \beta$-estradiol, on oxidation of LDL by HUV endothelial cells. The inhibition of oxidation by vitamin E, BPB and glutathione was less than expected from previous publications (Steinbrecher et al, 1984; Morel et al, 1984) and may reflect different experimental design.

There are many possible explanations for the inability of estrogen to inhibit HUV endothelial cell oxidation of LDL. Firstly, perhaps the modification of LDL by copper and HUV endothelial cells involves different free radical processes. Parthasarathy et al (1989) demonstrated that oxidation of LDL by endothelial cells involves lipoxygenases rather than superoxide anions since they were able to inhibit LDL oxidation by lipoxygenase inhibitors rather than superoxide dismutase. Superoxide dismutase has been shown to almost totally inhibit oxidation of LDL induced by copper in the absence of cells, and also inhibit smooth muscle cell oxidation of LDL by $60 \%$ (Heinecke et al, 1986; Parthasarathy et al, 1989). 17ß-estradiol may not inhibit lipoxygenase pathways and therefore in a system with a low metal ion concentration inhibition of LDL oxidation may not be observed. Secondly, precise control of experimental conditions for measurement of LDL oxidation by HUV endothelial cell appears critical. Steinbrecher et al (1984) found that certain media favoured cell induced LDL oxidation. Endothelial cells incubated in Ham's F10 medium induced rapid LDL oxidation while endothelial cells in 
DMEM did not. Triau et al (1988) showed that oxidation of LDL was greater in Medium MEM than in Medium DMEM. This difference was thought to be due to the higher levels of cysteine (a primary antioxidant) and tryptophan (an oxygen radical quencher) in DMEM than in MEM. Medium 199 contains no copper, but does contain iron, much more tryptophan and less cysteine than Ham's F10 medium. Other potential antioxidants in Medium 199 include glutathione, l-ascorbic acid, p-aminobenzoic acid and D-L- $\alpha$-tocopherol phosphate. Phenol red sodium salt, another known antioxidant commonly present in culture media was omitted from the Medium 199 preparation used in the studies reported here. Phenol red can also interfere in the TBA assay by producing background absorption (Esterbauer et al, 1990). It would appear that the copper and iron concentrations in the medium (Heinecke et al, 1984) and the concentration of antioxidants all have a significant effect on the extent to which LDL is oxidatively modified by incubation with cells.

In conclusion, the studies reported in this Chapter have shown that native LDL has a significant proliferative effect on HUV endothelial cells, while oxidized LDL inhibits proliferation, presumably because it is cytotoxic to HUV endothelial cells. The inhibition of HUV endothelial cell proliferation by high concentrations of sex steroids was overcome by native LDL in a concentration dependent manner. These same sex steroids augmented the inhibition of HUV endothelial cell proliferation by oxidized LDL. Low concentrations of sex steroids were unable to alter the effects of LDL on endothelial cell proliferation. $17 \beta$-estradiol $\left(10^{-10} \mathrm{M}\right)$ was able to retard LDL oxidation by copper ions but not by HUV endothelial cells. In contrast, 17קestradiol $\left(10^{-5} \mathrm{M}\right)$ caused increased oxidation of LDL within the first $9 \mathrm{~h}$ in the presence of copper sulphate but not at $18 \mathrm{~h}$ or $24 \mathrm{~h}$.

The studies reported in this Chapter therefore suggest that high concentrations of $17 \beta$-estradiol $\left(10^{-5} \mathrm{M}\right)$ may promote LDL oxidation and 
inhibit the proliferative effect of native LDL on HUV endothelial cells. 17קestradiol $\left(10^{-5} \mathrm{M}\right)$ and oxidized LDL together also caused a marked inhibition of HUV endothelial cell proliferation. In contrast, low concentrations of $17 \beta$ estradiol (10-10 M) do not appear to modify the action of native or oxidized LDL on endothelial cell proliferation, although low concentrations of $17 \beta-$ estradiol may have a significant antioxidant effect on LDL. The antioxidant effect of low concentrations of $17 \beta$-estradiol may help to explain the lower serum lipid peroxide levels found in premenopausal women compared to men (Yagi, 1982) and the loss of this difference after 50 years of age. Interestingly, the oral contraceptive, which contains a higher concentration of estrogen, has been associated with increased serum lipid peroxide levels (Wynn et al, 1979, Ciavatti et al, 1989; Ciavatti and Renaud, 1991). The effect of hormone replacement therapy on serum lipid peroxide levels remains to be fully assessed.

\subsection{SUMMARY}

The present studies demonstrate that, under the experimental conditions used:

(1) Native and minimally modified LDL can increase HUV endothelial cell proliferation in the physiological range while oxidized LDL at these same concentrations inhibits HUV endothelial cell proliferation;

(2) The proliferative effects of native and oxidized LDL can be modified by FCS in a concentration dependent manner;

(3) Low concentrations of sex steroids do not modify the proliferative effect of LDL; 
(4) High concentrations of sex steroids augment the inhibition of proliferation seen with oxidized LDL, and at lower concentrations of native LDL reduce its proliferative effect;

(5) 17 -estradiol $\left(10^{-10} \mathrm{M}\right)$ can inhibit oxidation LDL by copper sulphate over $18 \mathrm{~h}$, while $17 \beta$-estradiol (10-5 $\mathrm{M}$ ) appears to initially promote LDL oxidation; and

(6) The sex steroids do not appear to alter LDL modification by HUV endothelial cells. 


\section{Figure 6.1.}

GLC fatty acid profile of native LDL. The fatty acid methyl esters derived from $3 \mathrm{mg}$ of LDL were dissolved in methylene chloride/ butylated hydroxytoluene solution. The attenuation was $4 \times 1$.

A: This chromatogram illustrates a typical profile of a freshly prepared sample of native LDL (batch 9) with relatively high concentrations of $20: 4$ and 18:2. B: Illustrates a typical reference chromatogram where $1=$ methylene chloride, $2=\mathrm{BHT}$, $3=18: 2,4=18: 3$, and $5=20: 4$.

Figure 6.2.

GLC fatty acid profile of minimally modified LDL. The attenuation was $4 \times 1$.

A: Illustrates a typical profile of minimally modified LDL (batch 9 which has been stored at $4^{\circ} \mathrm{C}$ for 3 months with antioxidants). There is a small reduction in $18: 2$ and $20: 4$ relative to the fresh sample (refer to Figure 6.1). B: Illustrates a typical reference chromatogram as described in the legend to Figure 6.1.

\section{Figure 6.3.}

GLC fatty acid profile of oxidized LDL. The attenuation was $4 \times 1$. A: Illustrates a typical profile of oxidized LDL (batch 2, which is the oxidized preparation from native LDL batch 9) with the loss of $20: 4$ and reduction of $18: 2$ relative to the native preparation. B: Illustrates a typical reference chromatogram as described in the legend to Figure 6.1. 


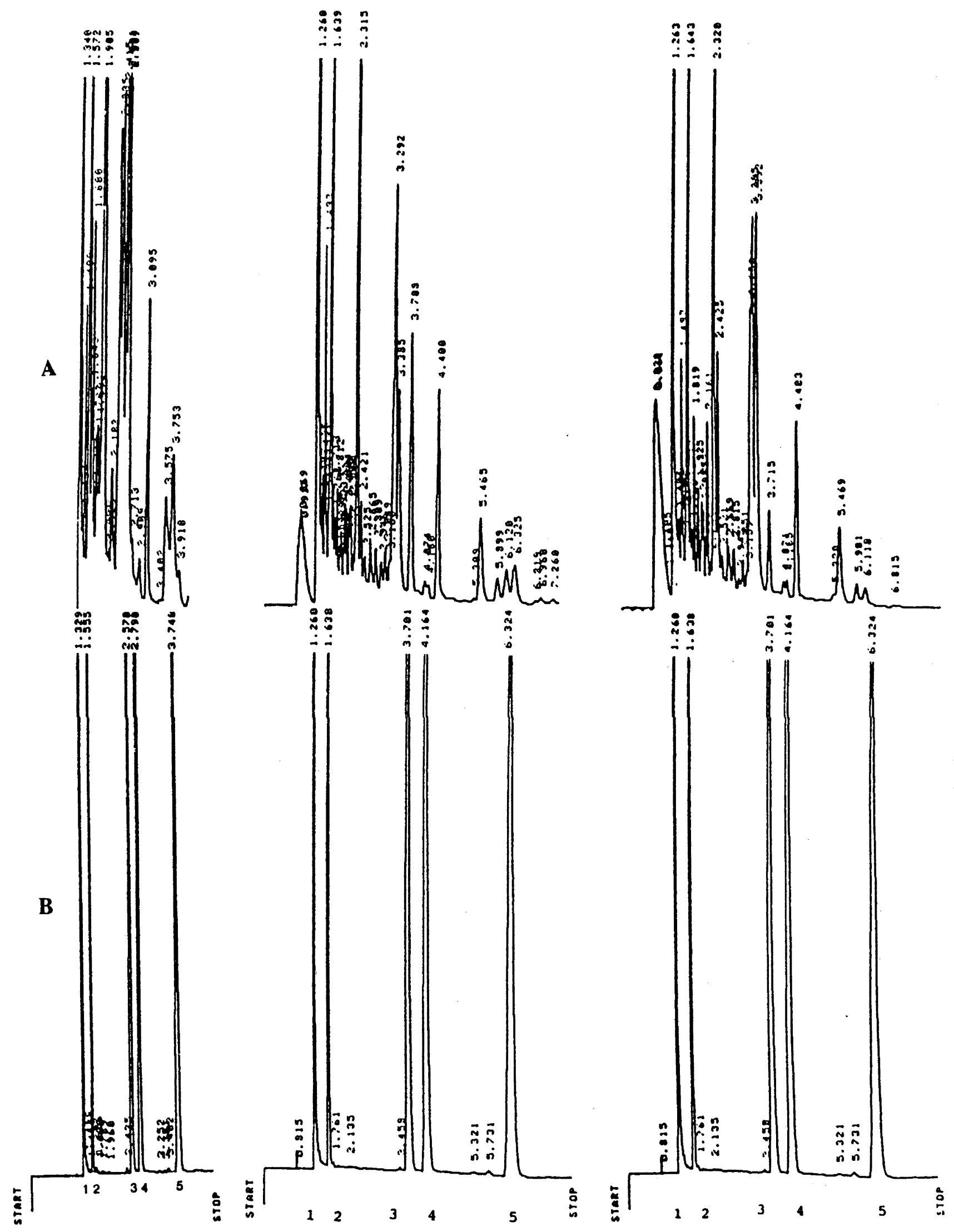

Figure 6.1

Figure 6.2

Figure 6.3 


\section{Figure 6.4.}

A $1 \%$ agarose gel electrophoretogram of a pooled LDL preparation (batch 3) exposed to copper sulphate at $4^{\circ} \mathrm{C}$ for up to 5 days. This was stained with Coomassie blue R-250. $10 \mu \mathrm{g}$ of protein were loaded for each LDL sample. The MDA content (nmol MDA /mg protein) and the presence of conjugated dienes (absorbance units) in the samples were also assessed. Lane 1 is the native LDL preparation, Lane 2 represents the mobility of the protein after $4 \mathrm{~h}$ oxidation, Lane 3 represents $9 \mathrm{~h}$ oxidation, Lanes 4,5 and 6 represent $18 \mathrm{~h}$, $24 \mathrm{~h}$ and 2 days oxidation of the LDL preparation respectively, while Lanes 7,8 and 9 represent 3,4 and 5 days oxidation of the same prepation of LDL. Lane 10 represents the mobility of human serum albumin $(5 \mu \mathrm{g})$. 


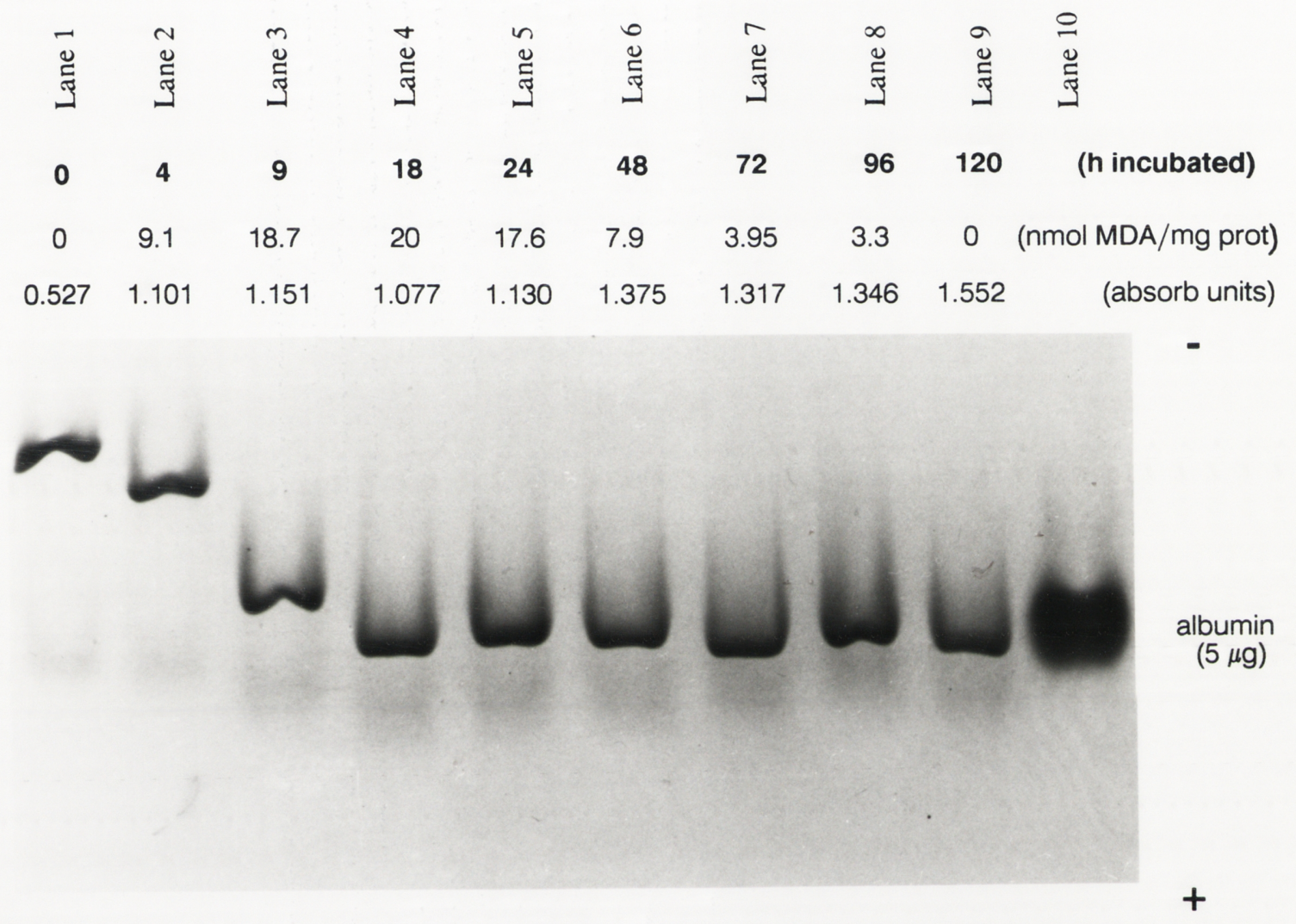




\section{Figure 6.5.}

Upper: A $1 \%$ agarose gel electrophoretogram of various LDL preparations stained with Coomassie blue R-250. $10 \mu \mathrm{g}$ of protein was loaded for each sample. Oxidized LDL samples (batches 1 and 3 ) are represented in Lanes 1 and 2, minimally modified LDL (batches 9, 10 and 7) in Lanes 3, 4 and 5, normal serum albumin in Lane 6, while native LDL samples (batches 11 and 12) are shown in Lanes 7 and 8. Note the difference in the ablumin content between the two LDL preparations.

Lower: A $1 \%$ agarose gel electrophoretogram of LDL samples stained with Sudan black. $10 \mu \mathrm{g}$ of protein was loaded for each sample. Oxidized LDL samples (batches 1 and 3) are represented in Lanes 1 and 2, minimally modified LDL (batches 9, 10 and 7) in Lanes 3, 4 and 5, normal serum albumin in Lane 6 (which did not stain for lipid), while native LDL preparations (batches 11 and 12) are shown in Lanes 7 and 8 . 


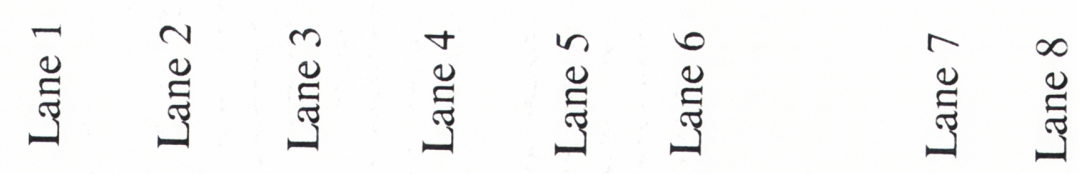
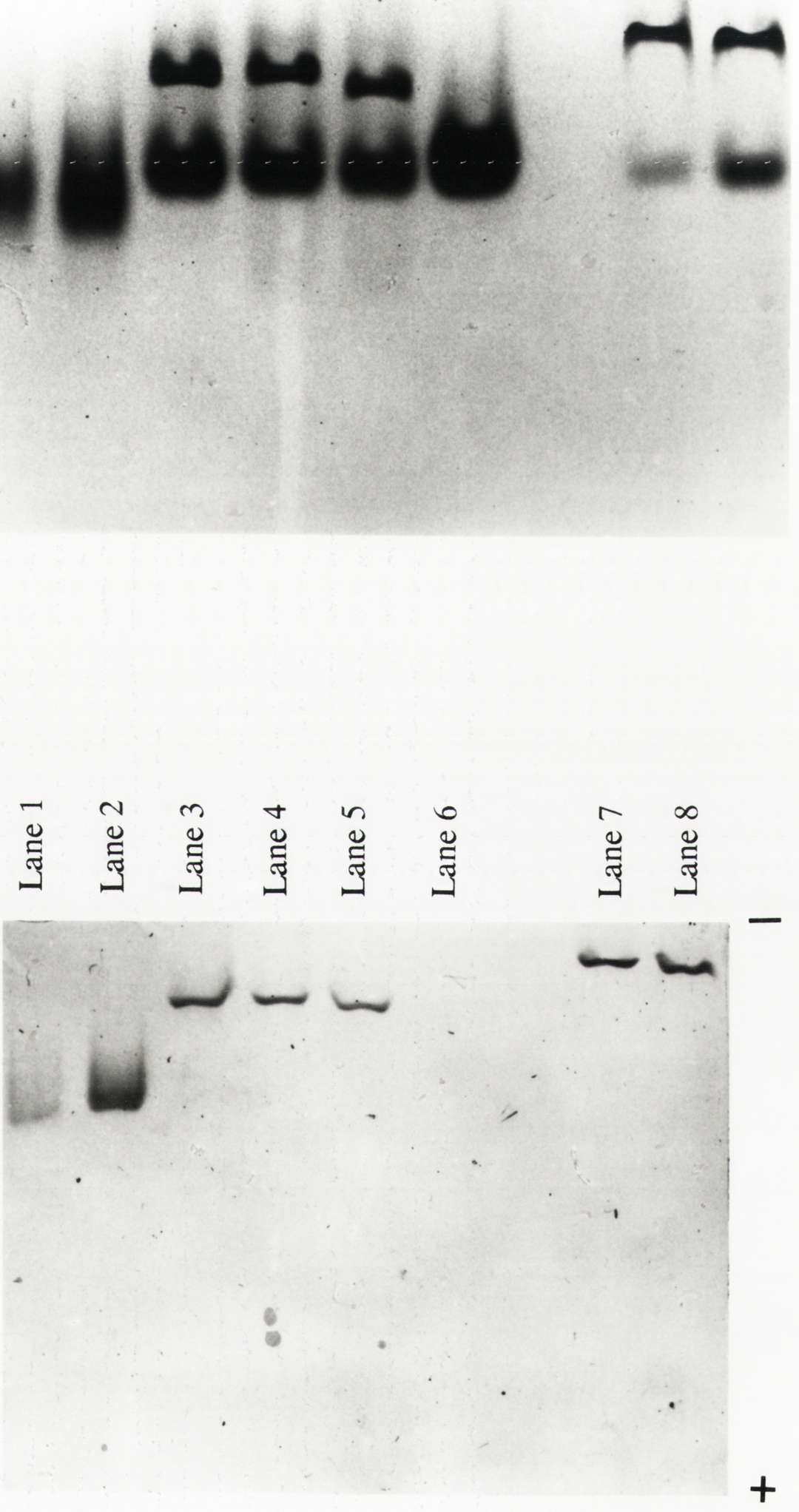
Figure 6.6.

SDS polyacrylamide gel $(10 \%)$ electrophoretograms of lipoprotein preparations. $10 \mu \mathrm{g}$ of protein was loaded for each lipoprotein sample and $5 \mu \mathrm{g}$ for normal serum albumin. Lane 1 is a native LDL preparation (batch 12). The major protein component of native LDL is apo B-100 (MW of approximately 500 000). Lane 2 is a copper sulphate oxidized LDL preparation (batch 3). Lane 4 is a native HDL preparation while Lane 5 is an oxidized HDL preparation. Native HDL (Lane 4) contains the apolipoproteins apo A-1 (26 000-28 $3000 \mathrm{MW}$ ) and apo E (37 000 MW approximately). Lane 7 is human serum albumin. High and low molecular weight markers are in Lanes 3 and 6 . The high molecular weight markers are: myosin (200 000), E. coli $\beta$ galactosidase (116 250), rabbit muscle phosphorylase b (97 400), bovine serum albumin $(66200)$ and hen egg white ovalbumin (42 699). The low molecular weight markers are: rabbit muscle phosphorylase $b$, bovine serum albumin, hen egg white ovalbumin, bovine carbonic anhydrase (31 000), soybean trypsin inhibitor (21 500) and hen egg white lysozyme (14 400).

\section{Figure 6.7.}

SDS polyacrylamide gel (10 \%) electrophoretograms of lipoprotein preparations. $10 \mu \mathrm{g}$ of protein was loaded for each sample. Lanes 1 and 2 are native LDL preparations (batches 11 and 12). Lanes 4, 5 and 6 are minimally modified preparations of LDL (batches $9,7,10$ ), while Lanes 8 and 9 are copper sulphate modified preparations of LDL (batches 2 and 5). Oxidization of LDL results in a breakdown of apo B. The high and low molecular weight markers are in Lanes 3 and 7 respectively and are described in the legend to Figure 6.6. 

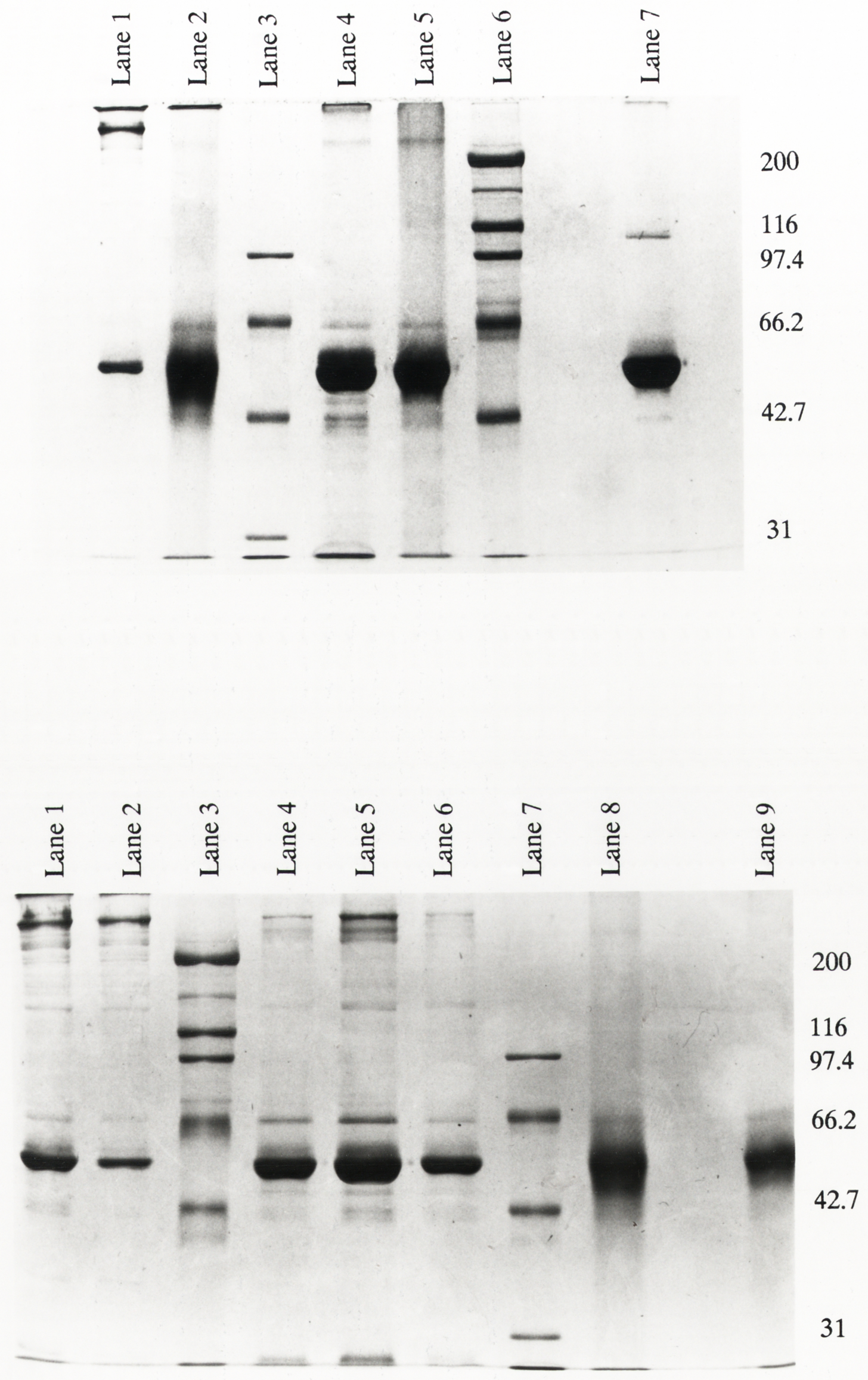
Figure 6.8.

Effect of FCS on HUV endothelial cell proliferation in the presence of LDL. Endothelial cells $\left(6 \times 10^{3}\right.$ cells /well, passage 9) were incubated for $18 \mathrm{~h}$ with FCS (1 to $50 \%$ ) and LDL (batch 8) $(1 \mathrm{mg} / \mathrm{ml})$. The "y" axis represents incorporation of $\left[{ }^{3} \mathrm{H}\right]$-thymidine by endothelial cells while the " $x$ " axis represents different concentrations of FCS. This graph is representative of 3 experiments performed in triplicate. ${ }^{*}=p<0.05$. 
EFFECT OF FCS CONCENTRATION ON ENDOTHELIAL CELL PROLIFERATION

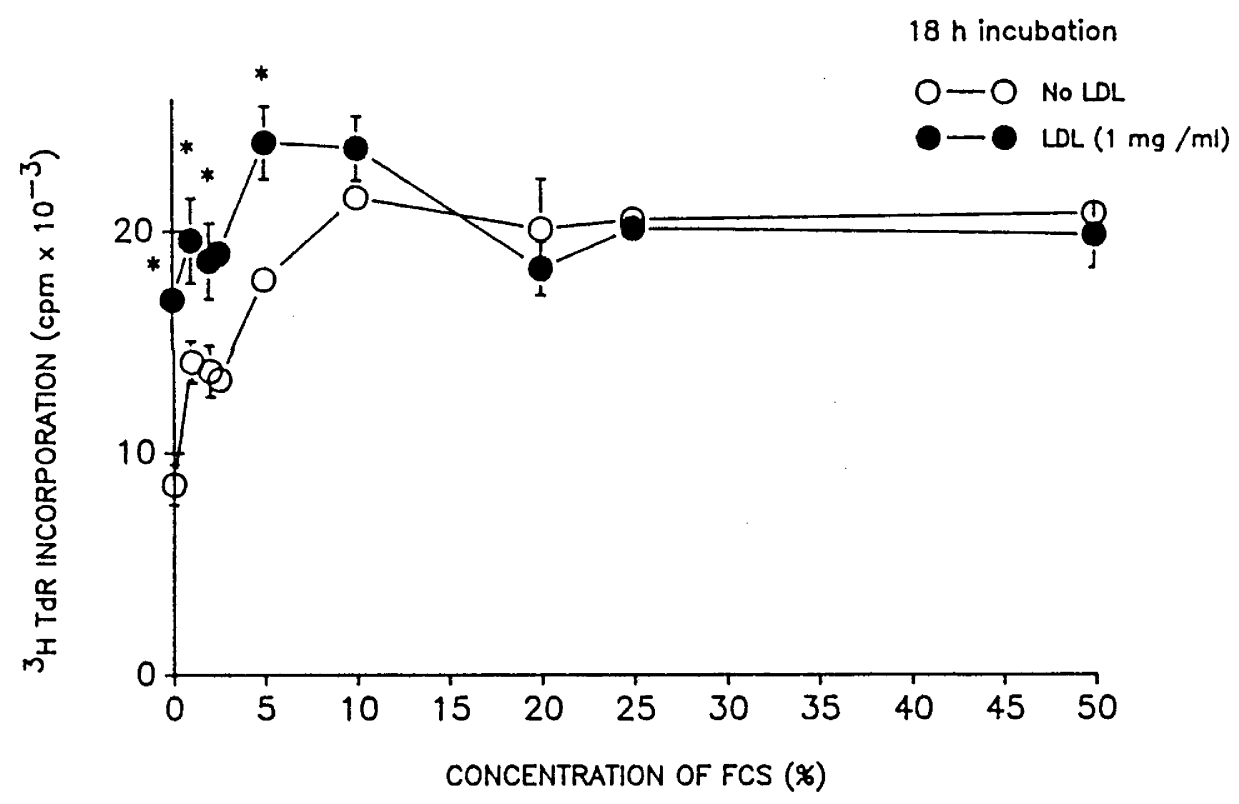




\section{Figure 6.9.}

Effect of native LDL on HUV endothelial cell proliferation in the presence of $20 \%$ FCS. Endothelial cells $\left(4 \times 10^{3}\right.$ cells /well, passage 8) were incubated for $15 \mathrm{~min}, 1 \mathrm{~h}$ or $6 \mathrm{~h}$ with 0.25 to $2 \mathrm{mg} / \mathrm{ml} \mathrm{LDL}$ (batch 1). $\left[{ }^{3} \mathrm{H}\right]$-thymidine was then added for $18 \mathrm{~h}$, in the presence of $20 \%$ FCS. This graph is representative of 3 experiments performed in triplicate.

\section{Figure 6.10.}

Effect of native LDL on HUV endothelial cell proliferation in the presence of $20 \%$ FCS. Endothelial cells $\left(5 \times 10^{3}\right.$ cells /well, passage 6) were incubated for $18 \mathrm{~h}$ or $42 \mathrm{~h}$ with 0.25 to $2 \mathrm{mg} / \mathrm{ml} \mathrm{LDL}$ (batch 10). [ $\left.{ }^{3} \mathrm{H}\right]$-thymidine was then added for $18 \mathrm{~h}$, in the presence of $20 \%$ FCS. This graph is representative of 3 experiments performed in triplicate. ${ }^{\star}=p$ $<0.05$. 
EFFECT OF NATIVE LDL ON ENDOTHELIAL CELL PROLIFERATION

$20 \%$ FCS

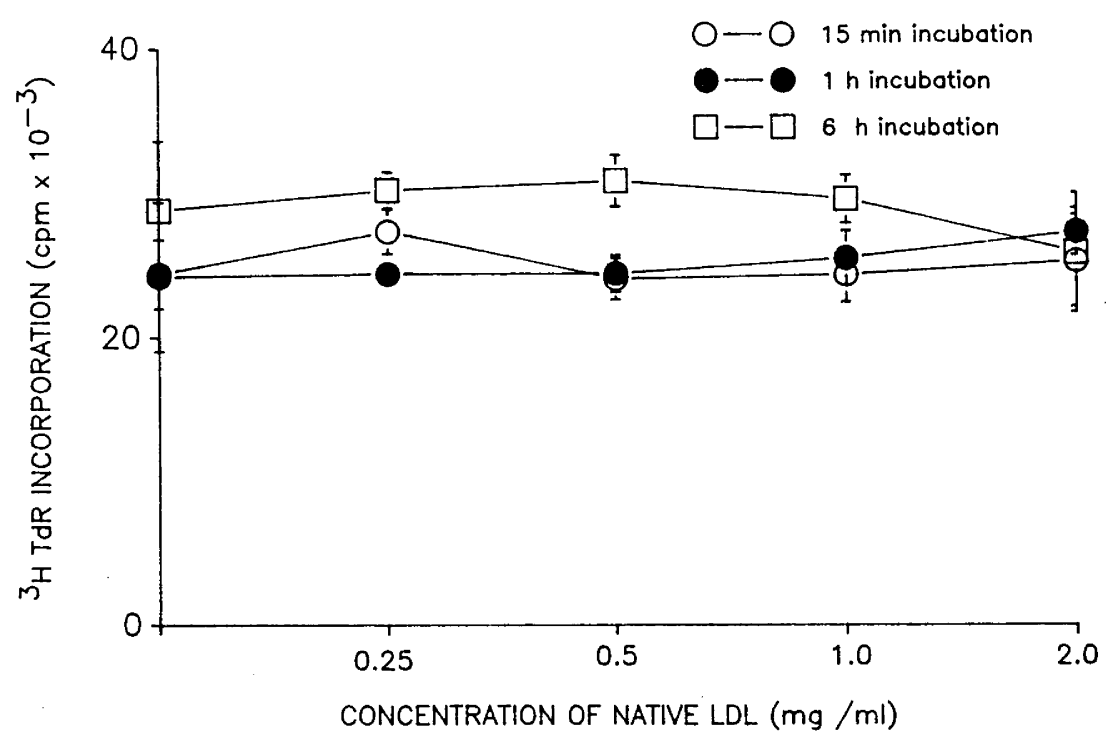

EFFECT OF NATIVE LDL ON ENDOTHELIAL CELL PROLIFERATION

$20 \%$ FCS

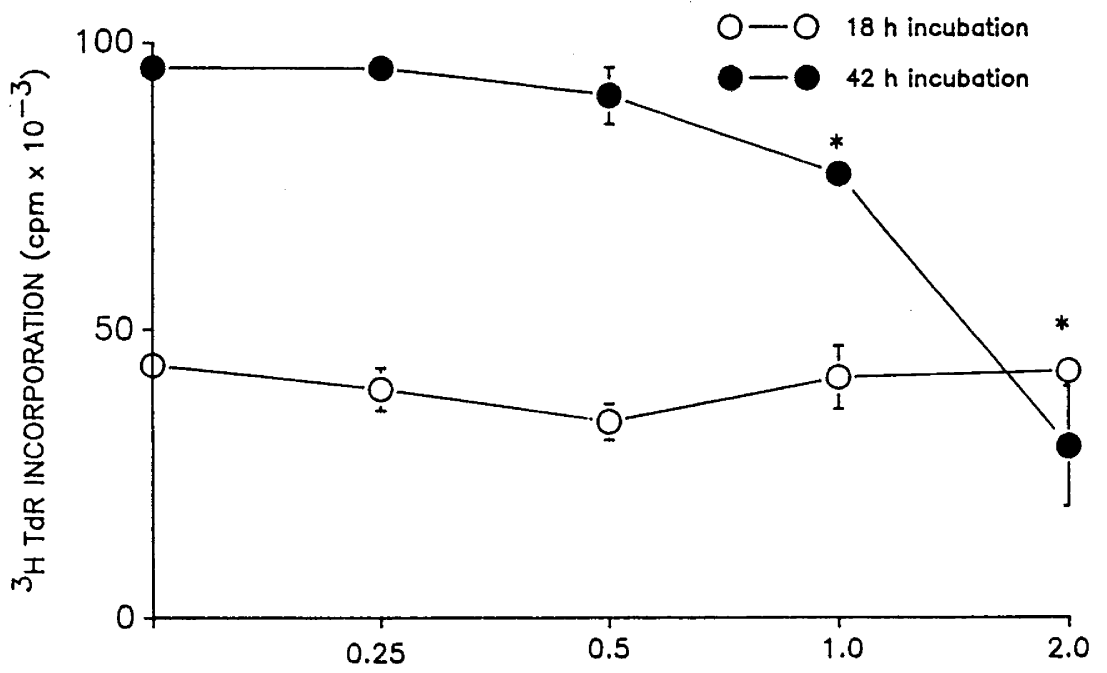

CONCENTRATION OF NATIVE LDL $(\mathrm{mg} / \mathrm{ml})$ 


\section{Figure 6.11.}

Effect of oxidized LDL on HUV endothelial cell proliferation in the presence of $20 \%$ FCS. Endothelial cells $\left(5 \times 10^{3}\right.$ cells /well, passage 6) were incubated for $15 \mathrm{~min}$ or $1 \mathrm{~h}$ with different concentrations of oxidized LDL (batch 3 ). [ [ $\left.{ }^{3} \mathrm{H}\right]-$ thymidine was then added for $18 \mathrm{~h}$ in the presence of $20 \%$ FCS. This graph is representative of 2 experiments performed in triplicate. ${ }^{*}=p<0.05$.

\section{Figure 6.12.}

Effect of oxidized LDL on HUV endothelial cell proliferation in the presence of $20 \%$ FCS. Endothelial cells $\left(5 \times 10^{3}\right.$ cells /well, passage 6) were incubated for $18 \mathrm{~h}$ or $42 \mathrm{~h}$ with different concentrations of oxidized LDL (batch 3 ). $\left[{ }^{3} \mathrm{H}\right]$ thymidine was then added for $18 \mathrm{~h}$ in the presence of $20 \%$ FCS. Note the difference in scale of the "y" between Figures 11 and 12. This graph is representative of 2 experiments performed in triplicate. ${ }^{*}=p<0.05$. 
EFFECT OF OXIDIZED LDL ON ENDOTHELIAL CELL PROLIFERATION

$20 \%$ FCS

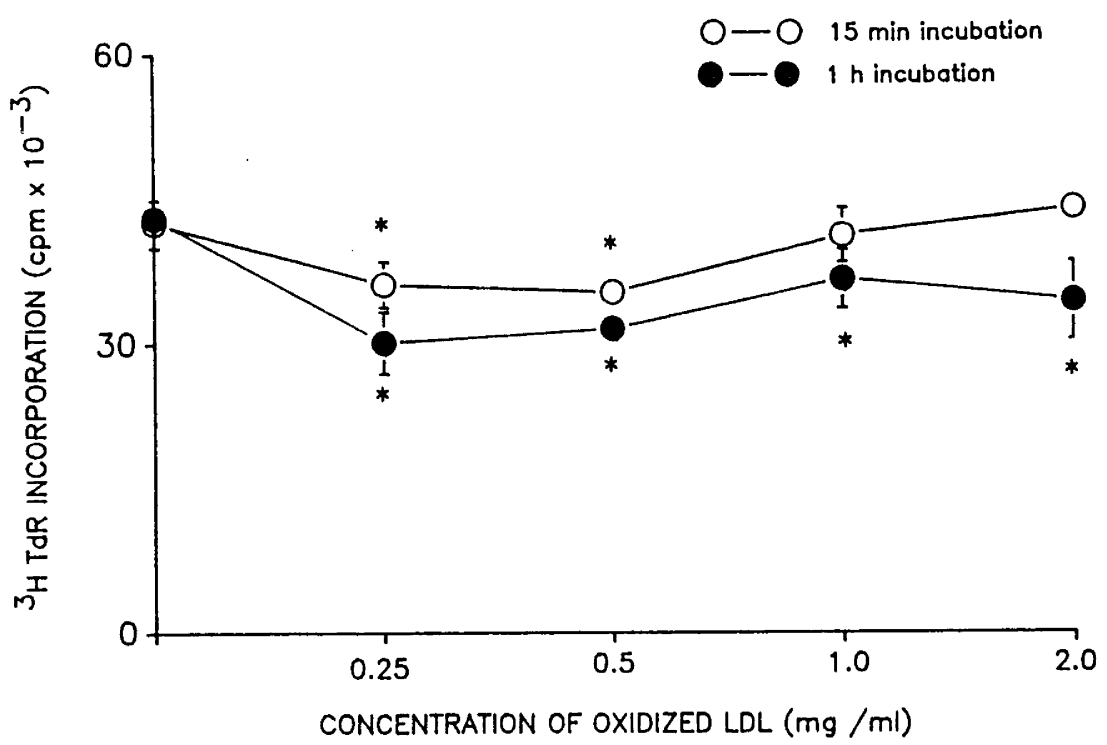

EFFECT OF OXIDIZED LDL ON ENDOTHELIAL CELL PROLIFERATION $20 \%$ FCS

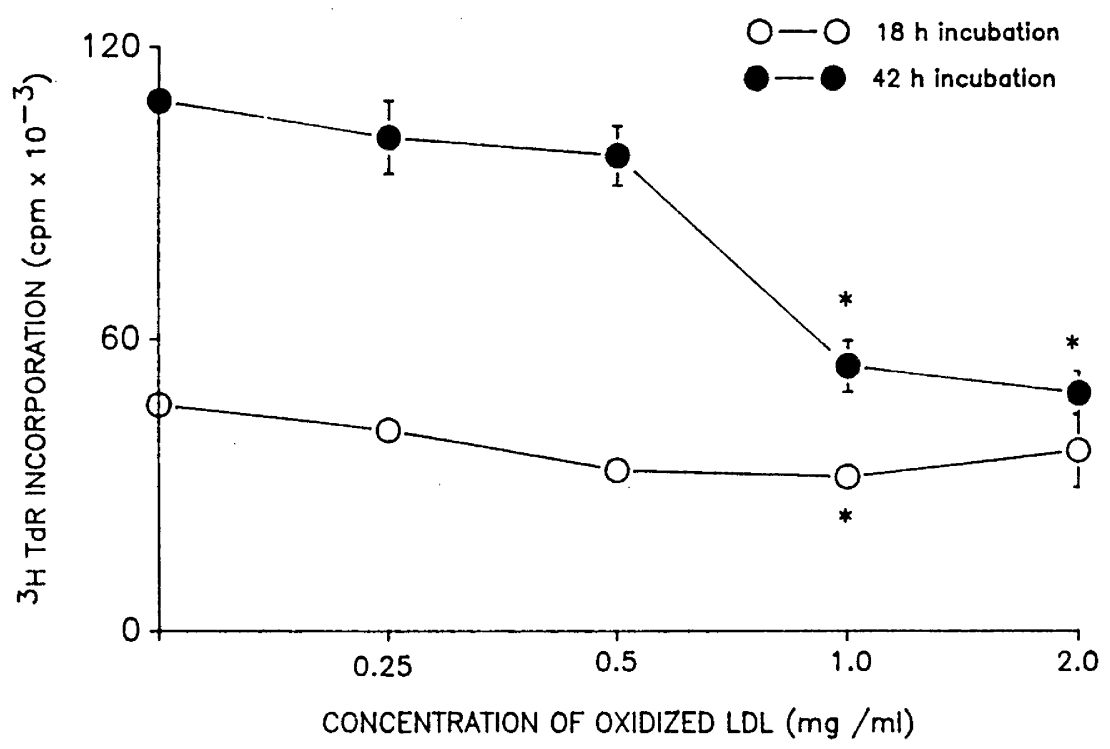




\section{Figure 6.13.}

Effect of native LDL on HUV endothelial cell proliferation in the presence and absence of FCS. Endothelial cells $\left(6 \times 10^{3}\right.$ cells /well, passage 9) were incubated for $18 \mathrm{~h}$ with different concentrations of native LDL (batch 8 ) in the presence or absence of $2 \%$ FCS. This graph is representative of 3 experiments performed in triplicate. ${ }^{*}=p<0.05$.

\section{Figure 6.14.}

Effect of oxidized LDL on HUV endothelial cell proliferation in the presence and absence of FCS. Endothelial cells $(6.3 \mathrm{x}$ $10^{3}$ cells /well, passage 8 ) were incubated for $18 \mathrm{~h}$ with different concentrations of oxidized LDL (batch 4) in the presence or absence of $2 \%$ FCS. This graph is representative of 3 experiments performed in triplicate. ${ }^{*}=p$ $<0.05$. 


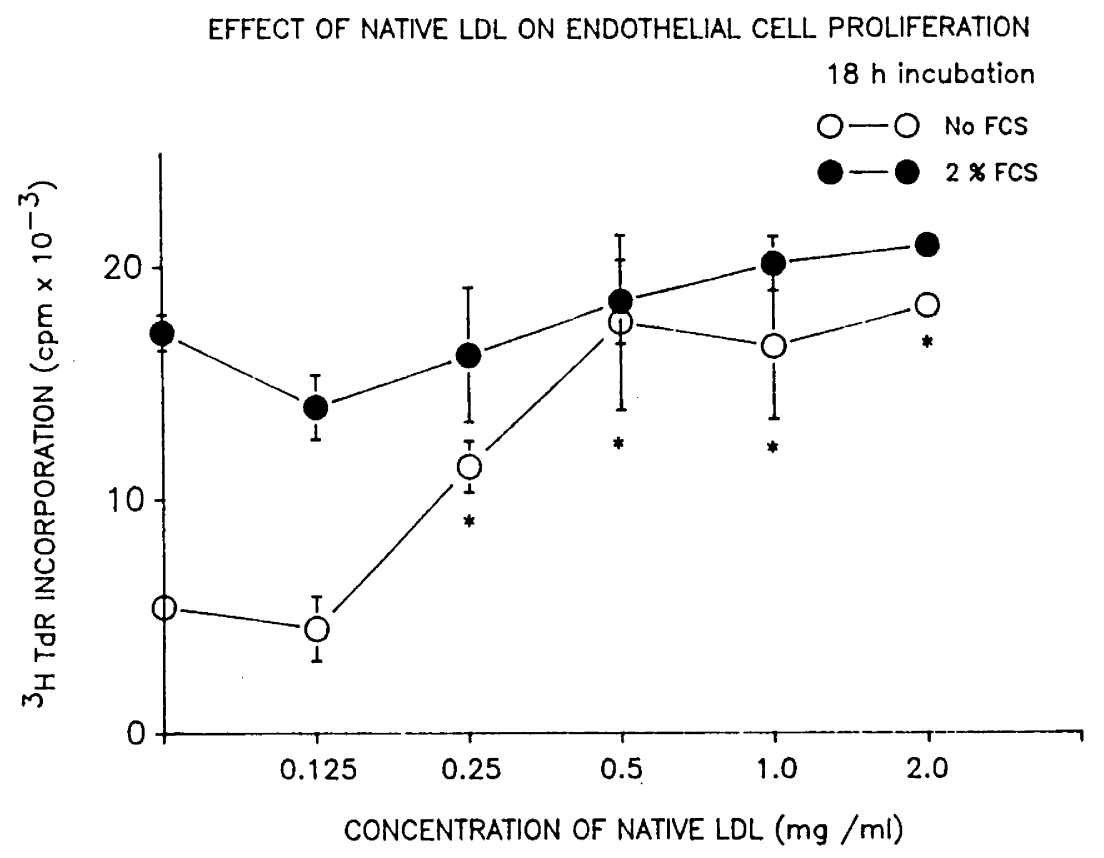

EFFECT OF OXIDIZED LDL ON ENDOTHELIAL CELL PROLIFERATION

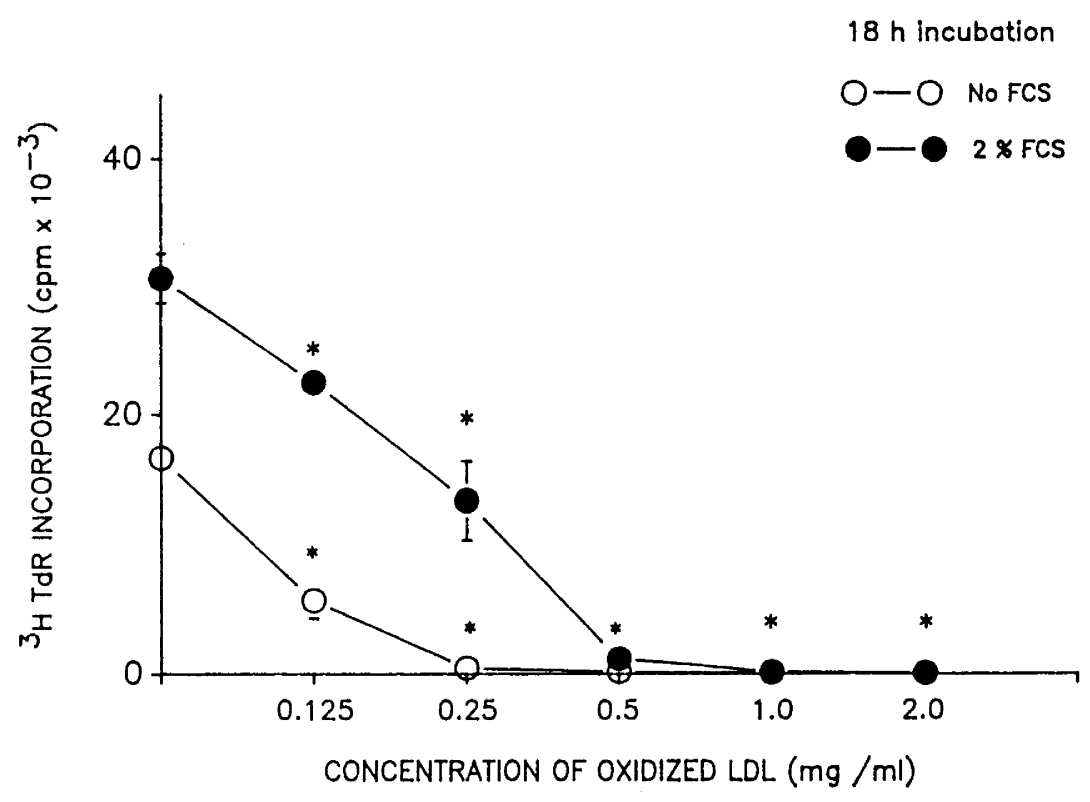


Figure 6.15.

Effect of native and minimally modified LDL on HUV endothelial cell proliferation in the absence of FCS. Endothelial cells (1.25 × $10^{4}$ cells /well, passage 8) were incubated for $18 \mathrm{~h}$ with different batches of native LDL (batches 11 and 12) (symbols joined by dotted lines) and minimally modified LDL (batches 9, 7 and 10) (symbols joined by solid lines). The control is Medium 199 alone. The "x" axis is logarithmic. This graph is representative of 2 experiments. Each point is a single value.

Figure 6.16.

Effect of oxidized LDL on HUV endothelial cell proliferation in the absence of FCS. Endothelial cells $\left(1.2 \times 10^{4}\right.$ cells /well, passage 7) were incubated for $18 \mathrm{~h}$ with different batches of LDL (batches 2, 3 and 5). Note the differences in the scale of the "y" axis between Figures 6.15 and 6.16. This graph is representative of 2 experiments. Each point is a single value. 
EFFECT OF MINIMALLY MODIFIED LDL AND NATIVE LDL ON ENDOTHELIAL CELL PROLIFERATION

$18 \mathrm{~h}$ incubation

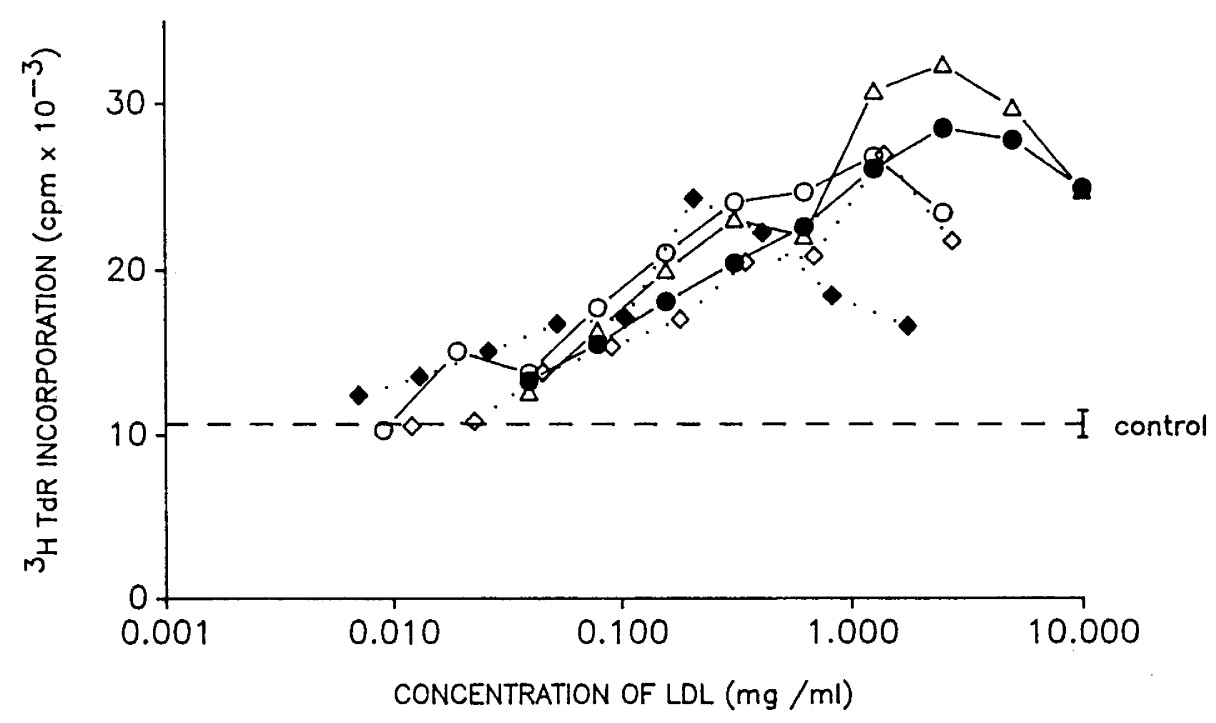

EFFECT OF OXIDIZED LDL ON ENDOTHELIAL CELL PROLIFERATION

$18 \mathrm{~h}$ incubation

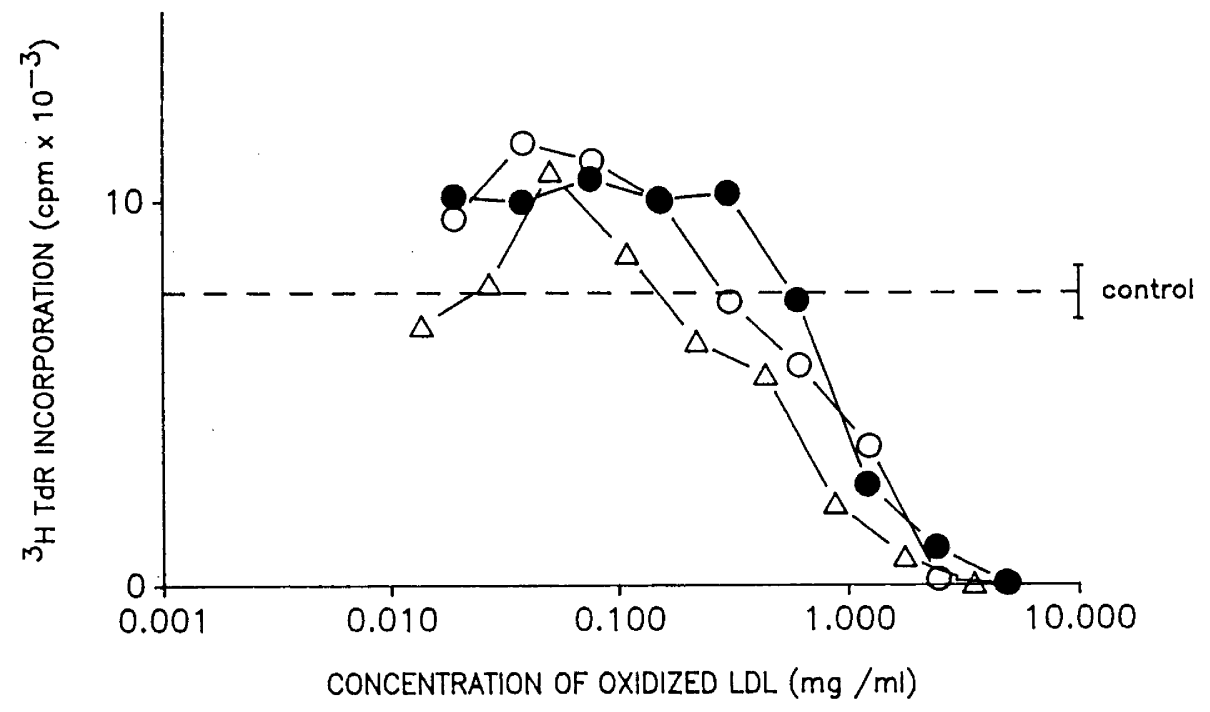




\section{Figure 6.17.}

Effect of native LDL on HUV endothelial cell proliferation in the presence of sex steroids and in the absence of FCS. Endothelial cells $\left(6.3 \times 10^{3}\right.$ cells /well, passage 8$)$ were incubated for $18 \mathrm{~h}$ in the presence of $17 \beta$-estradiol $\left(10^{-5} \mathrm{M}\right.$, $10^{-7} \mathrm{M}$ or $10^{-10} \mathrm{M}$ ) or PRF Medium 199 (control), and native LDL (batch 9). This graph is representative of 3 experiments performed in triplicate.

Figure 6.18.

Effect of native LDL on HUV endothelial cell proliferation in the presence of sex steroids and in the absence of FCS. Endothelial cells $\left(6.3 \times 10^{3}\right.$ cells /well, passage 8$)$ were incubated for $18 \mathrm{~h}$ in the presence of progesterone $\left(10^{-5} \mathrm{M}\right.$ or $10^{-9} \mathrm{M}$ ) or PRF Medium 199 (control), and native LDL (batch 9). This graph is representative of 3 experiments performed in triplicate. 
EFFECT OF SEX STEROIDS AND NATIVE LDL ON ENDOTHELIAL CELL PROLIFERATION

$18 \mathrm{~h}$ incubation

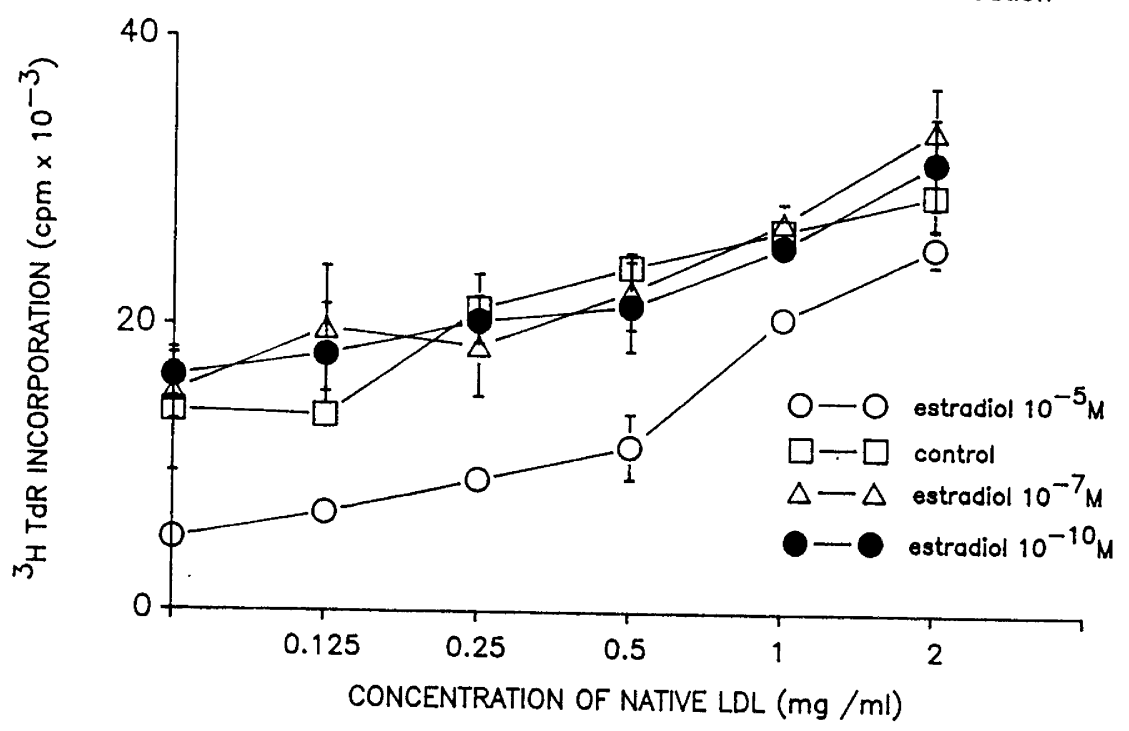

EFFECT OF SEX STEROIDS AND NATIVE LDL ON ENDOTHELIAL CELL PROLIFERATION

$18 \mathrm{~h}$ incubation

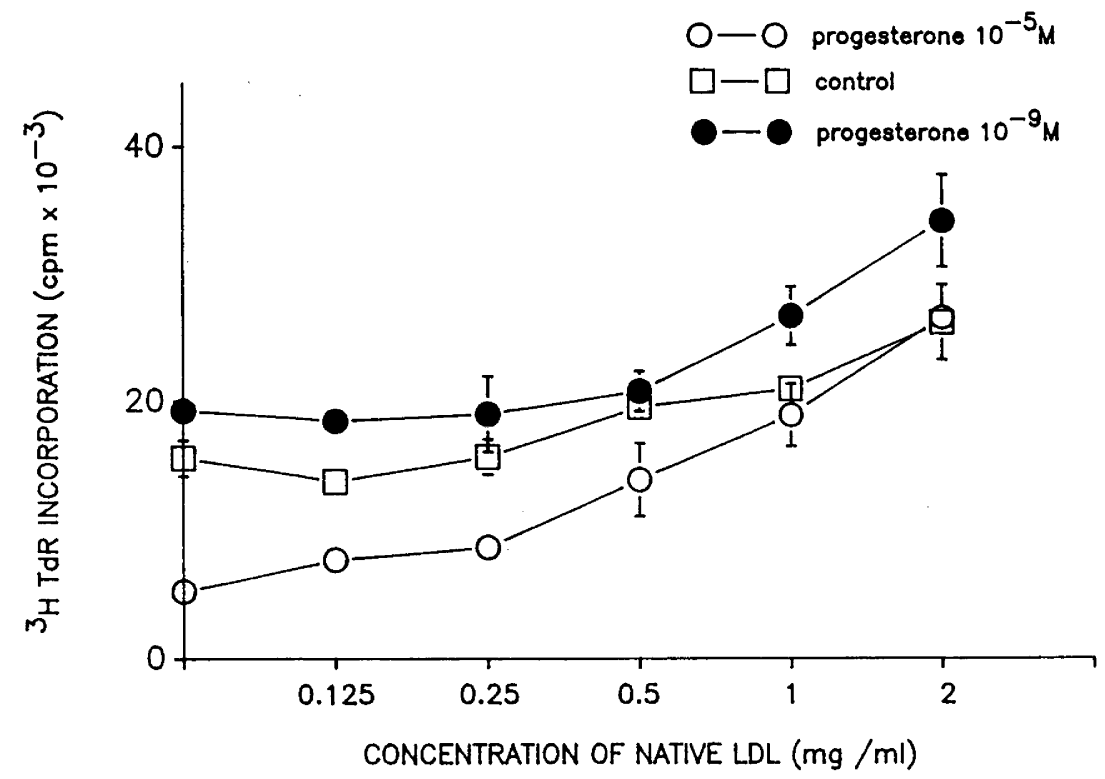


Figure 6.19.

Effect of native LDL on HUV endothelial cell proliferation in the presence of sex steroids and in the absence of FCS. Endothelial cells $\left(6.3 \times 10^{3}\right.$ cells /well, passage 8$)$ were incubated for $18 \mathrm{~h}$ in the presence of combinations of sex steroids or PRF Medium 199 (control), and native LDL (batch 9). This graph is representative of 3 experiments performed in triplicate.

\section{Figure 6.20.}

Effect of oxidized LDL on HUV endothelial cell proliferation in the presence of sex steroids and in the absence of FCS. Endothelial cells $\left(6 \times 10^{3}\right.$ cells /well, passage 9$)$ were incubated for $18 \mathrm{~h}$ in the presence of $17 \beta$-estradiol $\left(10^{-5} \mathrm{M}\right.$, $10^{-7} \mathrm{M}$ and $10^{-10} \mathrm{M}$ ) or PRF Medium 199 (control), and oxidized LDL (batch 1). This graph is representative of 3 experiments performed in triplicate. 
EFFECT OF SEX STEROIDS AND NATIVE LDL ON ENDOTHELIAL CELL PROLIFERATION $18 \mathrm{~h}$ incubation

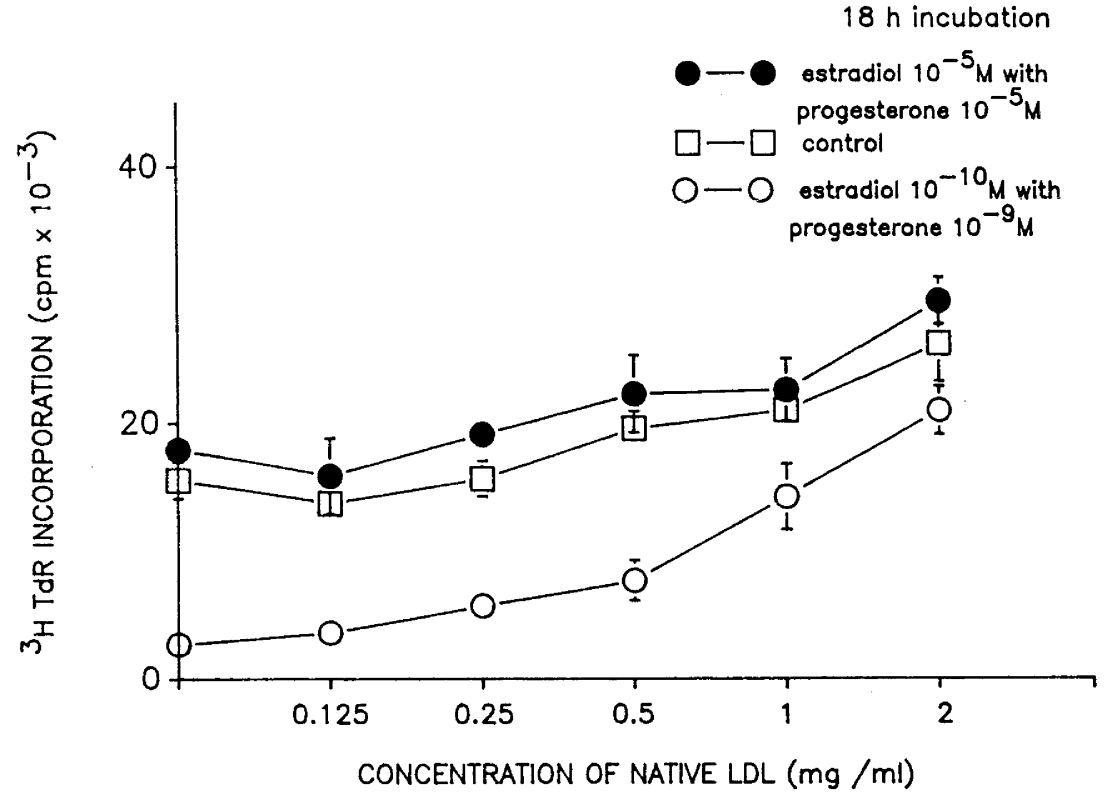

EFFECT OF SEX STEROIDS AND OXIDIZED LDL ON ENDOTHELIAL CELL PROLIFERATION

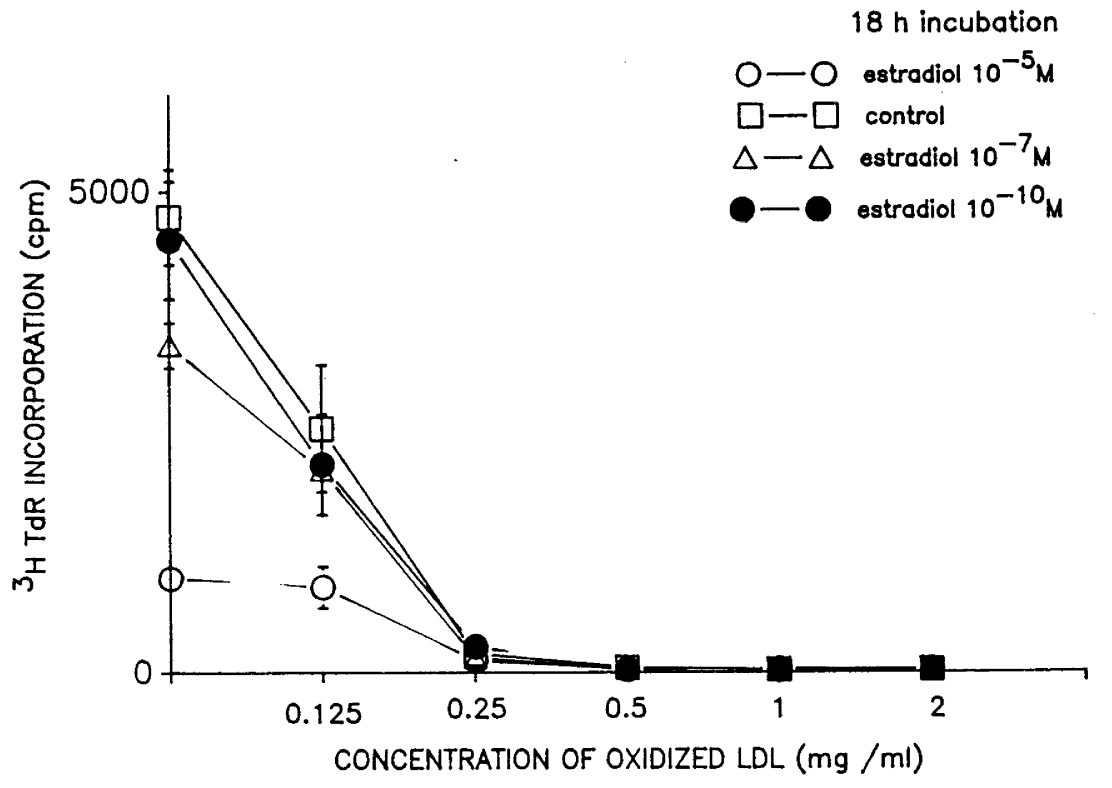




\section{Figure 6.21.}

Effect of oxidized LDL on HUV endothelial cell proliferation in the presence of sex steroids and in the absence of FCS. Endothelial cells $\left(6 \times 10^{3}\right.$ cells /well, passage 9) were incubated for $18 \mathrm{~h}$ in the presence of progesterone $\left(10^{-5} \mathrm{M}\right.$ and $10^{-9} \mathrm{M}$ ) or PRF Medium 199 (control), and oxidized LDL (batch 1). This graph is representative of 3 experiments performed in triplicate.

Figure 6.22.

Effect of oxidized LDL on HUV endothelial cell proliferation in the presence of sex steroids and in the absence of FCS. Endothelial cells $\left(6 \times 10^{3}\right.$ cells /well, passage 9) were incubated for $18 \mathrm{~h}$ in the presence or absence of combinations of sex steroids or PRF Medium 199 (control), and oxidized LDL (batch 1). This graph is representative of 3 experiments performed in triplicate. 
EFFECT OF SEX STEROIDS AND OXIDIZED LDL ON ENDOTHELIAL CELL PROLIFERATION

$$
18 \mathrm{~h} \text { incubation }
$$

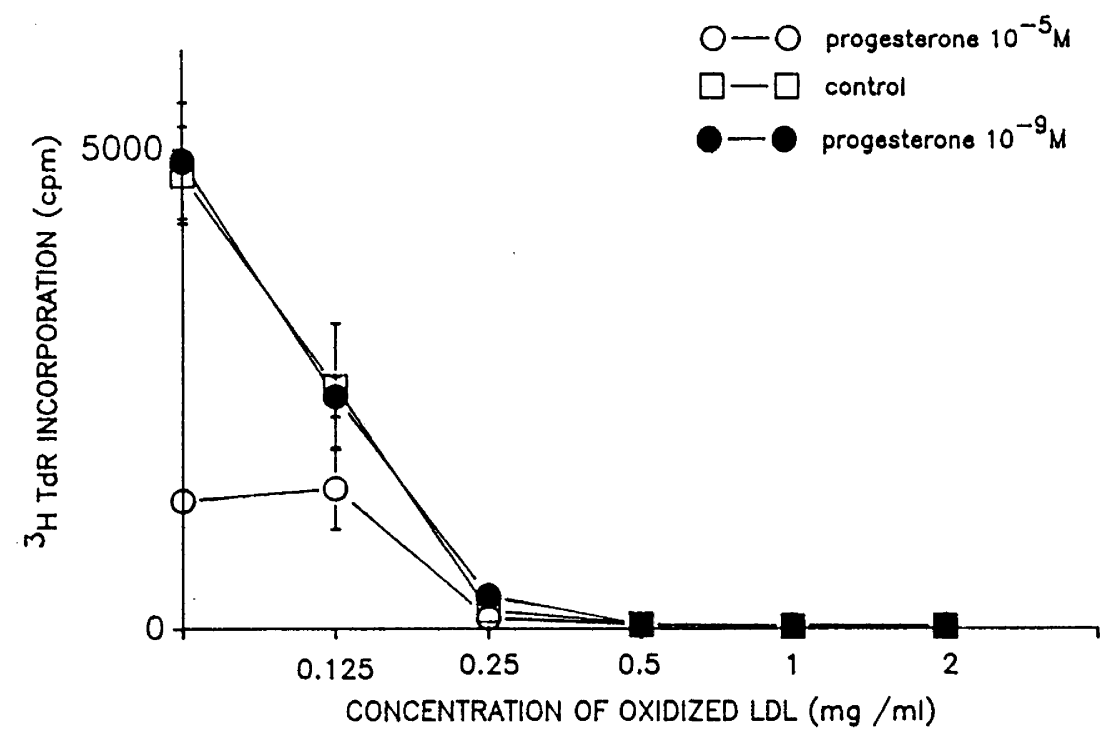

EFFECT OF SEX STEROIDS AND OXIDIZED LDL ON ENDOTHELIAL CELL PROLIFERATION

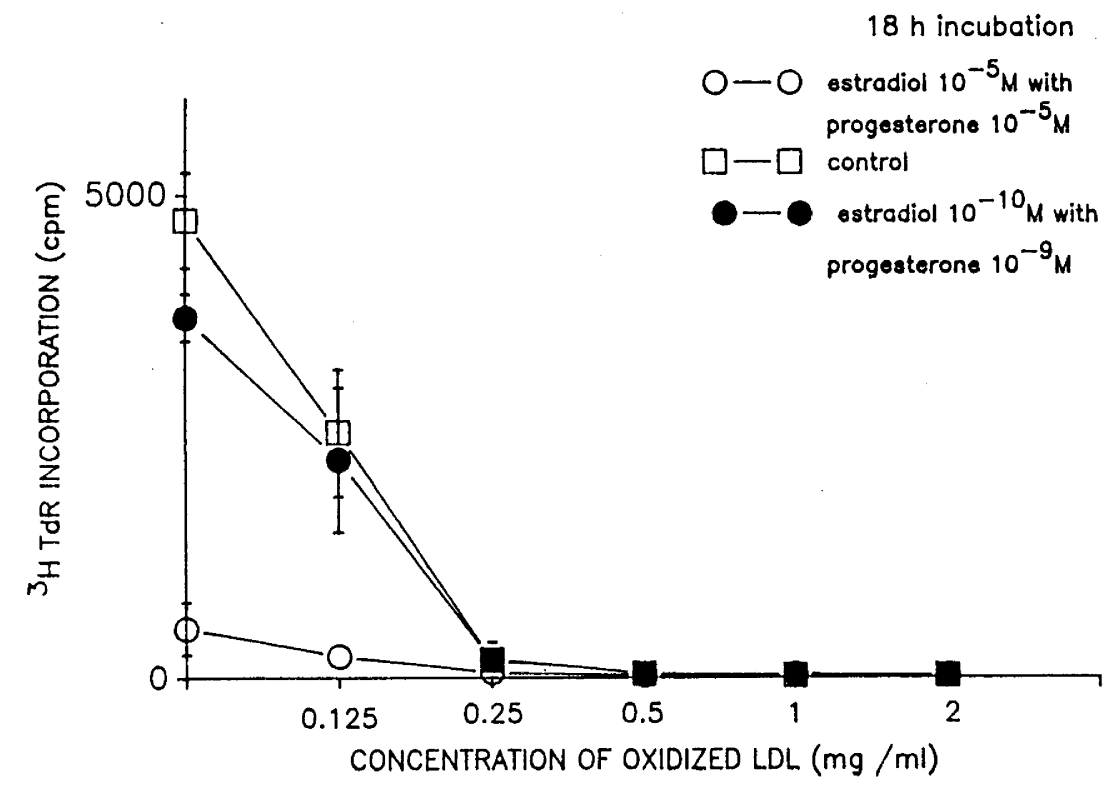




\section{Figure 6.23.}

Malondialdehyde content of LDL following incubation with copper sulphate in the presence of various treatments. LDL was oxidized in copper sulphate for 9,18 or $24 \mathrm{~h}$ at $4^{\circ} \mathrm{C}$. The "y" axis shows the percentage change in malondialdehyde content in LDL treated in various ways relative to untreated native LDL. The average MDA content of native LDL was $7.4 \pm 4.3 \mathrm{nmol} \mathrm{MDA} / \mathrm{mg}$ protein at $9 \mathrm{~h}, 7.95 \pm$ $2.9 \mathrm{nmolMDA} / \mathrm{mg}$ protein at $18 \mathrm{~h}$ and $5.22 \pm 3.1 \mathrm{nmol}$ MDA $/ \mathrm{mg}$ protein at $24 \mathrm{~h}$. This graph is the mean of 5 experiments performed in duplicate for $18 \mathrm{~h}$, and the mean of 3 experiments performed in duplicate for 9 and $24 \mathrm{~h}$ for all treatments except $17 \beta$-estradiol $\left(10^{-10} \mathrm{M}\right)$ in combination with progesterone $\left(10^{-10} M\right)$ where $n=2 .^{*}=p<0.05$.

\section{Figure 6.24.}

Modification of LDL by HUV endothelial cells in the presence of sex steroids. LDL was incubated with endothelial cells for $24 \mathrm{~h}$ at $37^{\circ} \mathrm{C}$ in the presence of various treatments. The "y" axis represents the percentage change in malondialdehyde content of samples relative to untreated LDL (average MDA content being $8.26 \pm 3.4 \mathrm{nmol} \mathrm{MDA} / \mathrm{mg}$ protein). The amount of MDA generated from the cells alone in the absence of added LDL was $1.8 \pm 0.8 \mathrm{nmol} \mathrm{MDA} / \mathrm{mg}$ protein in the first experiment, $1.26 \pm 0.99 \mathrm{nmol}$ MDA / $\mathrm{mg}$ protein in the second and $3.26 \pm 0.38 \mathrm{nmol} \mathrm{MDA} / \mathrm{mg}$ protein in the third. This graph is the mean of 3 experiments performed in duplicate. 


$$
\begin{aligned}
& \text { Hilin } \\
& \text { nillini }
\end{aligned}
$$




\section{Figure 6.25.}

A $1 \%$ agarose gel electrophoretogram of LDL samples stained with Sudan black. $8 \mu \mathrm{g}$ of protein was loaded for each sample. Lane 1 represents the mobility of native LDL (batch 12) while Lanes 2, 3 and 4 represent LDL incubated with copper sulphate for $9 \mathrm{~h}$. Lanes 5,6 and 7 represent $18 \mathrm{~h}$ incubation of LDL with copper sulphate and 8,9 and 10 represent $24 \mathrm{~h}$ exposure of preparations to copper sulphate. Lanes 2, 5 and 8 represent LDL incubated with 17 $\beta$-estradiol $\left(10^{-10} \mathrm{M}\right)$ while Lanes 3,6 and 9 represent LDL incubated with vitamin E. LDL alone exposed to copper sulphate for various times is represented is Lanes 4,7 and 10. 


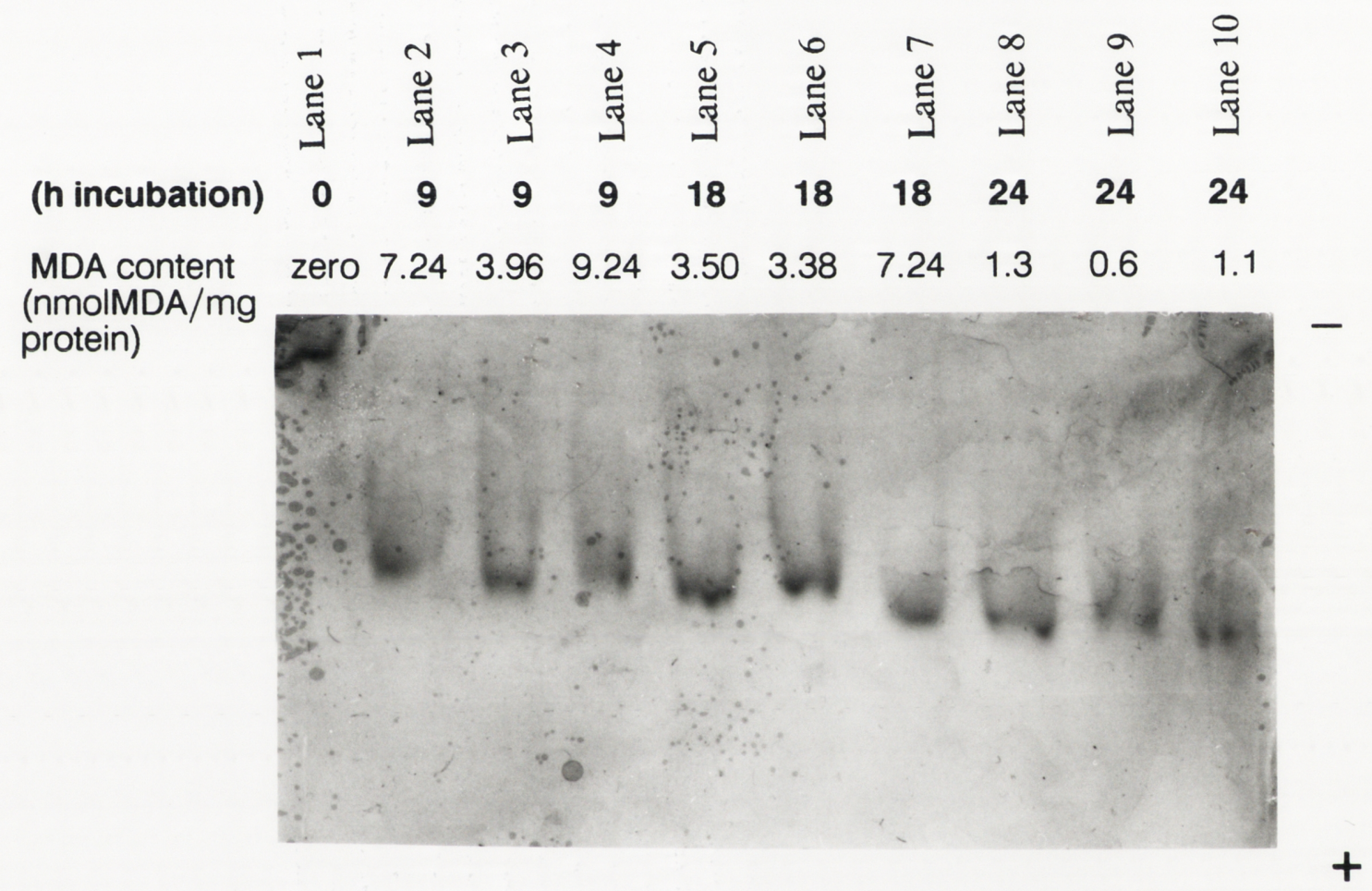


CHAPTER 7

SEX HORMONES AND ATHEROSCLEROSIS: THE CURRENT STUDIES IN PERSPECTIVE 
In earlier years studies of atherosclerosis ignored the endothelial cell, which was considered to be an inert barrier between the blood and tissues (Lefer and Sedar, 1991). Only in recent years has its active role in atherosclerosis and thrombosis begun to be addressed. Likewise, the gender difference in $\mathrm{CHD}$, although well established, has not been extensively investigated. This thesis has examined possible ways in which two sex steroids, $17 \beta$-estradiol and progesterone, can influence some endothelial cell functions of possible importance in atherosclerosis and thrombosis. The studies have also examined the effects of sex steroids on monocyte binding and on oxidation of LDL.

The main conclusions drawn from the experiments described in this thesis are that:

(a) HUV endothelial cells are responsive to $17 \beta$-estradiol and progesterone and that these sex hormones can modulate several aspects of endothelial cell function relevant to the atherosclerotic process and thrombosis;

(b) The effects of $17 \beta$-estradiol and progesterone on HUV endothelial cell function, monocyte binding and oxidation of LDL generally depend on the concentration and duration of exposure of these sex steroids to the cell or molecule of interest; and

(c) Progesterone and 17 $\beta$-estradiol can modify each other's effects on certain cell functions.

This discussion focuses on the relevance of the current findings in terms of atherogenesis and thrombosis. Particular points to be addressed include: the use of HUV endothelial cells as a model of endothelial cell function; the effects of concentration and time on the actions of $17 \beta$-estradiol and progesterone; the interaction between 17 $\beta$-estradiol and progesterone; the implications of the current findings in relation to the increased incidence of $\mathrm{CHD}$ in women 
after the menopause, hormone replacement therapy and oral contraceptive use; and finally, future directions of research.

\subsection{HUV endothelial cells as a model of arterial endothelial cell function}

HUV endothelial cells are widely used in vitro as a model for arterial endothelial cells, as discussed in Chapter 2, because they appear to have many characteristics of endothelial cells in vivo, and they are considered a suitable model for studying endothelial cells in an "injured" state (Ross 1986). From the current studies it is apparent that there is variation in response of endothelial cells to $17 \beta$-estradiol and progesterone. This is not surprising as the source of the umbilical cords was heterogeneous. The use of HUV endothelial cells from different donors may have increased the standard error of observations but it is seems more valid to use a heterogeneous human cell than non-human cells or an endothelial cell line. An endothelial cell line could have been used but as discussed in Chapter 3 long term cultures of endothelial cells are often associated with major changes in the properties of the cells.

Can the in vitro experiments described in this thesis be extrapolated to the in vivo situation? Attempts to do so should be made with caution, since there are many compounding factors, e.g. the effect of FCS concentration, the type of additives, the media used to maintain cultures and other factors such as LDL concentration. In the present studies these were all found to influence the proliferative response of HUV endothelial cells to $17 \beta$-estradiol and progesterone. As discussed in Chapter 1 , human or animal studies on $\mathrm{PGI}_{2}$ and vWf release are even more complex because of the possible effects of many more factors in vivo. At the very least, in vitro studies provide a technique which allows one to focus attention on a particular problem and to control for some of the variables. 


\subsection{Effects of $17 \beta$-estradiol and progesterone on HUV endothelial cell}

functions: The importance of concentration and time

As discussed in Chapter 3, serum concentrations of sex hormones may not reflect the actual concentrations of the hormone available at the endothelial or monocyte cell membrane. A large percentage of estradiol and progesterone is transported in association with proteins such as albumin and sex hormone binding globulin and therefore is probably not readily available to cells. Conversion of these hormones within the endothelial cell also occurs and therefore the intracellular concentration may be quite different to the extracellular concentration. Other hormones such as testosterone and cortisone, or disease states such as diabetes, can also alter endothelial cell function and possibly the interaction of progesterone and 17 $\beta$-estradiol with cells. It is well documented that humans have a widely varying capacity to metabolize sex hormones resulting in a great variation in serum concentrations. It was therefore necessary to study a range of concentrations of sex steroids to allow for variation in the concentration to which endothelial cells, macrophages and LDL may be exposed in vivo. As discussed in Chapter 3 the low concentrations of $17 \beta$-estradiol (10-8 $M-10^{-10}$ $\mathrm{M})$ and progesterone $\left(10^{-7} \mathrm{M}-10^{-9} \mathrm{M}\right)$ used in experiments approximate to the concentrations of sex steroids to which endothelial cells in coronary arteries are exposed. It is not known whether the higher concentrations of sex steroids (progesterone $\left(10^{-5} \mathrm{M}-10^{-6} \mathrm{M}\right)$ and $17 \beta$-estradiol $\left(10^{-5} \mathrm{M}-10^{-6} \mathrm{M}\right)$ ) used in experiments reflect levels seen at the endothelial cell surface in patients on the contraceptive pill or patients undergoing management of hormonally sensitive cancers, but these levels are commonly used in in vitro experiments designed to examine the pharmacological effects of sex steroids. 
Some of the observed effects of $17 \beta$-estradiol and progesterone on endothelial cell function such as inhibition of platelet binding to HUV endothelial cells and $\mathrm{PGI}_{2}$ release were time dependent. This probably reflects the proposed mechanism of action of sex steroids which is to regulate expression of genes in endothelial cells as discussed in Chapter 1. Preincubation of endothelial cells with cylcoheximide, a protein synthesis inhibitor, was shown prevent the response of endothelial cells to sex steroids.

Low concentrations of $17 \beta$-estradiol $\left(10^{-8} \mathrm{M}-10^{-10} \mathrm{M}\right.$ ) increased HUV endothelial cell proliferation but only when the concentration of ECGS was reduced. $17 \beta$-estradiol $\left(10^{-10} \mathrm{M}\right)$ did not appear to influence platelet binding, $\mathrm{PGI}_{2}$ release, the proliferative effect of native and oxidized LDL on HUV endothelial cells, and vWf expression by HUV endothelial cells or its release into the circulation. This failure to demonstrate such alterations to endothelial cell function is nevertheless an important negative observation. Similarly, low concentrations of progesterone $\left(10^{-7} \mathrm{M}-10^{-9} \mathrm{M}\right)$ altered only $\mathrm{vWf}$ expression on HUV endothelial cells. The significance of this is uncertain since the amount of vWf released from endothelial cells was not altered at this concentration of progesterone. The lack of effects of the sex steroids on these parameters, at these concentrations, may reflect the fact that these cells were already exposed to sex steroids prior to isolation and also in FCS in which the cells are cultured. $17 \beta$-estradiol $\left(10^{-10} \mathrm{M}\right)$ and progesterone (10$9 \mathrm{M}$ ) both increased mRNA for PDGF. The importance of these observations is as yet uncertain because of the need to determine whether the sex steroids also cause an increase in PDGF release from HUV endothelial cells. This could have important implications for the role of sex steroids in atherosclerosis as discussed in Chapter 1.

In contrast, high concentrations of $17 \beta$-estradiol $\left(10^{-5} \mathrm{M}\right)$ and progesterone $\left(10^{-5} \mathrm{M}\right)$ both separately and together altered a number of endothelial cell 
functions. $17 \beta$-estradiol $\left(10^{-5} \mathrm{M}\right)$ and progesterone $\left(10^{-5} \mathrm{M}\right)$ separately and together inhibited HUV endothelial cell proliferation. Together they increased mRNA expression for PDGF A and PDGF B. 17 $\beta$-estradiol (10-5 M) alone inhibited $\mathrm{PGI}_{2}$ release while also reducing platelet binding to endothelial cells which illustrates that reducing $\mathrm{PGI}_{2}$ release does not lead to increased platelet binding to endothelial cells as demonstrated by other investigators (refer to Chapter 4). Progesterone $\left(10^{-5} \mathrm{M}\right)$ alone and with 17 $\beta$-estradiol (10-5 M) inhibited VWf release and expression on HUV endothelial cells. 17kestradiol $\left(10^{-5} \mathrm{M}\right)$ and progesterone $\left(10^{-5} \mathrm{M}\right)$ also inhibited the proliferative effects of native LDL on HUV endothelial cells and increased the inhibitory effect of oxidized LDL on HUV endothelial cell proliferation. Some of these findings have the potential to promote atherogenesis while others could inhibit atherogenesis or thrombosis.

The combined effect of the two sex steroids also appears interesting. Firstly, progesterone $\left(10^{-9} \mathrm{M}\right)$ was shown to inhibit the proliferative effects of $17 \beta$-estradiol $\left(10^{-10} \mathrm{M}\right)$ in the current studies. Secondly, progesterone $\left(10^{-5}\right.$ M) was shown to inhibit the effect of $17 \beta$-estradiol in reducing the release of $\mathrm{PGI}_{2}$ from HUV endothelial cells induced by $17 \beta$-estradiol $\left(10^{-5} \mathrm{M}\right)$. Thirdly, reduced binding of platelets to endothelial cells caused by exposure of these cells to $17 \beta$-estradiol $\left(10^{-5} \mathrm{M}\right)$ was inhibited by progesterone $\left(10^{-5} \mathrm{M}\right)$. Fourthly, the combination of progesterone $\left(10^{-5} \mathrm{M}\right)$ with $17 \beta$-estradiol $\left(10^{-5}\right.$ M) did not alter monocyte binding to endothelial cells while separately each of these steroids increased monocyte binding. Fifthly, the combination of $17 \beta$ estradiol $\left(10^{-5} \mathrm{M}\right)$ with progesterone $\left(10^{-5} \mathrm{M}\right)$ caused a much more marked inhibition of HUV endothelial cell proliferation than achieved by these hormones separately. As discussed in earlier Chapters, other studies have also demonstrated the interaction of estrogen and progesterone in relation to cell functions concerned with atherosclerosis (Fischer, 1972; Fischer et al, 1985b; Armstrong, 1988; Kushwaha et al, 1991). This is of relevance 
because endothelial cells are exposed to a combination of sex steroids in vivo.

\subsection{Effect of $17 \beta$-estradiol and progesterone on monocyte binding and LDL oxidation}

$17 \beta$-estradiol $\left(10^{-10} \mathrm{M}\right)$ increased monocyte binding to endothelial cells by altering an adhesion property of the monocyte rather than by altering HUV endothelial cell function. Low concentrations of $17 \beta$-estradiol $\left(10^{-10} \mathrm{M}\right)$ also inhibited oxidation of LDL. $17 \beta$-estradiol $\left(10^{-10} \mathrm{M}\right)$ may therefore promote monocyte binding to endothelial cells but it is uncertain whether this would automatically result in increased migration into the subendothelial cell space. Even if $17 \beta$-estradiol did affect monocyte migration into the subendothelial cell space there may not be an increase in foam cell formation since $17 \beta$-estradiol has also been shown to inhibit LDL modification which is necessary for foam cell formation. On the other hand, the inhibitory effect of $17 \beta$-estradiol on LDL oxidation was only seen in the presence of copper but not in the presence of cells and therefore may not occur in the microenvironment of the vessel wall where oxidation of LDL may be essential for monocytes to clear lipid. On the other hand inhibition of LDL oxidation in the circulation by $17 \beta$-estradiol may be important in preventing atherosclerosis. Progesterone also increased monocyte binding to HUV endothelial cells but did not inhibit the oxidation of LDL. Thus, progesterone could conceivably promote fatty streak formation by promoting monocyte binding and perhaps migration into the subintimal space, and allowing modified LDL to be taken up by macrophages. 


\subsection{Implications for the use of $17 \beta$-estradiol and progesterone in}

hormone replacement therapy and oral contraceptive use

From available clinical and epidemiological studies (supported by the current studies) it would appear that low concentrations of $17 \beta$-estradiol (10$10 \mathrm{M}$ ) alone do not promote atherosclerosis and may even protect against the development of atherosclerosis. The protective effect is probably complex and is attributable in part to beneficial changes in the lipid profile e.g. lowered LDL-cholesterol and increased HDL-cholesterol levels. However, estrogen also appears to have direct beneficial effects on vessel wall physiology including inhibition of collagen synthesis, and increasing phagocytosis and immune reactivity by macrophages (Foegh, 1987). The current studies also indicate that $17 \beta$-estradiol can inhibit oxidation of LDL, which appears to be very important in fatty streak development. These studies suggest that the addition of progestin may attenuate or eliminate some beneficial effects of estrogen particularly in the pharmacological range. As suggested by some epidemiological studies (refer to Chapter 1) the addition of progestins to estrogens in hormone replacement therapy may reduce the beneficial effects of estrogen alone on CHD by not only reducing the favourable lipid profile but also by altering the balance of factors determining platelet adhesion in the event of endothelial injury.

High concentrations of estrogens used to prevent recurrent myocardial infarction or control prostatic carcinoma in men have been associated with increased risk of CHD (McGill and Stern, 1979). Similarly, high concentrations of estrogen used in the contraceptive pill have also been associated with increased CHD risk. This is thought to be mediated by mechanisms affecting the hemostatic system rather than by enhanced atherosclerosis. However, in the presence of exogenous estrogen, endothelial cell injury may further promote atherosclerosis because of slower 
regeneration of the endothelium. This would allow increased platelet adhesion and aggregation to the subendothelial space which may be further increased by the lower level of $\mathrm{PGl}_{2}$ released from endothelial cells. The release of platelet contents following aggregation may lead to increased PDGF release which could promote vasoconstriction and migration of smooth muscle cells into the intima with subsequent fibrous plaque formation. Thus, high concentrations of $17 \beta$-estradiol may be involved in the progression of atherosclerosis.

\subsection{Future directions of research}

The studies reported in this thesis open up many avenues of research. Amongst the more important would be experiments aimed at a better understanding of the molecular basis of the interactions between progesterone and $17 \beta$-estradiol in relation to endothelial and monocyte cell function. One approach would be to study steroid regulation of genes associated with mitogen release from endothelial cells and the regulation of mitogen receptors in target cells by $17 \beta$-estradiol and progesterone. Further studies on the regulation of mRNA expression of PDGF and possible release of PDGF-like mitogens from cells would be of particular interest. Demonstating that antibodies to PDGF could completely block the mitogenic response of $17 \beta$-estradiol would further characterize the present observations. These investigations would not only have important implications in atherosclerosis but also for many other diseases, such as breast cancer, where sex hormones appear to have an important regulatory role. Secondly, it would be interesting to know whether sex steroids influence endothelial cell migration as well as proliferation. Other factors such as hyperlipidemic serum selectively inhibit movement of endothelial cells (Schwartz et al, 1981) while others such as tumour necrosis factor- $\alpha$ induce migration (Pober and Cotran, 1990) but inhibit endothelial cell proliferation 
(Hajjar et al, 1987; Saegusa et al, 1990; Shimada et al, 1990). The effect of sex steroids on migration and proliferation of endothelial cells would also have important implications in angiogenesis.

There is a need for a better understanding of the clinical effects of sex hormones on atherosclerosis and thrombosis because of the increasing use of estrogens and progestins in Western society in the form of oral contraceptives, hormone replacement therapy, and in management of certain malignancies. There is still a great need for randomized controlled trials of sex hormone therapy especially in post menopausal women, to compare groups taking estrogen alone and the combination of estrogen and progestin, since many studies have failed to assess the impact of the combination of these sex steroids on CHD risk (Kalin and Zumoff, 1990), or the importance of different types of estrogens and progestins currently available. Partly as a result the clinical conundrum concerning the use of hormone replacement therapy remains. Hormone replacement therapy may not only affect the development of CHD (the most common cause of death in women of this age) but also influence the development of breast cancer, osteoporosis (a major cause of morbidity in women), perimenopausal symptoms (a leading cause of discomfort in women), and also one of the major issues of preventive medicine in women, viz. screening to detect gynecologic cancer (Goldman and Tosteson, 1991).

Finally, a greater understanding of the effects of sex hormones on atherosclerosis and, in particular, the cellular and molecular events involved may ultimately provide insight into the fundamental abnormalities which lead to arterial disease in both men and women. Knowledge gained from such studies may lead to improvements in treatment and prevention of atherosclerosis, a major cause of morbidity and mortality in Western society. 
APPENDIX 1

COMPOSITION OF TISSUE CULTURE MEDIA (MEDIUM 199)

INGREDIENT

L-Alanine

L-Arginine $\mathrm{HCl}$

L-Aspartic acid

L-Cysteine $\mathrm{HCl}$

L-Cystine

L-Glutamic acid

L-Glutamine

Glutathione

Glycine

L-Histidine.HCl.H2O

L-Hydroxyproline

L-Isoleucine

L-Leucine

L-Lysine $\mathrm{HCl}$

L-Methionine

L-Phenylalanine

L-Proline

L-Serine

L-Threonine

L-Tryptophan

L-Tyrosine disodium salt

L-Tyrosine $\mathrm{HCl}$

L-Valine

L-Ascorbic acid

Biotin

Calciferol

D-calcium pantothenate

Choline chloride

Folic acid

i-Inositol

menaphthone sodium

bisulphite $3 \mathrm{H}_{2} \mathrm{O}$

Nicotinic acid

Nicotinamide

p-Aminobenzoic acid

Pyridoxal $\mathrm{HCl}$

Pyridoxine $\mathrm{HCl}$

Ribroflavin

Thiamin $\mathrm{HCl}$

DL-a tocopherol phosphate disodium salt

Vitamin A acetate

Calcium chloride

Ferric nitrite

Potassium chloride

Magnesium sulphate
FLOW LABORATORY

$\mathrm{mg} / \mathrm{L}$

25.00

70.00

30.00

0.0987

23.66

66.82

omitted

0.05

50.00

21.88

10.00

20.00

60.00

70.00

15.00

25.00

40.00

25.00

30.00

10.00

49.72

25.00

0.05

0.01

0.10

0.01

0.50

0.01

0.05

0.019

0.025

0.025

0.05

0.025

0.025

0.01

0.01

0.01

0.1147

264.9

0.72

400

200
CYTOSYSTEMS

$\mathrm{mg} / \mathrm{l}$

25.00

70.00

30.00

0.11

26.00

75.00

omitted

0.05

50.00

21.88

10.00

20.00

60.00

70.00

15.00

25.00

40.00

25.00

30.00

10.00

48.00

25.00

0.05

0.01

0.10

0.01

0.50

0.01

0.05

0.019

0.025

0.05

0.05

0.01

0.01

0.01

0.14

200

0.1

400

97.7 
Sodium chloride

Sodium di-hydrogen ortho-phosphate

Sodium hydrogen carbonate

Adenine sulphate

5'-AMP

ATP, disodium salt

Cholesterol

2-deoxribose

D-glucose

Guanine $\mathrm{HCl}$

Hypoxanthine

D-ribose

Sodium acetate
6800

6800

158.3

omitted

140

10.00

0.20

0.20

0.2

0.5

1000

0.3

0.3

0.5

36.71 omitted

10.00

0.20

10

0.2

0.5

1000

0.3

0.354

0.5

50.00 


\section{APPENDIX 2}

\section{EFFECT OF SEX STEROIDS ON THE REQUIREMENT OF HUV ENDOTHELIAL CELLS FOR FCS, ECGS AND HEPARIN}

\section{METHODS}

Effect of sex steroids on the requirement of HUV endothelial cell proliferation for FCS

A 1 in 2 dilution of FCS was made in PRF Medium 199 or phenol containing Medium 199. The initial concentration of FCS was $20 \%$. To this the sex steroids were added and then the HUV endothelial cells $\left(2.5 \times 10^{3}\right.$ cells /well). The cells were incubated for $48 \mathrm{~h}$, and then $\left[{ }^{3} \mathrm{H}\right]$-thymidine $(0.5 \mu \mathrm{Ci} /$ well) was added and the cells incubated for a further $18 \mathrm{~h}$ in $5 \% \mathrm{CO}_{2}-95 \%$ air atmosphere at $37{ }^{\circ} \mathrm{C}$ before the supernatant was removed and the cells washed, trypsinized and harvested as described in Section 2.3.7.

Effect of sex steroids on the requirement of HUV endothelial cell proliferation for ECGS

The effect of different concentrations of ECGS on HUV endothelial cells ( $5 x$ $10^{3}$ cells /well) was assessed in the presence of sex steroids in PRF Medium 199 over $66 \mathrm{~h}$ following the same protocol as outlined in 2.3.10. The highest concentration of ECGS was either 200 or $400 \mu \mathrm{g} / \mathrm{ml}$, depending on the experiment. 
Effect of sex steroids on the requirement of HUV endothelial cell proliferation for heparin

The effects of the sex steroids on heparin requirements for optimal proliferation of HUV endothelial cells was assessed in the presence of $50 \mu \mathrm{g}$ $/ \mathrm{ml}$ of ECGS using the same protocol as described in Section 2.3.11.

In a second series of experiments the concentration of heparin was varied $(0-40 \mathrm{U} / \mathrm{ml})$ in the presence of different concentrations of ECGS and sex steroids as it has been demonstrated that the proliferative effects of some cytokines (e.g. IL-1 and tumour necrosis factor- $\alpha$ ) is modulated by the presence of ECGS and heparin (Saegusa et al, 1990). The HUV endothelial cells were then incubated for $48 \mathrm{~h}$ in $5 \% \mathrm{CO}_{2}-95 \%$ air atmosphere at $37^{\circ} \mathrm{C}$, $\left[{ }^{3} \mathrm{H}\right]$-thymidine $(0.5 \mu \mathrm{Ci} /$ well) was then added and the cells incubated for a further $18 \mathrm{~h}$ before being washed, trypsinized and harvested as described in Section 2.3.7.

\section{RESULTS}

Effect of sex steroids on the requirement of HUV endothelial cell proliferation for FCS

There was an increased requirement for FCS for optimal proliferation when HUV endothelial cells were exposed to high concentrations of $17 \beta$-estradiol $\left(10^{-5} \mathrm{M}\right)$, progesterone $\left(10^{-5} \mathrm{M}\right)$ and the combination of these two steroids. The addition of low concentrations of $17 \beta$-estradiol $\left(10^{-10} \mathrm{M}\right)$ or progesterone $\left(10^{-9} \mathrm{M}\right)$ or the combination of these two did not alter the requirement for FCS for optimal proliferation (Figures 1, 2, 3, 4, 5, 6). In PRF or phenol containing Medium 199, 10-20 \% FCS appeared to be the optimal concentration for 
maximal HUV endothelial cell proliferation. The relative proliferative effect of the sex steroids compared to Medium 199 alone (control) is evident. This is presented in more detail in Section 3.4.2.

Effect of sex steroids on the requirement of HUV endothelial cell proliferation for ECGS

The addition of sex steroids did not significantly alter the optimal requirement of HUV endothelial cells for ECGS, compared to Medium 199 alone (control) (Figures 7, 8 and 9).

Effect of sex steroids on the requirement of HUV endothelial cell proliferation for heparin

The addition of the sex steroids to HUV endothelial cells generally caused small shifts in the requirement for heparin relative to Medium 199 alone (control) (Figures 10, 11, and 12). The optimal concentration for heparin for HUV endothelial cell proliferation in the presence of $17 \beta$-estradiol $\left(10^{-5} \mathrm{M}\right.$ and $10^{-10} \mathrm{M}$ ) was between 6.25 and $12.5 \mathrm{U} / \mathrm{ml}$ (Figure 10) and between 12.5 and $25 \mathrm{U} / \mathrm{ml}$ with progesterone $\left(10^{-5} \mathrm{M}\right.$ and $\left.10^{-9} \mathrm{M}\right)$ (Figure 11). The proliferative effect of these sex steroids relative to Medium 199 alone (control) is also evident.

In the presence of sex steroids there was no change in the requirement for heparin when cells were exposed to $17 \beta$-estradiol $\left(10^{-10} \mathrm{M}\right)$ or progesterone $\left(10^{-9} \mathrm{M}\right)$, although when cells were exposed to a combination of $17 \beta$-estradiol $\left(10^{-10} \mathrm{M}\right)$ and progesterone $\left(10^{-9} \mathrm{M}\right)$ an optimal proliferative response was observed in the presence of $20 \mathrm{U} / \mathrm{ml}$ heparin rather than 5 or $10 \mathrm{U} / \mathrm{ml}$ (Figures 14,16 and 18$)$. In the presence of $17 \beta$-estradiol $\left(10^{-5} \mathrm{M}\right)$ and heparin 
$20 \mathrm{U} / \mathrm{ml}$ there was a lower level of HUV endothelial cell proliferation compared to proliferation with heparin concentrations of 5 or $10 \mathrm{U} / \mathrm{ml}$ in 2 of the three experiments (Figure 13). In the presence of progesterone $\left(10^{-5} \mathrm{M}\right)$ and the combination of $17 \beta$-estradiol $\left(10^{-5} \mathrm{M}\right)$ with progesterone $\left(10^{-5} \mathrm{M}\right)$ there was no consistent change in the requirement for heparin (Figures 15 and 17). 


\section{Figure 1.}

Effect of FCS on HUV endothelial cell proliferation in the presence of $17 \beta$-estradiol in PRF Medium 199. 1 in 2 dilutions of FCS (maximum concentration $20 \%$ ) were made to which $17 \beta$-estradiol $\left(10^{-5} \mathrm{M}\right.$ or $10^{-}$ 10 M) or PRF Medium 199 (control) was added and then incubated with endothelial cells (passage 7) for $66 \mathrm{~h}$. The background counts for endothelial cell proliferation in the absence of FCS for cells exposed to $17 \beta$-estradiol $\left(10^{-5} \mathrm{M}\right.$ and $10^{-10} \mathrm{M}$ ) were $95 \mathrm{cpm}$ and 22 $\mathrm{cpm}$ respectively, while the control was $26 \mathrm{cpm}$. Different concentrations of FCS are shown on the " $x$ " axis. Each point represents a single value of one experiment.

\section{Figure 2.}

Effect of FCS on HUV endothelial cell proliferation in the presence of $17 \beta$-estradiol in phenol red containing Medium 199. The same method as that described in Figure 2 was followed except that phenol containing Medium 199 was used. The background counts for endothelial cell proliferation (passage 7) in the absence of FCS for cells exposed to $17 \beta$-estradiol $\left(10^{-5} \mathrm{M}\right.$ and $10^{-10} \mathrm{M}$ ) were $16 \mathrm{cpm}$ and $10 \mathrm{cpm}$ respectively, while the control was $12 \mathrm{cpm}$. The key to this figure is the same as for Figure 1. Each point represents a single value of one experiment. 
EFFECT OF FCS ON ENDOTHELIAL CELL PROLIFERATION

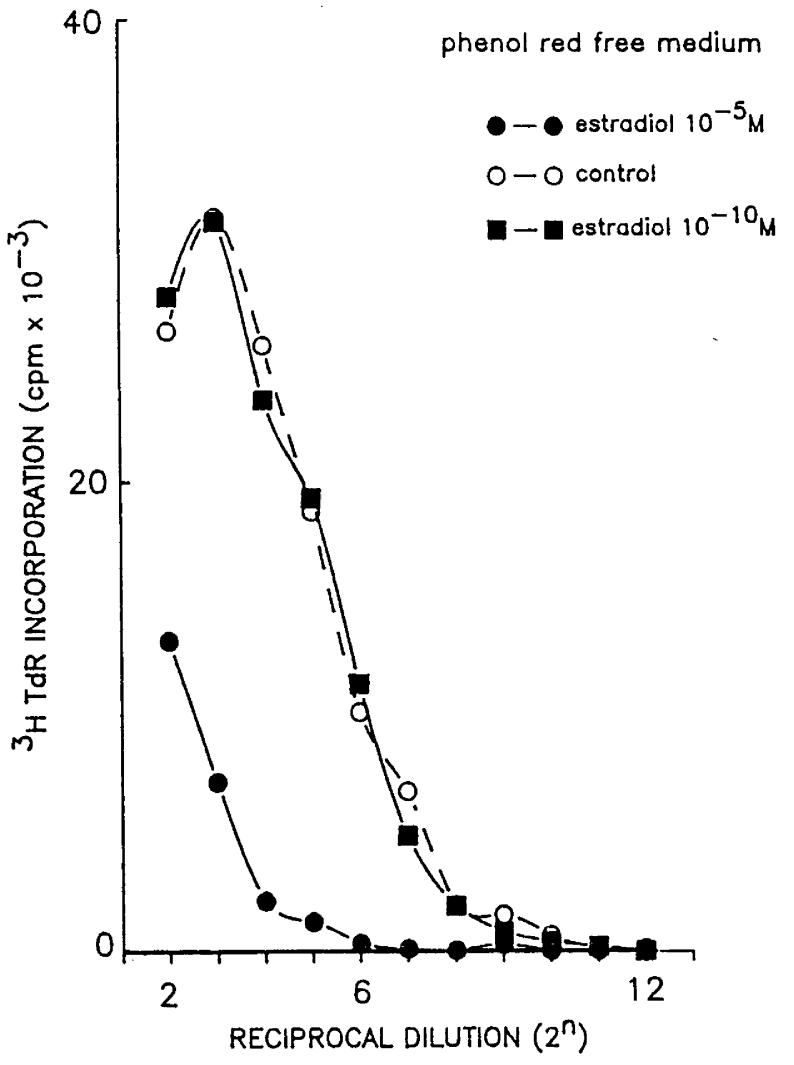

EFFECT OF FCS ON ENDOTHELIAL CELLL PROLIFERATION

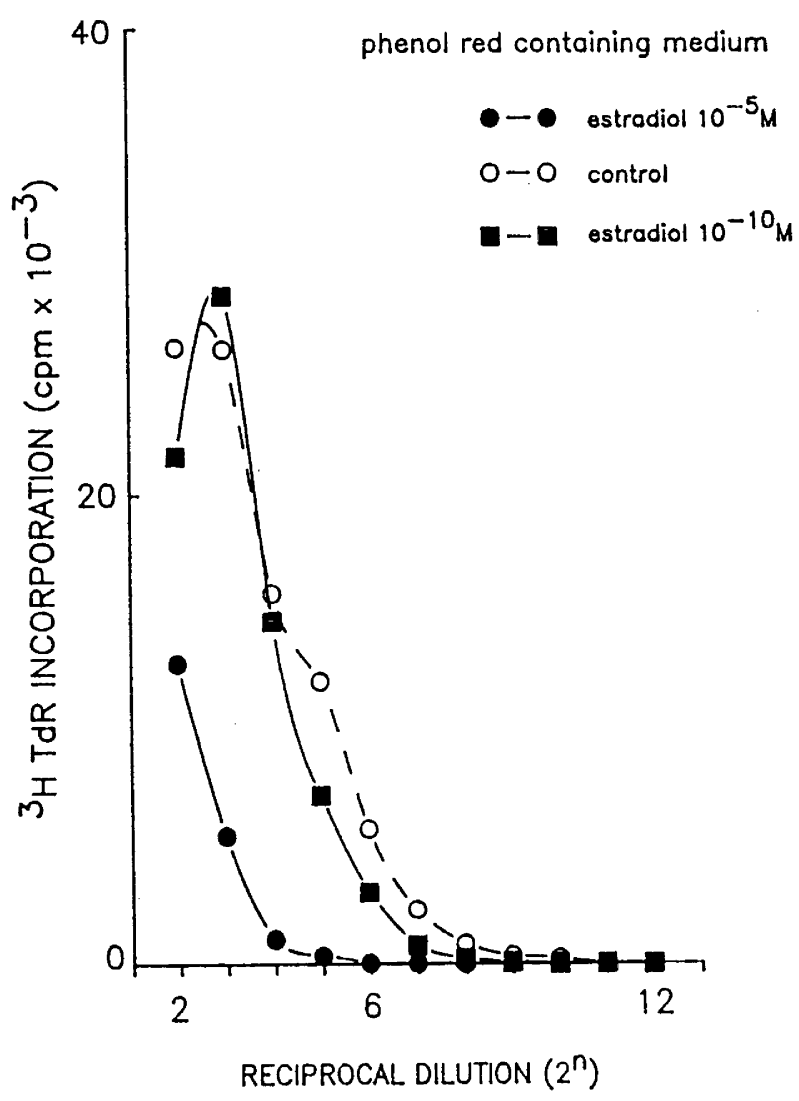




\section{Figure 3.}

Effect of FCS on HUV endothelial cell proliferation in the presence of progesterone in PFR Medium 199. 1 in 2 dilutions of FCS (maximum concentration $20 \%$ ) were made to which progesterone $\left(10^{-5} \mathrm{M}\right.$ or $10^{-}$ 9 M) or Medium 199 (control) was added and then incubated with endothelial cells for $66 \mathrm{~h}$. The background counts for endothelial cell proliferation (passage 7 ) in the absence of FCS for cells exposed to progesterone $\left(10^{-5} \mathrm{M}\right.$ and $\left.10^{-9} \mathrm{M}\right)$ were $12 \mathrm{cpm}$ and $16 \mathrm{cpm}$ respectively, while the control was $26 \mathrm{cpm}$. The key to this figure is the same as for Figure 1. Each point represents a single value of one experiment.

\section{Figure 4.}

Effect of FCS on HUV endothelial cell proliferation in the presence of progesterone in phenol red containing Medium 199. The same method as that described in the legend to Figure 3 was followed except that phenol red containing Medium 199 was used. The background counts for endothelial cell proliferation (passage 7) in the absence of FCS for cells exposed to progesterone $\left(10^{-5} \mathrm{M}\right.$ and $10^{-9}$ M) were $11 \mathrm{cpm}$ and $13 \mathrm{cpm}$ respectively, while the control was 12 $\mathrm{cpm}$. The key to this figure is the same as for Figure 1. Each point represents a single value of one experiment. 
EFFECT OF FCS ON ENDOTHELIAL CELL PROLIFERATION

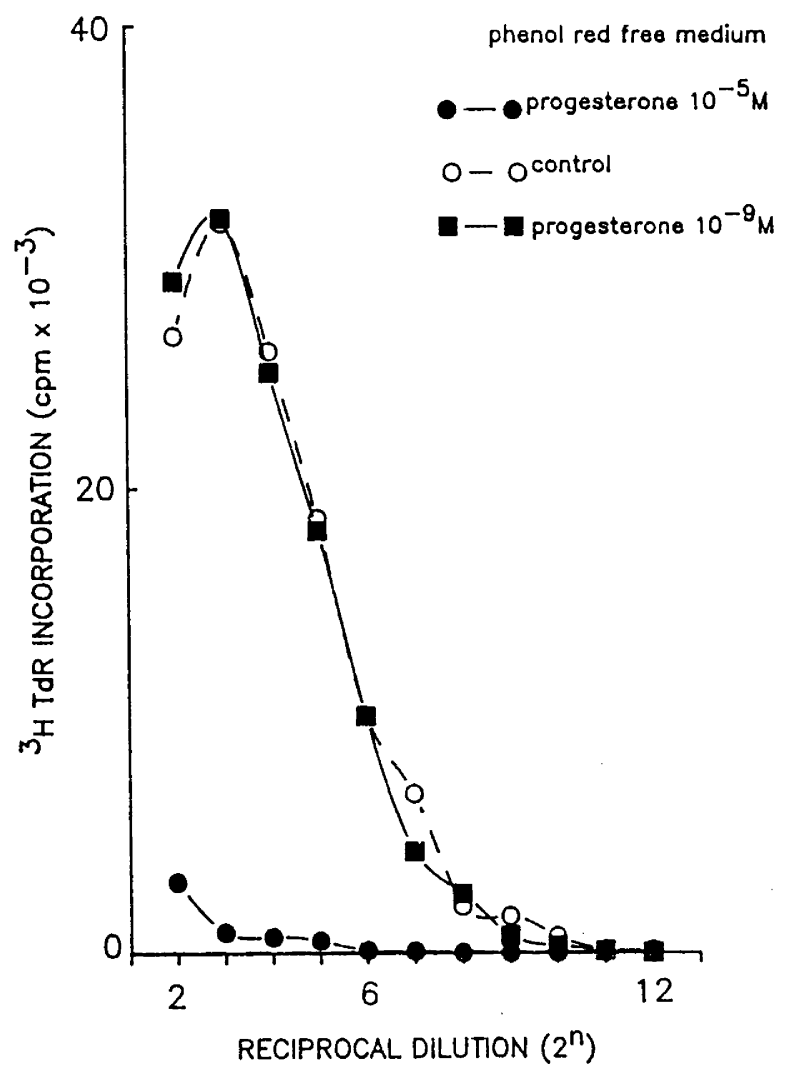

EFFECT OF FCS ON ENDOTHELIAL CELL PROLIFERATION

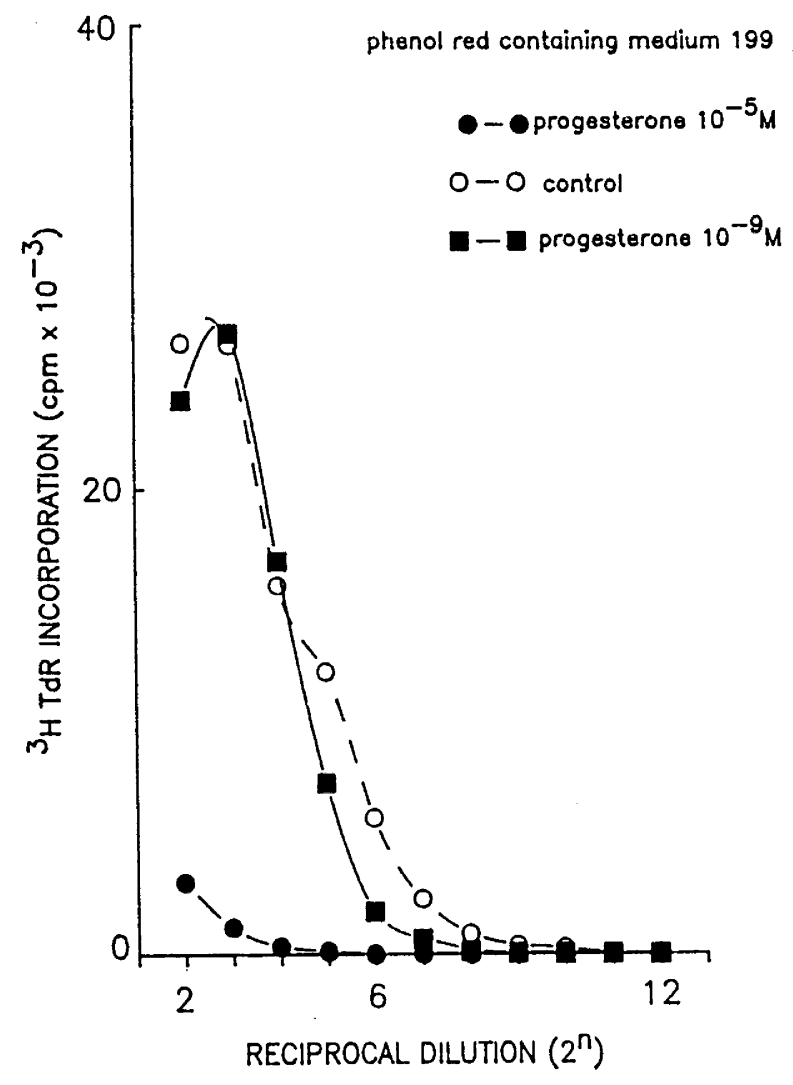




\section{Figure 5.}

Effect of FCS on HUV endothelial cell proliferation in the presence of combinations of sex steroids in PFR Medium 199. 1 in 2 dilutions of FCS (maximum concentration $20 \%$ ) were added to the combination of $17 \beta$-estradiol $\left(10^{-10} \mathrm{M}\right)$ with progesterone $\left(10^{-9} \mathrm{M}\right)$, or $17 \beta$-estradiol $\left(10^{-5} \mathrm{M}\right)$ in combination with progesterone $\left(10^{-5} \mathrm{M}\right)$, or PFR Medium 199 (control) and then incubated with endothelial cells (passage 7) for $66 \mathrm{~h}$. The background counts for endothelial cell proliferation in the absence of FCS for cells exposed to the combination of $17 \beta$-estradiol $\left(10^{-10} \mathrm{M}\right)$ with progesterone $\left(10^{-9} \mathrm{M}\right)$ or $17 \beta$-estradiol $\left(10^{-5} \mathrm{M}\right)$ with progesterone $\left(10^{-5} \mathrm{M}\right)$ were $21 \mathrm{cpm}$ and $8 \mathrm{cpm}$ respectively while the control was $26 \mathrm{cpm}$. The key to this figure is the same as for Figure 1. Each point represents a single value of one experiment.

\section{Figure 6.}

Effect of FCS on HUV endothelial cell proliferation in the presence of combinations of sex steroids in phenol red containing Medium 199. The same method as that described in Figure 5 was followed except that phenol red containing Medium 199 was used. The background counts for endothelial cell proliferation (passage 7) in the absence of FCS for cells exposed to the combination of $17 \beta$-estradiol $\left(10^{-10} \mathrm{M}\right)$ with progesterone $\left(10^{-9} \mathrm{M}\right)$, or $17 \beta$-estradiol $\left(10^{-5} \mathrm{M}\right)$ in combination with progesterone $\left(10^{-5} \mathrm{M}\right)$ were $15 \mathrm{cpm}$ and $13 \mathrm{cpm}$ respectively while the control was $12 \mathrm{cpm}$. The key to this figure is the same as for Figure 1. Each point represents a single value of one experiment. 
EFFECT OF FCS ON ENDOTHELIAL CELLL PROLIFERATION

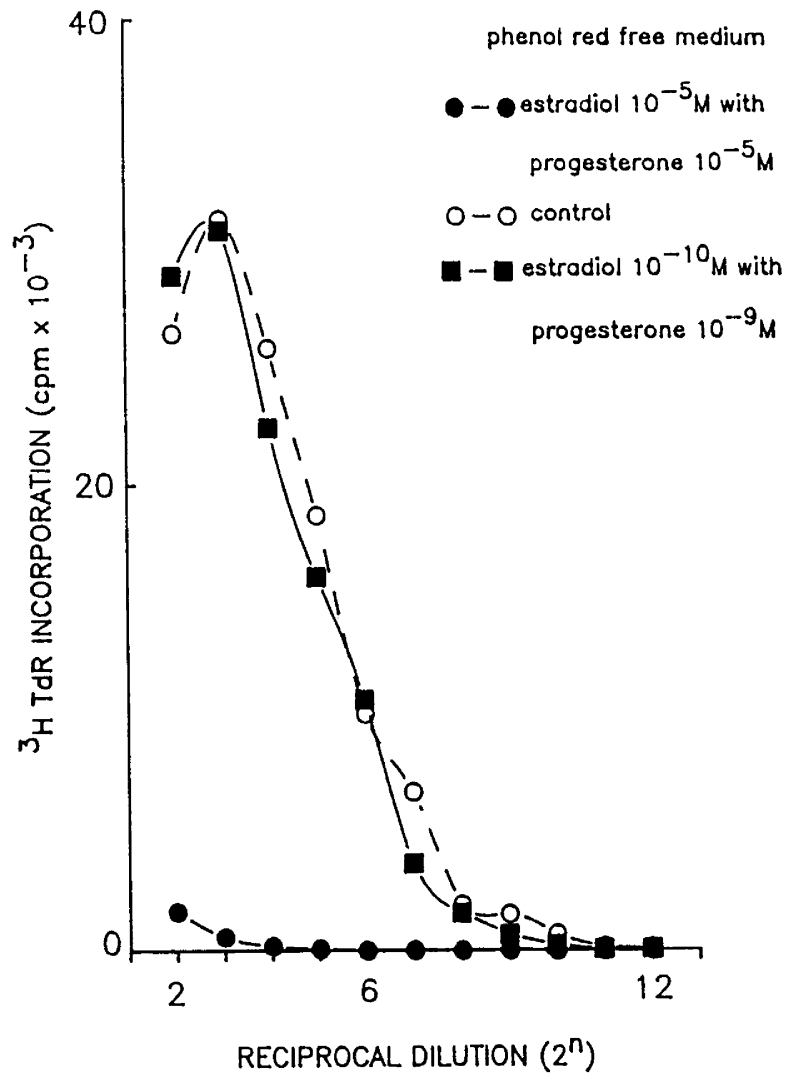

EFFECT OF FCS ON ENDOTHELIAL CELL PROLIFERATION

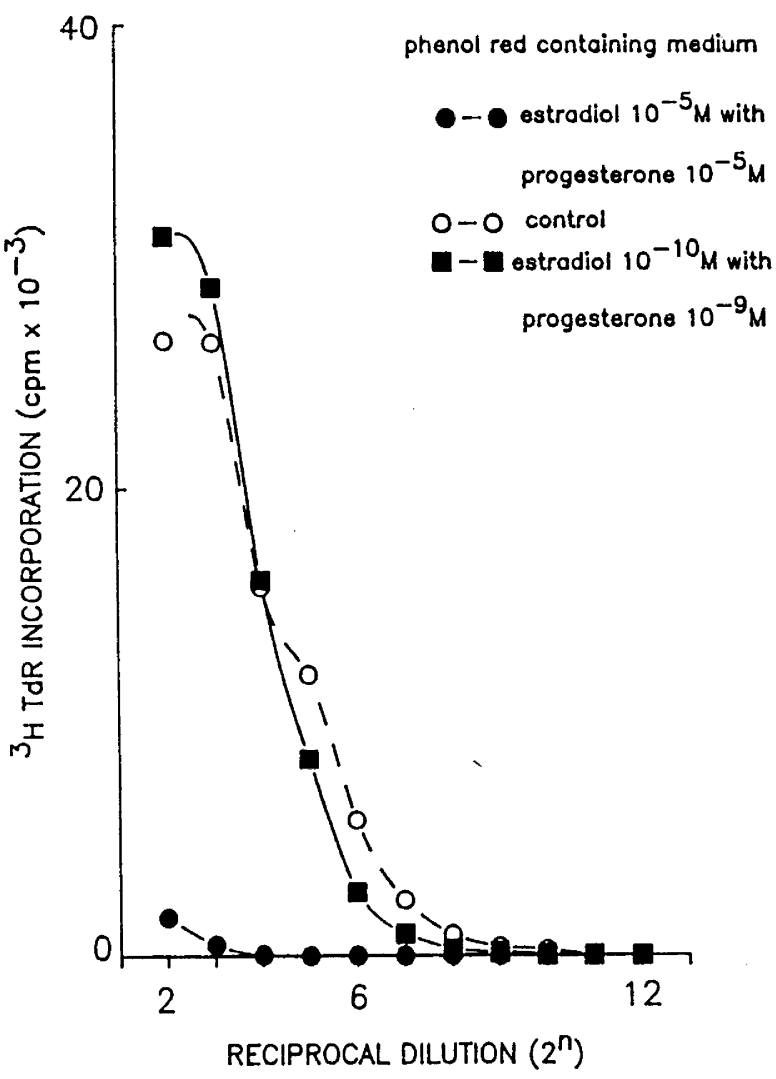




\section{Figure 7.}

Effect of different concentrations of ECGS on HUV endothelial cell proliferation in the presence of $20 \%$ FCS and $17 \beta$-estradiol. Endothelial cells (passage 7) were exposed to 1 in 2 dilutions of ECGS (maximum concentration $400 \mu \mathrm{g} / \mathrm{ml}$ ) over $66 \mathrm{~h}$ in the presence of $20 \% \mathrm{FCS}$, heparin $20 \mathrm{U} / \mathrm{ml}$ and $17 \beta$-estradiol $\left(10^{-5} \mathrm{M}\right.$ or $\left.10^{-10} \mathrm{M}\right)$ or control (Medium 199 alone). Different concentrations of ECGS are shown on the "x" axis.. This graph is representative of 2 experiments. Each point is a single value.

\section{Figure 8.}

Effect of different concentrations of ECGS on HUV endothelial cell proliferation in the presence of $20 \%$ FCS and progesterone. Endothelial cells (passage 7) were exposed to 1 in 2 dilutions of ECGS (maximum concentration $400 \mu \mathrm{g} / \mathrm{ml}$ ) over $66 \mathrm{~h}$ in the presence of $20 \% \mathrm{FCS}$, heparin $20 \mathrm{U} / \mathrm{ml}$ and progesterone $\left(10^{-5} \mathrm{M}\right.$ or $\left.10^{-9} \mathrm{M}\right)$. The key to this figure is the same as for Figure 7 . This graph is representative of 2 experiments. Each point is a single value. 
EFFECT OF ECGS ON ENDOTHELIAL CELL PROLIFERAION

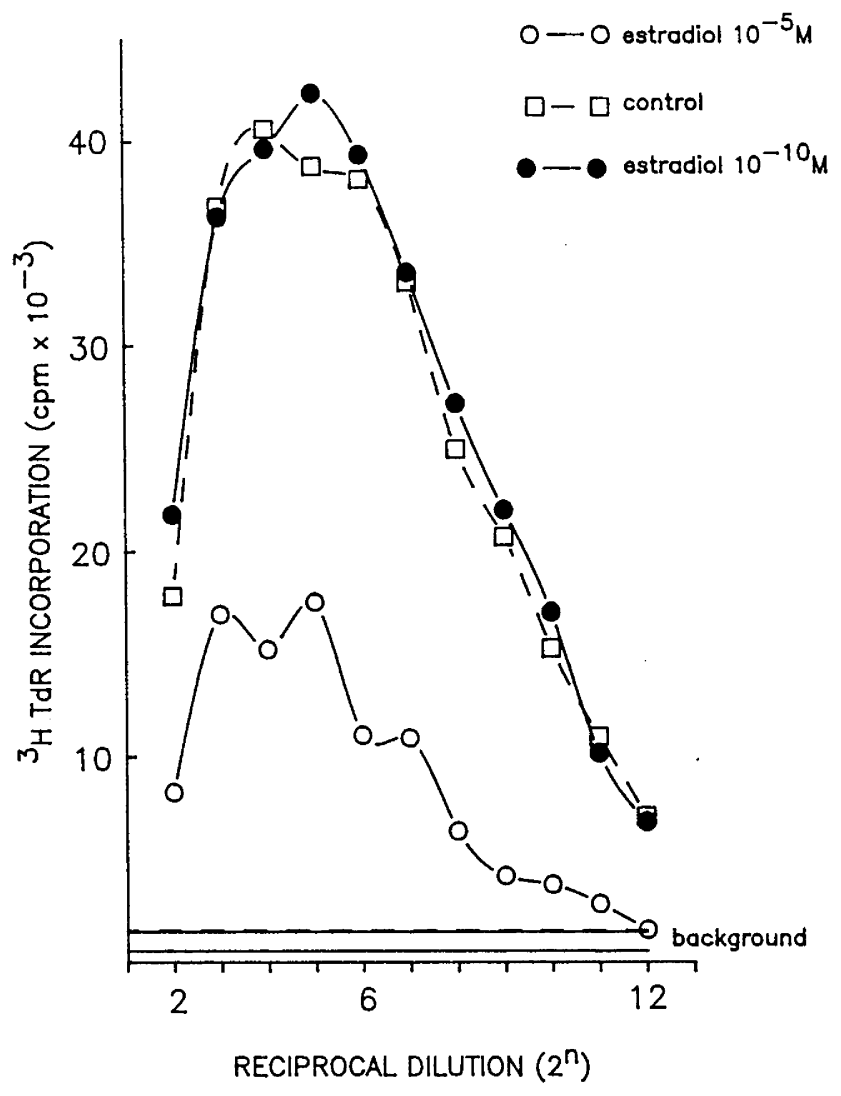

EFFECT OF ECGS ON ENDOTHELIAL CELL PROLIFERATION

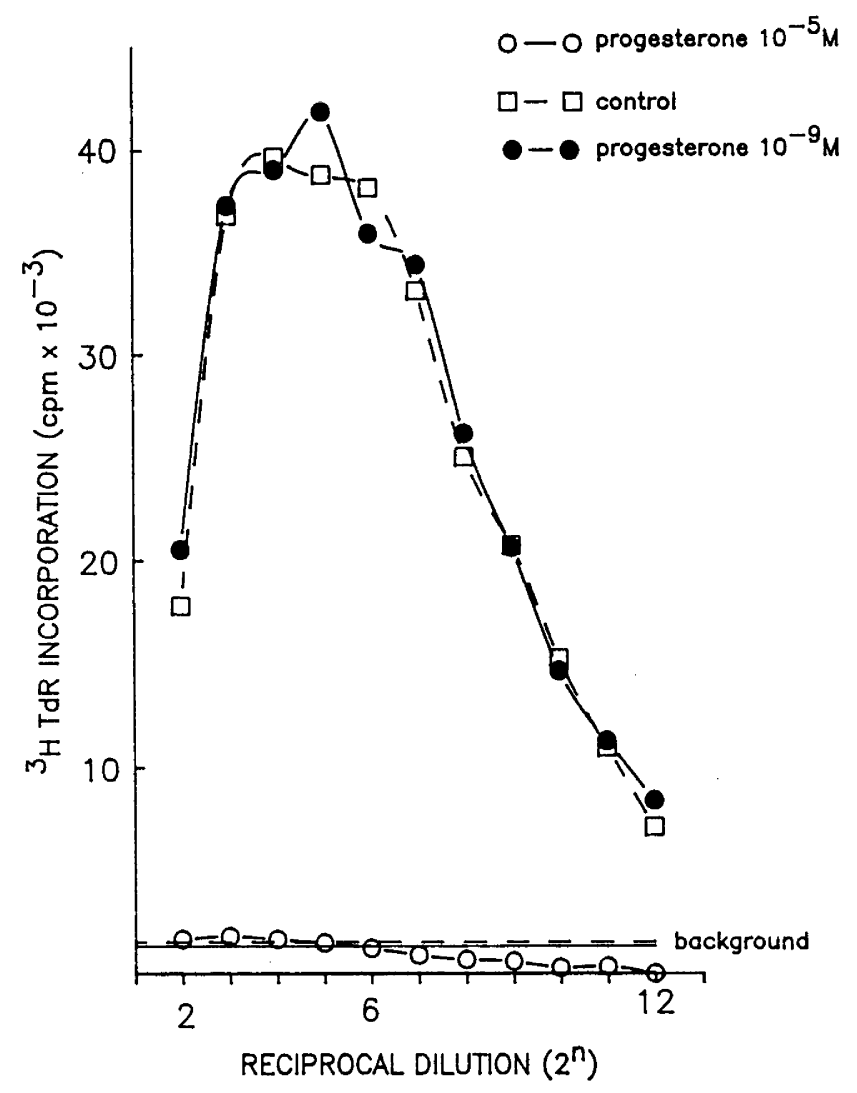




\section{Figure 9.}

Effect of different concentrations of ECGS on HUV endothelial cell proliferation in the presence of $20 \%$ FCS and combinations of $17 \beta$ estradiol and progesterone. Endothelial cells (passage 7) were exposed to 1 in 2 dilutions of ECGS (maximum concentration $400 \mu \mathrm{g}$ $/ \mathrm{ml}$ ) over $66 \mathrm{~h}$ in the presence of $20 \% \mathrm{FCS}$, heparin $20 \mathrm{U} / \mathrm{ml}$ and the combination of $17 \beta$-estradiol $\left(10^{-5} \mathrm{M}\right)$ with progesterone $\left(10^{-5} \mathrm{M}\right)$, or $17 \beta$-estradiol $\left(10^{-10} \mathrm{M}\right)$ in combination with progesterone $\left(10^{-9} \mathrm{M}\right)$. The key to this figure is the same as for Figure 7 . This graph is representative of 2 experiments. Each point is a single value.

\section{Figure 10.}

Effect of different concentrations of heparin on HUV endothelial cell proliferation in the presence of $17 \beta$-estradiol. Endothelial cells (passage 7) were exposed to 1 in 2 dilutions of heparin (maximum concentration $200 \mathrm{U} / \mathrm{ml}$ ) in Medium 199 (with $20 \%$ FCS, gentamicin $60 \mathrm{U} / \mathrm{ml}$ and ECGS $50 \mu \mathrm{g} / \mathrm{ml}$ ) with $17 \beta$-estradiol $\left(10^{-5} \mathrm{M}\right.$ or $\left.10^{-10} \mathrm{M}\right)$ and incubated for $66 \mathrm{~h}$. Different concentrations of heparin are shown on the " $x "$ axis. The background (straight lines) represents endothelial cells not exposed to heparin but exposed to $17 \beta$-estradiol or Medium 199 alone. This graph is representative of 2 experiments. Each point is a single value. 
EFFECT OF ECGS ON ENDOTHELIAL CELL PROLIFERATION

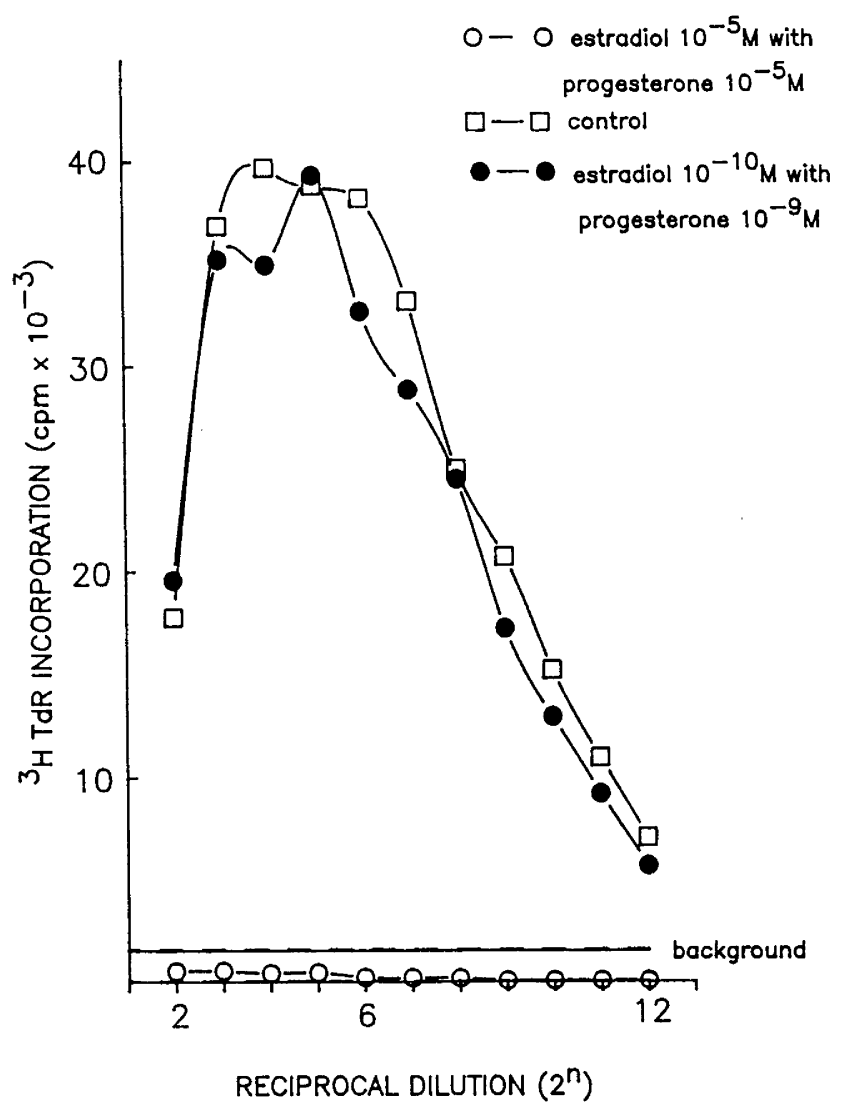

TITRATION OF HEPARIN IN PRESENCE OF SEX STEROIDS

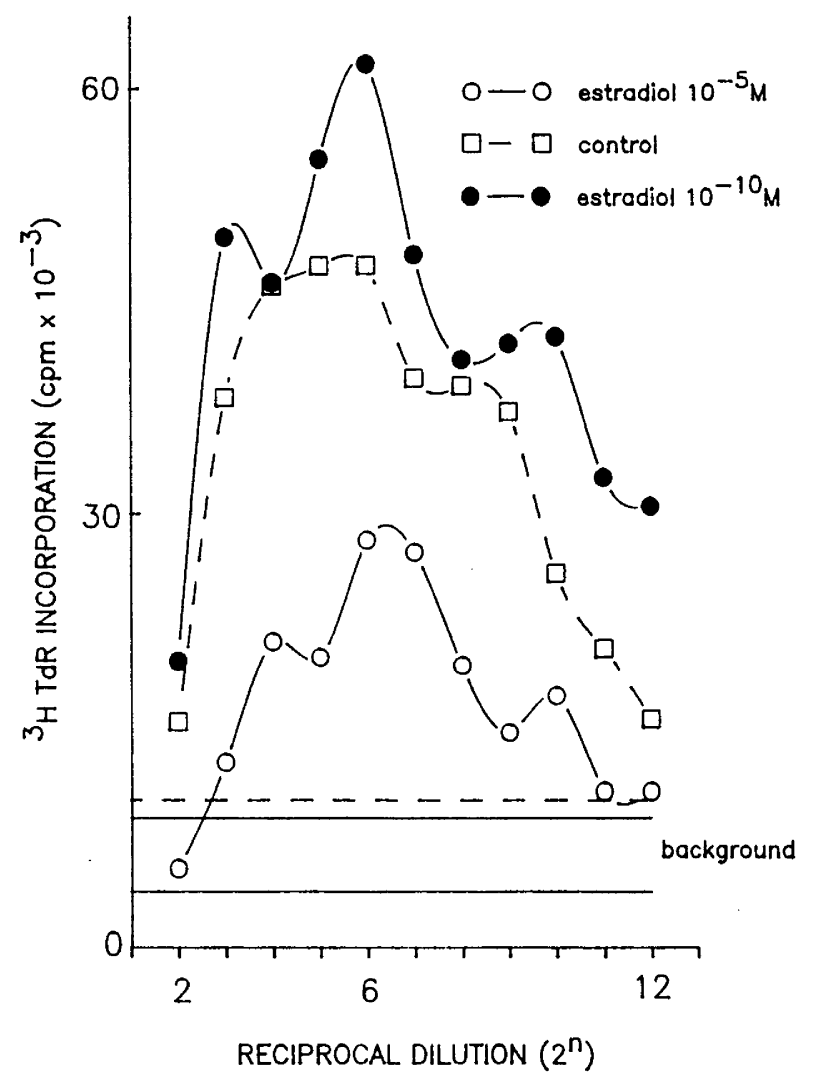




\section{Figure 11.}

Effect of different concentrations of heparin on HUV endothelial cell proliferation in the presence of progesterone. Endothelial cells (passage 7) were exposed to 1 in 2 dilutions of heparin (maximum concentration $200 \mathrm{U} / \mathrm{ml}$ ) in Medium 199 (with $20 \%$ FCS, gentamicin $60 \mathrm{U} / \mathrm{ml}$ and ECGS $50 \mu \mathrm{g} / \mathrm{ml})$ in the presence of progesterone $\left(10^{-5}\right.$ $\mathrm{M}$ or $10^{-9} \mathrm{M}$ ) and incubated for $66 \mathrm{~h}$. The key to this figure is the same as for Figure 10. The background (straight lines) represents endothelial cells not exposed to heparin but exposed to progesterone or Medium 199 alone. This graph is representative of 2 experiments. Each point is a single value.

\section{Figure 12.}

Effect of different concentrations of heparin on HUV endothelial cell proliferation in the presence of the combination of $17 \beta$-estradiol with progesterone. Endothelial cells (passage 7) were exposed to 1 in 2 dilutions of heparin (maximum concentration $200 \mathrm{U} / \mathrm{ml}$ ) in Medium 199 (with $20 \%$ FCS, gentamicin $60 \mathrm{U} / \mathrm{ml}$ and ECGS $50 \mu \mathrm{g} / \mathrm{ml}$ ), in the presence of the combination of $17 \beta$-estradiol $\left(10^{-10} \mathrm{M}\right)$ with progesterone $\left(10^{-9} \mathrm{M}\right)$, or $17 \beta$-estradiol $\left(10^{-5} \mathrm{M}\right)$ in combination with progesterone $\left(10^{-5} \mathrm{M}\right)$ and incubated for $66 \mathrm{~h}$. The key to this figure is the same as for Figure 10. The background (straight lines) represents endothelial cells not exposed to heparin but exposed to $17 \beta$-estradiol $\left(10^{-10} \mathrm{M}\right)$ with progesterone $\left(10^{-9} \mathrm{M}\right)$, or $17 \beta$-estradiol $\left(10^{-5} \mathrm{M}\right)$ in combination with progesterone $\left(10^{-5} \mathrm{M}\right)$, or Medium 199 alone. This graph is representative of 2 experiments. Each point is a single value. 
EFFECT OF HEPARIN ON ENDOTHELIAL CELL PROLIFERATION

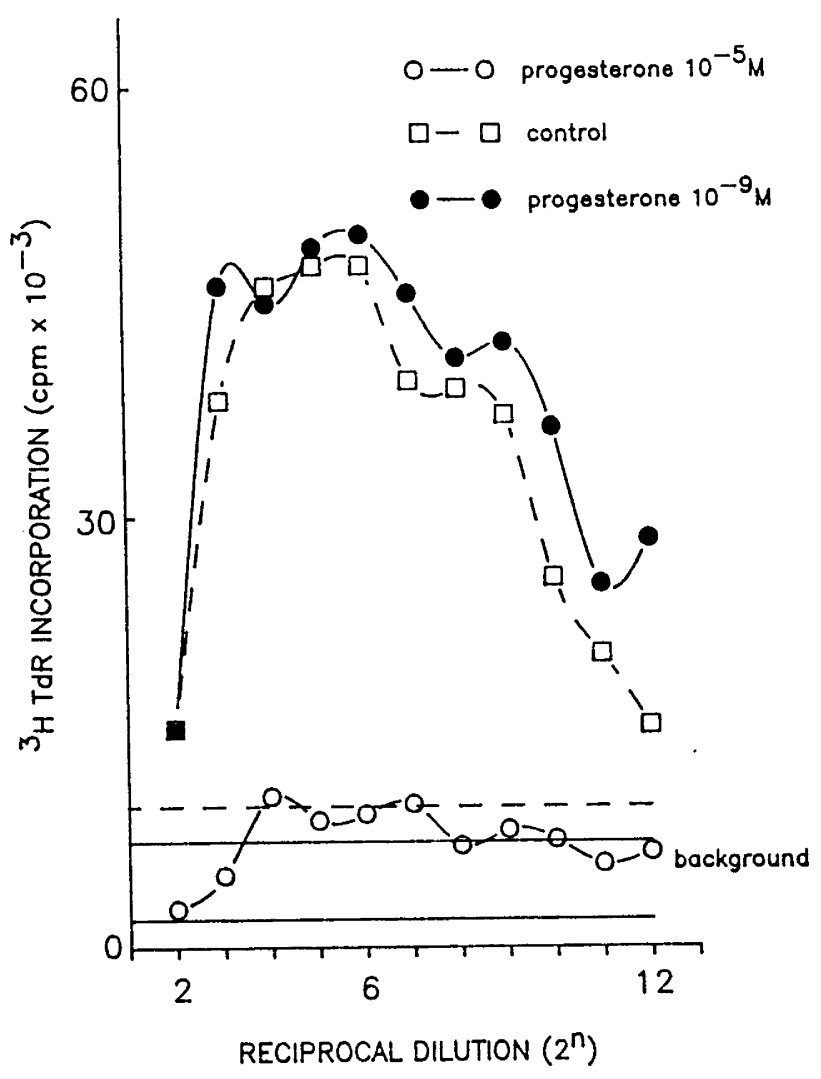

EFFECT OF HEPARIN ON ENDOTHELIAL CELL PROLIFERATION

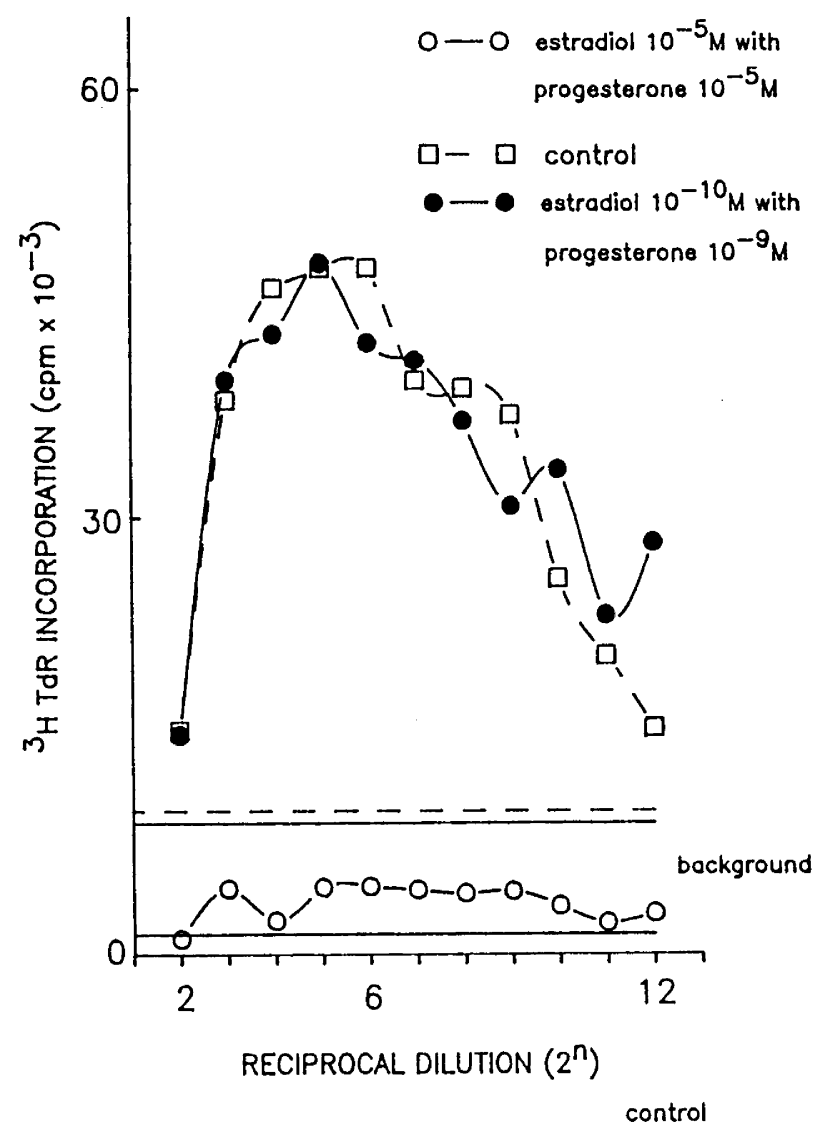




\section{Figure 13.}

Effect of different concentrations of ECGS on HUV endothelial cell proliferation in the presence of $20 \% \mathrm{FCS}, 17 \beta$-estradiol $\left(10^{-5} \mathrm{M}\right)$ and different concentrations of heparin. Endothelial cells (passage 7) were exposed to 1 in 2 dilutions of ECGS (maximum concentration $400 \mu \mathrm{g} / \mathrm{ml})$ over $66 \mathrm{~h}$ in the presence of $20 \% \mathrm{FCS}$, heparin (0-20 U $/ \mathrm{ml}$ ) and $17 \beta$-estradiol $\left(10^{-5} \mathrm{M}\right)$. The background count for endothelial cell proliferation in the absence of ECGS for cells exposed to $17 \beta$-estradiol $\left(10^{-5} \mathrm{M}\right)$ averaged $772 \mathrm{cpm}$. Different concentrations of ECGS are shown on the "x" axis. This graph is representative of 3 experiments. Each point is a single value.

\section{Figure 14.}

Effect of different concentrations of ECGS on HUV endothelial cell proliferation in the presence of $20 \% \mathrm{FCS}, 17 \beta$-estradiol (10-10 M) and different concentrations of heparin. Endothelial cells (passage 7) were exposed to 1 in 2 dilutions of ECGS (maximum concentration $400 \mu \mathrm{g} / \mathrm{ml}$ ) over $66 \mathrm{~h}$ in the presence of $20 \% \mathrm{FCS}$, heparin (0-20 U $/ \mathrm{ml}$ ) and $17 \beta$-estradiol $\left(10^{-10} \mathrm{M}\right)$. The background count for endothelial cell proliferation in the absence of ECGS for cells exposed to $17 \beta$-estradiol $\left(10^{-10} \mathrm{M}\right)$ averaged $2357 \mathrm{cpm}$. The key to this figure is the same as for Figure 13. This graph is representative of 2 experiments. Each point is a single value. 
EFFECT OF ECGS ON ENDOTHELIAL CELL PROLIFERATION

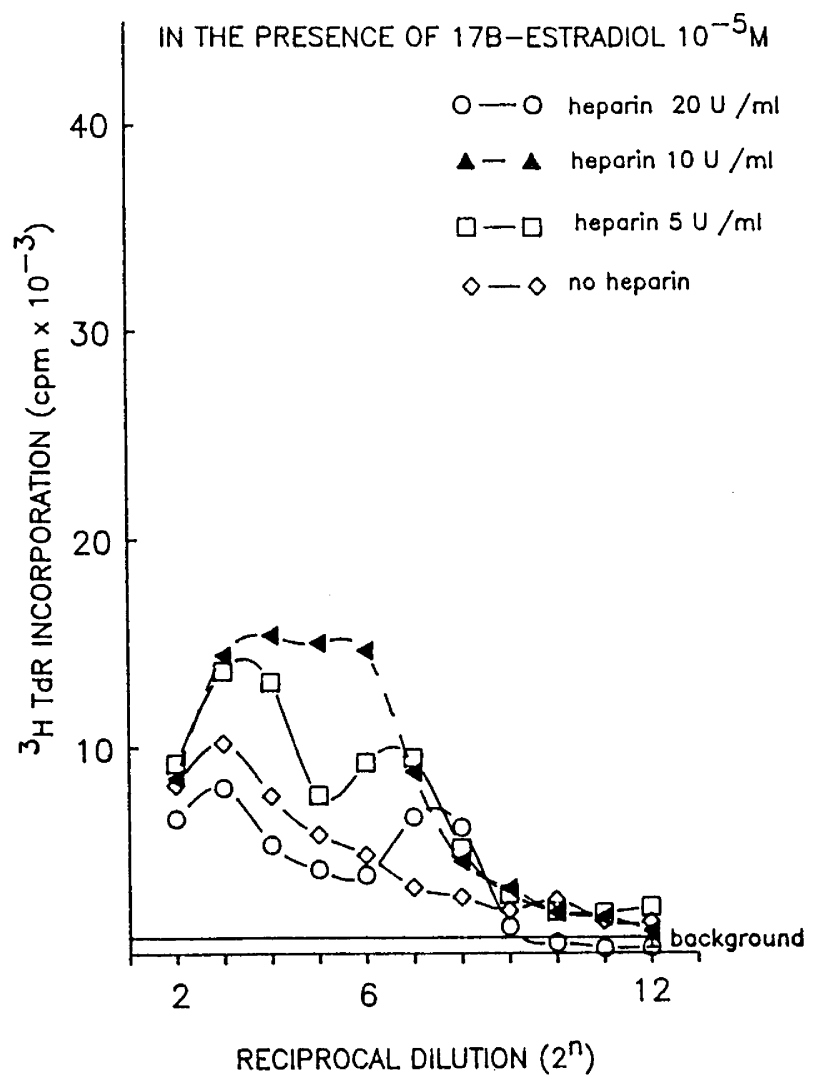

EFFECT OF ECGS ON ENDOTHELLAL CELL PROUFERATION

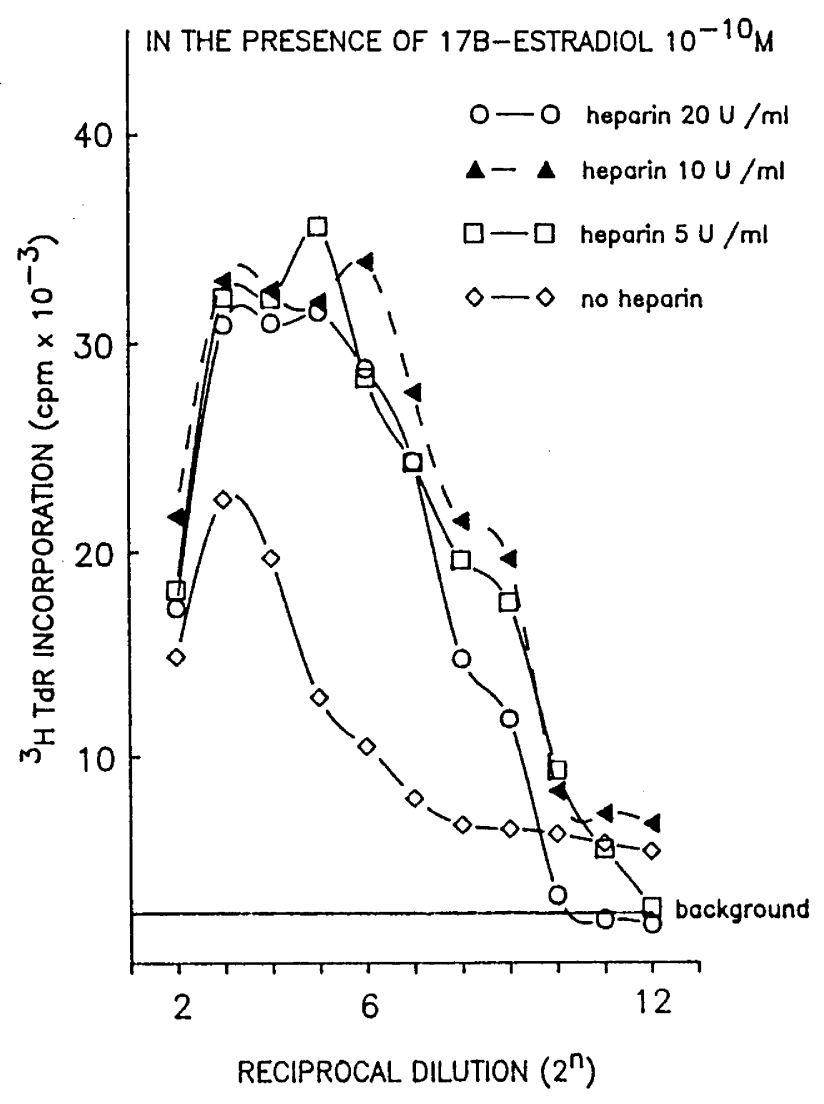




\section{Figure 15.}

Effect of different concentrations of ECGS on HUV endothelial cell proliferation in the presence of $20 \% \mathrm{FCS}$, progesterone $\left(10^{-5} \mathrm{M}\right)$ and different concentrations of heparin. Endothelial cells (passage 7) were exposed to 1 in 2 dilutions of ECGS (maximum concentration $400 \mu \mathrm{g} / \mathrm{ml})$ over $66 \mathrm{~h}$ in the presence of $20 \% \mathrm{FCS}$, heparin (0-20 U $/ \mathrm{ml}$ ) and progesterone $\left(10^{-5} \mathrm{M}\right)$. The background count for endothelial cell proliferation in the absence of ECGS for cells exposed to progesterone $\left(10^{-5} \mathrm{M}\right)$ averaged $1190 \mathrm{cpm}$. The key to this figure is the same as for Figure 13. This graph is representative of 3 experiments. Each point is a single value.

\section{Figure 16.}

Effect of different concentrations of ECGS on HUV endothelial cell proliferation in the presence of $20 \% \mathrm{FCS}$, progesterone $\left(10^{-9} \mathrm{M}\right)$ and different concentrations of heparin. Endothelial cells (passage 7) were exposed to 1 in 2 dilutions of ECGS (maximum concentration $400 \mu \mathrm{g} / \mathrm{ml}$ ) over $66 \mathrm{~h}$ in the presence of $20 \% \mathrm{FCS}$, heparin (0-20 U $/ \mathrm{ml}$ ) and progesterone $\left(10^{-9} \mathrm{M}\right)$. The background count for endothelial cell proliferation in the absence of ECGS for cells exposed to progesterone $\left(10^{-9} \mathrm{M}\right)$ averaged $1877 \mathrm{cpm}$. The key to this figure is the same as for Figure 13. This graph is representative of 2 experiments. Each point is a single value. 
EFFECT OF ECGS ON ENDOTHELIAL CELL PROLIFERATION IN THE PRESENCE OF PROGESTERONE $10^{-5} \mathrm{M}$

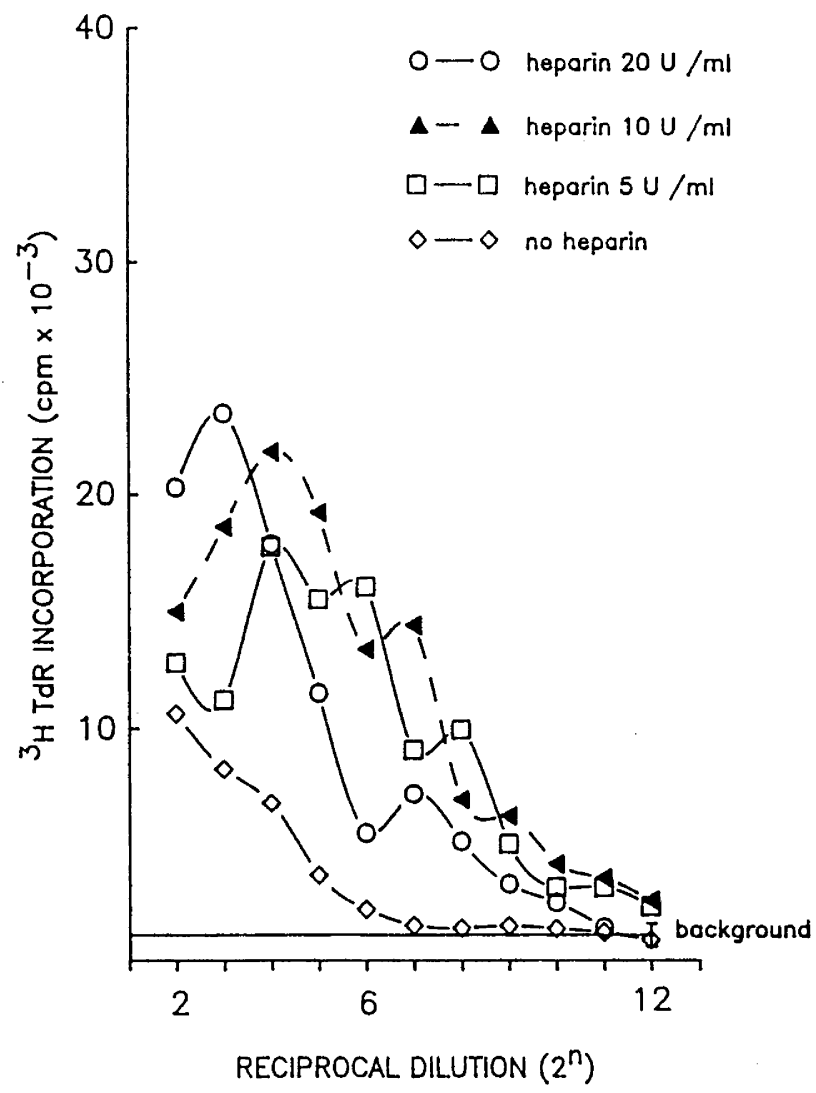

EFFECT OF ECGS ON ENDOTHELIAL CELL PROLIFERATION

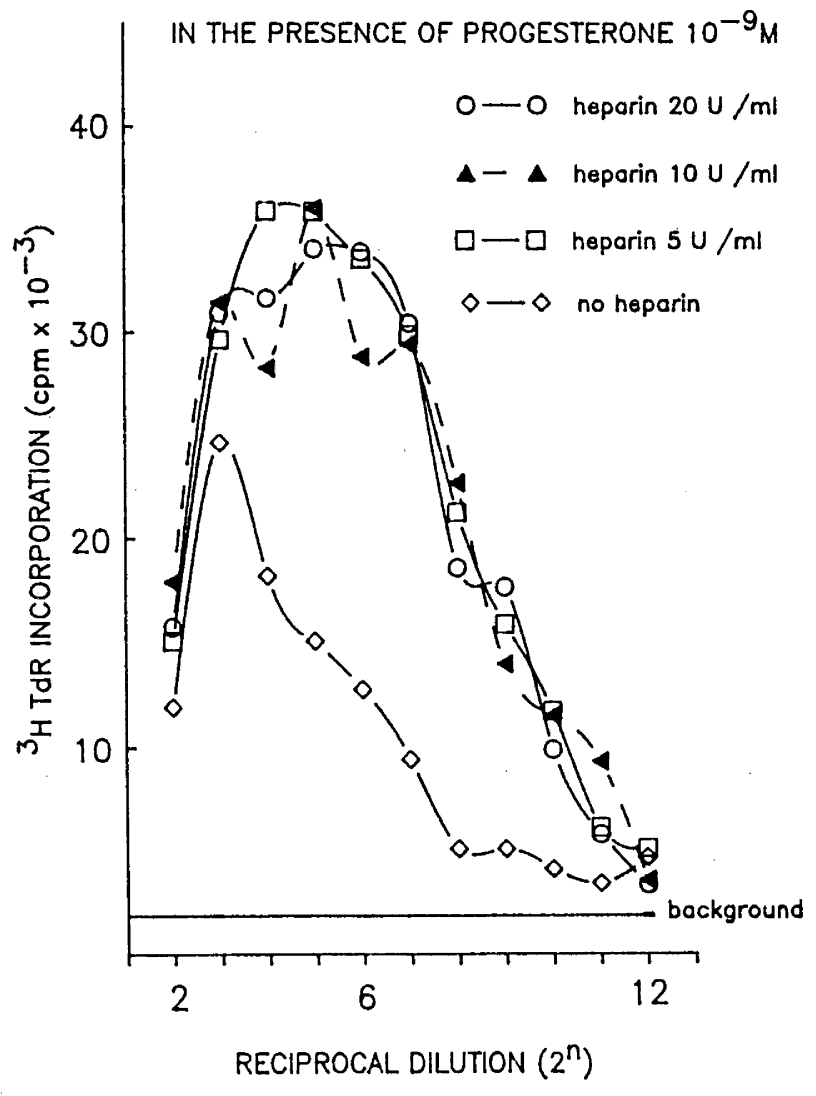




\section{Figure 17.}

Effect of different concentrations of ECGS on HUV endothelial cell proliferation in the presence of $20 \% \mathrm{FCS}, 17 \beta$-estradiol $\left(10^{-5} \mathrm{M}\right)$ in combination with progesterone $\left(10^{-5} \mathrm{M}\right)$ and different concentrations of heparin. Endothelial cells (passage 7) were exposed to 1 in 2 dilutions of ECGS (maximum concentration $400 \mu \mathrm{g} / \mathrm{ml}$ ) over $66 \mathrm{~h}$ in the presence of $20 \% \mathrm{FCS}$, heparin $(0-20 \mathrm{U} / \mathrm{ml}), 17 \beta$-estradiol $\left(10^{-5}\right.$ $\mathrm{M})$ in combination with progesterone $\left(10^{-5} \mathrm{M}\right)$ and variable concentrations of heparin. The background count for endothelial cell proliferation in the absence of ECGS for cells exposed to $17 \beta$-estradiol $\left(10^{-5} \mathrm{M}\right)$ in combination with progesterone $\left(10^{-5} \mathrm{M}\right)$ averaged 619 $\mathrm{cpm}$. The key to this figure is the same as for Figure 13. This graph is representative of 3 experiments. Each point is a single value.

\section{Figure 18.}

Effect of different concentrations of ECGS on HUV endothelial cell proliferation in the presence of $20 \%$ FCS, the combination of $17 \beta$ estradiol $\left(10^{-10} \mathrm{M}\right)$ with progesterone $\left(10^{-9} \mathrm{M}\right)$ and different concentrations of heparin. Endothelial cells (passage 7) were exposed to 1 in 2 dilutions of ECGS (maximum concentration $200 \mu \mathrm{g}$ $/ \mathrm{ml})$ over $66 \mathrm{~h}$ in the presence of $20 \% \mathrm{FCS}$, heparin $(0-20 \mathrm{U} / \mathrm{ml})$ and $17 \beta$-estradiol $\left(10^{-10} \mathrm{M}\right)$ with progesterone $\left(10^{-9} \mathrm{M}\right)$. The background count for endothelial cell proliferation in the absence of ECGS for cells exposed to the combination of $17 \beta$-estradiol $\left(10^{-10} \mathrm{M}\right)$ with progesterone $\left(10^{-9} \mathrm{M}\right)$ averaged $2579 \mathrm{cpm}$. The key to this figure is the same as for Figure 13. Note the difference in the scale of the "y" axis between Figures 17 and 18 . This graph is representative of 2 experiments. Each point is a single value. 
EFFECT OF ECGS ON ENDOTHELIAL CELL PROLIFERATION IN THE PRESENCE OF ESTRADIOL $10^{-5} \mathrm{M}$ IN COMBINATION

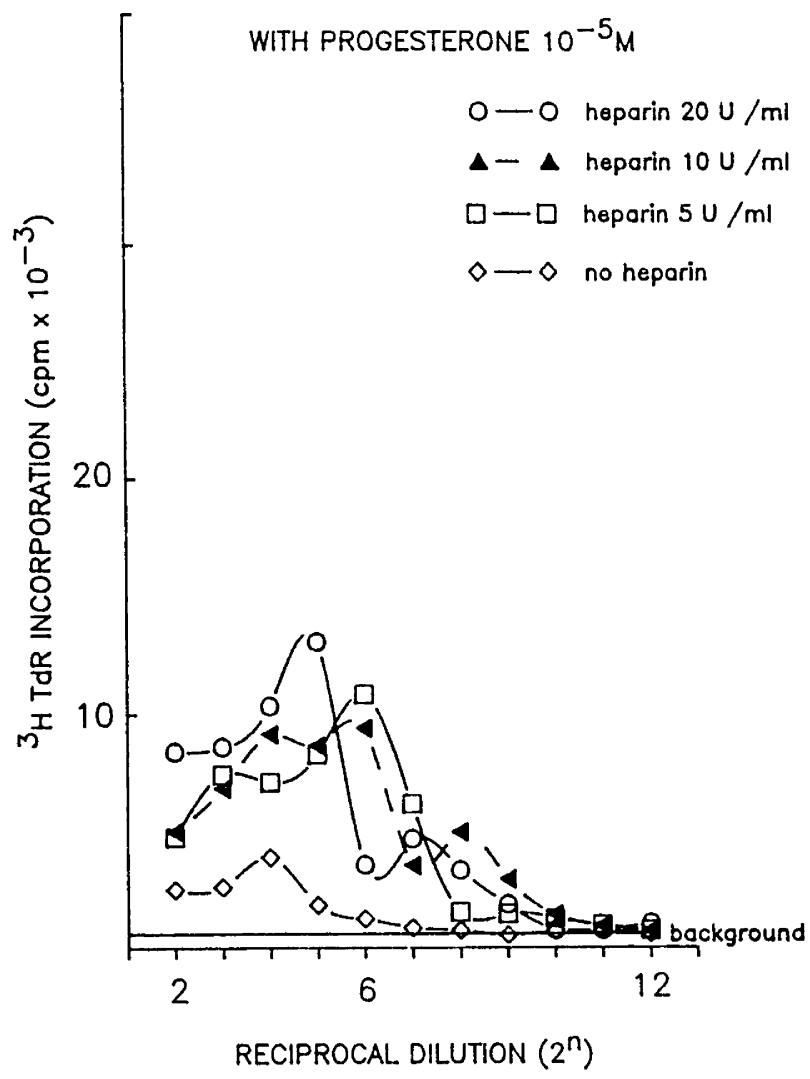

EFFECT OF ECGS ON ENDOTHELLAL CELL PROLIFERATION IN THE PRESENCE OF ESTRADIOL $10^{-10} \mathrm{M}$ IN

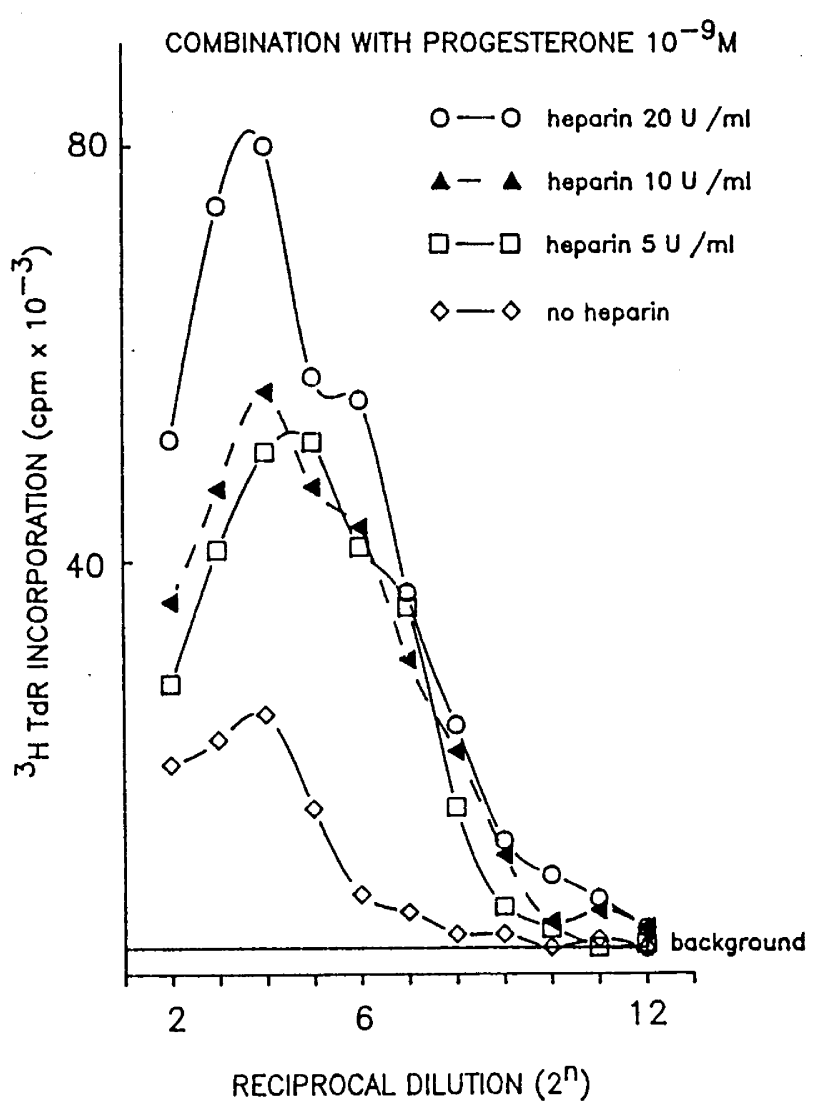




\section{BIBLIOGRAPHY}


Adams, M.R., Kaplan J.R., Koritnik D.R. and Clarkson T.B Pregnancyassociated inhibition of coronary artery atherosclerosis in monkeys Arteriosclerosis 1987; 7: 378-384

Adams, M.R., Clarkson, T.B., Kaplan, J.R., Koritnik, D.R. Experimental evidence in monkeys for beneficial effects of estrogen on coronary artery atherosclerosis Transplant Proc. 1989; 21: 3662-3664 (b)

Adams, M.R., Kaplan, J.R., Koritnik, D.R., Parkes, J.S., Wolfe, M.S. and Clarkson, T.B. Inhibition of coronary artery atherosclerosis (CAA) by estrogen or estrogen-progesterone replacement therapy in monkeys Circulation 1989; 84(Suppl): II 331 (a)

Adams, M.R., Kaplan, J.R., Manuck, S.B., Koritnik, D.R., Parks, J.S., Wolfe, M.S. and Clarkson, T.B. Inhibition of coronary artery atherosclerosis by 17-beta estradiol in ovariectomized monkeys Arteriosclerosis 1990; 10: 1051-1057

Adams, S., Williams, V. and Vessey, M.P. Cardiovascular disease and hormone replacement treatment: a pilot case-control study B $\mathbf{M} \mathbf{J}$ 1981; 282: 1277-1278

Adelman, S.J., Prozialeck, D.H., Mar-Lee McKean and Clark, D.E. Estrogens protect low density lipoprotein (LDL) from modification by $\mathrm{Cu}++$ and by rabbit aortic endothelial cells and J774 mouse macrophages Abstract from 9th International Symposium on Atherosclerosis 1991; 125

Ager, A., Gordon, J.L., Moncado, S., Pearson, J.D., Salmon, J.A. and Trevethick, M.A. Effects of isolation and culture on prostaglandin synthesis by porcine aortic endothelial and smooth muscle cells J. Cell. Physiol. 1982; 110: 9-16

Ajabor, L.N., Tsai, C.C., Vela, P. and Yen, S.S.C. Effect of exogenous estrogen on carbohydrate metabolism in postmenopausal women Am. J. Obstet. Gynecol. 1972; 113: 383-387

Adelman, S.J., Prozialeck, D.H., McKean, M-L. and Clark, D.E. Estrogens proect low density lipoprotein (LDL) from modification by $\mathrm{Cu}^{++}$and by rabbit aortic endothelial cells and $J 774$ mouse macrophages 9th International Symposium on Atherosclerosis, International Atherosclerosis Society 1991; 125

Alderson, L., Endemann, G., Lindsey, S., Prenezuk, A., Hoover, R. and Hayes, K. LDL enhances monocyte adhesion to endothelial cells in vitro Am. J. Pathol. 1986; 123: 334-342

Alexander, J.J., Hoenig, M., Graham, D. and Imbembo, A. Effect of estradiol on low density lipoprotein uptake by bovine aortic endothelial cells J. Surg. Res. 1989; 46: 537-542 (a)

Alexander, J.J., Hoenig, M., Graham, D. and Imbembo, A.L. The effect of estrogen on low-density lipoprotein binding kinetics in aortic endothelial cells Surgery 1989; 106: 386-390 (b)

Alfin-Slater, R.B. and Aftergood, L. Lipids and the pill Lipids 1971; 6: 693-705 
Alhenc-Gelas, F., Tsai, S.J., Callahan, K.S., Campbell, W.B. and Johnson, A.R. Stimulation of prostaglandin formation by vasoactive mediators in cultured human endothelial cells Prostaglandins 1982; 24: 723742

Ali, A.E., Barrett, J.C. and Eling, T.E. Prostaglandin and thromboxane production by fibroblasts and vascular endothelial cells Prostaglandins 1980; 20: 607-688

Almen, T., Hartel, M., Nylander, G. and Olivecrona, H. The effect of estrogen on the vascular endothelium and its possible relation to thrombosis Surg. Gynecol. Obstet. 1975; 140: 938-940

Alperin, J.B. Estrogens and surgery in women with von Willebrand's disease Am. J. Med. 1982; 73: 367-371

Anderson, D.C. Sex-hormone-binding globulin Clin. Endocrinol. 1974; 3: 6996

Anderson, J.R. Blood vessels and lymphatics In: Muir's Textbook of pathology (11th edition) Edward Arnold Ltd, London 1980; : 360-370

Antoniades, H.N., Pantazis, P. and Owen, A.J. Human platelet-derived growth factor and the sis/PDGF-2 gene In: Oncogenes Genes and Growth Factors, Guroff, $\mathrm{G}$ (ed.), Wiley-Interscience Publication 1987; : 1-40

Applebaum-Bowden, D., McLean, P., Sleinmetz, A., Fontana, D., Matthys, C., Warnick, G.R., Cheung, M., Albers, J.J. and Hazzard, W.R. Lipoprotein, apolipoprotein, and lipolytic enzyme changes following estrogen administration in post menopausal women $\mathrm{J}$. Lipid Res. 1989; 30: 1896-1906

Arai, H., Kita, T., Yokode, M., Narumiya, S. and Kawai, C. Multiple receptors for modified low density lipoproteins in mouse peritoneal macrophages: different uptake mechanisms for acetylated and oxidized low density lipoproteins Biochem. Biophys. Res. Commun. 1989; 153: 1375-1382

Ardlie, N.G., Selley, M.L. and Simons, L.A. Platelet activation by oxidatively modified low density lipoproteins Atherosclerosis 1989; 76: 117-124

Armstrong, B.K. Oestrogen therapy after the menopause-boon or bare Med. J. Aust. 1988; 148: 213-214

Arnaout, M.A., Lanier, L.L. and Faller, D.V. Relative contribution of the leukocyte molecules Mo1, LFA-1 and p 150,95 (LeuM5), in adhesion of granulocytes and monocytes to vascular endothelium is tissue and stimulus-specific J. Cell. Physiol. 1988; 137: 305-309

Aro, J., Haaplianienen, R., Sane, T., Rannikko, S., Pelkonen, R. and Alfthan, O. Effects of orchiectomy and polyestradiol phosphate therapy on serum lipoprotein lipids and glucose tolerance in prostatic cancer patients Eur. Virol. 1990; 17: 229-235 
Aurell, M., Cramer, K. and Rybo, G. Serum lipids and lipoproteins during longterm administration of an oral contraceptive Lancet 1966; i: 291293

Aviram, M., Dankner, G. and Brook, J.G. Platelet secretary products increase low density lipoprotein oxidation, enhance its uptake by macrophages, and reduce its fluidity Arteriosclerosis 1990; 10: 559-563

Babiy, A.V., Gebicki, J.M. and Sullivan, D.R. Vitamin E content and low density lipoprotein oxidizability induced by free radicals Atherosclerosis 1990; 81: 175-182

Bain, C., Willett, W., Hennekens, C.H., Rosner, B., Belanger, C. and Speizer, F.E. Use of postmenopausal hormones and risk of myocardial infarction Circulation 1981; 64: $42-46$

Baird, A. and Durkin, T. Inhibition of endothelial cell proliferation by type Btransforming growth factor interaction with aortic and basic fibroblast growth factors Biochem. Biophys. Res. Commun. 1986; 138: 476-482

Baird, A., Mormede, P. and Bohlen, P. Immunoreactive fibroblast growth factor in cells of peritoneal exudate suggests its identity with macrophage-derived growth factor Biochem. Biophys. Res. Commun. 1985; 126: 358-364

Bar, R.S., Boes, M., Booth, B.A., Dake, B.L., Henley, S. and Hart, M.N. The effects of platelet-derived growth factor in cultured microvessel endothelial cells Endocrinology 1989; 124: 1841-1848

Barak, V., Biran, S., Halimi, M. and Treves, A.J. The effect of estradiol on human myelomonocytic cells. II. Mechanism of enhancing activity of colony formation J. Reprod. Immunol. 1986; 9: 355-363

Barrett, T.B. and Benditt, E.P. Platelet-derived growth factor gene expression in human atherosclerotic plaques and normal arterial wall Proc. Natl. Acad. Sci. USA 1988; 85: 2810-2814

Barrett, T.B., Gajdusek, C.M., Schwartz, S.M., McDougall, J.K. and Benditt, E.P. Expression of the sis gene by endothelial cells in culture and in vivo Proc. Natl. Acad. Sci., USA 1984; 81: 6772-6774

Barrett-Connor, E., Wingard, D.L. and Criqui, M.H. Post menopausal estrogen use and heart disease risk factors in the 1980s JAMA 1989; 261: 2095-2100

Barzu, T., van Rijn, J., Petitous, M., Molho, P., Tobelem, G. and Caen, J. Endothelial binding sites for heparin Biochem. J. 1986; 238: 847-854 (a)

Barzu, T., van Rijn, J.L.M.L., Rosenbaun, J. and Tobelem, G. Endothelial cell reactions with heparin Med. J. Aust. 1986; 144: HS15-17 (b)

Basdevant, A., de Lignieres, B. and Guy-Grand, B. Differential lipemic and hormonal responses to oral and parenteral 17 beta estradiol in post menopausal women Am. J. Obstet. Gynecol. 1983; 147: 77-81 
Baungartner, H.R. and Born, G.V.R. Effect of 5-hydroxytryptamine on platelet aggregation Nature 1968; 218: 137-141

Baynes, J.W. Role of oxidative stress in development of complications in diabetes Diabetes 1991; 40: 405-412

Beaumont, V. and Beaumont J.-L. Oral contraception and thrombosis: implication of anti sex-steroid hormone antibodies. In: Myocardial Infarction in Women, Oliver, M.F., Vedin, A. and Wilhelmsson, C. (eds.), Churchill Livingstone, UK 1986; : 190-199

Beaumont, V. and Beaumont, J.-L. Myocardial infarction in women on oral contraceptives. In: Expanding Horizons in Atherosclerosis Research, Schlierf, G. and Mrl, H. (eds.), Springer-Verlag, Berlin, Heidelberg 1987; 151-160

Becker, R.C. and Corrac, J.M. Cardiovascular disease in women: scope of the problem Cardiology 1990; 77(Suppl.2): 6-7

Beekhuizer, H., Corsel-van Tilburg, A.J. and van Furth, R. Characterization of monocyte adherence to human macrovascular and microvascular endothelial cells J. Immunol. 1990; 145: 510-518

Beischer, N.A. and Mackay, E.V. Obstetrics and the new born for midwives and medical students Holt-Saunders Pty Ltd, Australia 1976; 394

Beledekas, J.C., Smith, R. and Franzblau, C. The effects of $17 \beta$-estradiol on the biosynthesis of collagen in cultured bovine aortic smooth muscle cells Circulation 1979; 59/60(Suppl.II): 219

Bell, L. and Madri, J.A. Effect of platelet factors on migration of cultured bovine aortic endothelial and smooth muscle cells Circulation Res. 1989; 65: 1057-1065

Beller, F.K. and Ebert, C. Effects of oral contraceptive on blood coagulation. A review Obstet. Gynecol. Surv. 1985; 40: 425-436

Benditt, E.P. The origin of atherosclerosis Sci. Am. 1977; 236: 74-85

Benditt, E.P. and Benditt, J.M. Evidence for a monoclonal origin of human atherosclerotic plaques Proc. Natl. Acad. Sci., USA 1973; 70: 1753-1756

Bengtsson, C. Ischaemic Heart Disease in Women Acta Med. Scand.(Suppl) 1973; 549: 1-128

Bengtsson, C., Lapidus, L. and Lindquist, O. Association between menopause and risk factors for ischaemic heart disease. In: Myocardial Infarction in Women, Oliver, M.F., Vedin, A. and Wilhelmsson, C. (eds.), Churchill Livingstone, UK 1986; : 93-102

Berge, L.N., Hansen, J.-B., Svensson, B., Lyngmo, V. and Nordoy, A. Female sex hormones and platelet/endothelial cell interactions Haemostasis 1990; 20: 313-320 
Berk, B.C., Alexander, R.W., Brock, T.A., Gimbrone, M.A. Jr. and Webb. R.C. Vasonconstriction - $A$ new activity for platelet-derived growth factor Science 1986; 232: 87-90

Berliner, J.A., Territo, M.C., Sevanian, A., Ramin, S., Kim, J.A., Bamshad, B., Esterson, M. and Fogelman, A.M. Minimally modified low density lipoproteins stimulates monocyte endothelial interactions J. Clin. Invest. 1990; 85: 1260-1266

Berthois, Y., Dong, X.F. and Martin, P.M. Regulation of epidermal growth factorreceptor by estrogen and antiestrogen in the human breast cancer cell line MCF-7 Biochem. Biophys. Res. Commun. 1989; 159: 126-131

Berthois, Y., Katzenellenbogen, J.A. and Katzenellenbogen, B.S. Phenol red in tissue culture media is a weak estrogen: implications concerning the study of estrogen-responsive cells in culture Proc. Natl. Acad. Sci., USA 1986; 83: 2496-2500

Betsholtz, C., Bergh, J., Bywater, M., Pettersson, M., Johnsson, A., Heldin C.-H., Ohlsson, R., Knott, T.J., Scott, J., Bell, G.I., Heldin C.-H. and Westermark, B. Expression of multiple growth factors in a human lung cancer cell line Int. J. Cancer 1987; 39: 502-507

Betsholtz, C., Heldin, C.-H, Nister, M., Ek, B., Wasteson, A. and Westermark, B. Synthesis of a PDGF-like growth factor in human glioma and arcoma cells suggest the expression of the cellular homologue to the transforming protein of simian sarcoma virus Biochem.

Biophys. Res. Commun. 1983; 117: 176-182

Betsholtz, C., Johnsson, A., Heldin, C.-H., Westermark, B., Lind, M. and Urdea, M.S., Eddy, R., Shows, T.B., Philpott, K., Mellor, A.L., Knott, T.J. and Scott, J. cDNA sequence and chromosomal localization of human platelet-derived growth factor A-chain and its expression in tumor cell line Nature 1986; 320: 695-699

Bevilacqua, M.P. and Gimbrone, M.A. Jr. Inducable endothelial functions in inflammation and coagulation Semin. Thromb. Hemost. 1987; 113: $425-433$

Bevilacqua, M.P., Pober, J.S., Wheeler, M.E., Cotran, R.S. and Gimbrone, M.A. Jr. Interleukin 1 acts on cultured human vascular endothelium to increase the adhesion of polymorphonuclear leukocytes, monocytes and related leukocyte cell lines J. Clin. Invest. 1985; 76: 2003-2011

Bevilacqua, M.P., Pober, J.S., Mendrick, D.L., Cotran, R.S. and Gimbrone, M.A. Jr. Identification of an inducible endothelial-leukocyte adhesion molecule Proc. Natl. Acad. Sci. USA 1987; 84: 9238-9242

Bevilacqua, M.P., Pober, J.S., Majeau, G.R., Cotran, R.S. and Gimbrone, M.A. Jr. Interleukin 1 induces biosynthesis and cell surface expression of procoagulant activity on human vascular endothelial cells J. Exp. Med. 1984; 160: 618-623

Bevilacqua, M.P., Pober, J.S., Wheeler, M.E., Cotran, R.S. and Gimbrone, M.A. Jr. Interleukin-1 (IL-1) activation of vascular endothelium Am. J. Pathol. 1985; 121: 393-403 
Bevilacqua, M.P., Stengelin, S, Gimbrone, M.A. Jr. and Seed, B. Endothelia/ leukocyte adhesion molecule 1: an inducible receptor for neutrophils related to complement regulatory proteins and lectins Science 1989; 243: 1160-1165

Bierenbaum, M.L., Fleischman, A.I., Stier, A., Watson, P., Somol, H., Naso, A.M. and Binder, $M$. Increased platelet aggregation and decreased high-density lipoprotein cholesterol in women on oral contraceptives Am. J. Obstet. Gynecol. 1979; 134: 638-641

Bjorkerud, S. and Bondjers, G. Arterial repair and atherosclerosis after mechanical injury. I. Permeability and light microscopic characteristics of endothelium in non-atherosclerotic and atherosclerotic lesions Atherosclerosis 1971; 13: 355-363

Blair, I.A, Barrow, S.E., Waddell, K.A., Lewis, P.J. and Dollery, C.T. Prostacyclin is not a circulating hormone in man Prostaglandins 1982; 23: 579-589

Blanc, J.J., Boschat, J., Morin, J.F., Clavier, J. and Penther, P. Menopause and myocardial infarction Am. J. Obstet. Gynecol. 1977; 127: 353355

Blankenhorn, D.H., Nessim, S.A., Johnson, R.L., Sammarco, M.E., Azen, S.P. and Cashin-Hemphill, L. Beneficial effects of combined colestipol-niacin therapy on coronary atherosclerosis and coronary venous bypass grafts JAMA 1987; 257: 3233-3240

Blose, S.H. and Chacko, S. In vitro behavior of guinea pig arterial and venous endothelial cells Dev. Growth Diff. 1975; 17: 153-165

Boeynaems, J.-M, Ramboer, I. and Pirotton, S. Palmitoyl--carnitine increases the release of prostacyclin from vascular endothelial cells Biochem. Pharm. 1989; 38: 3261-3266

Bolhuis, P.A., Sakariassen, K.S., Sander, H.L., Bouma, B.N. and Sixma, J.J. Binding of factor VIII-von Willebrand factor to human arterial subendothelium precedes increased platelet adhesion and enhances platelet spreading J. Lab. Clin. Med. 1981; 97: 568576

Bonfanti, R., Furie, B.C., Furie, B. and Wagner, D.D. PADGEM (GMP140) is a component of Weibel-Palade bodies of human endothelial cells Blood 1989; 73: 1109-1112

Bonin, P.D. and Singh, J.P. Modulation of interleukin-1 receptor expression and interleukin-1 response in fibroblasts by platelet-derived growth factor J. Biol. Chem. 1988; 263: 11052-11055

Bonin, P.D., Fici, G.J. and Singh, J.P. Interleukin-I promotes proliferation of vascular smooth muscle cells in coordination with PDGF or a monocyte derived growth factor Exp. Cell Res. 1989; 181: 475482

Bonnar, J., Haddon, M., Hunter, D.H., Richards, D.H. and Thornton, C. Coagulation system changes in post-menopausal women receiving oestrogen preparations Postgrad. Med. J. 1976; 52(suppl 6): 30-34 
Bonthron, D.T., Morton, C.C., Orkin, S.H. and Collins, T. Platelet-derived growth factor A chain: gene structure, chromosomal localization, and basis for alternative mRNA Proc. Natl. Acad. Sci., USA 1988; 85: 1492-1496

Booyse, F.M., Bell, S., Sedlack, B. and Rafelson, M.E. Development of an in vitro vessel wall model for studying certain aspects of platelet-vessel (endothelial) interaction Artery 1975; 1: 518-539

Booyse, F.M., Osikowicz, G. and Feder, S. Effects of various agents on ristocetin-von Willebrand factor activity in long-term culture of von Willebrand and normal human umbilical vein endothelial cells Thromb. Haemost. 1981; $46: 688$ (a)

Booyse, F.M., Osikowicz, G. and Quarfoot, A.J. Effects of chronic oral consumption of nicotine on the rabbit aortic endothelium Am. J. Pathol. 1981; 102: 229-238 (b)

Bфrsum, T. Biochemical properties of vascular endothelial cells Virchows Arch. B. Cell Pathol. 1991; 60: 279-286

B $\phi$ rsum, T., Henriksen, T. and Carlander, B. LDL induced cell injury, an effect of $L D L$ independent of specific binding and uptake of $L D L$ Thromb. Haemost. 1981; 46: 220

Boston Collaborative Drug Surveillance Programme Oral contraceptives and venous thromboembolic disease, surgically confirmed gallbladder disease, and breast tumours Lancet 1973; 1: 1399-1404

Boullion, R., van Baden, H. and der Moor, P. Physiology and pathophysiology of vitamin D-binding protein. In: Binding Proteins of Steroid Hormones Colloque INSERM, Joh Libbey Eurotext Ltd 1986; 149: 333-356

Boutherin-Falson, O. and Blaes, N. Decreased prostacyclin production by human umbilical vein endothelial cell growth factor and heparin Thromb. Res. 1989; 54: 487-492

Bowen-Pope, D.F. and Ross, R. Platelet-derived growth factor J. Biol. Chem. 1982; 257: 5161-5171

Bowen-Pope, D.F. and Ross, R. Methods of studying the platelet-derived growth factor receptor Methods Enzymol. 1985; 109: 69-100

Boyd, H.C., Gown, A.M., Wolfbauer, G. and Chait, A. Direct evidence of a protein recognized by a monoclonal antibody against oxidatively modified LDL in atherosclerotic lesions from a Watanabe heritable hyperlipietemic rabbit Am. J. Pathol. 1989; 135: 815-829

Bradley, D.D., Wingerd, J., Petitti, D.B., Krauss, R.M. and Ramcharan, S. Serum high-density-lipoprotein cholesterol in women using oral contraceptives, estrogens and progestins N. Engl. J. Med. 1978; 299: 17-20

Braquet, P., Bourgain, R. and Mencia-Huerta, J.M. Effect of platelet-activating factor on platelets and vascular endothelium Semin. Thromb. Hemost. 1989; 15: 184-196 
Breit, S.N. and Green, I. Modulation of endothelial cell synthesis of von Willebrand factor by mononuclear cell products Haemostasis 1988; 18: 137-145

Breviario, F., Proserpio, P., Bertocchi, F., Lampugnani, M.G., Mantovani, A. and Dejana, E. Interleukin-1 stimulates prostacyclin production by cultured human endothelial cells by increasing arachidonic acid mobilization and conversion Arteriosclerosis 1990; 10: 129-134

Bronzert, D.A., Pantazis, P., Antoniades, H.N., Kasid, A., Davidson, N., Dickson, R.B. and Lippman, M.E. Synthesis and secretion of plateletderived growth factor by human breast cancer cell lines Proc. Natl. Acad. Sci., USA 1987; 84: 5763-5767

Broudy, V.D., Kaushansky, K., Segal, G.M., Harlan, J.M. and Adamson, J.W. Tumor necrosis factor type a stimulates human endothelial cells to produce granulocyte-macrophage colony-stimulating factor Proc. Natl. Acad. Sci., USA 1986; 83: 7467-7471

Brown, M.S. and Goldstein, J.L. Lipoprotein metabolism in the macrophage: implications for cholesterol deposition in atherosclerosis Ann. Rev. Biochem. 1983; 52: 223-262

Brown, S.A., Boerwinkle, E., Kashanian, F.K., Swanson, N. and Patsch, W. Variation in concentration of lipids, lipoprotein lipids, and apolipoprotein $A-1$ and $B$ in plasma from healthy women Clin. Chem. 1990; 36: 207-210

Brown, Z., Neild, G.H. and Lewis, G.P. Inhibition of prostacyclin formation by cyclosporin is not due to reduced availablity of arachidonic acid in membrane phospholipids of cultured human endothelial cells Biochem. Pharm. 1990; 39: 1136-1138

Bruggeman, C.A., Debie, W.H.M., Muller, A.D., Schutte, B., van Dam-Mieras, M.C.E. Cytomegalovirus alters the von Willebrand factor content in human endothelial cells Thromb. Haemost. 1988; 59: 264268

Brunner, N., Bronzert, D., Vindelov, L.L., Rygaard, K., Spang-Thomsen, M. and Lippman, M.E. Effect on growth and cell cycle kinetics of estradiol and tamoxifen on MCF-7 human breast cancer cells grown in vitro and in nude mice Cancer Res. 1989; 49: 15151520

Buchanan, M.R., Vazquez, M. and Gimbrone, M.A. Jr. Arachadonic acid metabolism and the adhesion of human polymorphonuclear leukocytes to cultured vascular endothelial cells Blood 1983; 62: 889-895

Buchanan, M.R., Haas, T.A., Lagarde, M. and Guichardant, M. 13hydroxyoctadecadienoic acid is the vessel wall chemorepellant factor, LOX J. Biol. Chem. 1985; 260: 16056-16059

Buege, J.A. and Aust, S.D. Microsomal lipid peroxidation Methods Enzymol. 1978; LII: 302-310 
Bulusu, N.V., Lewis, S.B., Das, S. and Clayton, W.F. Jr. Serum lipid changes after estrogen therapy in prostatic carcinoma Urology 1982; 20: 147-150

Buonassisi, V. and Colburn, P. Hormone and surface receptors in vascular endothelium Adv. Microcirc. 1980; 9: 76-94

Buonassisi, v. and Venter, C. Neurotransmitter receptors in an established endothelial cell line Proc. Natl Acad. Sci. USA 1976; 731612

Burch, J.C., Byrd, B.F. Jr. and Vaughn, W.K. The effects of long-term estrogen on hysterectomized women Am. J. Obstet. Gynecol. 1974; 118: 778-782

Burdon, R.H. and von Knippenberg, P.H. In: Laboratory techniques in biochemistry and molecular biology Campbell, A.M.(ed.), Elsevier, Amsterdam, The Netherlands 1984; 13: 108

Burgess, W.H., Mehlman, T., Marshak, D.A., Fraser, B.A. and Maciag, T. Structural evidence that endothelial cell growth factor is the precursor of both Endothelial Cell Growth Factor $\alpha$ and Acidic Fibroblast Growth Factor Proc. Natl. Acad. Sci. USA 1986; 83: 7216-7220

Burgess, W.H., Mehlman, T., Friesel, R., Johnson, W.V. and Maciag, T. Multiple forms of endothelial cell growth factor J. Biol. Chem. 1985; 260: 11389-11392

Bus, J.S. and Gibson, J.E. Lipid peroxidation and its role in toxicology. In: Reviews in Biochemical Toxicology, Hadgen, E., Bend, J.R. and Philpot, R.M. (eds.), Elsevier, North Holland. 1979; 125-149

Bush, T.L. The adverse effects of hormonal therapy Cardiol. Clin. 1986; 4: 145152 (b)

Bush, T.L. The epidemiology of cardiovascular disease in postmenopausal women Ann. N.Y. Acad. Sci. 1990; 592: 263-271

Bush, T.L., Cowan, L.D., Barrett-Connor, E., Criqui, M.H., Kaon, J.M., Wallace. R.B., Tyroler. H.A. and Rifkind, B.M. Estrogen use and all cause mortality. Preliminary results from the Lipid Research Clinic Program Follow-up Study JAMA 1983; 249: 903-906

Bush, T.L., Rifkind, B.M., Criqui, M.H., Cowan, L.D., Barrett-Connor, E., Karon, J.M., Wallace, R.B. and Tyroler, H.A. Postmenopausal oestrogen use and cardiovascular mortality in women. In: Myocardial Infarction in Women, Oliver, M.F., Vedin, A. and Wilhelmsson, C, (eds.), Churchill Livingstone, UK 1986; 130-149 (a)

Bussolino, F., Breviario, F., Tetta, C., Aglietta, M., Mantovani, A., and Dejana, E. Interleukin 1 stimulates platelet-activating factor production in cultured human endothelial cells J. Clin. Invest. 1986; 77: 20272033

Bussolino, F., Wang, J.M., Defilippi, P., Turrini, F., Sanavio, F., Edgell, C.-J., Aglietto, M., Arese, P. and Mantovani, A. Granulocyte- and granuloctye-macrophage-colony stimulating factors induce 
human endothelial cells to migrate and proliferate Nature 1989; 337: 471-473 (a)

Bussolino, F., Wang, J.M., Turrini, F., Alessi, D., Ghigo, D., Costamagna, C., Pescarmona, G., Mantovani, A. and Bosia, A. Stimulation of the $\mathrm{Na}+/ \mathrm{H}+$ exchanger in human endothelial cells activated by granulocyte and granulocyte-macrophage-colony-stimulating factor J. Biol. Chem. 1989; 264: 18284-18287 (b)

Buster, J.E. and Simon, J.A. Placental hormones, hormonal preparation for and control of parturition, and hormonal diagnosis of pregnancy Endocrinology 1989; 3: 2043-2073

Byar, D.P. The Veterans Administration Cooperative Urological Research Group's Studies of cancer of the prostate Cancer 1973; 32: 1126-1130

Bylock, A.L. and Gerrity, R.G. Visualization of monocyte recruitment into atherosclerotic arteries using fluorescent labelling Atherosclerosis 1988; 71: 17-25

Bywater, M., Rorsman, F., Bongcam-Rudloff, E., Mark, G., Hammacher, A., Heldin, C.-H., Westermark, B. and Betsholtz, C. Expression of recombinant platelet-derived growth factor $A$ - and $B$-chain homodimers in rat-1 cells and human fibroblasts reveal differences in protein processing and autocrine effects Mol. Cell. Biol. 1988; 8: 2753-2762

Campbell, G.R. and Campbell, J.H. Smooth muscle phenotypic changes in arterial wall homeostasis: implications for pathogenesis of atherosclerosis Exp. Mol. Pathol. 1985; 42: 139-162

Campbell, J.H. and Campbell, G.R. Endothelial cell influences on vascular smooth muscle phenotype Ann. Rev. Physiol. 1986; 48: 295306

Caplan, B.A. and Schwartz, C.J. Increased endothelial cell turnover in areas of in vivo Evans Blue uptake in the pig aorta Atherosclerosis 1973; 17: 401-417

Carew, T.E. Role of biologically modified low density lipoprotein in atherosclerosis Am. J. Cardiol. 1989; 64: 189-229

Carew, T.E., Schwenke, D.C. and Steinberg, D. Antiatherogenic effect of probucol unrelated to its hypocholesterolemic effect: evidence that antioxidants in vivo can selectively inhibit low density lipoprotein degradation in macrophage-rich fatty streaks slowing the progression of atherosclerosis in the WHHL rabbit Proc. Natl. Acad. Sci., USA 1987; 84: 7725-7729

Cariou, R., Harousseau, J.L. and Tobelem, G. Inhibition of human endothelial cell proliferation by heparin and steroids Cell Biol. Int. Rep. 1988; 12: 1037-1047

Carlos, T.M., Dobrino, A., Ross, R. and Harlan, J.M. Multiple receptors on human monocytes are involved in adhesion to cultured human endothelial cells J. Leuk. Biol. 1990; 48: 451-456 
Carr, B.R. and Wilson, J.D. Disorders of the ovary and female reproductive tract. In: Harrison's Principles of Internal Medicine, 12th edition, Wilson, J.D., Braunwald, E., Isselbacher, K.J., Petersdorf, R.G., Martin, J.B., Fauci, A.S. and Root, R.K. (eds.), 1989; : 1776-1782

Castelli, W.P., Doyle, J.T., Gordon, T., Haines, C.G., Hjortland, M.C., Hulley, S.B., Kagan, A. and Zukel, N.J. HDL cholesterol and other lipids in coronary heart disease. The cooperative lipoprotein phenotyping study Circulation 1977; 55: 767-772

Cathcart, M.K., Morel, D.W. and Chisolm, G.M. Monocytes and neutrophils oxidize low density lipoprotein making it cytotoxic J. Leukoc. Biol. 1985; 38: 341-350

Cauley, J.A., Gutai, J.P., Kuller, L.H. and Powell, J.G. The relation of endogenous sex steroid hormone concentration to serum lipid and lipoprotein levels in post menopausal women Am. J. Epidemiol. 1990; 132: 884-894

Cavailles, V., Augereau, P., Garcia, M. and Rochefort, H. Estrogen and growth factors induce the MRNA of the $52 \mathrm{~K}$ pro-cathepsin D secreted by breast cancer cells Nucleic Acids Res. 1988; 16: 1903-1919

Chait, A., Ross, R., Albers, J.J. and Bierman, E.L. Platelet-derived growth factor stimulates low density lipoprotein receptor activity Proc. Natl. Acad. Sci., USA 1980; 77: 4084-4088

Chamley-Campbell, J.H., Campbell, G.R. and Ross, R. Phenotype-dependent response of cultured aortic smooth muscle to serum mitogens $\mathrm{J}$. Cell Biol. 1981; 89: 379-383 (a)

Chamley-Campbell, J.H. and Campbell, G.R. What controls smooth muscle phenotype Atherosclerosis 1981; 40: 347-357 (b)

Chan, L., Jackson, R.L., O'Malley, B.W., Means, A.R. Synthesis of very low density lipoproteins in the cockerel. Effects of estrogen J. Clin. Invest. 1976; 58: 368-379

Chang, C., Kokontis, J. and Liao, S. Molecular cloning of human and rat complementary DNA encoding androgen receptors Science 1988; 240: 324-326

Chang, W.-C. Nakao, J., Orimo, H. and Murota, S.-I. Stimulation of prostacyclin biosynthetic activity by estradiol in rat aortic smooth muscle cells in culture Biochim. Biophys. Acta 1980; 619: 107-118 (a)

Chang, W.-C., Nakao, J., Neichi, T., Orimo, H. and Murota, S.-I. Effects of estradiol on the metabolism of arachidonic acid by aortas and platelets in rats Biochim. Biophys. Acta 1981; 664: 291-297

Chang, W.-C., Nakao, J., Orimo, H. and Murota, S.-I. Stimulation of prostaglandin cycloxygenase and prostacyclin synthetase activity by estradiol in rat aortic smooth muscle cells Biochim. Biophys. Acta 1980; 620: $472-482$ (b)

Chen, Y. and Rabinovitch, P.S. Platelet-derived growth factor, epidermal growth factor, and insulin-like growth factor 1 regulate specific cell-cycle 
parameters of human diploid fibroblasts in serum-free cultures $\mathbf{J}$. Cell. Physiol. 1989; 140: 59-67

Cheresh, D. Human endothelial cells synthesize and express an Arg-Gly-Aspdirected adhesion receptor involved in attachment to fibrinogen and von Willebrand factor Proc. Natl. Acad. Sci. USA 1987; 184: 6471-6475

Chesterman, C.N. and Berndt, M.C. Platelet and vessel wall interaction and the genesis of atherosclerosis Clin. Haematol. 1986; 15: 323-353

Chesterman, C.N., Ager, A. and Gordon, J.L. Regulation of prostaglandin production and ectoenzyme activities in cultures aortic endothelial cells J. Cell. Physiol. 1983; 116: 45-50

Chesterman, C.N. Vascular endothelium, haemostasis and thrombosis Blood Rev. 1988; 2: 88-94

Chirgwin, J.M., Przybyla, A.E., MacDonald, R.J. and Rutter, W.J. Isolation of biologically active ribonucleic acid from sources enriched in ribonuclease Biochemistry 1979; 18: 5294-5299

Chisolm, G.M., Hessler, J.R., Morel, D.W. and Lewis, L.J. In vitro autooxidation renders low density lipoprotein toxic to cells in culture Arteriosclerosis 1981; 1: 359a

Christensen, B.C. and Garbarsch, C. Repair on arterial tissue. A scanning election microscopic (SEM) and light microscopic study on the endothelium of rabbit thoracic aorta following a single dilation injury Virchows Arch. 1973; 360: 93-106

Christensen, M.S., Hagen, C., Christiansen, C. and Transbol, I. Dose-response evaluation of cyclic estrogen/gestagen in postmenopausal women: Placebo-controlled trial of its gynecologic and metabolic actions Am. J. Obstet. Gynecol. 1982; 144: 873-879

Chung-Welch, N., Shepro, D., Dunham, B. and Hechtman, H.B. Prostacyclin and prostaglandin $E_{2}$ secretion by bovine pulmonary microvessel endothelial cells are altered by changes in culture conditions $\mathrm{J}$. Cell. Physiol. 1988; 135: 224-234

Ciavatti, M. and Renaud, S. Oxidative status and oral contraceptive. Its relevance to platelet abnormalities and cardiovascular risk Free Radic. Biol. Med. 1991; 10: 325-338

Ciavatti, M., Blache, D. and Renaud, S. Dietary fats modulate hormonal contraceptive induced changes in platelet aggregation, composition and lipid biosynthesis in female rats Nutrition Res. 1987; 7: 589-599

Ciavatti, M., Blache, D. and Renaud, S. Hormonal contraceptive increases plasma lipid peroxides in female rats Arteriosclerosis 1989; 9: 84-89

Claesson-Welsh, L., Eriksson, A., Moren, A., Severinsson, L., Ek, B.M., Ostman, A., Betsholtz, C. and Heldin, C.-H. cDNA cloning and expression of a human platelet-derived growth factor (PDGF) receptor 
specific for $\beta$-chain-containing PDGF molecules Mol. Cell. Biol. 1988; 8: 3476-3486

Claesson-Welsh, L., Eriksson, A., Westermark, B. and Heldin, C.-H. cDNA cloning and expression of human A-type platelet-derived growth factor (PDGF) receptor establishes structural similarity to the $B$ type PDGF receptor Proc. Natl. Acad. Sci., USA 1989; 86: 4917-4921

Clark, M.A., Chen, M-J., Crooke, T.S. and Bomalaski, J.S. Tumor necrosis factor (cachectin) induces phospholipase $A_{2}$ activity and synthesis of a phospholipase $A_{2}$-activating protein in endothelial cells Biochem. J. 1988; 250: 125-132

Clarkson, T.B., Adams, M.R., Kaplan, J.R. and Koritnik, D.R. Gonadal steroids in cardiac atherosclerosis in primates Transplant. Proc. 1987; X1X: 85-89

Clarkson, T.B., Shively, C.A., Morgan, T.M., Koritnik, D.R., Adams, M.R. and Kaplan, J.R. Oral contraceptives and coronary artery atherosclerosis of cynomolgus monkeys Obstet. Gynecol. 1990; 75: 217-222

Coetzee, G.A., Sanan, D.A., Strumpfer, A.E. and van de Westhuyzen, D.R. Native and acetylated low density lipoprotein and metabolism in proliferating and quiescent endothelial cells Arteriosclerosis $1984 ; 4: 536 a$

Coetzee, G.A., Stein, O. and Stein, Y. Uptake and degradation of low density lipoproteins (LDL) by confluent, contact-inhibited bovine and human endothelial cells exposed to physiological concentrations of $L D L$ Atherosclerosis 1979; 33: 425-431

Cohen, S. Isolation of a mouse submaxillary gland protein accelerating incisor eruption and eyelid opening in the new-born animal J. Biol. Chem. 1962; 237: 1555-1562

Colburn, P. and Buonassisi, V. Estrogen-binding sites in endothelial cells Science 1978; 210: 817-819

Colditz, G.A., Willett, W.C., Stampfer, M.J., Rosner, B., Speizer, F.E. and Hennekens, C.H. Menopause and the risk of coronary heart disease in women New Engl. J. Med. 1987; 316: 1105-10

Collaborative Group for the Study of Stroke in Young Women Oral contraception and increased risk of cerebral ischemia or thrombosis N. Engl. J. Med. 1973; 288: 871-878

Collen, D. Natural inhibitors of fibrinolysis J. Clin. Pathol. Suppl. 1980; 14: 2430

Collins, T., Bonthron, D.T. and Orkin, S.H. Alternative RNA splicing affects function of encoded platelet-derived growth factor $A$-chain Nature 1987; 328: 621-624

Collins, T., Ginsburg, D., Boss, J.M., Orkin, S.H. and Pober, J.S. Cultured human endothelial express platelet-derived growth factor $B$-chain: cDNA cloning and structural analysis Nature 1985; 316: 748-750 
Colotta, F., Lampugnani, M.G., Polentarutti, N., Dejana, E. and Mantovani, A. Interleukin-1 induces c-fos protooncogene expression in cultured human endothelial cells Biochem. Biophys. Res. Commun. 1988; 152: 1104-1110

Colvin, P.L. Jr., Auerbach, B.J., Koritnik, D.R., Hazzard, W.R. and ApplebaumBowden, D. Differential effects of oral estrone verses $17 \beta$ estradiol on lipoproteins in post menopausal women J. Clin. Endrocrinol. Metab. 1990; 70: 1568-1573

Consensus Conference Lowering blood cholesterol to prevent heart disease JAMA 1985; 253: 2080-2086

Coomber, B.L. and Gotlieb, A.I. In vitro endothelial wound repair. Interaction of cell migration and proliferation Arteriosclerosis 1990; 10: 215222

Coope, J., Thomson, J.M. and Poller, L. Effects of 'natural oestrogen' replacement therapy on menopausal symptoms and blood clotting BMJ 1975; 4: 139-143

Coronary Drug Project Research Group Coronary Drug Project - finding Leading to discontinuation of the $2.5 \mathrm{mg} /$ day estrogen group JAMA 1973; 226: 652-657

Corrao, J.M., Becker, R.C., Ockene, I.S. and Hamilton, G.A. Coronary heart disease risk factors in women Cardiology 1990; 77(suppl.2): 824

Corsini, A., Granata, A., Bernini, F., Maggi, E.M., Fumagalli, R. and Catapano, A..L Progesterone modulates the expression of $H D L$ binding sites in human skin fibroblasts Atherosclerosis 1988; 74: 107-113

Corvazier, E., Dupuy, E., Dosne, A.M. and Maclouf, J. Minimal effect of estrogens on endothelial cell growth and production of prostacyclin Thromb. Res. 1984; 34: 303-310

Croft, P. and Hannaford, P.C. Risk factors for acute myocardial infarction in women: evidence from the Royal College of General Practitioners' oral contraception study BMJ 1989; 298: 165-168

Crona, N., Silfverstolpe, G. and Samsioe, G. The influence of hormonal replacement therapy on lipid and lipoprotein metabolism Acta Obstet. Gynecol. Scand. Suppl. 1985; 130: 49-52

Cross, G.E., Halliwell, B., Borish, E.T., Pryer, W.A., Ames, B.N., Saul, R.L., McCord, J.M. and Harman, D. Oxygen radicals and human Disease Ann. Intern. Med. 1987; 107: 526-545

Crouse, J.R. III Progress in coronary artery disease risk-factor research: what remains to be done Clin. Chem. 1984; 30: 1125-1127

Crowell, E.B., Clatenoff, O.V. and Keikhofer, W. The effect of oral contraceptives on factor VIII levels J. Lab. Clin. Med. 1971; 77: 551-557

Crum, R. Szabo, S. and Folkman, J. A new class of steroids inhibits angiogenesis in the presence of heparin or a heparin fragment Science 1985; 230: 1375-1378 
Cullingford, T.E. and Pollard, J.W. RU 486 completely inhibits the action of progesterone on cell proliferation in the mouse uterus J. Reprod. Fert 1988; 83: 909-914

Cunha, G.R., Chung, L.W.K., Shannon, J.M., Tagucshi, O. and Fujii, H. Hormone-induced morphogenesis and growth: role of mesechymal-epithelial interactions Recent Prog. Horm. Res. 1983; 39: 559-598

Curtiss, L.K., Black, A.S., Takagi, Y. and Plow, E.F. New mechanism for foam cell generation in atherosclerotic lesions J. Clin. Invest. 1987; $80: 367-373$

Curwen, K.D., Kim, H.-Y., Vazquez, M., Handin, R.I. and Gimbrone, M.A. Jr. Platelet adhesion to cultured vascular endothelial cells J. Lab. Clin. Med. 1982; 100: 425-436

Curwen, K.D., Gimbrone, M.A. Jr. and Handin, R.I. The role of prostacyclin $\left(\mathrm{PGI}_{2}\right)$ in platelet adhesion to cultured normal and virally transformed human Vascular Endothelial Cells Lab. Invest. 1980; 42: 366-374

Cushing, S.D., Berliner, J.A., Valente, A.J., Territo, M.C., Navab, M., Parhami, F., Gerrity, R., Scwartz, C.J. and Fogelman, A.M. Minimally modified low density lipoprotein induces monocyte chemotactic protein 1 in human endothelial cells and smooth muscle cells Proc. Natl. Acad. Sci. USA 1990; 87: 5134-5138

Czervionke, R.L., Smith, J.B., Hoak, J.C., Fry, G.L. and Haycraft, D.L. Inhibition of prostacyclin by treatment of endothelium with aspirin J. Clin. Invest. 1979; 63: 1089-1092 (a)

Czervionke, R.L., Hoak, J.C. and Fry, G.L. Effect of aspirin on thrombin-induced adherence of platelets to cultured cells from the blood vessel wall J. Clin. Invest. 1978; 62: 847-856 (b)

Czervionke, R.L., Smith, J.B., Hoak, J.C., Fry, G.L. and Haycraft, D.L. Use of a radioimmunoassay to study thrombin-induced release of $P G I_{2}$ from cultured endothelium Thromb. Res. 1979; 14: 781-786

Dacie, J.V. and Lewis, S.M. Practical haematology Churchill Livingstone, N.Y. 1975: 13

Danel, L., Souweire, G., Monier, J.C. and Saez, S. Specific estrogen binding sites in human lymphoid cells and thymic cells J. Steroid Biochem. 1983; 18: 559-563

Daniel, T.O., Gibbs, V.C., Milfay, D.F., Garovoy, M.R. and Williams, L.T. Thrombin stimulates C-sis gene expression in microvascular endothelial cells J. Biol. Chem. 1986; 261: 9579-9582

Danielpour, D. and Sirbasku, D. A. Estrogen-inducible autostimulatory growth factors found in human breast cancer cells J. Cell Biol. 1983; 95: 394a

Darbre, P., Yates, J., Curtis, S. and King, R.J.B. Effect of estradiol on human breast cancer cells in culture Cancer Res. 1983; 43: 349-359 
Daugherty, A., Zweifel, B.S., Sobel, B.E. and Schonfeld, G. Isolation of low density lipoprotein from atherosclerotic vascular tissue of Watanabe heritable hyperlipemic rabbits Arteriosclerosis 1988; 8: 768-777

David, M., Griesmacher, A. and Muller, M.M. $17 \alpha$-ethinyl estradiol decreases production and release of prostacyclin in cultured human umbilical vein endothelial cells Prostaglandins 1989; 38: 431438

Davies, P.F. Biology of disease: vascular cell interactions with special reference to the pathogenesis of atherosclerosis Lab. Invest. 1986; 55: 5-23

Davies, P.F. and Ross, R. Medication of pinocytosis in cultured arterial smooth muscle and endothelial cells by platelet-derived growth factor $\mathrm{J}$. Cell Biol. 1978; 79: 663-671

Davies, M.J. and Thomas. A.C. Plaque fissuring- the cause of acute myocardial infarction, sudden ischaemic death, and crescendo angina $\mathrm{Br}$. Heart J. 1985; 53: 363-373

Davis, L.G., Dibner, M.D. and Baltey, J.F. "Dot Blot" hybridization of labeled probe to DNA or RNA samples In: Basic Methods in Molecular Biology Elsevier, NY, 1986; : 147-149

Davison, P.M., Bensch, K. and Karasek, M.A. Growth and morphology of rabbit marginal vessel endothelium in cell culture J. Cell Biol. 1980; 85: 187-198

Day, J.H. and Solum, N.O. Fibrinogen associated with subcellular platelet particles Scand. J. Haematol. 1973; 10: 136-143

de Bono, D.P. and Green, C. The adhesion of different cell types to cultured vascular endothelium: effect of culture density and age $\mathrm{Br} . \mathrm{J}$. Exp. Pathol. 1984; 65: 145-154

de Groot, P.G., Verweij, C.L., Nawroth, P.P., de Boer, H.C., Stern, D.M. and Sixma, J.J. Interleukin-l inhibits the synthesis on von Willebrand factor in endothelial cells, which results in a decrease reactivity of their matrix toward platelets Arteriosclerosis 1987; 7: 605-611

de Nucci, G., Gryglewski, R.J., Warner, I.D. and Vane, J.R. Receptor-mediated release of endothelium-derived relaxing factor and prostacyclin from bovine aortic endothelial cells is coupled Proc. Natl. Acad. Sci. USA 1988; 85: 2334-2338

de Witt, D.L., Day, J.S., Sonnenburg, W.K. and Smith W.L. Concentration of prostaglandin endoperoxide synthase and prostaglandin 12 synthase in the endothelium and smooth muscle of bovine aorta J. Clin. Invest. 1983; 72: $1882-1888$

Dear, H.D. and Jones, W.B. Myocardial infarction associated with the use of oral contraceptives Ann. Intern. Med. 1971; 74: 236-239

Dejana, E., Brevario, F., Erroi, A., Bussolino, F., Mussoni, L., Gramse, M., Pintucci, G., Casali, B., Dinarello, C.A., van Damme, J. and 
Mantovani, A. Modulation of endothelial cell function by different molecular species of interleukin-1 Blood 1987; 69: 695-699

Dejana, E., Cazenave, J.-P., Groves, H.M., Kinlough-Rathbone, R.L., Richardson, M., Packam, M.A. and Mustard, J.F. The effect of aspirin inhibition of $\mathrm{PGI}_{2}$ production on platelet adherence to normal and damaged rabbit aortae Thromb. Res. 1980; 17: 453464

Dejana, E., Lampugnani, M.G., Giorgi, M., Gaboli, M., Federici, A.B., Ruggeri, Z.M. and Marchisio, P.C. Von Willebrand factor promotes endothelial cell adhesion via an Arg-Gly-Asp-dependent mechanism J. Cell Biol. 1989; 109: 367-375

Del Boccio, G., Lapenna, D., Porreca, E., Pennelli, A., Savini, F. Feliciani, P., Ricci, G. and Cuccurullo, F. Aortic antioxidant defence mechanisms: time-related changes in cholesterol fed rabbits Atherosclerosis 1990; 81: 127-135

Demers, L.M. and Derck, D.D. A radioimmunoassay for 6-keto prostoglandin $F_{1 \alpha}$. In: Advances in Prostaglandin and Thromboxane Research, Samuelsson, B., Ramwell, P.W. and Paoletti, R. (eds.), Raven Press Ltd, NY 1980; 6: 193-199

Denis, N., Corcos, D., Kruh, J. and Kitzis, A. A rapid and accurate method for quantitating total RNA transferred during Northern blot analysis Nucleic acid Res. 1988; 16: 2354

Depres, J.-P., Moorjani, S., Lupien, P.J., Tremblay, A., Nadeau, A. and Bouchard, C. Regional distribution of body fat, plasma lipoproteins, and cardiovascular disease Arteriosclerosis 1990; 10: $497-511$

Derynck, R. Transforming growth factor- $\alpha$ Cell 1988; 54: 593-595

Dewey, W.C. and Highfield, D.P. $G_{2}$ block in Chinese hamster cells induced by $x$-irradiation, hyperthermia, cycloheximide, or actinomycin- $D$ Radiat. Res. 1976; 65: 511-528

Dickinson, E.S. and Slakey, L.L. Plasma-derived serum as a selective agent to obtain endothelial cultures from swine aorta In Vitro 1982; 18: 63-70

Dickson, R.B. and Lippman, M.E. Estrogen regulation of growth and polypeptide growth factor reaction in human breast carcinoma Endocr. Rev. 1987; 8: 29-43

Dickson, R.B., Bates, Valverius, E., Knabbe, C., Salomon, D., Huff, K.K., Bronzert, D., Walker-Jones, D., Freter, C., Favoni, R., Yee, D., Zugmaier, G., Ennis, B., Clarke, R., Kern, F., Rosen, N. and Lippman, M.E. Estrogen and antiestrogen regulation of mitogenic growth factors in human breast cancer cells. In: Progess in Cancer Research and Therapy, vol.35: Hormones and Cancer 3, Bresciani, F., King, R.J.B., Lippman, M.E. and Raynaud, P. Jr. (eds.), Raven Press Ltd, NY 1988; 217-220

Dickson, R.B., Huff, K.K., Spencer, E.M. and Lippman, M.E. Induction of epidermal growth factor-related peptides by $17 \beta$-estradiol in MCF- 
7 human breast cancer cell Endocrinology 1986; 118: 138-142 (b)

Dickson, R.B., McManaway, M.E. and Lippman, M.E. Estrogen-induced factors of breast cancer cells partially replace estrogen to promote tumor growth Science 1986; 232: 1540-1543 (a)

DiCorleto, P.E. and Bowen-Pope, D.F. Cultured endothelial cells produce a platelet-derived growth factor-like protein Proc. Natl. Acad. Sci. USA 1983; 80: 1919-1923

DiCorleto, P.E. and Chisolm, G.M. Participation of the endothelium in the development of the atherosclerotic plaque Prog. Lipid Res. 1986; 25: 365-374

DiCorleto, P.E. and de la Motte, C.A. Thrombin causes increased monocytic-cell adhesion to endothelial cells through a protein kinase cdependent pathway Biochem. J. 1989; 264: 71-77

DiCorleto, P.E. and de la Motte, C.A. Characterization of the adhesion of the human monocytic cell line U-937 to cultured endothelial cells Arteriosclerosis 1984; 4: 537a

DiCorleto, P.E. and de la Motte, C.A. Characterization of the adhesion of the human monocyte cell line U-937 to cultured endothelial cells J. Clin. Invest. 1985; 75: 1153-1161

DiCorleto, P.E. and Fox, P.L. Growth factor production by endothelial cells. In: Endothelial Cells, Volume II, Ryan, U.S.(ed.) CRC Press, Boca Raton, Florida 1988; : 51-61

DiCorleto, P.E., Gajdusek, C.M., Schwartz, S.M. and Ross, R. Biochemical properties of the endothelium-derived growth factor: comparison to other growth factors J. Cell. Physiol. 1983; 114: 339-345

Dieterle, Y., Ody, C., Ehrensberger, A., Stalder, H. and Junod, A.F. Metabolism and uptake of adenosine triphosphate and adenosine by porcine aortic and Pulmonary Emdothelial Cells and Fibroblasts in Culture Circ. Res. 1978; 42: 869-876

Dilley, R. McGeachie, J. and Prendergast, F. A review of the proliferative behaviour, morphology and phenotypes of vascular smooth muscle Atherosclerosis 1987; 63: 99-107

Dinarello, C. Biology of interleukin 1 FASEB J. 1988; 2 : 108-115

Doherty, D.E., Haslett, C., Tonnesen, M.G. and Henson, P.M. Human monocyte adherence: a primary effect of chemotactic factors on the monocyte to stimulate adherence to human endothelium $\mathbf{J}$. Immunol. 1987; 138: 1762-1771

Doherty, D.E., Zagarella, L., Henson, P.M. and Worthen, G.S.

Lipopolysaccharide stimulates monocyte adherence by effects on both the monocyte and the endothelial cell J. Immunol. 1989;

143: $3673-3679$ 
Donahue, R.P., Orchard, T.J., Becker, D.J., Kuller, L.H. and Drash, A.L. Sex differences in the coronary heart disease risk profile: a possible role for insulin Am. J. Epidemiol. 1987; 125: 650-657

Doolittle, R.F., Hunkapiller, M.W., Hood, L.E., Devare, S.G., Robbins, K.C., Aaronson, S.A. and Antoniades, H.N. Simian sarcoma virus oncogene, $v$-sis, is derived from the gene (or genes) encoding a platelet-derived growth factor Science 1983; 221: 275-277

Drouet, L.O., Rhee, C.Y., Cintron, J.R. and Spaet, T.H. Estrogen inhibition of experimental arteriosclerosis in the rabbit Fed. proc. 1978; 37: 474

Dugdale, M. and Masi, A.T. Effect of oral contraceptives on blood clotting. In: Second Report on the Oral Contraceptives, Advisory Committee on Obsterics and Gyneocology Food and Drug Administration, Washington, DC 1969; 43-51

Duguid, J.B. Thromobsis as a factor in the pathogenosis of coronary atherosclerosis J. Pathol. Bact. 1946; 58: 207-212

Duthie, G.G., Arthur, J.R. and James, W.P.T. Effect of smoking and vitamin E on blood antioxidant status Am. J. Clin. Nutr. 1991; 53: 1061510635

Dyerberg, J. and Hjorne, H. Plasma lipid and lipoprotein levels in a Danish population Acta. Med. Scand. 1972; 191: 413-421

Editor Coronary heart disease without risk factors Nutr. Rev. 1989; 47: 18-22

Edwards, D.P., Chamness, G.C. and McGuire, W.L. Estrogen and progesterone receptor proteins in breast cancer Biochim. Biophys. Acta 1979; 560: 457-486

El-Saadani, M., Esterbauer, H., El-Sayed, M., Goher, M., Nassar, A.Y. and Jurgens, G. A spectrophotometric assay for lipid peroxides in serum lipoproteins using a commercially available agent J. Lipid Res. 1989; 30: 627-630

Elam, M.B., Lipscomb, G.E., Chesney, C.M., Terragno, D.A. and Terragno, N.A. Effect of synthetic estrogen on platelet aggregration and vascular release of $\mathrm{PGI}_{2}$-like material in the rabbit Prostaglandins 1980; 20: 1039-1051

Eldor, A., Falcone, D.J., Hajjar, D.P., Minick, C.R. and Weksler, B.B. Recovery of prostacyclin production by deendothelized rabbit aorta: critical role of the neointimal smooth muscle cells J. Clin. Invest. 1981; 67: 735-741

Eldor, A., Fuks, Z., Matzner, Y., Witte, L.D. and Vlodavsky, I. Perturbation of endothelial functions by ionizing irradiation: effects on prostaglandins, chemoattractants and mitogens Semin. Thromb. Hemost. 1989; 15: 215-225

Eldor, A., Vlodavsky, I., Hy-am, E., Atzman, R., Weksler, B.B., Raz, A. and Fuks, Z. Cultured endothelial cells increase their capacity to synthesize prostacyclin following formation of a contact-inhibited cell monolayer J. Cell. Physiol. 1983; 114: 179-183 (b) 
Eldor, A., Vlodavsky, I., Hy-am, E., Atzmon, R. and Fuks, Z. The effect of radiation on prostacyclin $\left(P G I_{2}\right)$ production by cultured endothelial cells Prostaglandins 1983; 25: 263-279 (a)

Elgjo, R.F., Henriksen, T. and Evensen, S.A. Ultra structural identification of umbilical cord vein endothelium in situ and in culture Cell Tissue Res. 1975; 162: 49-59

Elliott, M.J., Vadas, M.A., Cleland, L.G., Gamble, J.R. and Lopez, A.F. IL-3 and granulocyte-macrophage colony-stimulating factor stimulate two distinct phases of adhesion in human monocytes J. Immunol. 1990; 145: 167-176

Ellis, E.F., Oelz, O., Roberts, II L.J., Payne, A., Sweetman, B.J., Nies, A.S. and Oates, J.A. Coronary arterial smooth muscle contraction by a substance released from platelets: evidence that it is thromboxane $A_{2}$ Science 1976; 193: 1135-1137

Elveback, L.R., Connolly, D.C., and Melton, L.J. III Coronary heart disease in residents of Rochester Minnesota VII. Incidence 1950-1982 Mayo Clin. Proc. 1986; 61: 896-900

Elwood, P.C., Sweetman, P., Mahler, R., Moore, F. and Welsby, E. Association between circulating hemoglobin level, serum cholesterol and blood pressure Lancet 1970; 1: 589-590

Emeis, J.J. and Kooistra, T. Interleukin 1 and lipopolysaccharde induce an inhibitor of tissue-type plasminogen activator in vivo and in cultured endothelial cells J. Exp. Med. 1986; 163: 1260-1266

Emms, H. and Lewis, G.P. Sex hormonal influence on platelet sensitivity and coagulation in the rat $\mathrm{Br}$. J. Pharmacol. 1985; 86: 557-563

Endermann, G. Pronzcuk, A., Friedman, G., Lindsey, S., Alderson, L. and Hayes, K.C. Monocyte adherence to endothelial cells in vitro is increased by B-VLDL Am. J. Pathol. 1987; 126: 1-6

Enk, J., Crona, N., Samsioe, G. and Silfverstolpe, G. Dose and duration of estradiol valerate on serum and lipoprotein lipids Horm. Metab. Res. 1986; 18: 551-554

Eriksson, M., Berglund, L., Ruding, M., Henriksson, P., Angelin, B. Effect of estrogen on low density lipoprotein metabolism in males $\mathrm{J}$. Clin. Invest. 1989; 84: 802-810

Ernst, M., Schmid, C.H. and Froesch, E.R. Enhanced osteoblast proliferation and collagen gene expression by estradiol Proc. Natl. Acad. Sci. USA 1988; 85: 2307-2310

Ernst, M., Heath, J.K. and Rodan, G.A. Estradiol effects on proliferation, messenger ribonucleic acid for collagen and insulin- like growth factor-1, and parathyroid hormone-stimulated adenylate cyclase activity in osteoblastic cells from calvariae and long bones Endocrinology 1989; 125: 825-833

Escobedo, J.A. Navankasatussas, S. Cousens, L.S. Coughlin, S.R. Bell, G.I. and Williams, L.T. A common PDGF receptor Is activated by 
homodimeric $A$ and $B$ forms of PDGF Science 1988; 240: 15321534

Escobedo, J.A. and Williams, L.T. A PDGF receptor domain essential for mitogenesis but not for many other responses to PDGF Nature 1988; 335: 85-87

Eskin, S.G., Sybers, H.D., Trevino, L., Lie, J.T. and Chimoskey, J.E. Comparison of tissue-cultured bovine endothelial cells from aorta and saphenous vein In Vitro 1978; 14: 903-910

Esmon, C.T. and Esmon, N.L. Protein C activation Semin. Thromb. Hemost. 1984; 10: 122-130

Esterbauer, H., Dieber-Rotheneder, M.D., Waeg, G., Striegl, G. and Jurgens, G. Biochemical, structural and functional properties of oxidized lowdensity lipoprotein Chem. Res. Toxicol. 1990; 3: 77-92

Esterbauer, H., Jurgens, G., Quenhenberger, O. and Koller, E. Autoxidation of human low density lipoprotein: loss of poly unsaturated fatty acids and vitiamin E and generation of aldehydes J. Lipid Res. 1987; 28: 495-509

Esterbauer, H., Quehenberger, O. and Jurgens, G. Oxidation of human low density lipoprotein with special attention to aldehydic lipid peroxidation products Free Rad. Method. Concepts 1988; : 243-268

Etingin, O.R. and Hajiar, D.P. Evidence for cytokine regulation of cholesterol metabolism in herpes virus-infected arterial cells by the lipoxygenase pathway J. Lipid Res. 1990; 31: 299-305

Etoh, T., Takehara, K., Igarashi, A. and Ishibashi, Y. The effects of various growth factors on endothelial cell survival in vitro Biochem. Biophys. Res. Commun. 1989; 162: 1010-1016

Evans, C.E., Billington, D. and McEvoy, F.A. Prostacyclin production by confluent and non-confluent human endothelial cells in culture Prostagland. Leukot. Med. 1984; 14: 255-266

Evensen, S.A., Galdal, K.S. and Nilsen, E. LDL-induced cytotoxicity and its inhibition by anti-oxidant treatment in cultured human endothelial cells and fibroblasts Atherosclerosis 1983; 49: 23-30

Fabricant, C.G., Fabricant, J., Litrenta, M.M. and Minick, C.R. Virus-induced atherosclerosis J. Exp. Med. 1978; 148: 335-339

Faggiotto, A. and Ross, R. Studies of hypercholesterolemia in the non human primate. II. Fatty streak conversion to fibrous plaque Arteriosclerosis 1984; 4: 341-356

Faggiotto, A., Ross, R. and Harker, L. Studies of hypercholesterolemia in the non human primate. I. Changes that lead to fatty streak formation Arteriosclerosis 1984; 4: 323-340

Fayle, D. Sim, P. Invine, D. and Doe, W. Isolation of plasma membrane from human blood monocytes Eur. J. Biochem. 1985; 147: 409-419 
Ferreira, S.H. and Vane, J.R. Prostaglandins: their disappearance from the release into the circulation Nature 1967; 216: 868-873

Fielding, P.E. and Fielding, C.J. Role of lipoproteins in the regulation of cultured endothelial cell cholesterol metabolism. In: Biology of Endothelial Cells, Jaffe, E.A. (ed.), Martinus Nijhoff Publishers, Boston 1984; 356-364

Fink, K.E., Wieber, E., Woloschak, G.E. and Spelsberg, T. C. Rapid regulation of c-myc protooncogene expression by progesterone in the avian oviduct Proc. Natl. Acad. Sci. USA 1988; 85: 1796-1800

Fisch, I.R. and Frank, J. Oral contraceptives and blood pressure JAMA 1977; 237: 2499-2503

Fischer, G.M. Bashey, R.I. Rosebaum, H. and Lyttle, C.R. A possible mechanism in aterial wall for mediation of sex differences in atherosclerosis

Exp. Mol. Pathol. 1985; 43: 288-29.6

Fischer, G.M. In vivo effects of estradiol on collagen and elastin dynamics in rat aorta Endocrinology 1972; 91: 1227-1232

Fischer, G.M. and Swain, M.L. Effects of sex hormones on blood pressure and vascular connective tissue in castrated and noncastrated male rats Am. J. Physiol. 1977; 232: H617-H621

Fischer, G.M. and Swain, M.L. Effect of estradiol and progesterone on the increased synthesis of collagen in atherosclerotic rabbit aortas Atherosclerosis 1985; 54: 177-185

Fischer, G.M., Cherian, K. and Swain, M.L. Increased synthesis of aortic collagen and elastin in experimental atherosclerosis. Inhibition by contraceptive steroids Atherosclerosis 1981; 39: 463-467

Fischer-Dzoga, K., Wissler, R.W. and Vesselinovitch, D. The effect of estradiol on the proliferation of rabbit aortic medial tissue cultures cells induced by hyperlipemic serum Exp. Mol. Pathol. 1983; 39: 355-363

Florentin, R.A., Nam, S.C., Lee, K.T. and Thomas, W.A. Increased ${ }^{3}$ H-thymidine incorporation into endothelial cells of swine fed cholesterol for 3 days Exp. Mol. Pathol. 1969; 10: 250-255

Flynn, A. Stimulation of interleukin-1 production from placental monocytes Lymphokine Res. 1984; 3: 1-5

Flynn, A. Expression of la and the production of interleukin 1 by peritoneal exudate macrophages activated in vivo by steroids Life Sci. 1986; 38: 2455-2460

Foegh, M.L. Khirabadi, B.S. Vargas, R., Nakanishi, T. and Ramvell, P.W. Estradiol protects against experimental cardiac transplant atherosclerosis Transplant. Proc. 1987; X1X: 90-95

Fogelberg, M., Vesterqvist, O., Diczfalusy, U. and Henriksson, P. Experimental atherosclerosis: effects of oestrogen and atherosclerosis on thromboxane and prostacyclin formation Eur. J. Clin. Invest. 1990; 20: 105-110 
Fogelman, A.M., Shechter, I., Seager, J., Hokom, M., Child, J.S. and Edwards, P.A. Malondialdehyde alteration of low density lipoproteins leads to cholesteryl ester accumulation in human monocyte-

macrophages Proc. Natl. Acad. Sci., USA 1980; 77: 2214-2218

Folkman, J. How is blood vessel growth regulated in normal and neoplastic tissue? Cancer Res. 1986; 46: 467-473

Folkman, J., Langer, R., Linbardt, R.J., Haudenschild, C.C. and Taylor, S. Angiogenesis inhibition and tumor regression caused by heparin or a heparin fragment in the presence of cortisone Science 1983; 221: 719-725

Fong, L.G., Parthasarathy, S., Witztum, J.L. and Steinberg, D. Nonenzymatic oxidative cleavage of peptide bonds in apoprotein B-100 J. Lipid Res. 1987; 28: 1466-1477

Ford, J.W., Burkel, W.E. and Kahn, R.H. Isolation of adult canine venous endothelium for tissue culture In Vitro 1981; 17: 44-50

Fowler, S., Shio, H. and Haley, N.J. Characterization of lipid-laden aortic cells from cholesterol fed rabbits. $N$ investigation of macrophage-like properties of aortic cell populations Lab. Invest. 1979; 41: 372378

Fox, P.L. and DiCorleto, P.E. Modified low density lipoprotein suppress production of a platelet-derived growth factor-like protein by cultured endothelial cells Proc. Natl. Acad. Sci. USA 1986; 83: 4774-4800

Fox, P.L. and DiCorleto, P.E. Regulation of production of a platelet-derived growth factor-like protein by cultured bovine aortic endothelial cells J. Cell. Physiol. 1984; 121: 298-308

Fox, P.L. and DiCorleto, P.E. Acetylated low density lipoprotein suppresses production of platelet-derived growth factor by cultured endothelial cells J. Cell Biol. 1985; 101: 107a

Foxall, T.L. and Shwaery, G.T. Effect of dietary fish oil and butter fat on serum lipids and monocyte and platelet interactions with aortic endothelial cells Atherosclerosis 1990; 80: 171-179

Fraater-Schroder, M., Muller, G., Birchmeier, W. and Bohlen, P. Transforming growth factor-beta inhibits endothelial cell proliferation Biochem. Biophys. Res. Commun. 1986; 137: 295-302

Franchi, A.M., Gimeno, A.L. and Gimeno, M.A.F. Influences of estradiol and of catechol and non catechol estrogens on the output of prostaglandins in uteri from spayed rats Prostaglandins Leukot. Essent. Fatty Acids 1989; 36: 25-29

Francos, J.A., Eskin, S.G., McIntire, L.V. and Ives, C.L. Flow effects on prostacyclin production by cultured human endothelial cells Science 1985; 227: 1477-1479

Freeman, B.A., Rosen, G.M. and Barber, M.J. Superoxide perturbation of the organization of vascular endothelial cell membranes J. Biol. Chem. 1986; 216: 6590-6593 
Friedman, M. Type A behaviour pattern: some of its pathophysiological components Bull. NY Acad. Med. 1977; 53: 593-604

Friedman, M. and Rosenman, R.H. Type A behavior pattern: Its association with coronary diseases Ann. Clin. Res. 1971; 3: 300-312

Friedman, M., Thoresen, C.E.,Gill, J.J., Ulmer, D., Thompson, L., Powell, L., Price, V., Elek, S.R., Rabin, D.D., Breall, W.S., Piaget, G., Dixon, T., Bourg, E., Levy, R.A. and Tasto. D.L. Feasibility of altering type A behaviour pattern after myocardial infarction. Recurrent Coronary Prevention Project Study: Methods, baseline results and preliminary findings Circulation 1982; 66: 83-92

Friedman, R.J., Stemerman, M.B., Wenz, B., Moore, S., Gauldie, J., Gent, M., Tiell, M.L. and Spaet, T.H. The effect of thrombocytopenia on experimental atherosclerotic lesion formation in rabbits. Smooth muscle cell proliferation and re-endothelialization J. Clin. Invest. 1977; 60: 1191-1201

Frostegard, J., Nilsson, J., Haegerstrand, A., Hamsten, A., Wigzell, H. and Gidlund, M. Oxidized low density lipoprotein induces differentiation and adhesion of human monocytes and the monocytic cell line U937 Proc. NatI. Acad. Sci., USA 1990; 87: 904-908

Fryer, D.G., Birnbaum, G. and Luttrell, C.N. Human endothelium in cell culture J. Athero. Res. 1966; 6: 151-163

Fujii-Hanamoto, H., Crossman, C.J., Roselle, G.A., Mendenhall, C.L. and Seiki, $\mathrm{K}$. Nuclear progestin receptors in rat thymic tissue Thymus 1990; 15: $31-45$

Furman, R.H. Are gonadal hormones (estrogen and androgens) of significance in the development of ischomic heart disease? Ann. NY Acad. Sci. 1986; 149: 822-833

Furman, R.H., Howard, R.P., Norcia, L.N. and Keaty, E.C. The influence of androgens, estrogens and related steroids on serum lipids and lipoproteins. Observations in hypogonadal and normal human subjects Am. J. Med. 1958; 24: 80-97

Fuster, V., Bowie, E.J.W., Lewis, J.C., Fass, D.N., Owen, Jr. C.A. and Brown, A.L. Resistance to arteriosclerosis in pigs with von Willebrand's disease J. Clin. Invest. 1978; 61: 722-730

Gajdusek, C., Carbon, S., Ross, R., Nawroth, P. and Stern, D. Activation of coagulation releases endothelial cell mitogens J. Cell Biol. 1986; 103: $419-428$

Gajdusek, C.M, DiCorleto, P.E., Ross, R. and Schwartz, S.M. An endothelial cell-derived growth factor J. Cell Biol. 1980; 85: 467-472

Gamble, J.R. and Vadas, M.A. Endothelial adhesiveness for blood neutrophils is inhibited by transforming growth factor- $\beta$ Science 1988; 242: 9798 (a) 
Gamble, J.R. and Vadas, M.A. A new array for the measurement of the attachment of neutrophils and other cells types to endothelial cells J. Immunol. Meth. 1988; 109: 175-18 (b)

Gamble, J.R., Elliott, M.J., Jaipargas, E., Lopez, A.F. and Vadas, M.A. Regulation of human monocyte adherence by granulocytemacrophage colony-stimulating factor Proc. Natl. Acad. Sci., USA 1989; 86: 7169-7173

Gardner, E., Gray, D. and O'Rahilly, R. Anatomy. A Regional Study of Human Stucture (4th ed.), Sauders Company, USA 1975; : 41

Gardner, R.M., Verner, G., Kirkland, J.L. and Stancel, G.M. Regulation of uterine epidermal growth factor (ECF) receptors by estrogen in mature rate and during the estrous cycle J. Steroid Biochem. 1989; 32: 339-343

Garrison, R.J., Wilson, P.W., Cestell, W.P., Feinleib, M., Kannel, W.B. and McNamara, P.M. Obesity and lipoprotein cholesterol in the Framingham off spring study Metabolism 1980; 29: 1053-1060

Gaunt, S.D. and Pierce, K.R. Effects of estradiol on hematopoietic and marrow adherent cells of dogs Am. J. Vet Res. 1986; 47: 906-909

Gay, C.G. and Winkles, J.A. Heparin-binding growth factor-1 stimulation of human endothelial cells induced platelet-derived growth factor Achain gene expression. J. Biol. Chem. 1990; 265: 3284-3292

Gerrity, R.G. The role of monocytes in atherogenesis: I. transition of bloodborne monocytes into foam cells in fatty lesions Am. J. Pathol. 1981; 103: 181-190 (a)

Gerrity, R.G. The role of the monocyte in atherosclerosis: II. migration of foam cells from atherosclerotic lesions Am. J. Pathol. 1981; 103: 191200 (b)

Gerrity, R.G. and Naito, H.K. Ultrastructural identification of monocyte-derived foam cells in fatty streak lesions Artery 1980; 8: 208-214 (a)

Gerrity, R.G. and Naito, H.K. Lipid clearance from fatty streak lesions by foam cell migration Artery 1980; 8: 215-219 (b)

Gerrity, R.G. and Schwartz, C.J. Endothelial cell injury in early mild hypercholesterolemia Prog. Biochem. Pharmacol. 1977; 13: 213-219

Gerrity, R.G., Goss, J.A. and Soby, L. Control of monocyte recruitment by chemotactic factor (s) in lesion-prome areas of swine aorta Arteriosclerosis 1985; 5: 55-66

Gerrity, R.G., Naito, H.K., Richardson, M. and Schwartz, C.J. Dietary induced atherogenesis in swine Am. J. Pathol. 1979; 95: 775-793

Gimbrone, M.A. Jr. Culture of vascular endothelium. In: Prog. Hemost. Thromb., T.H. Spaet (ed.), Grune and Stratton Inc., NY 1976; 3: $1-28$ 
Gimbrone, M.A. Jr. Vascular endothelium and atherosclerosis. In: Vascular Injury and Atherosclerosis, Moore, S. (ed.), M. Dekker, Inc., NY $1981 ;:$ :25-53

Gimbrone, M.A. Jr. and Cotran, R.S. Human vascular smooth muscle in culture: growth and ultrastructure Lab. Invest. 1975; 33: 16-27

Gimbrone, M.A. Jr. and Fareed, G.C. Transformation of cultured human vascular endothelium by SV40 DNA Cell 1976; 9: 685-693

Gimbrone, M.A., Jr. Cotran, R.S. and Folkman, J. Human vascular endothelial cells in culture: growth and DNA synthesis J. Cell Biol. 1974; 60: 673-684

Gimbrone, M.A. Jr., Martin, B.M., Baldwin, W.M., Unanue, E.R. and Cotran, R.S. Stimulation of vascular cell growth by macrophage products. In: Pathobiology of the Endothelial Cell, Nossel, H.L. and Vogel, H.J. (eds.), Academic Press, NY 1982; 3-17

Gladwin, A.M., Hassall, D.G., Martin, J.F. and Booth, R.F. MAC-1 mediates adherence of human monocytes to endothelium via a protein kinase $C$ dependent mechanism Biochim. Biophys. Acta 1990; 1052: 166-172

Glassberg, M.K., Bern, M.M., Coughlin, S.R., Haudenschild, C.C., Hoyer, L.W., Antoniades, H.N. and Zetter, B.R., Cultured endothelial cells derived from the human iliac arteries In Vitro 1982; 18: 859-866

Glanvind, J., Hartmann, S., Clemmesen, J., Jessen, K.E. and Dam, H. Studies on the role of lipoperoxides in human pathology II. the presence of peroxidized lipids in the atherosclerotic aorta Acta Pathol. 1952; 30: 1-6

Glomset, J.A. The plasma lecithin: cholesterol acyltransferase reaction J. Lipid Res. 1968; 9: 155-167

Glueck, C.J., Scheel, D., Fishback, J. and Steiner, P. Progestagens, anabolicandrogenic compounds, estrogens: effects on triglycerides and postheparin lipolytic enzymes Lipids 1972; 7: 110-113

Goerdt, S., Zwadlo, G., Schlegel, R., Hagemeier, H-H and Sorg, C. Characterization and expression kinetics of an endothelial cell activation antigen present in vivo only in acute inflammatory tissues Exp. Cell. Biol. 1987; 55: 117-126

Gofman, J.W., Jones, H.B., Lindgren, F.T., Lyon, T.P., Elliot, H.A. and Strisower, B. Blood lipids and human atherosclerosis Circulation 1950; 2: 161-178

Goldberg, R. J., Gore, J. M., Zive, M., Brady, P., Klaiber, E., Broverman, D., Ockene, I. S. and Dalen, J. E. Serum estradiol and coronary artery disease Am. J. Med. 1987; 82 : 1-4

Goldman, L. and Tosteson, A.N.A. Uncertainity about post menopausal estrogen N. Engl. J. Med. 1991; 325: 800-802

Goldstein, J.L. and Brown, M.S. Atherosclerosis: the low density lipoprotein receptor hypothesis Metab. Clin. Exp. 1977; 26: 1257-1275 
Goldstein, J.L., Helgeson, J.A.S. and Brown, M.S. Inhibition of cholesterol synthesis with compaction renders growth of cultured cells dependent on low density lipoprotein receptors J. Biol. Chem. 1979; 254: 5403-5409 (a)

Goldstein, J.L., Ho, Y.K., Basu, S.K. and Brown, M.S. Binding site on macrophages that mediates uptake and degradation of acetylated low density lipoprotein, producing massive cholesterol deposition Proc. Natl. Acad. Sci., USA 1979; 76: 333-337 (b)

Goldstein, J.L., Ho, Y.K., Brown, M.S., Innerarity, T.L. and Mahley, R.W. Cholesteryl ester accumulations in macrophages resulting from receptor-mediated uptake and degradation of hypercholesterolemic canine very low density lipoproteins $\mathbf{J}$. Biol. Chem. 1980; 225: 1839-1848

Goldstein, J.L., Hoff, H.L., Ho, Y.K., Basu, S.K. and Brown, M.S. Stimulation of cholesteryl ester biosynthesis in macrophages by extracts of atherosclerotic human aortas and complexes of albumin/cholesteryl esters Arteriosclerosis 1981; 1: 210-226

Goode, T.B., Davies, P.F., Reidy, M.A. and Bowyer, D.E. Aortic endothelial cell morphology observed in situ by scanning election microscopy during atherogenesis in the rabbit Atherosclerosis 1977; 27: 235-251

Gordon, T. and Kannel, W.B. Multiple risk functions for predicting coronary heart disease: the concepts, accuracy and application Am. Heart J. 1982; 103: 1031-1039

Gordon, T., Kannel, W.B., Hjortland, M.C. and McNamara, P.M. Menopause and coronary heart disease: Framingham study Ann. Intern. Med. 1978; 89: 157-161

Gordon, T., Castelli, W.P., Hjortland, M.C., Kannel, W.B. and Dawber, T.R. High density lipoprotein as a protective factor against coronary heart disease. the Framingham study Am. J. Med. 1977; 62: 707-714

Gorski, J. and Gannon, F. Current models of steroid hormone action: a critique Ann. Rev. Physiol. 1976; 38: 425-450

Gorski, J., Toft, D., Shyamala, G., Smith, D. and Notides, A. Hormone receptors: studies on the interaction of estrogen with the uterus Recent Prog. Horm. Res. 1968; 24: 45-80

Gospodarowicz, D. Purification of a fibroblast growth factor from bovine pituitary J. Biol. Chem. 1975; 250: 2515-2520

Gospodarowicz, D., Bialecki, H. and Greenburg, G. Purification of the fibroblast growth factor activity from bovine brain J. Biol. Chem. 1978; 253: 3736-3743 (b)

Gospodarowicz, D., Brown, K.D., Birdwell, C.R. and Zetter, B.R. Control of proliferation of human vascular endothelial cells.

Characterization of the response of human umbilical vein endothelial cells to fibroblast growth factor, epidermal growth factor, and thrombin J. Cell Biol. 1978; 77: 774-88 (a) 
Gospodarowicz, D., Greenburg, G., Bialecki, H. and Zetter, B.R. Factors involved in the modulation of cell proliferation in vivo and in vitro: the role of fibroblast and epidermal growth factors in the proliferative response of mammalian cells In Vitro 1978; 14: 85118

Gospodarowicz, D., Moran, J.S., Braun, D.L. and Birdwell, C.R. Clonal growth of bovine vascular endothelial cells: fibroblast growth factor as a survival agent Proc. Natl. Acad. Sci., USA 1976; 73: 4120-4124

Gospodarowicz, D., Moran, J. and Braun, D.L. Control of proliferation of bovine vascular endothelial cells J. Cell. Physiol. 1977; 91: 377-386

Gotlieb, A.I., Spector, W., Desai, K. and Steiner, G. The effect of low density lipoprotein on in vitro porcine endothelial cell reendothelialization Artery 1983; 12: 132-144

Gotzos, V., Cappelli-Gotzos, B. and Bovet, R. In vitro responses of proliferating and non-proliferating cells to high doses of 17 beta-estradiol: cytochemcial study Acta Histochem. (Jena) 1981; 69: 40-49

Goustin, A.S., Betsholtz, C., Pfeifer-Ohlsson, S., Persson, H., Rydnert, J., Bywater, M., Holmgren, G., Heldin, C.-H., Westermark, B. and Ohlsson, R. Coexpression of the sis and myc proto-oncogenes in developing human placenta suggests autocrine control of trophoblast growth Cell 1985; $41: 310-312$

Gower, D.B. The biosynthesis of steroid hormones: an up-date. In: Hormones and their Action, Part 1, Cooke, B.A., King, R.J.B., van der Molen, H.J. (eds.), 1988; 3-28

Gray, J.M. and Wade, G.N. Cytoplasmic progestin binding in rat adipose tissue Endocrinology 1979; 104: 1377-1382

Gray, T.K., Flynn, T.C., Gray, K.M. and Nabell, L.M. 17ק-estradiol acts directly on the clonal osteoblastic cell line UMR106 Proc. Natl. Acad. Sci. USA 1987; 84: 6267-6271

Green, S., Walter, P., Kumar, V., Krust, A., Bornert, J.-M., Argos, P. and Chambon, $\mathrm{P}$. Human oestrogen receptor CDNA sequence, expression and homology to v-erb-A Nature 1986; 320: 134-139

Greene, G.L., Gilna, P., Waterfield, M. Baker, A., Hort, Y. and Shine, J. Sequence and expression of human estrogen receptor complementary DNA Science 1986; 231: 1150-1154

Griffin, J.E. and Wilson, J.D. Disorders of the testis. In: Harrison's Principles of Internal Medicine, 12th edition, Wilson, J.D., Braunwald, E., Isselbacher, K.J., Petersdorf, R.G., Martin, J.B., Fauci, A.S. and Root, R.K. (eds.), McGraw-Hill, Inc., Sydney 1989; 1765-1770

Gronwald, R.G., Grant, F.J., Haldeman, B.A., Hart, C.E., O’Hara, P.J., Hagen, F.S., Ross, R., Bowen-Pope, D.F. and Murray, M.J. Cloning and expression of a cDNA coding for the human platelet-derived growth factor receptor: evidence for more than one receptor class Proc. Natl. Acad. Sci., USA 1988; 85: 3435-3439 
Gryglewski, R.J., Botting, R.M. and Vane, J.R. Mediators produced by the endothelial cells Hypertension 1988; 12: 530-548

Gulshan, S., McCruden, A.B. and Stimson, W.H. Oestrogen receptors in macrophages Scand. J. Immunol. 1990; 31: 691-697

Gurney, T. One-dimensional electrophoresis of nucleic acids in agarose using denaturation with formaldehyde and identification of ${ }^{3} \mathrm{H}$-labeled RNA by fluorography In: Methods in Molecular Biology, Walker, J.M. (ed.), Humana Press, New Jersey 1984; 2: 77-92

Gustafson, A., and Svanborg, A. Gonadal steroid effects on plasma lipoproteins and individual phospholipids J. Clin. Endocrinol. Metab. 1972; 35: 203-207

Haarbo, J., Hassager, C., Jensen, S.B., Riis, B.J. and Christiansen, C. Serum lipids, lipoproteins and apolipoproteins during post menopausal estrogen replacement therapy combined with either 19nortestosterone derivatives or 17-hydroxy progesterone derivatives Am. J. Med. 1991; 90: 584-489 (a)

Haarbo, J., Leth-Espensen, P., Stender, S. and Christiansen, C. Estrogen monotherapy and combined estrogen-progestogen replacement therapy attenuate aortic accumulation of cho;estero; in ovariectomized cholesterol-fed rabbits J. Clin. Invest. 1991; 87: 1274-1279 (b)

Haberland, M.E. Physiological modification of arterial lipoproteins by malondialdehyde In: Oxidative Damage and Repair Davies, K.J. (ed.), Pergamon Press, 1991; 65

Haberland, M.E. and Fogelman, A.M. The role of altered lipoproteins in the pathogenesis of atherosclerosis Am. Heart J. 1987; 113: 573577

Haberland, M.E., Fong, D. and Cheng, L. Malondialdehyde-altered protein occurs in atheroma of Watanabe hertitable hyperlipidemic rabbits Science 1988; 241: 215-218

Hajjar, K.A., Hajjar, D.P., Silverstein, R.L. and Nachman, R.L. Tumor necrosis factor mediated release of platelet-derived growth factor from cultured endothelial cells J. Exp. Med. 1987; 166: 235-245

Hallam, T.J., Pearson, J.D. and Needham, L.A. Thrombin-stimulated elevation of human endothelial-cell cytoplasmic free calcium concentration causes prostacyclin production Biochem. J. 1988; 251: 243-249

Halliwell, B. Oxidants and human disease: some new concepts FASEB J. 1987; 1: 358-364

Halliwell, B. and Gutteridge, J.M.C. Free radicals in biology and medicine Oxford University Press, NY 1986; 20-187

Ham, E.A., Cirillo, V.J., Zanetti, M.E. and Kuehl, F.A. Jr. Estrogen-directed synthesis of specific prostaglandins in uterus Proc. Natl. Acad. Sci., USA 1975; 72: 1420-1424 
Hammond, C.B., Jelovsek, F.R., Lee, K.L., Greasman, W.T. and Parker, R.T. Effects of long-term estrogen replacement therapy. I. Metabolic effects Am. J. Obstet. Gynecol. 1979; 133: 525-536

Hampel, G., Watanabe, K., Weksler, B.B. and Jaffe, E.A. Selenium deficiency inhibits prostacyclin release and enhances production of platelet activating factor by human endothelial cells Biochim. Biophys. Acta 1989; 1006: 151-158

Han, P., Boatwright, C. and Ardlie, N. Verapamil and collagen induced platelet reactions - evidence for a role for intracellular calcium in platelet activation Thromb. Haemost. 1983; 50: 537-540

Hanash, K.A., Kottke, B.A., Greene, L.F. and Titus, J.L. Effects of conjugated estrogens on spontaneous atherosclerosis in pigeons Arch. Pathol. 1972; 93: 184-189

Hannan, R.L., Kourembanas, S., Flanders, K.C., Rogelj, S.J., Roberts, A.B., Faller, D.V. and Klagsbrun, M. Endothelial cells synthesize basic fibroblast growth factor and transforming growth factor beta Growth Factors 1988; 1: 7-17

Hannink, M. and Donoghue, D.L. Structure and function of platelet-derived growth factor (PDGF) and related proteins Biochim. Biophys. Acta 1989; 989: 1-10

Hansson, G.K. and Bondjers, G. Endothelial dysfunction and injury in atherosclerosis Acta Med. Scand. 1987; 715 (Suppl): 11-17

Harats, D., Ben-Naim, M., Dahach, Y., Hollander, G., Stein, O. and Stein, Y. Cigarette smoking renders $L D L$ susceptible to peroxidative modification and enhanced metabolism by macrophages Atherosclerosis 1989; 79: 245-252

Harder, D.R. and Coulson, P.B. Estrogen receptors and effects of estrogen on membrane electrical properties of coronary vascular smooth muscle J. Cell. Physiol. 1979; 100: 375-382

Harker, L.A., Ross, R., Slichter, S.J. and Scott, C.R. Homocystine-induced arteriosclerosis: the role of endothelial cells injury and platelet responses in its genesis J. Clin. Invest. 1976; 58: 731-741

Harker, L., Slichter, S.J., Scott, C.R. and Ross, R. Homocystinemia: vascular injury and arterial thrombosis N. Engl. J. Med. 1974; 291: 537543

Harlan, J.M., Killen, P.D., Harker, L.A., Striker, G.E and Wright, D.G. Neutrophilmediated endothelial injury in vitro. Mechanism of cell attachment J. Clin. Invest. 1981; 68: 1394-1403

Harlan, J.M., Thompson, P.J., Ross, R. and Bowen-Pope, D.F. Alpha-thrombin induces release of platelet-derived growth factor-like molecule(s) by cultured human endothelial cells J. Cell Biol. 1986; 103: 1129-1133

Harrison, H.E., Reece, A.H. and Johnson, M. Decreased vascular prostacyclin in experimental diabetes Life Sci. 1978; 23: 351-356 
Harrison, R.L. and McKee, P.A. Estrogen stimulates von Willebrand factor production by cultures endothelial cells Blood 1984; 63: 657$665(a)$

Harrison, R.L. and McKee, P.A. The effect of estrogen on von Willibrand factor production and endothelial cell number in culture Thromb. Haemost. 1984; 46: 20 (b)

Hart, C.E., Forstrom, J.W., Kelly, J.D., Seifert, R.A., Smith, R.A., Ross, R., Murray, M. and Bowen-Pope, D.F. Two classes of PDGF receptor recognize different isoforms of PDGF Science 1988; 240: 15291531

Hasegawa, N., Yamamoto, M. and Yamamoto, K. Stimulation of cell growth and inhibition of prestacyclin production by heparin in human umbilical vein endothelial cells J. Cell. Physiol. 1988; 137: 603607

Hashemi, S., Tackaberry, E.S., Palmer, D.S., Rock, G. and Ganz, P.R. DDAVPinduced release of von Willebrand factor from endothelial cells in vitro: the effects of plasma and blood cells Biochim. Biophys. Acta 1990; 1052: 63-70

Hassall, D.G., Martin, J.F. and Booth, R.F.G. Prostacyclin inhibits monocyteendothelial cell interactions Suppl. Circulation 1988; 79: 453

Hatch, F.T. and Lees, R.S. Practical methods for plasma lipoprotein analysis Adv. Lipid Res. 1968; 6: 1-68

Haudenschild, C.C. Pathogenesis of atherosclerosis-state of the art Cardiovascular drugs and therapy 1990; 4: 993-1004

Haudenschild, C.C., Cotran, R.S., Gimbrone, M.A. Jr. and Folkman, J. Fine structure of vascular endothelium in culture J. Ultrastruc. Res. 1975; 50: 22-32

Haudenschild, C.C., Zahniser, D., Folkman, J. and Klagsburn, M. Human vascular endothelial cells in culture: lack of response to serum growth factors Exp. Cell Res. 1976; 98: 175-183

Hauge, B. A cautionary note on the use of blot hybridization for RNA size determination Gene 1988; 1: 225-230

Haust, M.D. Pathogensis of atherosclerosis. In: Expanding Horizons in Atherosclerosis Research, Schlierf, G. and Mrl, H. (eds.) Springer-Verlag, Berlin, Heidelberg 1987; 3-12

Haust, M.D. Injury and repair in the pathogensis of atherosclerotic lesions. In: Atherosclerosis, Proceedings of the Second International Symposium, Jones, R.J. (ed.), Springer, NY, Heidelberg, Berlin. 1970; 12-20

Hayman, E.G., Pierschbacher, M.D. and Ruoslahti, E. Detachment of cells from culture substrate by soluble fibronectin peptides. J. Cell Biol. 1985; 100: 1948-1954

Haynes, S.S. Type A behavior, employment status, and coronary heart disease in women - a review. In: Myocardial Infarction in Women, 
Oliver, M.F., Vedin, A. and Wilhelmsson, C. (eds.), Churchill Livingstone, London 1986; 66-92

Hazzard W.R. Estrogen replacement and cardiovascular disease: serum lipids and blood pressure effects Am. J. Obstet. Gynecol. 1989; 161: 1847-1853 (b)

Hazzard, W.R. Atherosclerosis and Aging: a scenario in flux Am. J. Cardiol. 1989; 63: 20H-24H (b)

Hazzard, W.R. Biological basis of the sex diiferential in longevity J. Am. Geriatr. Soc. 1986: 34: 455-471

Heimark, R.L., Twardzik, D.R. and Schwartz, S.M. Inhibition of endothelial regeneration by type-beta transforming growth factor from platelets Science 1986; 233: 1078-1080

Heinecke, J.W. Free radical modification of low density lipoproteins: mechanisms and biological consequences Free Radic. Biol. Med. 1987; 3: 65-73

Heinecke, J.W., Rosen, H. and Chait, A. Iron and copper promote modification of LDL by human arterial smooth muscle cells in culture J. Clin. Invest. 1984; 74: 1890-1984

Heinecke, J.W., Baker, L., Rosen, H. and Chait, A. Superoxide-mediated modification of low density lipoprotein by arterial smooth muscle cells J. Clin. Invest. 1986; 77: 757-761

Heinecke, J.W., Rosen, H. and Chait, A. Iron and copper promote modification of low density lipoprotein by human arterial muscle cells in culture J. Clin. Invest. 1984; 74: 1890-1894

Heinle, H. and Liebich, H. The influence of diet-induced hypercholesterolemia on the degree of oxidation of glutathione in rabbit aorta Atherosclerosis 1980; 37: 637-640

Heldin, C.-H. and Westermark, B. PDGF-like growth factors in autocrine stimulation of growth J. Cell. Physiol. 1987; supp 5: 31-34

Heldin, C.-H, Westermark, B. and Wasteson, A. Chemical and biological properties of a growth factor from osteosarcoma cell: resemblance with platelet-derived growth factor J. Cell. Physiol. 1980; 105: 235-246

Heldin, C.-H, Westermark, B. and Wasteson, A. Platelet-derived growth factor. Purification and partial characterization Proc. Natl. Acad. Sci., USA 1979; 76: $3722-3726$

Heldin, C.-H., Westermark, B. and Wasteson, A. Specific receptors for plateletderived growth factors on cells derived from connective tissue and glia Proc. Natl. Acad. Sci.USA 1981; 78: 3664-3668

Heldin, N.-E., Gustavssen, B., Claesson-Welsh, L, Hammacker, A., Mark, J., Heldin, C.-H. and Westermark, B Aberrant expression of receptors for platelet-derived growth factor in an anaplastic thyroid carcinoma cell line. Proc. Natl. Acad. Sci. USA 1988; 85: 9302-9306 
Helgason, S., Damber, J.E., Damber, M.G., von Scoultz, B., Selstrom, G. and Sodergard, R. A comparative longitudional study on sex hormones binding globulin capacity during estrogen replacement therapy Acta Obstet. Gynecol. Scand. 1982; 61: 97-100

Helin, P., Lorenzen, I., Garbarsch, C. and Mattiesen, M.E. Arteriosclerosis in rabbit aorta induced by mechanical dilation: biochemical and morphological studies Atherosclerosis 1971; 13: 319-331

Heller, R.F. and Jacobs, H.S. Coronary heart disease in relation to age, sex and menopause BMJ 1978; 1: 472-474

Heller, R.F., Jacobs, H.S., Vermeulen, A. and Deslypere, J.P. Androgens, oestrogens and coronary heart disease BMJ 1981; 282: 438-439

Hennig, B. and Chow, C.K. Lipid peroxidation and endothelial cell injury: Implications in atherosclerosis Free Radic. Biol. Med. 1988; : 99-106

Hennig, B., Shasby, D.M. and Spector, A.A. Exposure to fatty acid increases human low density lipoprotein transfer across cultured endothelial monolayers Circ. Res. 1985; 57: 776-780

Henriksen, T., Evensen, S.A. and Carlander, B. Injury to human endothelial cells in culture induced by low density lipoproteins Scand. J. Clin. Lab. Invest. 1979; 39: 369-375 (a)

Henriksen, T., Evensen, S.A. and Carlander, B. Injury to cultured endothelial cells induced by low density lipoproteins: protection by high density lipoproteins Scand. J. Clin. Lab. Invest. 1979; 39: 369375 (b)

Henriksen, T., Evensen, S.A. and Carlander, B. Injury of endothelial cells in culture by low density lipoproteins Thromb. Haemost. 1977; 38: 138 (c)

Henriksen, T., Evenson, S.A. and Carlender, B. Injury of endothelial cells in culture induced by low density lipoproteins Int. Cong. Throm. Haematol. 1979 ; $1: 139$

Henriksen, T., Mahoney, E.M. and Steinberg, D. Enhanced macrophage degradation of LDL previously incubated with cultured endothelial cells: recognition by recepter for acetylated low density lipoproteins Proc. Natl. Acad. Sci. USA 1981; 78: 6499-6503

Henriksen, T., Mahoney, E.M. and Steinberg, D. Interactions of plasma lipoproteins with endothelial cells Ann. NY Acad. Sci. 1982; 401: 102-116 (a)

Henriksen, T., Mahoney, E.M. and Steinberg. D. Enhanced macrophage degradation of biologically modified low density lipoprotein Arteriosclerosis 1983; 3: 149-159 (b)

Henriksson, P., Stamberger, M., Eriksson, M., Rudling, M., Diczfalusy, U., Berglund, L. and Angelin, B. Oestrogen-induced changes in lipoprotein metabolism: role in prevention of atherosclerosis in the cholesterol fed rabbit Eur. J. Clin. Invest. 1989; 19: 395-403 
Henry, J. Mechanisms by which stress can lead to coronary heart disease Postgrad. Med. J. 1986; 62: 687-693

Hermansson, M., Nister, M., Betsholtz, C., Heldin, C.-H., Westermark, B. and Funa, K. Endothelial cell hyperplasia in human glioblastoma: coexpression of mRNA for platelet-derived growth factor (PDGF) $B$ chain and PDGF receptor suggests autocrine growth stimulation Proc. Natl. Acad. Sci. USA 1988; 85: 7748-7752

Hessel, L.W. Combined oral contraceptive: from risk to benefit?. In: Expanding Horizons in Atherosclerosis Research, Schlierf, G. and Mrl, $\mathrm{H}$. (eds.), Springer-Verlag, Berlin, Heidelberg 1987; 161-164

Hessler, J.R., Morel, D.W., Lewis, L.J. and Chisolm, G.M. Lipoprotein oxidation and lipoprotein-induced cytotoxicity Arteriosclerosis 1983; 3: 215-222

Hessler, J.R., Robertson, A.L. Jr. and Chisolm, G.M. LDL induced cytotoxicity and its inhibition by HDL in human vascular smooth muscle and endothelial cells in culture Atherosclerosis 1979; 32: 213-229

Higuchi, Y. Lipid peroxides and alpha-tocopherol in rat streptozotocin-induced diabetes mellitus Acta. Med. Okayama 1982; 36: 165-175

Hiramatsu, K., Rosen, H., Heinecke, J.W., Wolfbauer, G. and Chait, A. Superoxide initiated oxidation of low density lipoproteins by human monocytes Arteriosclerosis 1987; 7: 55-58

Hirsch, L.J. and Mazzone, T. Dexamethasone modulates lipoprotein metabolism in cultured human monocyte-derived macrophages. Stimulation of scavenger receptor activity J. Clin. Invest. 1986; 77: 485-490

Hjortland, M.C., McNamara, P.M. and Kannel, W.B. Some atherogenic concomitants of menopause: the Framingham study Am. J. Epidemiol. 1976; 103: 304-311

Hoff, H.F. and Gaubatz, J.W. Isolation, purification, and characterization of a lipoprotein containing $A$ poB from the human aorta Atherosclerosis 1982; 42: 273-297

Hoff, H.F. and Morton, R.E. Lipoproteins containing apoB extracted from human aortas: structure and function Ann. NY Acad. Sci. 1985; 454: 183-194

Hoff, H.F., O'Neil, J., Chisolm III, G.M., Cole, T.B., Quenhenberger, O., Esterbauer, H. and Jurgens, G. Modification of low density lipoprotein with 4-hydroxynoneral induces uptake by macrophages Arteriosclerosis 1989; 9: 538-549

Holland, J.A., Pritchard, K.A., Rogers, W.J. and Stemerman, M.B. Pertubation of cultured human endothelial cells by atherogenic levels of LDL Am. J. Pathol. 1988; 132: 474-478

Hollenberg, S.M., Weinberger, C., Ong, E.S., Cerelli, G., Oro, A., Lebo, R., Thompson, E.B., Rosenfeld, M.G. and Evans, R.M. Primary structure and expression of a functional human glucocorticoid receptor cDNA Nature 1985; 318: 635-641 
Hong, S.L. Effect of bradykinin and thrombin on prostacyclin synthesis in endothelial cells from calf and pig aorta and human umbilical cord vein Thromb. Res. 1980; 18: 787-795

Hope, W., Martin, T.J., Chesterman, C.N. and Morgan, F.J. Human betathromboglobulin inhibits PGI2 production and binds to a specific site in bovine aortic endothelial cells Nature 1979; 282: 210-212

Hopkins, P.N. and Williams, R.R. Identification and relative weight of cardiovascular risk factors Cardiol. Clin. 1986; 4: 3-31

Hopkins, P.N. and Williams, R.R. A survey of 246 suggested coronary risk factors Atherosclerosis 1981; 40: 1-52

Horwitz, K.B. and Horwitz, L.D. Canine vascular tissues are targets for androgens, estrogens, progestins and glucocorticoids J. Clin. Invest. 1982; 69: 750-758

Houdijk, W.P.M., de Groot, P.G., Nievelstein, P.F.E.M., Sakariassen, K.S. and Sixma, J.J. ubendothelial proteins and platelet adhesion Arteriosclerosis 1986; 6: 24-33

Hough, J.F. and Silversmith, D.B. Effect of $17 \beta$ - estradiol on aortic cholesterol content and metabolism in cholesterol-fed rabbits Arteriosclerosis 1986; 6: 57-63

Howard, M.A. and Firkin, B.G. Ristocetin-a new tool in the investigation of platelet aggregation Thromb. Diathesis Haem. 1971; 2: 586588

Hoyer, L.M. de los, Santos, R.P. and Hoyer, J.R. Antihemophilic factor antigen localization in endothelial cells by immunofluorescent microscopy J. Clin. Invest. 1973; 52: 2737-2744

Hsu, S.-M., Raine, L. and Fanger, H. Use of avidin-biotin-peroxidase complex $(A B C)$ in immunoperoxidase techniques J. Histochem. Cytochem. 1981; 29 577-580

Hu, S.K., Mitcho, Y.L. and Rath, N.C. Effect of estradiol on interleukin-1 synthesis by macrophages Int. J. Immunopharmacol. 1988; 10: 247-252

Huang, N., Lineberger, B. and Steiner, M. Alpha-tocopherol, a potent modulator of endothelial cell function Thromb. Res. 1988; 50: 547-557

Huber, L.A., Scheffler, E., Poll, T., Ziegler, R. and Dresel, H.A. 17 Beta-estradiol inhibits LDL oxidation and cholesteryl ester formation in cultured macrophages Free Radic. Res. Commun. 1990; 8: 167-173

Hull, R.W., Hasbargen, J.A., Fall, S. and O'Barr, T.P. Conjugated estrogens reduce endothelial prostacyclin production and fail to reduce post by pass blood loss Chest 1991; 99: 1116-1119

Hunt, K., Vessey, M. and McPherson, K. Mortality in a cohort of long-term users of hormone replacement therapy Br. J. Obstet. Gynaecol. 1990; 97: 1080-1086 
Ingegno, M.D., Money, S.R., Thelmo, W., Greene, G.L., Davidian, M., Jaffe, B.M. and Pertschuk, L.P. Progesterone receptors in the human heart and great vessels Lab. Invest. 1988; 59: 353-356

Inman, W.H.W. and Vessey, M.P. Oral contraceptives and thrombosis BMJ 1968; 2: 193-199

Inman, W.H.W., Vessey, M.P., Westerholm, B. and Engelund, A. Thromboembolic disease and the steroidal content of oral contraceptives - a report to the committee on safety of drugs BMJ 1970; 2: 203-209

Ishii, Y., Shinoda, M. and Shikita, M. Specificty of the suppressive action of glucocorticoids on the proliferation of monocyte/macrophages in the CSF-stimulated cultures of mouse bone marrow Exp. Haematol. 1983; 11: 178-186

Ishikawa, F., Miyazono, K., Hellman, U., Drexler, H., Wernstedt, C., Hagiwa, G.K., Usuki, K., Takaku, F., Risau, W. and Heldin, C.-H. Identification of angiogenic activity and the cloning and expression of platelet-derived endothelial cell growth factor Nature 1989; 338: 557-562

Ishiwata, I., Nozawa, S and Okumura, H. Effects of $17 \beta$-estradiol and progesterone on growth and morphology of human endometrial carcinoma cells in vitro Cancer Res. 1977; 37: 4246-4249

Jaffe, E. A., Nachman, R .L., Becker, C.G. and Minick, C.R. Culture of human endothelial cells derived from umbilical veins J. Clin. Invest. 1973; 52: 2745-2756 (a)

Jaffe, E.A., Hoyer, L.W. and Nachman, R.L. Synthesis of antihemophilic factor antigen by cultured human endothelial cells J. Clin. Invest. 1973; 52: 2757-2764 (b)

Jaffe, E.A. Culture and identification of large vessel endothelial cells In: Biology of Endothelial Cells, Jaffe, E.A. (ed.), Martinus Nijhoff Publishers, Boston 1984; : 1-13

Jaffe, E.A. Endothelial cells and the biology of factor VIII N. Engl. J. Med. 1977; 296: 377-383

Jaffe, E.A. Physiologic functions of normal endothelial cells Ann. NY Acad. Sci. 1985; 454: 279-291

Jaffe, E.A. Synthesis of factor VIII by endothelial cells. In: Biology of Endothelial Cells, Jaffe, E.A. (ed.), Martinus Nijhoff Publishers, Boston 1984; : 209-214

Jaffe, E.A., Hoyer, L.W. and Nachman, R.L. Synthesis of von Willebrand factor by cultured human endothelial cells Proc. Natl. Acad. Sci., USA 1974; 71: 1906-1909

Jaffe, E.A., Nachman, R.L. and Becker, C.G. Culture of human endothelial cells derived from umbilical cord veins J. Clin. Invest. 1972; 51: 46A (a) 
Jaffe, E.A., Nachman, R.L., Becker, C.G. and Minick, C.R. Culture of human endothelial cells derived from human umbilical cord veins Circ. 1972 197; 46 (Suppl. II): 252 (b)

Jaye, M., Howk,R., Burgess, W.H., Ricca, G.A., Chiu. I.-M., Ravera, M.W., O'Brien, S.J., Modi, W.S., Maciag, T. and Drohan, W.N. Human endothelial cell growth factor: cloning nucleotide sequence, and chromosome localization Science 1986; 233: 541-545

Jaye, M., McConathy, E., Drohan, W., Tong, B., Deuel, T. and Maciag, T. Modulation of the sis gene transcript during endothelial differentiation in vitro Science 1985; 22: 882-885

Jeltsch, J.M., Krozowski, Z., Quirin-Stricker, C., Gronemeyer, H., Simpson, R.J., Garnier, J.M., Krust, A., Jacob, F. and Chambon, P. Cloning of the chicken progesterone Proc. Natl. Acad. Sci. USA 1986; 83: 5424-5428

Jensen, E.V. and de Sombre, E.R. Estrogenic hormones effect transformation of specific receptor proteins to a biochemically functional form Science 1973; 182: 126-134

Jensen, E.V., Suzuki, T., Kawashima, T., Stumpf, W.E., Jungblut, P.W. and de Sombre, E.R. A two-step mechanism for the interaction of estradiol with rat uterus Proc. Natl, Acad. Sci., USA 1968; 59: 632-638

Jensen, J. and Christiansen, C. Dose-response effects on serum lipids and lipoproteins following combined oestrogen-progestogen therapy in post-menopausal women Maturitas 1987; 9: 259-266

Jerome, W.G. and Lewis, J.C. Early atherogenosis in white corneau pigeons: I leucocyte margination and endothelial alterations at the celiac bifurcation Am. J. Pathol. 1984; 116: 56-68

Jessup, W., Rankin, S.M., de Whalley, C.V., Hoult, J.R.S., Scott, J. and Leake, D.S. a-tocopherol consumption during low-density-lipoprotein oxidation Biochem. J. 1990; 2665: 399-405

Jialal, I. and Grundy, S.M. Preservation of the endogeneous antioxidants in low density lipoprotein by ascorbate but not probucol during oxidative modification J. Clin. Invest. 1991; 87: 597-601

Jick, H., Dinan, B. and Rothman, K.J. Noncontraceptive estrogens and nonfatal myocardial infarction JAMA 1978; 239: 1407-1408

Jick, H., Dinan, B., Herman, R. and Rothman, K.J. Myocardial infarction and other vascular diseases in young women JAMA 1978; 240: 2548-2552

Jick, H., Slone, D., Westerholm, B., Inman, W.H.W., Vessey, M.D., Shapiro, S., Lewis, G.P. and Worcester, J. Venous thromboembolic disease and $A B O$ blood type: a cooperative study Lancet 1969; 1: 539542

Johannisson, E. Effects of oestradiol and progesterone on the synthesis of DNA and the anti-haemophilic factor VIII antigen in human endometrial 
endothelial cells in vitro: a pilot study Human Reprod. 1986; 1: 207-212

Johansson, S., Vedin, A. and Wilhelmsson, C. Myocardial infarction in Gteborg, Sweden. A community study of mortality and incidence, 1968-1977 Am. J. Epidemiol. 1984; 119: 603-609

Johnson, A.R., Revtyak, G. and Campbell, W.B. Arachidonic acid metabolites and endothelial injury: studies with cultures of human endothelial cells Fed. Proc. 1985; 44: 19-24

Johnson, A.R. Human pulmonary endothelial cells in culture: activities of cells from arteries and cells from vieins J. Clin. Invest. 1980; 65: 841850

Johnson, M., Ramey, E. and Ramwell, P.W. Sex differences in human platelet aggregation Nature 1975; 253; 355-357

Johnson, M., Ramey, E. and Ramwell, P.W. Androgen-mediated sensitivity in platelet aggregation Am. J. Physiol. 1977; 232: H381-H385

Johnsson, A., Heldin, C.-H, Wasteson, A., Westermark, B., Deuel, T.F., Huang, J.S., Seeburg, D.H., Gray, E., Ullrich, A., Scrace, G., Stroobant, P. and Waterfield, M.D. The $C$-sis gene encodes a precursor of the $B$ chain of platelet-derived growth factor EMBO J. 1984; 3: 921928

Joris, I.Z. and T., Nunnari, J.J., Krolikowski, F.J. and Majno, G. Studies on the pathogenesis of atherosclerosis: adhesion and emigration of mononuclear cells in the aorta of hypercholesterolemic rats Am. J. Pathol. 1983; 113: 341-358

Joseph-Silverstein, J. and Rifkin, D. Endothelial cell growth factors and the vessel qalls Semin. Thromb. Hemost. 1987; 13: 504-513

Jozan, S., Faye, J.C., Tournier, J.F., Tauber, J.-P., David, J.F. and Bayard, F. Interaction of estradiol and high density lipoproteins on proliferation of the human breast cancer cell line MCF-7 adapted to grow in serum free conditions Biochem. Biophys. Res. Commun. 1985; 133: 105-112

Judd, H.L., Judd, G.E., Lucas, W.F. and Yen, S.S.C. Endocrine function of the postmenopause ovary J. Clin. Endocrinol. Metab. 1974; 39: 1020-1024

Jullien, G., Gichtenaere, J.C. and Gerard, R. Coronary insufficiency in the female: possible effect of menopause Nouv. Presse. Med. 1977; 6: 1125-1128

Jurgens, G., Ashy, A. and Esterbauer, H. Detection of new epitopes formed upon oxidation of low-density lipoprotein, lipoprotein (a) and very low-density lipoprotein Biochem. J. 1990; 265: 605-608

Kalin, M.F. and Zumoff, B. Sex hormones and coronary disease : a review of clinical studies Steroids 1990; 55: 330-352 
Kalinyak, J.E., Dorin, R.I., Hoffman, A.R. and Perlman, A.J. Tissues-specific regulation of glucocorticoid receptor $m R N A$ by dexamethasone J. Biol. Chem. 1987; 262: 10441-10444

Kamp, D.W., Bauer, K.D., Rubin, D.B. and Dunn, M.M. Tumor promoting phorbol esters inhibit monocyte adherence to endothelial cells $\mathrm{J}$. Appl. Physiol. 1989; 66: 437-442

Kannel, W.B. Metabolic risk factors for coronary heart disease in women Am. Heart J. 1987; 114: 413-419

Kannel, W.B., Hjortland, M.C. and McNamara, P. Menopause and risk of cardiovascular disease: Framingham study Ann. Intern. Med. 1976; 85: 447-452

Kannel, W.B. Coronary risk factors. II. Prospects for prevention of atherosclerosis in the young Aust. NZ J. Med. 1976; 6: 410-419

Kannel, W.B. Metabolic risk factors for coronary heart disease in women: perspective from the Framingham study Am. Heart J. 1987; 114: 413-419

Kannel, W.B. and Abbott, R.D. Incidence and prognosis of myocardial in farction in women: the Framingham study In: Coronary Heart Disease in Women. Proceedings of an NIH workshop Wenger, N.K. (ed) N.Y., Haymarket, Doyma 1987; 208-214

Kannel, W.B. and Sytkowski, P.A. Atherosclerosis risk factors Pharmac. Ther. 1987; 32: 207-235

Kannel, W.B., Castell, W.P. and Gordon, T. Cholesterol in the prediction of atherosclerotic disease: new perspectives based on the Framingham study Ann. Intern. Med. 1979; 90: 85-91

Kannel, W.B., Doyle, J.T. and Ostfeld, A.M. Optimal resources for primary prevention of atherosclerotic disease Circulation 1984; 70: 157A-205A

Kao, K-J., Pizzo, S.V. and McKee, P.A. Demonstration and characterization of specific binding sites for factor VIII/von Willebrand factor on human platelets J. Clin. Invest. 1979; 63: 656-664

Kaplan, D.R., Chao, F.C., Stiles, C.D., Antoniades, H.N. and Scher, C.D. Platelet a granules contain a growth factor for fibroblasts Blood 1979; 53: 1043-1052

Kaplan, J.E., Moon, D.G. Weston, L.K., Minnear, F.L., Del Vecchio, P.J., Shepard, J.M. and Fenton II, J.W. Platelets adhere to thrombintreated endothelial cells in vitro Am. J. Physiol. 1989; 257: H423-H433

Kaplan, J.R., Adams, M.R., Clarkson, T.B. and Koritnik, D.R. Psychosocial influences on female 'protection' among cynomoglus macaques Atherosclerosis 1984; 53: 283-295

Kardutsch, A.A., Chen, H.W. and Heiniger, H.J. Biological activity of some oxygenated sterols Science 1978; 201: 498-501 
Karpati, L., Chow, F.P.R., Woollard, M.L., Hutton, R.A. and Dandona, P. Prostacyclin-like activity in the female rat thoracic aorta and the inferior vena cava after ethinyloestradiol and norethisterone Clin. Sci. 1980; 59: 369-372

Katzenellenbogen, B.S. Dynamics of steroid hormone recepter action Ann. Rev. Physiol. 1980; 42: 17-35

Katzenellenbogen, B.S and Norman, M.J. Multihormonal regulation of the progesterone receptor in MCF-7 human breast cancer cells: interrelationships among insulin/insulin-like growth factor-l, serum and estrogen Endocrinology 1990; 126: 891-898

Kaushansky, K. Control of granulocyte-macrophage colony-stimulating factor production in normal endothelial cells by positive and negative regulatory elements J. Immunol. 1989; 143: 2525-2529

Kawakami, M., Ishibashi, S., Ogawa, H., Murase, T.; Takaku, F. and Shibata, S. Cachectin/TNF as well as interleukin-1 induces prostacyclin synthesis in cultured vascular endothelial cells Biochem. Biophys. Res. Commun. 1986; 141: $482-487$

Kazlauskas, A. and DiCorleto, P.E. Cultured endothelial cells do not respond to platelet-derived growth factor like protein in an autocrine manner Biochem. Biophys. Acta 1985; 846: 405-412

Keck, J.P., Hausen, S.D., Krivi, G., Sanzo, K., Warren, T., Feder, J. and Connolly, D.T Vascular permeability factor, on endothelial cell mitogen related to PDGF Science 1989; 246: 1309-1312

Keizer, G.P., Te Velde, A.A., Schwarting, R., Figdor, C.G. and de Vries, J.E. Role of p150, 95 in adhesion migration, chemotoxis and phagocytosis of human monoctyes Eur. J. Immunol. 1987; 9: 1317-1322

Keller, R., Silbert, J.E., Furthmayer, H. and Madri, J.A. Aortic endothelial cell proteoheparan sulphates: isolation and characterization of plasma membrane-associated and extracellular species Am. J. Pathol. 1987; 128: 286-298

Kelly, K., Cochran, B.H., Stiles, C.D. and Leder, P. Cell-specific regulation of the $c$-myc gene by lymphocyte mitogens and platelet-derived growth factor Cell 1983; 35: 603-610

Kim, R.S. and LaBella, F.S. Comparison of analytical methods for monitoring autoxidation profiles of authentic lipids J. Lipid Res. 1987; 28: 1110-1117

Kirkeby, K., Ingvaldsen, P. and Bjerkedal, I. Fatty acid composition of serum lipids in men with myocardial infarction Acta Med. Scand. 1972; 192: $513-519$

Kishi, Y. and Numano, F. In vitro study of vascular endothelial injury by activiated platelets and its prevention Atherosclerosis 1989; 76: 95-101

Kita, T., Nagano, Y., Yokode, M., Ishii, K., Kume, N., Ooshima, A., Yoshida, H. and Kawai, C. Probucol prevents the progression of atherosclerosis in Watanabe heritable hyperlipidemic rabbit, an 
animal model for familial hypercholesterolemia Proc. Natl. Acad. Sci., USA 1987; 84: 5928-5931

Klagsbrun, M. and Edelman, E.R. Biological and biochemical properties of fibroblast growth factors: implications for the pathogenesis of atherosclerosis Arteriosclerosis 1989; 9: 269-278

Klaiber, E.L., Broverman, D.M., Haffajee, C.I., Hochman, J.S., Sacks, G.M. and Dolen, J.E. Serum estrogen levels in men with acute myocardial infarction Am. J. Med. 1982; 73: 872-881

Klimov, A.N., Zubzhitsky, Y.N. and Nagornev, V.A. Immunochemical aspects of atherosclerosis. In: Atherosclerosis Reviews, Paoletti, R. and Gotto, A.M. (eds.), Raven Press Ltd, NY 1979; 4: 119-156

Knight, J.A., Pieper, R.K. and McCiellan, L. Specificity of the thiobarbituric acid reaction. Its use in studies of lipid peroxidation Clin. Chem. 1988; 34: 2433-2438

Knopp, R.H., Walden, C.E., Wahl, P.W., Hoover, J.J., Warnick, G.R., Albers, J.J., Ogilvie, J.T. and Hazzard, W.R. Oral contraceptive and postmenopausal estrogen effects on lipoprotein triglyceride and cholesterol in an adult female population: relationships to estrogen and progestin potency J. Clin. Endocrinol. Metab. 1981; 53: 1123-1132

Knopp, R.H., Walden, C.E., Wahl, P.W. and Hoover, J.J. Effects of oral contraceptives on lipoprotein triglyceride and cholesterol: relationships to estrogen and progestin potency Am. J. Obstet. Gynecol. 1982; 142: 725-731

Kooistia, T., Bosma, P.J., Jespersen, J. and Kluft, C. Studies on the mechanism of action of oral contraceptives with regard to fibrinolytic variables Am. J. Obstet. Gynecol. 1990; 163: 404-413

Kosugi, K., Morel, D.W., DiCorleto, P.E. and Chisolm, G.M. Toxicity of oxidized low-density lipoprotein to cultured fibroblasts is selective for $S$ phase of the cell cycle J. Cell. Physiol. 1987; 130: 311-320

Kovanen, P.T., Brown, M.S. and Goldstein, J.L. Increased binding of low density lipoprotein to liver membrances from rats treated with $17 \alpha$-ethinyl estradiol J. Biol. Chem. 1979; 254: 11367-11373

Kruth, H.J., Avigan, J., Gamble, W. and Vaughan, M. Effect of cell density on binding and uptake of low density lipoprotein by human fibroblasts J. Cell Biol. 1979; 83: 588-594

Kushwaha, R.S., Lewis, D.S., Carey, K.D. and McGill, H.C. Jr. Effects of estrogen and progesterone on plasma lipoproteins and experimental atherosclerosis in the baboon (Papio sp.) Arteriosclerosis Thromb. 1991; 11: 23-31

Kuzuya, M., Naito, M., Funaki, C., Hayashi, T., Asai, K. and Kuzuya, F. Lipid peroxide and transition metals are required for the toxicity of oxidized low density lipoprotein to cultured endothelial cells Biochim. Biophys. Acta 1991; 1096: 155-161 
Kuzuya, M., Naito, M., Funaki, G., Hayashi, T., Asai, K. and Kuzuya, F. Protective role of intracellular glutathione against oxidized low density lipoprotein in cultured endothelial cells Biochem. Biophys. Res. Commun. 1989; 163: 1466-1472

Kuzuya, M., Naito, M., Yamada, K., Funaki, C., Hayashi, T., Asai, K. and Kuzuya, $\mathrm{F}$. Involvement of intracellular iron in the toxicity of oxidized low density lipoprotien to cultured endothelial cells Biochem. Internat. 1990; 22: 567-574

La Vecchia, C., Decarli, A., Franceschi, S., Gentile, A., Negri, E. and Parazzini, F. Menstrual and reproductive factors and the risk of myocardial infarction in women under fifty-five years of age Am. J. Obstet. Gynecol. 1987; 157: 1108-1112

Laemmli, U.K. Cleavage of structural proteins during the assembly of the head of bacteriophage T4 Nature 1970; 227: 680-685

Lafferty, F.W. and Helmuth, D.O. Post-menopausal estrogen replacement: the prevention of osteroporosis and systemic effects Maturitas 1985; 7: 147-159

Lahoua, Z., Astruc, M.E. and Crastes de Paulet, A. Serum-induced arachidonic add release and prostaglandin biosynthesis are potentiated by oxygenated serols in NRK $49 \mathrm{~F}$ cells Biochem. Biophys. Acta 1988; 958: 396-404

Landolfi, R. and Steiner, M. Ethanol raises prostacyclin in vivo and in vitro Blood 1984; 64: 679-689

Landolfi, R., Castellano, M.A.C., de Cristofaro, R., de Candia, E. and Bizzi, B. Effect of picotamide on prostacyclin production by human endothelial cells Thromb. Haemost. 1988; 60: 529

Lansing, A.I., Alex, M. and Rosenthal, T.B. Calcium and elastin in human arteriosclerosis J. Gerontol. 1950; 5: 112-119

Larocca, L.M., Piantelli, M., Leone, G., Sica, S., Teofili, L., Panici, P.B., Scambia, G., Mancuso, S., Capelli, A. and Ranelletti, F.O. Type II oestrogen binding sites in acute lymphoid and myeloid leukaemias: growth inhibitory effect of oestrogen and flavonoids $\mathrm{Br}$. J. Haematol. 1990; 75: 489-495

Lapidus, L., Bengtsson, C., Pennert, K., Rybo. E. and Sjostrom, L. Distribution of adipose tissue and risk of cardiovascular disease and death: $A 12$ year follow up of participants in the population study of women in Gothenburg, Sweden BMJ 1984; 289: 1257-1261

Larsson, B., Svardsudd, K., Welin, L. Wilhelmen, L. Bjorntorp, P. and Tibblin, G. Abdominal adipose tissue distribution, obesity, and risk of cardiovascular disease and death 13 year follow up of the participants on the study of men born in 1913 BMJ 1984; 288: 1401-1404

Last, J.M. (ed.) A dictionary of epidemiology Oxford University Press, NY 1983; : 
Laurell, C.-B. Quantitative estimation of proteins by electrophoresis in agarose gel containing antibodies Analyt. Biochem. 1966; 15: 45-52

Lee, D.M. Malondialdehyde formation in stored plasma Biochem. Biophys. Res. Commun. 1980; 95: 1663-1672

Lefer, A.M. and Sedar, A.W. Endothelial alterations in hypercholesterolaemia and atherosclerosis Pharmacol. Res. 1991; 23: 1-12

Leone, G., Moneta, E. and Paparatti, G. Von Willebrand's disease in pregnancy N. Engl. J. Med. 1975: 293: 456

Leszczynski, D.E. and Schafer, R.M. Metabolic conversion of six steroid hormones by human plasma high-density lipoprotein Biochim. Biophys. Acta 1991; 103: 18-28

Leszczynski, D. and Kakatnur, M. Serum sex hormones binding globulin and high density lipoprotein cholesterol in male patients at risk for Coronary Heart Disease Med. Sci. Res. 1987; 15: 575-576

Leung, D.W., Cachlanes, G., Kuang, W.-J., Goeddel, D.V. and Ferrara, N. Vascular endothelial growth factor is a secreted angiogenic mitogen Science 1989; 246: 1306-1309

Levene, C.I. and Poole, J.C.F. The collagen content normal and atherosclerotic human aortic intima Br. J. Exp. Pathol. 1962; 43: 469-471

Levin, R.I., Weksler, B., Marcus, A. and Jaffe, E.A. Prostacyclin production by endothelial cells. In: Biology of Endothelial Cells, Jaffe, E.A. (ed.), Martinus Nijhoff Publishers, Boston 1984; : 228-247

Levine, J.D., Harlan, J.M., Harker, L.A., Joseph, M. and Counts, R.B. Thrombin induced release of factor VIII antigen from human endothelial cells Blood 1981; 58: 235a

Levine, J.D., Harlan, J.M., Harker, L.A., Joseph, M.L. and Counts, R.B. Thrombin-mediated release of factor VIII antigen from human umbilical vein endothelial cells in culture Blood 1982; 60: 531534

Levy, D., Wilson, P.W.F., Anderson, K.M. and Castelli, W.P. Stratifying the patient at risk from coronary disease: new insights from the Framingham Heart Study Am. Heart J. 1990; 119: 712-717

Lewis, G.D., Campbell, W.B. and Johnson, A.R. Inhibition of prostaglandin synthesis by glucocorticoids in human endothelial cells Endocrinology 1986; 119: 62-69

Lewis, J.C. and Kottke, B.A. Endothelial damage and thrombocyte adhesion in pigeon atherosclerosis Science 1977; 196: 1007-1009

Lewis, J.C., Taylor, R.G., Jones, N.D., St Clair, R.W. and Cornhill, J.F. Endothelial surface characteristics in pigeon coronary artery atherosclerosis. I. Cellular alterations during the initial stages of dietry cholesterol challenge Lab. Invest. 1982; 46: 123-138

Lewis, L.A. and Page, I.H. An unusual serum lipoprotein-globulin complex in a patient with hyperlipemia Am. J. Med. 1965; 38: 286-297 
Liao, F., Berliner, J.A., Mehrabian, M., Navab, M., Demer, L.L., Lusis, A.J. and Fogelman, A.M. Minimally modified low density lipoprtoein is biologically active in vivo in mice J. Clin. Invest. 1991; 87: 22532257

Liebovich, S.J. and Ross, R. A macrophage-dependent factor that stimulates the proliferation of fibroblasts in vitro Am. J. Pathol. 1976; 84: 501513

Liehr, J.G. and Roy, D. Free radical generation by redox cycling of estrogens Free Radic. Biol. Med. 1990; 8: 415-439

Lim, Y.L., Lumbers, E.R., Walters, W.A.W. and Whelan, R.F. Effects of oestrogens on the human circulation Br. J. Obstet. Gynaecol. 1970; 77: 349-355

Lin, A L., McGill, H.C. Jr. and Shain, S.A. Hormone receptors of the baboon cardiovascular system Atherosclerosis 1981; 1: 257-264

Lin, A.L., Gonzalez, R. Jr., Carey, K.D. and Shain, S.A. Estradiol-17 $\beta$ effects estrogen receptor distribution and elevates progesterone receptor content in baboon aorta Arteriosclerosis 1986; 6: 495-504

Lin, A.L., McGill, H.C. Jr. and Shain, S.A. Hormone receptors of the baboon cardiovascular system Circ. Res. 1982; 50: 610-616

Lin, S-J., Jan, K.M., Weinbaum, S. and Chien, S. Transendothelial transport of low density lipoprotein in association with cell mitosis in rate aorta Arteriosclerosis 1989; 9: 230-236

Lindberg, U.B., Crona, L., Stigendal, L., Teger-Nilsson, A.C., Silfverstople, G. A comparison between effects of estradiol valerate and low dose ethinyl estradiol on haemostasis parameters Thromb. Haemost. 1989; 61: 65-69

Linder, D. and Gartler, S.M. Glucose-6-phosphate dehydrogenase mosaicism: ultilization as a cell marker in the study of leiomyomas Science 1965; 150: 67-68

Lindner, V., Reidy, M.A. and Fingerle, J. Regrowth of arterial endothelium Lab. Invest. 1989; 61: 556-563

Lingham, R.B., Stancel, G.M. and Loose-Mitchell, D.S. Estrogen regulation of epidermal growth factor receptor messenger ribonucleic acid Mol. Endocrinol. 1988; 2: 230-235

Loesberg, C., Gonsalves, M.D., Zandbergen, J., Willems, C., van Aken, W.G., Stel, H.V., van Mourik, J.A. and de Groot, P.G. The effect of calcium on the secretion of factor VIII-related antigen by cultured human endothelial cells Biochem. Biophys. Acta 1983; 76: 160-168

Lonchampt, M.O., Romquin, N., Bonne, C. and Regnault, F. The effect of angiotensin II on PGI(2) production by endothelial cells in culture Thromb. Haemost. 1981; $46: 39$ 
Longcope, C., Kato, T. and Horton, R. Conversion of blood androgens to estrogens in normal adult men and women J. Clin. Invest. 1969; 48: 2191-2201

Lonning, P.E., Dowsett, M., Schem, J.B., Hardy, J. and Powles. T.J. Lack of diurnal variation in plasma levels of androstenedione, testosterone, estrone and estradiol in postmenopausal women $\mathbf{J}$. Steroid Biochem. 1989; 34: 551-553

Lowry, O., Rosebrough, Farr, A. L. and Randall, R. J. Protein measurement with the folin phenol reagent J. Biol. Chem. 1951; 193: 265-275

Luria, M. H., Johnson, M. W., Pego, R., Seue, C.A., Manuben, S.J., Weiland, M.R. and Weiland, R.G. Relationship between sex hormones, myocardial infarction and occlusive coronary disease Arch. Inter. Med. 1982; 142: 42-44

Maca, R.D., Fry, G.L. and Hoak, J.C. The effect of glucocorticoids on cultured human endothelial cells Br. J. Haematol. 1978; 38: 501-509

Macarak, E.J., Kirby, E., Kirk, T. and Kefalides, N.A. Synthesis of cold-insoluble globulin by cultured calf endothelial cells Proc. Natl. Acad. Sci., USA 1978; 75: 2621-2625

Machlin, L. and Bendich, A. Free radical tissue damage: protective role of antioxidant nutrients FASEB J. 1987; 1: 441-445

Malczak, H.T. and Buck, R.C. Regeneration of endothelium in rat aorta after local freezing. A scanning electron microscopic study Am. J. Pathol. 1977; 86: 133-148

Maciag, T., Cerundolo, J., llsley, S., Kelley, P.R. and Forard, R. An endothelial cell growth factor from bovine hypothalamus: identification and partial characterization Proc. Natl. Acad. Sci., USA 1979; 76: 5674-5678

Maciag, T., Hoover, G.A. and Weinstein, R. High and low molecular weight forms of endothelial cell growth factor J. Biol. Chem. 1982; 257: 5333-5336

Maciag, T., Hoover, G.A., Stemerman, M.B. and Weinstein, R. Factors which stimulate the growth of human umbilical vein endothelial cells in vitro, In: Biology of Endothelial Cells, Jaffe, E.A. (ed.), Martinus Nijhoff Publishers, Boston 1984; 87-96

Mackintosh, D. and Mason, R.M. Pharmacological actions of 17 $\beta$-estradiol on articular cartilage chondrocytes and chonrosarcoma chondrocytes in the absence of estrogen receptors Biochim. Biophys. Acta 1988; 964: 295-302

Mader, S., Kumar, V., de Verneuil, H. and Chambon, P. Three amino acids of the estrogen receptor are essential to its ability to distinguish an estrogen from a glucocorticoid-responsive element Nature 1989; 338: $271-274$

Mahley, R.W. Development of accelerated atherosclerosis concepts derived from cell biology and animal studies Arch. Pathol. Lab. Med. 1983; 107: 393-399 
Mahley, R.W. Atherogenic hyperlipoproteinemia: the cellular and molecular biology of plasma lipoproteins altered by dietary fat and cholesterol Med. Clin. North. Am. 1982; 66: 375

Mahley, R.W., Innerarity, T.L., Weisgraber, K.H. and On, S.Y. Altered metabolism (in vivo and vitro) of plasma lipoprotein after selective chemical modification of lysine residues of apoprotein J. Clin. Invest. 1979; 64: 743-750

Makila, U.-M., Wahlberg,L., Viinikka, L. and Ylikorkala. O. Regulation of prostacyclin and thromboxane production by human umblicial vessels: the effect of estradiol and progesterone in a superfusion model Prostaglandins Leuk. Med. 1982; 8: 115-124

Malden, L.T., Chait, A., Raines, E.W. and Ross, R. The influence of oxidatively modified low density lipoprotein on expression of platelet-derived growth factor by human monocyte-derived macrophages J. Biol. Chem. 1991; 266: 13901-13907

Maniatis, T., Fritsch, E. F. and Sambrook, J. In: Molecular Cloning: A Laboratory Manual, Cold Spring Harbor Laboratory 1982; $202-$ $203 ; 383$

Mann, J.I. and Inman, W.H.W. Oral contraceptives and death from myocardial infarction BMJ 1975; 2: 245-248 (b)

Mann, J.I., Thorogood, M., Waters, W.E., Powell, C. Oral contraceptives and myocardial infarction in young women: a further report BMJ 1975; 3: 631-632

Mann, J.I., Vessey, M.P., Thorogood, M. and Doll, R. Myocardial infarction in young women with special reference to oral contraceptive practice BMJ 1975; 2: 241-245 (a)

Mannucci, P.M., Pareti, F.I., Ruggeri, Z.M. and Capitanio, A. 1-deamino-8-Darginine vasopressin: a new pharmocological approach to the management of hemophilia and von Willibrand's disease Lancet 1977; 1: 869-872

Manson, J.E., Colditz, G.A., Stampfer, M.J., Willett, W.C., Rosner, B., Monson, R.R., Speizer, F.E. and Hennekens, C.H. A prospective study of obesity and risk of coronary heart disease in women $\mathbf{N}$. Engl. J. Med. 1990; 322: 882-889

Mantovani, A. and Dejana, E. Cytokines as communication signals between leukocytes and endothelial cells Immunol.Today 1989; 10: 370375

Manuck, S.B., Kaplan, J.R. and Matthews, K.A. Behavioural antecedents if coronary heart disease and atheroscleroisis Arteriosclerosis 1986; 6: 2-14

Maoz, H., Kaiser, N., Halimi, M., Barak, V., Haimovitz, A., Weinstein, D., Simon, A., Yagel, S., Biran, S. and Treves, A.J. The effect of estradiol on human myelomonocytic cells. 1. Enhancement of colony formation J. Reprod. Immunol. 1985; 7: 325-335 
Marcum, J.A., McKenney, J.B. and Rosenberg, R.D. Acceleration of thrombin. Antithrombin complex formation in rat hindquarters via heparin like molecules bound to the endothelium J. Clin. Invest. 1984; 74: 341-350

Marmorston, J., Moore, F.J., Hopkins, C.E., Kuzma, O.T. and Weiner, J. Clinical studies of long-term estrogen therapy in men with myocardial infarction Proc. Soc. Exp. Biol. Med. 1962; 110: 400-408

Martin, B.M., Gimbrone, M.A. Jr., Unanue, E.R. and Cotran, R.S. Stimulation of nonlymphoid mesenchymal cell proliferation by a macrophagederived growth factor J. Immunol. 1981; 126: 1510-1515

Maruyama, Y. The human endothelial cell in tissue culture Z. Zellforsch. Mikrosk. Anat. 1963; 60: 69-79

Master, A.M., Dack, S. and Jaffe, H.L. Age, sex and hypertension in myocardial infarction due to coronary occlusion Arch. Intern. Med. 1939; 64: 767-786

Matsui, T., Heidaran, M., Miki, T., Popescu, N., Rochelle, W.L., Kraus, M., Pierce, $\mathrm{J}$. and Aaronson, $\mathrm{S}$. Isolation of a novel receptor CDNA established the existence of two PDGF receptor genes Science 1989; 243: 800-805

Matthews, K.A., Meilahn, E., Kuller, L.H., Kelsey, S.F., Caggiula, A.W. and Wing, R.R. Menopuase and risk factors for coronary heart disease New Engl. J. Med. 1989; 321: 641-6

Maziere, C., Auclair, M., Ronveaux, M.-F., Salmon, S., Santus, R. and Maziere, J.-C. Estrogens inhibit copper and cell-mediated modification of low density lipoprotein Atherosclerosis 1991; 89: 175-182

McEver, R.P., Beckstead, J.H., Moore, K.L., Marshall-Carlson, L. and Bainton, D.F. GMP-140, a platelet - $\alpha$-granule membrane protein, is also synthesized by vascular endothelial cells and is localised in weibel-palade bodies J. Clin. Invest. 1989; 84: 92-99

McFarland, K.F., Boniface, M.E., Harnung, C.A., Earnhardt, W. and Humphries, J.O. Risk factors and non contraceptive estrogen use in women with and without coronary disease Am. Heart J. 1989; 117: 1209-1214

McGill, H.C. Jr. and Sheridan, P.J. Nuclear uptake of sex steroid hormones in the cardiovascular system of the baboon Circ. Res. 1981; 48: 238-244

McGill, H.C. Jr. and Stern, M.P. Sex and atherosclerosis. In: Atherosclerosis Reviews Paoletti, R. and Gotta, A.M. Jr.(eds.), Raven Press 1979; 4: 157-235

McGill, H.C. Jr., Axelrod, L.R., McMahon, C.A., Wigodsky, H.S. and Mott, G.E. Estrogens and experimental atherosclerosis in the baboon Circulation 1977; 56: 657-662

McGookin, R. Gel electrophoresis of RNA in agarose and polyacryamide under non denaturing conditions In: Methods in Molecular Biology 
Walker, J.M. (ed) Humana Press, Clifton, New Jersey, 1984; 2: 93-100

McMaster, G.K. and Carmichael, G.C Analysis of single-and double-stranded nucleic acid on polyacrylamide and agarose gels by using gyloxal and acridine orange Proc. Natl. Acad. Sci. USA 1977; 74: 48354838

McNeil, P.L., Muthukrishnan, L., Warder, E. and D'Amore, P.A. Growth factors are released by mechanically wounded endothelial cells J. Cell Biol. 1989; 109: 811-822

Meade, T.W., Imeson, J.D., Haines, A.P., Stirling, Y. and Thompson, S. G. Menopausal status and haemostatic variables Lancet 1983; 1: 22-24

Meade, T.W., Dyer, S., Howarth, D.J., Imeson, J.D. and Stirling, Y. Antithrombin III and procoagulant activity: sex differences and effects of the menopause Br. J. Haematol. 1990; 74: 74-81

Meade, T.W., Greenberg, G. and Thompson, S.G. Progestogens and cardiovascular reations associated with oral contraceptives and a comparison of the safety of 50 and $300 \mu \mathrm{g}$ oestrogen preparations BMJ 1980; 280: 1157-1161 (a)

Meade, T.W., North, W.R.S., Chakrabarti, R., Stirling, Y., Haines, A.P. and Thompson, S.G. Haemostatic function and cardiovascular death; early results of a prospective study Lancet 1980; 1: 1050-1053 (b)

Mehta, P., Mehta, J. and Hay, D. Thromboxane and prostacyclin generation by intact human vessels in response to balloon catheter trauma Prostagland. Leukot. Med. 1982; 9: 539-548

Mentzer, S.J., Crimmin, M.A.V., Burakoff, S.J. and Faller, D.V. Alpha and beta subunits of LFA-1 (CDW 18) membrane molecule are involved in human monocyte-endothelial cell adhesion J. Cell. Physiol. 1987; 130: 410-415

Merke, J., Milde, P., Lewicka, S., Hugel, U., Klaus, G., Mangelsdorf, D.J. and Haussler, M.R. Identification and regulation of 1,25 dihydroxy vitamin $D_{3}$ receptor activity and biosynthesis of 1,25 dihydroxy vitamin $D_{3}$ J. Clin. Invest. 1989; 83: 1903-1915

Migliorisi, G., Folkes, E., Pawlowski, N. and Cramer, E.B. In vitro studies of human monocyte migration across endothelium in response to leukotriene $B_{4}$ and $f-M e t-L e u-P h e$ Am. J. Pathol. 1987; 127: 157-167

Milewich, L., Kaimal, V. and Johnson, A.R. Steroid 5 alpha-reductase activity in endothelial cells from human unbilical cord vessels J. Steroid Biochem. 1987; 26: 561-567

Milo, G.E., Malarkey, W.B., Powell, J.E., Blakeslee, J.R. and Yohn, D.S. Effect of steroid hormones in fetal bovine serum on plating and cloning of human cells in vitro In Vitro 1976; 12: 23-30 
Milton, J.G. and Frojmovic Sex-related differences in platelet morphology in whole blood (WB) and platelet-rich plasma (PRP) Thromb. Res. 1983; 30: 595-607

Minick, C.R., Murphy, G.E. and Campbell, W.G. Experimental induction of atherosclerosis by the synergy of allergic injury to arteries and lipid rich diet. I. Effect of repeated injections of horse serum in rabbits fed a dietary cholesterol supplement J. Exp. Med. 1966; 124: 635-651

Mitchell, H.C. and Williams, M.A. Effect of estrogens and a progestogen on platelet adhesiveness and aggregation in rabbits J. Lab. Clin. Med. 1974; 83: 79-89

Miyazono, K., Okabe, T., Urabe, A., Takahu, F. and Heldin C.-H. Purification and properities of endothelial cell growth factor from human platelets J. Biol. Chem. 1987; 262: 4098-4103

Molenaar, R., Visser, W.J., Verkerk, A., Koster, J.F. and Jongkind, J.F. Peroxidative stress and in vitro ageing of endothelial cells increases the monocyte endothelial cell adherence in a human in vitro system Atherosclerosis 1989; 76: 193-202

Moncado, S. and Vane, J.R. Pharmacology and endogenous roles of prostaglandin endoperoxides, thromboxane $A_{2}$ and Prostacyclin Pharmacol. Rev. 1979; 30: 293-331

Moore, S., Friedman, R.J., Singal, D.P., Gauldie, J., Blajchman, M.A. and Roberts, R.S. Inhibition of injury induced thromboatherosclerotic lesions by anti-platelet serum in rabbits Thromb. Haemost. 1976; 35: 70-81

Mordes, D.B., Lazarchick, J., Colwell, J.A. and Sens, D.A. Elevated glucose concentrations increase factor VIII R:Ag levels in human umbilical vein endothelial cells Diabetes 1983; 32: 876-878

Morel, D.W. and Chisolm, G.M. Antioxidant treatment of diabetic rats inhibits lipoprotein oxidation J. Lipid Res. 1989; 30: 1827-1834

Morel, D.W., Cathcart, M.K. and Chisolm, G.M. Cytotoxicity of low density lipoprotein oxidized by cell generated free radicals J. Cell Biol. 1983; 97: 427a (b)

Morel, D.W., DiCorleto, P.E. and Chisolm, G.M. Endothelial and smooth muscle cells alter low density lipoprotein in vitro by free radical oxidation Arteriosclerosis 1984; 4: 357-364

Morel, D.W., Hessler, J.R. and Chisolm, G.M. Low density lipoprotein cytotoxicity induced by free radical peroxidation of lipid J. Lipid Res. 1983; 24: 1070-1976 (a)

Morikawa, M., Kojima, T., Inoue, M. and Tsuboi, M. Sex difference in the inhibitory effect of aspirin on prostacyclin production of rat aorta Jpn J. Pharmacol. 1984; 35: 1-7

Morton, R.E., West, G.A. and Hoff, H.F. A low density lipoprotein-sized particle isolated from human atherosclerotic lesions is internalized by 
macrophages via a non-scavenger-receptor mechanism J. Lipid Res. 1986; 27: 1124-1134

Mosher, D.F., Williams, E.C. and McKeoun-Longo, P.J. Metabolism of thrombospondin and fibronectin by endothelial cells. In: Biology of Endothelial Cells, Jaffe, E.A. (ed.), Martinus Nijhoff Publishers, Boston 1984; 155-160

Mowri, H., Chinen, K., Ohkuma, S. and Takano, T. Peroxidized lipid isolated by HPLC from atherosclerotic aortic Biochem. Int. 1986; 12: 347352

Mueller, S.N., Thomas, K.A., Di Salvo, J. and Levine, E.M. Stabilization by heparin of acidic fibroblast growth factor mitogenicity for human endothelial cells in vitro J. Cell. Physiol. 1989; 140: 439-448

Mueller, R., Bravo, R., Burdshardt, J. and Curran, T. Induction of c-fos gene and protein by growth factors precedes activation of $c$-myc Nature 1984; 312: 716-720

Mulvihill, E.R. and Palmiter, R.D. Relationship of nuclear progesterone receptors to induction of ovalbumin and conalbumin mRNA in chick oviduct J. Biol. Chem. 1980; 255: 2085-2091

Murphy, L. J. and Friesen, H.G. Differential effects of estrogen and growth hormone on uterine and hepatic insulin-like growth factor I gene expression in the ovariectomized hypophysectomized rat Endocrinology 1988; 122: 325-332

Murphy, L.J., Murphy, L.C. and Friesen, H.G. Estrogen induction of N-myc and C-myc proto-oncogene expression in the rat uterus Endocrinology 1987; 120: 1882-1888

Mustard, J.F., Perry, D.W., Ardlie, N.G. and Packman, M.A. Preparation of suspensions of washed platelets from humans Br. J. Haematol. 1972; 22: 193-204

Mustard, J.F., Groves, H.M., Kinlough-Rathbone, R.L., Packham, M.A. Thrombogenic and non thrombogenic biological surfaces Ann. NY Acad. Sci. 1987; 516: 12-21

Mustard, J.F., Packham, M.A. and Kinlough-Rathbone, R.L. Platelets, atherosclerosis, and clinical complication. In: Vascular Injury and Atherosclerosis, Moore, S. (ed.), Marcel Dekker, NY 1981; 9-110

Myers, P.R., Guerra, R. Jr. and Harrison, D.G. Release of NO and EDRF from cultured bovine aortic endothelial cells Am. J. Physiol. 1989; 256: H1030-H1037

Nachtigall, L.E., Nachtigall, R.H., Nachtigall, R.D., Beckman, E.M. Estrogen replacement therapy II: a prospective study in the relationship to carcinoma and cardiovascular and metabolic problems Obstet. Gynecol. 1979; 54: 74-79

Nagelkerke, J.F., Havekes, L., van Hinsbergh, V.W.M. and van Berkel, T.J.C. In vivo catabolism of biologically modified $L D L$ Arteriosclerosis 1984; 4: 256-264 
Nakano, M., Sugioka, K., Naito, I., Takekoshi, S. and Niki, E. Novel and potent biological antioxidants on membrane phospolipid peroxidation: 2hydroxy estrone and 2-hydroxy estradiol Biochem. Biophys. Res. Commun. 1987; 142: 919-924

Nakao, J., Chang, W.-C., Murato, S.-I. and Orimo, H. Estradiol binding site in rat aortic smooth muscle cells in culture Atherosclerosis 1981; 38: 75-80

Nakao, J., Chang, W.-C., Murota, S.-I. and Orimo, H. Testosterone inhibits prostacyclin production by rat aortic smooth muscle cells in culture Atherosclerosis 1981; 39: 203-209

Nawroth, P.P. and Stern, D.M. Endothelial cell procoagulant properties and the host response Semin. Thromb. Hemost. 1987; 13: 391-397

Nawroth, P.P., Handley, D.A., Esmon, C.T. and Stern, D.M. Interleukin 1 induces endothelial cell procoagulant while suppressing cellsurface anticoagulant activity Proc. NatI. Acad. Sci., USA 1986; 83: 3460-3464

Needleman, S.W. and Parks, W.M. Catechol estrogens and thrombosis: differential effect of 2-hydroxyestradiol and estradiol on prostacyclin release Contraception 1982; 26: 317-320

Newby, A.C. and Henderson, A.H. Stimulus-secretion coupling in vascular endothelial cells Ann. Rev. Physiol. 1990; 52: 661-674

Newman, H.A.I., Murad, T.M. and Geer, J.C. Foam cells of rabbit atheromatous lesion: identification and cholesterol uptake in isolated cells Lab. Invest. 1971; 25: 586-595

Nichols, A.V., Rehnborg. C.S. and Lindgren, F.T. Gas chromatographic analysis of fatty acids from dialyzed lipoproteins J. Lipid Res. 1961; 2: 203-207

Nichols, W.W., Buynak, E.B., Bradt, C., Hill, R., Aronson, M., Jarrell, B.E., Mueller, S.N. and Levine, E. M. Cyogenetic evaluation of human endothelial cell cultures J. Cell. Physiol. 1987; 132: 453-462

Nievelstein, P.F.E.M. and de Groot, P.G. Interaction of blood platelets with the vessel wall Hemostasis 1988; 18: 342-359

Niewiarowski, S. and Thomas, D.P. Platelet factor 4 and adenosine dephosphate release during human platelet aggregation Nature 1969; 222: 1269-1270

Niki, E. Antioxidants in relation to lipid peroxidation Chem. Phys. Lipids 1987; 44: 227-253 (a)

Niki, E. Lipid antioxidants: how they may act in biological systems Br. J. Cancer 1987; 8: 153-157 (b)

Niki, E. and Nakano, M. Estrogens as antioxidants Methods Enzymol. 1990; 186: 330-333

Nikkila, E.A., Tikkaren, M.J. and Kuusi, T. Gonadal hormones, lipoprotein metablism and coronary heart disease. In: Myocardial Infarction 
in Women, Oliver, M.F., Vedin, A. and Wilhelmsson, C. (eds.), Churchill Livingstone, London 1986; 34-45

Nilsson, J. Growth factors and pathogenesis of atherosclerosis Atherosclerosis 1986; 62: 185-199

Nilsson, J. Smooth muscle Cells in the atherosclerotic process Acta. Med. Scand. 1987; 715 (suppl): 25-31

Nilsson, J., Svensson, J., Hamsten, A. and de Faire, U. Increased plateletderived mitogenic activity in plasma of young patients with coronary atherosclerosis Atherosclerosis 1986; 61: 237-253

Nilsson, J., Sjolurd, M., Palmberg, L., Thyberg, J. and Heldin, C.-H Arterial smooth muscle cells in primary culture produce a platelet-derived growth factor-like protein Proc. Natl. Acad. Sci., USA 1985; 82: 4418-4422

Nishizawa, Y., Sato, B., Miyashita, Y., Tsukado, S., Hirose, T., Kishimoto, S. and Matsumoto, K. Autocrine regulation of cell proliferation by estradiol and hydroxy tamoxifen of transformed mouse leydig cells in serum-free culture Endocrinology 1988; 122: 227-235

Nister, M., Libermann, T.A., Betsholtz , C., Pettersson, M., Claesson-Welsh, L., Heldin, C.-H., Schlessinger, J. and.Westermark, B. Expression of messenger RNAs for platelet-derived growth factor and transforming growth factor-alpha and their receptors in human malignant glioma cell lines Cancer Res. 1988; 48: 3910-3918 (a)

Nister, M., Hammacher, A., Mellstrom, K., Siegbahn, A., Ronnstrand, L., Westermark, B. and Heldin, C.-H. A glioma-derived PDGF A chain homodimer has different functional activities from a PDGF $A B$ heterodimer purified from platelets Cell 1988; 52: 791-799 (b)

Noble, R.P. Electophoretic separation of plasma lipoproteins in agarose gel J. Lipid Res. 1968; 9: 693-700

Nordoy, A., Svensson, B., Wiebe, D. and Hoak, J.C. Lipoproteins and the inhibitory effect of human endothelial cells on platelet function Circ. Res. 1978; 43: 527-34

Norioka, K., Hara, M., Kitani, A., Hirose, T., Hirose, W., Harigai, M., Suzuki, K., Kawakami, M., Tabata, H., Kawagoe, M and Nakamura, $H$. Inhibitory effect of human recombinant interleukin-1A and $B$ on growth of human vascular endothelial cells Biochem. Biophys. Res. Commun. 1987; 145: 969-975

Norris, J.S., Cornett, L.E., Hardin, J.W., Kohler, P.O., MacLeod, S.L., Srivastava, A., Syms, A.J. and Smith, R.G. Autocrine regulation of growth, Part 2 (Glucocorticoids inhibit transcription of c-sis oncogenespecific RNA transcripts) Biochem. Biophys. Res. Commun. 1984; 122: 124-128

Novak, E.R. and Williams, T.J. Autopsy comparison of cardiovascular changes in castrated and normal women Am. J. Obstet. Gynecol. 1960; 80: 803-872 
Okabe, T. and Takaku, F. A macrophage factor that stimulates the proliferation of vascular endothelial cells Biochem. Biophys. Res. Commun. 1986; 134: 344-350

Oliver, M.F. Oral contraceptives and myocardial infarction BMJ 1970; 2: 210213

Oliver, M.F. What is the difference between women and men? In: Myocardial Infarction in Women, Oliver, M.F., Vedin, A. and Wilhelmsson (eds.), Churchill Livingstone, UK 1986; 15-22 (a)

Oliver, M.F. Prevention of coronary heart disease-propaganda, promises, problems, and prospects Circulation 1986; 73: 1-9 (b)

Oliver, M.F. and Boyd, G.S Effect of bilaterial ovariectomy on coronary-artery disease and serum-lipid levels Lancet 1959; 2: 690-694

Oliver, M.F. and Boyd, G.S. The influence of sex hormones on the circulating lipids and lipproteins in coronary sclerosis Circulation 1956; 13: 82-91

Olson, R.E. Is there an optimum diet for the prevention of coronary heart disease? In: Nutrition, Lipids and Coronary Heart Disease, Levy, R.I. Rifkind, R.B.M., Dennis, B.H. and Ernst, N. (eds.) NY, Raven Press 1979; 1: 349-364

Ontko, J.A. Physical and chemical changes in isolated chylomicrons: prevention by EDTA J. Lipid Res. 1970; 11: 367-375

Oppenheimer-Marks, N., Davis, L.S. and Lipsky, P.E. Human T lympocyte adhesion to endothelial cells and transendothelial status of both the T cell and the endothelial cell J. Immunol. 1990; 145: 140148

Orekhov, A.N., Tertov, V.V., Mukhin, D.N. and Mikhailenko, I.A. Modification of low density lipoprotein by desialylation causes lipid accumulation in cultured cells Biochem. Biophys. Res. Commun. 1989; 162: 200-211

Orekhov, A.N., Tertov, V.V., Mukhin, D.N., Koteliansky, V.E., Glukhova, M.A., Frid, M.G., Sukhova, G.K. Khashimov, K.A. and Smirnov, V.N. Insolubilization of low density lipoproteins induces cholesterol accumulation in cultured subendothelial cells of human aorta Atherosclerosis 1989; 79: 59-70

Okret, S., Poellinger, L., Dong, Y., Gustafsson, J.A. Down-regulation of glucorticoid receptor mRNA by glucocorticoid hormones and recognition by the receptor of a specific binding sequence within a receptor cDNA clone Proc. Natl. Acad. Sci. USA 1986; 83: 5899-5903

Owen, A.J., Pantazis, P. and Antoniades, H.N. Simian sarcoma virustransformed cells secreted a mitogen identical to platelet-derived growth factor Science 1984; 225: 54-56

Pacifici, R., Rifas, L., McCracken, R., Vered, I., McMurtry, C., Avioli, L.V. and Peck, W.A. Ovarian steroid treatment blocks a post menopausal 
increase in blood monocyte interleukin-1 release Proc. Natl.

Acad. Sci. USA 1989; 82: 2398-2402

Page, M.J., Field, J.K., Everett, N.P. and Green, C.D. Serum regulation of the estrogen responsiveness of the human breast cancer cell line MCF-7 Cancer Res. 1983; 43: 1244-1250

Palinski, W., Rosenfeld, M.E, Yla-Herttuala, S., Gurtner, G.C., Socher, S.S., Bulter, S.W., Parthasarathy, S., Carew, T.E. Steinberg, D. and Witztum, J.L. Low density lipoprotein undergoes oxidative modification in vivo Proc. Natl. Acad. Sci., USA 1989; 86: 13721376

Palmer, R.M.J., Ferrige, A.G. and Moncado, S. Nitric oxide release accounts for biological activity of endothelium-derived relaxing factor Nature 1987; 327: 524-526

Pals, S.T., Hogervorst, F., Keizer, G., Thepen, T., Horst, E. and Figdor, C.C. Identification of a widely distributed $90 \mathrm{~K}$ Da glycoprotein that is homologous to the hermes-1 human lymphocyte homing receptor J. Immunol. 1989; 143: 851-857

Pantazis, P., Pelicci, P.G., Dalla-Favera, R. and Antoniades, H.N. Synthesis and secretion of proteins resembling platelets derived growth factor by human glioblastoma and fibrosarcoma cells in culture Proc. Natl. Acad. Sci., USA 1985; 82: 2404-2408

Pantazis, P., Sariban, E., Kufe, D. and Antoniades, H.N. Induction of C-sis gene expression and synthesis of platelet-derived growth factor in human myeliod leukemia cells during monocytic differentiation Proc. Natl. Acad. Sci. USA 1986; 83: 6455-6459

Parikh, I., Rajendran, K.G., Su, J.L., Lopez, T. and Sar, M. Are estrogen receptors cytoplasmic or nuclear? Some immunocytochemical and biochemical studies J. Steroid Biochem. 1987; 27: 185-192

Parker, F. and Odland, G.F. A correlative histochemical, biochemcial and electron microscopic study of experimental atherosclerosis in the rabbit aorta with special reference to myo-intimal cell Am. J. Pathol. 1966; 48: 197-239

Parrish, H.M., Carr, C.A., Hall, D.G. and King, T.M. Time interval from castration in premenopausal women to development of excessive coronary atherosclerosis Am. J. Obstet. Gynecol. 1967; 99: 155-162

Parry, E.W. and Abramovich, D.R. The ultra structure of human umbilical vessel endothelium from early pregnancy to full term J. Anat. 1972; 111: 29-42

Parthasarathy, S., Fong, L.G., Quinn, M.T. and Steinberg, D. Oxidative modification of LDL: comparison between cell-mediated and copper-mediated modification Eur. Heart J. 1990; Suppl.E: 8387

Parthasarathy, S., Printz., D.J., Boyd, D., Joy, L. and Steinberg, D. Macrophage oxidation of low density lipoprotein generates a modified form recognized by the scavenger receptor Arteriosclerosis 1986; 6: 505-510 (a) 
Parthasarathy, S., Quinn, M.T., Schwenke, D.C., Carew, T.E. and Steinberg, D. Oxidative modification of Beta- very low density lipoprotein : potential role in monocyte recrutiment and foam cell formation Arteriosclerosis 1989; 9: 398-404 (a)

Parthasarathy, S., Steinbrecher, U.P., Barnett, J., Witztum., J.L. and Steinberg, D. Essential role of phospholipase $A_{2}$ activity in endothelial cellinduced modification of low density lipoprotein Proc. Natl. Acad. Sci., USA 1985; 82: 3000-3004

Parthasarathy, S., Wieland, E. and Steinberg, D. A role for endothelial cell lipoxygenase in the oxidative modification of low density lipoprotein Proc. Natl. Acad. Sci., USA 1989; 86: 1046-1050 (b)

Parthasarathy, S., Young, S.G., Witztum, J.L., Pittman, R.C. and Steinberg, D. Probucol inhibits oxidative modification of low density lipoprotein J. Clin. Invest. 1986; 77: 641-644 (a)

Patterson, M.K. Jr. Measurement of growth and viability of cells in culture Methods Enzymol. 1979; LVIII: 141-152

Pawlowski, N.A., Abraham, E.L., Pontier, S., Scott, W.A. and Cohn, Z.A. Human monocyte-endothelial cell interaction in vitro Proc. Natl. Acad. Sci. USA 1985; 82: 8208-8212

Pawlowski, N.A., Kaplan, G., Abraham, E. and Cohn, Z.A. The selective binding and transmigration of monocytes through the junctional complexes of human endothelium J. Exp. Med. 1988; 168: 1865-1882

Pearson, J.D. and Gordon, J.L. Vascular endothelial and smooth muscle cells in culture selectively releases adenine nucleotides Nature 1979; 281: 384-387

Pearson, T.A., Dillman, J.M., Solex, K. and Heptinstall, R.H. Clonal markers in the study of the origin and growth of human atherosclerotic lesions Circ. Res. 1978; 43: 10-18

Pearson, T.A., Kramer, E.C., Solez, K. and Heptinstall, R.H. The human atherosclerotic plaque Am. J. Pathol. 1977; 86: 657-664

Peek, M., Markham, R. and Fraser, I.S. A comparison of fetal and new born bovine serum components (from Commonwealth Serum Laboratories) Am. J. Med. Lab. Sci. 1989; 10: 40-41

Peres, R., Betsholtz, C., westermark, B. and Heldin C.-H. Frequent expression of growth factors for mesenchymal cells in human mammary carcinoma cell lines Cancer Res. 1987; 47: 3425-3429

Peri, G., Chiaffarino, F., Bernasconi, S., Padura, I.M. and Mantovani, A. Cytotoxicity of activated macrophages on endothelial cells $\mathbf{J}$. Immunol. 1990; 144: 1444-1448

Petitti, D.B., Wingerd, J., Pellegrin, F. and Ramcharan, S. Risk of vascular disease in women: smoking, oral contraceptives, noncontraceptive estrogens, and other factors JAMA 1979; 242: 1150-1154 
Pfeiffer, R.W. and Patterson, R.M. Modulation of lymphokine-induced macrophage activation by estrogen metabolites $\mathbf{J}$. Immunopharmacol. 1985; 7: 247-263

Phillips, G.B., Castelli, W.P., Abbott, R.D. and McNamar, P.M. Association of hyperestrogenemia and coronary heart disease in men in the Framingham cohort Am. J. Med. 1983; 74: 863-869

Phillips, G.B. Evidence for hyperoestrognaemia as a risk factor for myocardial infarction in man Lancet 1976; 2: 14-18

Pick, R., Stamler, J., Rodbard, S. and Katz, L.N. The inhibition of coronary atherosclerosis by estrogens in cholesterol-fed chicks Circulation 1952; 6: 276-280

Pierschbacher, M., Hayman, E.G. and Ruoslahti, E. Synthetic peptide with cell attachment activity of fibronectin Proc. Natl. Acad. Sci. USA 1983; 80: 1224-1227

Pierce, G.F., Mustoe, T.A., Lingelbach, J., Masakowski, V.R., Griffin, G.L., Senior, R.M. and Deuel, T.F. Platelet-derived growth factor and transforming growth factor-B enhance tissue repair activities by unique mechanisms J. Cell Biol. 1989; 109: 429-440

Pinto, S., Coppo, M., Paniccia, R., Prosco, D., Gori, A.M., Attanasio, M. and Abbate, $\mathrm{R}$. Sex related differences in platelet TXA2 generation Prostaglandins Leuko. Essent. Fatty Acids 1990; 40: 217-221

Piotrowski, J.J., Hunter, G.C., Eskelson, C.D., Dubick, M.A. and Bernhard, V.M. Evidence for lipid peroxidation in atherosclerosis Life Sci. 1990; 46: 715-721

Pober, J.S. Cytokine-mediated activation of vascular endothelium Am. J. Pathol. 1988; 133: 426-433

Pober, J.S. and Cotran, R.S. Cytokines and endothelial cell biology Physiol. Rev. 1990; 70: 427-451

Pohlman, T.H., Staness, K.A., Beatty, P.G., Ochs, H.D. and Harlan, J.M. An endothelial cell surface factor(s) induced in vitro by lipopolysaccharide interleukin 1 and tumor neorosis factora increases neutrophil adherence by a CDN18 dependent mechanism J. Immunol. 1985; 136: 4548-4553

Polan, M.L., Daniele, A. and Kuo, A. Gonadal steroids modulate human monocyte interleukin-1 (IL-1) activity Fertil. Steril. 1988; 49: 964968

Polan, M.L., Loukides, J., Nelson, P., Carding, S., Diamond, M., Walsh, A. and Bottomly, K. Progesterone and estradiol modulate interleukin-1 beta messenger ribonucleic acid levels in cultured human peripheral monocytes J. Clin. Endocrinol. Metab. 1989; 69: 1200-1206

Poller, L., Priest, C.M. and Thomson, J.M. Platelet aggregation during oral contraception BMJ 1969; 4: 273-274 
Pologe, L.G., Cramer, E.B., Pawlowski, N.A., Abraham, E., Cohn, Z.A. and Scott, W.A. Stimulation of human endothelial cell prostacyclin synthesis by selected leukotrienes J. Exp. Med. 1984; 160: 1043-1053

Pomerantz, K., Maddox, Y., Maggi, F., Ramey, E. and Ramwell, P. Sex and hormonal modification of 6-Keto-PGF1a Life Sci. 1980; 27: 12331236

Poole, J.C.F., Sanders, A.G. and Florey, H.W. The regeneration of aortic endothelium J. Pathol. Bact. 1958; 75: 133-143

Poole, J.C.F. and Florey, H.W. Changes in the endothelium of the aorta and the behaviour of macrophages in experiemtnal atheroma of rabbits $\mathbf{J}$. Pathol. Bact. 1958; 75: 245-253

Poumay, Y. and Ronveaux-Dupal, M.F. Incubation of endothelial cells in superoxide-generating system: impaired low-density lipoprotein receptor-mediated endocytosis J. Cell. Physiol. 1988; 136: 289296

Powers, M.S., Schenkel, L., Darley, P.E., Good, W.R., Balestra, J.C. and Place, V.A. Pharmacokinetics and pharmacodynamics of transdermal dosage forms of 17 $\beta$-estradiol: comparison with conventional oral estrogens used for hormone replacement Am. J. Obstet. Gynecol. 1985; 152: 1099-1106.

Prasad, K., Kalra, J., Chan, W.P. and Chaudhary, A.K. Effect of oxygen free radicals on cardiovascular function at organ and cellular levels Am. Heart J. 1989; 117: 1196-1202

Prescott, S.M., Zimmerman, G.A. and Mclntyre, T.M. Human endothelial cells in culture produce platelet activating factor (1-alkyl-2-acetyl-SNglycero-phosphoryt choline) when stimulated with thrombin Proc. Natl. Acad. Sci., USA 1984; 81: 3534-3538

Presta, M. Sex hormones modulate the synthesis of basic fibroblast growth factor in human endometrial adenocarcinoma cells: implications for the neovascularization of normal and neoplastic endothelium J. Cell. Physiol. 1988; 137: 593-597

Prichard, R.W., Clarkson, T.B. and Lofland, H.B. Estrogen in pigeon atherosclerosis: estradiol valerate effects at several doses levels on cholesterol-fed male white carneau pigeons Arch. Pathol. 1966; 82: 15-17

Pritchard, K.A. Jr., Tota, R.P., Stemerman, M.B. and Wong, P.Y. 14, 15epoxyeicosatrienoic acid promotes endothelial cell dependent adhesion of human monocytic tumor U937 cells Biochem. Biophys. Res. Commun. 1990; 167: 137-142

Pyorala, K. and Laakso, M. Macrovascular disease in diabetes mellitus. In: Diabetes in Epidemiological Perspective, Mann, J.I., Pyrl, K. and Teuscher, A. (eds.), Churchill Livingstone, London 1983; 183-247

Quinn, M.T., Parthasarathy, S. and Steinberg, D. Endothelial cell-derived chemalactic activity for main peritoneal macrophages and the 
effects of modified forms of low density lipoproetins Proc. NatI. Acad. Sci., USA 1985; 82: 5949-5953

Quinn, M.T., Parthasarathy, S. and Steinberg, D. Lysophosphatidylcholine: a chemotactic factor for human monocytes and its potential role in atherogenesis Proc. Natl. Acad. Sci., USA 1988; 85: 2805-2809

Quinn, M.T., Parthasarathy, S., Fong. L.G. and Steinberg, D. Oxidatively modified low density lysoproteins: a potential role in recruitment and retention of monocyte/macrophages during atherogenesis

Proc. Natl. Acad. Sci., USA 1987; 84: 2995-2998

Radomski, M.W., Palmer, R.M. and Moncado, S. Endogenous nitric oxide inhibits human platelet adhesion to vascular endothelium Lancet 1987; 2: 1057-1058

Raines, E.W., Downer, S.K. and Ross, R. Interleukin -1 mitogenic activity for fibroblasts and smooth muscle cells is due to PDGF-AA Science 1989; 243:393-396

Rajavashisth, T.B., Andalibi, A., Territo, M.C., Berliner, J.A., Navab, M., Fogelman, A.M. and Lusis, A.J. Induction of endothelial cell expression of granulocyte and macrophage colony-stimulating factors by modified low-density lipoproteins Nature 1990; 344: 254-257

Rajendran, K. G., Lopez, T. and Parikh, I. Estrogenic effect of phenol red in MCF-7 Cells is achieved through activation of estrogen receptor by interacting with a site distinct from the steroid binding site Biochem. Biophys. Res. Commun. 1987; 142: 724-731

Ramcharan, S. and Pellegrin, F.A. Some findings in the walnut creek contraceptive study after 5 years Am. J. Epidemiol. 1975; 102: 459

Ramsay, M.M., Walker, L.N. and Bowyer, D.E. Narrow superficial injury to rabbit aortic endothelium Atherosclerosis 1982; 43: 233-243

Rand, J.H., Sussman, I.I., Gordon, R.E., Chu, S.V. and Solomon, V. Localization of factor VIII-related antigen in human vascular subendothelium Blood 1980; 55: 752-756

Rand, M.L., Vickers, J.D., Kinlough-Rathbone, R.L., Packham, M.A. and Mustard, J.F. Thrombin-induced inosital triphosphate production by rabbit platelets is inhibited by ethanol Biochem. J. 1988; 251: 279-284 (a)

Rand, M.L., Packham, M.A., Kinlough-Rathbone, R.L. and Mustard, J.F. Effect of ethanol on pathways or platelet aggregation in vitro Thromb. Haemost. 1988; 59: 383-387 (b)

Rankin, S.M., Parthasarathy, S. and Steinberg, D. Evidence for a dominant role of lipoxygenase(s) in the oxidation of LDL by mouse peritoneal macrophages J. Lipid Res. 1991; 32: $449-456$

Reckless, J.P.D., Weinstein, W.B. and Steinberg, D. Lipoprotein and cholesterol metabolism in rabbit arterial endothelial cells in culture Biochem. Biophys. Acta 1978; 529: 475-487 
Recknagel, R.O. and Ghoshal, A.K. Quantitative estimation of peroxidative degeneration of rat liver microsomal and mitochondrial lipids after carbon tetrachloride poisoning Exp. Mol. Pathol. 1966; 5: 413426

Ree, A.H., Landmark, B.F., Eskild, W., Levy, F.O., Lahooti, H., Johnson, T., Aakuaag, A. and Hansson, V. Autologous down-regulation of messenger ribonucleic acid and protein levels for estrogen receptors in MCF- 7 cells: an inverse correlation to progesterone receptor levels Endocrinology 1989; 124: 2577-2583

Reed, D.M., Strong, J.P., Resch, J. and Hayashi, T. Serum lipids and lipoproteins as predictors of atherosclerosis. An autopsy study Arteriosclerosis 1989; 9: 560-564

Reidy, M.A. and Silver, M. Endothelial regeneration, Part 7. (Lack of intimal proliferation after defined injury to rat aorta) Am. J. Pathol. 1985; 118: 173-177

Reilly, C.F. and Broski, J.E., Differential effects of PPGF and PDGF-BB on vascular smooth muscle cells Biochem. Biophys. Res. Commun. 1989; 160: 1047-1054

Reunanen, A. Diabetes as a risk factor for coronary heart disease. In: Myocardial Infarction in Women, Oliver, M.F., Vedin, A. and Wilhelmsson, C. (eds.), Churchill Livingstone, London 1986; 4653

Rhee, C.Y., Drouet, L.O., Spaet, T.H. and Geiger, C.H. Growth inhibition of cultured vascular smooth cells by estradiol Fed. Proc. 1978; 37: 474

Rhee, C.Y., Spaet, T.H., Gaynor, E., Lajam, F., Shiang, H.H., Caruso, E. and Litwak, R.S. Suppression of surgically induced vascular intimal hypertrophy by estrogen Circulation 1974; 49(suppl III): 92

Rice, G.E., and Bevilacqua, M.P. An inducible endothelial cell surface glycoprotein mediates melanoma adhesion Science 1989; 246: 1303-1306

Rissanen, A.M. Familial occurrence of coronary heart disease and major risk factors. In: Myocardial Infarction in Women, Oliver, M.F., Vedin, A. and Wilhelmsson, C. (eds.), Churchill Livingstone, London 1986; 18-31

Ristimaki, A. Transforming growth factor alpha stimulates prostacyclin production by cultured human vascular endothelial cells more potently than epidermal growth factor Biochem. Biophys. Res. Commun. 1989; 160: 1100-1105

Ristimaki, A., Ylikorkala, O., Perheentupa, J. and Viinikka, L. Epidermal growth factor stimulates prostacyclin production by cultured human umbilical vein endothelial cells Thromb. Haemost. 1988; 59: 248-250

Ritterband, A.B., Jaffe, I.A., Densen, P.M., Magagna, J.F. and Reed, E. Gondal function and the development of coronary heart disease

Circulation 1963; 27: 237-251 
Roberts, W.C. Factors linking cholesterol to atherosclerotic plaques Am J. Cardiol. 1988; 62: 495-499

Robertson, W.B. and Strong, J.P. Atherosclerosis in persons with hypertension and diabetes melitus Lab. Invest. 1968; 18: 538-551

Robinson, R.W., Higano, N. and Cohen, W.D. Increased incidence of coronary heart disease in women castrated prior to the menopause Arch. Intern. Med. 1959; 104; 82-87

Robinson, R.W. and LeBeau, R.J. Effect of conjugated equine estrogens on serum lipids and the clotting mechanism Atherosclerosis Res. 1965; 5: 120-124

Rodgers, G. Hemostatic properties of normal and perturbed vascular cells FASEB J. 1988; 2: 116-123

Roncaglioni, M., Minno, G., Meyers, I., Gaetano, G. and Donati, M. Increased prostacyclin-like activity in vascular tissues from rats on long-term treatment with an oestrogen-progestagen combination Thromb. Res. 1979; 14: 793

Rosenbaum, J., Tobelem, G., Molho, P., Barzu, T. and Coen, J.P. Modulation of endothelial cell growth induced by heparin Cell Biol. Int. Rep. 1986; 10: $437-446$

Rosenberg, L., Armstrong, B. and Jick, H. Myocardial infarction and estrogen therapy in premenopausal women N. Engl. J. Med. 1976; 294: 1290-1291 (a)

Rosenberg, L., Armstrong, B. and Jick, H. Myocardial infarction and estrogen therapy in post-menopausal women N. Engl. J. Med. 1976; 294: 1256-1259 (b)

Rosenberg, L., Hennekens, C.H., Rosner, B., Belanger, C., Rothman, K.J. and Speizer, F.E. Early menopause and the risk of myocardial infarction Am. J. Obstet. Gynecol. 1981; 139: 47-51

Rosenberg, L., Miller, D.R. and Kaufman, D.W. Myocardial infarction in women under 50 years of age JAMA 1983; 250: 2801-2806

Rosenberg, L., Palmer, J.R., Lesko, S.M. and Shapiro, S. Oral contraceptive use and the risk of myocardial infarction Am. J. Epidemiol. 1990; 131: 1009-1016

Rosenberg, L., Slone, D., Shapiro, S., Kaufman, D.W., Stalley, P.D. and Miettinen, O.S. Noncontraceptive estrogens and myocardial infarction in young women JAMA 1980; 244: 339-342

Rosenfeld, M.E., Tsukada, T., Chait, A., Bierman, E.L., Gown, A.M. and Ross, R. Fatty streak expansion and maturation in the WHHL and comparably hypercholesterolemic fat-fed rabbits Arteriosclerosis 1987; 7: 24-34 (b)

Rosenfeld, M.E., Tsukada, T., Gown, A.M. and Ross, R. Fatty streak initiation in the WHHL and comparably hypercholesterolemic fat-fed rabbits Arteriosclerosis 1987; 7: 9-23 (a) 
Rosengart, T.K., Kupferschmid, J.P., Ferrans, V.J., Casscells, W., Maciage, T. and Clark, R.E. Heparin-binding Growth Factor-1 (endothelial cell growth factor) binds to endothelium in vivo J. Vasc. Surg. 1988; 7: 311-317

Rosenman, R.H., Brand, R.J., Jenkins, C.D., Friedman, M., Straus, R. and Wurm, $M$. Coronary heart disease in the western collaborative group study. Final follow-up experience of 8 1/2 years JAMA 1975; 233: 872-877

Ross, R. Platelet-derived growth factor Ann. Rev. Med. 1987; 38: 71-79

Ross, R. The pathogenesis of atherosclerosis- an update New Engl. J. Med. 1986; 314: 488-500

Ross, R. Platelet-derived growth factors Lancet 1989; 1: 1179-1182

Ross, R. Atherosclerosis - a problem of the biology of arterial cells and their interaction with blood components Arteriosclerosis 1981; 1: 293-311 (c)

Ross, R. Smooth muscle cells and atherosclerosis. In: Vascular Injury and Atherosclerosis, Moore, S. (ed.), Marcel Dekker Inc., NY 1981; 53-77 (b)

Ross, R. and Glomset, J.A. The pathogenesis of atherosclerosis N. Engl. J. Med. 1976; 295: 369-377; 420-425

Ross, R. and Harker, L. Hyperlipidemia and atherosclerosis Science 1976; 193: $1094-1100$

Ross, R. and Vogel, A. The platelet-derived growth factor Cell 1978; 14: 203210

Ross, R.K., Paganini-Hill, A., Mack, T. and Hendersen, B.E. Cardiovascular benefits of estrogen replacement therapy Am. J. Obstet. Gynecol. 1989; 160: 1301-6

Ross, R.K., Paganini-Hill, A., Mack, T.M., Arthur, M. and Henderson, B.E. Menopausal oestrogen therapy and protection from death from ischemic heart disease Lancet 1981; 1: 858-860 (a)

Ross, R., Raines, E.W. and Bowen-Pope, D.F. The biology of platelet-derived growth factor Cell 1986; 46: 155-169

Rossi, V., Breviario, F., Ghezzi, P., Dejana, E. and Mantovani, A. Prostacyclin synthesis induced in vascular cells by interleukin-1 Science 1985; 229: 174-176

Roy, D. and Leihr, J.G. Changes in activities of free radical detoxifying enzymes in kidneys of male Syrian hamsters treatment with estradiol Cancer Res. 1989; 49: 1475-1480

Royal College of General Practitioners' Oral Contraception Study. Mortality among oral-contraceptive users Lancet 1977; 2: 727-731 
Royal College of General Practitioners' Oral Contraception Study. Oral contraceptive, venous thrombosis and varicose veins J. R. Coll. Gen. Pract. 1978; 28: 393-399

Royall, J.A., Berkow, R.L., Beckman, J.S., Cunningham, M.K., Matalon, S. and Freeman, B.A. Tumor necrosis factor and interleukin I. increase vascular endothelial permeability Am. J. Physiol. 1989; 257: L399-L410

Rubin, D.B., Mason, R.J. and Dobbs, C.J. Angiotensin converting enzyme substrates hydrolzed by fibroblasts and vascular endothelial cells Exp. Lung Res. 1982; 3: 137-146

Rudy, D.R. Hormone replacement therapy Post Grad. Med. 1990; 88: 157-164

Russ, E.M., Eder, H.A. and Barr, D.P. Protein-lipid relationships in human plasma. I. In normal individuals Am. J. Med. 1951; 11: 468-479

Russ, E.M., Eder, H.A. and Barr, D.P. Influence of gonadal hormones on protein-lipid relations in human plasma Am. J. Med. 1955; 19: 424

Ryan, J.W. and Ryan, U.S. Endothelial Metabolism In: Microcirculation, Effros, R.M., Schmid-Shonbem, H. and Ditzel, J. (eds.), Academic Press Inc 1981; 147-169

Ryan, U.S., Clements, E., Habliston, D. and Ryan, J.W. Isolation and culture of pulmonary artery endothelial cells Tissue Cell 1978; 10: 535554

Rybak, M.E., Gimbrone, M.A. Jr., Davies, P.F. and Handin, R.I. Interaction of platelet factor four with cultured vascular endothelial cells Blood 1989; 73: 1534-1539

Saba, S.R., Zucker, W.H., Mason, R.G. Some properties of endothelial cells isolated from human umbilical cord vein Ser. Haemat. 1973; VI: 456-468

Sabra, A and Bonnar, J. Hemostatic system changes induced by 50 micrograms and 30 micrograms estrogen/progestogen oral contraceptives. Modification of estrogen effects by levonorgestrel J. Reprod. Med. 1983; 28: 85-89

Saceda, M., Lippman, M.E., Chambon, P., Lindsey, R.L., Ponglikitmongkol, M., Puente, M. and Martin, M.B. Regulation of the estrogen receptor in MCF-7 cell by estradiol Mol. Endocrinol. 1988; 2: 1157-1162

Sacks, T., Moldow, C.F., Craddock, P.R., Bowers, T.K. and Jacobs, H.S. Oxygen radical mediate endothelial cell damage by complementstimulated granulocytes. An in vitro model of immune vascular damage J. Clin. Invest. 1978; 61: 1161-1167

Saegusa, Y, Ziff, M., Welkovich, L. and Cavender, D. Effect of inflammatory cytokines on human endothelial cell proliferation J. Cell.

Physiol. 1990; 142: 488-495 
Saegusa, Y., Cavender, D. and Ziff, M. Stimulation of mononuclear cell binding to human endothelial cell monolayers by thrombin J. Immunol. 1988; 141: 4140-4145

Sage, $\mathrm{H}$. Collagen synthesis by endothelial cells in culture In: Biology of Endothelial Cells Jaffe, E.A. (ed.), Martinus-Nijhoff, Boston 1984: 161-177

Sakamoto, N., Tanaka, N. G., Tohgo, A., Osada, Y. and Ogawa, H. Inhibitory effects of heparin plus cortisone actelate on endothelial cell growth both in cultures and in tumor masses J. Natl. Cancer Inst. 1987; 78: 581-585

Sanchez, A., Chesterman, C.N. and Sleigh, M.J. Novel human PDGF A gene transcripts derived by alternative mRNA splicing Gene 1991; 98: 295-298

Santos, G., Scott, G., Lee, W., Liv, E. and Benz, C. Estrogen-induced post transcriptional modulation of c-myc proto-onogene expression in human breast cancer cells J. Biol. Chem. 1988; 263: 9565-9568

Saphir, P.O., Ohringer, L. and Wong, R. Changes in the intramural coronary branches in coronary arteriosclerosis Arch. Pathol. 1965; 62: 159-170

Sartwell, P.E., Masi, A.T., Arthes, F.G., Greene, G.R. and Smith, H.E. Thromboembolism and oral contraceptives: an epidemiologiccase-control study Am. J. Epidemiol. 1969; 90: 365-380

Sato, B., Maeda, Y., Nishizawa, Y., Noma, K., Kishimoto, S. and Matsumoto, K. Effects of tamoxifen on estrogen-induced enhancement or inhibition of tumor growth in mouse Leydig cell tumor lines Cancer Res. 1984; 44: 4386-4391

Sato, Y. and Rifkin, D.B. Autocrine activities of basic fibroblast growth factor: regulation of endothelial movement, plasminogen activator synthesis, and DNA synthesis J. Cell Biol. 1988; 107: 1199-1205

Sato, Y., Hotta, N., Sakamoto, N., Matsuoka, S., Ohishi, N. and Yagi, K. Lipid peroxide level in plasma of diabetic patients Biochem. Med. 1979; 21: 104-107

Scarpati, E.M. and Sadler, J.E. Regulation of endothelial cell coagulant properties J. Biol. Chem. 1989; 264: 20705-20713

Schaefer, E.J. and Levy, R.I. Pathogenesis and management of lipoprotein disorders N. Engl. J. Med. 1985; 312: 1300-1310

Schaefer, E.J., Foster, D.M., Zech, L.A., Lindgren, F.T., Brewer, H.B. Jr. and Levy, R.I. The effects of estrogen administration on plasma lipoprotein metabolism in premenopausal females J. Clin. Endocrinol. Metab. 1983; 57: 262-267

Schafer, A.I., Rodirguez, R., Loscalzo, J. and Gimbrone, M.A. Jr. Inhibition of vascular endothelial cell prostacyclin synthesis by plasmin Blood 1989; 74: 1015-1020 
Schaffner, T., Taylor, K., Bartucci, E.J., Fischer-Dzoga, K., Beeson, J.A., Glagov, S. and Wissler, R.W. Arterial foam cells with destinctive immunomorphologic and histochemical features of macrophages Am. J. Pathol. 1980; 100: 57-73

Schiffman, S. and Rapaport, S.J. Increase factor VIII levels in suspected carriers of hemophilia A taking contraceptives by mouth N. Engl. J. Med. 1966; 275: 599

Schleef, R.R., Bevilacqua, M.P., Sawdey, M., Gimbrone, M.A. Jr. and Loskutoff, D.J. Cytokine activation of vascular endothelium J. Biol. Chem. 1988; 263: 5797-5803

Schmid, C., Ernst, M., Zapf, J. and Froesch, E.R. Release of insulin-like growth factor carrier proteins by osteoblasts: stimulation by estradiol and growth hormone Biochem. Biophys. Res. Commun. 1989; 160: 788-794

Schmidt, K., Mayer, B. and Kukovetz, W.R. Effect of calcium on endotheliumderived relaxing factor formation and CGMP levels in endothelial cells Eur. J. Pharm. 1989; 170: 157-166

Schmitz, G., Niemann, R., Brennhausen, B., Krause, R. and Assman, G. Regulation of high density lipoprotein receptors in cultured macrophages: role of acyl-COA: cholesterolacyl-COA EMBO J. 1985; 4: 2773-2779

Schorer, A.E., Moldow, C.F. and Rick, M.E. Interleukin 1 or endotoxin increases the release of von Willebrand factor from human endothelial cells Br. J. Haematol. 1987; 67: 193-197

Schreiber, A.B., Kenney, J. and Kowalski, W.J. Interaction of endothelial cell growth with heparin: characterization by receptor and antibody recognition Proc. Natl. Acad. Sci., USA 1985; 82: 6138-6142 (a)

Schreiber, A.B., Winkler, M.E. and Derynck, R. Transforming growth factor a: A more potent angiogenic mediator than epidermal growth factor Science 1986; 232: 1250-1253

Schrieber, A.B., Kenney, J., Kowalski, J., Thomas, K.A., Gimenez-Gallego, G., Rios-Candelore, M., Di Salvo, J., Barritault, D., Courty, J., Courtois, Y., Moenner, M., Loret, C., Burgess, W.H., Mehlam, T., Friesel, R., Johnson, W. and Maciag, T. A unique family of endothelial cell polypeptide mitogens: The antigenic and receptor crossactivity of bovine endothelial cell growth factor, brain derived acidic fibroblast growth factor, and eye- derived growth factor-11 J. Cell Biol. 1985; 101: 1623-1626 (b)

Schuff-Weiner, P., Claus, G., Armstrong, V.W., Kostering, H. and Seidel, D. Enhanced procoagulatary activity (PCA) of human monocytes/macrophages after in vitro stimulation with chemically modified LDL Atherosclerosis 1989; 78: 109-1112

Schuh, J., Fairclough, G.F. Jr. and Haschemeyer, R.H. Oxygen-mediated heterogeneity of apo- low-density lipoprotein Proc. Natl. Acad. Sci., USA 1978; 75: 3173-3177 
Schumann, R.R., van der Bosch, J. van der, Ruller, S., Ernst, M. and Schlaak, M. Monocyte long-term cultivation on microvascular endothelial cell monolayers: morphologic and phenotypic charecterization and comparison with monocytes cultured on tissue culture plastic Blood 1989; 73: 818-826

Schwartz, S.M. Selection and characterization of bovine aortic endothelial cells In Vitro 1978; 14: 966-980

Schwartz, S.M. and Benditt, E.P. Selection and characterization of bovine aortic endothelium Proc. Natl. Acad. Sci., USA 1976; 73: 651-653

Schwartz, S.M. and Benditt, E.P. Aortic endothelia cell replication, Part 1 (Effects of age and hypertension in rat) Circ. Res. 1977; 41: 248255

Schwartz, S.M., Gajdusek, C.M., Reidy, M.A., Selden, S.C. and Haudenschild, C.C. Maintenance of integrity in aortic endothelium Fed. Proc. 1980; 39: 2618-2625

Schwartz, S.M., Gajdusek, C.M., Selden, S.C. III Vascular wall growth control: the role of the endothelium Arteriosclerosis 1981; 1: 107-126

Schwartz, S.M., Stemerman, M.B. and Benditt, E.P. The aortic intima. II. Repair of the aortic lining after mechanical denudation Am. J. Pathol. 1975; 81: 15-30

Schweiger, L., Neufeld, G., Friedman, J., Abraham, J. A., Fieldes, J. C. and Gospodarowicz, D. Capillary endothelial cells express basic fibroblast growth factor, a mitogen that promotes their own growth Nature 1987; 325: 257-259

Scott, J. Thrombogenesis linked to atherogenesis at last Nature 1989; 341: 2223

Seegers, J.C., Aveling, M.L., von Aswegen, C.H., Cross, M., Koch, F. and Joubert, W.S. The cytotoxic effects of estradiol-17 $\beta$, catecholestradiols and methoxyestradiols on dividing MCF-7 and HeLa cells J. Steroid Biochem. 1989; 32: 797-809

Seely, S. The gender gap: Why do women live longer than men? Int. J. Cardiol. 1990; 29: 113-119

Seifert, R.A., Schwartz, S.M. and Bowen-Pope, D.F. Developmentally regulated production of platelet-derived growth factor-like molecules Nature 1984; 311: 669-671

Seillan, C., Ody, C., Russo-Marie, F. and Duval, D. Differential effects of sex steroids on prostaglandin secretion by male and female cultured piglet endothelial cells Prostaglandins 1983; 26: 3-12

Sengel, A. and Stoebner, P. Golgi origins of tubular inclusions in endothelial cells J. Cell Biol. 1970; 44: 223-226

Setiadi, H., Wautier, J.L., Courillan-Mallet, A., Passa, P. and Caen, J. Increased adhesion to fibronectin and Mo-1 expression by diabetic monocytes J. Immunol. 1987; 138: 3230-3234 
Shakhov, lu A., Dorian, B., Daret, D., Desgranges, C. and Schcherbakova, I.A. Lipoprotein-dependent synthesis of prostacyclin in smooth muscle cells Biokhimiia 1988; 53: 118-125

Shalaby, M.R., Laegreid, W.W., Ammann, A.S. and Liggitt, H.D. Tumor necrosis factor -a -associated uterine endothelial injury in vivo: influence of dietary fat Lab. Invest. 1989; 61: 564-570

Shapiro, S., Slone, D., Rosenberg, L., Kaufman, D.W., Stolley, P.D. and Miettinen, O.S. Oral contraceptive use in relation to myocardial infarction Lancet 1979; 1: 743-747

Shasby, D.M., Lird, S.E., Shasby, S.S., Goldsmith, J.S. and Hunninghake, G.W. Reversible oxidant-induced increase in albumin transfer across cultured endothelium: alterations in cell shape and calcium homeostasis Blood 1985; 65: 605-614

Shaude, N., Sussman, I.I., Rosner, F. and Pacholuk, V. Oral contraception and platelet aggregatation Am. J. Obstet. Gynecol. 1978; 132: 303306

Shearman, R.P. Oral contraceptive agents Med. J. Aust. 1986; 144: 201-205

Shechter, I., Fogelman, A.M., Haberland, M.E., Seager, J., Hokom, M.E. and Edwards, P.A. The metabolism of native and malondialdehydealtered low density lipoproteins by human monocytesmacrophages J. Lipid Res. 1981; 22: 63-71

Shelton, J. Prostacyclin from the uterus and woman's cardiovascular advantage Prostaglandins Leukot. Med. 1982; 8: 459-466

Shibata, H., Matsuzaki, T. and Hatano, S. Relationship of relevant factors of atherosclerosis to menopause in japanese women Am. J. Epidemiol. 1979; 109: 420-424

Shimado, Y., Kajo, K., Ito, H., Noda, K. and Matsuo, M. Growth-inhibiting effect of tumor neorosis factor on human umbilical vein endothelial cells is enhanced with advancing age in vitro J. Cell. Physiol. 1990; 142: $31-38$

Shimizu, Y., van Seventer, G.A., Siraganian, R., Wahl, L. and Shaw, S. Dual role of the CD44 moledule in T cell adhesion and activation $\mathrm{J}$. Immunol. 1989; 143: 2457-2463

Shimokado, K., Raines, E.W., Madtes, D.K., Barrett, T.B., Benditt, E.P. and Ross, R. A significant part of macrophage-derived growth factor consists of at least two forms of PDGF Cell 1985; 43: 277-286

Shingu, M., Yoshioka, K., Nobunaga, M. and Yoshida, K. Human vascular smooth muscle cells and endothelial cells lack catalase activity and are susceptible to hyrogen peroxide Inflammation 1985; 9: 309-320

Sieff, C.A. Hematopoietic Growth Factors J. Clin. Invest. 1987; 79: 1549-1557 (a)

Sieff, C.A., Niemeyer, C.M. and Faller, D.V. The role of endothelial cells in haematopoiesis Prog. Clin. Biol. Res. 1987; 251: 13-21 (b) 
Sieff, C.A., Tsai, S. and Faller, D.V. Interleukin 1 induces cultured human endothelial cell production of granulocyte-macrophage colonystimulating factor J. Clin. Invest. 1987; 79: 48-51

Silberbauer, K., Schernthaner,G., Sinzinger, G., Piza-Katzer,H. and Winter, M. Decreased vascular prostacyclin in juvenile onset diabetes $\mathbf{N}$. Engl. J. Med. 1979; 300: 366-367

Silverstein, R.L., Leung, L.L.K. and Nachman, R.L. Thrombospondin: A versatile multi functional glycoprotein Arteriosclerosis 1986; 6: 245-253

Sirbasku, D.A., Officer, J.B., Leiand, F.E. and lio, M. Evidence of a new role for pituitary-derived hormones and growth factors in mammary tumor cell growth in vivo and in vitro Cold Spring Harbor Conf. Cell Prolif. 1982; 9: 763-778

Sitaras, N.M., Sariban, E., Pantazis, P., Zetter, B. and Antoniades, H.N. Human iliac artery endothelial cells express both genes encodong the chains of platlelet-derived growth factor(PDGF) and synthesize PDGF-like mitogen J. Cell. Physiol. 1987; 132: 376-380

Slack, J. Genetic influences on coronary heart disease in women In: Myocardial Infarction in Women, Oliver, M.F., Vedin, A. and Wilhelmsson, C. (eds.), Churchill Livingstone, UKK 1986; 9-17

Slack, J. and Evans, K.A. The increased risk of death of ischaemic heart disease in first degree relatives of 121 men and 96 women with ischaemic heart disease J. Med. Genet. 1966; 3: 239-257

Slater, D.N. and Sloan, J.M. The porcine endothelial cell in tissue culture Atherosclerosis 1975; 21: 259-272

Slater, T. Free-radical mechanisms in tissue injury Biochem. J. 1984; 222: 115

Smart, J.V. Elements of Medical Statistics, Staples Press, London 1970; 8099, 115-121

Smith, E.B. and Staples, E.M. Intimal and medial protein concentrations and endothelial function Atherosclerosis 1982; 41: 295-308

Smith, E..B. and Staples, E.M. Distribution of plasma proteins across the human aortic wall Atherosclerosis 1980; 37: 579-590

Smith, W. Prostaglandins biosynthesis and its compartmentation in vascular smooth muscle and endothelial cells Ann. Rev. Physiol. 1986; 48: 251-62

Sneddon, J.M. and Vane, J.R. Endothelium-derived relaxing factor reduces platelet adhesion to bovine endothelial cells Proc. Natl. Acad. Sci. USA 1988; 85: 2800-2804

Solberg, L.A. and Strong, J.P. Risk factors and atherosclerotic lesions Arteriosclerosis 1983; 3: 187-198

Sonnendecker, E.W.W., Polakow, E.S., Benade, A.J.S. and Simchowitz, E. Serum lipoprotein effects of conjugated estrogen and a sequential conjugated estrogen medrogestone regimen in hysterectomized 
post menopausal women Am. J. Obstet. Gynecol. 1989; 160: 1128-34

Soto, A.M. and Sonnenschein, C. Mechanism of estrogen action on cellular proliferation: evidence for indirect and negative control on cloned breast tumor cells Biochem. Biophys. Res. Commun. 1984; 122: $1097-1103$

Soto, A.M. and Sonnenschein, C. The role of estrogens on the proliferation of human breast tumor cells (MCF-7) J. Steroid Biochem. 1985; 23: 87-94

Soto, A.M. and Sonnenschein, C. Cell proliferation of oestrogen-sensitive cells: the case for negative control Endocr. Rev. 1987; 8: 44-52

Souadjian, J.V., Kottke, B.A. and Titus, J.L. Estrogen effect on spontaneous atherosclerosis Arch. Pathol. 1968; 85: 463-467

Soule, H.D. and McGrath, C.M. Estrogen responsive proliteration of clonal human breast carcinoma in athymic mice Cancer Lett. 1980; 10: 177-189

Spaet, T.H., Stemerman, M.B., Veith, F.J. and Lejneiks, I. Intimal injury and regrowth in the rabbit aorta Circ. Res. 1975; 36: 58-70

Span, A.H., Endert, J., van Boven, C.P. and Bruggeman, C.A. Virus induced adherence of monocytes to endothelial cells FEMS Microbiol. Immunol. 1989; 4: 237-244

Spellacy, W.N. and Birk, S.A. The effect of intra-uterine devices, oral contraceptives, estrogens, and progestogens on blood pressure Am. J. Obstet. Gynecol. 1972; 112: 912-919

Sporn, I.A., Rubin, P., Marder, V.J. and Wagner, D.D. Irradiation induces releases of von Willebrand protein from endothelial cells in culture Blood 1984; 64: 567-570

Sprague, E.A., Moser, M., Edwards, E.H. and Schwartz, C.J. Stimulation of receptor-mediated low density lipoprotein endocytosis in neuraminidase-treated cultured bovine aortic endothelial cells $\mathbf{J}$. Cell. Physiol. 1988; 137: 251-262

Stack, J. Genetic influences on coronary heart disease in women. In: Myocardial Infarction in Women, Oliver, M.F., Vedin, A. and Wilhelmsson, C. (eds.), Churchill Livingstone, London 1986; 9-17

Stadel, B.V. Oral contraceptives and cardiovascular disease N. Engl. J. Med. 1981; 305: 612-618 (a)

Stadel, B.V. Oral contraceptives and cardiovascular disease N. Engl. J. Med. 1981; 305: 672-678 (b)

Stadel, B.V., Sternthal, P.M., Schlesselman, J.J., Douglas, M.B., Hall, W.D., Kaul, L.and Ahluwalia, B. Variation of ethinylestradiol blood levels among healthy women using oral contraceptives Fertil. Steril. 1980; 33: 257-260 
Staiano-Coico, L., Hajjar, D.P., Hefton, J.M., Hajjar, K.A. and Kimmel, M. Interactions of aterial cells:111. stathmokinetic analyses of smooth muscle cell co-cultured with endothelial cells J. Cell. Physiol. 1988; 134: 485-490

Stamler, J., Pick, R., Katz, L.N., Pick, A., Kaplan, B.M., Berkson, D.M. and Century, D. Effectiveness of estrogens for therapy of myocardial infarction in middle-age men JAMA 1963; 183: 632-638

Stampfer, M.J. Smoking, estrogen and prevention of heart disease in women Mayo Clin. Proc. 1990; 64: 1553-1557

Stampfer, M.J., Colditz, G.A., Willett, W.C., Manson, J.E., Rosner, B., Speizer, F.E. and Hennekens, C.H. Postmenopausal estrogen therapy and cardiovascular disease N. Engl. J. Med. 1991; 325: 756-762

Stampfer, M.J., Sacks, F.M., Salvini, S., Willett, W.C. and Hennekens, C.H. A prospective study of cholesterol, apolipoproteins, and the risk of myocardial infarction N. Engl. J. Med. 1991; 325: 373-381

Stampfer, M.J., Willett, W.C., Colditz, G.A., Rosner, B., Speizer, F.E. and Hennekens, C.H. A prospective study of post menopausal estrogen therapy and coronary heart disease N. Engl. J. Med. 1985; 313: 1044

Stampfer, M.J., Willett, W.C., Colditz, G.A., Speizer, F.E. and Hennekens, C.H. A prospective study of past use of oral contraceptive agents and risk of cardiovascular diseases N. Engl. J. Med. 1988; 319: 13131317

Starksen, N.F., Harsh, G.R., Gibbs, V.C. and Williams, L.T. Regulated expression of the platelet-derived growth factor A chain gene in microvascular endothelial cells J. Biol. Chem. 1987; 262: 14381-14384

Stary, H.C. What are initial, early and intermediate stages of atherosclerotic lesions Newsletter, Council on Arteriosclerosis, Am. Heart Assoc. 1991; : 15-16

Staunton, D.E., Dustin, M.L. and Springer, T.A. Functional cloning of ICAM-2, a cell adhesion ligand for LFA-1 homologous to ICAM-1 Nature 1989; 339: 61-64

Stein, O. and Stein, Y. Bovine aortic endothelial cells display macrophage-like properties towards acetylated 125/-labelled low density lipoprotein Biochim. Biophys. Acta 1980; 620: 631-635

Stein, O. and Stein, Y. High density lipoproteins reduce the uptake of low density lipoproteins by human endothelial cells in culture Biochem. Biophys. Acta 1976; 431: 363-368

Stein, O., Stein, Y. and Eisenberg, S. A radioautographic study of the transport of $125 \mathrm{I}$-labeled serum lipoproteins in rat aorta $\mathrm{Z}$. Zellforsch 1973; 138: 223-237

Stein, $Y$ and Stein, O. Growth factors, cytokines and the development of atheroma Lipid Rev. 1990; 4: 57-62 
Steinberg, D. Antioxidants and atherosclerosis Circulation 1991; 84: 14201425

Steinberg, D Atherosclerosis from the view point of cell biology In:

Atherosclerosis-Development, Complications and Treatment, Elsevier Science, B.V. 1987; 3-20 (a)

Steinberg, D. Lipoproteins and atherosclerosis: a look back and a look ahead Arteriosclerosis 1983; 3: 283-301

Steinberg, D. Lipoproteins and the pathogenesis of atherosclerosis Circulation 1987; 76: 508-514 (b)

Steinberg, D. Metabolism of lipoproteins and their role in the pathogenesis of atherosc/erosis. Atherosclerosis Reviews 1988; 18:

Steinberg, D., Parthasarathy, S., Carew, T.E., Khoo, J.C. and Witztum, J.L. Beyond Cholesterol: modifications of low-density lipoprotein that increase its atherogenicity New Engl.J. Med. 1989; 320: 915924

Steinbrecher, U.P., Lougheed, M., Kwan, W.-C. and Dirks, M. Recognition of oxidized low density lipoprotein by the scavenger receptor of macrophages results from derivatization of apolipoprotein $B$ by products of fatty acid peroxidation J. Biol. Chem. 1989; 64: 15216-15223

Steinbrecher, U.P., Zhang, H. and Lougheed, M. Role of oxidatively modified $L D L$ in atherosclerosis Free Radic. Biol. Med. 1990; 9: 155-168

Steinbrecher, U.P., Parthasarathy, S., Leake, D.S., Witztum, J.L. and Steinberg, D. Modification of low density lipoprotein by endothelial cells involved lipid peroxidation and degradation of low density lipoprotein phospholipids Proc. Natl. Acad. Sci. USA 1984; 81: 3883-3887

Steinbrecher, U.P., Witztum, J.L., Parthasarathy, S. and Steinberg, D. Decreases in reactive amino groups during oxidation of endothelial cell modification of LDL Atherosclerosis 1987; 7: 135-143

Stemerman, M.B. and Ross, R. Experimental arteriosclerosis. I. Fibrous plaque formation in primates: An electron microscope study J. Exp. Med. 1972; 136: 769-789

Sternby, N.H. Sex differences in atherosclerosis. In: Myocardial Infarction in Women, Oliver, M.F., Vedin, A. and Wilhelmsson, C. (eds.), Churchill Livingstone, UK 1986; 166-172

Stock, J.L., Coderre, J.A., McDonald, B. and Rosenwasser, L.J. Effects of estrogen in vivo and in vitro on spontaneous interleukin-1 release by monocytes from postmenopausal women J. Clin. Endocrinol. Metab. 1989; 68: 364-368

Stocker,R., Bowry, V.W amd Frei, B. Ubiquinol-10 protects human low density lipoprotein more efficiently against lipid peroxidation than does alpha-tocopherol Proc. Nati. Acad. Sci. USA 1991; 88: 16461650 
Stokes, J. III, Kannel, W.B., Wolf, P.A., Cupples, L.A. and D'agostino, R.B. The relative importance of selected risk factors for various manifestations of cardiovascular disease among men and women from 35 to 64 . 30 years of follow-up in the Framingham Study Circulation 1987; 75(suppl.V): V65-V73

Stokes, T. and Wynn, V. Serum-lipids in women on oral contraceptives Lancet 1971; 2: 677-680

Stolley, P.D., Tonascia, J.A., Tockman, M.S., Sartwell, P.E., Rutledge, A.H. and Jacobs, M.P. Thrombosis with low-estrogen oral contraceptives Am. J. Epidemiol. 1975; 102: 197-208

Streeton, E.A., Ornberg, R., Curcio, F., Sakaguchi, K., Marx, S., Aurbach, G.D., Brandi, M.L. Cloned endothelial cells from fetal bovine bone Proc. Natl. Acad. Sci., USA 1989; 86: 916-920

Strieter, R.M.,Kunkel, S.L., Showell, H.J., Remick, D.G., Phan, H., Ward, P.A. and Marks, R.M. Endothelial cell gene expression of a neutrophil chemotactic factor by TNF- $\alpha$, LPS and IL-1 $\beta$ Science 1989; 243: 1467-1469 (a)

Strieter, R.M., Wiggins, R., Phan, S.H., Wharram, B.L., Showell, H.J .,Remick, D.G., Chensue, S.W. and Kunkel, S.L. Monocyte chemotactic protein gene expression by cytokine-treated human fibroblasts and endothelial cells Biochem. Biophys. Res. Commun. 1989; 162 : $694-700$ (b)

Stumpf, W.E. Steroid hormones and the cardiovascular system: direct actions of estradiol, progesterone, testosterone, gluco-and mineral corticoids and soltriol (vitamin D) on central nervous regulatory and peripheral tissues Experientia 1990; 46: 13-25

Subbiah, M.T.R. Effect of estrogens in the activities of cholesteryl ester synthetase and cholesteryl ester hydrolases in pigeon aorta Steroids 1977; 30: 259-265

Subbiah, M.T.R., Deitemeyer, D., Yunker, R. and Gallon, L. Effects of specific estrogens on prostaglandin synthesis in aorta and thrombocytes of female pigeons Proc. Soc. Exp. Biol. Med. 1981; 166: 300304

Sugioka, K., Shimosegaw, Y. and Nakano, M. Estrogens as natural antioxidants of membrane phospholipid peroxidation FEBS Lett. 1987; 210: 37-39

Suits, A.G., Chait, A., Aviram, M. and Heinecke, J.W. Phagocytosis of aggregated lipoprotein by macrophages: low density lipoprotein receptor-dependent foam-cell formation Proc. Natl. Acad. Sci. USA 1989; 86: 2713-2717

Sullivan, J.L. Iron and the sex difference in heart disease risk Lancet 1981; 1: 1293-1294

Suzuki, H., Shibano, K., Okane, M., Kono, I., Matsui, Y. Yamane, K. and Kashiwagi, $\mathrm{H}$. Interferon- $Y$ modulates messenger $R N A$ levels of $C$ sis (PDGF-B chain), PDGF-A chain, and IL-I $\beta$ genes in human vascular endothelial cells Am. J. Pathol. 1989; 134: 35-43 
Svanborg, A. and Vikrot, O. Plasma lipids during the menstrual cycle Acta Med. Scand. 1967; 181: 93-96

Svensson, J. and Fredholm, B.B. Vasoconstrictor effect of thromboxane $A_{2}$ Acta. Physiol. Scand. 1977; 101: 366-368

Syms, A.J., Norris, J.S. and Smith, R.G. Autocrine regulation of growth I . glucocorticoid inhibition is overcome by exogenous plateletderived growth factor Biochem. Biophys. Res. Commun. 1984; 122: 68-74

Sytkowski, P.A., Kannel, W.B. and D'Agostino, R.B. Changes in risk factors and the decline in mortality from cardiovascular disease: the Framingham Heart Study N. Engl. J. Med. 1990; 322: 1635-1641

Szczeklik, A. and Gryglewski, R.J. Low density lipoproteins $(L D L)$ are carriers for lipid peroxides and inhibit prostacyclin $\left(\mathrm{PGI}_{2}\right)$ biosynthesis in arteries Artery 1980; 7: 488-495

Szondy, E., Horvath, M., Mezey, Z., Szaekely, J., Lengyel, E., Fust, G. and Gero, S. Free and complexed antilipoprotein antibodies in vascular diseases Atherosclerosis 1983; 49: 69-77

Tabibzadeh, S.S., Santhanam, U., Sehgal, P.B. and May, L.T. Cyotkine-induced production of IFN-B2/IL-6 by freshly explanted human endometrial stromal cells. Modulation by estradiol-17 $\beta$ J. Immunol. 1989; 142: 3134-3139

Takehara, K., LeRoy, E.C. and Grotendorst, G.R. TGF- $\beta$ inhibition of endothelial cell proliferation: alteration of EGF binding and EGF induced growth-regulatory /competence gene expression Cell 1987; 49: 415-422

Tamura, H., Tanaka, S., Obayashi, T., Yoshida, M. and Kawai, T. A new sensitive method for determining endotoxin in whole blood Clinica. Chimica. Acta 1991; 200: 35-42

Tappel, A.L. Lipid peroxidation damage to cell components Fed. Proc. 1973; 32: 1870-1874

Tappel, A.L. In: Free Radicals in Biology Pryor, W.A. (ed.), Academic Press, N.Y. 1980; 4: 1-47

Tauber, J.-P., Cheng, J. and Gospodarowicz, D. Effect of high and low density lipoproteins on proliferation of culture bovine vascular endothelial cells J. Clin. Invest. 1980; 66: 696-708

Taylor, R.G. and Lewis, J.C. Endothelial cell proliferation and monocyte adhesion to atheroscerlotic lesions of white carneau pigeons Am. J. Pathol. 1986; 125: 152-160

Territo, M., Berliner, J., Ramirez, R. and Fogelman, A. Effect of lipoprotein on endothelial binding of monocytes Arteriosclerosis 1987; 7: 496a

Territo, M.C., Berliner, J.A., Almada, L., Ramirez, R. and Fogelman, A.M. BVLDL pretreatment of endothelial monolayers increases monocyte but not neutrophil adhesion Arteriosclerosis 1989; 9: 824-828 
Tertov, V.V., Orekhov, A.N. Martsenyuk, O.V., Perova, N.V. and Smirnov, V.N. Low-density lipoprotein isolated from the blood of patients with coronary heart disease induce the accumulation of lipids in human aortic cells Exp. Mol. Pathol. 1989; 50: 337-347 (a)

Tertov, V.V., Sobenin, I.A., Gabbasov, Z.A., Popov, E.G. and Orekhov, A.N. Lipoprotein aggregration as an essential condition of intracellular lipid accumulation caused by modified low density lipoproteins Biochem. Biophys. Res. Commun. 1989; 163: 489-494 (b)

TeVelde, A.A., Keizer, G.D. and Figdor, C.G. Differential function of LFA-1 family molecules (CD11 and CD18) in adhesion of human monocytes to melanoma and endothelial cells Immunology 1987; 61: 261-267

The Coronary Drug Project Research Group. The Coronary Drug Project Findings leading to discontinuation of the $2.5 \mathrm{mg} /$ day estrogen group JAMA 1973; 226: 652-657

The Coronary Drug Project Research Group. The Coronary Drug Project Initial findings leading to modifications of its research protocol JAMA 1970; 214: 1303-1313

The Lipid Research Clinics Program Epidemiology Committee Plasma lipid distributions in selected North American populations. The Lipid Research Clinics Program Prevalence Study Circulation 1979; 60: $4267-439$

Thelle, D. Women and coronary heart disease: a review with special emphasis on some risk factors Lipid Rev.; 4: 33-39

Thomas, K.A., Rios-Candelore, M., Gimenez-Gallego, G., Disalvo, J., Bennett, C., Rodkey, J. and Fitzpatrick, S. Pure brain-deived acid fibroblast growth factor is a potent angiogenic vascular endothelial cell mitogen with sequence homology to interleukin 1 Proc. Natl. Acad. Sci. USA 1985; 82: 6409-6413

Thompson, E.A. Steroid regulation of rRNA synthesis In: Steroid and Sterol Hormone Action, Spelsberg, T.C. and Kumar, R. (eds.), Martinus Nijhoff Publishing, UK 1987; 227-249

Thorgeirsson, G. and Robertson, A.L. Jr. Platelet factors and the human vascular wall Atherosclerosis 1978; 30: 67-78

Thorgeirsson, G., Robertson, A.L. Jr. and Cowan, D.H. Migration of human vascular endothelial and smooth muscle cells Lab. Invest. 1979; 41: 51-62

Thornton, S.C., Mueller, S.N. and Levine, E.M. Human endothelial cells: use of heparin in cloning and long-term serial cultivation Science 1983; 222: 623-625

Thyberg, J., Ostman, A., Bsackstrsom, G., Westermark, B. and Heldin, C.-H. Localization of platelet-derived growth factor (PDGF) in $\mathrm{CHO}$ cells trransfected with PDGF A- or B-chain CDNA: retention of PDGF$B B$ in the endoplasmic reticulum and golgi complex J. Cell Sci. 1990: 97; 219-229 
Tikkanen, M.J. and Nikkila, E.A. Regulation of hepatic lipase and serum lipoproteins by sex steroids Am. Heart J. 1987; 113: 562

Tikkanen, M.J., Nikkil, E.A., Kuusi, T. and Sipinen, S. Different effects of two progestins on plasma high density lipoproteins $\left(\mathrm{HDL}_{2}\right)$ and postheparin plasma hepatic lipase activity Atherosclerosis 1981; 40: 365-369

Tikkanen, M.J., Nikkil, E.A., Vartiainen, E. Natural oestrogen as an efective treatment for type-Il hyperlipoproteinaemia in postmenopausal women Lancet 1978; 2: 490-491

Tong, B.D., Auer, D.E., Jaye, M., Kaplow, J.M., Ricca, G., McConathy, E., Drohan, W. and Deuel,T.F. cDNA clones reveal differences between human glial and endothelial cell platelet-derived growth factor A-chains Nature 1987; 328: 619-621

Tracey, G.P., Brown, D.E., Johnson, L.W. and Gottlieb, A.J. Radiation induced coronary artery disease JAMA 1974; 228: 1660-1662

Triau, J.E., Meydani, S.N. and Schaefer, E.J. Oxidized low density lipoprotein stimulates prostacyclin production by adult human vascular endothelial cells Arteriosclerosis 1988; 8: 810-818

Tschopp, T.B., Weiss, H.J. and Baumgartner, H.R. Decreased adhesion of platelets to subendothelium in von Willebrand's disease J. Lab. Clin. Med. 1974; 83: 296-300

Tsuchida, M., Miura, T., Mizutani, K. and Aibara, K. Fluroescent substances in mouse and human sera as a parameter of in vivo lipid peroxidation Biochim. Biophys. Acta 1985; 834: 196-204

Tuddenham, E.G.P., Lazarchick, J. and Hoyer, L.W. Synthesis and release of factor VIII by cultured human endothelial cells Br. J. Haematol. $1981 ; 47: 617-626$

Tuszynski, G.P., Rothman, V., Murphy, A., Siegler, K., Smith, L., Smith, S., Karczewski, J. and Knudsen, K. A. Thrombospondin promotes cell-substratum adhesion Science 1987: 236: 1570-1573

Tzeng, D.Y., Deuel, T.F., Huang, J.S. and Bachner, R.L. Platelet-derived growth factor promotes human peripheral monocyte activation Blood 1985; 66: 179-183

Usuki, K., Heldin, N.-E., Miyazono, K., Ishikawa, F., Takaku, F., Westermark, B. and Heldin, C.-H. Production of platelet-derived endothelial cell growth factor by narrow and transformed human cells on culture Proc. Natl. Acad. Sci., USA 1989; 86: 7427-7431

Utermann, G. The mysteries of lipoprotein (a) Science 1989 246; 904-910

Valente, A.J., Sprague, E.A., Nassiri, M.R., Wadington, S., Kelley, J.L., Suenram, C.A. and Schwartz, C.J. Influence of plasma proteins on blood monocyte adherence to vascular endothelium Arteriosclerosis 1984; 4: 540a

Van der Burg, B., de Laat, S.W., van Zoelen, E.J.J. Mitogenic stimulation of human breast cancer cells in a growth factor-defined medium: 
synergistic action of insulin and estrogens. In: Progress in Cancer Research and Therapy, : Hormones and Cancer 3, Bresciani, F., King, R.J.B., Lippman, M.E. and Raynaud, P. Jr.(eds.), Raven Press Ltd, NY 1988; 35: 231-233

Van Erpecum, K.L., van Berge Henegouwen, G.P., Verschoor, L., Stoelwinder, B. and Willekens, F.L.H. Different hepatobiliary effects of oral and transdermal estradiol in post menopausal women

Gastroenterology 1991; 100: 482-488

Van Hinsbergh, V.W.M., Binnema, D., Scheffer, M.A., Sprengers, E.D. Kooistra, T. and Rijken, D.C. Production of plasminogen activators and inhibitors by serially propagated endothelial cells from adult human blood vessels Arteriosclerosis 1987; 7: 389-700

Van Hinsbergh, V.W.M., Havekes, L., Emeis, J.J., van Corven, E. and Scheffer, M. Low density lipoprotein metabolism by endothelial cells from human umbilical cord arteries and veins Arteriosclerosis 1983; 3: 547-559

Van Hinsbergh, V.W.M., Scheffer, M., Havekes, L. and Kempon, H.J.M. Role of endothelial cells and their products in the modification of lowdensity lipoproteins Biochem. Biophys. Acta 1986; 878: 49-64

Vane, J.R, Anggard, E.E. and Botting, R.M. Regulatory functions of the vascular endothelium New Engl. J. Med. 1990; 323: 27-36

Vane, J.R., Bunting, S. and Moncado, S. Prostacyclin in physiology and pathophysiology Int. Rev. Exp. Pathol. 1982; 23: 161-207

Vasile, E., Simionescu, M. and Simionescu, N. Visualization of the binding, endocytosis and transcytosis of low density lipoproteins in the arterial endothelium in situ J. Cell Biol. 1983; 96: 1677-1689

Vedeckis, W. V., Eastman-Reks, S. B., Lapointe, M. C. and Reker, C. E. Glucocorticoid regulation of protooncogene expression and cellular proliferation In: Steroid and Sterol Hormone Action Spelsberg, T.C. and Kumar, R. (eds.), Martinus Nijhoff Publishing, Boston 1987; : 213-226

Vercellotti, G.M., Dodson, M., Schorer, A.E. and Moldow, C.F. Endothelial cell heterogencity: antioxidant profiles determine vulnerability to oxidant injury Proc. Soc. Exp. Biol. Med. 1988; 187: 181-189

Vernon-Roberts, B. The effects of steroid hormones on macrophage activity Int. Rev. Cytol. 1969; 25: 131-159

Vessey, M.P. and Doll. R. Investigation of the relation between use of oral contraceptives and thromboembolic disease BMJ 1968; 2: 199205

Vessey, M.P. and Doll, R. Investigation of relationship between use of oral contraceptives and thromboembolic disease: a further report BMJ 1969; 2: 651-657

Vessey, M.P. and Mann, J.I. Female sex hormones and thrombosis. Epidemiological aspects Br. Med. Bull. 1978; 34: 157-162 
Vessey, M.P., McPherson, K. and Johnson, B. Mortality among women participating in the Oxford family planning association contraceptive study Lancet 1977; 2: 731-733

Vignon, F., Capony, F., Chambon, M., Freiss, G., Garcia, M. and Rochefert, H. Autocrine growth stimulation of the MCF-7 breast cancer cells by the estrogen-regulated 52K protein Endocrinology 1986; 118: 1537-1545

Vijayagopal, P., Srinivasan, S.R., Radnakrishnamurthy, B. and Berenson, G.S. Factors regulating the metabolism of low-density lipoproteinproteoglycan complex in macrophages Biochim. Biophys. Acta 1990; 1042: 204-209

Vilette, D., Setiadi, H., Wautier, M.-P., Caen, J. and Wautier, J.-L. Identification of an endothelial cell growth-inhibitory activity produced by human monocytes Exp. Cell Res. 1990; 188: 219-225

Vlodavsky, I. Fridman, R., Sullivan, K., Sasse, J. and Klagsbrun, M. Aortic endothelial cells synthesize basic fibroblast growth factor which remains cell-associated and PDGF-like protein which is secreted J. Cell. Physiol. 1987; 131: $402-408$ (a)

Vlodavsky, I., Folkman, J., Sullivan, R., Fridman, R., Ishai-Michael, R., Sasse, J. and Klagsbrun, M. Endothelial cell-derived basic fibroblast growth factor: synthesis and deposition into subendothelial extracellular matrix Proc. NatI. Acad. Sci. USA 1987; 84: 22922296 (b)

Wagner, D.D., Olmsted, J.B. and Marder, V.J. Immunolocalization of von Willebrand protein in Weibel-Palade bodies of human endothelial cells J. Cell Biol. 1982; 95: 355-360

Wagner, J.D., Clarksen, T.B., St Clair, R.W., Schwenke, D.C. and Adams, M.R. Estrogen replacement therapy (ERT) and coronary artery (CA) atherogenesis in surgically post menopausal cynomolgus monkeys Circulation (Suppl) 1989; 84: 11-331

Wahl, P.W., Walden, C.E. and Knopp, R.H., Hoover, J., Wallace, R., Heiss, G. and Rifkind, B. Effects of estrogen/progestin potency on lipid/lipoprotein cholesterol N. Engl. J. Med. 1983; 308: 862867

Wakasugi, M., Noguchi, T., Kazama, Y.-I., Kanemaru, Y. and Onaya, T. The effects of sex hormones on the synthesis of prostacylin (PG/2) by vascular tissues Prostaglandins 1989; 37: 401-410

Walker, L.N. and Bowyer, D.E. Endothelial healing in the rabbit aorta and the effect of risk factors for atherosclerosis Arteriosclerosis 1984; 4: 479-489

Walker, P., Weichsel, M.E., Hoath, S.B., Poland, R.E. and Fisher, D.A. Effect of thyroxine testosterone, and corticosterone on nerve growth factor (NGF) and epidermal growth factor (EGF) concentrations in adult female mouse submaxillary gland: dissociation of NGF and EGF responses Endocrinology 1981; 109: 582-587 
Wall, R.T., Harker, L.A., Quadracci, L.J. and Striker, G.E. Factors influencing endothelial cell proliferation in vitro J. Cell. Physiol. 1978; 96: 203-214

Wallace, M.R. Oral contraceptives and severe hypertension Aust. NZ J. Med. 1971; 1 : 49-52

Wallace, R.B., Hoover, J., Barrett-Connor, E., Rifkind, B.M., Hunninghake, D.B., Mackenthun, A. and Heiss, G. Altered plasma lipid and lipoprotein levels associated with oral contraceptive and oestrogen use. Report from the medications working group of the lipid research clinic program Lancet 1979; 2: 112-115 (a)

Wallace, R.B., Hoover, J., Sandler, D., Rikind, B.M. and Tyroler, H.A. Altered plasma-lipids associated with oral contraceptive or oestrogen consumption. The lipid research clinic program Lancet 1977; 2: 11-14 (b)

Walmsley, T.A., Grant, S. and george, P.M. Effect of plasma triglyceride concentrations on the accuracy of immunoturbidimetric assays of apolipoprotein B Clin. Chem. 1991; 37: 748-753

Wang, J., Zhen, E., Guo, Z. and Lu, Y. Effect of hyperlipidemic serum on lipid peroxidation synthesis of prostacyclin and thromboxane by cultured endothelial cells: protective effect of antioxidants Free Radic. Biol. Med. 1989; 7: 243-249

Warso, M.A. and Lands, W.E.M. Lipid peroxidation in relation to prostacyclin and thromboxane physiology and pathophysiology Br. Med. Bull. 1983; 39: 277-280

Waterfield, M.D., Scrace, G.T., Whittle, N., Stroobant, P., Johnsson, A., Wasteson, A., Westermark, B., Heldin, C.-H, Huang, J.S. and Deuel, T.F. Platelet-derived growth factor is structurally related to the putative transforming protein p28( $v$-sis) of simian sarcoma virus Nature 1983; 304: 35-39

Watkins, M.R., Sharefkin, J.B. and Zajtchuk, R. Adult human saphenous vein endothelial cells: assessment of their reproductive capacity for use in endothelial seeding of vascular prostheses J. Surg. Res. 1984; 36: 588-596

Wechezak, A.R., Mansfield, P.B. and Way, S.A. Platelet interaction with cultured endothelial cells following in vitro injury Artery 1975; 1: 507-517

Weibel, E.R. and Palade, G.E. New cytoplasmic components in arterial endothelial cells J. Cell Biol. 1964; 23: 101-112

Weigensberg, B.I., Lough, J. and More, R.H., Katz, E., Pugash, E. and Peniston, C. Effects of estradiol on myointimal thickenings from catheter injury and on organizing white mural non-occlusive thrombi Atherosclerosis 1984; 52: 253-265

Weinberg, K.S., Douglas, W.H.J., MacNamee, D.R., Lanzillo, J.J. and Fanburg, B.L. Angiotensin 1-converting enzyme localization on cultured fibroblasts by immunofluorescence In Vitro Cell Dev. Biol. 1982; 18: $400-406$ 
Weinstein, R. and Weng, K. Growth factor responses of human arterial endothelial cells in vitro In Vitro Cell Dev. Biol. 1986; 22: 549556

Weir, R.J. and Robertson, J.I.S. Oral contraception: hormonal influences on blood pressure. In: Myocardial Infarction in Women, Oliver, M.F., Vedin, A. and Wilhelmsson, C. (eds.), Churchill Livingstone, London 1986; 54-64

Weisgraber, K.H., Innerarity, T.L. and Mahley, R.W. Role of the lysine residues of plasma lipoproteins in high affinity binding to cell surface receptors on human fibroblasts J. Biol. Chem. 1978; 253: 90539062

Weiss, S.J., Young, J., Lobuglio, A.F., Slivka, A. and Nimeh, N.F. Role of hydrogen peroxide in neutrophil-mediated destruction of cultured endothelial cells J. Clin. Invest. 1981; 68: 714-721

Weisz, A. and Bresciani, F. Estrogen induces expression of c-fos and c-myc protooncogenes in rat uterus Mol. Endocrinol. 1988; 2: 816-24

Weisz, A., Martini, P.E., Scalana, M., Cicatiello, L. and Bresciani, F. Rapid sequential induction of $c$-fos and c-myc gene expression in rat uterus by estrogen. In: Progress in Cancer Research and Therapy: Hormones and Cancer 3, Bresciani, F., King, R.J.B., Lippman, E. and Raynaud, P. Jr. (eds.), Raven Press Ltd, NY 1988; 35: 122-129

Weksler, B.B., Ley, C.W. and Jaffe, E.A. Stimulation of endothelial cell prostacyclin produced by thrombin, trypsin and the ionophore A23187 J. Clin. Invest. 1978; 62: 923-930

Weksler, B.B., Marcus, A.J. and Jaffe, E.A. Synthesis of prostaglandin $I_{2}$ (prostacyclin) by cultured human and bovine endothelial cells Proc. Natl. Acad. Sci., USA 1977; 74: 3922-3926

Werko, L. The enigma of coronary heart disease and its prevention Acta Med. Scand. 1987; 221: 323-33

Westermark, B. and Heldin, C.-H. Structure and function of platelet-derived growth factor Acta Med. Scand. 1986; 715: 19-23

Westermark, B. and Heldin, C.-H. Platelet-derived growth factor: structural and functional aspects J. Intern. Med. 1989; 225: 55-58

Westley, B. and Rochefort, H. A secreted glycoprotein induced by estrogen in human breast cancer cell lines Cell 1980; 20: 352-362

Whatley, R.E., Zimmerman, G.A., Mcintyre, T.M., Taylor, R. and Prescott, S.M. Production of platelet-activating factor by endothelial cells Semin. Thromb. Hemost. 1987; 13: 445-453

Whatley, R.E., Nelsen, P., Zimmerman, G.A., Stevens, D.L., Parker, C.J. Mclntyre,T.M. and Prescott, S.M. The regulation of plateletactivating factor production in endothelial cells J. Biol. Chem. 1989; 264: 6325-6333 
Whatley, R.E., Zimmerman, G.A., McIntyre, T.M. and Prescott, S.M. Lipid metabolism and signal transduction in endothelial cells Prog. Lipid Res. 1990; 29: 45-63

Wheeler, M.E., Luscinskas, F.W., Bevilacqua, M.P. and Gimbrone, M.A. Jr. Cultured human endothelial cells stimulated with cytokines or endotoxin produce an inhibitor of leukocyte adhesion J. Clin. Invest. 1988; 82: 1211-1218

Whisler, R.L., Cornwell, D.G., Proctor, K.V.W. and Downs, E. Bacterial lipopolysaccharide acts on human endothelial cells to enhance the adherence of peripheral blood monocytes J. Lab. Clin. Med. 1989; 114: 708-716

Wight, T.N. and Ross, R. Proteoglycans in primate arteries J. Cell Biol. 1975; 67: $660-674$

Wilhelmsen, L., Svardsudd, K., Korsan-Bengtsen, K., Larsson, B., Welin, L. and Tibblin, G. Fibrinogen as a risk factor for stroke and myocardial infarction N. Engl. J. Med. 1984; 311: 501-505

Williams, J.K., Adams, M.R. and Klopfenstein, H.S. Estrogen modulates responses of atherosclerotic coronary arteries Circulation 1990; 81: 1680-1687

Wilson, J.E. and Foster, D.W. Special endocrine tests. In: Williams, Textbook of Endocrinology, 7th edition, W.B. Saunders Company, Sydney 1985; back page

Wingard, D.L. Sex differences and coronary heart disease Circulation 1990; 81: 1710-1712

Winkles, J.A., Friesel, R., Burgess, W.H., Howk, R., Mehlman, T., Weinstein, R. and Maciag, T. Human vascular smooth muscle cells both express and respond to heparin-binding growth factor 1 (endothelial cell growth factor) Proc. Natl. Acad. Sci., USA 1987; 84: 7124-7128

Wiseman, L.R., Wakeling, A.E., May, F.E.B. and Westley, B.R. Effects of the antioestrogen, IC1 164,384 on oestrogen induced RNAs in MCF-7 cells J. Steroid Biochem. 1986; 33: 1-6

Wissler, R. Update on the pathogenesis of Atherosclerosis Am. J. Med. 1991; 91 (suppl 1B): 3S-9S

Witteman, J.C.M., Grobbee, D.E., Kok, F.J., Hofman, A. and Valkenburg, H.A. Increased risk of atherosclerosis in women after the menopause BMJ 1989; 298: 642-644

Witter, F.R. and DiBlasi, M.C. Effect of steroid hormones on arachidonic acid metabolites on endothelial cells Obstet. Gynecol. 1984; 63: 747751

Wolfe, B.M. and Huff, M.W. Effects of combined estrogen and progestin administration of plasma lipoprotein metabolism in post menopausal women J. Clin. Invest. 1989; 83: 40-45 
Wolinsky, $\mathrm{H}$. Effects of estrogen treatment on the oophorectomized female rate aorta Proc. Soc. Exp. Biol. Med. 1973; 144: 864-867

Wood, D.A., Bulter, S., Riemersa, R.A., Thomson, M., Oliver, M.F., Fulton, M., Birthwhistle, A. and Elton, R. Adipose tissue and platelet fatty acids and coronary heart disease in Scottish men Lancet 1984; 2: 117-121

World Health Organization Classification of atherosclerotic lesions WHO Tech. Rep. Ser. 1958; 143

Wren, B.G. Minimal dose combined hormonal replacement therapy for menopausal women Med. J. Aust. 1981; 4: 353-356

Wren, B.G., Brown, L.B. and Routledge, D.A. Differential clinical response to estrogens after menopause Med. J. Aust. 1982; 2: 329-232

Wright, S.D. and Jong, M.T.C. Adhesion-promoting receptors on human macrophages recognize escherichia coli by binding to lipopolysaccharide J.Exp. Med. 1986; 164: 1876-1888

Wu, K.K., Hatzakis, H., Lo, S.S., Seong, D.C. and Sanduja, S.K. and Tai, H.H. Stimulation of de novo synthesis of prostaglandin $\mathrm{G} / \mathrm{H}$ synthase in human endothelial cells by phorbol esters J. Biol. Chem. 1988; 263: 19043-19047

Wynn, V. Adverse metabolic effects of oral contraceptives. In: Myocardial Infarction in Women, Oliver, M.F., Vedin, A. and Wilhelmsson, C. (eds.) Churchill Livingstone, London 1986; 103-118

Wynn, V. Effect of progesterone and progestins on carbohydrate metabolism. In: Progesterone and Progestins, Bardin, C.W., Milgrm, E. and Mauvais-Jarvis, P. (eds.), Raven Press Ltd, NY 1983; 395-410

Wynn, V., Doar, J.W.H. and Mills, G.L. Some effects of oral contraceptives on serum-lipid and lipoprotein levels Lancet 1966; 2: 720-723

Wynn, V., Godstand, I. and Nithyananthan, R. Comparison of effects of different combined oral-contraceptives on carbohydrate and lipid metabolism Lancet 1979; 1: 1045-1049

Yagi, K. and Komura, S. Inhibitory effect of female hormones on lipid peroxidation Biochem. Int. 1986; 13: 1051-1055

Yam, L.T., Li, C.Y. and Crosby, W.H. Cytochemical identification of monocytes and granutocytes Am. J. Clin. Pathol. 1971; 55: 283-290

Yamamoto, K.R. Steroid receptor regulated transcription of specific genes and gene networks Ann. Rev. Genet. 1985; 19: 209-252

Yarden, Y., Escodedo, J.A., Kuang, W.-J., Yang-Feng, T.L., Daniel, T.O., Tremble, P.M., Chen, E.Y., Ando, M.E., Harkins, R.N., Francke, U., Fried, V.A., Ullrich, A. and Williams, L.T. Structure of the receptor of platelet-derived growth factor helps define a family of closely related growth factor receptors Nature 1986; 323: 22632 
Yla-Herttuala, S., Lipton, B.A., Rosenfeld, M.E., Sarkioja, T., Yoshimura, T., Leonard, E.J., Witztum, J.L. and Steinberg, D. Expression of monocyte chemoattractant protein 1 in macrophage-rich areas of human and rabbit atherosclerotic lesions Proc. Natl. Acad. Sci. USA 1991; 88: 5252-5256

Ylikorkala, O., Hirvonen, E., Saure, A. and Viinikka, L. Urinary excretion of prostacyclin and thromboxane metabolities in climacteric women: effect of estrogen-progestin replacement therapy Maturitas 1990; 39: 33-37

Ylikorkala, O., Puolakka, J. and Viinikka, L. The effect of oral contraceptives on antiaggregatory prostacyclin and proaggregatory thromboxane $A_{2}$ in humans Am. J. Obstet. Gynecol. 1982; 142: 573-576

Yokode, M., Kita, T., Arai, H., Kawai, C., Narumiya, S. and Fujiwara, M. Cholesteryl ester accumulation in macrophages incubated with low density lipoprtoein preotreated with cigarette smoke extract Proc. Nati. Acad. Sci. USA 1988; 85: 2344-2348

Young, S.G. Recent progress in understanding apolipoprotein B Circulation 1990; 62: 1574-1594

Zava, D.T. and McGuire, W.L. Androgen action through estrogen receptor in a human breast cancer cell line Endocrinology 1978; 103: 624631

Zavoico, G.B., Ewenstein, B.M., Schaefer, A.I. and Pober, J.S. IL-1 and related cytokines enhance thrombin-stimulated $P G I_{2}$ production in cultured endothelial cells without affecting platelet-activating factor biosynthesis J. Immunol. 1989; 142: 3993-3999

Zawadzki, Z., Milne, R.W. and Marcel, Y.L. An immunochemical marker of low density oxidation J. Lipid Res. 1989; 30: 885-891

Zeldis, S.M., Nemerson, Y., Pitlick, F.A. and Lentz, T.L. Tissue factor (thromboplastin): localization to plasma membranes by peroxidase-conjugated antibodies Science 1972; 175: 766-768

Zetter, B.R., Johnson, L.K., Shuman, M.A. and Gospodarowicz, D. The isolation of vascular endothelial cell lines with altered cell surface and platelet binding properties Cell 1978; 14: 501-509

Zhou, D., Ding, J., Picker, L., Bargatze, R., Butcher, E. and Goedell, D. Molecular cloning and expression of PgP-1 J. Immunol. 1989; 143: $3390-3395$

Zoja, C., Furci, L., Ghilardi, F., Zilio, P., Benigni, A. and Remuzzi, G. Cyclosporininduced endothelial cell injury Lab. Invest. 1986; 55: 455-462

Zucker, M.B. and Nachmias, V.T. Platelet activation Arteriosclerosis 1985; 5: 2-18

Zucker, M.B. and Vroman, L. Platelet adhesion induced by fibrinogen absorbed onto glass Proc. Soc. Exp. Biol. Med. 1969; 131: 318-320 
Zullo, J.N and Faller, D.V. p21 v-ras inhibits induction of c-myc and c-fos expression by platelet-derived growth factor Mol. Cell. Biol. 1988; 8: 5080-5085

Zumoff, B. The role of endogenous hormonal factors in coronary disease: a review Milit. Med. 1981; 146: 755-760

Zwaginga, J.J., de Boer, H.C., ljsseldijk, M.J.W., Muller-Berghaus, G., Gruhlichhenn, J., Sixma, J.J. and de Groot, P.G. Thrombogenicity of vascular cells: comparison between endothelial cells isolated from different sources and smooth muscle cells and fibroblasts Arteriosclerosis 1990; 10: 437-448

Zwierzina, W., Kunz, R., Kogelnig, R. and Herold, M. Sex-related differences in platelet aggregation in native whole blood Thromb. Res. 1987; 48: 161-171 